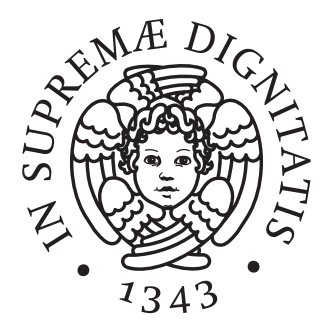

Università di Pisa

DIPARTIMENTO DI INGEGNERIA CIVILE E INDUSTRIALE SEZIONE AEROSPAZIALE

Corso di Laurea Magistrale in Ingegneria Aerospaziale

Tesi di Laurea Magistrale

\title{
Aerodynamic optimization of a large PrandtlPlane configuration
}

Relatori:

Prof. Ing. Aldo Frediani

Dott. Ing. Emanuele Rizzo

Ing. Fabrizio Oliviero

Dott. Ing. Vittorio Cipolla
Candidati:

Lorenzo Cappelli

Giulio Costa 

Alla mia Famiglia, Lorenzo

A mio nonno Giuliano,

Giulio 



\section{Ringraziamenti}

Questa Tesi è stata realizzata grazie al Prof. Ing. Aldo Frediani, il quale con grande spirito scientifico e ingegneristico ha intrapreso un percorso di innovazione nel panorama aeronautico che ci ha da subito affascinato: si vuole sottolineare il continuo entusiasmo e la passione per il proprio lavoro che ci ha trasmesso ad ogni incontro. Ricordiamo anche con grande piacere le opportunità, oltre alla presente Tesi, che il Professore ci ha offerto nel corso di questo ultimo anno, in particolare le esperienze all'Aeroporto di Tassignano. Vogliamo ringraziare anche l'Ing. Fabrizio Oliviero e il Dott. Ing. Emanuele Rizzo, che ci hanno seguito continuamente in modo puntuale con infinita pazienza e disponibilità. Un ulteriore contributo è stato dato dal Dott. Ing. Vittorio Cipolla, con commenti ed idee che ci hanno permesso di affrontare in modo più efficace il lavoro. Con le persone sopracitate ci siamo sempre sentiti come parte del loro team e mai dei semplici studenti: per questo li ringraziamo sinceramente.

Lorenzo Cappelli, Giulio Costa 



\section{Ringraziamenti}

Innanzitutto, un grazie a tutti gli amici e compagni di studi che mi sono stati vicini, a cui ho raccontato e condiviso ogni istante di questo percorso.

Un particolare ringraziamento è rivolto a Giulio, compagno di studi nonché amico, con cui ho affrontato insieme questo percorso, dimostrando di essere una persona unica, con una dedizione al lavoro ed un amore per la Scienza inesauribili.

Voglio ringraziare Martina, che in questi anni mi ha appoggiato in ogni momento, sia di fronte alle difficoltà, che nei momenti di felicità.

Infine, ringrazio tutta la mia Famiglia che, con immensi sacrifici, affetto e sostegno, mi ha permesso di concludere questo percorso di studi: senza, non sarebbe stato possibile.

Lorenzo Cappelli 



\section{Ringraziamenti}

I miei ringraziamenti personali sono rivolti in primo luogo ai miei genitori, comprensivi e incondizionatamente presenti durante il mio percorso di studio. Mio fratello Gabriele merita una significativa nota di riconoscimento per aver sopportato i miei umori nei periodi di stress intenso. Laura non ha perso occasione in questi anni per dimostrarmi il suo prezioso appoggio e per trasmettermi la sua serenità, insostituibile in svariati momenti. Tutta la famiglia mi è sempre stata vicino ma un ringraziamento particolare è dedicato agli zii Pierluigi e Simona. Ricordo con il sorriso i miei compagni di studi, ormai soprattutto amici, Giovanni, Alessandro e Francesco. Per Paolo e Antonio non bastano queste poche righe ad esprimere la profonda stima che nutro per loro. Infine, desidero elogiare Lorenzo, fedele compagno di avventura di questo ultimo anno, con il quale sono state affrontate le quotidiane difficoltà, sempre con grande dedizione ed abnegazione.

Giulio Costa 



\section{Contents}

$\begin{array}{ll}\text { Abstract } & 23\end{array}$

1 Introduction $\quad 25$

1.1 The PrandtlPlane ${ }^{\circledR}$ Concept . . . . . . . . . . . . . . . . . . . . 25

1.2 Long term forecast of air cargo . . . . . . . . . . . . . . . . . 28

1.3 PrandtlPlane ${ }^{\circledR}$ Freighter Conceptual Design . . . . . . . . . . . . . . . . . 29

1.4 Preliminary estimate of Weights and Performances . . . . . . . . . . . 31

1.5 Employment of a third lifting surface . . . . . . . . . . . . . 34

2 3D shape design of freighter aircraft $\quad 41$

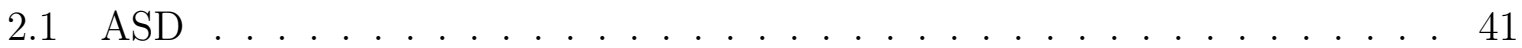

2.2 Body . . . . . . . . . . . . . . . . . . . . . 42

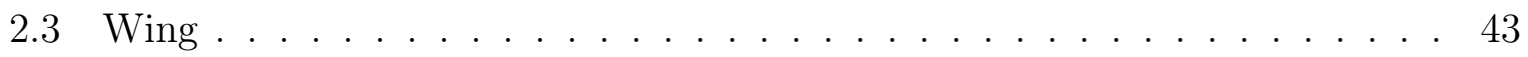

2.4 Bulk . . . . . . . . . . . . . . . . . . . . . 44

2.5 Fillet window . . . . . . . . . . . . . . . . . . . 44

2.6 Add/Modify Wround . . . . . . . . . . . . . . . . . 45

2.7 Airfoil Manager . . . . . . . . . . . . . . . . . . . . . . . . . . . . . . . . . . . . 46

2.8 Surface mesher . . . . . . . . . . . . . . . . . . . . . . 46

2.9 Other features . . . . . . . . . . . . . . . . . . . 47

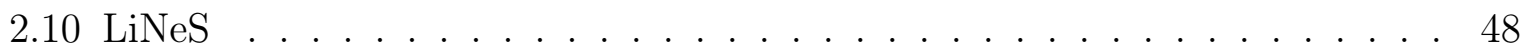

2.11 Example of ASD surfaces generation . . . . . . . . . . . 50

3 AEROSTATE $\quad 51$

3.1 Software description . . . . . . . . . . . . . . . . 51

3.2 Optimization methods . . . . . . . . . . . . . . . 52

3.2.1 Unconstrained optimization theory . . . . . . . . . . . . . 53

3.2.1.1 Abstract convergence Theorem (Orthega) . . . . . . . . 53

3.2.1.2 Armijio's Theorem . . . . . . . . . . . . . . . 54

3.2 .2 Second Order Methods . . . . . . . . . . . . . . . 54

3.2.2.1 Quasi-Newton methods . . . . . . . . . . . 55

3.2.3 Constrained optimization theory . . . . . . . . . . . . . 55

3.2.3.1 Karush-Kuhn-Tucker Theorem . . . . . . . . . . . 56

3.2.3.2 Second order sufficient optimality conditions . . . . . . . 57

3.2.4 Constrained optimization algorithms . . . . . . . . . . . . 57 
3.2.4.1 Sequential Quadratic Programming (SQP) Method . . . . 57

3.2.4.2 LOCSMOOTH algorithm . . . . . . . . . . . . . 61

3.2.5 AEROSTATE - software structure . . . . . . . . . . . . 63

3.3 AVL software . . . . . . . . . . . . . . . . 76

3.3.1 The Vortex Lattice Method . . . . . . . . . . . . . . . . . 76

3.3.2 AVL: a general overview . . . . . . . . . . . . . 82

3.3.3 AEROSTATE and AVL .................. 86

4 INTERFACE $\quad 93$

4.1 Conversion function . . . . . . . . . . . . . . . . . . . . 95

5 Research of preliminary optimum configurations $\quad 105$

5.1 A preliminary study: front wing loading variation . . . . . . . . . . . 106

5.1.1 Parameters setting and starting geometry file . . . . . . . . . 108

5.1 .2 Analysis and Results . . . . . . . . . . . . . . . . . . 111

5.2 The bulk parameter setting . . . . . . . . . . . . . . . . . 121

5.3 Validation of the front wing loading study . . . . . . . . . . . . . 126

5.4 The rear wing loading . . . . . . . . . . . . . . . . . . 126

5.5 Sensitivity to the wing span and to the fin height . . . . . . . . . 133

5.5.1 Fin height sensitivity . . . . . . . . . . . . . . . 133

5.5.2 Wing span sensitivity . . . . . . . . . . . . . . . 142

5.6 The height-wingspan ratio . . . . . . . . . . . . . . . . . 150

5.7 Advancement of the rear wing . . . . . . . . . . . . . . . 159

5.8 The reference analysis . . . . . . . . . . . . . . . . . 165

6 Low Speed Design $\quad 195$

6.1 Landing conditions - NASA CR 4746 method . . . . . . . . . . . . . 195

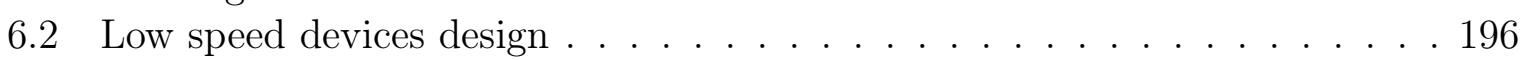

6.2.1 The low speed analytical procedure . . . . . . . . . . . . 196

6.2.2 Leading edge high lift devices . . . . . . . . . . . . . . . . . . 198

6.2 .3 Trailing edge flaps . . . . . . . . . . . . . . . . . . 200

6.3 Results . . . . . . . . . . . . . . . . . . . 208

6.3.1 The main design quantities . . . . . . . . . . . . . 208

$\begin{array}{ll}\text { Conclusions } & 225\end{array}$

$\begin{array}{lr}\text { Appendix A } & 229\end{array}$

$\begin{array}{ll}\text { Appendix B } & 237\end{array}$

$\begin{array}{ll}\text { Appendix C } & 243\end{array}$

$\begin{array}{lr}\text { Appendix D } & 247\end{array}$

$\begin{array}{ll}\text { Appendix E } & 255\end{array}$ 
Contents

Bibliography

258 



\section{List of Figures}

1.1 Lift distribution on the BWS . . . . . . . . . . . . 26

1.2 Best Wing System Efficiency . . . . . . . . . . . . . . . . 26

1.3 Prandtl Plane Freighter . . . . . . . . . . . . . . . . . . . . . . . . . . . . . . . . . 37

1.4 Cargo disposition . . . . . . . . . . . . . . . . . . . . . 30

1.5 Fuselage cross section . . . . . . . . . . . . . . . . . . . 30

1.6 Design Point . . . . . . . . . . . . . . . . . . . . . 33

1.7 Beam scheme . . . . . . . . . . . . . . . . . . . . . 35

1.8 Comparison between displacements . . . . . . . . . . . . . . . . . . . . . . . . . . . . . 37

1.9 Comparison between bending moment . . . . . . . . . . . . 38

1.10 Comparison between shear forces . . . . . . . . . . . . . 38

1.11 Example of a new-kind configuration with a canard . . . . . . . . . 39

2.1 ASD main window. . . . . . . . . . . . . . . . . . . . . 42

2.2 ASD Add/Modify Body window. . . . . . . . . . . . . . . . 43

2.3 ASD Add/Modify Wing window. . . . . . . . . . . . . . . . . . 43

2.4 ASD Add/Modify Bulk window. . . . . . . . . . . . . . . . . . 44

2.5 ASD Add/Modify Fillet window. . . . . . . . . . . . . . . . . . . 44

2.6 ASD Add/Modify TFillet window. . . . . . . . . . . . . . . . . 45

2.7 ASD Add/Modify Wround window. . . . . . . . . . . . . . . . 45

2.8 ASD AIRFOIL MANAGER. . . . . . . . . . . . . . . . . . . 46

2.9 ASD Surface Mesher window. . . . . . . . . . . . . . . . . 47

2.10 ASD Surfaces Viewer window. . . . . . . . . . . . . . . . 47

2.11 LiNeS side view window. . . . . . . . . . . . . . . . . . . . . 48

2.12 LiNeS top view window. . . . . . . . . . . . . . . . . . . 48

2.13 Example of freighter aircraft surfaces generation, with ASD software. . . . 50

3.1 An example of a function with a funnel structure and its step function. . . 61

3.2 geo variable. . . . . . . . . . . . . . . . . . . . 64

3.3 Example of lifting surface variable into geo. . . . . . . . . . . . . 65

3.4 res variable. . . . . . . . . . . . . . . . . . 66

3.5 res variable - detail. . . . . . . . . . . . . . . . 66

3.6 AEROSTATE main diagram. . . . . . . . . . . . . . . . . 68

3.7 Blocks definitions. . . . . . . . . . . . . . . . . . . . . . 69

3.8 outfunNEW.m output graphics. . . . . . . . . . . . . . . . 73 
3.9 outfunNEW.m output graphic. . . . . . . . . . . . . . . . . . 73

3.10 LOCSMOOTH global optimization process. . . . . . . . . . . . . 75

3.11 Generic airfoil in steady, inviscid, irrotational flow . . . . . . . . . . 77

3.12 Semi-infinite vortex and finite vortex . . . . . . . . . . . . . . 79

3.13 The "horseshoe" vortex . . . . . . . . . . . . . . . . . . 80

3.14 VLM on a general wing . . . . . . . . . . . . . . . . . 81

3.15 AVL spacing possibilities . . . . . . . . . . . . . . . . 83

3.16 AVL input file . . . . . . . . . . . . . . . . . . . . 83

3.17 AVL surface definition . . . . . . . . . . . . . . . . . . 84

3.18 AVL PrandtPlane configuration . . . . . . . . . . . . . . 86

3.19 AVL starting data . . . . . . . . . . . . . . . . . . . . 86

3.20 AVL front wing and fuselage . . . . . . . . . . . . . . . . 87

3.21 AVL front wing and fuselage - Detail . . . . . . . . . . . . . . . 88

3.22 AVL rear wing- Detail . . . . . . . . . . . . . . . . . 88

3.23 AVL rear wing . . . . . . . . . . . . . . . . . . . . . 89

3.24 AVL winglet . . . . . . . . . . . . . . . . . . . . . . . . 90 90

3.25 AVL winglet - Detail . . . . . . . . . . . . . . . . . . . 90

3.26 AVL bulk . . . . . . . . . . . . . . . . . . . . . . 91

3.27 AVL bulk - Detail . . . . . . . . . . . . . . . . . . 91

4.1 INTERFACE window. . . . . . . . . . . . . . . . . . . . . . 94

4.2 INTERFACE window. . . . . . . . . . . . . . . . . . . . . . . 95

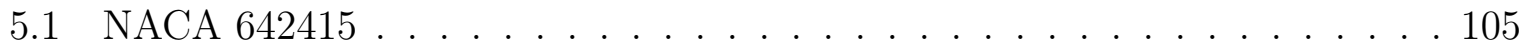

5.2 NACA 642415 - Aerodynamic features - Source: Abbott . . . . . . . . . . 106

5.3 Wing Loading vs MTOW for current aircraft . . . . . . . . . . . . . . . . 107

5.4 Starting Geometry . . . . . . . . . . . . . . . . . . . . 108

5.5 Lift distribution . . . . . . . . . . . . . . . . . . . . . . . . . . . . . . . . 109

5.6 Check on the equilibrium condition. . . . . . . . . . . . . . 111

5.7 Global CL vs global Wing Loading. . . . . . . . . . . . . . . . . . . . . . 112

5.8 Efficiency vs front Wing Loading. . . . . . . . . . . . . . . . . . . . 113

5.9 Efficiency vs global Wing Loading. . . . . . . . . . . . . . . . . . . . 114

5.10 Efficiency vs global Aspect Ratio. . . . . . . . . . . . . . . . . . . 115

5.11 Lift of rear wing and auxiliary wing. . . . . . . . . . . . 116

5.12 Surface of rear wing and auxiliary wing. . . . . . . . . . . . 117

5.13 Wing Loading: auxiliary wing - front wing relationship. . . . . . . . . . . . 118

5.14 CD contributions. . . . . . . . . . . . . . . . . 118

5.15 CLtot trends. . . . . . . . . . . . . . . . . . . . . 119

5.16 CL trends. . . . . . . . . . . . . . . . . . . . . . 120

$5.17 C_{L}$ aux wing vs $K_{\text {bulk }}$ parameter. . . . . . . . . . . . . . . . . 122

5.18 Efficiency and Bulk Length. . . . . . . . . . . . . . . . . . . 123

5.19 Lift Contribution vs $K_{\text {bulk }}$ parameter. . . . . . . . . . . . . . . . . . 124

5.20 Wing Loading vs $K_{\text {bulk }}$ parameter. . . . . . . . . . . . . . . . . . . . 125

$5.21 C_{L}$ tot vs Global wing loading - Rear wing analysis. . . . . . . . . . . 127 
5.22 Efficiency vs Global wing loading - Rear wing analysis. . . . . . . . . . . . 128

5.23 Efficiency vs Rear wing loading - Rear wing analysis. . . . . . . . . . . . . 128

5.24 Margin of Stability vs Rear wing loading- Rear wing analysis. . . . . . . . 129

5.25 Rear Lift vs CG-rear wing distance - Rear wing analysis. . . . . . . . . . . 130

5.26 CG-front wing distance vs CG-rear wing distance - Rear wing analysis. . . 130

$5.27 C_{L}$ tot trends - Rear wing analysis. . . . . . . . . . . . . . 132

5.28 Generalities (part 1) - H sensitivity. . . . . . . . . . . . . 134

5.29 Efficiency vs $\mathrm{H}-\mathrm{H}$ sensitivity. . . . . . . . . . . . . . . 135

5.30 AR gen vs $\mathrm{H}-\mathrm{H}$ sensitivity. . . . . . . . . . . . . . . . . . . . . . . . . . . . . . . . . . .

5.31 Generalities (part 2) - H sensitivity. . . . . . . . . . . . . 136

5.32 Front wing lift and surface - $\mathrm{H}$ sensitivity. . . . . . . . . . . . 137

5.33 Auxiliary wing lift and surface - H sensitivity. . . . . . . . . . . 138

5.34 AR behaviour $-\mathrm{H}$ sensitivity. . . . . . . . . . . . . . . . 139

5.35 CL behaviour (part 1) - H sensitivity. . . . . . . . . . . . . . . 140

5.36 Total lift coefficient and total drag coefficient - H sensitivity. . . . . . . . . 141

5.37 CL tot vs Global wing loading - B sensitivity . . . . . . . . . . . . . 143

5.38 AR gen vs Global wing loading - B sensitivity . . . . . . . . . . . . . 143

5.39 Efficiency - B sensitivity . . . . . . . . . . . . . . . . . 144

$5.40 A R$ gen - B sensitivity . . . . . . . . . . . . . . . . . . 144

5.41 Efficiency vs $A R$ gen - B sensitivity . . . . . . . . . . . . . . . 145

5.42 Efficiency vs Global wing loading - B sensitivity . . . . . . . . . . . 145

5.43 Margin of Stability vs Global wing loading - B sensitivity . . . . . . . . . 146

5.44 Drag coefficients - B sensitivity . . . . . . . . . . . . . 146

5.45 Front wing lift and surface - B sensitivity . . . . . . . . . . . . 147

$5.46 C L$ behaviour - B sensitivity . . . . . . . . . . . . . . . . . 148

5.47 Wing loadings (part 2) - B sensitivity . . . . . . . . . . . 149

5.48 CLtot vs Global wing loading $-h / b$ constant . . . . . . . . . . . . . 150

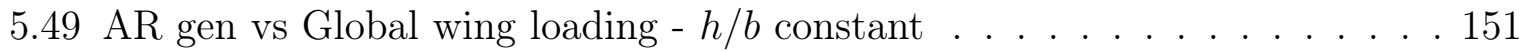

5.50 Efficiency vs Global wing loading $-h / b$ constant . . . . . . . . . . . . . . 151

5.51 Efficiency vs ARgen $-h / b$ constant . . . . . . . . . . . . . . . . . . . . . . . . . 152

5.52 CD trends $-h / b$ constant . . . . . . . . . . . . . . . 152

5.53 Front Wing behaviour $-h / b$ constant . . . . . . . . . . . 153

5.54 Rear Wing behaviour $-h / b$ constant . . . . . . . . . . . . . . . 154

5.55 Auxiliary Wing behaviour $-h / b$ constant . . . . . . . . . . . . . . 155

5.56 Auxiliary Wing wingspan $-h / b$ constant . . . . . . . . . . . . . 156

5.57 Auxiliary Wing root chord $-h / b$ constant . . . . . . . . . . . 156

5.58 CLtot trends $-h / b$ constant . . . . . . . . . . . . . . 157

5.59 CL trends $-h / b$ constant . . . . . . . . . . . . . . . . 158

5.60 Example of configuration with rear wing advancement . . . . . . . . . 159

5.61 Rear wing location vs rear wing loading (rear wing advancement) . . . . . 160

5.62 Auxiliary wing location vs rear wing loading (rear wing advancement) . . . 160

5.63 Margin of Stability vs rear wing loading (rear wing advancement) . . . . . 161

5.64 Lift contributions (rear wing advancement) . . . . . . . . . . . . . 162

5.65 Auxiliary wing loading vs rear wing loading (rear wing advancement) . . . 163 
5.66 Efficiency vs rear wing loading (rear wing advancement) . . . . . . . . . 163

5.67 Efficiency vs global wing loading (rear wing advancement) . . . . . . . . . 164

5.68 Analysis Results: test5trisCurr0. . . . . . . . . . . . . . . . . 167

5.69 Analysis Results: test5trisCurr1. . . . . . . . . . . . . . . . 169

5.70 Analysis Results: test5trisCurr3. . . . . . . . . . . . . . . . 171

5.71 Analysis Results: OPTIMUM. . . . . . . . . . . . . . . . . . 173

5.72 Analysis Results: test5trisCurr6. . . . . . . . . . . . . . . . . . . 175

5.73 Analysis Results: test5trisCurr7. . . . . . . . . . . . . . . . . . . 177

5.74 Analysis Results: test5trisCurr8. . . . . . . . . . . . . . . . 179

5.75 Analysis Results: test5trisCurr9. . . . . . . . . . . . . . . 181

5.76 Analysis Results: test5trisCurr10. . . . . . . . . . . . . . . . 183

5.77 Analysis Results: test5trisCurr12. . . . . . . . . . . . . . . . . 185

5.78 Analysis Results: test5trisCurr13. . . . . . . . . . . . . . . . . 187

5.79 Analysis Results: test5trisCurr14. . . . . . . . . . . . . . . . . 189

5.80 Analysis Results: test5trisCurr15. . . . . . . . . . . . . . . . . 191

5.81 Analysis Results: test5trisCurr16. . . . . . . . . . . . . . . 193

6.1 Lift edge high lift devices. . . . . . . . . . . . . . . . . . . . . . . 198

6.2 Lift curve with and without high lift devices. . . . . . . . . . . . . . 198

6.3 Flap deflection effects on the lift curve. . . . . . . . . . . . . . . . . . . . 201

6.4 Parameters definition for flaps deflection. . . . . . . . . . . . . . . 201

6.5 Plain flap. . . . . . . . . . . . . . . . . . . . . . 202

6.6 Plain flap efficiency. . . . . . . . . . . . . . . . . . . . . . 202

6.7 Single slotted flap. . . . . . . . . . . . . . . . . . . . 203

6.8 Fowler flap. . . . . . . . . . . . . . . . . . . . . . . . . . . . . . 203

6.9 Single slotted flap and Fowler flap efficiency. . . . . . . . . . . . . . . . . 204

6.10 Double slotted flap with fixed vane. . . . . . . . . . . . . . . . 204

6.11 Double slotted flap with variable geometry. . . . . . . . . . . . . . 205

6.12 Double slotted flap efficiency. . . . . . . . . . . . . . . . . . 205

6.13 Chord extension. . . . . . . . . . . . . . . 206

6.14 test5trisb75Curr0. . . . . . . . . . . . . . . . 209

6.15 test5trisb75Curr0 - High lift devices. . . . . . . . . . . . . . 210

6.16 test5trisb75Curr0 - Lifting distributions. . . . . . . . . . . . . . 211

6.17 test5trisCurr0. . . . . . . . . . . . . . . . . . . . 212

6.18 test5trisCurr0 - High lift devices. . . . . . . . . . . . . . . . . 213

6.19 test5trisCurr0 - Lifting distributions. . . . . . . . . . . . . . . . 214

6.20 test5trisCurr8. . . . . . . . . . . . . . . . . 215

6.21 test5trisCurr8 - High lift devices. . . . . . . . . . . . . . 216

6.22 test5trisCurr8 - Lifting distributions. . . . . . . . . . . . . . 217

6.23 test5trisCurr6. . . . . . . . . . . . . . . . . . . 218

6.24 test5trisCurr6 - High lift devices. . . . . . . . . . . . . . . . . . . 219

6.25 test5trisCurr6 - Lifting distributions. . . . . . . . . . . . . . . 220

6.26 test5trish11Curr18. . . . . . . . . . . . . . . . 221

6.27 test5trish11Curr18 - High lift devices. . . . . . . . . . . . . . . 222 
6.28 test5trish11Curr18 - Lifting distributions. . . . . . . . . . . . . 223

A.1 $C_{L}$ tot vs Global wing loading $-K_{\text {bulk }}=0.8 \ldots \ldots \ldots \ldots \ldots$

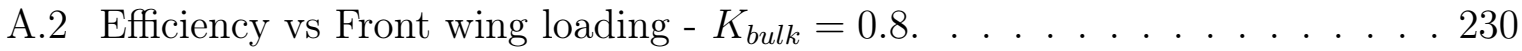

A.3 Efficiency vs Global wing loading $-K_{\text {bulk }}=0.8 \ldots \ldots \ldots \ldots$

A.4 Lift of rear wing and auxiliary wing $-K_{b u l k}=0.8 \ldots \ldots \ldots . \ldots . \ldots 231$

A.5 Surface of rear wing and auxiliary wing $-K_{\text {bulk }}=0.8 \ldots \ldots . \ldots . . .232$

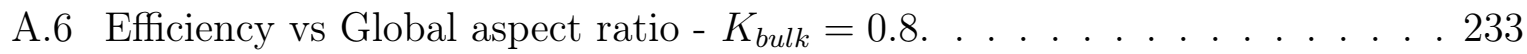

A.7 Auxiliary wing loading vs Front wing loading $-K_{b u l k}=0.8 \ldots \ldots . \ldots . .233$

A.8 $C_{L}$ tot trends $-K_{b u l k}=0.8 \ldots \ldots \ldots \ldots \ldots \ldots \ldots \ldots \ldots \ldots \ldots \ldots \ldots$

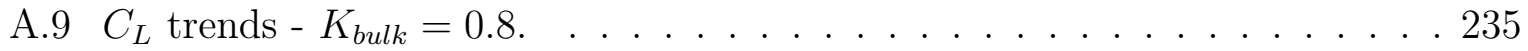

A.10 $C_{D}$ contributions $-K_{\text {bulk }}=0.8 \ldots \ldots \ldots \ldots \ldots$

B.1 Rear wing behaviour - Rear wing analysis. . . . . . . . . . . . 237

B.2 Auxiliary wing behaviour - Rear wing analysis. . . . . . . . . . . . 238

B.3 Front wing behaviour - Rear wing analysis. . . . . . . . . . . . . . 239

B.4 Global Aspect Ratio effects - Rear wing analysis. . . . . . . . . . . . . . . 240

B.5 Rear Aspect Ratio effects - Rear wing analysis. . . . . . . . . . . . . . . 241

B.6 $C_{L}$ trends - Rear wing analysis. . . . . . . . . . . . . . . 242

C.1 Rear wing lift and surface - H sensitivity. . . . . . . . . . . . . 243

C.2 Wing loadings - H sensitivity. . . . . . . . . . . . . . . . . . 244

C.3 Induced drag coefficient and viscosity drag coefficient - H sensitivity. . . . . 245

C.4 CL behaviour (part 2) - H sensitivity. . . . . . . . . . . . . 246

D.1 Rear wing lift and surface - B sensitivity . . . . . . . . . . . . 247

D.2 Auxiliary wing lift and surface - B sensitivity . . . . . . . . . . . 248

D.3 AR behaviour - B sensitivity . . . . . . . . . . . . . . . . . 249

D.4 CL behaviour (part 2) - B sensitivity . . . . . . . . . . . . 250

D.5 Wing loadings (part 1) - B sensitivity . . . . . . . . . . . 251

D.6 Auxiliary wing span - B sensitivity . . . . . . . . . . . . . 252

D.7 Auxiliary wing $A R$ - B sensitivity . . . . . . . . . . . . . . 252

D.8 Global wing loading - B sensitivity . . . . . . . . . . . . . 253

D.9 Induced drag coefficient - B sensitivity . . . . . . . . . . . . . . . 253

D.10 Viscosity drag coefficient - B sensitivity . . . . . . . . . . . . . 254

E.1 CLtot trends (rear wing advancement) . . . . . . . . . . . . . . 255

E.2 CL trends (rear wing advancement) . . . . . . . . . . . . . . 256

E.3 Surface trends (rear wing advancement) . . . . . . . . . . . . 257 



\section{List of Tables}

1.1 Weight results . . . . . . . . . . . . . . . . 33

1.2 Main quantities . . . . . . . . . . . . . . . . . . . . . 35

1.3 Boundary Conditions . . . . . . . . . . . . . . . 36

2.1 Input for the line in the side view of the Fuselage. . . . . . . . . . . . 49

2.2 Input for the line in the top view of the Fuselage. . . . . . . . . . . 49

5.1 Front Wing loading Lower Boundaries (LB) and Upper Boundaries (UB) . 108

5.2 Configuration features $\ldots \ldots \ldots \ldots \ldots$

5.3 Wing loadings $-K_{\text {bulk }}$ Analyses. . . . . . . . . . . . . . . . 121

$5.4 \quad K_{\text {bulk }}$ Analyses. . . . . . . . . . . . . . . . . . . 121

5.5 Front wing loading Lower Boundaries and Upper Boundaries $-K_{b u l k}=0.8 .126$

5.6 Rear wing loading Lower Boundaries and Upper Boundaries. . . . . . . . 126

5.7 Wing span and fin height for each analyses. . . . . . . . . . . . 133

5.8 Comparison between analysis 31,46 and $47 \ldots \ldots \ldots \ldots$

5.9 Analysis Results: test5trisCurr0. . . . . . . . . . . . . . . . . 166

5.10 Analysis Results: test5trisCurr1. . . . . . . . . . . . . . . . . 168

5.11 Analysis Results: test5trisCurr3. . . . . . . . . . . . . . . . . . 170

5.12 Analysis Results: OPTIMUM. . . . . . . . . . . . . . . . . . . . . . 172

5.13 Analysis Results: test5trisCurr6. . . . . . . . . . . . . . . . . . 174

5.14 Analysis Results: test5trisCurr7. . . . . . . . . . . . . . . . . 176

5.15 Analysis Results: test5trisCurr8. . . . . . . . . . . . . . . . 178

5.16 Analysis Results: test5trisCurr9. . . . . . . . . . . . . . . . . . . 180

5.17 Analysis Results: test5trisCurr10 . . . . . . . . . . . . . . . . . . 182

5.18 Analysis Results: test5trisCurr12 . . . . . . . . . . . . . . . . 184

5.19 Analysis Results: test5trisCurr13. . . . . . . . . . . . . . . . . 186

5.20 Analysis Results: test5trisCurr14. . . . . . . . . . . . . . . . . . . 188

5.21 Analysis Results: test5trisCurr15 . . . . . . . . . . . . . . . . . . 190

5.22 Analysis Results: test5trisCurr16 . . . . . . . . . . . . . . . . . 192

6.1 test5trisb75Curr0 - Geometry . . . . . . . . . . . . . . . . . 209

6.2 test5trisb75Curr0 - Low Speed Analysis . . . . . . . . . . . . . 210

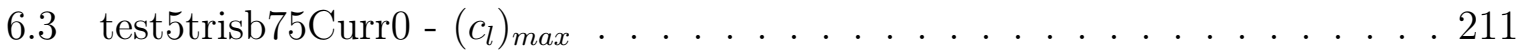

6.4 test5trisCurr0 - Geometry . . . . . . . . . . . . . . . . . 212 
6.5 test5trisCurr0 - Low Speed Analysis . . . . . . . . . . . . . . . . . 213

6.6 test5trisCurr0 $-\left(c_{l}\right)_{\max } \ldots \ldots \ldots \ldots \ldots \ldots$

6.7 test5trisCurr8-Geometry . . . . . . . . . . . . . . 215

6.8 test5trisCurr8 - Low Speed Analysis . . . . . . . . . . . . . . . 216

6.9 test5trisCurr8 $-\left(c_{l}\right)_{\max } \ldots \ldots \ldots \ldots . \ldots \ldots$

6.10 test5trisCurr6 - Geometry . . . . . . . . . . . . . . . . 218

6.11 test5trisCurr6 - Low Speed Analysis . . . . . . . . . . . . . . . . . 219

6.12 test5trisCurr6 $-\left(c_{l}\right)_{\max } \ldots \ldots \ldots \ldots \ldots$

6.13 test5trish11Curr18 - Geometry . . . . . . . . . . . . . . 221

6.14 test5trish11Curr18 - Low Speed Analysis . . . . . . . . . . . . . . . . 222

6.15 test5trish11Curr18 $-\left(c_{l}\right)_{\max } \ldots \ldots \ldots \ldots . \ldots \ldots 222$ 


\section{Abstract}

The present Thesis deals with the application of optimization methods to the preliminary design of a large PrandtlPlane ${ }^{\circledR}$ freighter and with graphical tools to visualize the most interesting configurations.

The conceptual design of the PrandtlPlane ${ }^{\circledR}$ freighter has been developed by the Department of Civil and Industrial Engineering - Aerospace Section of the University of Pisa and it is summarized in Chapter 1; the installation of a third lifting surface is described and its effects are foreseen for a large aircraft: improving the maneuverability and the stability is the main aim, without penalizing aerodynamic efficiency and weights.

The preliminary study needs a graphical software package (ASD) and an aerodynamic optimizer (AEROSTATE): both of them have been performed using MATLAB ${ }^{\circledR}$ routines. Chapter 2 briefly describes ASD. Local and global optimization methods, together with their implementation in AEROSTATE, are shown in Chapter 3. The aerodynamic solver (AVL) is based on the Vortex lattice Method; despite of its simplicity, the reliability of the results on a PrandtlPlane ${ }^{\circledR}$ configuration has been proved in previous studies: thus, this method is the compromise between accuracy and computational simplicity.

AEROSTATE provides results as MATLAB ${ }^{\circledR}$ structured variables and they are transferred to ASD by means INTERFACE, a MATLAB ${ }^{\circledR}$ environment that is developed in this thesis and it is presented in Chapter 4.

In Chapter 5, analyses and results are shown: sensitivity studies have been made to the front wing loading, to the rear wing loading, to the bulks length, to the wingspan and to the fins height. The purpose of these studies is to understand how the designer can improve the aerodynamic efficiency in cruise and to obtain a feasible structure.

Finally, Chapter 6 explains that many configurations, with different wings arrangements, satisfy low speed constraints, i.e. the landing is possible with low angles of attack, and the trim conditions on the longitudinal plane are met, when flaps and slats are deflected, by means suitable elevators. Conclusions and future developments are collected at the end of this paper. 



\section{Chapter 1}

\section{Introduction}

\subsection{The PrandtlPlane ${ }^{\circledR}$ Concept}

The document "European Aeronautic: a vision for 2020" was published in 2001 by the European Commission and it sets the main goals the aeronautical industry has to reach in the perspective of 2020. Great improvements are requested in terms of environmental aspects: the hardest purpose is to halve the $\mathrm{CO}_{2}$ emission per ATK and to cut 80 percent of nitrogen oxide emission. Furthermore, the document deals with other aspects, as improvements of "Quality and affordability", "Safety" and "Air Transport Systems". They all imply an enormous technological effort for conventional monoplane configurations because forefront outstanding aircraft (e.g. A380) are already optimized. The gap between demand and actual available technology has pushed modern aeronautical research towards new configurations.

The PrandtlPlane ${ }^{\circledR}$ configuration originated in 1924 by L. Prandtl's studies, who started comparing the drag induced by a monoplane and a biplane. The optimum monoplane has an elliptical distribution of lifting forces along the wing span while, keeping the total lift constant, the optimum biplane has an equally distributed aerodynamic load on the two wings. Keeping constant the wing span $(b)$, higher is the vertical distance between the two biplane wings (call it $h$ ), lower is the induced drag.

Prandtl considered a new wing system, which is very close to a biplane, but with linked wings; this box-wing shape has total lift equally distributed on the two horizontal wings. Each horizontal wing has a superposition of a constant and an elliptic distribution of lifting forces. In case of double symmetry vertical bulks, the distribution of aerodynamic lift is butterfly-shaped (so the total lift developed by the bulks is zero), refer to Fig.1.1.

Prandtl called this new configuration "Best Wing System" (BWS) [6] and he gave an approximated expression of the induced drag:

$$
\frac{D_{B W S}}{D_{M O N}}=\frac{1+0.45 \frac{h}{b}}{1.04+2.81 \frac{h}{b}}
$$




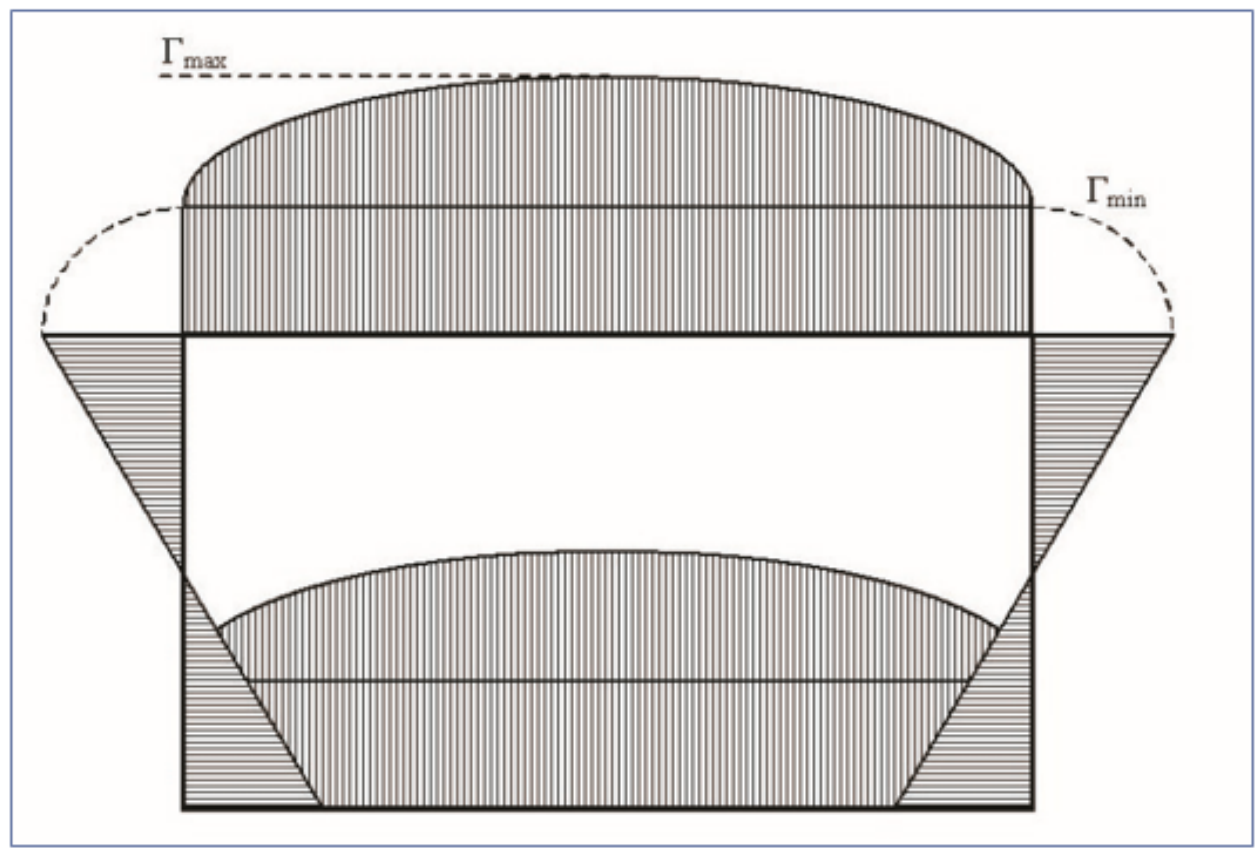

Figure 1.1. Lift distribution on the BWS

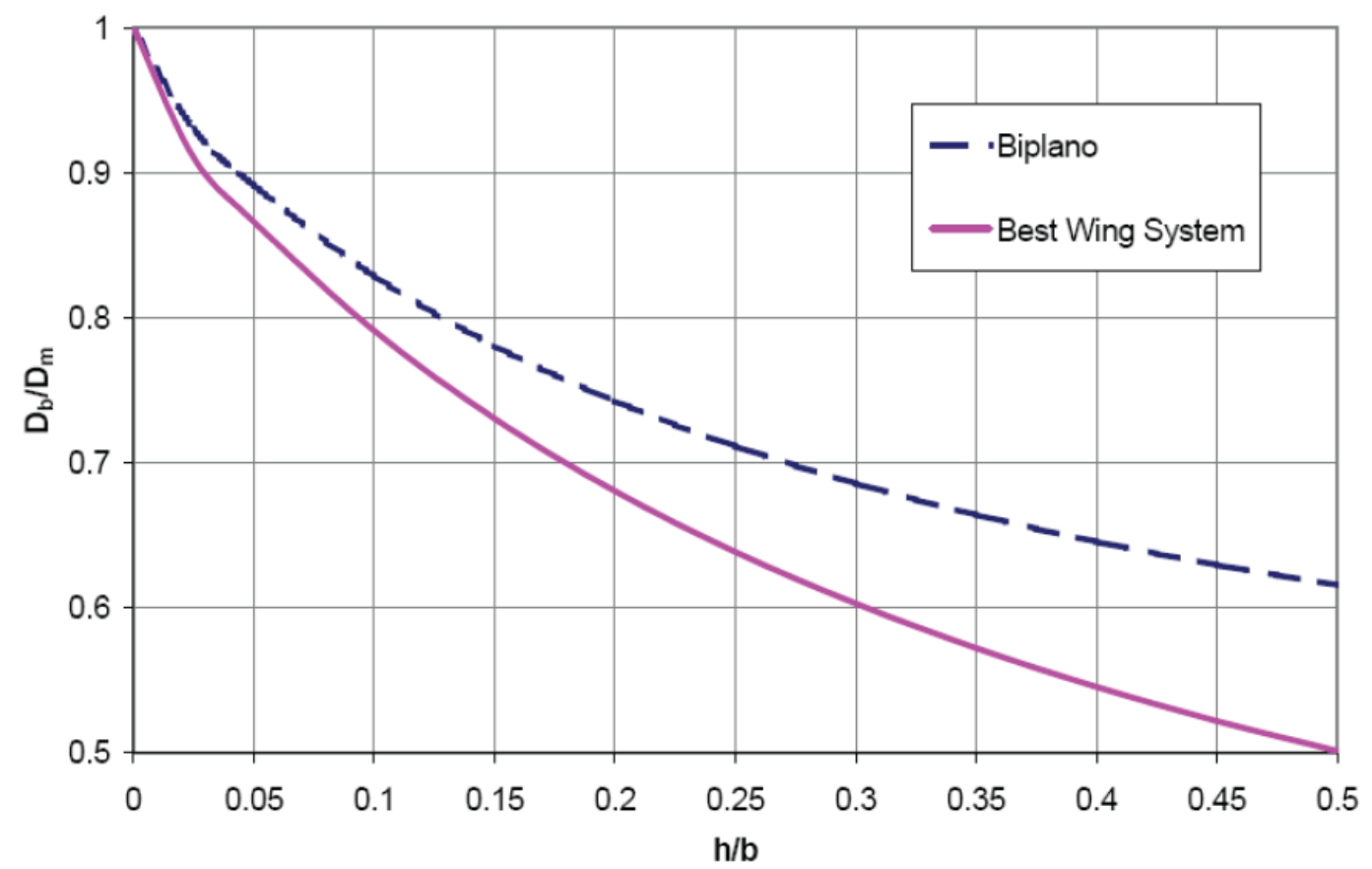

Figure 1.2. Best Wing System Efficiency 
In 1999 the previous law was confirmed for $0<\frac{h}{b}<0.2$, while Prandtl's prediction is not conservative for higher values of $\frac{h}{b}$ (refer to [7]). Anyway this is not a real problem because the actual field of interest is $0<\frac{h}{b}<0.2$. Too high wing gap will introduce friction drag and structural problems: vertical supports for a very high horizontal wing need a stiff structure and it implies a rise of weights. In the considered range of $\frac{h}{b}$, the induced drag is reduced by 20-30 percent and the total drag is reduced by 8-13 percent during cruise, but a more significant advantage is obtained during takeoff and landing, where the induced drag is about 70 percent or more of the total drag. Moreover the induced drag is independent by the sweep angle (Munk's theorems), thus Prandtl's BWS is a worth solution also for transonic transport aircraft.

The application of the BWS to an air freighter is the main aim of this thesis and the final result would be an optimized version of the configuration represented in Fig.1.3.

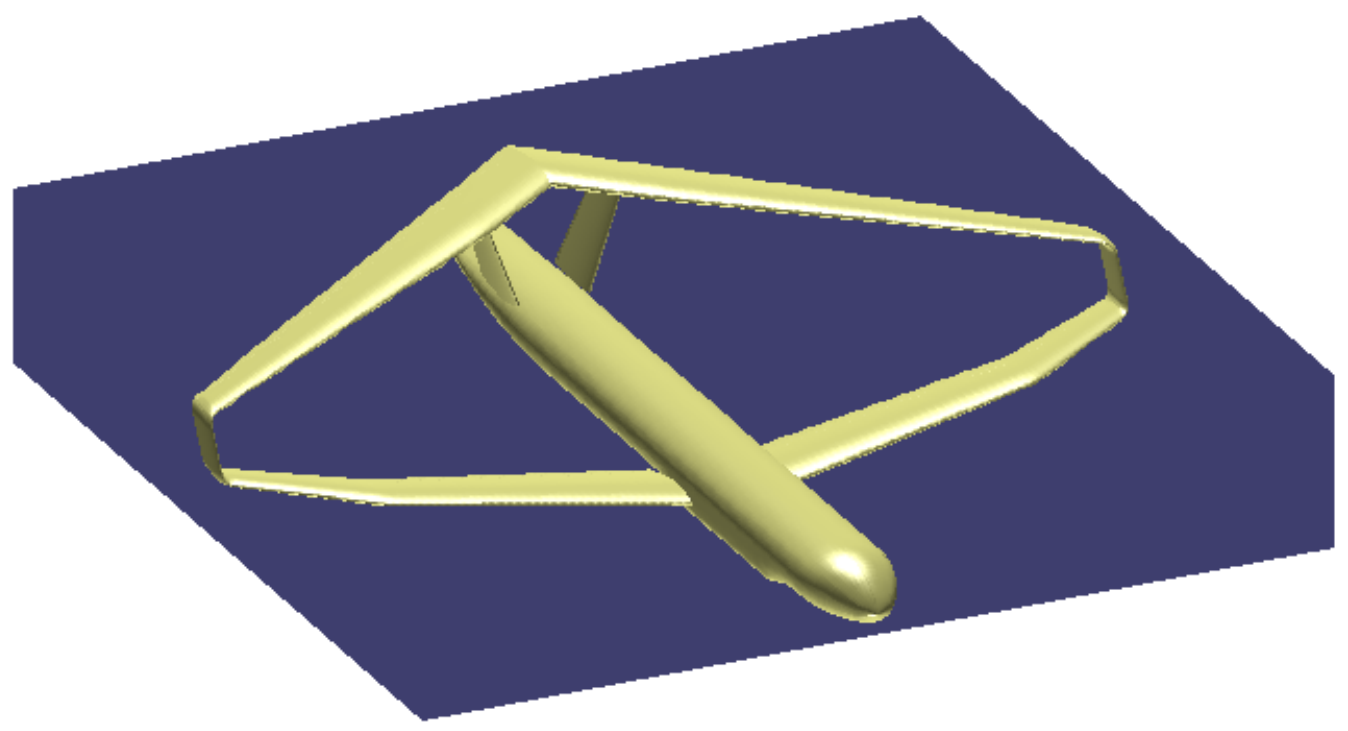

Figure 1.3. Prandtl Plane Freighter 
The main features of the new PrandtlPlane ${ }^{\circledR}$ can be summarized below.

- The mechanical model of the lifting system is overconstrained to the fuselage and, thus, the global stiffness of the wing system is improved and, at the same time, aeroelastic phenomena are limited when the double fin support for the rear wing is applied. The forward sweep angle of the rear wing is not dangerous because of the lateral bulks, which link the wings and increase stability towards divergence.

- The Margin of Stability can be easily controlled by distributing lift forces properly. Besides, pitch control could be obtained by means of two elevators positioned on the front and rear wing roots, so that a pure torque moment is applied on the aircraft and only small forces are necessary because of the large distance between them. Lateral stability and control could be obtained by means a double fin and rudder, while roll control is obtained by ailerons on both wings or flaperons on the rear wing only. Low speed performances are satisfied.

- The fuselage, contrary to conventional monoplanes, is enlarged to position the two fins: then, the section of the fuselage is elliptical. From a structural point of view, the fuselage is a double supported beam and, as shown later on, the new fuselage setting allows an efficient payload distribution.

\subsection{Long term forecast of air cargo}

Economical interests in a new kind of air freighter are well explained in [5]; the main items are summarized below.

Although the importance of air freight is negligible compared to other means of transport in terms of quantity of carried goods, $(0.2-0.5 \%$ of the total transported tons), the air freight can reach the $30 \%$ of the global cargo system. The very high cost of air cargo is the main reason of this particular phenomenon. This indicates that only high-value goods can be carried by air. From an economical point of view, it is not convenient to carry goods of less than $15 € / \mathrm{kg}$. On the other hand, air freighters present benefits, as high transport speed, independence of geographical obstacles and elevated reliability. Most of the air freighters derive from a conversion of airlines passenger aircraft and, despite they have a low acquisition cost, they undergo an increasing operative cost because of the old technology and maintenance operations of aircraft, and, besides, this kind of aircraft have relatively short operative life.

The most important task is to build up a new aircraft that is optimized for its cargomission. As it is shown in many financial reports, the air freight fleet is intended to double from 2010 to 2030 and there is a big interest in large wide-body aircraft: they have superior reliability and relatively low operating costs, which can overcome the acquisition cost advantages enjoyed by conversions. Hence, design parameters, as wing span $b$ and fuselage length $L_{f u s}$, are chosen as the maximum allowed:

$$
L_{\text {fus }}=80 m \quad b=80 m
$$


The geographical areas which will provide the development of markets in the next fifteen years are, most of all, into the Asian continent. Asia is a centre of interest for regional and inter-continental air cargo transports. Africa could be an interesting new market because there are not enough infrastructures and building up a new airport for load/unload operations will be less expensive than building up new roads or railways. All these aspects affect the design range choice. A design range of $3000 \mathrm{~nm}$ is considered a good compromise because it allows to have an efficient continental transport and some intercontinental transport, eventually needing an intermediate landing.

Payload is strictly connected to the choice of the container: IATA M-2-AGA containers are lighter than ISO containers and at the same time they are intermodal, so load/unload operations enjoy a saving of time. IATA containers are not utilized in usual monoplane because they badly occupy the Fuselage spaces. The design gross payload is

$$
W_{\text {pay }}=250000 \mathrm{~kg}
$$

The propulsion system equips particular turboprop engines, Open Rotors Engines, which are the best choice according to a cruise Mach of 0.6-0.7 and a maximum cruise altitude of 6000-7000 m. The Mach number and altitude are chosen in order to reduce the specific fuel consumption and to limit the transonic field at the tip of the blades. Ivchenko Progress D27 is a possible example of installed engine.

The required field length for take off and landing must respect regulation limits. Today the biggest airports ensure a runway length longer than $4000 \mathrm{~m}$, so we assume:

$$
\text { Maximum Runway Length }=4000 \mathrm{~m} \text {. }
$$

\subsection{PrandtlPlane ${ }^{\circledR}$ Freighter Conceptual Design}

The new box wing configuration suggests not only a revolutionary design of wings, but also the fuselage has to be designed in order to allocate two lateral fins for the optimized disposition of containers. The number of containers is obtained by estimating an average bulk density $\left(\rho_{\text {bulk }}\right)$ and the assigned payload [5].

$$
\rho_{\text {bulk }}=220 \div 250 \mathrm{~kg} / \mathrm{m}^{3} \quad N_{\text {cont }}=25
$$

The fuselage has to be designed specifically for containers transportation. The main differences between the freighter fuselage and usual aircraft fuselage are:

1. There is no pressurization, apart from the cockpit area; thus, a structural weight saving is possible because membrane and bending stress are absent.

2. No windows are required (cockpit area excepted).

3. Systems are adapted only for cargo holding: it means a simpler configuration than the passengers one.

4. Simple cabin set. 
5. Floor has to be designed in order to allow simple cargo movement; this Fuselage needs also efficient locking systems to ensure that containers do not move during the flight.

The cargo disposition is widely discussed in [5] and the final containers arrangement is sketched in Fig.1.4. The Fuselage cross section design results directly from containers

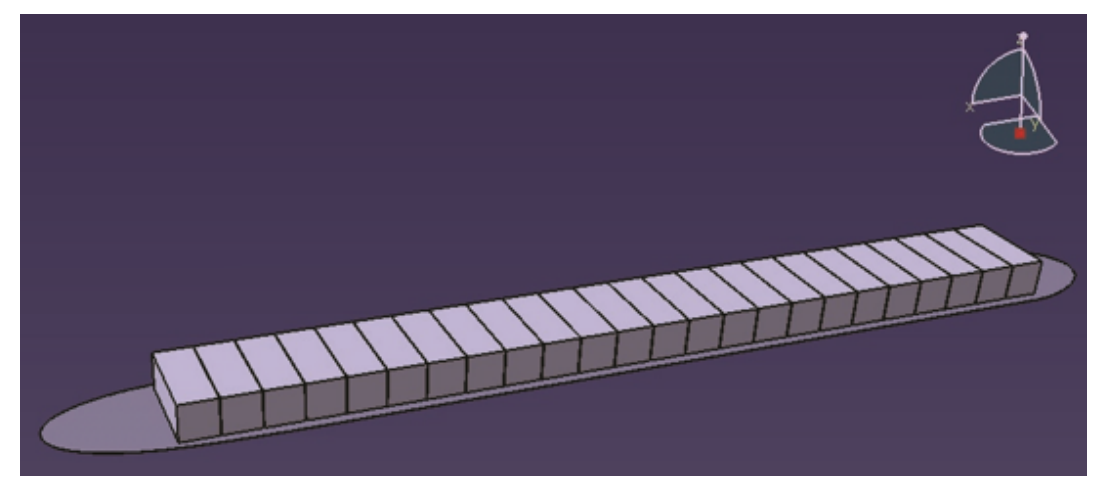

Figure 1.4. Cargo disposition

disposition (Fig.1.5). The cross section has two symmetry axes and it is composed by four circumferences arches. The centre of circumferences is located on the vertical axis for the top and bottom arches, while it is located on the horizontal axis for the lateral arches. The contour of the fuselage section has the same first derivative in the connection points, while the second derivative (the curvature) is different. The consequence is a localized stress concentration with the need of a local reinforcement.

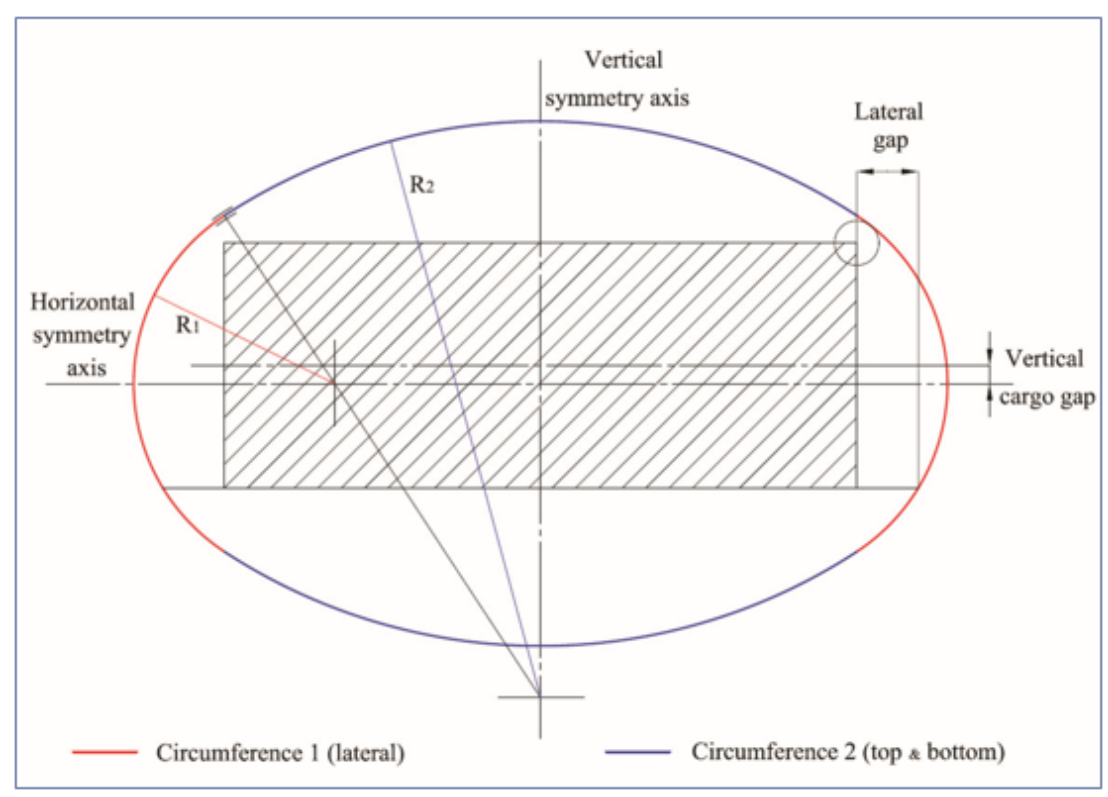

Figure 1.5. Fuselage cross section 
The overall height $(H)$ has to ensure an appropriate bending stiffness to the fuselage and the total width $(W)$ guarantees a proper clearance between containers and external shape. The space under the deck allows wing crossing and landing gears dispositions without any interference; the main lengths of the cross section are:

$$
W=7799 \mathrm{~mm} \quad H=5030 \mathrm{~mm}
$$

A plausible design philosophy can be summarized by Torenbeek [8]: "The fuselage should be designed from the inside to the outwards and the skin should envelope the load in such a way that the wetted area is minimum, thus avoiding break away of the airflow as far as possible".

The loading doors are located close to the rear and to the front fuselage (two doors for any side) in order to limit the local stress (refer to [5]).

\subsection{Preliminary estimate of Weights and Performances}

The take off weight of the PrandtlPlane ${ }^{\circledR}$ is:

$$
W_{\text {to }}=W_{\text {pay }}+W_{\text {crew }}+W_{\text {eo }}+W_{\text {fuel }}
$$

$W_{t o}=$ Maximum Take Off Weight MTOW

$W_{\text {pay }}=$ Gross payload

$W_{\text {crew }}=95 \mathrm{Kg}$ for each unit (AEA requirements).

$W_{e o}=$ Empty Operative Weight.

$W_{\text {fuel }}=$ Weight of fuel required for the nominal mission.

The previous equation can be written as follows:

$$
W_{t o}=\frac{W_{\text {pay }}+W_{\text {crew }}}{1-\frac{W_{e o}}{W_{\text {to }}}-\frac{W_{\text {fuel }}}{W_{\text {to }}}} ;
$$

it can be solved by using an iterative procedure. The hardest difficulty is to have a suitable estimation of $\left(\frac{W_{e o}}{W_{t_{o}}}\right)$. ( $\left(\frac{W_{f u e l}}{W_{t_{o}}}\right)$ is found out by using Breguet's formula and it needs to fix a "design point" in terms of wing loading $\left(\frac{W}{S}\right)$ and horsepower-to-weight $\left(\frac{h p}{W}\right)$.

The "design point" is necessary to define efficiency in flight condition and the fuel consumption in cruise and loiter; it is assumed in such a way that all operational requirements are achieved in each mission segment. 
- TAKE OFF: the horse-to-power ratio is related to the wing loading through the Take Off Parameter according to FAR 25. It depends on the Balanced Field Length, assumed as $4000 \mathrm{~m}$ in this analysis; it also depends on the value of $C_{L t o}$ coefficient:

$$
C_{\text {Lmaxto }}=2.5
$$

$C_{\text {Lmaxto }}$ is higher than conventional aircraft because both wings equip high lift devices, and the assumption is:

$$
C_{\text {Lto }}=1.1 \frac{C_{\text {Lmaxto }}}{1.21}
$$

where the 1.1 factor takes into account an expected increase of $10 \%$ because of the effect of propelled propulsion on flaps.

- CLIMB: FAR 25 appoints the minimum Climb Gradient Rate to be taken during climb operations with one engine inoperative. The $\left(\frac{h p}{W}\right)$ vs $\left(\frac{W}{S}\right)$ relationship is found out by using the standard formula that relates available power and necessary power.

$$
C G R=\frac{P_{d}-P_{n}}{V W}
$$

$P_{d}$ is the available power, while $P_{n}$ is the necessary power. $V$ is the climb speed (it is assigned in FAR 25).

- CRUISE: Cruise condition is simply studied with equilibrium equations. Significant parameters used during the calculation are shown below:

$$
\begin{gathered}
\frac{W_{c r}}{W_{t o}}=0.975 \quad(\text { from statistics on historical trends }) \\
\frac{h p_{t o}}{h p_{c r}}=2 \quad(\text { from propfan avaliable data })
\end{gathered}
$$

where $h p_{t o}$ is the horsepower in take off conditions and $h p_{c r}$ is the horsepower in the cruise condition.

- APPROACH: The approaching speed and $C_{L \max }$ are set as below and $\frac{W}{S}$ can be directly calculated because it does not depend on $\frac{h p}{W}$.

$$
\begin{gathered}
V_{\text {app }}=75 \quad \mathrm{~m} / \mathrm{s} \\
C_{\text {Lmax }}=2.9 \\
\left.\frac{W}{S}\right|_{\text {app }}=555 \quad \mathrm{Kg} / \mathrm{m}^{2}
\end{gathered}
$$




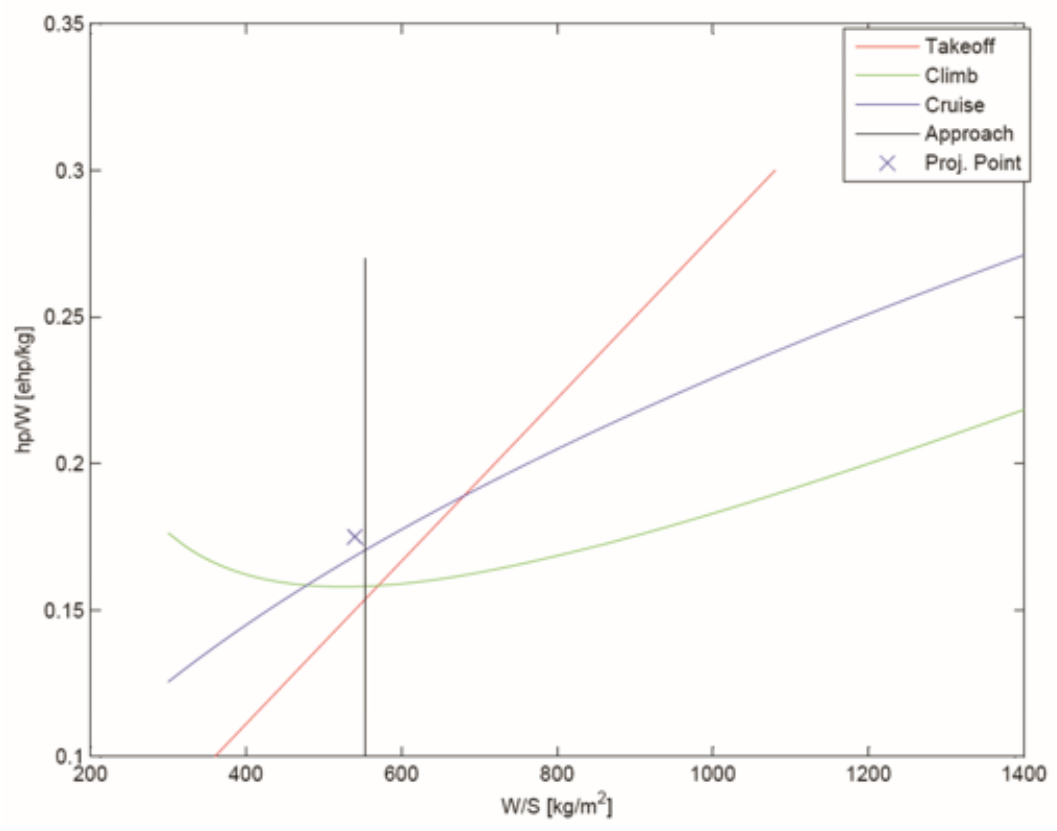

Figure 1.6. Design Point

Combining previous information, the Design Point is obtained and it is shown in Fig.1.6 as "Project Point":

$$
\begin{cases}\frac{W}{S}=540 & \mathrm{~kg} / \mathrm{m}^{2} \\ \frac{h p}{W}=0.175 & \mathrm{ehp} / \mathrm{kg}\end{cases}
$$

It is necessary to underline that the previous parameters have been estimated as a first index of the aircraft. They will change during the design process: in particular, in this Thesis the wing loading will be deeply investigated, as it will be widely explained in the following chapters. The empty weight fraction is estimated by Jane's models. The only difference is about the fuselage empty weight: as it has been explained in the previous paragraph, the fuselage enjoys a significant weight saving, so the Jane's prediction is decreased by $30 \%$. Results of the whole analysis are reported in the next table:

\begin{tabular}{lcc}
\hline Weight & Value $[\mathbf{K g}]$ & Weigth fraction \\
\hline$W_{t o}$ & 624883 & 1 \\
\hline$W_{\text {eo }}$ & 250420 & 0.4011 \\
\hline$W_{\text {fuel }}$ & 124460 & 0.1943 \\
\hline$W_{\text {pay }}$ & 250000 & 0.4046 \\
\hline
\end{tabular}

Table 1.1. Weight results 


\subsection{Employment of a third lifting surface}

In general, when the Maximum Take Off Weight increases and the dimensions are limited by the regulations, a feasible wing plan configuration is difficult to be found. Specially, the lift generation becomes in contrast with both the flight stability and structural requirements; also the maneuvrability tends to be critic for very large aircraft. The Box Wing configuration could provide proper solution for all the contrasting requirements. In particular, previous studies (refer to [14]) remarked that the PrandtPlane ${ }^{\circledR}$ satisfies requirement on the longitudinal stability and dynamics with a lower value of the Margin of Stability, if compared to a conventional configuration; these aspects have benefits on the overall maneuvrability and on the sizing of the elevators. Nevertheless, when the Maximum Take Off Weight is very high, the stability and the lift generation can reduce again the feasible solution space and other solutions in terms of wings configuration should be introduced. For this reason, in the preliminary design, a third wing is introduced (it will be called auxiliary wing in the following chapters), so that many effects can be observed; they are summarized in the following points:

- Even if this auxiliary wing leads to an increase of the structural weight, other components (such as the fuselage) may have benefits and thus, the global structural weight can be considered constant;

- Fuselage save weight does not lead to a significant cut of the section moment of inertia: the average thickness (skin and stringers) will decrease but the middle line remains unchanged;

$$
I_{x}=\pi R_{e q}^{3 \bar{t}}
$$

According to the section moment of inertia formula, it will decrease linearly with $\bar{t}$;

- The new weight disposition (with a new wing and a lighter fuselage) allows more efficient flight mechanics because the new wing can move the neutral point of the whole configuration to a more suitable position.

- If the new wing is set far enough from the aircraft center of gravity, the pitch trim will be easier because of the rather big distance between applied force and the centroid itself. In this case the elevator does not need high angular to respect trim conditions, and saturation problems are deleted.

- A third wing obviously gives a new contribution of induced drag and friction drag. The induced drag contribution depends on aspect ratio of wings, while friction drag depends on wet surface. Even though these additional contributions are present, the two standard wings of the box wing configuration reduce their drag because they reduce their surface. The total surface of the lifting system probably will not be affected by the number of wings. The two starting wings have shorter chords when a third surface is set (because the wing span is imposed), so that they improve their aspect ratio and their surface decreases. 
All previous aspects need to be taken into account in quantitative manner. In this paragraph the evident decrease of bending moment along the fuselage will be shown and it will be considered a satisfactory proof of efficiency of the third lifting surface. Due to the position of the third wing along the fuselage, wings sizing and trim devices have to be carefully designed. The design process involves a graphic software $(A S D)$ and an aerodynamic optimizer (AEROSTATE): both have been developed by the Aerospace department of University of Pisa and they will be widely described in the following chapters.

The model that describes the fuselage mechanical behaviour, when it is supported by three wings, is extremely rough but it is exhaustive enough at this point of the design flow. It is considered as a three-point supported beam with a distributed load: the scheme is represented in Fig.1.7.

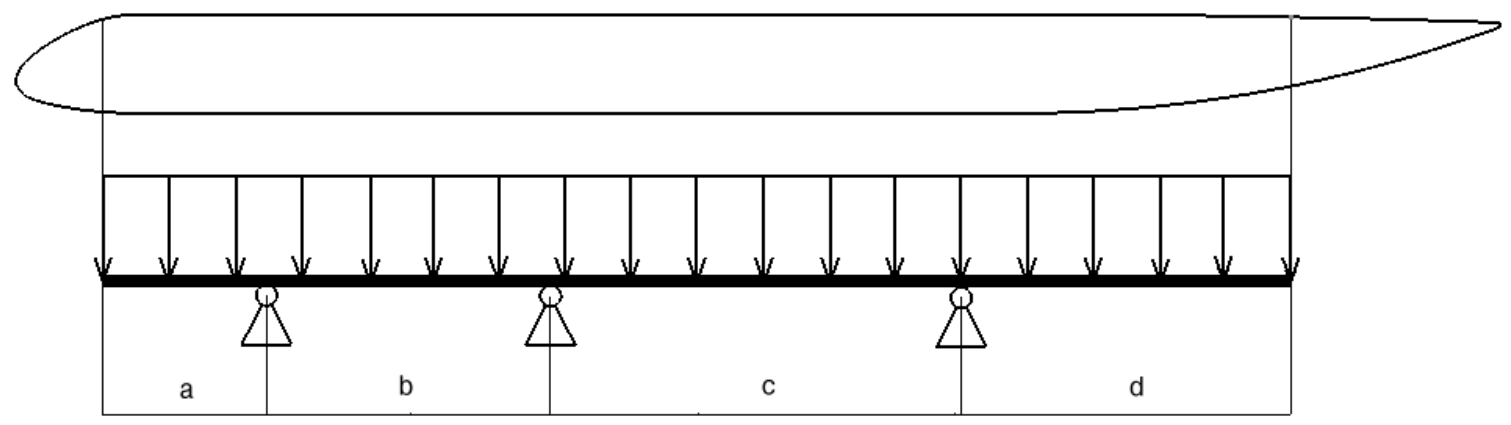

Figure 1.7. Beam scheme

Material properties, section properties, and loads are taken directly by [5] and they are summarized in the table below; note that the beam total length is not the fuselage total length because only the middle part is considered. Nose and tail need more attention during the design process: in fact the nose is pressurized and the tail has a particular bulkhead. However nose and tail are not supposed to bring payload, therefore they can be cut out by this analysis.

\begin{tabular}{lcc}
\hline Type & Symbol & Value \\
\hline Young Modulus $[\mathrm{MPa}]$ & $E$ & 73000 \\
\hline Total Length $[\mathrm{mm}]$ & $L_{\text {tot }}$ & 64000 \\
\hline Section Moment of Inertia $\left[\mathrm{mm}^{4}\right]$ & $I_{x}$ & $4.69 * 10^{11}$ \\
\hline Distribuited load $[\mathrm{N} / \mathrm{mm}]$ & $p$ & 102.5 \\
\hline
\end{tabular}

Table 1.2. Main quantities 
Let $v$ be the vertical displacement of the elastic line which represents the middle part of the fuselage. All the following derivatives are referred to an abscissa $s$ along the elastic line. Besides $a, b, c, d$ are the distances along the beam which identify the supports. The elastic line equation has to be written and solved for every part of the beam: that means there are four equations, like the following one

$$
E I_{x} v^{\prime \prime \prime \prime}(s)=p
$$

The general integration of the former equation is

$$
v(s)=\frac{p s^{4}}{24 E I_{x}}+A \frac{s^{3}}{6}+B \frac{s^{2}}{2}+C s+D .
$$

The four equations can be solved by introducing boundary conditions in terms of shear forces, momentum, rotational and vertical displacements. There are sixteen conditions to be met and they are all shown in Tab.1.3.

\begin{tabular}{lllr}
\hline 1) $v_{1}^{\prime \prime}(0)=0$ & 2) $v_{1}^{\prime \prime \prime}(0)=0$ & 3) $v_{1}(a)=0$ & 4) $v_{2}(0)=0$ \\
\hline 5) $v_{1}^{\prime}(a)=v_{2}^{\prime}(0)$ & 6) $v_{1}^{\prime \prime}(a)=v_{2}^{\prime \prime}(0)$ & 7) $v_{2}(b)=0$ & 8) $v_{3}(0)=0$ \\
\hline 9) $v_{2}^{\prime}(b)=v_{3}^{\prime}(0)$ & 10) $v_{2}^{\prime \prime}(b)=v_{3}^{\prime \prime}(0)$ & 11) $v_{3}(c)=0$ & 12) $v_{4}(0)=0$ \\
\hline 13) $v_{3}^{\prime}(c)=v_{4}^{\prime}(0)$ & 14) $v_{3}^{\prime \prime}(c)=v_{4}^{\prime \prime}(0)$ & 15) $v_{4}^{\prime \prime}(d)=0$ & 16) $v_{4}^{\prime \prime \prime}(d)=0$ \\
\hline
\end{tabular}

Table 1.3. Boundary Conditions

Unknown variables are gathered in a vector $\boldsymbol{X}$ while known coefficients are gathered in a vector $\boldsymbol{c}$. $\boldsymbol{A}$ is the matrix to be inverted to get the solution. The final expression of the problem, once it has been noted that there are five null unknown, is reported:

- $A_{1}=0$ from condition 1

- $B_{1}=0$ from condition 2

- $D_{2}=0$ from condition 4

- $D_{3}=0$ from condition 8

- $D_{4}=0$ from condition 12

- $A_{4}=-\frac{p d}{E I_{x}}$ from condition 16

$$
A X=c
$$




$$
\left[\begin{array}{cccccccccc}
a & 1 & 0 & 0 & 0 & 0 & 0 & 0 & 0 & 0 \\
1 & 0 & 0 & 0 & -1 & 0 & 0 & 0 & 0 & 0 \\
0 & 0 & 0 & 1 & 0 & 0 & 0 & 0 & 0 & 0 \\
0 & 0 & \frac{b^{3}}{6} & \frac{b^{2}}{2} & b & 0 & 0 & 0 & 0 & 0 \\
0 & 0 & \frac{b^{2}}{2} & b & 1 & 0 & 0 & -1 & 0 & 0 \\
0 & 0 & b & 1 & 0 & 0 & -1 & 0 & 0 & 0 \\
0 & 0 & 0 & 0 & 0 & \frac{c^{3}}{6} & \frac{c^{2}}{2} & c & 0 & 0 \\
0 & 0 & 0 & 0 & 0 & \frac{c^{2}}{2} & c & 1 & 0 & -1 \\
0 & 0 & 0 & 0 & 0 & c & 1 & 0 & -1 & 0 \\
0 & 0 & 0 & 0 & 0 & 0 & 0 & 0 & 1 & 0
\end{array}\right]\left[\begin{array}{c}
C_{1} \\
D_{1} \\
A_{2} \\
B_{2} \\
C_{2} \\
A_{3} \\
B_{3} \\
C_{3} \\
B_{4} \\
C_{4}
\end{array}\right]=\left[\begin{array}{c}
-\frac{p a^{4}}{24 E I_{x}} \\
p a^{3} \\
-\frac{6 E I_{x}}{p a^{2}} \\
\frac{2 E I_{x}}{p b^{4}} \\
-\frac{24 I_{x}}{24 b^{3}} \\
-\frac{p I_{x}}{6 E b^{2}} \\
-\frac{p b^{2}}{2 E I_{x}} \\
p c^{4} \\
-\frac{p I_{x}}{24 E} \\
-\frac{p c^{3}}{6 E I_{x}} \\
p c^{2} \\
-\frac{p I_{x}}{2 E I_{x}} \\
\frac{p d^{2}}{2 E I_{x}}
\end{array}\right]
$$

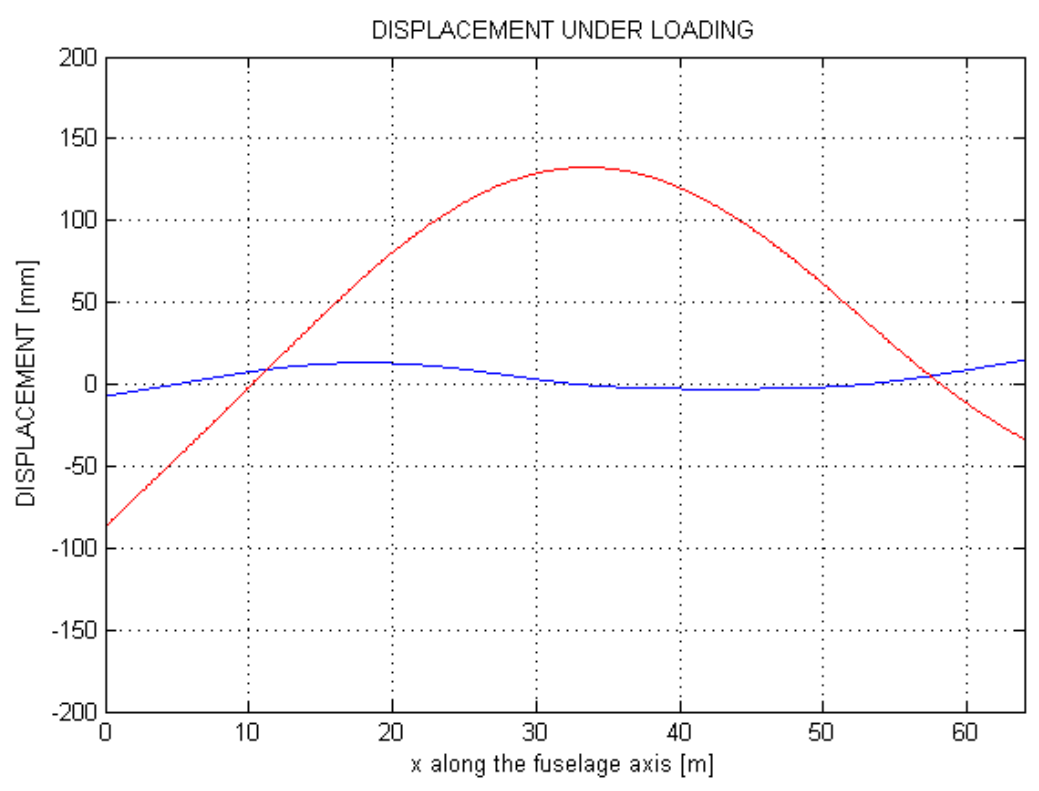

Figure 1.8. Comparison between displacements 


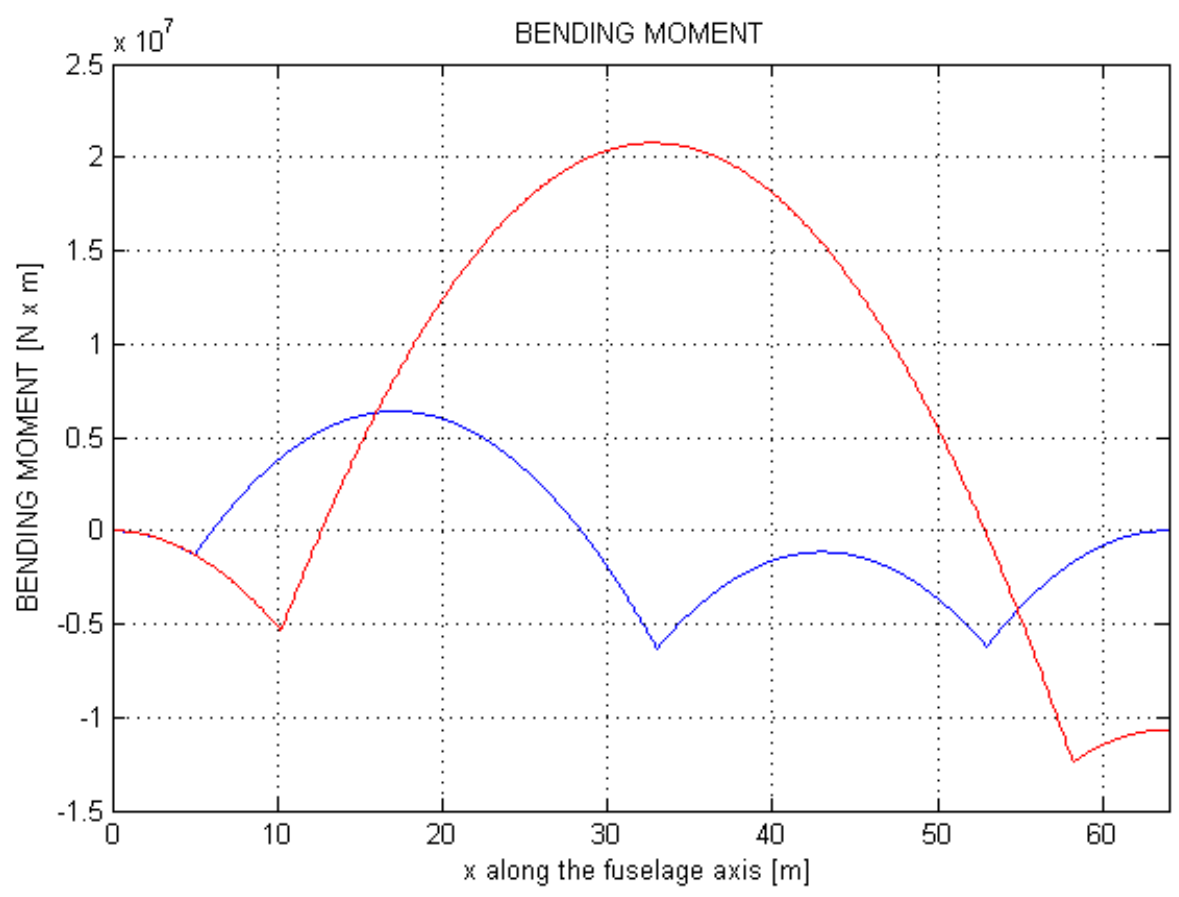

Figure 1.9. Comparison between bending moment

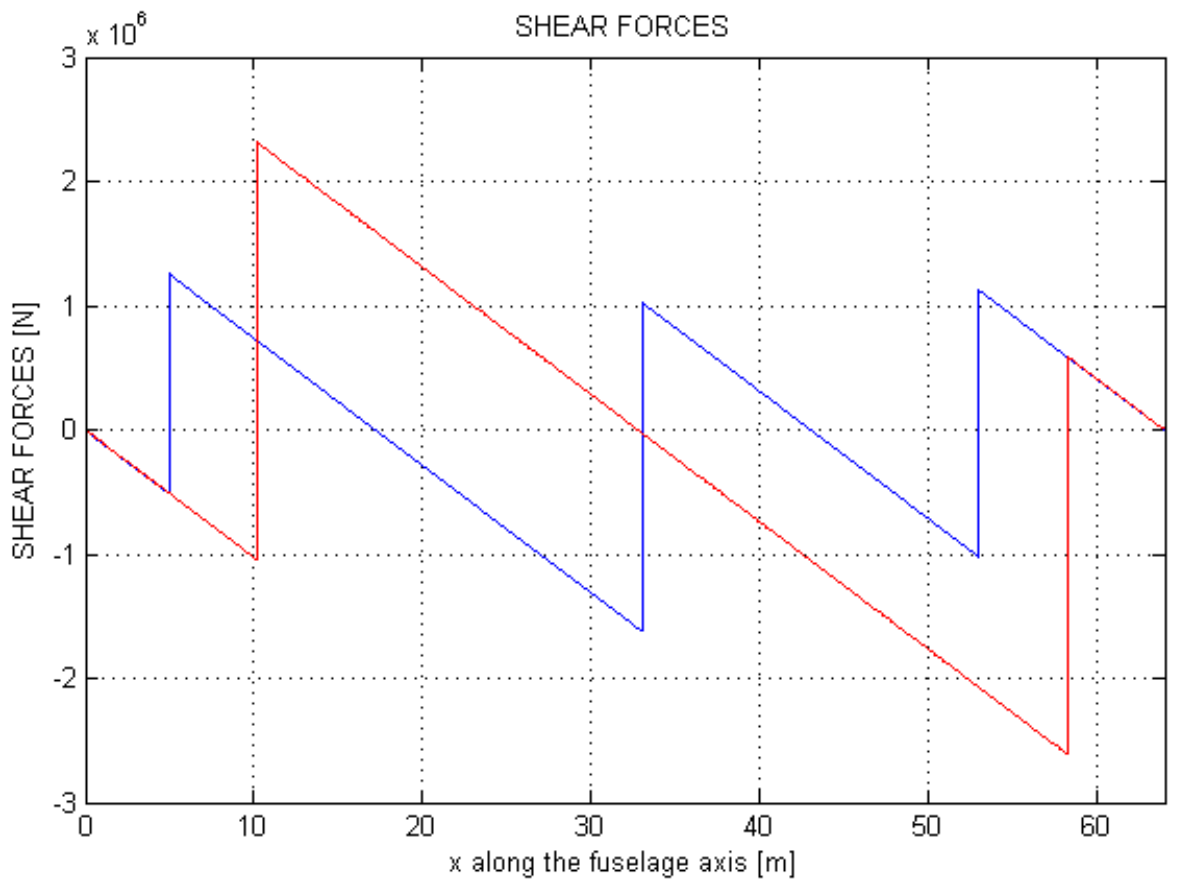

Figure 1.10. Comparison between shear forces 
The foregoing linear system 1.12 has been solved by using $M A T L A B^{\circledR}$ and the results are plotted: red curves represent the old solution with two support as blue curves represent the new solution with three lifting surfaces (Fig. $1.8-1.9-1.10$ ). The better efficiency of the new system is noticeable: as the section moment of inertia does not change, the first solution leads to great deformations of the fuselage axis, which correspond to high values of bending moment. Bending moment is the dominating sizing criterion for the fuselage, and the stress along the $\mathrm{x}$ direction is linearly dependent on it. Even though the weight saving is not directly linked to the bending moment decrease, we can conclude that the three lifting surface-configuration is worth. Besides, by examining the shear forces curves, the lift that each wing has to develop (that means the discontinuity among two curves) is less than the two-wing configuration. In this preliminary estimation, the trim conditions are unknown, so it is impossible to predict exactly how the new solution will be; however smaller surfaces are reasonably expected and that means smaller chords, higher aspect ratios and less wet surface. All these aspects are encouraging and next chapters will deal with this innovative aircraft kind (see Fig.1.11).

The optimization program can provide solutions for the new configuration and the position of the three wings along the fuselage axis is not known at now. Hence, in the previous calculation the three wings are all set as supports without distinguishing features: in the following chapters every support will become a backward swept wing, a forward swept wing or a third smaller wing.

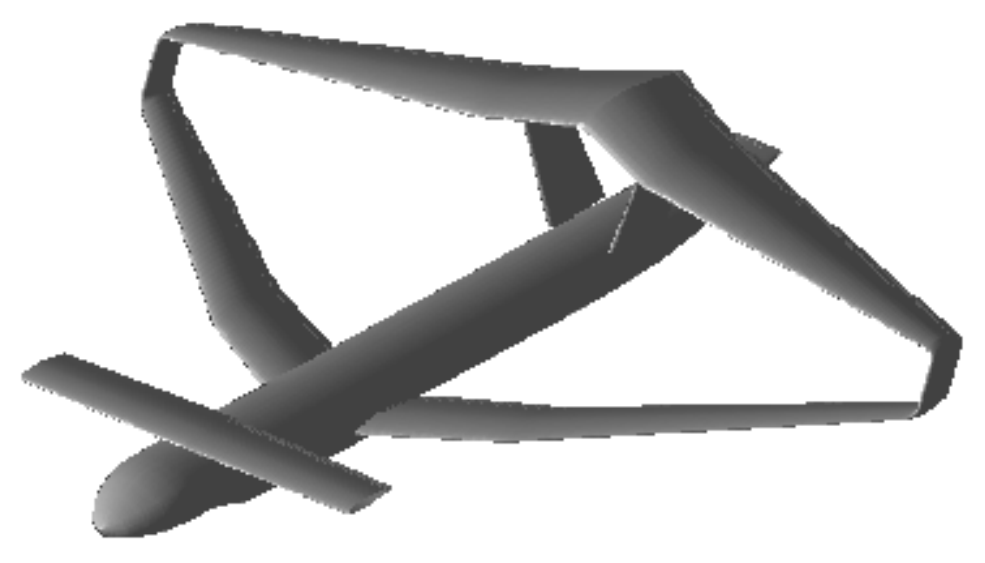

Figure 1.11. Example of a new-kind configuration with a canard 



\section{Chapter 2}

\section{D shape design of freighter aircraft}

The shape of every aircraft built in this Thesis, has been realized by using ASD (Aerodynamic Shape Designer). This software has been developed at Department of Aerospace Engineering of Pisa in collaboration with I.D.S. S.p.A.

In this Thesis, ASD has been modified in his GUI structure with the purpose of automating the software (reference to the chapter 4). In the following sections, principal properties of ASD are explained.

\section{$2.1 \quad$ ASD}

The purpose of ASD software package is to shape a complex surface, like an airplane, in a parametric, quick and modular way. The generation of aerodynamic surfaces is executed by the interpolation of N.U.R.B.S. curves [1] [2], acronym for Non-Uniform-Rational-BSpline. In particular, ASD is a MATLAB ${ }^{\circledR}$ Toolbox, aimed to set up a framework for aircraft conceptual design and optimization. The conceptual design needs to generate and analyze a huge number of slightly different configurations that are defined by a set of variable parameters.

The peculiarity of the software package is the capability to analyse non conventional configurations (for example, PrandtlPlane ${ }^{\circledR}$ aircraft configuration), whose aerodynamic shapes can not be easily designed using common CAD softwares.

ASD [3] incorporates a set of tools to create and edit various types of shapes, ranging from axis-symmetric bodies to wings and blended wing-body surfaces as well as many of fillet surfaces between them.

The output of the surface generator can be exported as a set of trimmed NURBS into an IGES file or fed into meshing tool that is built into the code. The meshing tool is capable of generating triangular or mixed quad-tri meshes, and to export the result in several formats, for different applications, ranging from electromagnetic to aerodynamic simulation codes. 
When the user runs ASD, the program interface appears and the main window is shown in Fig.2.1. Through this GUI (acronym for Graphical User Interface), the user is able to generate all the features of the aircraft.

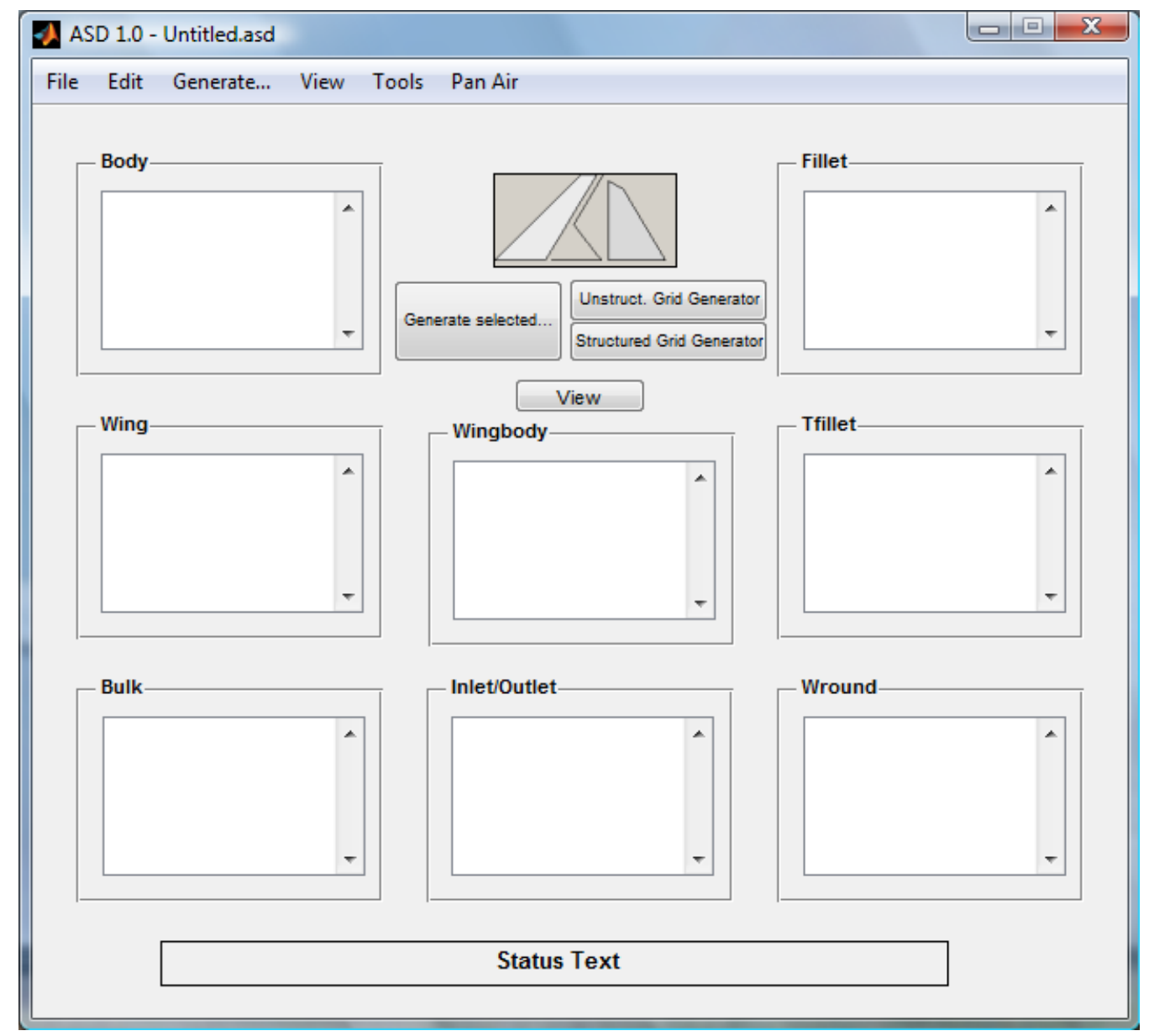

Figure 2.1. ASD main window.

\subsection{Body}

Using the feature "Add/Modify Body" (Fig.2.2), it is possible to generate a Body (for example the Fuselage of an aircraft), defining only the shape of sections and the principal sizes of it. In particular, the user must define four lines, as shown in Fig.2.2: Bottom line, Center line, Side line and Top line.

ASD requires a file for every line, with ".dat" extension, in which there must be the coordinates of them. 


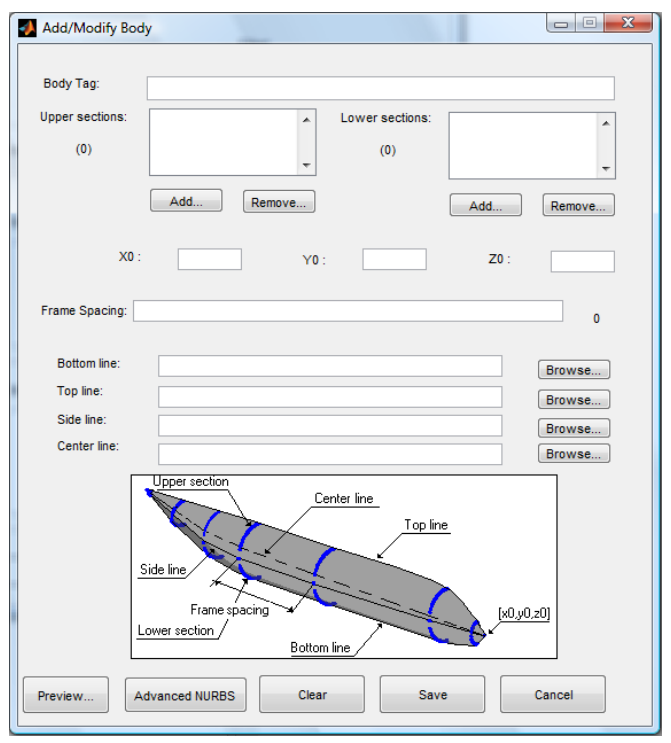

Figure 2.2. ASD Add/Modify Body window.

\section{$2.3 \quad$ Wing}

With regard to wings and tail surfaces, there is also the feature "Add/Modify Wing" (Fig. 2.3 ), in which the user can generate the aerodynamic surfaces, specifying the principal parameters of them (wingspan, aspect ratio, sweep and dihedron angles, etc.).

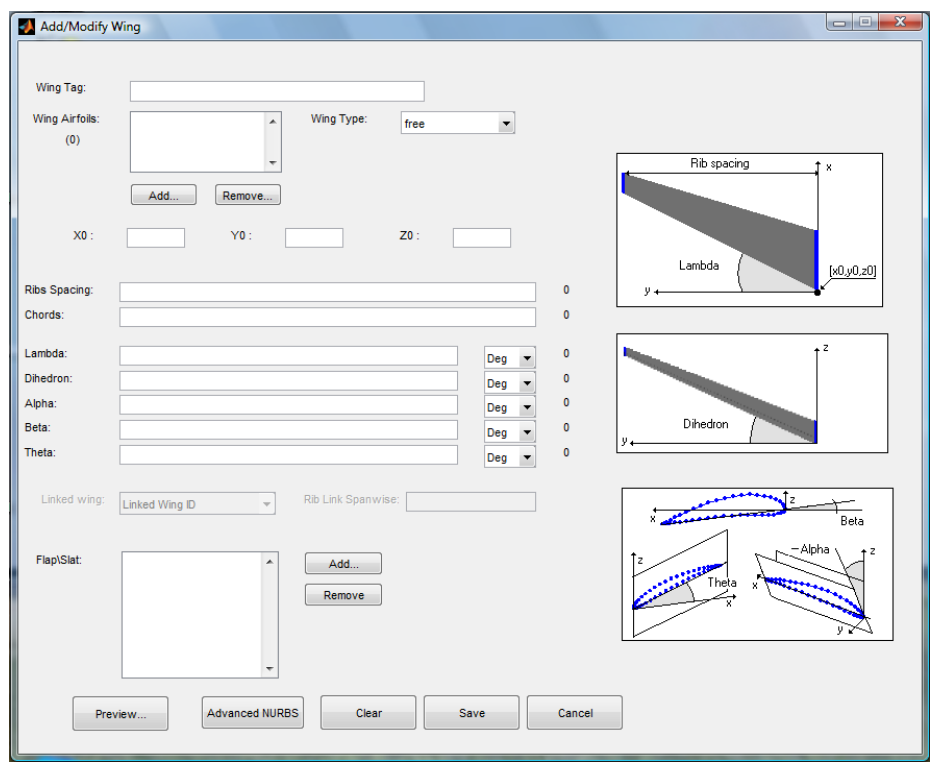

Figure 2.3. ASD Add/Modify Wing window. 


\subsection{Bulk}

This feature allows the user to define the bulk, which represents a connection between two wings: this is an unconventional aerodynamic configuration and it usually is a peculiarity of PrandtlPlane ${ }^{\circledR}$ aircraft configuration. The GUI control panel is plotted in Fig.2.4.

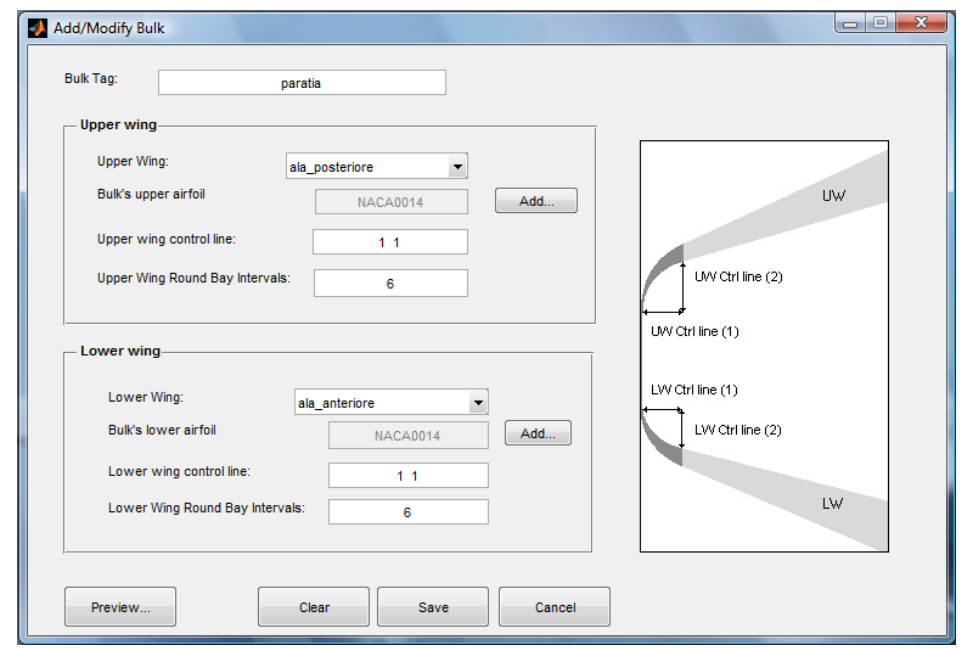

Figure 2.4. ASD Add/Modify Bulk window.

\subsection{Fillet window}

The "Add/Modify Fillet" interface lets the user specify all the parameters involved in the generation of a fillet feature, between a wing and a body. The interface of Add/Modify Fillet is represented in Fig.2.5.

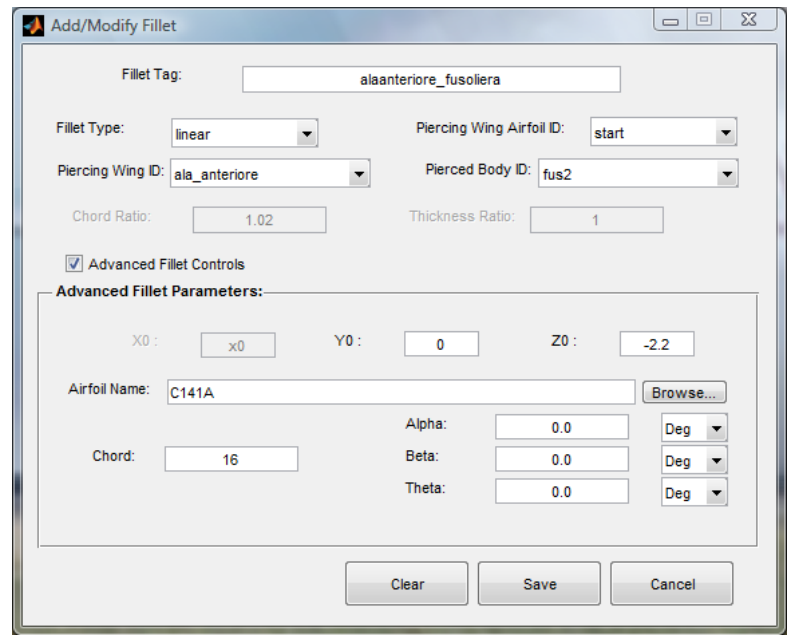

Figure 2.5. ASD Add/Modify Fillet window. 
Instead, the feature "Add/Modify TFillet" lets the user generate a fillet between two wings. The interface of "Add/Modify TFillet" is plotted in Fig.2.6.

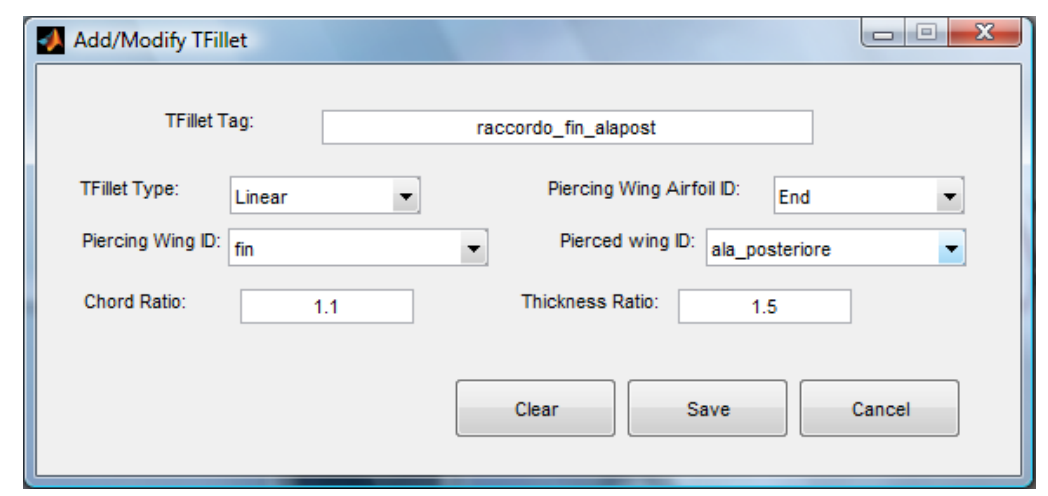

Figure 2.6. ASD Add/Modify TFillet window.

\subsection{Add/Modify Wround}

The "Add/Modify Wround" interface lets the user specify all the parameters involved the generation of a Wround feature. The interface for this feature is shown in Fig.2.7.

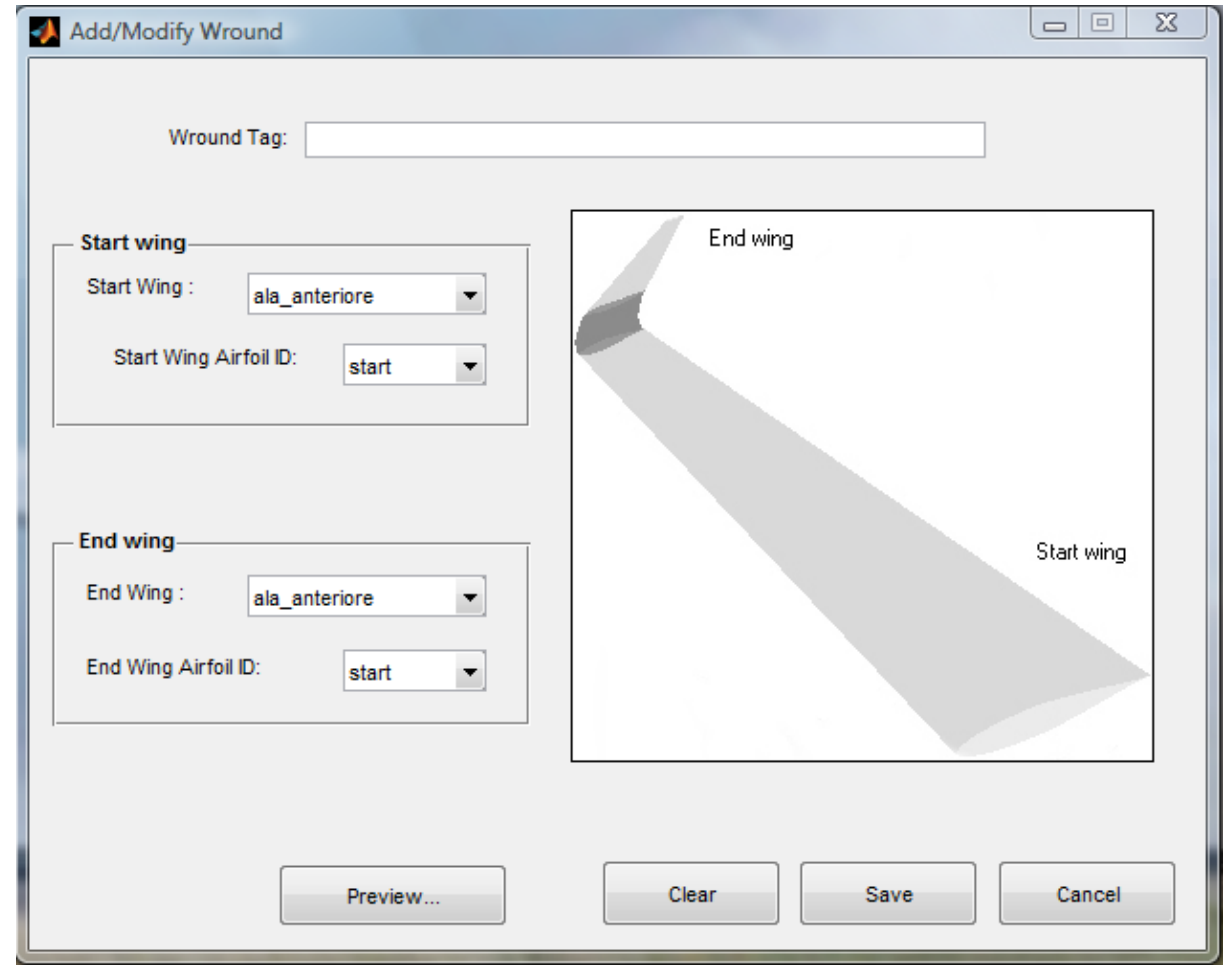

Figure 2.7. ASD Add/Modify Wround window. 


\subsection{Airfoil Manager}

AIRFOIL MANAGER (Fig.2.8) is a graphical user interface that gives the user the capability to visualize all the airfoil stored in database. It is a manage airfoil databases stored in ".dat" files.

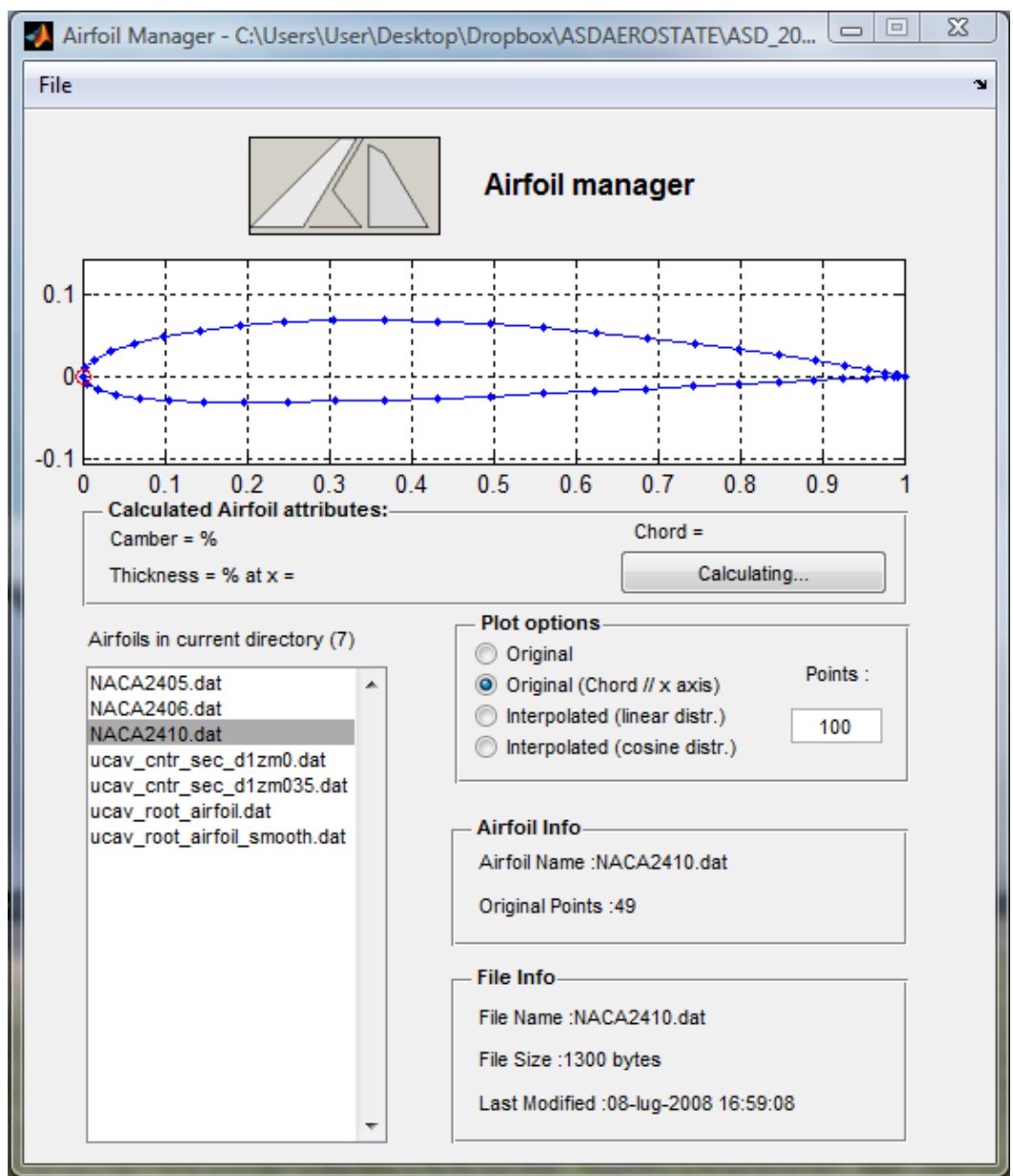

Figure 2.8. ASD AIRFOIL MANAGER.

\subsection{Surface mesher}

Moreover, the software ASD has a surface mesher. From the ASD main window (Fig.2.1), the user can launch the "ASD Surface Mesher" (Fig.2.9) on the selected features. The ASD Surface mesher is a graphical user interface to help the user manage all the parameters and functions underlying the process of mesh generation. The ASD mesher sub-function is specifically designed to generate triangular meshes with maximum chord distance and maximum element size specified by user. The interface also enables the user to perform different tasks on the mesh, ranging from Delaunay Flip to Triangular-to-Mixed mesh transform, plotting and analyzing meshes. 


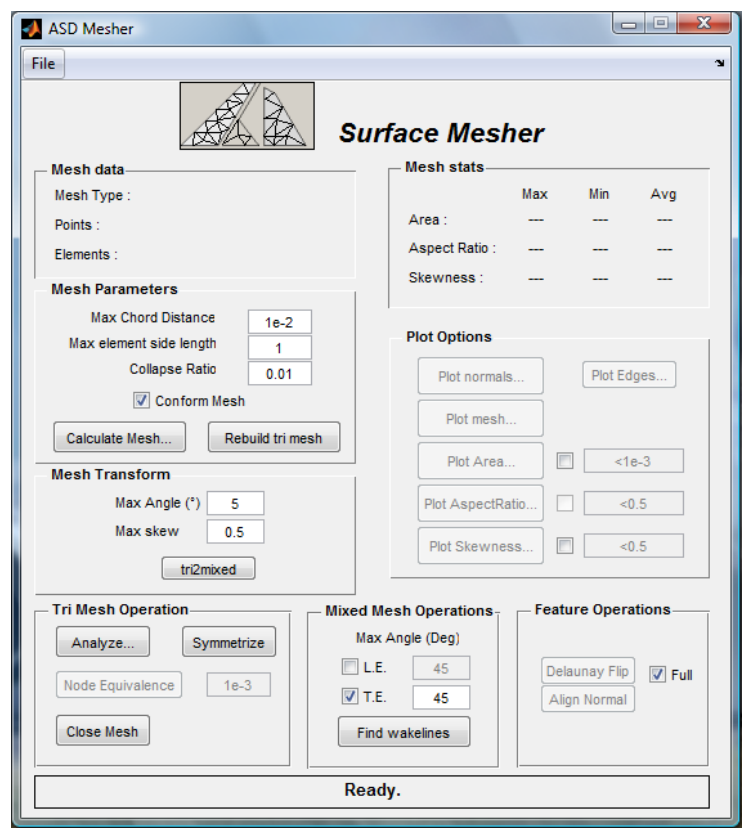

Figure 2.9. ASD Surface Mesher window.

\subsection{Other features}

In the previous sections the most important properties of the software have been shown. ASD contains also a NACA AIRFOIL GENERATOR, in which the user is able to create NACA 4-digit and NACA 5-digit airfoil series. ASD contains also a Flap Sketcher: it is an interface that gives the user the capability to create any Flap/Slat configuration starting from the base airfoil, or to modify an existing configuration.

To generate some or all the features selected, the "Generate Selected" pushbutton is used, as shown in Fig.2.1. Instead, the "View" pushbutton is used to view the plot of surfaces selected: in this way, ASD runs the "Surfaces Viewer" to plot all the surfaces (Fig.2.10).

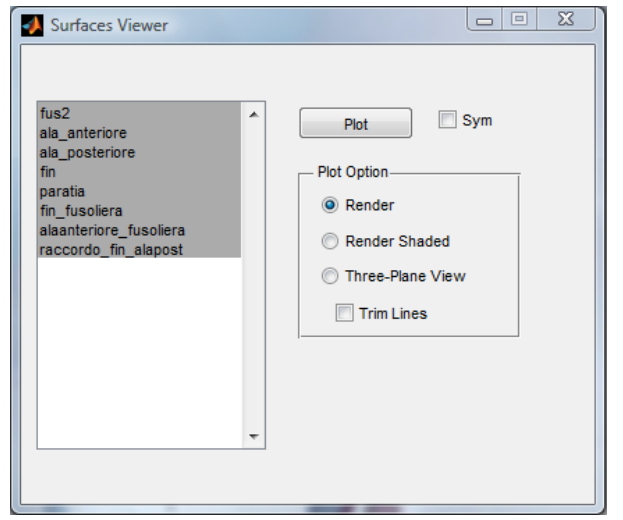

Figure 2.10. ASD Surfaces Viewer window. 


\section{$2.10 \quad \mathrm{LiNeS}$}

When the user needs to generate a Body, as shown in section 2.2, ASD requires ".dat" files containing the coordinates of four principal lines. The software LiNeS has been developed in MATLAB ${ }^{\circledR}$ environment to build these files.

The analytical definition of lines is reported in [4] and because of its complexity, the software has been realized with a graphical user interface (GUI). "LiNeS" is organized in two sections: the first concerns the definition of side view of the fuselage (Fig. 2.11), and the second deals with the fuselage top view, with the assumption of symmetrical fuselage (Fig. 2.12).

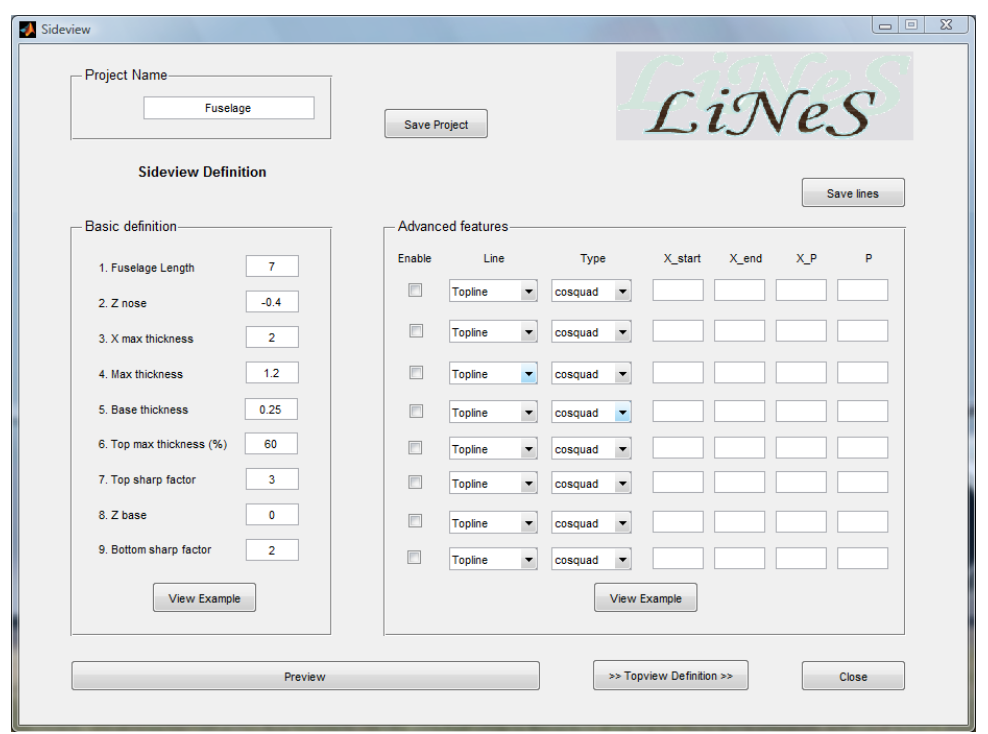

Figure 2.11. LiNeS side view window.

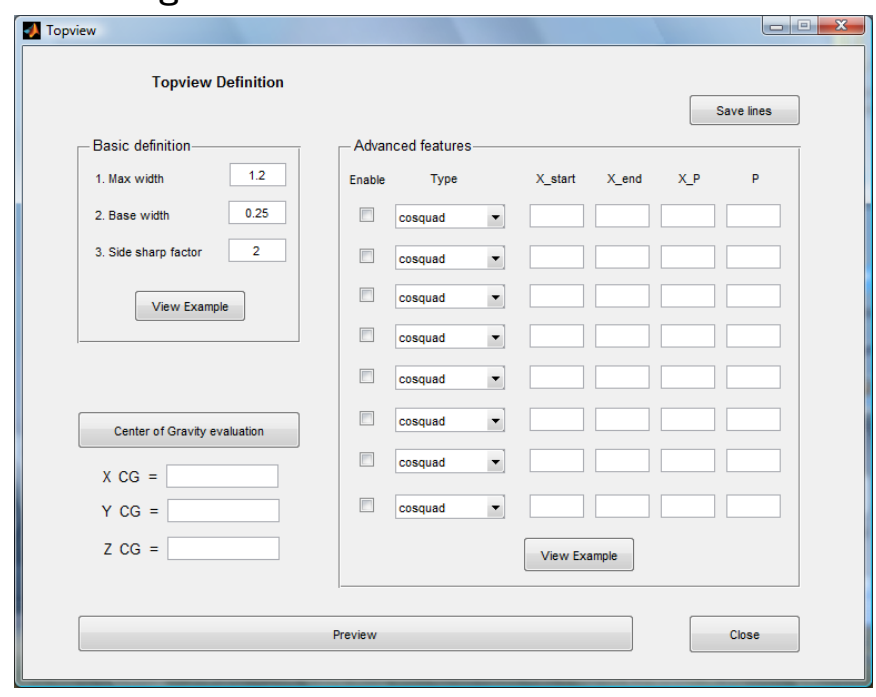

Figure 2.12. LiNeS top view window.

The side view is used to generate the top, center and bottom lines of the Fuselage; the 
basic quantities to be provided as input are summarized in Tab.2.1. Moreover, there are the "Advanced features", which are used to generate some other changes.

\begin{tabular}{ll}
\hline INPUT & DESCRIPTION \\
\hline$L_{\text {fus }}$ & Fuselage length \\
\hline$x_{\text {Tmax }}$ & Maximum coordinate thickness of Fuselage profile \\
\hline$T_{\text {max }}$ & Maximum thickness of Fuselage profile \\
\hline$T_{\text {base }}$ & Thickness of Fuselage profile at $x=L_{f u s e}$ \\
\hline$T_{\text {max }}^{\text {top }}$ & Percentage of maximum thickness to be placed toward the back \\
\hline$z_{\text {base }}$ & z coordinate axis of the base of Fuselage \\
\hline$k_{\text {up }}$ & "Sharp factor" of the lower part of the nose of Fuselage \\
\hline$k_{\text {down }}$ & "Sharp factor" of the upper part of the nose of Fuselage \\
\hline
\end{tabular}

Table 2.1. Input for the line in the side view of the Fuselage.

Instead, the top view is used to generate the side line of the Body; the basic quantities to be provided as input are summarized in Tab.2.2. Moreover, there are the "Advanced features", which are used to generate some other changes.

\begin{tabular}{ll}
\hline INPUT & DESCRIPTION \\
\hline$W_{\max }$ & Maximum Fuselage width \\
\hline$W_{\text {base }}$ & Width of the base of Fuselage \\
\hline$k_{\text {side }}$ & Lateral "Sharp factor" for the nose of Fuselage \\
\hline
\end{tabular}

Table 2.2. Input for the line in the top view of the Fuselage.

After the definition of the top view, it is possible to calculate a first evaluation of center of gravity of the Fuselage. This calculation is based on assigning to each section of the body a "weight" equal to the perimeter of the rectangle circumscribed to it, which has as its sides the height $h$ and the width $l$ of the Fuselage to the $x$ considered. For further information, refer to [4]. 


\subsection{Example of ASD surfaces generation}

This chapter ends with an example of PrandtlPlane ${ }^{\circledR}$ freighter aircraft, which surfaces are generated by the software ASD, as shown in Fig.2.13. This airplane is a preliminary configuration of non conventional PrandtlPlane ${ }^{\circledR}$ freighter aircraft: the shape of his body is described in ref.[5].

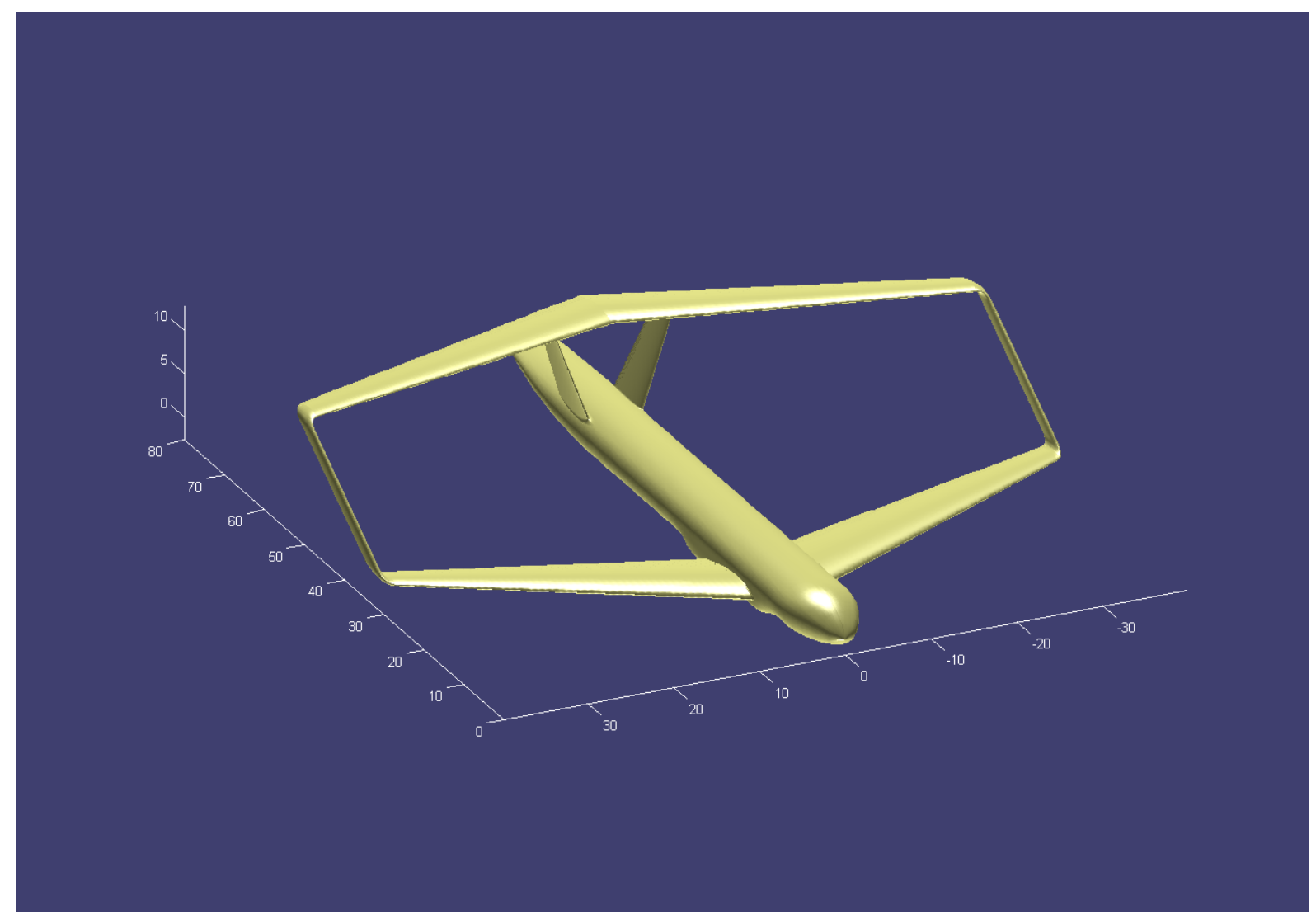

Figure 2.13. Example of freighter aircraft surfaces generation, with ASD software. 


\section{Chapter 3}

\section{AEROSTATE}

AEROSTATE (AERodynamic Optimization with STAtic stability and Trim Evaluator) is a software developed by the Department of Aerospace Engineering of Pisa and its theoretical basis are described in [9] and in [10]. It is a program capable of finding the optimum wing planforms of whatever configuration under geometrical and aerodynamic constraints in cruise condition.

\subsection{Software description}

The research of the optimum configuration in AEROSTATE is obtained by the implementation of a local and a global algorithm. There are two basic strategies to determine the optimum of a constrained or an unconstrained function: the first one is an analytical exact method, that computes the Hessian matrix to evaluate the gradient of the function; the second strategy implies using algorithms which contain a random component (e.g. genetic algorithms) and it is based on the so called "heuristic methods". Analytical methods can find out the local minimum, while heuristic methods determine the global minimum, sacrificing the solution speed. The present problem has many variables, many constraints and the objective function can not evaluated in analytical way: if a gradient method is used, probably the solution tends to a local minimum and not to the global minimum. Meanwhile, genetic algorithms are not efficient from a computational point of view. Thus, the employed research algorithm is composed of a local algorithm to find out local minima and a procedure to build an approximation of the objective function and, so, to determine the global minimum.

There are many procedures to find the global solution in literature: one way is to restart the search from a new solution, once a region has been extensively explored (multi start procedure), and it is the most know. Instead, the local algorithm allows to find a minimum of a function, in a small area of the solution space.

The local algorithm that is implemented in AEROSTATE is the SQP (Sequential Quadratic Programming) Method and it is described in section 3.2.4.1.

The global algorithm which is implemented in this software is called LOCSMOOTH (Local Optima Smoothing for Global Optimization) and it is described in section 3.2.4.2. 


\subsection{Optimization methods}

In this section a general overview of the optimization methods is presented.

Considering the functions $f(x), g(x)$ and $h(x)$ :

$$
\begin{gathered}
f: \mathbb{R}^{n} \rightarrow \mathbb{R}, f \in C^{1} \\
g: \mathbb{R}^{n} \rightarrow \mathbb{R}^{p}, g \in C^{1} \\
h: \mathbb{R}^{n} \rightarrow \mathbb{R}^{m}, h \in C^{1}
\end{gathered}
$$

The set $\Omega$ is defined as follows:

$$
\Omega=\left\{x \in \mathbb{R}^{n}: g(x) \leq 0, h(x)=0\right\} .
$$

Then, the optimization problems, that will be analysed, are in the form 3.5:

$$
\left\{\begin{array}{l}
\min f(x) \\
x \in \Omega \subset \mathbb{R}^{n}
\end{array}\right.
$$

There are three hypotheses that guarantee the existence of minima for the function $f$ in $\Omega$ :

1. $f$ is a lower semi-continuous function and $\Omega$ is a compact set;

2. $f$ is a lower semi-continuous function, it is coercive and $\Omega$ is a compact set;

3. $\mathrm{f}$ is continuous and the set $L_{0}=\left\{x \in \mathbb{R}^{n}: f(x) \leq f\left(x^{0}\right)\right\}$ is compact, where $x^{0}$ is the starting point.

From a different point of view, the first two hypotheses may be avoided if the existence of the minimum is demonstrated.

In general, an optimization algorithm generates a sequence of points $\left\{x_{k}\right\}$ along a descent direction:

$$
x^{k+1}=x^{k}+t_{k} d^{k}
$$

The previous sequence is generated by the algorithm $f\left(x^{k+1}\right) \leq f\left(x^{k}\right)$; $t_{k}$ represents the step length and $d^{k}$ is the search direction.

Now $x^{k}, d^{k}$ and a linear restriction of $f$ are considered (refer to 3.7):

$$
\varphi(t)=f\left(x^{k}+t d^{k}\right)
$$

Moreover, the first derivative of $\varphi(t)$, calculated in $t=0$ is get in equation 3.8:

$$
\varphi^{\prime}(0)=\nabla f\left(x^{k}\right) d^{k}
$$

Clearly, if $\varphi^{\prime}(0)<0, d^{k}$ is a descent direction and all the direction $d^{k}$ forming an angle with the gradient vector in the range $\left(-\frac{\pi}{2}, \frac{\pi}{2}\right)$. A generic descent direction is indicated in equation 3.9 as: 


$$
d^{k}=-H^{k} \nabla f\left(x^{k}\right)
$$

where $H^{k}$ is a generic square matrix.

In the following subsections, analytic algorithms with the property of descending directions at each iteration are considered.

\subsubsection{Unconstrained optimization theory}

If the optimization problem is unconstrained, $\Omega=\mathbb{R}^{n}$ and $x^{*}$ is a minimum point only if a necessary condition is satisfied:

$$
\nabla f\left(x^{*}\right)=0
$$

If the function $f \in C^{2}$, it can be added the condition that the Hessian $\nabla^{2} f\left(x^{*}\right)$ is positive semi-definite. Moreover, if $\Omega=\mathbb{R}^{n}$ and $f \in C^{2}$, a sufficient condition to ensure that $x^{*}$ is a minimum point, is that the Hessian of $f$ is positive definite.

The conditions of convergence of a generic algorithm, with the property of descending directions at each iteration, are given by the abstract convergence theorem due to Orthega (refer to 3.2.1.1).

\subsubsection{Abstract convergence Theorem (Orthega)}

Given the sequence $x^{k+1}=x^{k}+t_{k} d^{k}$, if the following hypotheses

1. $L_{0}$ is a compact set;

2. $f\left(x^{k+1}\right) \leq f\left(x^{k}\right)$;

3. $\nabla f\left(x^{k}\right) \neq 0, d^{k} \neq 0$ and

$$
\lim _{k \rightarrow+\infty} \frac{\nabla f\left(x^{k}\right) d^{k}}{\left\|d^{k}\right\|}=0
$$

4. $\exists \beta>0: \frac{\left|\nabla f\left(x^{k}\right) d^{k}\right|}{\left\|d^{k}\right\|} \geq \beta\left\|\nabla f\left(x^{k}\right)\right\|$

are observed, then either:

- $\exists \nu$ such that $x^{v} \in L_{0}$ and $\nabla f\left(x^{v}\right)=0$

or

- The sequence $\left\{x^{k}\right\} \subset L_{0}, \forall k$, has accumulation points in $L_{0}$, then, the sequence $\left\{f\left(x^{k}\right)\right\}$ convergences and

$$
\lim _{k \rightarrow+\infty}\left\|\nabla f\left(x^{k}\right)\right\|=0
$$

At least, the convergence to a minimum is guaranteed for whatever optimization algorithm satisfying the Orthega's Theorem conditions.

It is observed that the third Orthega's Theorem condition is more difficult to be satisfied: it is substituted by a sufficient condition and it is defined in the Armijio's Theorem (refer to 3.2.1.2). 


\subsubsection{Armijio's Theorem}

If $f \in C^{1}, L_{0}$ is a compact set, $\nabla f\left(x^{k}\right) d^{k}<0$ and if

$$
\left\|d^{k}\right\| \geq \rho \frac{\left|\nabla f\left(x^{k}\right) d^{k}\right|}{\left\|d^{k}\right\|}, \rho>0
$$

then

$$
\lim _{k \rightarrow \infty} \frac{\nabla f\left(x^{k}\right) d^{k}}{\left\|d^{k}\right\|}=0
$$

When the direction $d^{k}=-H^{k} \nabla f\left(x^{k}\right)$ is considered, it can be shown that the condition 3.11 is satisfied, as well as the third condition of the Orthega's Theorem.

\subsubsection{Second Order Methods}

The second order methods, also known as Newton Methods, assume a quadratic approximation of the non-linear objective function, refer to eq. 3.13, about the point $x^{k}$.

$$
q^{k}(s)=f\left(x^{k}\right)+\nabla f\left(x^{k}\right) s+\frac{1}{2} s \nabla^{2} f\left(x^{k}\right) s
$$

Then, according to necessary optimality condition, refer to eq. 3.10:

$$
\nabla q^{k}(s)=\nabla f\left(x^{k}\right)+\nabla^{2} f\left(x^{k}\right) s=0
$$

and solving respect to $\mathrm{s}$, the search direction is get:

$$
s=-\left[\nabla^{2} f\left(x^{k}\right)\right]^{-1} \nabla f\left(x^{k}\right)
$$

The term $\left[\nabla^{2} f\left(x^{k}\right)\right]^{-1}=H^{k}$ is the inverse of the Hessian matrix ; it must be nonsingular and for the Orthega's Theorem, it must be positive. The step size is chosen constant $\left(t_{k}=1\right)$.

The Newton-Kantorovich's Theorem asserts the following conditions for Newton's Method:

1. $f \in C^{2}$ and $\nabla^{2} f$ be Lipschitz or $f \in C^{3}$;

2. $\exists x^{*}$ such that $\nabla f\left(x^{*}\right)=0$;

3. $\nabla^{2} f$ is non singular at least at each point of iteration.

Then, $\exists \varepsilon>0$ such that $\forall x_{0} \in B\left(x^{*}, \varepsilon\right)$, the sequence $\left\{x^{k}\right\}$ converges to $x^{*}$ with a quadratic speed rate.

The main disadvantage of Newton's methods is the calculation of the inverse of the Hessian matrix at each step and it is very time consuming. Consequently, an Hessian matrix approximation can be introduced, as described in section 3.2.2.1. 


\subsubsection{Quasi-Newton methods}

The Newton's algorithms, in which an approximated expression for the inverse of the Hessian matrix is used, are called Quasi-Newton methods. The general structure of the algorithm remains the same with the only exception that the Hessian Matrix is computed by an iterative method:

$$
\begin{gathered}
H_{k}=\left[\nabla^{2} f\left(x^{k}\right)\right]^{-1} \\
\left\{\begin{array}{l}
H_{0}=I, \text { if } k=0 \\
H_{k+1}=H_{k}+\Delta H_{k}, \text { if } k \geq 1
\end{array}\right.
\end{gathered}
$$

in which:

$$
\Delta H_{k}=\frac{\delta^{k} \delta^{k}}{\delta^{k} \gamma^{k}}-\frac{\left(H_{k} \gamma^{k}\right)\left(H_{k} \gamma^{k}\right)}{\gamma^{k} H_{k} \gamma^{k}}+c H_{k} \gamma^{k} \nu^{k} \nu^{k}
$$

and

$$
\begin{aligned}
\delta^{k} & =x^{k}-x^{k+1} \\
\gamma^{k} & =\nabla f\left(x^{k+1}\right)-\nabla f\left(x^{k}\right) \\
\nu^{k} & =\frac{\delta^{k}}{\delta^{k} \gamma^{k}}-\frac{H_{k} \gamma^{k}}{\gamma^{k} H_{k} \gamma^{k}} \\
c & =\left\{\begin{array}{l}
0 \text { by Davidon-Fletcher-Powell } \\
1 \text { by Broyden-Fletcher-Goldfarb-Shanno }
\end{array}\right.
\end{aligned}
$$

There is also a theorem ensuring that if:

- $\frac{\left\|\gamma^{k}\right\|^{2}}{\gamma^{k} \delta^{k}} \leq \rho$

- $\rho \geq 0$

the sequence $\left\{x^{k}\right\}$ has at least one sub-sequence converging to a stationary point.

\subsubsection{Constrained optimization theory}

In this section, some results of constrained optimization theory are presented. They are useful to introduce the algorithms which are used in AEROSTATE.

The standard form of constrained problems is represented in eq. 3.20 and it is called "Nonlinear Programming problem".

$$
\left\{\begin{array}{l}
\min f(x) \\
g(x) \leq 0 \\
h(x)=0 \\
x \in \Omega \subset \mathbb{R}^{n}
\end{array}\right.
$$


The $f, g$ and $h$ functions are defined in eq. 3.1, 3.2 and 3.3; the set $\Omega$ is defined by the lower and upper boundaries. The equation system 3.20 is a general formulation, when:

- $\max f(x)=-\min [-f(x)]$

- $c(x) \geq 0 \Leftrightarrow g(x) \leq 0$, where $g(x)=-c(x)$

A point $x^{*} \in \Omega$ is a global solution of the problem 3.20 if

$$
f\left(x^{*}\right) \leq f(x), \forall x \in \Omega
$$

For the Weierstrass Theorem, a global solution exists if $\Omega$ is a compact set.

A point $x^{*} \in \Omega$ is a local solution of the problem 3.20, if exists an open neighborhood $B_{x^{*}}$ of $x^{*}$, such that $f\left(x^{*}\right) \leq f(x), \forall x \in \Omega \cap B_{x^{*}}$.

The constrained local solutions must satisfy necessary optimality conditions, which are defined by the Karush-Kuhn-Tucker Theorem (refer to 3.2.3.1).

\subsubsection{Karush-Kuhn-Tucker Theorem}

The definition of the generalized Lagrangian function is reported in eq. 3.22.

$$
L(x, \lambda, \mu)=f(x)+\sum_{i=1}^{p} \lambda_{i} g_{i}(x)+\sum_{j=1}^{m} \mu_{j} h_{j}(x)
$$

and assuming that:

- $x^{*}$ is a solution;

- A regularity condition of the feasible region holds at $x^{*}$;

- $\lambda_{i}^{*} \geq 0$ and $\mu^{*}$ exist, such that the equations system 3.23 is satisfied.

$$
\left\{\begin{array}{l}
\nabla_{x} L\left(x^{*}, \lambda^{*}, \mu^{*}\right)=0 \\
\lambda_{i}^{*} g_{i}\left(x^{*}\right)=0, i=1, \ldots, p \\
h_{j}\left(x^{*}\right)=0, j=1, \ldots, m
\end{array}\right.
$$

The equations system is a non linear system, in $n+p+m$ equations and unknowns. It is observed that the regularity condition is called constraint qualification. This condition asserts that the gradient of the active constraints at point $\tilde{x}, \nabla g_{i}(\tilde{x}), i \in\left\{1, \ldots, p: g_{i}(\tilde{x})=\right.$ $0\}$ and $\nabla h(\tilde{x})$, must be linearly independent.

A triplet $\left(x^{*}, \lambda^{*}, \mu^{*}\right)$ which satisfies the equations system 3.23 , is called a KKT point. 


\subsubsection{Second order sufficient optimality conditions}

The constraint solutions can satisfy also sufficient conditions for optimality conditions, which are described below.

- The $f, g$ and $h$ functions are twice continuously differentiable;

- The point $\left(x^{*}, \lambda^{*}, \mu^{*}\right)$ is a KKT point;

- The Hessian matrix of Lagrangian function $\nabla^{2} L\left(x^{*}, \lambda^{*}, \mu^{*}\right)$ is positive definite.

Then, the $x^{*}$ solution is a strict local solution of the problem 3.20.

\subsubsection{Constrained optimization algorithms}

The problem 3.20 is solved by two approaches. The first approach is to transform the constrained optimization problem into an equivalent unconstrained problem, which is described in section 3.2.1. The second approach consists in solving successive constrained quadratic subproblems.

The methods that transform the constrained problem into an unconstrained problem are called also "penalty methods". They are further divided in two subcategories:

- Asymptotic unconstrained penalty methods;

- Exact unconstrained penalty methods.

The asymptotic unconstrained penalty methods can be also divided in:

- External penalty methods;

- Interior penalty methods.

The latter division depends on the starting point position (respecively in or out of the region). In the sections 3.2.4.1 and 3.2.4.2, the algorithms, which are implemented in AEROSTATE, are presented. In particular, the local solver is the Sequential Quadratic Programming (SQP) Method, whereas the global solver is the LOCSMOOTH algorithm.

\subsubsection{Sequential Quadratic Programming (SQP) Method}

The SQP algorithm can be considered an extension of the Newton's method (valid for the unconstrained problems, refer to section 3.2.2), to the constrained problem. The basic idea is to move away from the current point by minimizing a quadratic model of the problem.

A constrained problem is now considered in eq. 3.24.

$$
\left\{\begin{array}{l}
\min _{x} f(x) \\
h_{j}(x)=0 j=1, \ldots, m
\end{array}\right.
$$


Then, the Lagrangian function is defined in eq. 3.25.

$$
L(x ; \mu)=f(x)+\mu^{T} h(x)
$$

A solution may be obtained by solving the equations system 3.23 , which is reported in 3.26 .

$$
\left\{\begin{array}{l}
\nabla_{x} L(x, \mu)=0 \\
h_{j}(x)=0, j=1, \ldots, m
\end{array}\right.
$$

The Newton iteration is given by:

$$
\left[\begin{array}{l}
x^{k+1} \\
\mu^{k+1}
\end{array}\right]=\left[\begin{array}{l}
x^{k} \\
\mu^{k}
\end{array}\right]+\left[\begin{array}{l}
d_{x}^{k} \\
d_{\mu}^{k}
\end{array}\right]
$$

Analogously to the Newton's method, refer to the eq. 3.14, the step is obtained by the following equation:

$$
\nabla_{x} L(x, \mu)+\nabla_{x}^{2} L\left(x^{k}, \mu^{k}\right) d_{x}=0
$$

from which:

$$
\begin{aligned}
& \nabla_{x}\left[f\left(x^{k}\right)+\mu^{k+1} h\left(x^{k}\right)\right]+\nabla_{x}^{2} L\left(x^{k}, \mu^{k}\right) d_{x}=0 \\
& \nabla_{x}^{2} L\left(x^{k}, \mu^{k}\right) d_{x}=-\left[\nabla_{x}\left(f\left(x^{k}\right)+\mu^{k+1} h\left(x^{k}\right)\right)\right] \\
& \nabla_{x}^{2} L\left(x^{k}, \mu^{k}\right) d_{x}=-\left[\nabla_{x} f\left(x^{k}\right)+\nabla_{x} h\left(x^{k}\right)\left[\mu^{k}+d_{\mu}\right]\right] \\
& \nabla_{x}^{2} L\left(x^{k}, \mu^{k}\right) d_{x}+\nabla_{x} h\left(x^{k}\right) d_{\mu}=-\nabla_{x} f\left(x^{k}\right)-\nabla_{x} h\left(x^{k}\right) \mu^{k}
\end{aligned}
$$

Therefore, the KKT system 3.26 becomes:

$$
\left\{\begin{array}{l}
\nabla_{x}^{2} L\left(x^{k}, \mu^{k}\right) d_{x}+\nabla_{x} h\left(x^{k}\right) d_{\mu}=-\nabla_{x} f\left(x^{k}\right)-\nabla_{x} h\left(x^{k}\right) \mu^{k} \\
h\left(x^{k}\right)+\nabla_{x} h\left(x^{k}\right) d_{x}=0
\end{array}\right.
$$

From the equations system 3.30 the step is obtained by the follow equations:

$$
\left[\begin{array}{cc}
\nabla_{x}^{2} L\left(x^{k}, \mu^{k}\right) & \nabla_{x} h\left(x^{k}\right) \\
\nabla_{x} h\left(x^{k}\right)^{T} & 0
\end{array}\right]\left[\begin{array}{l}
d_{x} \\
d_{\mu}
\end{array}\right]=-\left[\begin{array}{c}
\nabla_{x} f\left(x^{k}\right)+\nabla_{x} h\left(x^{k}\right) \mu^{k} \\
h\left(x^{k}\right)
\end{array}\right]
$$

By adding to both sides of the first equation of the system 3.31, the Newton's iteration becomes:

$$
\begin{gathered}
x^{k+1}=x^{k}+d^{k} \\
{\left[\begin{array}{cc}
\nabla_{x}^{2} L\left(x^{k}, \mu^{k}\right) & \nabla_{x} h\left(x^{k}\right) \\
\nabla_{x} h\left(x^{k}\right)^{T} & 0
\end{array}\right]\left[\begin{array}{c}
d_{x} \\
\mu^{k+1}
\end{array}\right]=-\left[\begin{array}{c}
\nabla_{x} f\left(x^{k}\right) \\
h\left(x^{k}\right)
\end{array}\right]}
\end{gathered}
$$

The iteration defined by eq. 3.32 and eq. 3.33 are the Newton-Lagrange method for solving the problem 3.24. 
The quadratic programming problem is now considered.

$$
\left\{\begin{array}{l}
\min _{s \in \mathbb{R}^{n}}\left[\frac{1}{2} s^{T} \nabla_{x}^{2} L\left(x^{k}, \mu^{k}\right) s+\nabla_{x} f\left(x^{k}\right)^{T} s\right] \\
\nabla_{x} h\left(x^{k}\right) s+h\left(x^{k}\right)=0
\end{array}\right.
$$

The first order necessary optimality conditions are:

$$
\left[\begin{array}{cc}
\nabla_{x}^{2} L\left(x^{k}, \mu^{k}\right) & \nabla_{x} h\left(x^{k}\right) \\
\nabla_{x} h\left(x^{k}\right)^{T} & 0
\end{array}\right]\left[\begin{array}{l}
s \\
\eta
\end{array}\right]=-\left[\begin{array}{c}
\nabla_{x} f\left(x^{k}\right) \\
h\left(x^{k}\right)
\end{array}\right]
$$

where $\eta$ is a Lagrange multiplier for the problem 3.34 .

Comparing the equations 3.33 and 3.35 , it is noted that $\left(d_{x}, \mu^{k+1}\right)$ and $(s, \eta)$ satisfy the same system. Therefore, in order to solve the problem 3.24, an iteration based on the solution of the quadratic problem 3.34 may be used.

The simplest form of an algorithm based on the SQP method is:

1. Choose an initial pair $\left(x^{0}, \mu^{0}\right)$;

2. If $\left(x^{k}, \mu^{k}\right)$ is a KKT point of the problem 3.24, stop;

3. Find $\left(s^{k}, \eta^{k}\right)$ as KKT point of the problem 3.34, by using the eq. 3.35;

4. Set $x^{k+1}=x^{k}+s^{k}, \mu^{k+1}=\eta^{k}, k=k+1$ and go to step 2 .

The SQP method is preferable to the Newton-Lagrange method because it satisfies both the first and the second order necessary optimality conditions. In the problem 3.34, the objective function is a quadratic approximation of the Lagrangian function and the constraint is a linear approximation of the equality constraint.

In general, problems are in the form:

$$
\left\{\begin{array}{l}
\min _{x} f(x) \\
g_{i}(x) \leq 0 \quad i=1, \ldots, p \\
h_{j}(x)=0 \quad j=1, \ldots, m
\end{array}\right.
$$

Linearizing the constraints and developing to the second order the Lagrangian function, it is obtained:

$$
\left\{\begin{array}{l}
\min _{s \in \mathbb{R}^{n}}\left[\frac{1}{2} s^{T} \nabla_{x}^{2} L\left(x^{k}, \lambda^{k}, \mu^{k}\right) s+\nabla_{x} f\left(x^{k}\right)^{T} s\right] \\
\nabla_{x} g\left(x^{k}\right)^{T} s+g\left(x^{k}\right) \leq 0 \\
\nabla_{x} h\left(x^{k}\right)^{T} s+h\left(x^{k}\right)=0
\end{array}\right.
$$

The SQP algorithm for the problem 3.36, is:

1. Choose an initial triplet $\left(x^{0}, \lambda^{0}, \mu^{0}\right)$; 
2. If $\left(x^{k}, \lambda^{k}, \mu^{k}\right)$ is a KKT point, stop;

3. Find $\left(s^{k}, \zeta^{k}, \eta^{k}\right)$ as KKT point of the problem 3.37;

4. Set $x^{k+1}=x^{k}+s^{k}, \lambda^{k+1}=\zeta^{k}, \mu^{k+1}=\eta^{k}, k=k+1$ and go to step 2 .

The sequence $\left(x^{k}, \lambda^{k}, \mu^{k}\right)$, which is generated by the SQP algorithm, converges with a quadratic rate to the solution $\left(x^{*}, \lambda^{*}, \mu^{*}\right)$ if:

- The functions $f, g$ and $h$ are twice differentiable with Lipschitz continuous second derivatives;

- $\left(x^{*}, \lambda^{*}, \mu^{*}\right)$ satisfies the second order sufficient conditions.

As in the Newton's methods, the SQP method requires to compute the second order derivatives; as in Newton's methods, this can be avoided by using an iterative QuasiNewton approximation, refer to sec. 3.2.2.1, $B^{k}$.

An update strategy for $B^{k}$ could be obtained as follows:

$$
\begin{aligned}
\delta^{k} & =x^{k+1}-x^{k} \\
\gamma^{k} & =\nabla L\left(x^{k+1}, \lambda^{k}, \mu^{k}\right)-\nabla L\left(x^{k}, \lambda^{k}, \mu^{k}\right)
\end{aligned}
$$

and updating the eq. 3.18 for unconstrained optimization, choosing the BroydenFletcher-Goldfarb-Shanno formulation:

$$
B^{k+1}=B^{k}-\frac{B^{k} \delta^{k}\left(\delta^{k}\right)^{T} B^{k}}{\left(\delta^{k}\right)^{T} B^{k} \delta^{k}}+\frac{\gamma^{k}\left(\gamma^{k}\right)^{T}}{\left(\gamma^{k}\right)^{T} \delta^{k}}
$$

It is observed that the positive definiteness of $B^{k}$ is no longer assured, but it may be overcome by modifying the term $\gamma^{k}$, as proposed by Powell:

$$
\bar{\gamma}^{k}=\tau \gamma^{k}+(1-\tau) B^{k} \delta^{k}
$$

where $\tau$ is defined as:

$$
\begin{cases}1 & \text { if }\left(\delta^{k}\right)^{T} \gamma^{k} \geq 0.2\left(\delta^{k}\right)^{T} B^{k} \delta^{k} \\ 0.8 \frac{\left(\delta^{k}\right)^{T} B^{k} \delta^{k}}{\left(\delta^{k}\right)^{T} B^{k} \delta^{k}-\left(\delta^{k}\right)^{T} \gamma^{k}} & \text { if }\left(\delta^{k}\right)^{T} \gamma^{k}<0.2\left(\delta^{k}\right)^{T} B^{k} \delta^{k}\end{cases}
$$

The disadvantage is the convergence rate that becomes superlinear, by a Quasi-Newton approximation.

The step-size for the SQP algorithm are two:

- Line search approach;

- Trust region approach. 
In the "line search approach", the sequence is given by:

$$
x^{k+1}=x^{k}+t_{k} s^{k}
$$

where the step-size $t_{k}$ is evaluated as to get a sufficient decrease of the merit function, which allows to reduce both the objective function and the constraint violations. A widely used merit function is the "I1 penalty function"; however, due to its non differentiability, it suffers of the "Maratos effect" that is a deteriorating effect on the converge rate near the solution.

This effect can be overcome by using the "Fletcher's merit function" or the merit function of the "Exact penalty methods".

This approach is implemented in the MATLAB ${ }^{\circledR}$ function fmincon, which is implemented in the local solver of the software AEROSTATE.

\subsubsection{LOCSMOOTH algorithm}

LOCSMOOTH is an algorithm for global optimization, which deals with the search of global optima and it is due to Addis, Locatelli and Schoen. It is supposed to search the global minimum $x^{*}$ of the function represented in continuous line in Fig. 3.1. The algorithm works properly when the starting function has a funnel structure, i.e. a superposition of an underlying structure (the dashed curve represented in the follows figure) and some perturbation around it.

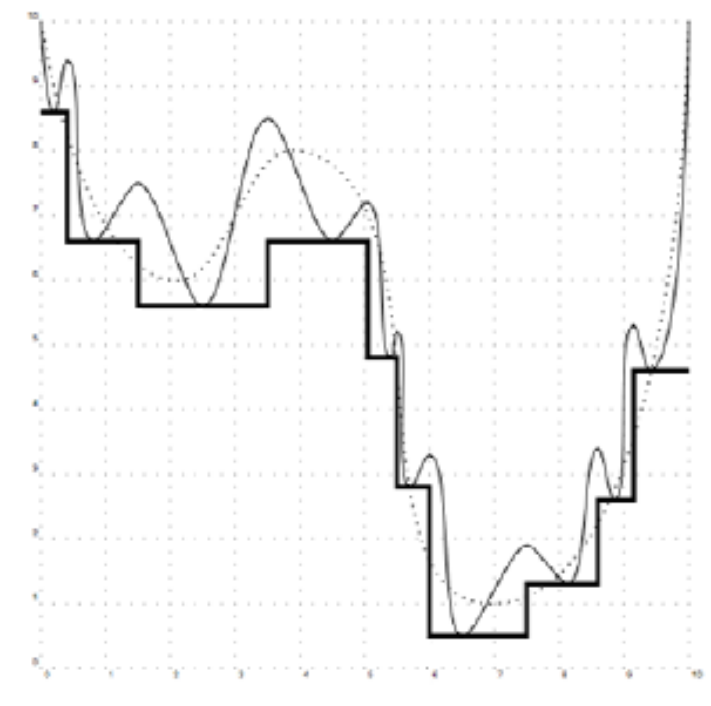

Figure 3.1. An example of a function with a funnel structure and its step function.

The local optimum $x_{1}$ depends on the starting point $x^{*}$; thus, the local optimum function $\mathrm{L}\left(\mathrm{x}^{*}\right)$ can be defined and reported in Fig. 3.1 with the tick line: this is a step function and a rapid convergence algorithms cannot be applied. For this reason, a Gaussian filtering smooths the thickest curve and the minimum point of the smoothed 
function is found. From this point the local minimum of the objective function is searched with a generic local algorithm. This logic is implemented in the LOCSMOOTH algorithm that is organized in two phases:

- Approximation phase;

- Displacement phase.

In the approximation phase, the local minimum $L\left(x_{h}\right)$, starting from the point $x_{h}$, is found. In particular, a uniform random sample of $\mathrm{K}$ points, inside a sphere of radius $R$ and centered at $x_{h}, S\left(x_{h}, R\right)$, is generated and the local minima $L\left(x_{k}\right), x_{k} \in S\left(x_{k}, R\right)$ are calculated. These values are used to generate an approximated filtering function $\hat{L}_{g}^{B}(x)$.

In the displacement phase, the minimum of $\hat{L}_{g}^{B}(x)$ is taken as the next starting point and the procedure is iterated. The procedure is stopped when no improvements are observed. Then, the procedure is now described in details, in the follows paragraph.

First of all, a reference configuration $x_{0}$ is considered; moreover, other parameters are introduced, which are: $R, K$, and MaxNoImp. Then an uniform random sample point $x$ is chosen in a sphere $S\left(x_{0}, R\right)$ and a local minimum point $x^{*}$ is computed starting from the sample point $x$; furthermore, it is set current $=$ record $=f\left(x^{*}\right)$.

Secondly the steps procedure is described below.

STEP 1 If NoImp $<$ MaxNoImp, $i$ is set to 0; else, the optimization process is ended.

STEP 2 If $i<K$ and record $\leq$ current, $i=i+1$ and an uniformly random point $y_{i}$ is sampled inside the sphere $S\left(x^{*}, R\right)$; moreover, a local minimum point of $y_{i}^{*}$ is computed starting from $y_{i}$. It is set current $=f\left(y^{*}\right)$.

STEP 3 If current < record then a new record has been found and record $=$ current is posed; moreover, the center of the sphere is changed $y^{*}$. Else, NoImp $=N o \operatorname{Im} p+K$ is set and the smoothed function is built using the stored local minima $y_{i}^{*}$. Then, $\hat{x}$, the minimum of the smoothed function, is found. Furthermore, the local minimum $y^{*}$ of the objective function is obtained, starting from $\hat{x}$ and it is set current $=f\left(y^{*}\right)$.

STEP 4 If current < record, a new record has been found. Therefore, record $=$ current is set and the sphere is moved in $y_{i}^{*}$ position $\left(x^{*}=y_{i}^{*}\right): S\left(y_{i}^{*}, R\right)$. The parameter NoImp is set equal to zero. Instead, $x^{*}=\hat{x}$, go to STEP 1 .

The global optimization ends when NoImp = MaxNoImp. The smoothed function $\hat{L}_{g}^{B}(x)$ is given by the model shown in eq. 3.43 .

$$
\hat{L}_{g}^{B}(x)=\frac{\sum_{i=1}^{K} L\left(y_{i}\right) g\left(\left\|y_{i}-x\right\|\right)}{\sum_{i=1}^{K} g\left(\left\|y_{i}-x\right\|\right)}
$$

where: 


$$
g(z)=e^{-\frac{z^{2}}{(2 \sigma)^{2}}}
$$

is the Gaussian kernel and the standard deviation $\sigma$ is:

$$
\sigma=r K^{-\frac{1}{n}}
$$

If this standard deviation definition is used, all the volume of the sphere (which is proportional to $r^{n}$ ) is covered by the Gaussian weight. Indeed, in order to obtain equal coverage for different radius values, the number of samples must be $K=\frac{r^{n}}{\sigma^{n}}$. This choice of standard deviation may be less effective when the variable have different range of variation. In order to avoid this, a different standard deviation is used, refer to eq. 3.46.

$$
\sigma=\left(\frac{\prod_{i=1}^{n} r_{i}}{K}\right)^{\frac{1}{n}}
$$

where $r_{i}$ are the radii along the different variable axes. The parameter $n$, which represents the number of points $n$ to be sampled, influences the quality of the approximated function; however it is time consuming, because of a local optimization must be performed for each sample.

The parameter $r_{i}$ influences the exploration algorithm aptitude of the algorithm: a too small radius limits the search of the solution, whereas a huge radius could give too dispersed data and a deterioration of the approximated function.

\subsubsection{AEROSTATE - software structure}

The software AEROSTATE has been developed in MATLAB ${ }^{\circledR}$ environment and it is made up of MATLAB ${ }^{\circledR}$ 's functions. The software AEROSTATE is used in the preliminary design of aircraft and it allows to define a wing plan form in such a way that a proper functional is minimized under the presence of several constraints.

The most important variables which are handled during the optimization procedure are geo, res and $X_{\text {memo }}$, and now they are described.

All geometric information about the configuration are gathered into the geo variable, that is a MATLAB ${ }^{\circledR}$ structured variable (refer to Fig. 3.2). The three lifting surfaces are named "ala_ant" (front wing), "ala_post" (rear wing) and "aletta" (auxiliary wing). Information about them are gathered into other structured variables which are called in the same way of the wing. The variable geo.ala_ant is reported in Fig. 3.3 to show which geometric features are managed. As it is shown, it is possible to set parameters in each control section of the wing (variables expressed by means an array of three components as chords and the twist angle theta) or in each bay (variables expressed by means an array of two components as the sweep angle $S W$ or the span). The coordinates $x, y$ and $z$ refer to the leading edge of each section. It is important to notice that every geometric quantity can be set as variable or constant by means the structured variable var. 


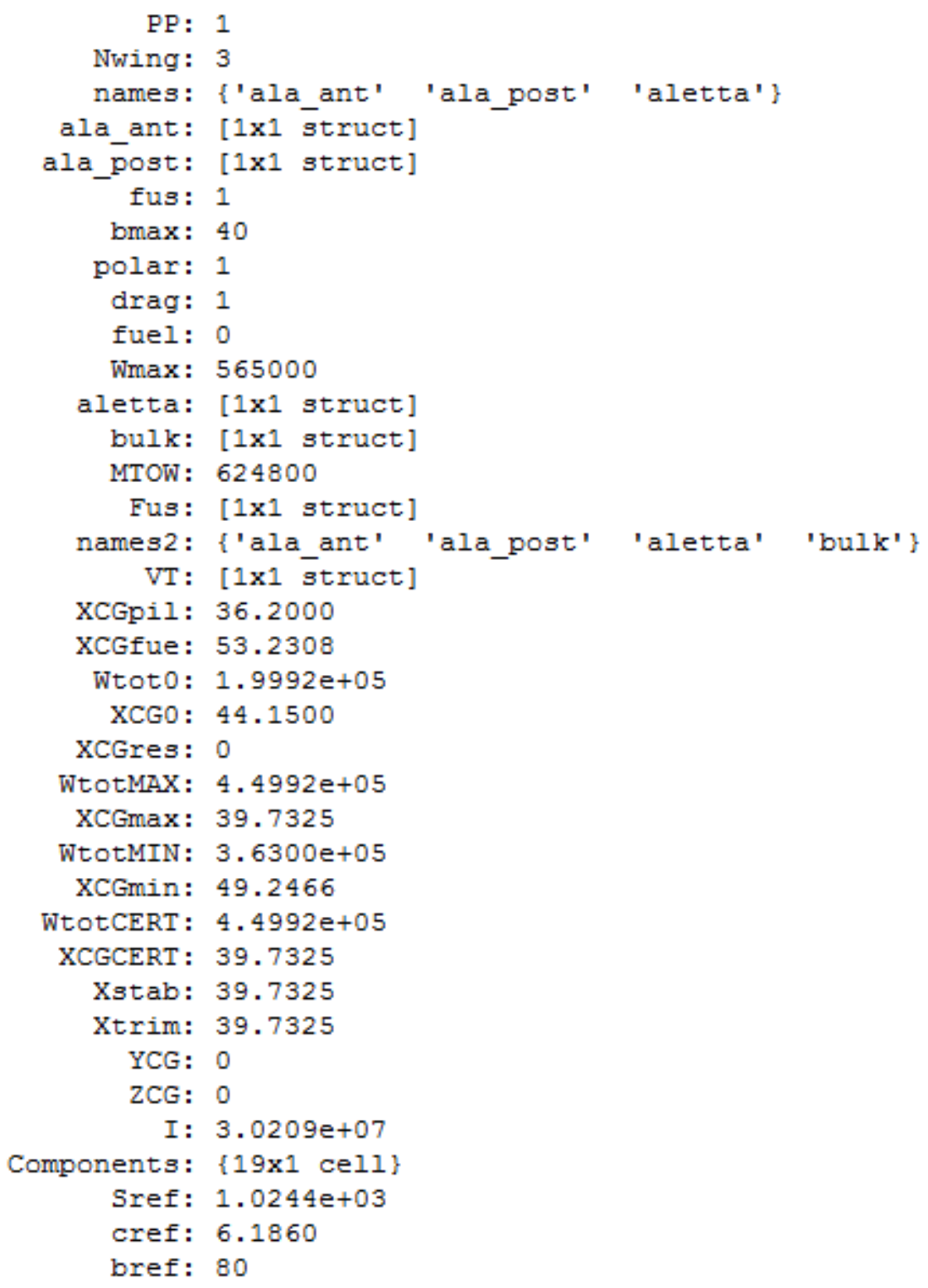

Figure 3.2. geo variable. 


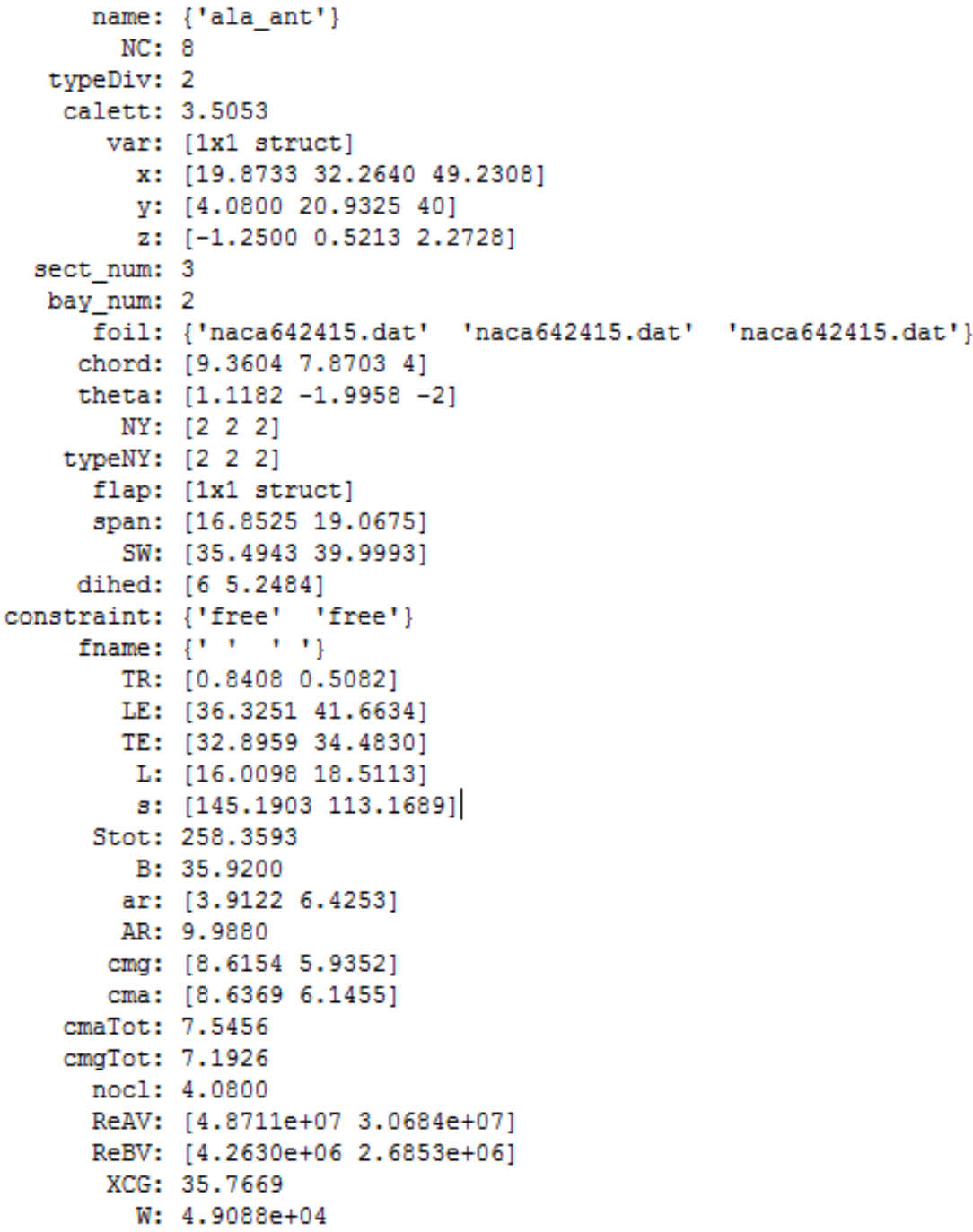

Figure 3.3. Example of lifting surface variable into geo.

The variable var is organized as the variable of Fig. 3.3 but the components of each vector are set " 1 " or " 0 ". If " 1 ", it means the specified quantity is a variable, so the user has to fix a suitable interval by means the lower and upper boundary. If "0", the specified quantity is constant, so it assumes always the same value of the starting geometry and it does not change during the optimization. It is possible to set the sub structured variable var for each lifting surface.

In the variable geo, there is also a sub structured variable "VT", that indicates the vertical tail, i.e. the two fins in the examined configurations.

During the optimizations progress, the variable geo is recalled many times: it has been decided to transform the variable geo, that is a complicated structured variable, in an array $\left(X_{\text {memo }}\right)$ in order to handle it immediately. Then, when the optimizer finds out a local minimum, it does not save the geometry directly in geo, but it stores all the information in the array $X_{m e m o}$. 
The res variable is a structured variable that contains all aerodynamic information, related to a high speed or cruise situation (refer to Fig. 3.4). As shown, res stores the lifting coefficient (CLtot), the induced drag and the friction drag coefficients (CDitot and $C D 0 t o t)$ and the momentum coefficient (CMtot) for the whole configuration; also the main aerodynamic derivatives are reported ( $C L a$ and $C M a$ which stand for $C_{L \alpha}$ and $\left.C_{M \alpha}\right)$ together with the position of the neutral point $(X P N)$ and the Margin of Stability $(M S)$. Every lifting surface and the lateral bulks have a dedicated sub variable into the

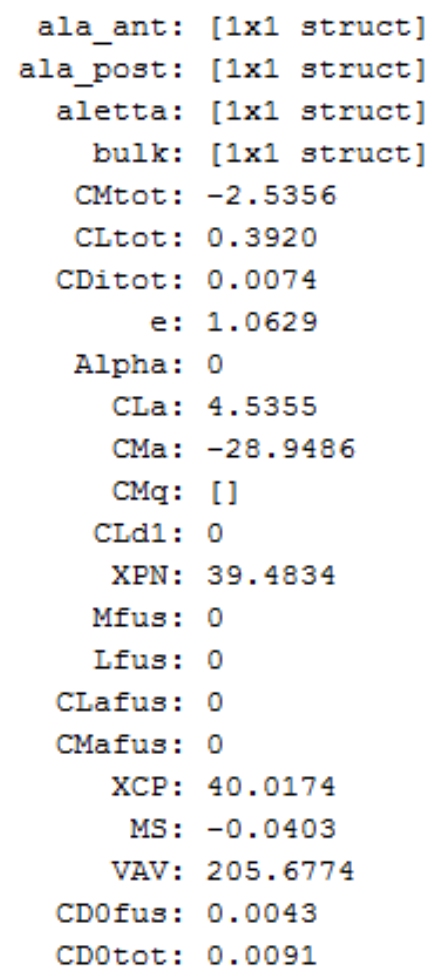

Figure 3.4. res variable.

res variable. For example, if the front wing is investigated, the following parameters appear (refer to Fig. 3.5). The most interesting result is the distribution of the lifting coefficient along the wing span: the array $c l$ is provided and the generic $c l(i)$ is located at $Y(i)$.

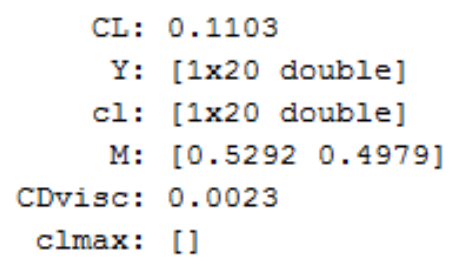

Figure 3.5. res variable - detail. 
Now, we can proceed to illustrate the AEROSTATE structure. Fig. 3.6 refers to a main diagramm and Fig. 3.7 shows the meaning of every block.

The functions are described below.

mainNEW.m It is the main script from which the program AEROSTATE is launched. The user is able to interact with an interface to define equality linear and inequality nonlinear constraints (both geometric and aerodynamic constraints). This script begins and launches the preproc.m function, in which the preprocessor activities analyze the initial configuration (set by the user) from geometric and aerodynamic point of view. At the end of the preprocessing phase, the software has generated a geometry that satisfies all the geometric boundaries and aerodynamic data are available. After the preprocessing phase, the optimization phase starts. First of all, equality linear and inequality nonlinear constraints are defined through loading of some suitable files. Afterwards, the software saves geo, res and $X_{\text {memo }}$ variables in directory RISULTATI; these variables represent geometry and aerodynamic data of the preliminary configuration. The script goes on and launches the obj.m function, in which the objective function is defined. Subsequently, confun.m function is recalled to build the constraint vector $c$ and the disegnaALA.m function is executed to plot an airplane sketch. The next step is to execute the gradient optimization. First of all, the software sets the properties for the algorithm used by the local solver, calling the optimoptions.m function, with $S Q P$ algorithm and the fmincon.m function; moreover, the tolerance for constraint violation is set to 0,01 and outfunNEW.m function is used to represent the behaviour of the optimization process. Secondly, optimize.m function is launched, specifying the main properties for global algorithm:

$$
\begin{aligned}
& R=\frac{u b-l b}{2} \text { (Radius of the sphere) } \\
& K=5 \text { (number of sample in the sphere of radius } \mathrm{R} \text { ) } \\
& \operatorname{MaxNoImp}=2 K
\end{aligned}
$$

where MaxNoImp is the number of times that are accepted without finding a smaller minimum. This script ends with a post-processing phase which is gotten by calling the post_pro.m function and POST.m script: in this phase all geometric and aerodynamic features of the configurations obtained during the optimization process are saved in .mat and .avl files.

preproc.m This script begins with an user interface whereby some main features (analysis name, starting geometry input file name) are defined; moreover, the script adds on all the bulk geometry features. Preliminarily, it introduces some main aerodynamic parameters (eq. 3.48) and a fuselage description (eq. 3.49): 


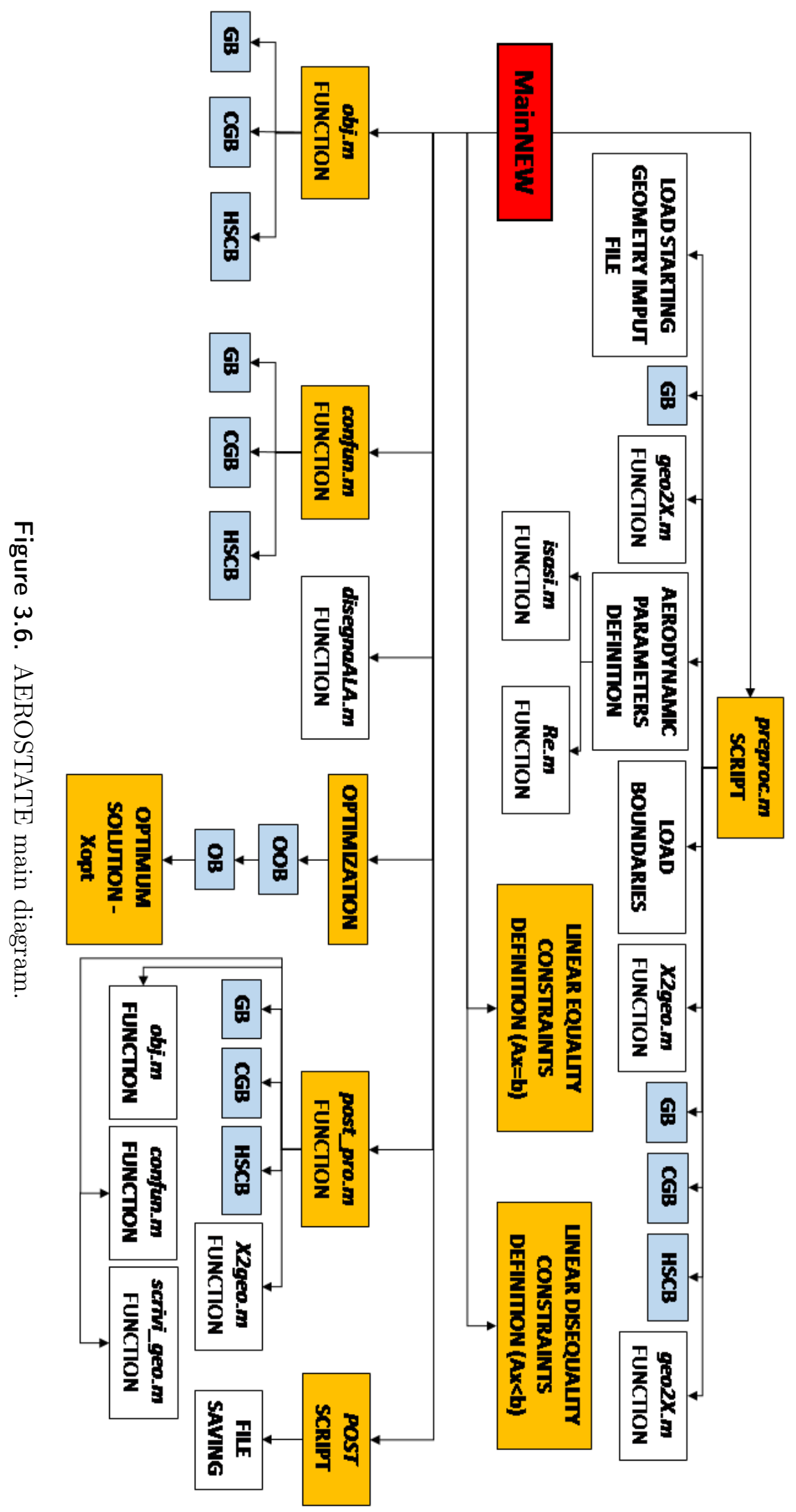



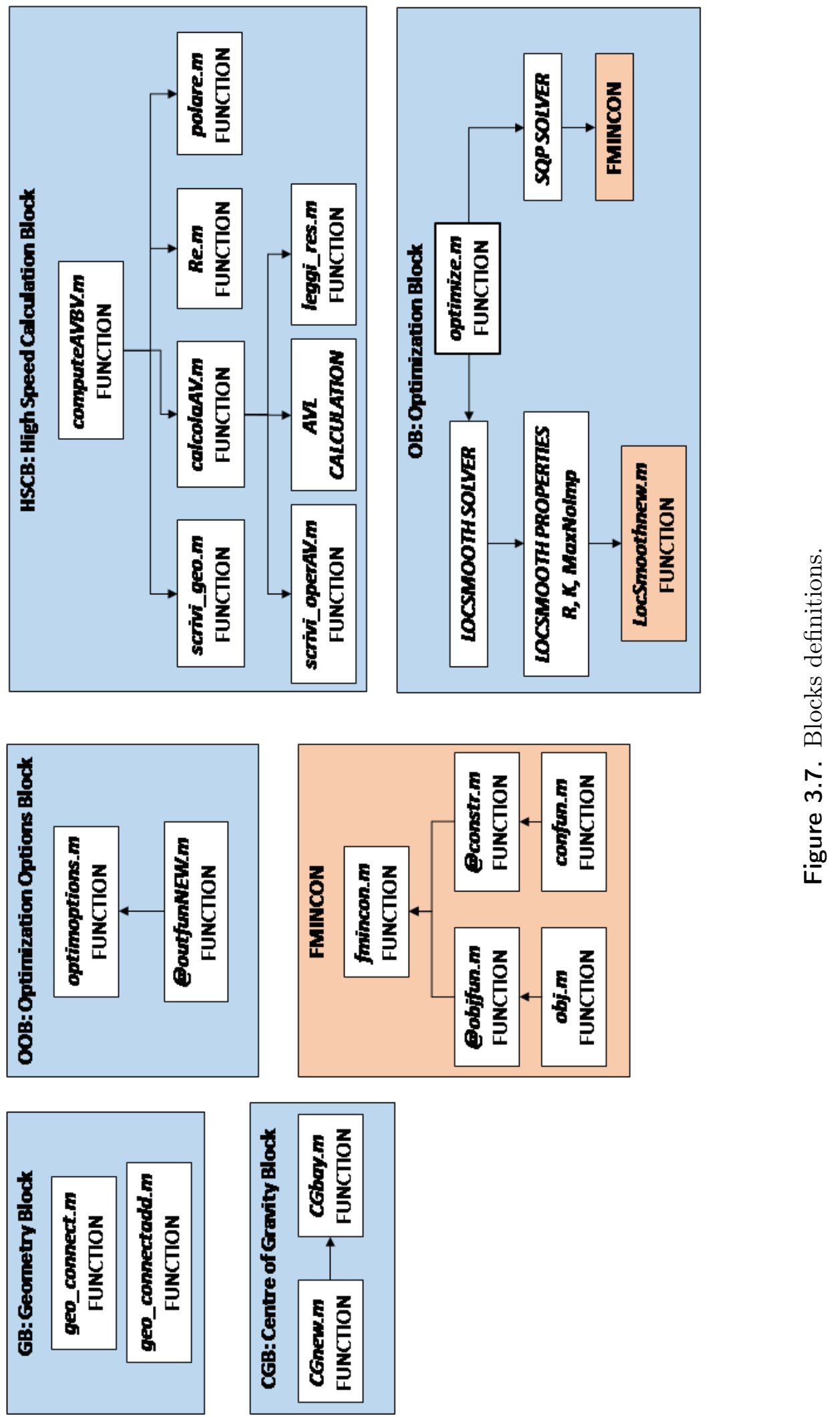


$$
\begin{aligned}
& W=565000 \mathrm{Kg} \text { (design weight) } \\
& M T O W=624800 \mathrm{Kg} \text { (maximum take off weight) } \\
& h_{A V}=6000 \mathrm{~m} \text { (high speed height) } \\
& \text { Mach }=0.65 \\
& \quad L_{\text {fus }}=74 \mathrm{~m} \\
& D_{\text {fus }}=4.08 * 2 \mathrm{~m} \\
& \quad S_{\text {wet }}=3.14 D_{\text {fus }} L_{\text {fus }} 0.9 \\
& Q=1.4 \text { (interference factor, by Raymer) }
\end{aligned}
$$

The next step is to calculate the Reynolds' number for each bay of the aircraft wing, by launching Re.m function. Moreover, the starting geometry input file is loaded and the boundaries are visualized on MATLAB ${ }^{\circledR}$ Command Window. Before proceeding with the aerodynamic calculation, the initial geometry is corrected by using the geometric boundaries and the center of gravity is calculated with the function $C G$ new.m. Then, the aerodynamic computation is obtained by launching the computeAVBV.m function. At the end the geometry is set and it satisfies all the geometric boundaries and all the aerodynamic data are available.

Re.m This function computes the Reynolds' number for each bay of the aircraft wing, using the formula:

$$
R e=V_{H S} \cos ^{2}(\Lambda) \frac{m a c}{\nu}
$$

where $V_{H S}$ is the design speed, $\Lambda$ is the sweep angle at the $25 \%$ of the chord, mac is the main aerodynamic chord and $\nu$ is the kinematic viscosity.

geo2X.m It is a function that converts the structured variable $g e o$, in a vector $X$ that contains all the features of the configuration.

X2geo It is a function that converts the vector $X$, in a structured variable geo that contains all the features of the configuration.

geo_connect.m This function computes all the principal parameters of the wings.

geo connectadd.m It is a function that adds the bulk geometric characteristics to the variable geo of the configuration.

CGnew.m This function calculates the position of center of gravity, considering a square meter model to compute the weight of all the lifting surfaces. First of all, some typical vertical tail geometric parameters are introduced; moreover the surface densities are defined: 


$$
\begin{aligned}
& \rho_{\text {ala ant }_{\text {ant }}}=\rho_{\text {ala }} \text { post } \\
& \rho_{\text {bulk }}=90 \mathrm{~kg} / \mathrm{m}^{2}
\end{aligned}
$$

Secondly the fuselage peculiarities are introduced:

$$
\begin{aligned}
& M_{\text {fus }}=58000 \mathrm{~kg} \\
& C G_{\text {fus }}=40 \mathrm{~m} \\
& M_{\text {payload }}=250000 \mathrm{~kg} \\
& C G_{\text {pay }}=36,2 \mathrm{~m} \\
& M_{\text {lgpost }}=30000 \mathrm{~kg} \\
& C G_{\text {lgpost }}=50 \mathrm{~m}
\end{aligned}
$$

The nose wheel mass and engines masses are not considered in this calculation. In this script, the wings centers of gravity are calculated by using the CGbay.m function.

CGbay.m This function computes the wings center of gravity positions, assuming constant the mass density.

computeAVBV.m First of all, this function has got the peculiarities of setting all the aerodynamic parameters which are necessary to launch the aerodynamic computation with AVL software (in particular, the maximum and the minimum incidence of the fuselage, refer section 3.3). Secondly, the AVL file input is built by using scrivi_geo.m function; then, the function calcolaAV.m is launched, so the aerodynamic calculation is made and all results are read. The next step is represented by the computation of the moment coefficient:

$$
\begin{aligned}
& M_{\text {tot }}=M_{\text {wing }}+M_{f u s} \\
& M_{\text {fus }}=0 \\
& C_{\text {ref }}=\frac{c_{1} S_{1}+c_{2} S_{2}}{S_{\text {reftot }}} \\
& C_{\text {mtot }}=\frac{M_{\text {tot }}}{q S_{\text {ref }} c_{\text {ref }}}
\end{aligned}
$$

The longitudinal position of the center of pressure is calculated by a moment equilibrium of the entire generalized forces agent on the aircraft. The Margin of stability is computed by using the rearmost position of the center of gravity.

$$
M S=\frac{X P N-X C G r e f}{c_{r e f}}
$$


At least, the drag aerodynamic coefficient $C D 0$ is calculated by estimating the Reynolds number both for wings and bulk then the polare.m function is launched. It is noted that the Mach number does not influence the calculation (it is set to Mach $=0.3$ ). The drag coefficients are obtained from the formulas:

$$
\begin{aligned}
& C_{D 0 \text { wing }}=\frac{\left(C d_{\text {airfoil } 1} \text { chord }_{1}+C d_{\text {airfoil } 2} \text { chord }_{2}\right) \text { span }}{S_{\text {reftot }}} \\
& C_{\text {D0bulk }}=\frac{\left(C d_{\text {airfoil } 1} \text { chord }_{1}+C d_{\text {airfoil } 2} \text { chord }_{2}\right) \text { span }}{S_{\text {reftot }}}
\end{aligned}
$$

As far as the fuselage drag coefficient is obtained with the following assumption:

$$
\begin{aligned}
& \text { Reynolds }_{\text {fus }}=\frac{V L_{f u s}}{\nu} \\
& C_{f}=\frac{0.455}{\log \left(\text { Reynold }_{f u s}\right)^{2.58}} \text { (shape coefficient) } \\
& f=\frac{L_{\text {fus }}}{D_{\text {fus }}} \\
& F F=1+\frac{60}{f^{3}}+\frac{f}{400} \\
& S_{f u s}=\pi D_{f u s} L_{f u s} 0.9 \text { (estimated fuselage surface) } \\
& Q=1.4(\text { interference factor) } \\
& C_{D 0 f u s}=Q C_{f} F F \frac{S_{f u s}}{S_{\text {ref }}} \\
& C_{D 0 t o t}=\sum_{i=1}^{K} C_{D 0_{i}}
\end{aligned}
$$

scrivi_geo.m This function builds the input file for AVL v3.3.5 software.

calcolaAV.m This function builds the file with the operation that AVL must perform. Moreover, this script launches AVL software with the purpose to execute the aerodynamic calculation.

leggi res.m This function reads the results given by AVL.

polare.m This function uses the polar curve of a profile to calculate the coefficient of viscous drag, in correspondence of a required $C_{L}$. It is obtained by using a cubic interpolation of the profile data and without considering the compressibility effect.

obj.m It is a script in which the objective function is defined. 
confun.m It is a function where the constraint vector is built starting from geometric and aerodynamic parameters of the current configuration. Moreover, this configuration is loaded by the temporary data, previously saved in the folder AEROSTATE $\mid$ RISULTATI.

disegnaALA.m This function executes a sketch of the considered aircraft.

outfunNEW.m It is a function that produces some graphical representations in which the behaviour of the optimization process is shown. The trends of the objective function $F_{v a l}$ and max constraint value are represented. In Fig. 3.8 and in Fig. 3.9, an example of these output graphics is shown.

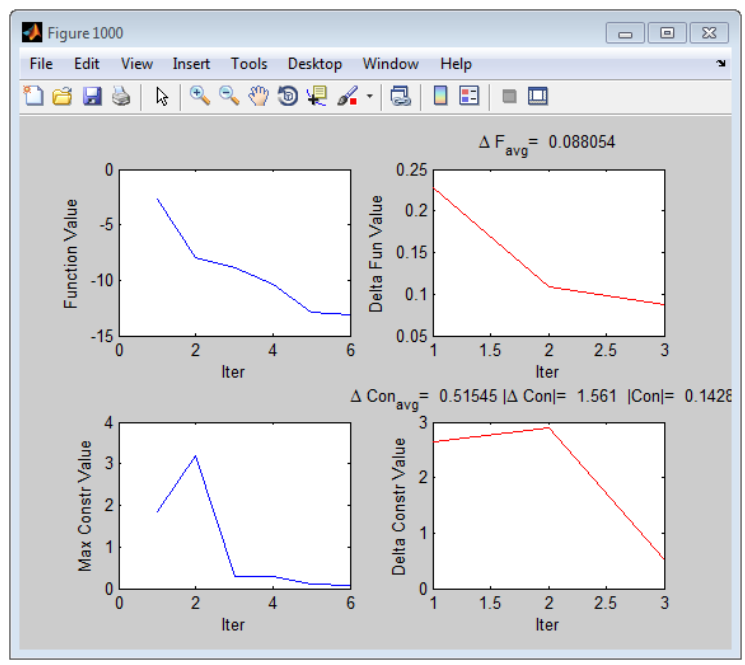

Figure 3.8. outfunNEW.m output graphics.

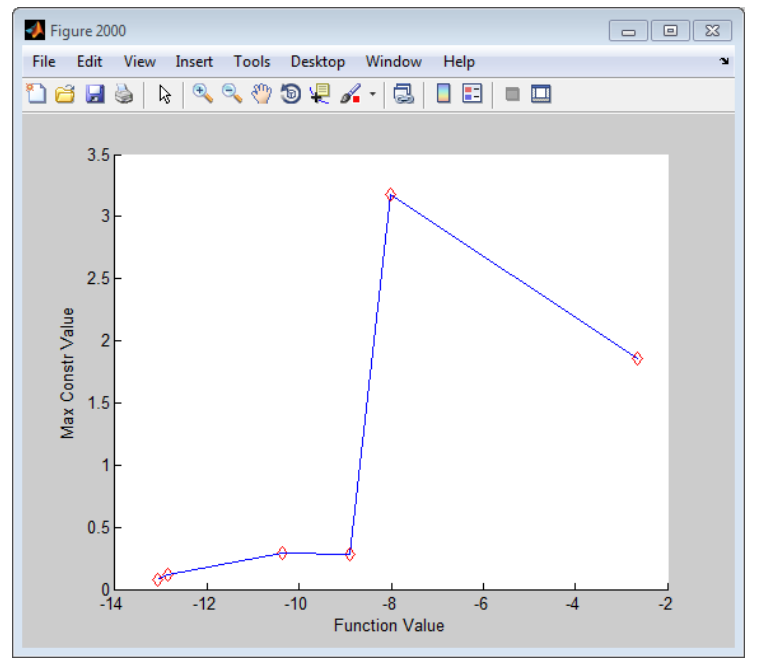

Figure 3.9. outfunNEW.m output graphic.

optimize.m This function has got the aim to set all the required parameter to run the solver of type LOCSMOOTH. The main parameters are: 


$$
\begin{aligned}
& R=\frac{u b-l b}{2} \text { (Radius of the sphere) } \\
& K=5 \text { (number of sample in the sphere of radius } \mathrm{R}) \\
& \operatorname{Max} N o \operatorname{Imp}=2 \mathrm{~K}
\end{aligned}
$$

Then, the LocSmoothenew. $m$ function is launched.

LocSmoothnew.m This function has got the aim to manage the global optimization algorithm that is the LOCSMOOTH Algorithm type. This Algorithm is realized in this function by an Initialization phase and next steps:

Initialization phase: First of all, a reference configuration $x_{0}$ is considered; furthermore, other parameters are introduced, which are: $R, K$, and MaxNoImp. Then an uniform random sample point $x$ is chosen in a sphere $S\left(x_{0}, R\right)$ and calling the optimizeSphere.m function, a local point of minimum $x^{*}$ is obtained; hence current $=$ record $=f\left(x^{*}\right)$ is set. Secondly the steps procedure is described below.

STEP 1 NoImp $<$ MaxNoImp, $i$ is set to 0; else, the optimization process is ended.

STEP 2 If $i<K$ and record $\leq$ current, $i=i+1$ and an uniformly random point $y_{i}$ is sampled inside the sphere $S\left(x^{*}, R\right)$ by calling the spheresamp.m function; moreover, a local point of minimum $y_{i}^{*}$ is computed by calling the optimizeSphere.m function. It is posed current $=f\left(y^{*}\right)$.

STEP 3 If current < record then a new record has been found and record=current is posed; moreover, the center of the sphere is changed $S\left(y^{*}, R\right)$. Else, NoImp $=$ $N o I m p+K$ is set and the smoothed function is built using the stored local minima $y_{i}^{*}$. Then, $\hat{x}$, the minimum of the smoothed function, is found by calling the optimizeSphereG.m function. Furthermore, the local minimum $y^{*}$ is obtained starting from $\hat{x}$, launching optimizeSphere. $m$ function and setting current $=f\left(y^{*}\right)$.

STEP 4 If current $<$ record, a new record has been found. Therefore, record=current is set and the sphere is moved in $y_{i}^{*}$ position $\left(x^{*}=y_{i}^{*}\right): S\left(y_{i}^{*}, R\right)$. The parameter NoImp is set equal to zero. Instead, $x^{*}=\hat{x}$, go to STEP1.

This global optimization process is shown in Fig. 3.10.

sphersamp.m This function generates a random configuration $x$ in a sphere $S\left(x_{0}, R\right)$, respecting the geometric boundaries, where $x_{0}$ is the starting point and $R$ is the radius.

optimizeSphere.m It is a script in which the local minimum of a specific function is obtained from a starting point $x_{0}$, minimizing a quadratic model of the problem. In particular, the local algorithm is SQP type and the fmincon.m function is called. 


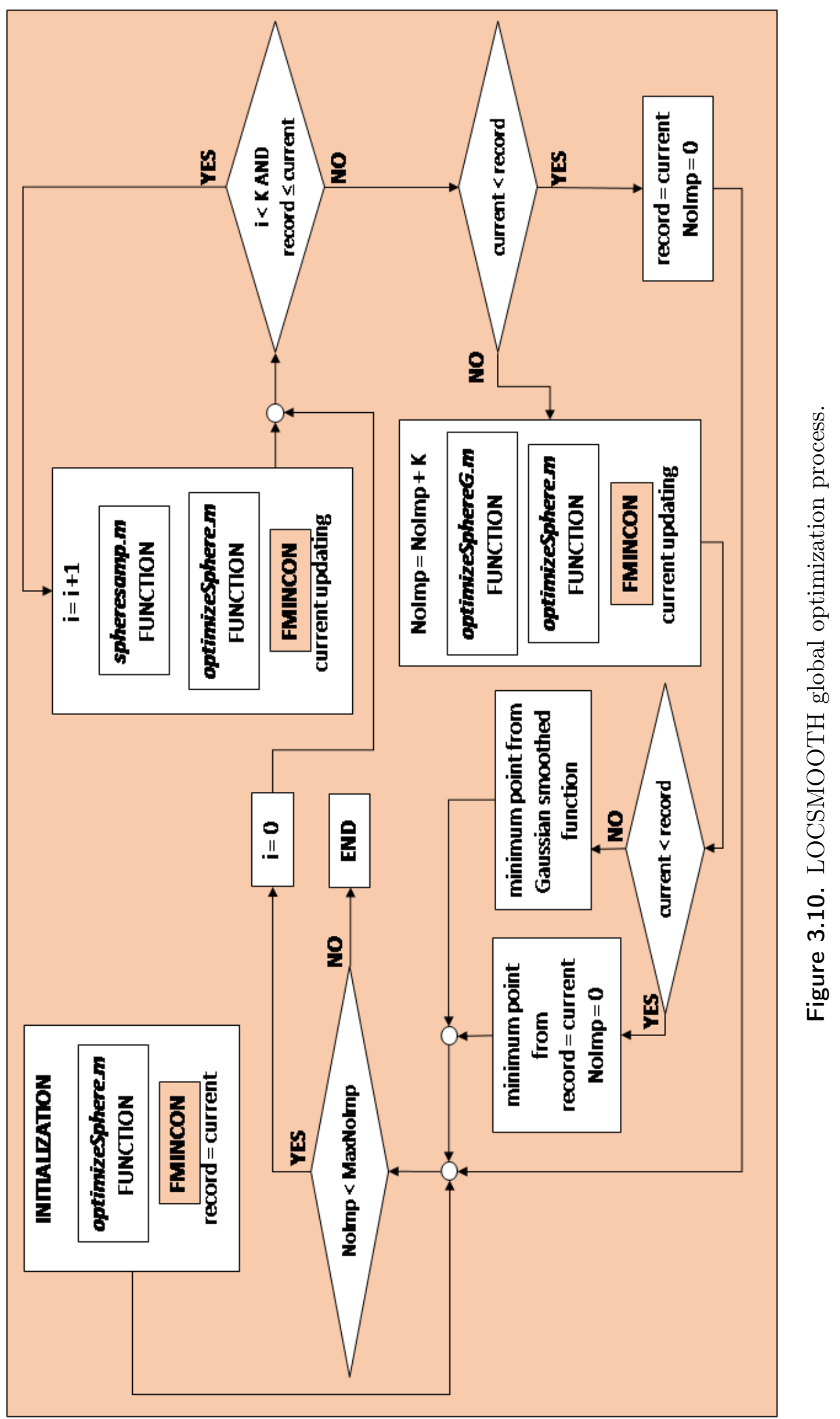


optimizeSphereG.m This function computes the point of minimum $\hat{x}$ of the smoothed function, starting from the configuration $x_{0}$ and using the SQP algorithm (the fmincon.m function is launched).

post_pro.m First of all, in this function all the geometric parameters of a configuration $\mathrm{x}$ are obtained, launching X2geo.m, geo_connect.m and geo_connencadd.m functions. Moreover, all the aerodynamic parameters are calculated by calling computeAVBV.m function. Secondly, the constraint vector is computed by the confun.m function and the value of the objective function is determined by using the obj.m function; in this way, the software is able to plot a sketch of the configuration containing the main parameters of it, just calling disegnaALA.m function. Finally, the geometric input file for AVL software is written by launching the scrivi_geo.m function.

POST.m This is a script in which the post processor is done. For each configuration, the post_pro.m function is called. Then, all the configurations are plotted and all the data are saved into the folder $\mid$ AEROSTATE $\mid$ out $\mid j o b_{-}$name_analisys.

\subsection{AVL software}

Athena Vortex Lattice $(A V L)$ is a program for the aerodynamic and flight-dynamic analysis of rigid aircraft of arbitrary configuration. It employs an extended vortex lattice model for the lifting surfaces, together with a slender-body model for fuselages and nacelles. Besides $A V L$ allows:

- to specify general nonlinear flight state;

- to linearize the aerodynamic model about any flight state;

- to consider mass properties.

Before dealing with the software, the vortex lattice method is briefly explained and the main hypotheses and characteristics are summarized in section 3.3.1. Thus, section 3.3.2 describes the software, paying particular attention to the models of wings and fuselage. Finally, section 3.3.3 explains how AVL is used by AEROSTATE.

\subsubsection{The Vortex Lattice Method}

The Vortex Lattice Method ( $V L M$ ) has been widely studied in Aerodynamics and there are many variations of it. However the intimate nature of the method is the same and the present discussion refers to [12]. The Vortex Lattice Method, as the panel method, is based on solutions to Laplace's Equation and there are many similarities: both place the singularities on the surface and the non-penetration condition has to be satisfied at a number of control points. Finally the differential problem becomes an algebric problem. The most important differences between the panel method and the vortex lattice method 
deal with the pressure coefficient and the airfoils thickness. The VLM does not compute the $C_{P}$ on the lower and the upper surface of the wing; it just provides the $\Delta C_{P}$. Moreover the thickness is not taken into account for the calculation.

The boundary condition is linearized and trasferred from the actual surface to a flat mean surface in VLM ("thin airfoil boundary condition approach"). Also the relationship between the pressure and the flow speed is simplified. Refer to a steady, inviscid and irrotational flow around an airfoil. The non-penetration condition in the exact form is shown in eq. 3.58 , where $\mathbf{V}$ is the flow speed and $\mathbf{n}$ the external normal vector of the body.

$$
\mathbf{V} \cdot \mathbf{n}=0
$$

The implicit form of the body equation is given in eq. 3.59 (refer to fig. 3.11).

$$
F(x, y)=y-f(x)=0
$$

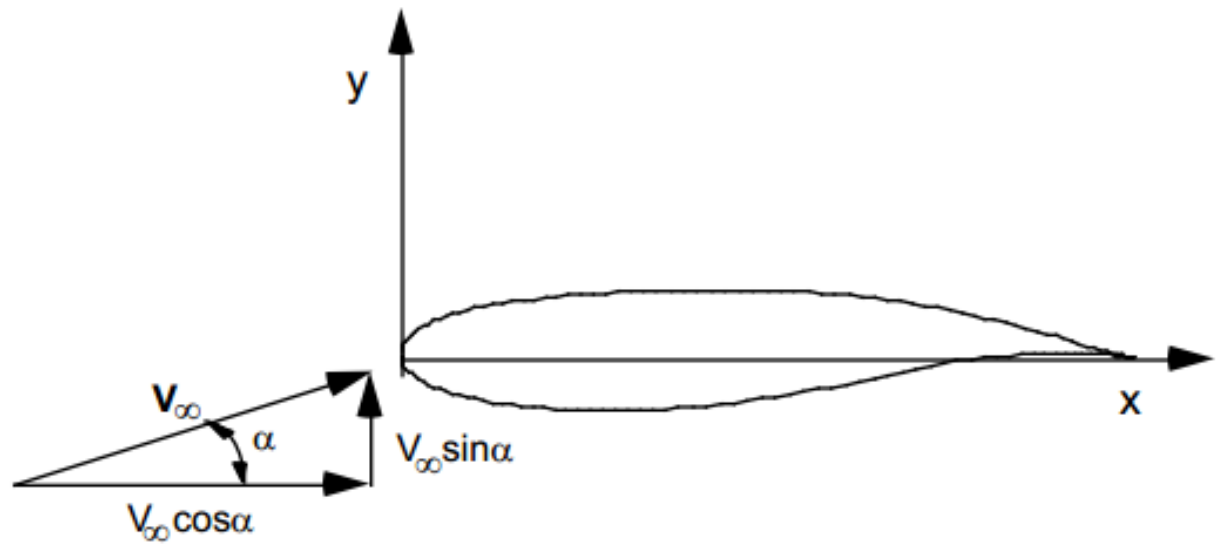

Figure 3.11. Generic airfoil in steady, inviscid, irrotational flow

The flow speed is the sum of $\mathbf{V}_{\infty}$ and $\mathbf{q}=(u, v)$, that is a disturbance velocity. All the hypotheses for the potential flow are respected, so the equation $\mathbf{q}=\nabla \phi$ can be written ( $\phi$ is the potential of velocity) and, according to fig. 3.11, the velocity components and the normal vector can be expressed as follows.

$$
\begin{gathered}
\left\{\begin{array}{l}
V_{x}=V_{\infty} \cos \alpha+u \\
V_{y}=V_{\infty} \sin \alpha+v
\end{array}\right. \\
\mathbf{n}=\frac{\nabla F(x, y)}{\|\nabla F(x, y)\|}
\end{gathered}
$$

So the exact, nonlinear, boundary condition is given by eq. 3.62 .

$$
v=\frac{d f}{d x}\left(V_{\infty} \cos \alpha+u\right)-V_{\infty} \sin \alpha
$$


The previous relation can not be used for an automatic computational method, so it needs to be simplified: the following equations are assumed:

$$
\left\{\begin{array}{l}
\frac{u}{V_{\infty}}<<1 \\
\frac{v}{V_{\infty}}<<1 \\
\cos \alpha \simeq 1 \\
\sin \alpha \simeq \alpha
\end{array}\right.
$$

The new, approximate, boundary condition is reported in eq. 3.64.

$$
\frac{v}{V_{\infty}}=\frac{d f}{d x}-\alpha
$$

This form of the boundary condition is not valid if the flow disturbance is elevated, compared to the freestream velocity: for aerodynamically streamlined shapes this is usually valid everywhere except at the leading edge of the airfoil, where a stagnation point exists $\left(u=-V_{\infty}\right)$ and the slope is infinite $(d f / d x=\infty)$. Thus, if the details of the flow at the leading edge are not important to the analysis, the linearized boundary condition can be used. A further simplification of the non-penetration condition can be obtained by using a Taylor series expansion of the $v$ component calculated on the body: assuming small airfoils and small perturbations, eq. 3.65 is obtained.

$$
v(x, y)=v(x, f(x))=v(x, 0)+\frac{\partial v(x, 0)}{\partial y} f(x)+\ldots \simeq v(x, 0)
$$

Now, let $f_{l}$ and $f_{u}$ be respectively the lower and the upper surface; consider $f_{c}$ the camber description and $f_{t}$ the semi-thickness description. It can be inferred that $f_{u / l}=f_{c} \pm f_{t}$, and the boundary condition splits as shown in eq. 3.66.

$$
\left\{\begin{array}{l}
\frac{v\left(x, 0^{+}\right)}{V_{\infty}}=\frac{d f_{c}}{d x}+\frac{d f_{t}}{d x}-\alpha \\
\frac{v\left(x, 0^{-}\right)}{V_{\infty}}=\frac{d f_{c}}{d x}-\frac{d f_{t}}{d x}-\alpha
\end{array}\right.
$$

If the thickness is neglected, the boundary condition becomes the same for the upper and the lower surface. The expression of the $C_{P}$ coefficient has to be consistent with the previous approximations: eq. 3.67 is obtained (refer to [12]).

$$
C_{P}=-\frac{2 u}{V_{\infty}}
$$

Using the superposition effect, it can be shown that $\Delta C_{P}$ is not affected by the airfoil thickness, hence the VLM does not consider it, because thickness does not contribute to lift to first order in the velocity disturbance:

$$
\Delta C_{P}=2\left(C_{\text {Pcamber }}+C_{P \alpha}\right)
$$

The conclusion of this analysis is that the lift is not affected by the surface thickness and the boundary condition can be imposed on a flat coordinate surface.

The core of the VLM is using vortex singularities, so a briefly overview on the vortextheorems (Kelvin-Helmotz) is reported below: 
- Along a vortex line, the circulation $(\Gamma)$ is constant.

- A vortex filament (or line) can not begin or end abruptly in a fluid. The vortex line must be closed or extend to infinity, or end at a solid boundary. Furthermore, the circulation $(\Gamma)$ about any section is the vortex strength.

- An initially irrotational, inviscid flow will remain irrotational.

The most suitable result of the previous theorems for the VLM points out that a sheet of vortices can support a jump in tangential velocity, while the normal velocity is continuous. Thus, a vortex sheet can represent a lifting surface.

The Biot-Savart formula is the most general result that can be used to compute the induced velocity $(\mathbf{w})$ at a point $P$ by a portion $(d \mathbf{l})$ of a three-dimensional vortex filament (strenght $\Gamma$ ) located at point $Q$ (refer to 3.69, where $\mathbf{r}_{Q P}$ is the vector from point $Q$ to point $P$ ).

$$
d \mathbf{w}=\frac{\Gamma}{4 \pi} \frac{d \mathbf{l} \wedge \mathbf{r}_{Q P}}{\left|\mathbf{r}_{Q P}\right|^{3}}
$$

Applying the Biot-Savart formula to two remarkable cases, the following relations are proved:

$$
\begin{gathered}
w=\frac{\Gamma}{4 \pi h}\left(1+\cos \theta_{0}\right) \\
w=\frac{\Gamma}{4 \pi h}\left(\cos \theta_{1}-\cos \theta_{2}\right)
\end{gathered}
$$

Equation 3.70 is valid for a semi-infinite vortex, while equation 3.71 is valid for a finite vortex (see fig. 3.12).
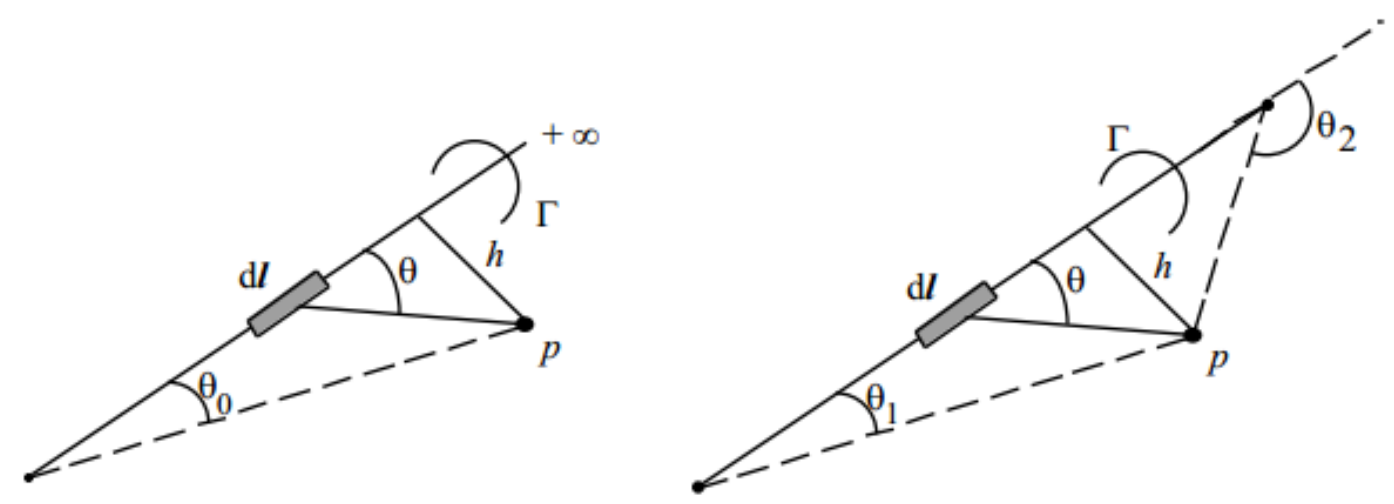

Figure 3.12. Semi-infinite vortex and finite vortex

There is a specific form of vortex used in the traditional VLM: it is a "horseshoe vortex", which extends from downstream infinity to a point in the field "A", then from point "A" to point "B," and another vortex from point "B" downstream to infinity. The velocity induced by this vortex is the sum of the three parts and it is computed by using 3.70 and 3.71 .

$$
\mathbf{w}=\mathbf{w}_{A B}+\mathbf{w}_{A \infty}+\mathbf{w}_{B \infty}
$$


Set $\Gamma_{n}$ the vortex strenght. The coordinates of the corner points of the vortex are $A=$ $\left(x_{1 n}, y_{1 n}, z_{1 n}\right)$ and $B=\left(x_{2 n}, y_{2 n}, z_{2 n}\right)$ (refer to fig. 3.13). The induced point is $C=$ $(x, y, z)$. The expression of the three parts of the induced velocity is given by the following equations:

$$
\begin{gathered}
\mathbf{w}_{A B}=\left(\frac{\Gamma_{n}}{4 \pi} \Omega\right) \Psi \\
\mathbf{w}_{A \infty}=\left(\frac{\Gamma_{n}}{4 \pi} \Omega_{A \infty}\right) \Psi_{A \infty} \\
\mathbf{w}_{B \infty}=-\left(\frac{\Gamma_{n}}{4 \pi} \Omega_{B \infty}\right) \Psi_{B \infty}
\end{gathered}
$$

The expressions of the vectors $\Psi, \Psi_{A \infty}, \Psi_{B \infty}$ and of the scalar quantities $\Omega, \Omega_{A \infty}$ and $\Omega_{B \infty}$ can be found in [12], as well as a further explaination about the geometric meaning of them.

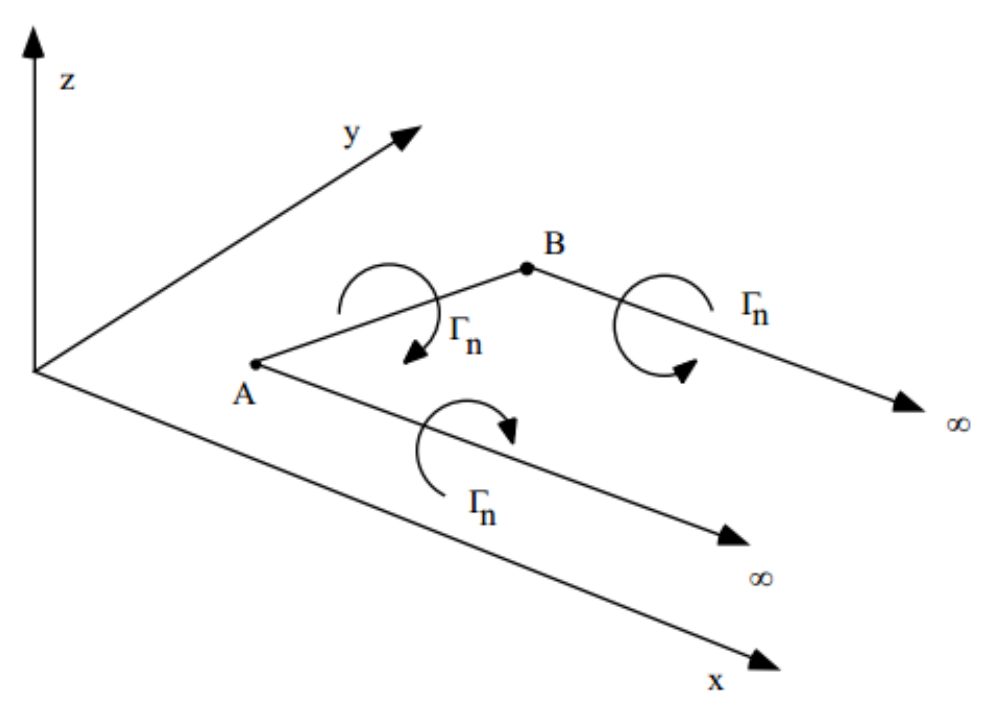

Figure 3.13. The "horseshoe" vortex

Finally, the general expression of the induced velocity at location $m$ due to a general vortex placed in $n$ is:

$$
\mathbf{w}_{m}=\mathbf{C}_{m n} \Gamma_{n}
$$

Since horseshoe vortices defined above are used to represent a lifting surface, the vortex location and the control point location (where the surface boundary condition is satisfied) are basic information. Tradition determines their locations by comparison with two-dimensional known results and they are directly applied to the three-domensional cases. The Flat Plate Case proves that the distance between the vortex and the control point should be one half of the chord; the Parabolic Camber Model demostrates that the vortex should be posed at $c / 4$. The two latter information are collected in the "1/4-3/4 
Rule" due to Enrico Pistolesi.

The next steps summarize the classical procedure to apply the VLM:

- Divide the planform up into a lattice of quadrilateral panels, and put a horseshoe vortex on the $1 / 4$ chord element line of each panel.

- Place the control point on the $3 / 4$ chord point of each panel at the midpoint in the spanwise direction.

- Assume a flat wake oriented to the upstream direction: the wake should not lift and this fact occurs if the velocity vector $\mathbf{V}$ and the circulation vector $\Gamma$ are parallel (in fact the Kutta-Joukowski Theorem asserts $L=\rho \mathbf{V} \wedge \Gamma$ ).

- Determine the strengths of each $\Gamma_{n}$ required to satisfy the boundary conditions by solving a system of linear equations.

The VLM provides reliable results about wing aerodynamics and aboout the interaction among more lifting surfaces. The VLM are widely used in the design process to evaluate the neutral point of a whole configuration, by considering the position of wing and tail or canard. Aerodynamic coefficients, as the drag coefficient $C_{D}$ and the slope of the lifting curve $C_{L \alpha}$, can be forecast by the VLM. Finally, the VLM can also estimate the effects of controls and their deflections.

Neglecting the sideslip angle and assuming a mainly 2D-surface, the bondary condition is given by equation 3.77 , where $\mathrm{N}$ is the total vortex number on a half wing and the only unknowns are the $\Gamma_{n}$.

$$
\sum_{n=1}^{2 N} \mathbf{C}_{m n}\left(\frac{\Gamma_{n}}{V_{\infty}}\right)=\left(\frac{d f_{c}}{d x}-\alpha\right)_{m}
$$

There are exacly $2 N$ control points, so $m=1, \ldots, 2 N$ and the problem is easily solvable. An implementation of the VLM on a general surface is reported in fig. 3.14.

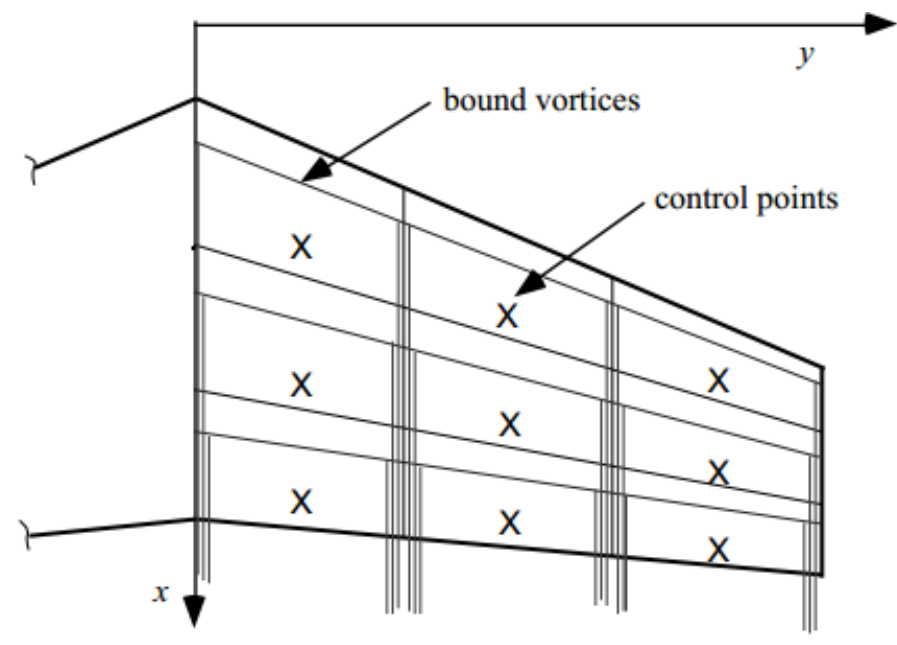

Figure 3.14. VLM on a general wing 


\subsubsection{AVL: a general overview}

$A V L$ has been conceived for lifting surfaces with thin airfoils, low angles of attack and sideslip. These surfaces and their trailing wakes are represented by a layer of "horseshoe" vortices, whose filaments are directed parallel to the $x$ axis. $A V L$ also allows to model slender bodies, such as fuselages and nacelles, by using sources and doublets: however the experience with this model is relatively limited, and hence modelling of bodies should be done with caution. Previous preliminary studies (refer to [14]) analysed the solution at varying the topology of fuselage modelling: when only the longitudinal plane stability and dynamics are taken into account, the most reliable results are obtained when the fuselage is modeled as part of the front lifting surface. Further information will be provided later on this paragraph.

Compressibility is treated in $A V L$ using the classical Prandtl-Glauert transformation, which converts the Prandtl-Glauert equation to the Laplace equation. This is equivalent to the compressible continuity equation, with the assumptions of irrotationality and linearization about the freestream. The forces are computed by applying the KuttaJoukowsky relation to each vortex, this remaining valid for compressible flow. The relative importance of compressible effects can be judged by the Prandtl-Glauert factor $1 / B$ (eq. 3.78), where $M$ is the freestream Mach number and $\Lambda$ is the wing sweep at $c / 4$.

$$
\frac{1}{B}=\frac{1}{\sqrt{1-(M \cos \Lambda)^{2}}}
$$

One of the most interesting aspect of $A V L$ is the paneling operation. The remarkable guidelines to panel the aerodynamic surfaces have been summarized in [4] and they are reported below:

1. Over a total number of 100 panels for a lifting surface the solution does not get better.

2. It is better to choose a wing span panel distribution such that panels are gathered at the tip of the wing.

3. It is better to choose a chord panel distribution such that panels are gathered at the leading edge.

The panel distribution can be chosen by setting a parameter in $A V L$, according to the scheme of fig. 3.15 .

As $A V L$ has to provide stabily and trim results, the effect of the panel distribution and the solution sensitivity to the number of panels have been studied (refer to [4]) in terms of neutral point and center of pressure. The neutral point is not affected by the panel number or distribution, while the center of pressure is well evaluated when at least 8 panels are used in the chord direction and they are distribuited as a sine function. The spanwise direction is not so influent, so 20 panels are chosen, distribuited as a cosine. 


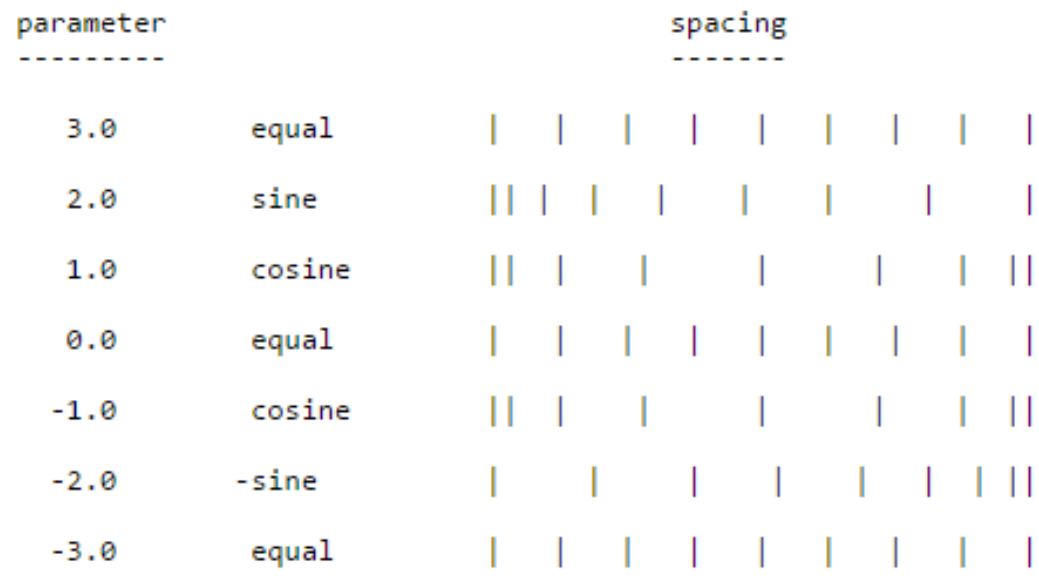

Figure 3.15. AVL spacing possibilities

\section{Starting an $A V L$ input file}

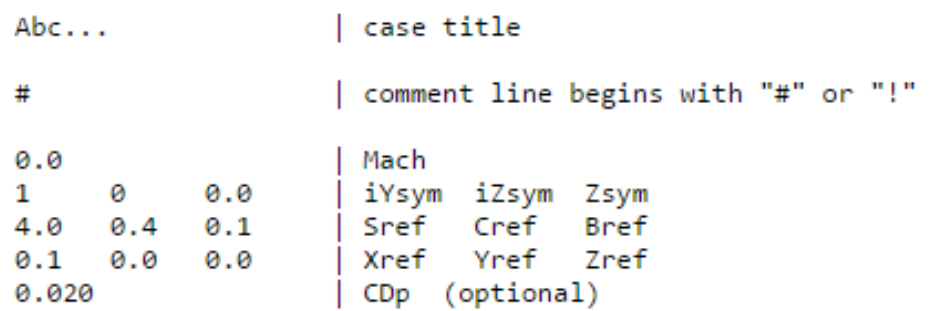

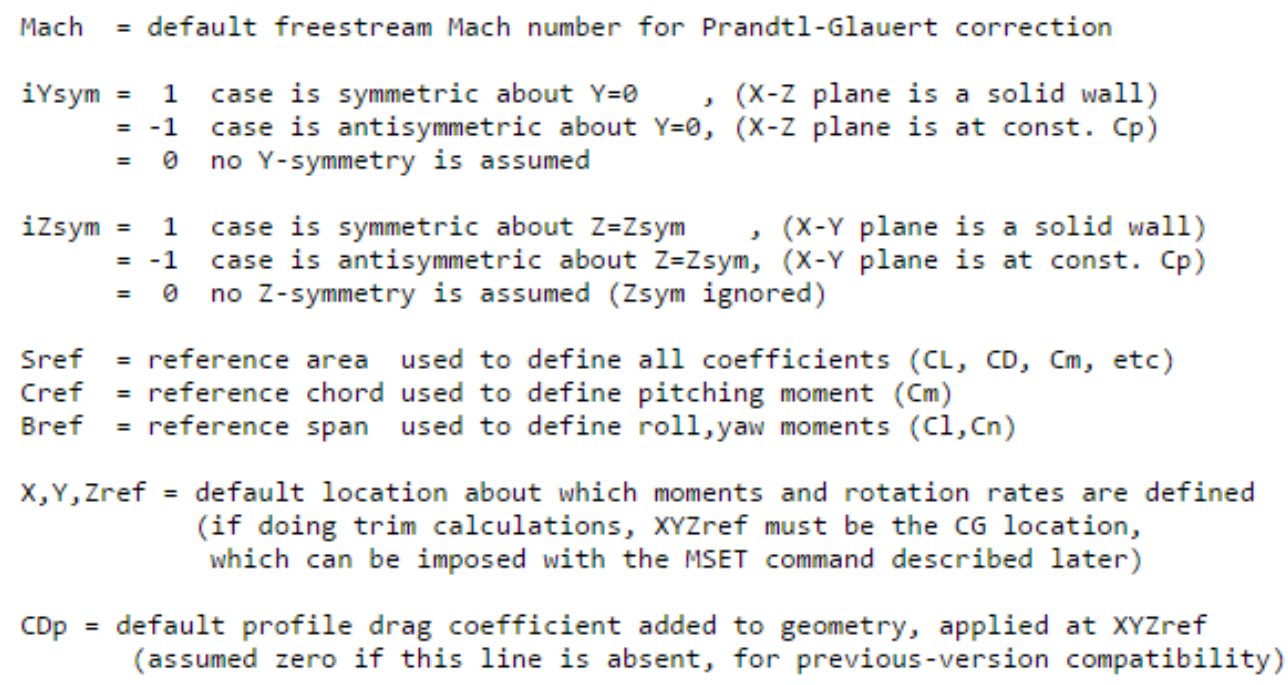

Figure 3.16. AVL input file 
The $A V L$ input file must refer to a coordinate system defined as follows: $x$ axis: from upstream to downstream;

$y$ axis: out the right wing;

$z$ axis: from down to up.

All the main information are summarized in fig. 3.16.

\section{The thin surfaces model}

It has been already spread out how a lifting surface is treated. In this paragraph the $A V L$ advanced options are considered in order to take into account the airfoil thickness and its curvature. The bay is always defined by means two sections, which are the boundaries of the bay; moreover, some quantities need to be introduced for each section:

- A ".dat" file, containing the airfoil coordinates, is specified to take into account the curvature.

- The thickness correction is obtained by means a factor $(C L A F)$, which has to multiply the airfoil $C_{L \alpha}$ (set $2 \pi$ by default).

$$
C L A F=1+0.77 \frac{t}{c}
$$

There are also other options to describe the control surfaces: refer to [13] and to [4] for further information. Fig. 3.17 shows a typical input file for $A V L$ : each line is explained in the figure itself.

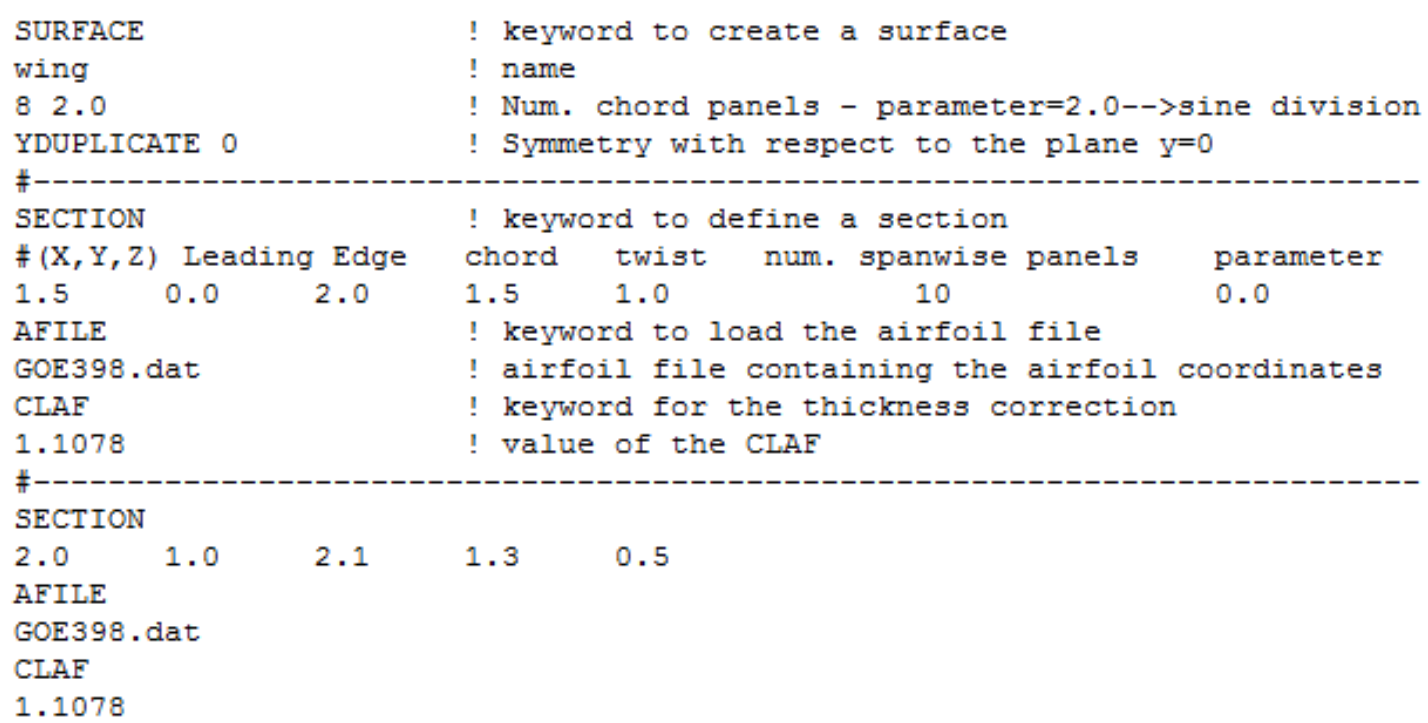

Figure 3.17. AVL surface definition 


\section{The fuselage model}

The best way to treat a fuselage is considering it as a portion of a wing. Thus, the fuselage is represented as a SURFACE element instead of a BODY element. The two-dimensional shape of the fuselage is the top view of the fuselage itself, while no airfoil are considered so that finally this part is modeled as a flat plate, continuously connected to the front wing. This aspect introduces some troubles, which will be deepened in Chapter 5 .

The fuselage induced drag is computed directly by $A V L$, yet the friction drag is added by AEROSTATE in the function computeAVBV.m: however this procedure is not so reliable, because of considering the fuselage as a wing. This difficult can be overcome by setting the fuselage angle of attack equal to zero: the whole fuselage angle of attack is not necessary equal to zero, but the twist angle of the sections that separate the fuselage and the wing can be set as the opposite of the fuselage angle of attack. This trick allows to take into account that the portion of the wing that crosses the fuselage does not contribute to the lift. 


\subsubsection{AEROSTATE and AVL}

The aim of this section is to show how an $A V L$ input file is built in order to obtain a PrandtlPlane configuration such as fig. 3.18 illustrates.

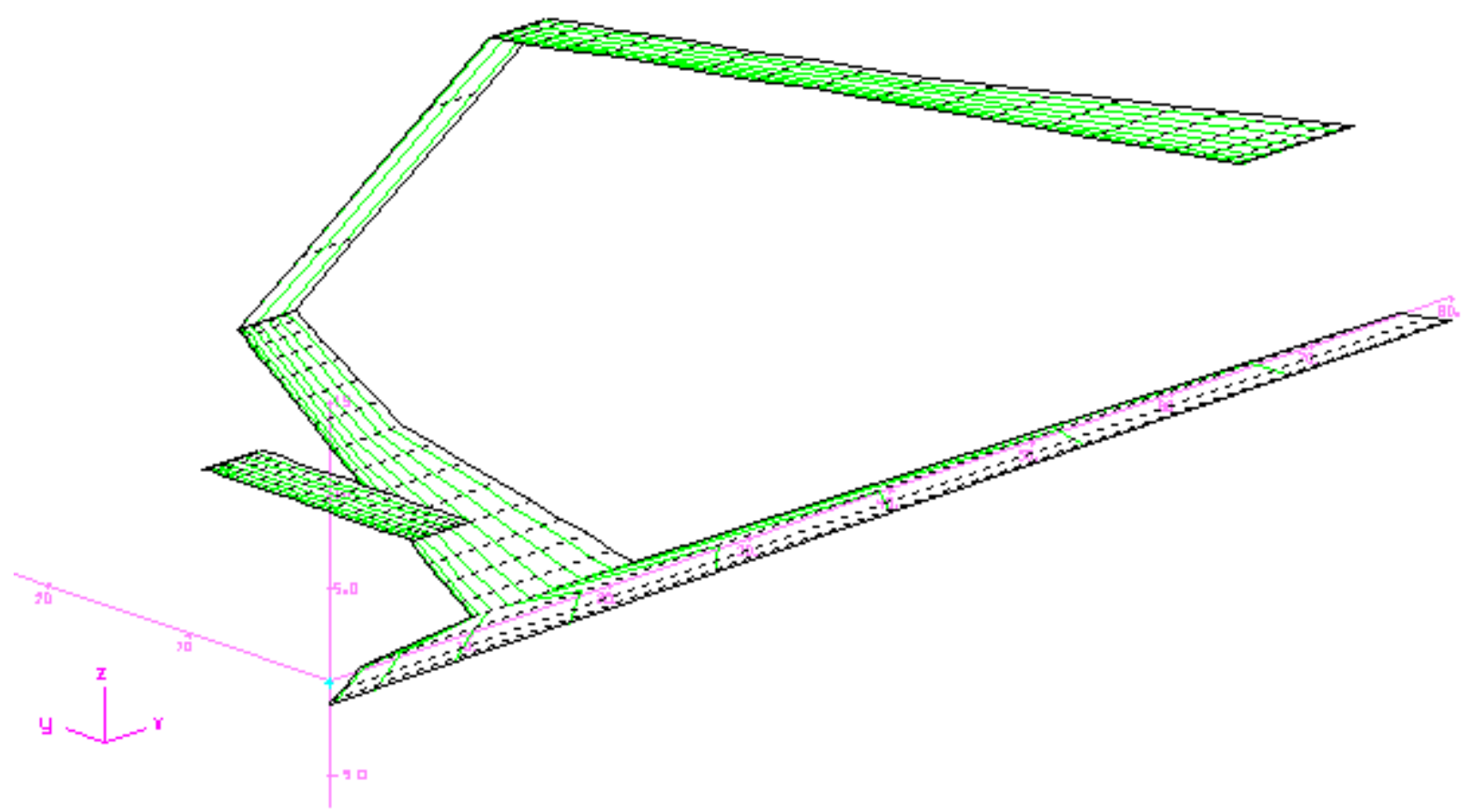

Figure 3.18. AVL PrandtPlane configuration

Referring to fig. 3.19, the calculation starts by defining the Mach number and the symmetry condition: as only half aircraft has been modeled, the symmetry with respect to the plane $y=0$ is active. The following line collect the main quantities (surface, chord and spanwise) used to compute the aerodynamic derivatives. Finally, the pole of the aerodynamic moments has to be specified.
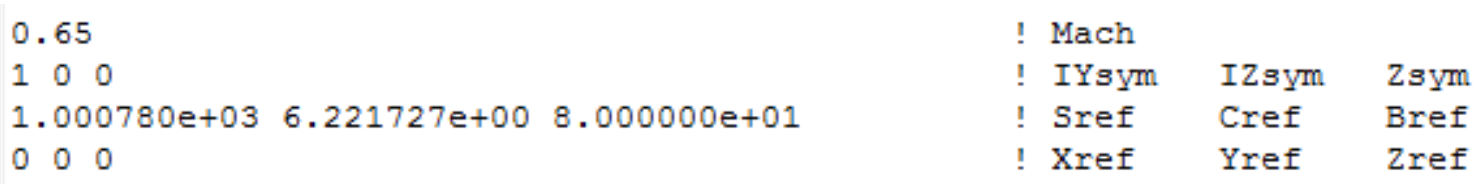

Figure 3.19. AVL starting data 
As it has been told in section 3.3.2, the front wing and the fuselage are gathered in an only lifting surface. Fig. 3.20 shows the main features of this surface. Note that in this case number panels in the spanwise direction and their distribution are declared at the beginning of the surface: there are 20 panels and the distribution follows a cosine trend. Thus, Nspanwise and Space are ignored in the following lines. The keyword ANGLE allows the user to set a pitch angle for all the surface, without changing the twist angles. The NACA642415 airfoil will be discussed in Chapter 5 .

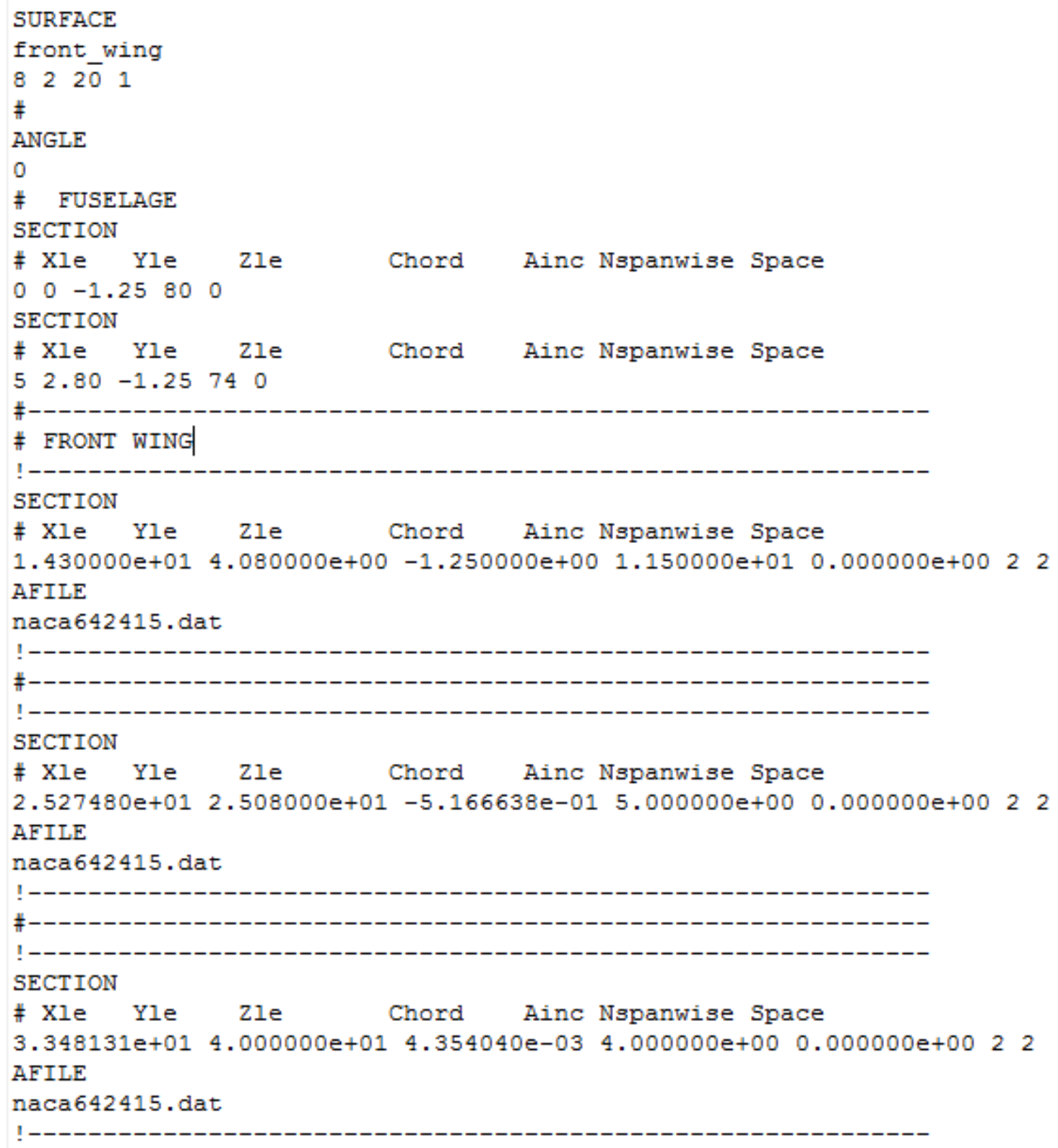

Figure 3.20. AVL front wing and fuselage 
Fig. 3.21 shows the front wing: the spanwise division and the chord division are evident.

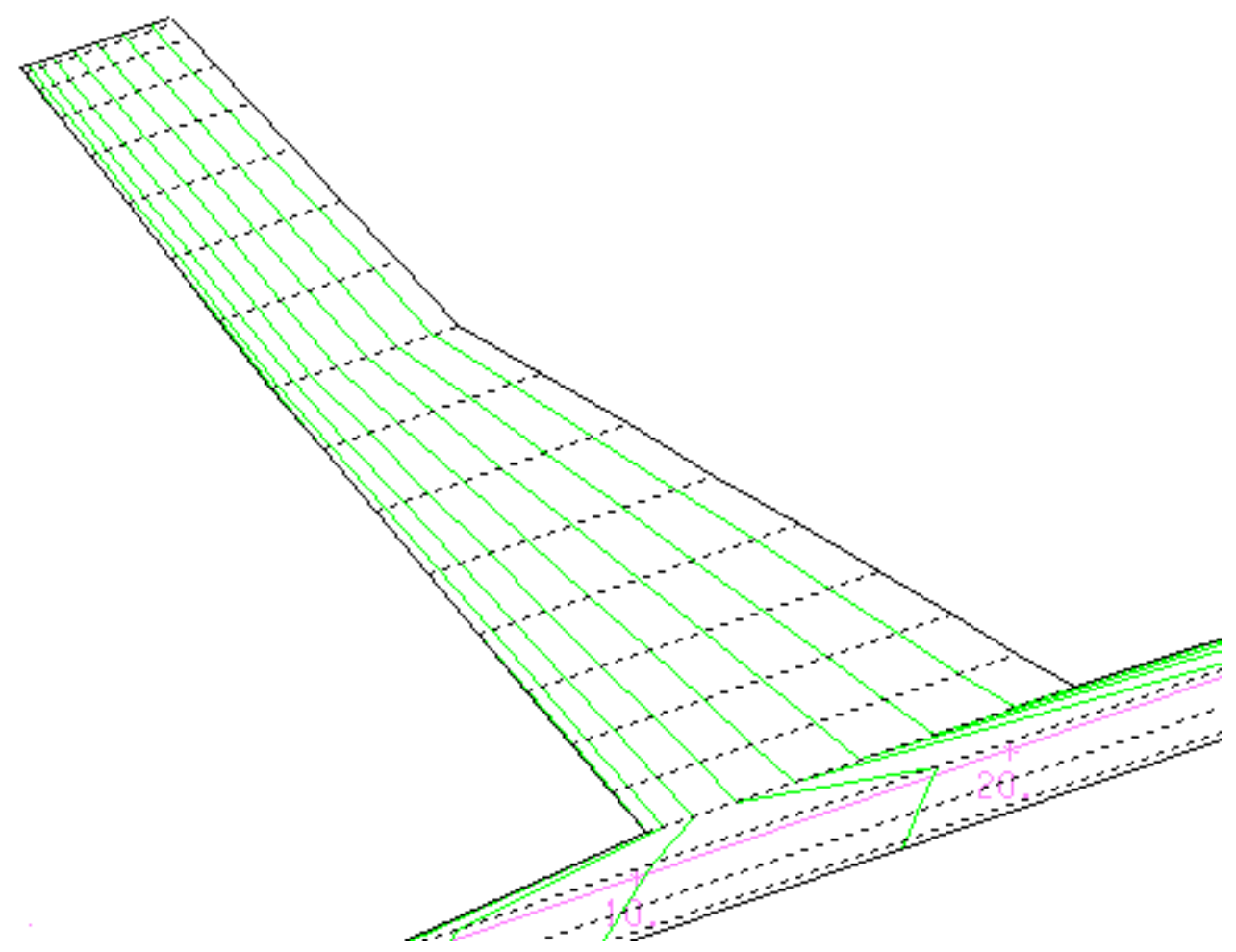

Figure 3.21. AVL front wing and fuselage - Detail

The rear wing is not affected by the fuselage because it has the root chord at the top of the two vertical fins. So there are just three section to define the rear wing (refer to fig. 3.23). The rear panels distribution is shown in fig. 3.22.

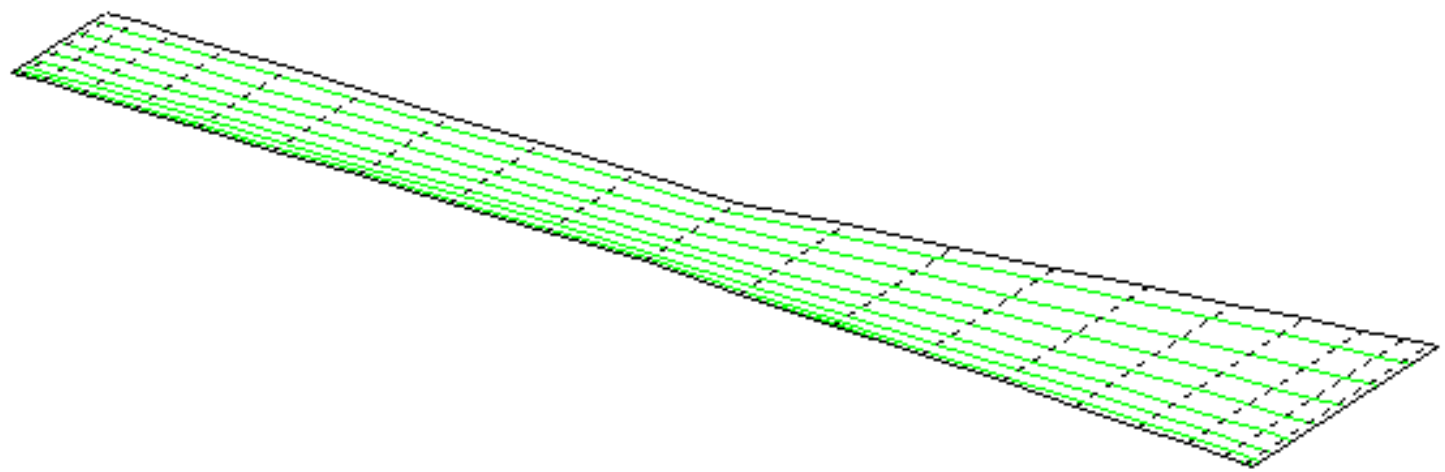

Figure 3.22. AVL rear wing - Detail 


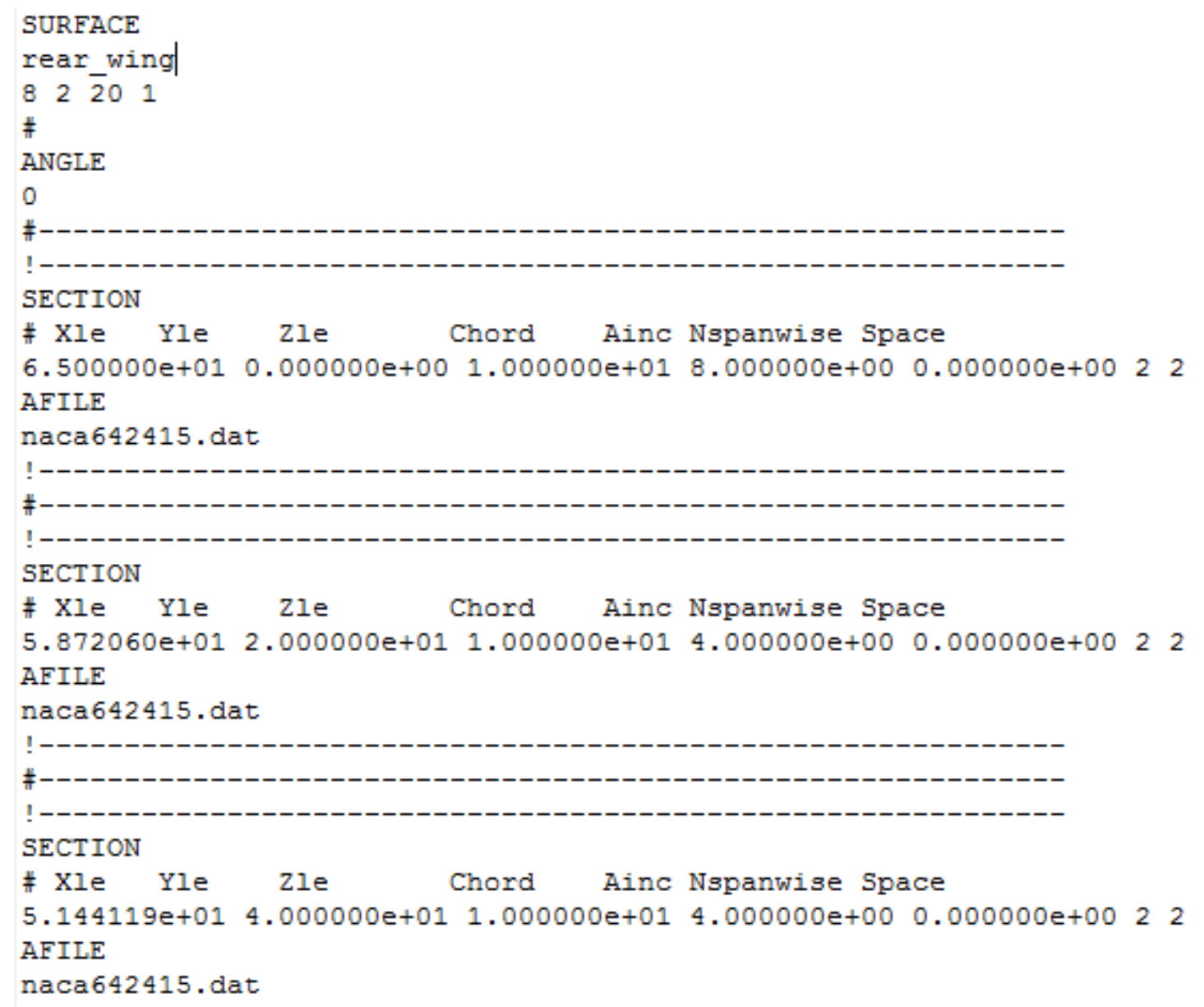

Figure 3.23. AVL rear wing

The adding wing is a simplier lifting surface than the front or the rear wing because it needs two only bays to be defined because a kink is not considered in this case (refer to fig. 3.24). Some structural devices will be designed in order to link the auxiliary wing to the top of the fuselage. This observation allows to consider the entire auxiliary wing span to generate lifting forces, because the auxiliary wing does not cross the fuselage. The third wing is shown in fig. 3.25.

The bulk is modeled in a different way: it has been shown in [4] that the panels distribution along the chord direction has negligible effects on the aerodynamic solution (especially in terms of neutral point and centre of pressure). So the number of vortices ( 3 vortices in the chord direction and 3 vortices in the spanwise direction) is due to the calculation times. However the vortices distribution is more important and it is preferable setting the distribution parameter at 1, i.e. a cosine distribution (refer to fig. 3.26).

The last comment deals with the vertical fins. They are not considered in the present computation because they do not contribute to lift, so they do not develop any induced drag contribution in ideal conditions (null sideslip angles, without side perturbations). 


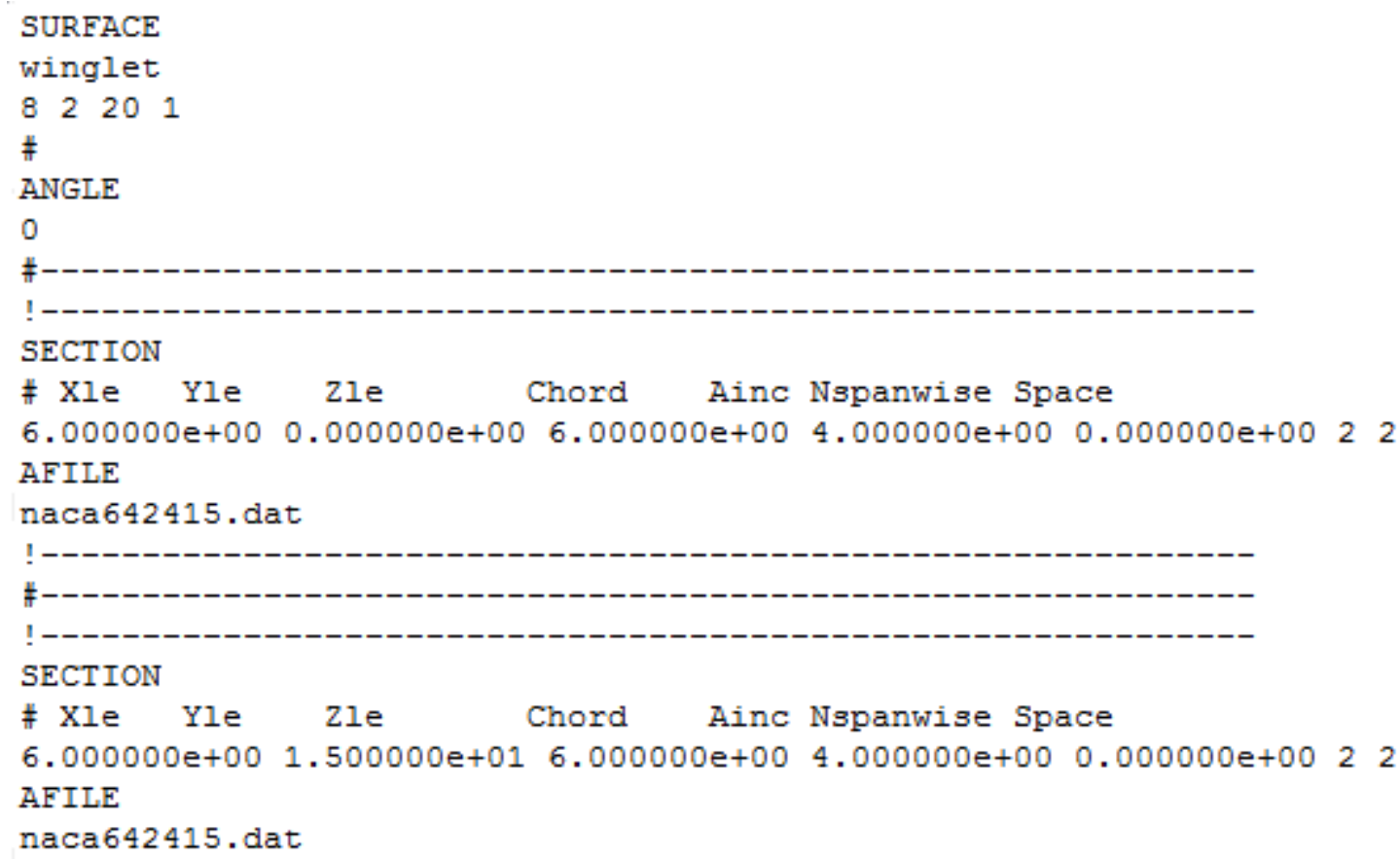

Figure 3.24. AVL winglet

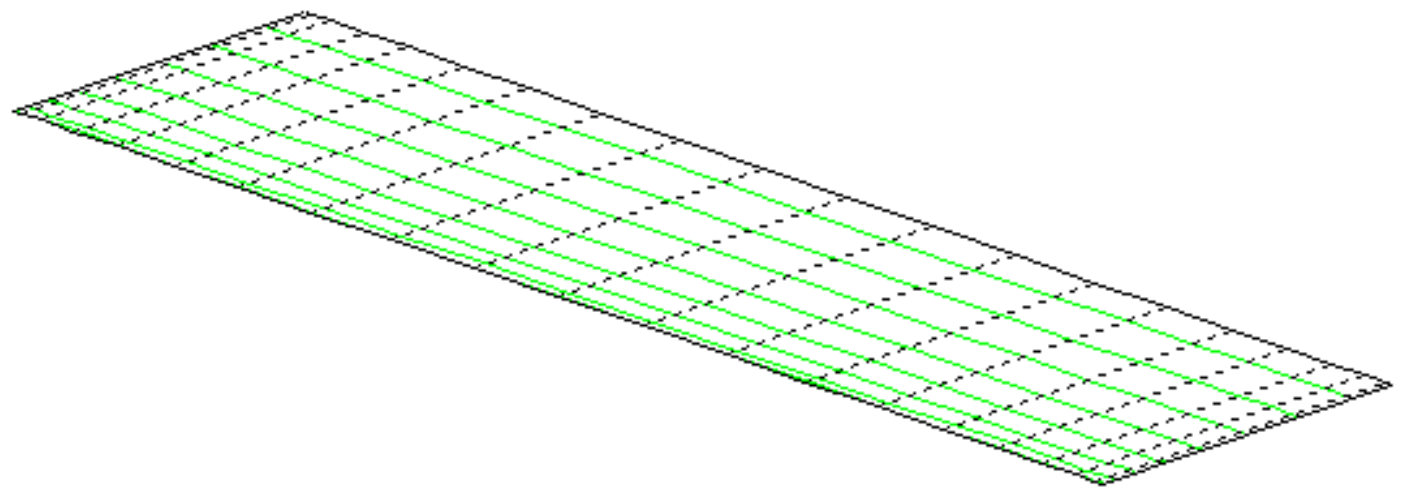

Figure 3.25. AVL winglet - Detail 


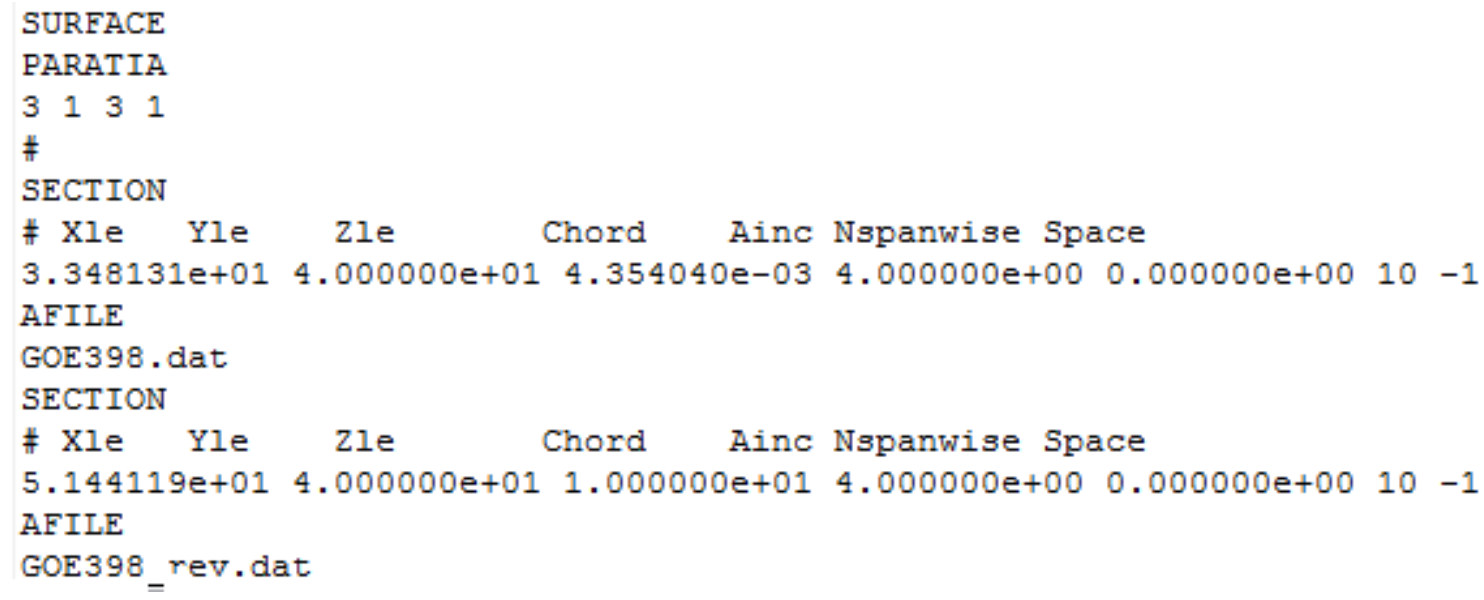

Figure 3.26. AVL bulk

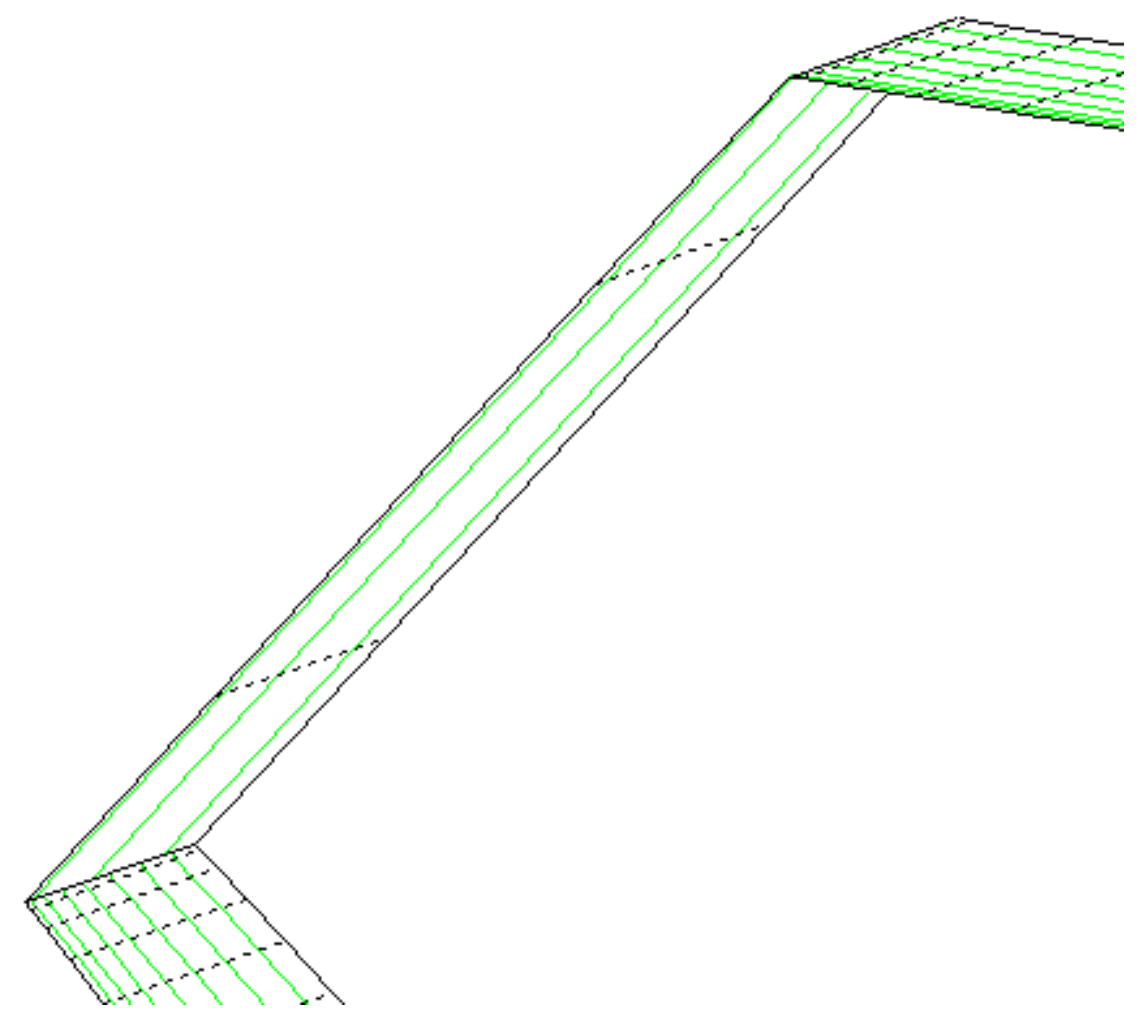

Figure 3.27. AVL bulk - Detail 



\section{Chapter 4}

\section{INTERFACE}

A part of the activities of the present work, has been dedicated to the development of the software called INTERFACE, which is able to link the optimization software AEROSTATE and the geometric software ASD each other: in particular, the geometric results, obtained from the high speed optimization, are converted into three dimensional $A S D$ configurations, in automatic way.

Both AEROSTATE, ASD and LiNeS have been integrated in INTERFACE, with the aim of obtaining a master software capable of to merge all these tools.

INTERFACE is a software developed in MATLAB ${ }^{\circledR} \mathrm{R} 2012 \mathrm{a}$ environment: in particular, it has been designed as a graphical user interface (GUI) and its master window is shown in Fig. 4.1.

In this window it is possible to observe the push buttons AEROSTATE, LiNeS and $A S D$ which launch the corresponding software. The 3D - Representation block contains all the necessary tools to make the conversion, from the AEROSTATE geometric information, to the 3D $A S D$ representation.

The text edit "Path name of results" needs the full path of the results folder (its name needs to be the same of the analysis name).

The analysis name is required in the text edit "Analysis name", while the starting geometry input file name needs to be inserted in the text edit "starting geometry input file name".

When all these informations are indicated to INTERFACE by the user, the software is able to convert the AEROSTATE results, following this sequence:

1. Click on the "Import geo" push button: in this way, all the AEROSTATE ".mat" results are copied into the folder:

... $\backslash$ ASDAEROSTATE $\backslash$ GUI_ASDAEROSTATE $\backslash$ INTERFACCIA $\backslash$ GEOFILE

2. Click on the "Aerostate to ASD" push button: the ".mat" result files are converted in ".asd" files, using the main function "conf.m", refer to section 4.1; then, they are saved into the folder:

... $\backslash$ ASDAEROSTATE $\backslash$ GUI_ASDAEROSTATE $\backslash$ INTERFACCIA $\backslash$ GEOSAVEASD 


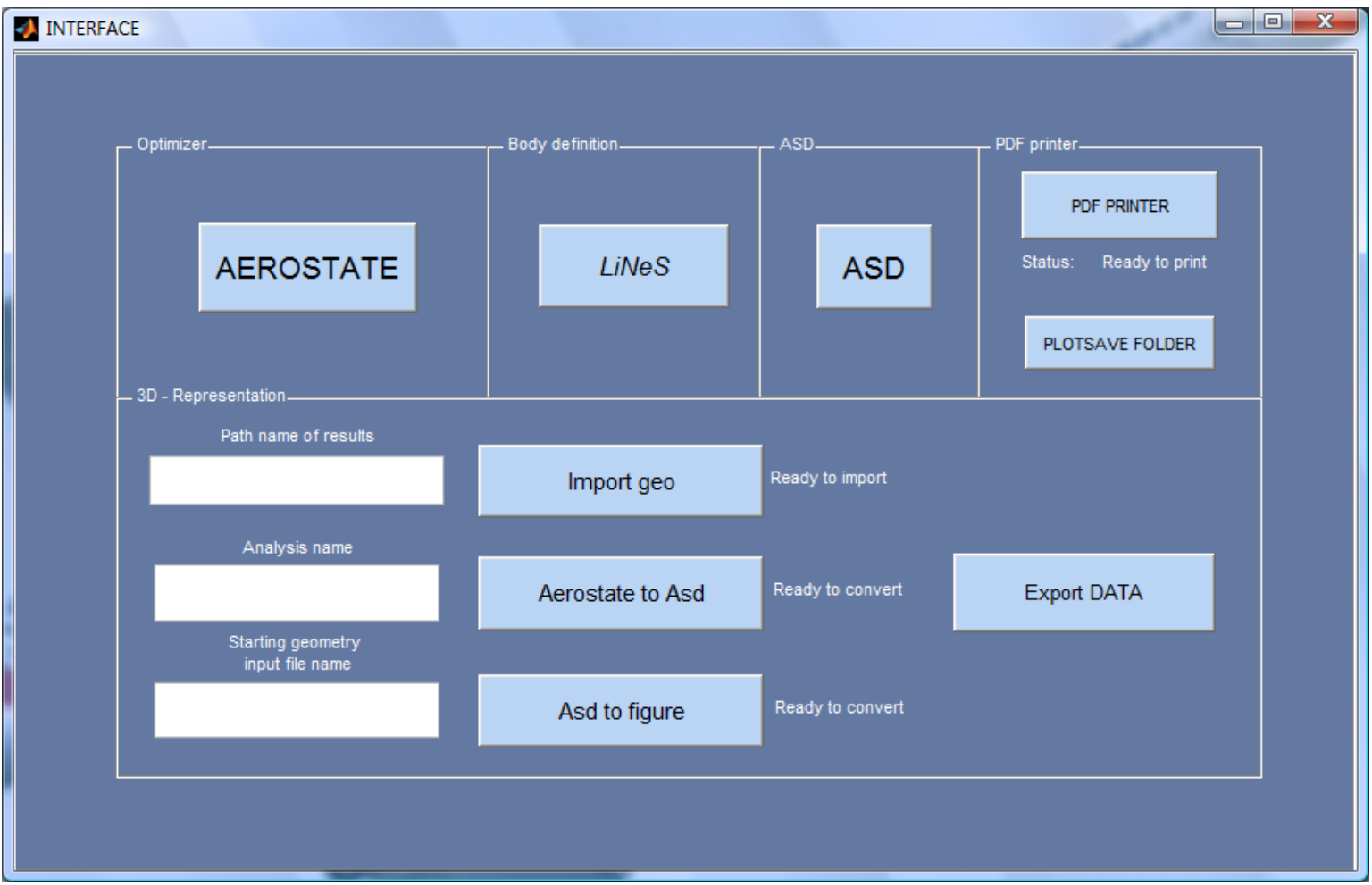

Figure 4.1. INTERFACE window.

3. Click on the "Asd to figure" push button: in this step, the $A S D$ configurations are generated by launching the $A S D$ software in a iterative way and all these configurations are saved in a ".fig" format, in the folder:

... $\backslash$ ASDAEROSTATE $\backslash$ GUI_ASDAEROSTATE $\backslash$ INTERFACCIA $\backslash$ PLOTSAVE This process has been realized modifying, in a suitable way, the $A S D$ software and it is placed into the folder:

... $\backslash$ ASDAEROSTATE $\backslash$ GUI_ASDAEROSTATE $\backslash$ ASD_2009_07_MODIFIED_2

4. Click on the "PDF PRINTER" push button: the function "PDF_PRINTER.m" is called and all the ".fig" files are convert in ".pdf" files. Them are saved in the folder: ... $\backslash$ ASDAEROSTATE $\backslash$ GUI_ASDAEROSTATE $\backslash$ INTERFACCIA $\backslash$ PLOTSAVE

5. Click on the "Export DATA" push button: all the generated files are moved into a new folder "INTERFACE_RESULTS", which is created into the initial analysis folder.

Moreover, the "PLOTSAVE FOLDER" push button is used to call the "PLOTSAVE" folder and it has been introduced for convenience.

In Fig. 4.2 is shown the main structure of the INTERFACE, to give a global reference for the user of this software. 


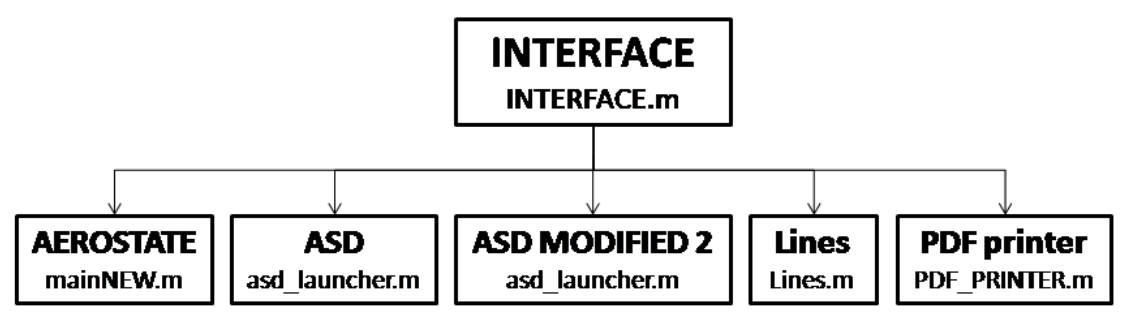

Figure 4.2. INTERFACE window.

\subsection{Conversion function}

In this section, the main conversion function "conf.m" is shown, with the purpose of presenting the function that allows to interface AEROSTATE and ASD, which is implemented in INTERFACE software.

The features of this $M A T L A B^{\circledR}$ function are listed below:

1. The $A S D$ software needs parametric data as input to generate the wing planforms: they are extrapolated from the $M A T L A B^{\circledR}$ structured variable geo, defined in AEROSTATE. The automatic procedure allows to take into account the possible presence of the kink;

2. Information on the fuselage are inserted in terms of shape of the transversal section, longitudinal lines (eventually developed with the software $L i N e S$ ) and longitudinal position of the section;

3. The twin vertical tails are positioned automatically depending on the position of the rear wing, with the purpose of a good representation;

4. Finally, the bulk parameters are set for the $A S D$ software, starting from the wings positions. 


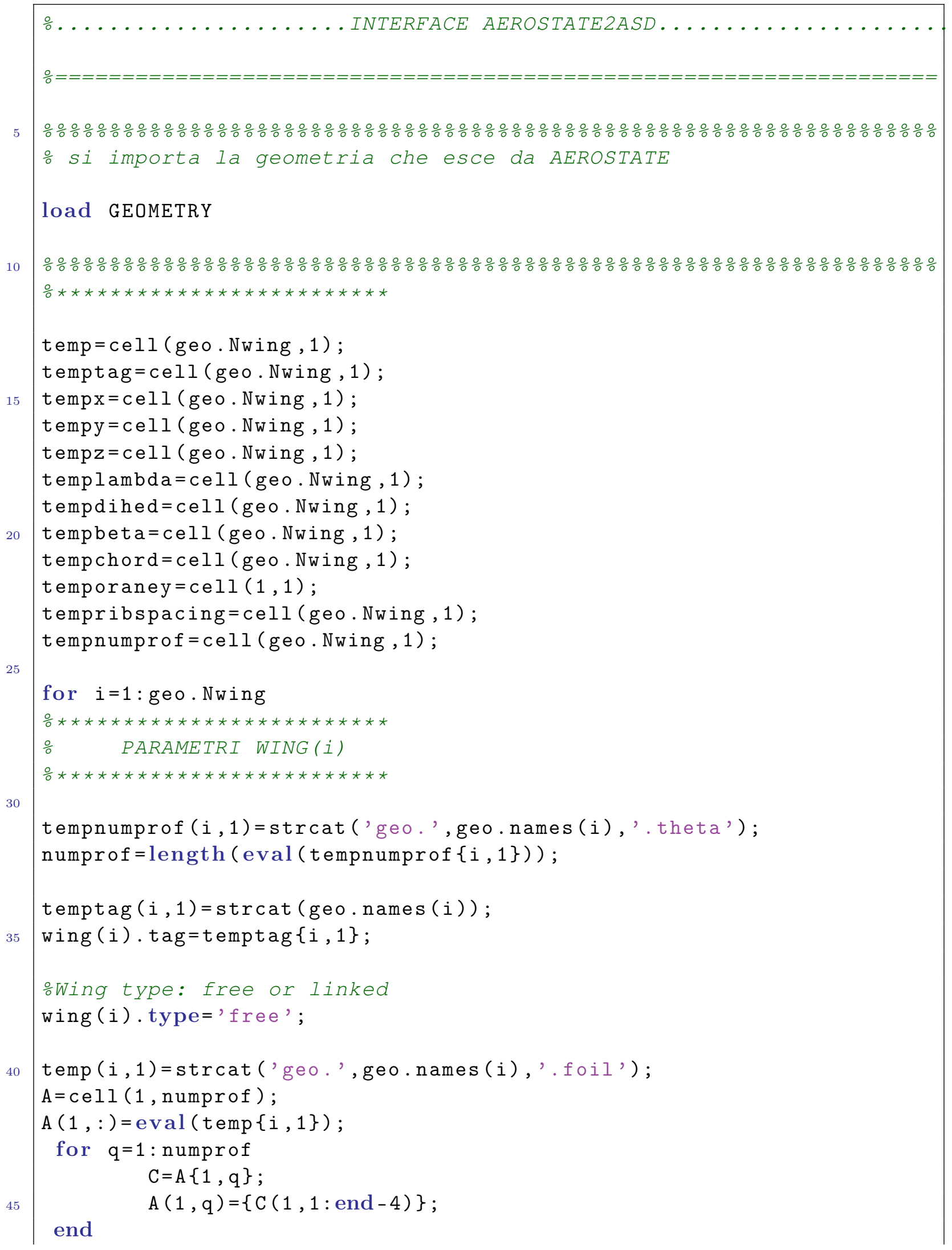




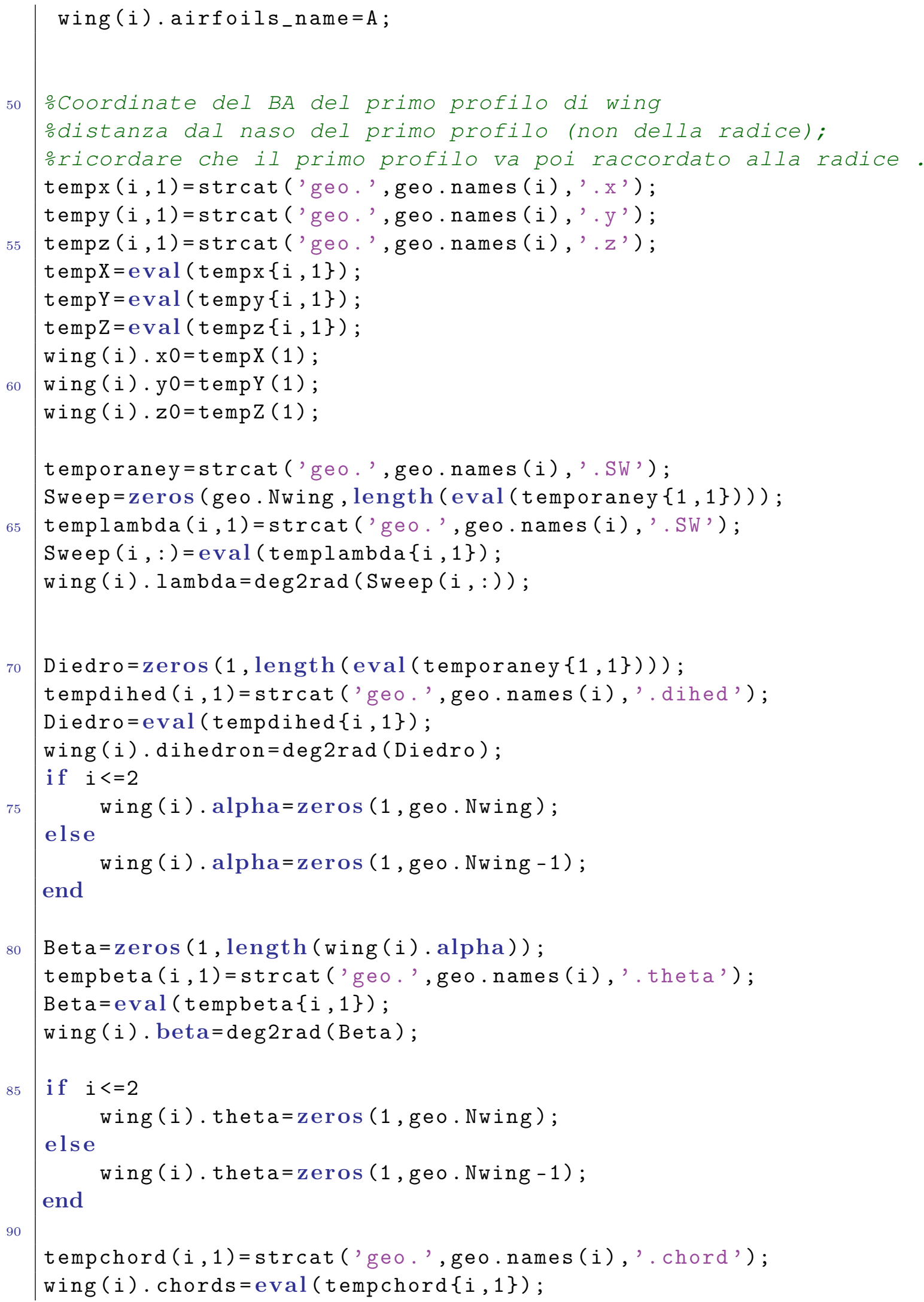


95 $y=\operatorname{eval}($ tempribspacing $\{i, 1\})$

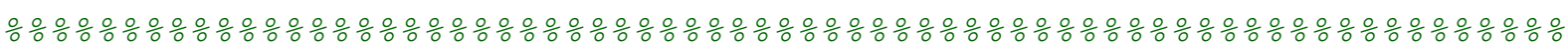
응 Determinazione del rib_spacing 


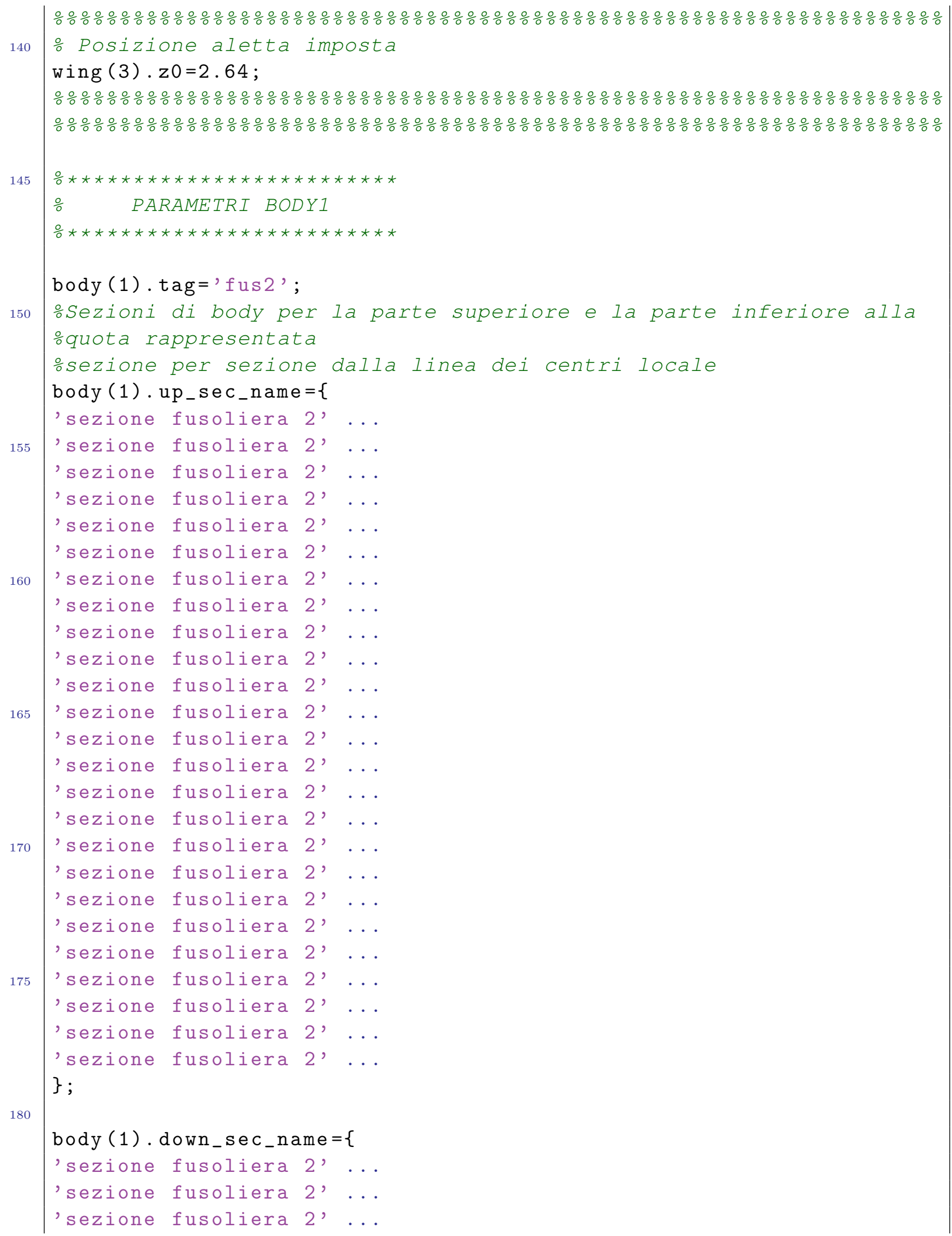




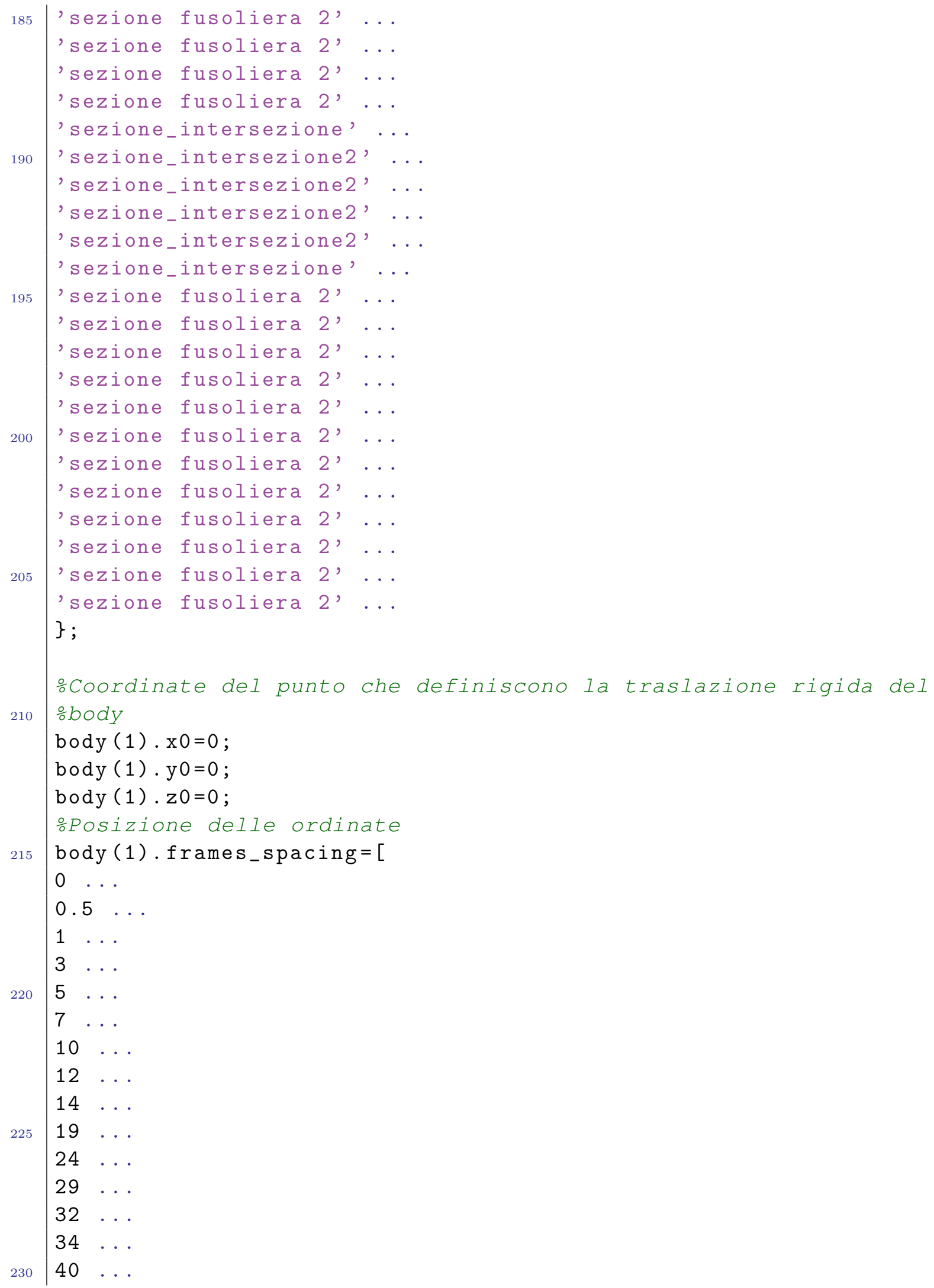




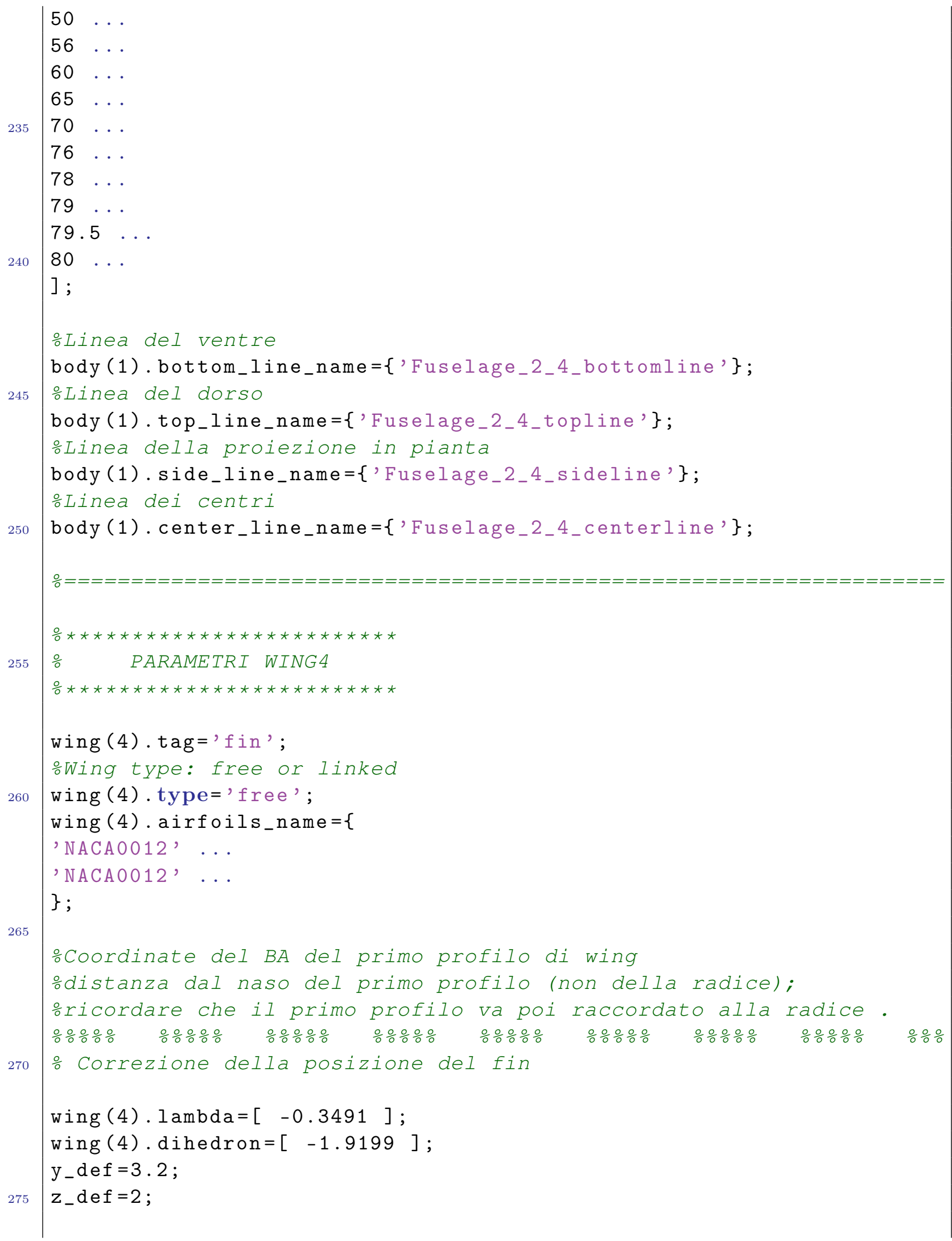


$y_{-}$star $=\left(y_{-}\right.$def $* \tan ($ wing $(4)$. dihedron $)-z_{-}$def + wing $\left.(2) . z 0\right) / \ldots$ 
bulk(1). ctrline_up_wing=[ $\left.\begin{array}{cc}1 & 1\end{array}\right]$; 



\section{Chapter 5}

\section{Research of preliminary optimum configurations}

AEROSTATE and ASD provide the mathematical and graphical tools to study new PrandtlPlane ${ }^{\circledR}$ configurations. INTERFACE is a suitable MATLAB ${ }^{\circledR}$ environment that allows ASD to get the necessary information from AEROSTATE to draw a 3D sketch of a particular aircraft. All these tools are extensively used in the present chapter to research a preliminary optimum configuration. Firstly, main design parameters are considered: the aim of the first analysis is to set an appropriate front wing loading range, in order to use it in the following analyses. Then the sensitivity to the "k bulk" parameter (see the objective function - Chapter 3) has been studied. Once the "k bulk" parameter has been set, the analysis about the front wing loading is repeated as validation. Hence, other analyses have been necessary in order to set the rear wing loading, the auxiliary wing loading, the fins height and the wingspan.

The chosen airfoil is a six digits NACA airfoil (NACA 642415) for each analysis: it ensures good performances in subsonic conditions because of a large laminar zone. The airfoil $c_{d}$ is lower and a suitable value of the airfoil $c_{l}$ can be selected during the optimizations process to increase the aerodynamic efficiency.

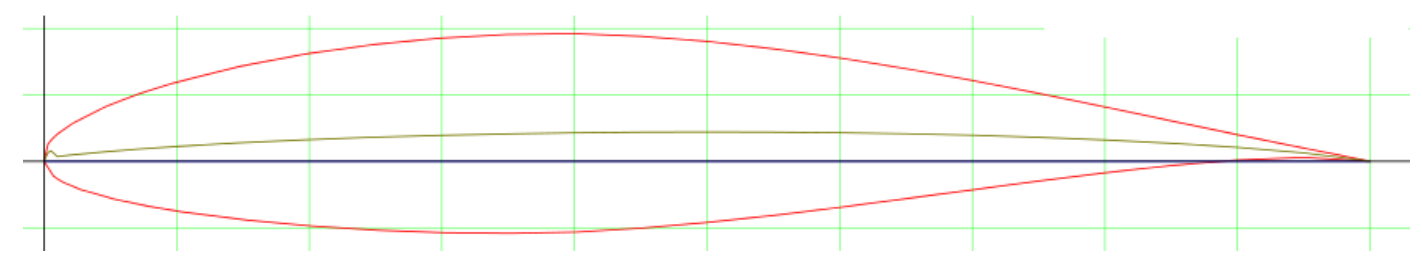

Figure 5.1. NACA 642415 
Aerodynamic features are shown in Fig. $5.2\left(\right.$ Reynolds $\left.=10^{6}\right)$.
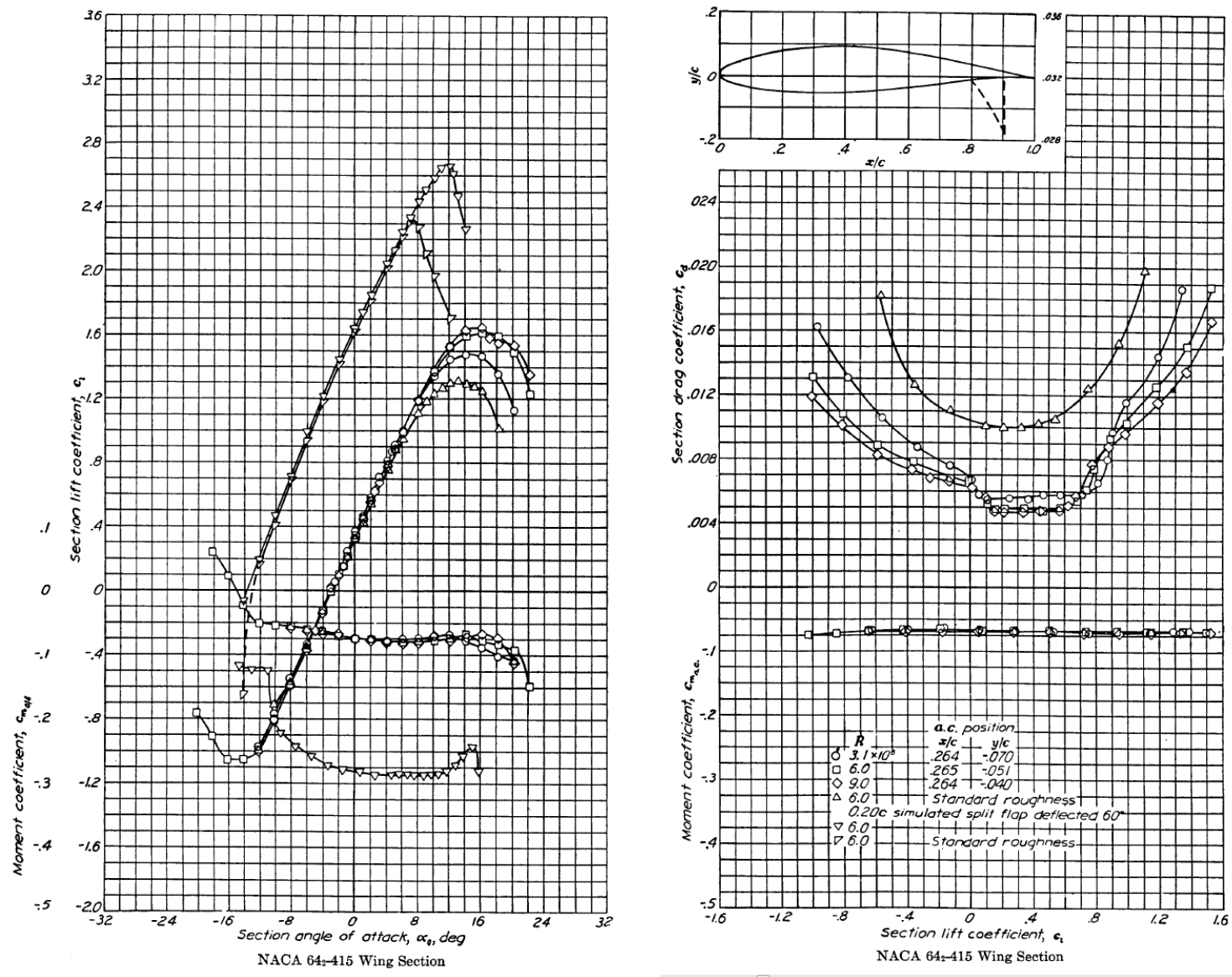

Figure 5.2. NACA 642415 - Aerodynamic features - Source: Abbott

\subsection{A preliminary study: front wing loading variation}

One of the most important aerodynamic parameters is the wing loading, that heavily affects performances, aerodynamics and flight mechanics. Wing loading for conventional planes identifies the aircraft class: Boeing 737-1 and Airbus 320 have similar wing loading (respectively $568 \mathrm{~kg} / \mathrm{m}^{2}$ and $636 \mathrm{~kg} / \mathrm{m}^{2}$ ) while Airbus 340 wing loading is higher than $860 \mathrm{~kg} / \mathrm{m}^{2}$ (refer to Fig. 5.3). The graph of wing loading vs MTOW has a growing trend, if the last two aircraft are not considered (they are A380 and AN225): thus, when MTOW is very high, the wing loading seems to not increase any more probably because of structural and stability limits. The conventional wing loading is computed considering the total aircraft weight in a specific mission point (e.g. take off or cruise design point) and the wing surface: the tail is not considered in this case because it is significantly smaller than the wing. Moreover the tail could lift or deport while the wing always lifts. This last aspect explains why the tail is neglected. 


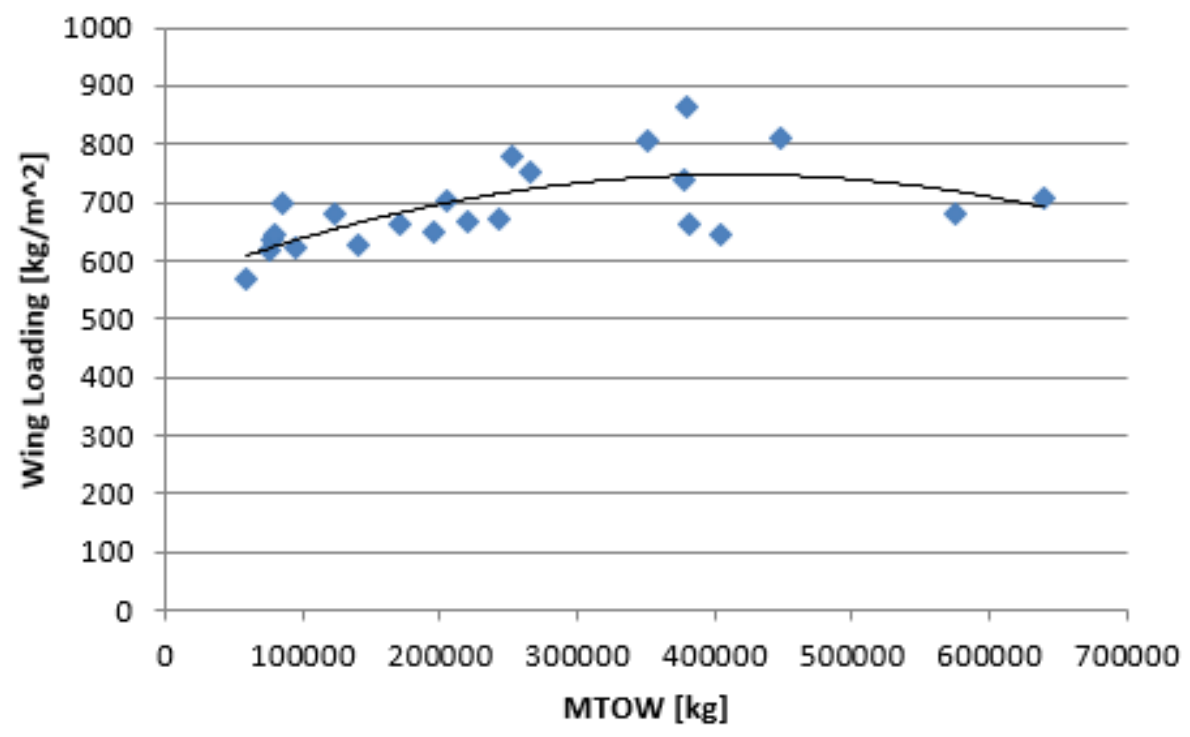

Figure 5.3. Wing Loading vs MTOW for current aircraft

In a PrandtlPlane ${ }^{\circledR}$ aircraft all surfaces contribute to the total lift; so, every wing has its proper wing loading defined as the portion of the weight on that wing divided by its surface (refer to eq. 5.1, where $W$ is the design weight considered at a quarter of the total cruise range).

$$
\left(\frac{W}{S}\right)_{i}=\frac{L_{i}}{S_{i}}
$$

The wing loading of the whole configuration is given by eq. 5.2 , where it can be observed that the front, the rear and the auxiliary wing loadings can also overcome the global wing loading because a scale factor $S_{i} / S_{r e f}$ is present $\left(S_{r e f}\right.$ is the sum of all wing surfaces).

$$
\left(\frac{W}{S_{\text {ref }}}\right)=\left(\frac{W}{S}\right)_{\text {front }}\left(\frac{S_{\text {front }}}{S_{\text {tot }}}\right)+\left(\frac{W}{S}\right)_{\text {rear }}\left(\frac{S_{\text {rear }}}{S_{\text {tot }}}\right)+\left(\frac{W}{S}\right)_{\text {aux }}\left(\frac{S_{\text {aux }}}{S_{\text {tot }}}\right)
$$

The first analysis deals with the front wing loadings in Tab. 5.1; the rear wing and the auxiliary wing loadings have been assumed to vary in a wide range, so that the resultant configuration depends only on the front wing loading. We assume (in $\mathrm{kg} / \mathrm{m}^{2}$ ):

The effects of the variation of the rear wing loading will be considered in the following sections.

$$
\begin{gathered}
0<\left(\frac{W}{S}\right)_{\text {rear }}<900 \\
0<\left(\frac{W}{S}\right)_{\text {auxwing }}<900
\end{gathered}
$$




\begin{tabular}{ccc}
\hline Analysis & $(W / S)_{\text {front }}-L B$ & $(W / S)_{\text {front }}-U B$ \\
\hline 1 & 400 & 600 \\
\hline 2 & 500 & 700 \\
\hline 3 & 600 & 800 \\
\hline 4 & 700 & 900
\end{tabular}

Table 5.1. Front Wing loading Lower Boundaries (LB) and Upper Boundaries (UB)

\subsubsection{Parameters setting and starting geometry file}

The starting shape is shown in Fig. 5.4 and its main characteristics are summarized in Tab. 5.2. In the code AEROSTATE just the half span of the wings is consider; there are three control sections for the front and the rear wings, two control sections for the auxiliary wing and, hence, there are two bays for front and rear wings (corresponding to arrays of two components, refer to Tab. 5.2) and only one bay for the auxiliary wing.

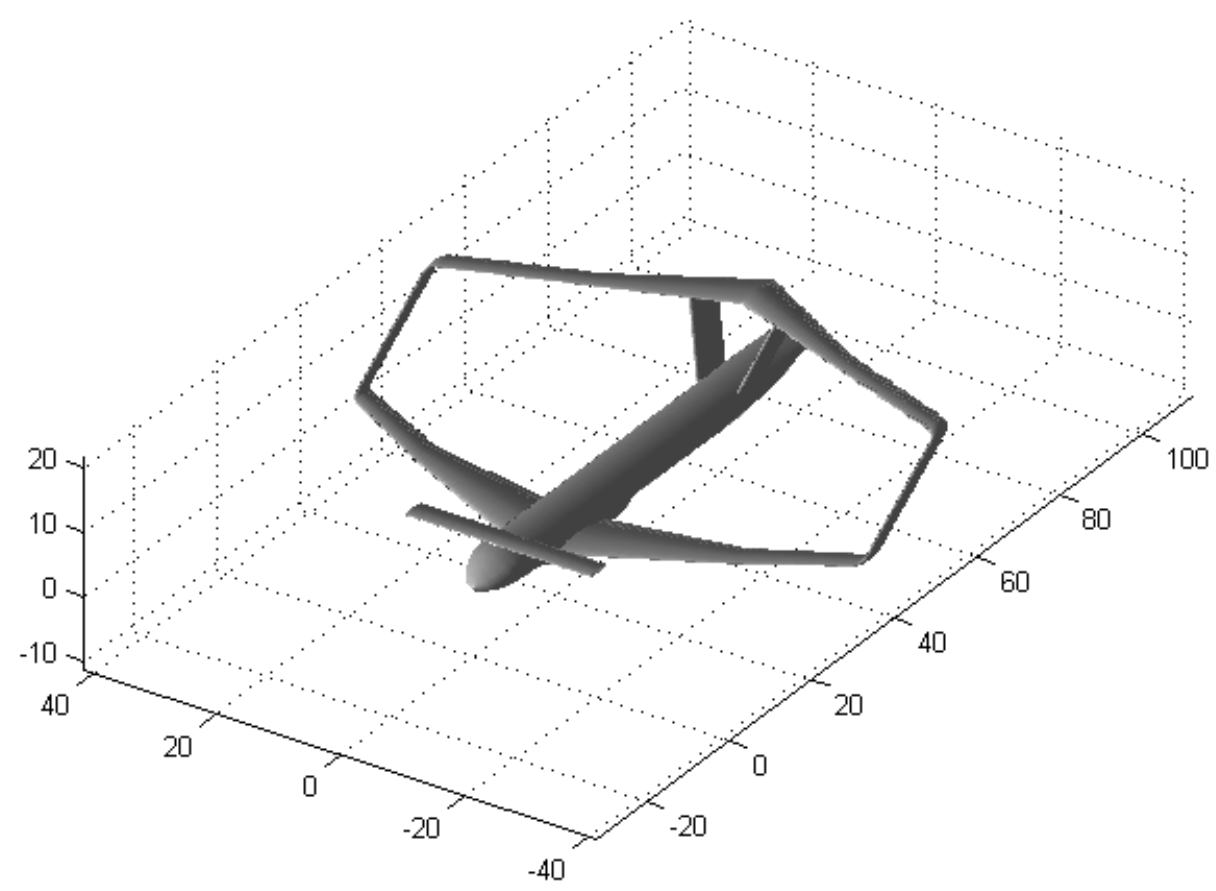

Figure 5.4. Starting Geometry 


\begin{tabular}{|c|c|c|c|c|}
\hline Type & Whole Configuration & Front Wing & Rear Wing & Winglet \\
\hline Design Weight $[k g]$ & 565000 & - & - & - \\
\hline $\mathbf{X C G}[m]$ & 38.97 & - & - & - \\
\hline $\mathbf{b}[m]$ & 80 & 80 & 80 & 30 \\
\hline MAC $[m]$ & 6.22 & 7.52 & 5.33 & 4 \\
\hline Stot $\left[m^{2}\right]$ & 1000.8 & 480.8 & 400 & 120 \\
\hline Aspect Ratio & 6.4 & 10.7 & 16 & 7.5 \\
\hline Pitch Angle $[d e g]$ & - & 0 & 0 & 0 \\
\hline Twist Angle $[\mathrm{deg}]$ & - & {$\left[\begin{array}{ll}0 & 0\end{array}\right]$} & {$\left[\begin{array}{ll}0 & 0\end{array}\right]$} & 0 \\
\hline Sweep Angle $[\mathrm{deg}]$ & - & [24 28.07] & {$[-20-20]$} & 0 \\
\hline Dihedral $[d e g]$ & - & {$\left[\begin{array}{ll}2 & 2\end{array}\right]$} & {$\left[\begin{array}{ll}0 & 0\end{array}\right]$} & 0 \\
\hline Taper Ratio & - & {$\left[\begin{array}{ll}0.4 & 0.8\end{array}\right]$} & {$\left[\begin{array}{ll}0.5 & 1\end{array}\right]$} & 1 \\
\hline
\end{tabular}

Table 5.2. Configuration features

The angle of attack of the fuselage, i.e. the angle between the flow direction and the fuselage axis, is set zero (in AEROSTATE we put "alpha $=0$ "), in order to minimize the induced drag of the fuselage. In fact, the fuselage is assumed as a plane plate in the aerodynamic model and, thus, induced drag should be added when alpha increases, but by putting alpha $=0$, the model is simpler and the calculation faster. Furthermore, the fuselage floor is horizontal during cruise and the fuselage products no lift. The front wing crosses the fuselage and precautionary, the lift distribution on the front wing in the fuselage is set zero (Fig. 5.5).

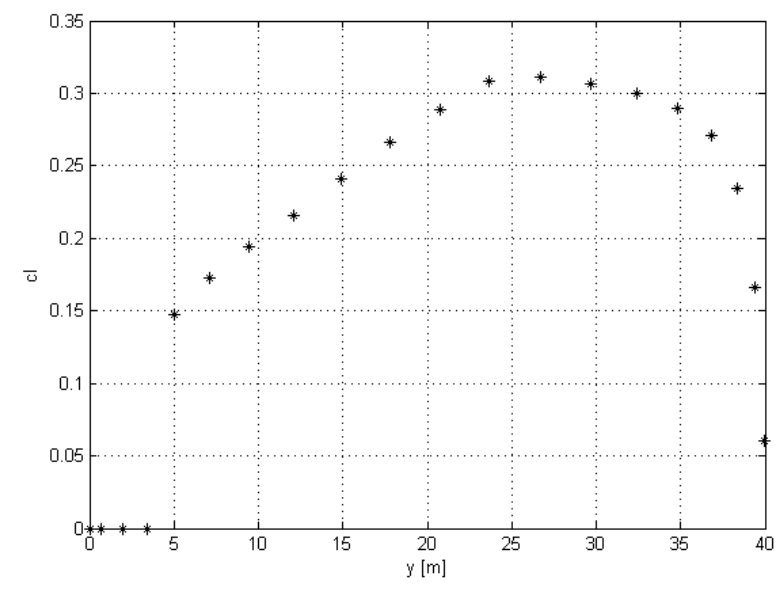

Figure 5.5. Lift distribution 
The Margin of Stability is limited to the range $(0 \%-3 \%)$, lower than conventional $(10 \%-15 \%)$ because, the PrandtlPlane ${ }^{\circledR}$ configuration needs lower Margins [14]: indeed, the $M_{q}$ derivative is very high in a PrandtlPlane ${ }^{\circledR}$ and it affects the short period frequency, according to eq. 5.5.

$$
\omega_{S P}=\sqrt{-M_{\alpha}+Z_{w} M_{q}}
$$

Good flight qualities need a proper range of $\omega_{S P}$ and hence, being $M_{q}$ high and negative, the only way to reduce the maximum value of $\omega_{S P}$ is to reduce $M_{\alpha}$, that is to reduce the Margin of Stability $(M S)$. The assumed range of $M S$ ensures sufficient stability and maneuverability. 


\subsubsection{Analysis and Results}

In the next figures, the results are examined; every result of AEROSTATE's analysis is a local or a global optimum configuration.
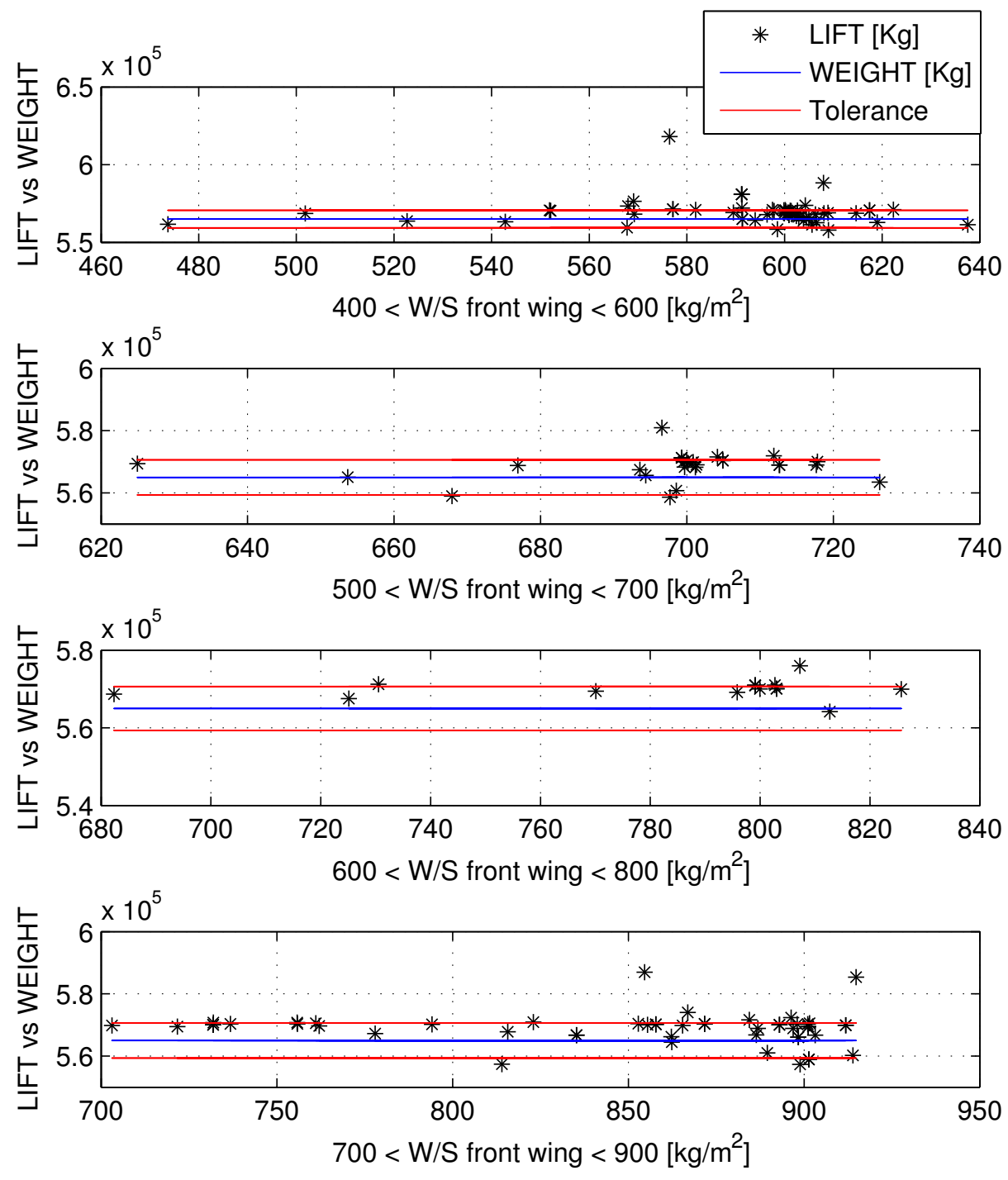

Figure 5.6. Check on the equilibrium condition.

The vertical equilibrium $W=L_{t o t}$ is introduced in the optimization by means of an inequality constraint:

$$
\left|W-L_{t o t}\right|<\epsilon
$$

In Fig. 5.6 the vertical equilibrium is evaluated for all the results of runs 1-4. The red line indicates the tolerance limits defined trough the coefficient $\epsilon( \pm 1 \%$ of $W)$ while each configuration is represented with the star symbol. 
It is possible that some configurations could violate some constraints, because, as a matter of fact, this situation is possible in AEROSTATE if the most constraints are fulfilled (thus, interesting configurations are not eliminated, to be checked next). The numerical violation of constraints allows to reach the global minima in a fast way.

Fig. 5.7 can be assumed as a check of the whole calculation in the optimization performed; from the equilibrium conditions, the relationship between $C_{L}$ and $\left(\frac{W}{S}\right)_{g e n}$ becomes

$$
C_{L}=\frac{1}{q}\left(\frac{W}{S}\right)_{g e n}=A\left(\frac{W}{S}\right)_{g e n}
$$

The coefficient $A$ has been computed theoretically $\left(A_{\text {theo }}=7.03 * 10^{-4} \mathrm{~m}^{2} / \mathrm{kg}\right)$ and from Fig. $5.7\left(A_{\text {graph }}=7.14 * 10^{-4} \mathrm{~m}^{2} / \mathrm{kg}\right)$, with an error less than $2 \%$.

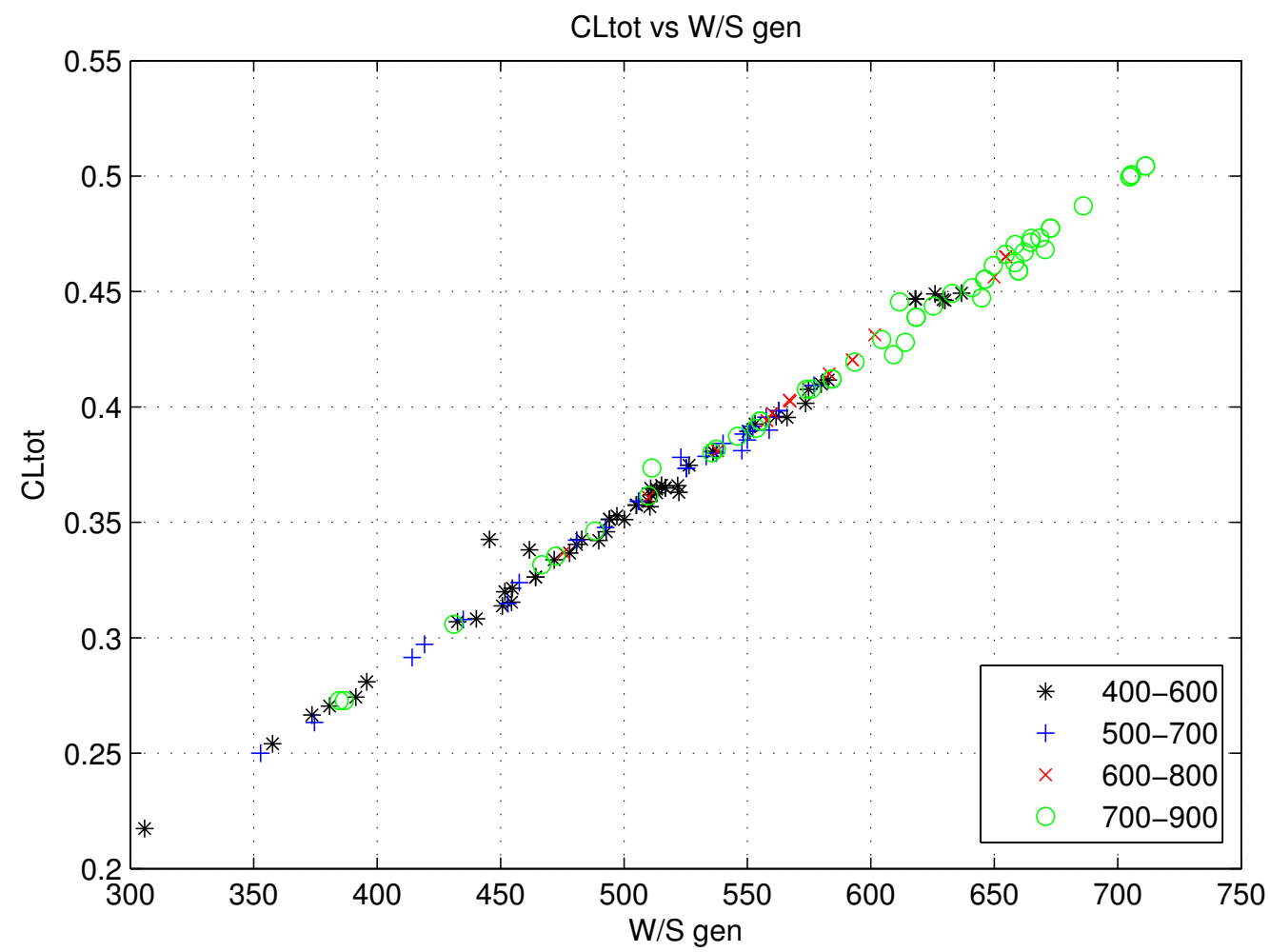

Figure 5.7. Global CL vs global Wing Loading.

Since the vertical equilibrium is respected, the role of the rear wing and of the auxiliary wing, varying the maximum wing loading, is reported in Fig. 5.11 and Fig. 5.12.

The most important aerodynamic parameter is the efficiency, E. Fig. 5.8 and Fig. 5.9 show how $E$ is affected by the front wing loading and by the wing loading of the whole configuration. If the front wing loading increases, the efficiency increases too; the wing loading limitations come from structural problems and low speed aerodynamics $\left(C_{\text {Lmax }}\right)$. It can be inferred that the optimizer tends to consider only the upper boundary of the front wing loading in order to reach high efficiencies. 
The definition of the global wing loading is the following:

$$
\left(\frac{W}{S}\right)_{g e n}=\frac{W_{\text {design }}}{S_{\text {ref }}}
$$

The efficiency $E$ is a growing function of the wing loading $\left(\frac{W}{S}\right)_{\text {gen }}$ (Fig. 5.9); the trend shown in Fig. 5.9 is justified by considering that:

$$
E=\frac{1}{q}\left(\frac{W}{S}\right)_{g e n} \frac{1}{C_{D}}
$$

where the dynamic pressure, $q$, is the same for all the configurations (the altitude and the Mach number are given and are $h=6000 \mathrm{~m}$ and $M=0.65$ respectively); $C_{D}$ depends on the $\left(\frac{W}{S}\right)_{g e n}$ and the relationship is nearly linear (Fig. 5.14).

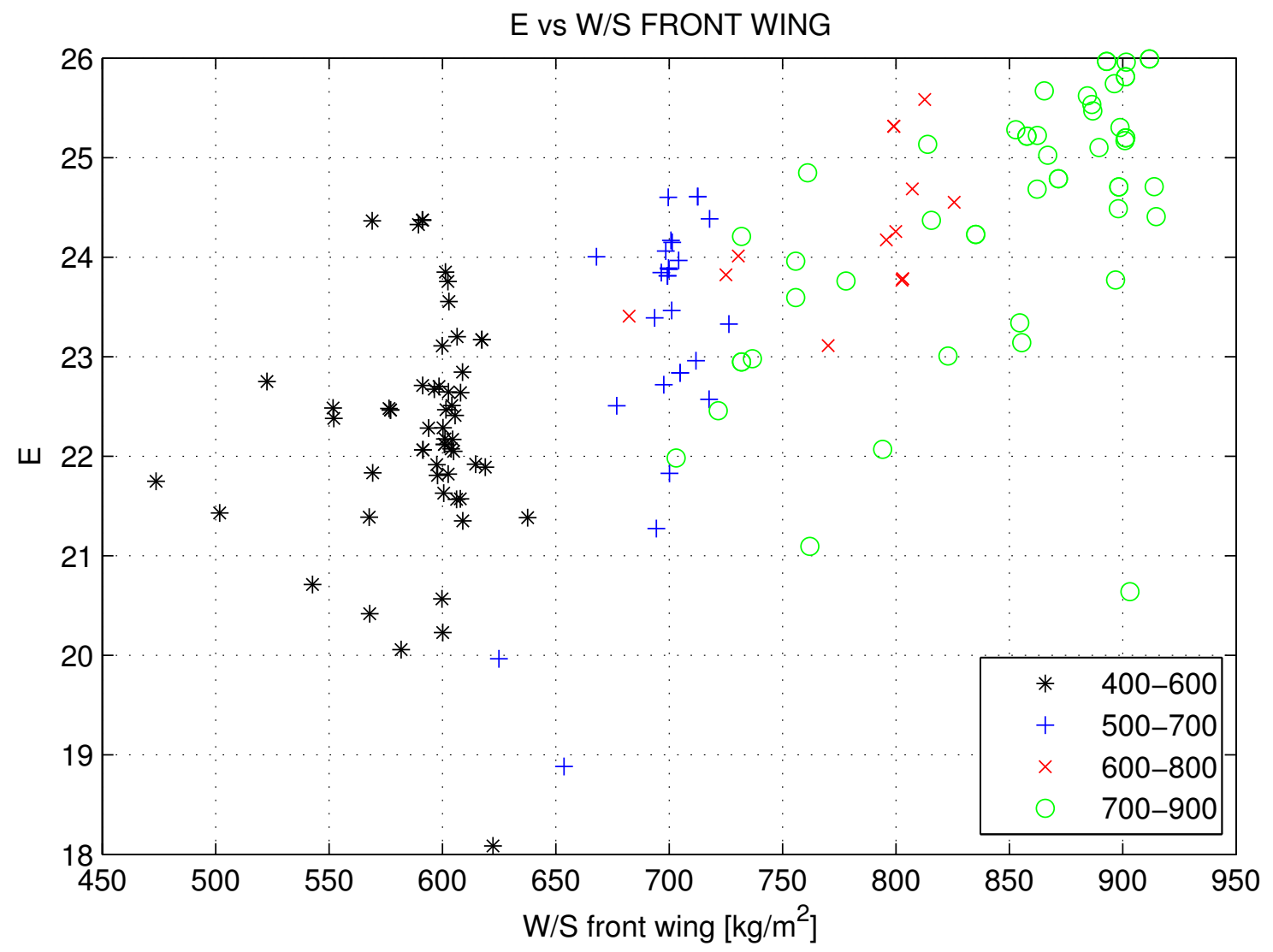

Figure 5.8. Efficiency vs front Wing Loading.

When the front wing loading varies between $400 \mathrm{~kg} / \mathrm{m}^{2}$ and $600 \mathrm{~kg} / \mathrm{m}^{2}$, the lift acting on the auxiliary wing and the surface of it are significant. When the front wing loading increases, the auxiliary wing tends to disappear: its surface becomes smaller and smaller and the lift of the aircraft is not affected at all by the presence of the auxiliary wing. It can be inferred by the previous results that the configurations with three lifting surfaces have no sense for wing loading increases over $800 \mathrm{~kg} / \mathrm{m}^{2}$. 


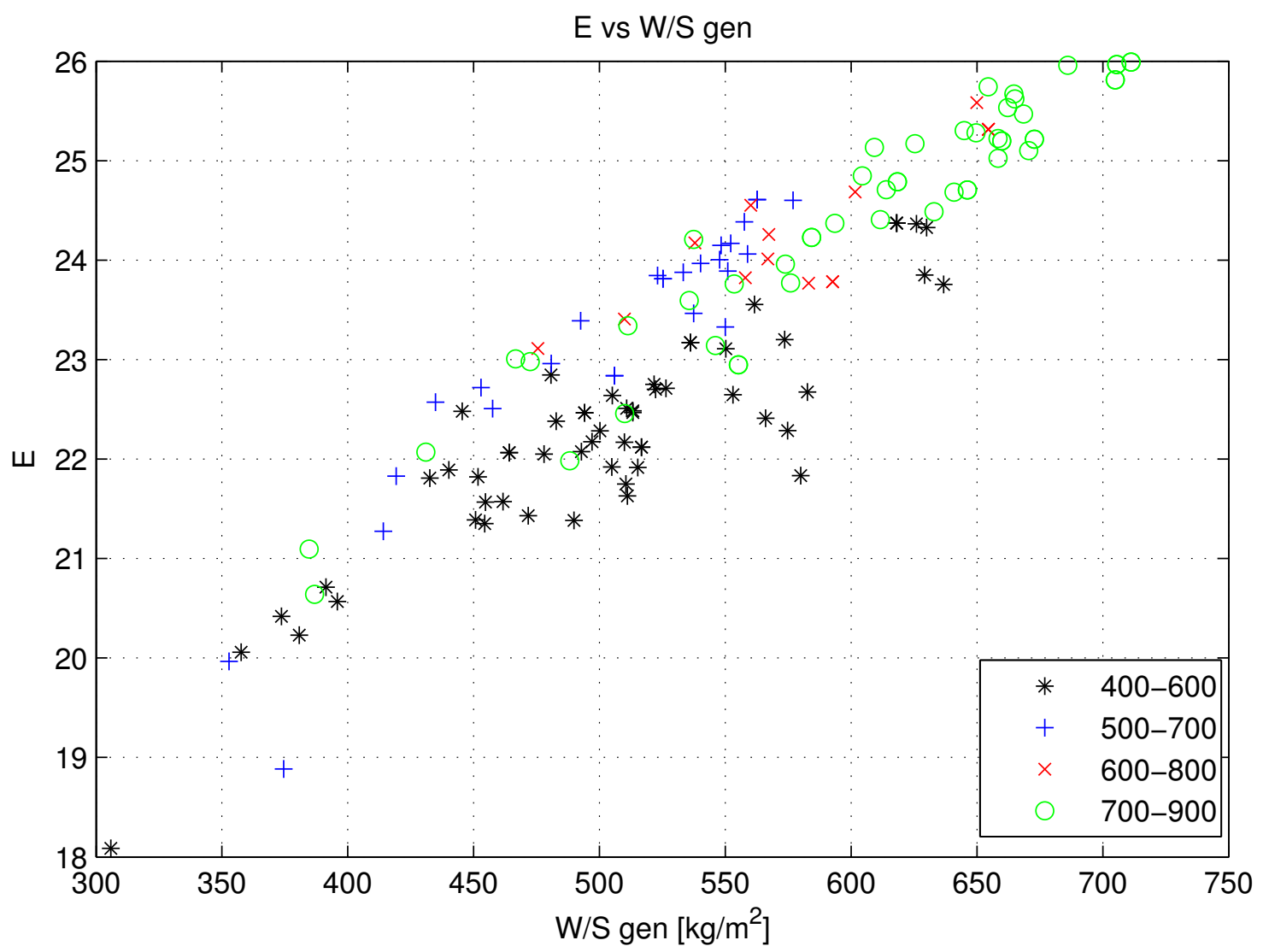

Figure 5.9. Efficiency vs global Wing Loading.

The rear wing characteristic are not influenced by the front wing loading: the average lift is between $10 \%$ and $40 \%$ of total and the surface is always between $30 \%$ and $50 \%$. The rear wing loading is not constrained in these preliminary analyses; thus, the main role of the rear wing is to ensure the right Margin of Stability, in the limits given before $(0-3 \%)$, which are fixed. Further remarks will be deepened afterwards.

Fig. 5.10 underlines the importance of the global Aspect Ratio in the PrandtlPlane ${ }^{\circledR}$ configuration, where, by definition,

$$
A R_{g e n}=\frac{b^{2}}{S_{t o t}}
$$

The trend shown in fig. 5.10 is easily explained by rearranging eq. 5.9 into eq. 5.11, to obtain:

$$
E=\frac{1}{q} \frac{W_{\text {design }}}{b^{2}} \frac{A R_{\text {gen }}}{C_{D}}
$$

where $q, W_{\text {design }}$ and $b$ are all constants. In a PrandtlPlane ${ }^{\circledR}$ configuration, all the surfaces give a positive lift and two of them are significant because of their sizes; instead, in a conventional monoplane configuration, the tail is used just for trim setting and for maneuvers without any contribution to the lift. 
The values of $A R_{g e n}$ in fig. 5.10 are lower than those for a conventional monoplane, because now, the whole lifting surface is considered, contrary to the conventional; it is very interesting to remark that the way to improve the efficiency is to decrease all the surfaces of the PrandtlPlane ${ }^{\circledR}$ aircraft and, thus, with a given span, all the wings need to be slender.

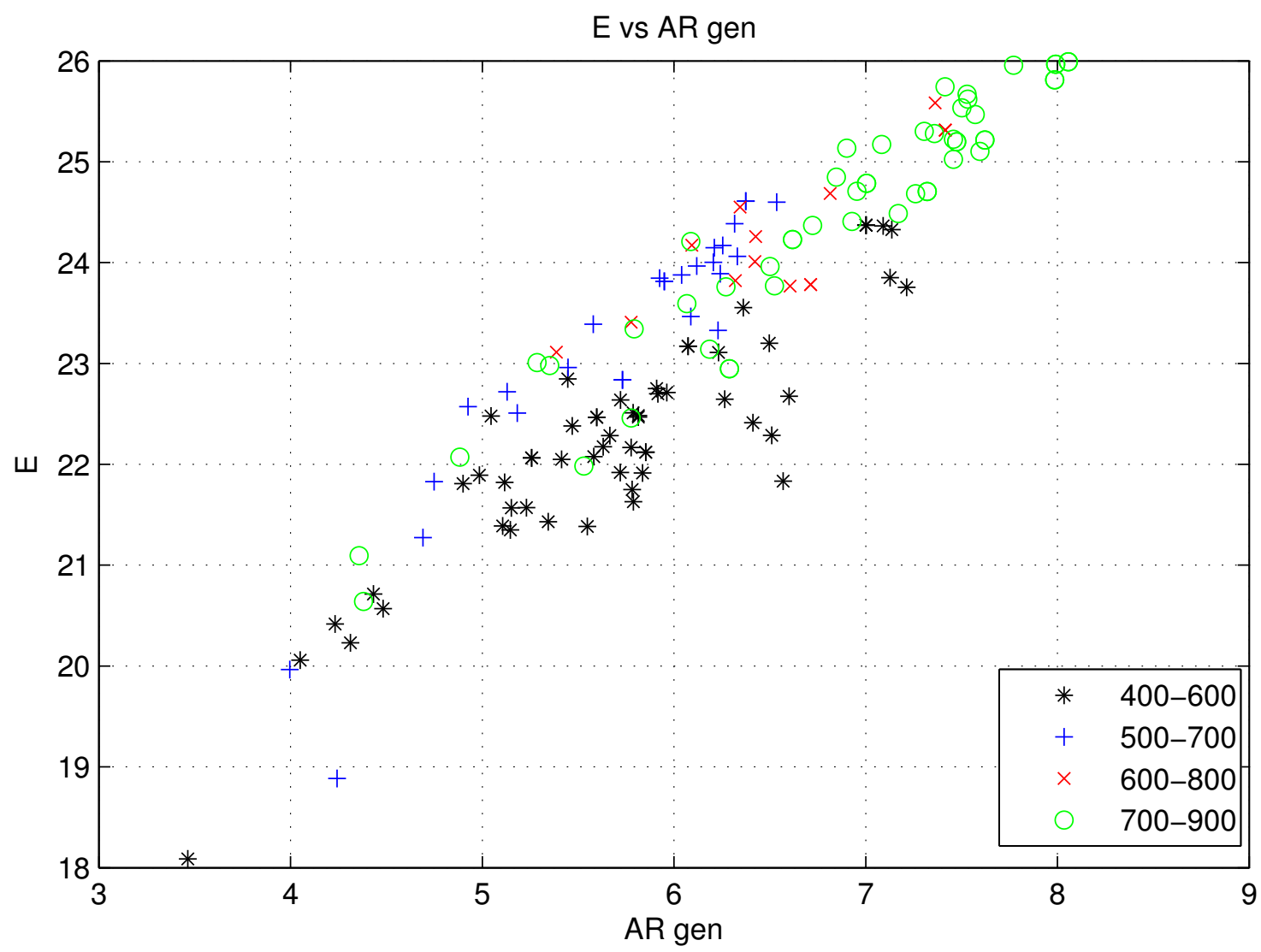

Figure 5.10. Efficiency vs global Aspect Ratio.

Fig. 5.15 and Fig. 5.16, together with Fig. 5.13, show how the auxiliary wing is not necessary with very high values of the front wing loading. Looking at the $C_{L}$ graphs (fig. 5.15 and fig. 5.16), it can be noticed that $C_{L}$ of the whole configuration increases as the front wing loading increases, while it decreases as the wing loading of the auxiliary wing increases. No evident relationship exists between the rear wing loading and the global $C_{L}$; this fact can be explained as follows: the rear wing can help the front wing to develop the necessary lift, but, at the same time, it is a relatively big surface far enough from the centre of gravity to affect the Margin of Stability significantly, according to the second graph of fig. 5.16. So, many solutions are not related to the global $C_{L}$. A high rear wing loading ensures higher efficiency; yet, it implies small chords, that means a reduced Margin of Stability; then, the optimizer works to meet the condition $0<M S<0.03$ acting in the rear wing chords. 

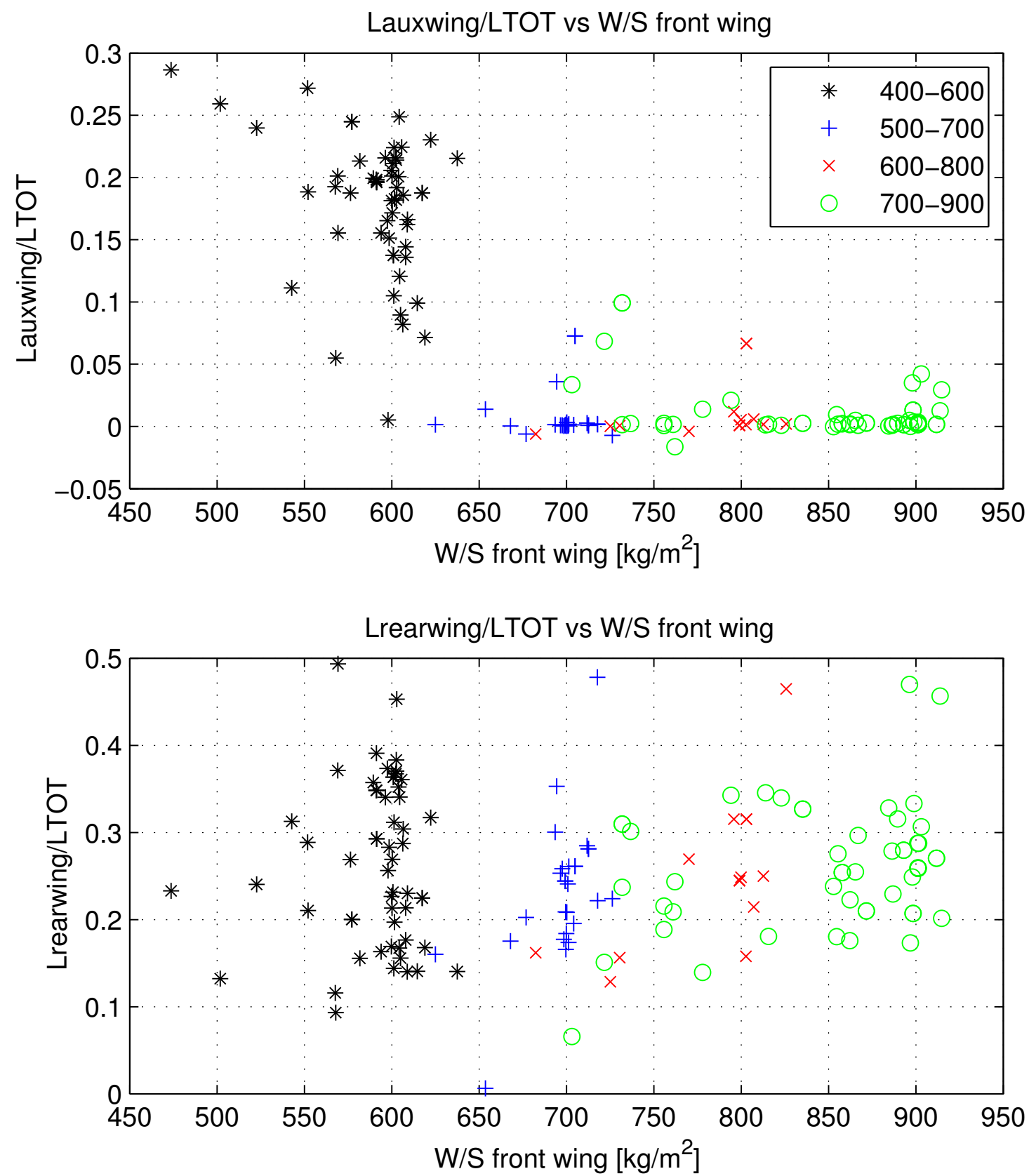

Figure 5.11. Lift of rear wing and auxiliary wing. 

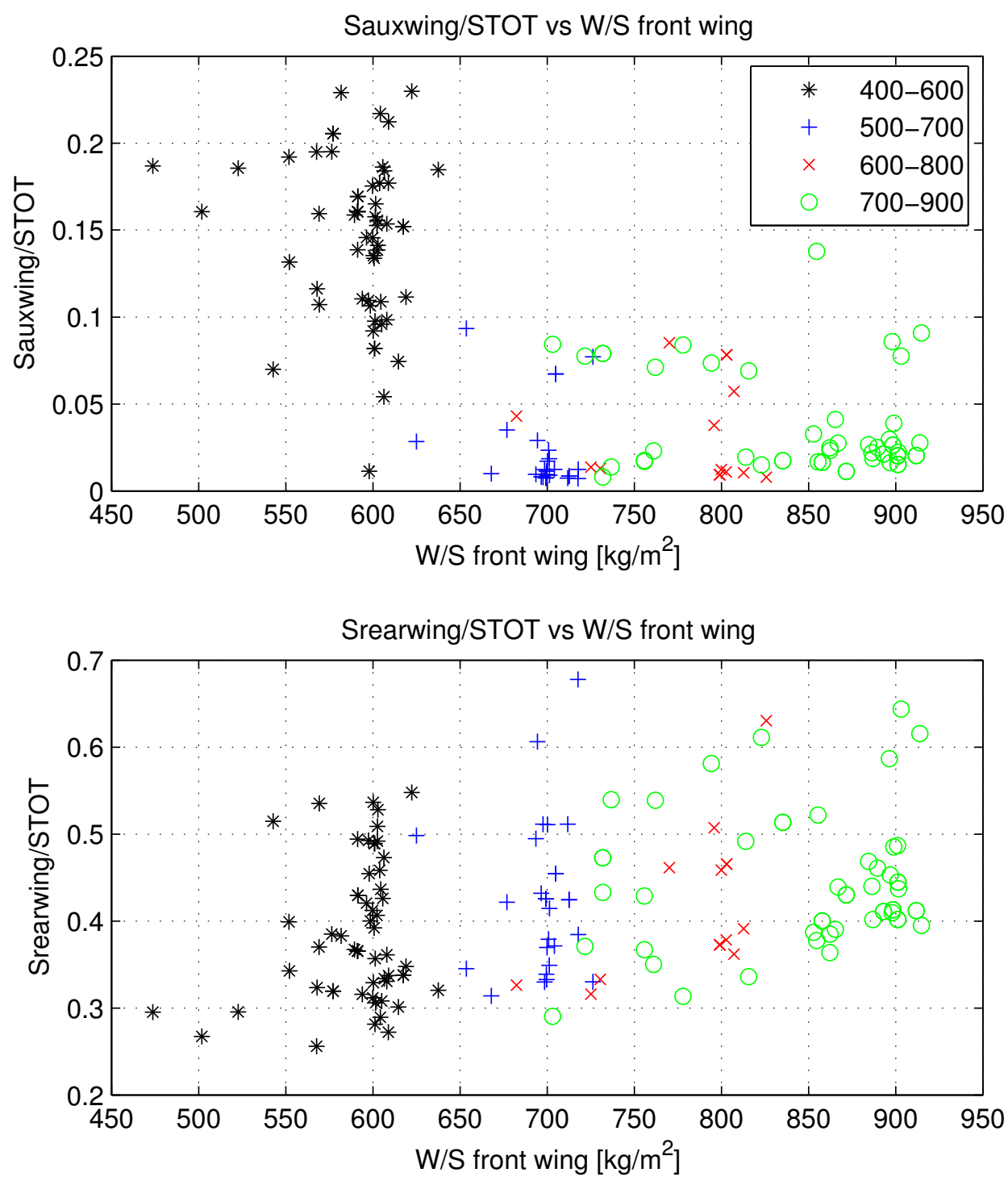

Figure 5.12. Surface of rear wing and auxiliary wing.

Finally, Fig. 5.14 shows that, for a high global wing loading, the induced drag and friction drag coefficients tend to assume the same values. Low global wing loading configurations have the friction drag coefficient about $50 \%$ higher than the induced drag coefficient (the total surface is large). However the slope of the induced drag curve is higher than the friction drag curve, thus, the induced drag is more sensitive to the global wing loading variation. The previous results on the configurations with different front wing loading lead to the choice of $400 \mathrm{~kg} / \mathrm{m}^{2}<\left(\frac{W}{S}\right)_{\text {front }}<600 \mathrm{~kg} / \mathrm{m}^{2}$. This range ensures good efficiency, not too high values of $C_{L}$ and structural feasibility. 


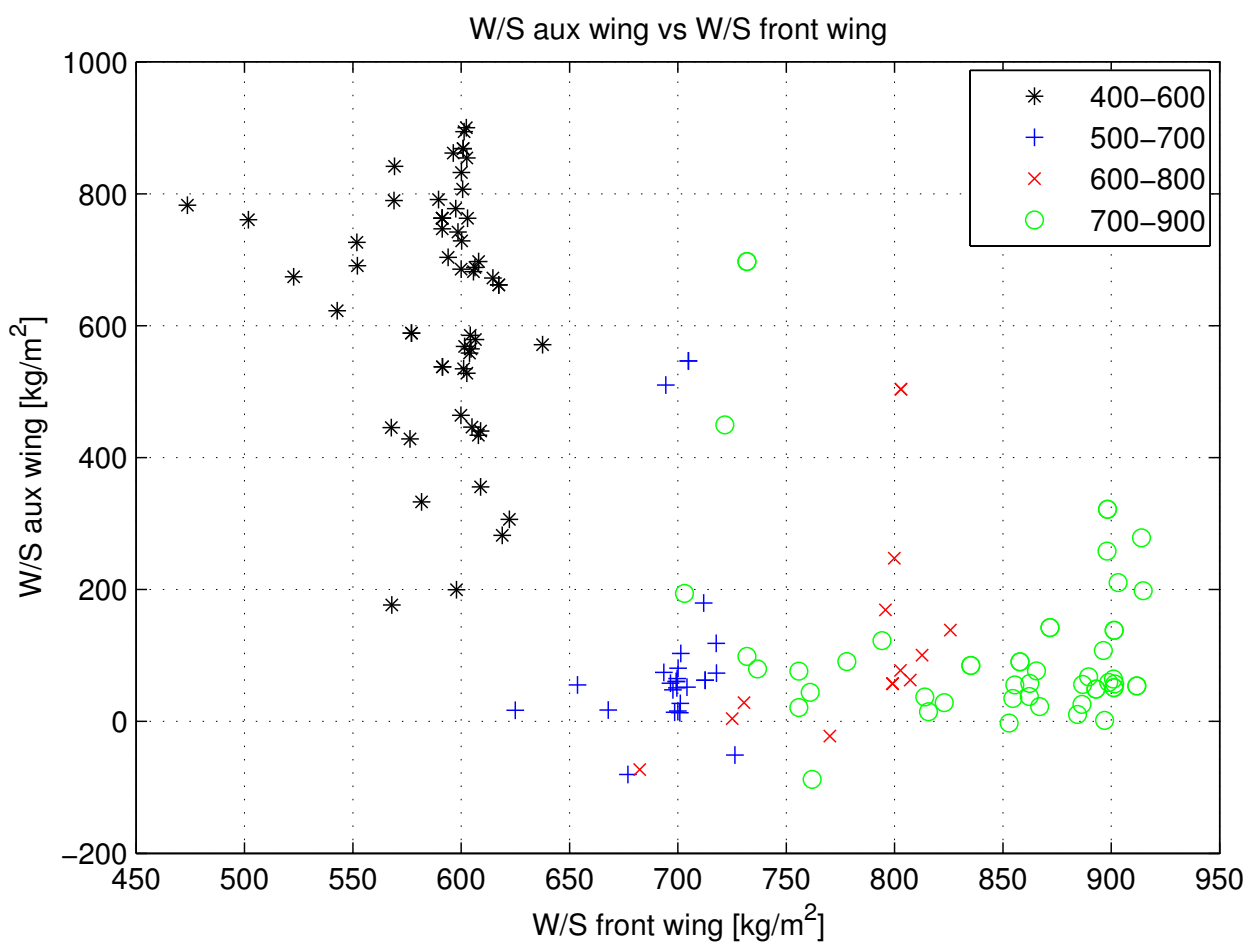

Figure 5.13. Wing Loading: auxiliary wing - front wing relationship.

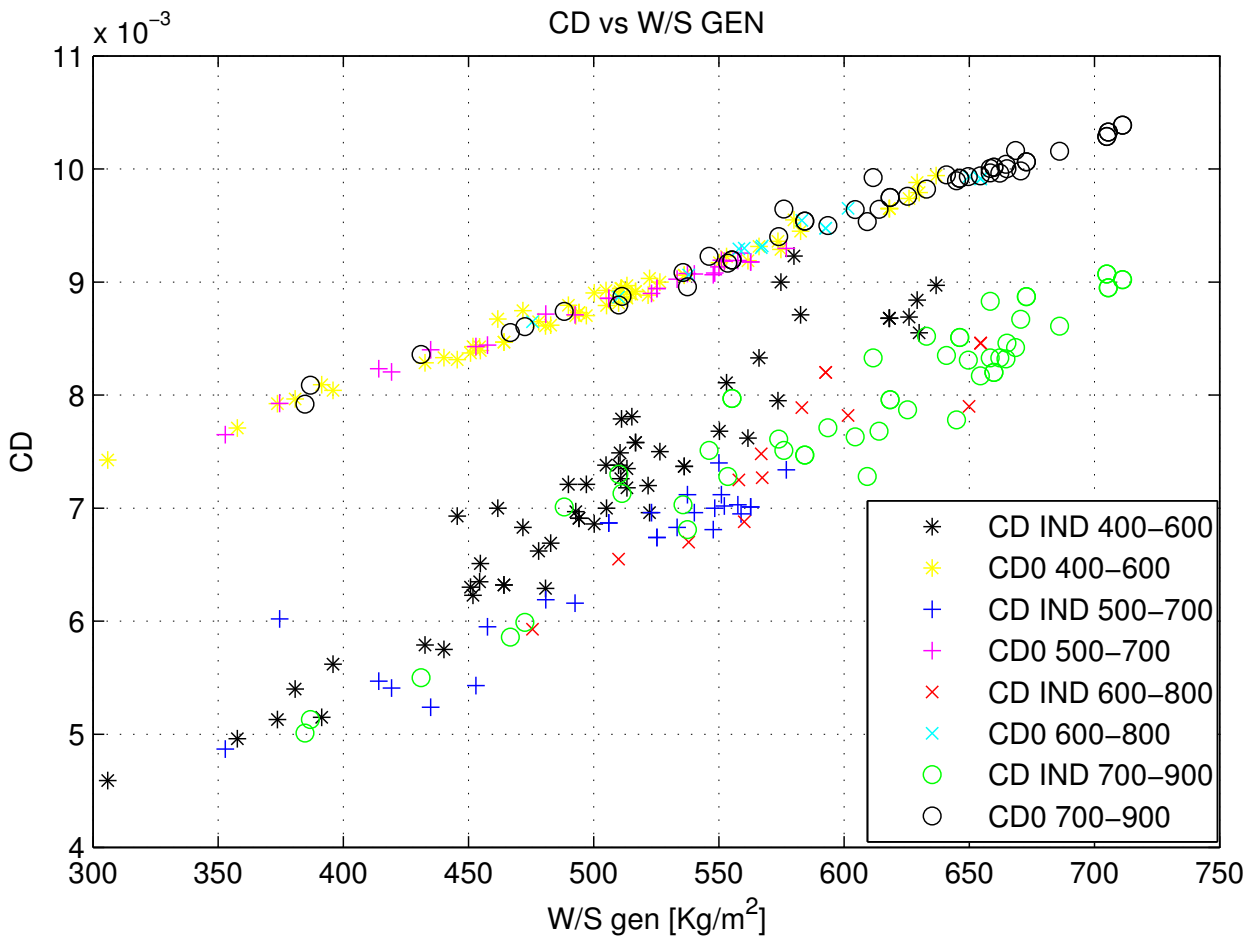

Figure 5.14. CD contributions. 

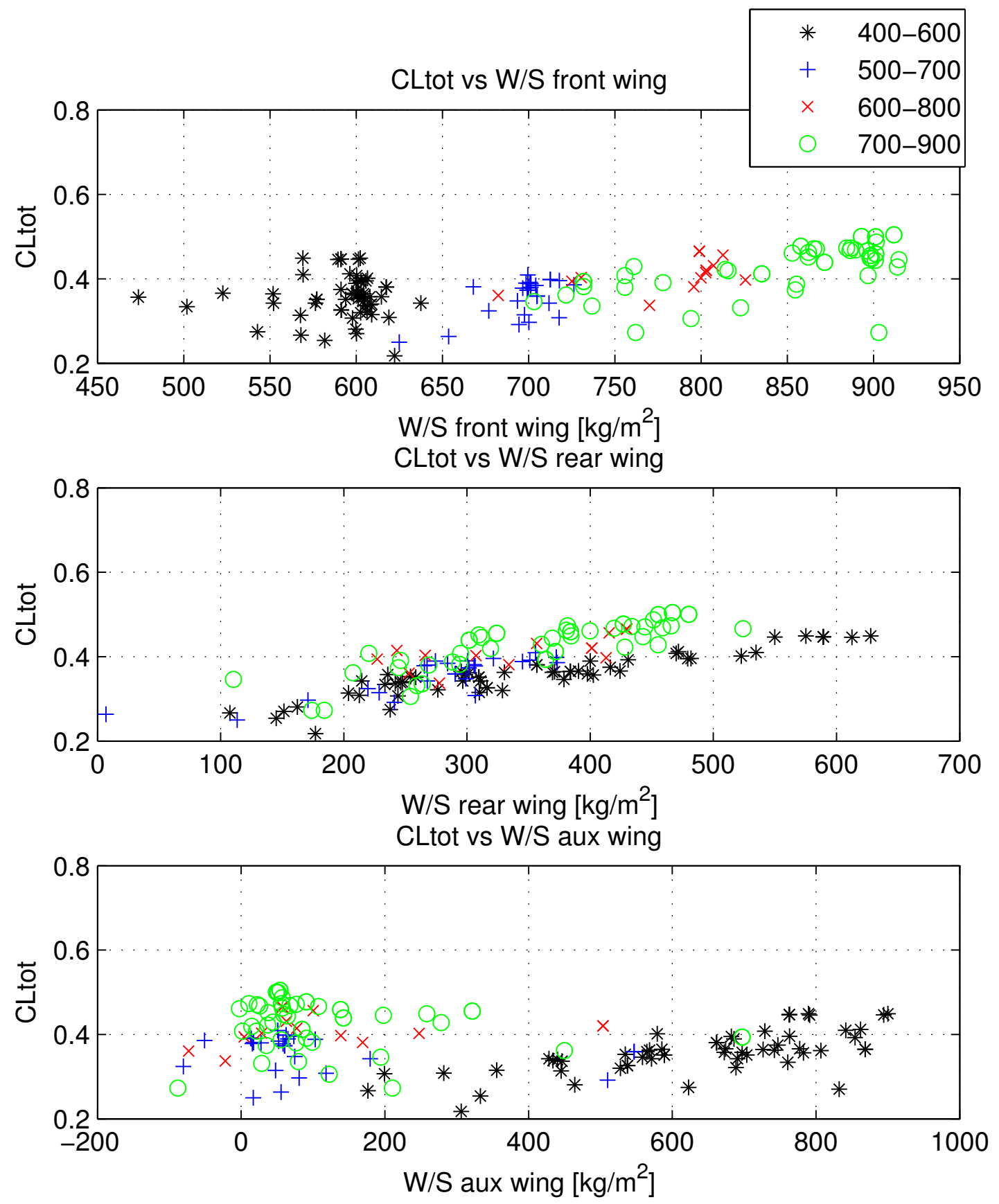

Figure 5.15. CLtot trends. 

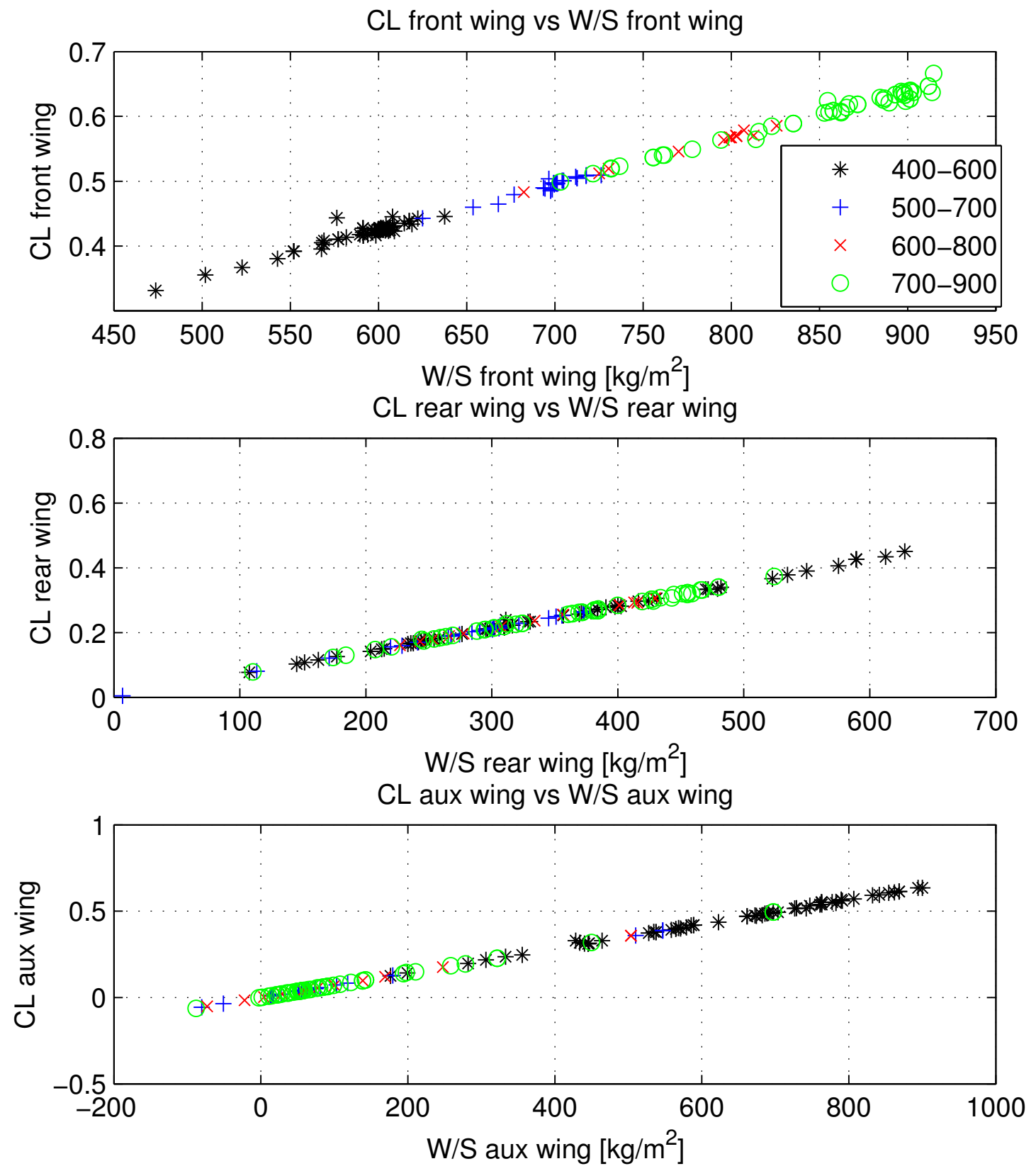

Figure 5.16. CL trends. 


\subsection{The bulk parameter setting}

The objective function of AEROSTATE is (refer to Chapter 3):

$$
O B J=-E+K_{b u l k} L_{b u l k}
$$

The aerodynamic efficiency has to be improved as more as possible and the bulk length has to be reduced, in order to avoid efficient configurations but with long bulks; if bulks are too long, buckling could occur, because they are thin beams from a structural point of view, loaded in compression in some conditions. Thus, the modified objective function, reported in eq. 5.12, allows to take some structural requirements into account in a very preliminary level; the following analyses have been conducted in order to study the effects of $K_{\text {bulk }}$.

The Margin of Stability and the value of alpha are set in the same way of the former analysis. The front wing loading is the one stated in the previous section and the wing loadings of the rear wing and of the auxiliary wing have been slightly modified with a reduction of the ranges of variation. Too low rear wing loadings lead to less efficient configurations and no interesting solutions exist for nearly unloaded auxiliary wings. Thus, new wing loading ranges are established and reported in Tab. 5.3.

\begin{tabular}{ccc}
\hline Lower Boundary $\left[\mathrm{kg} / \mathrm{m}^{2}\right]$ & Wing Loading & Upper Boundary $\left[\mathrm{kg} / \mathrm{m}^{2}\right]$ \\
\hline 400 & Front Wing & 600 \\
\hline 300 & Rear Wing & 700 \\
\hline 200 & Winglet & 900 \\
\hline
\end{tabular}

Table 5.3. Wing loadings - $K_{\text {bulk }}$ Analyses.

Different analyses have been performed by assuming the values of $K_{\text {bulk }}$, reported in Tab. 5.4.

\begin{tabular}{cccccccccccc}
\hline Analysis & 5 & 6 & 7 & 8 & 9 & 10 & 11 & 12 & 13 & 14 & 15 \\
\hline$K_{\text {bulk }}$ & 0 & 0.1 & 0.2 & 0.3 & 0.4 & 0.5 & 0.6 & 0.7 & 0.8 & 0.9 & 1 \\
\hline
\end{tabular}

Table 5.4. $K_{\text {bulk }}$ Analyses.

The most important variables are considered in the present analysis as function of $K_{\text {bulk }}$. Firstly, only the optimum configuration has been considered in each analysis and the result is the red continuous curve from Fig. 5.17 to Fig. 5.20).

It clearly appears that these results do not show a regular trend. In order to verify whether these results could be dependent on the optimal configuration (absolute minima), also local minima have been reported. For any $K_{b u l k}$ value, all local minima have 


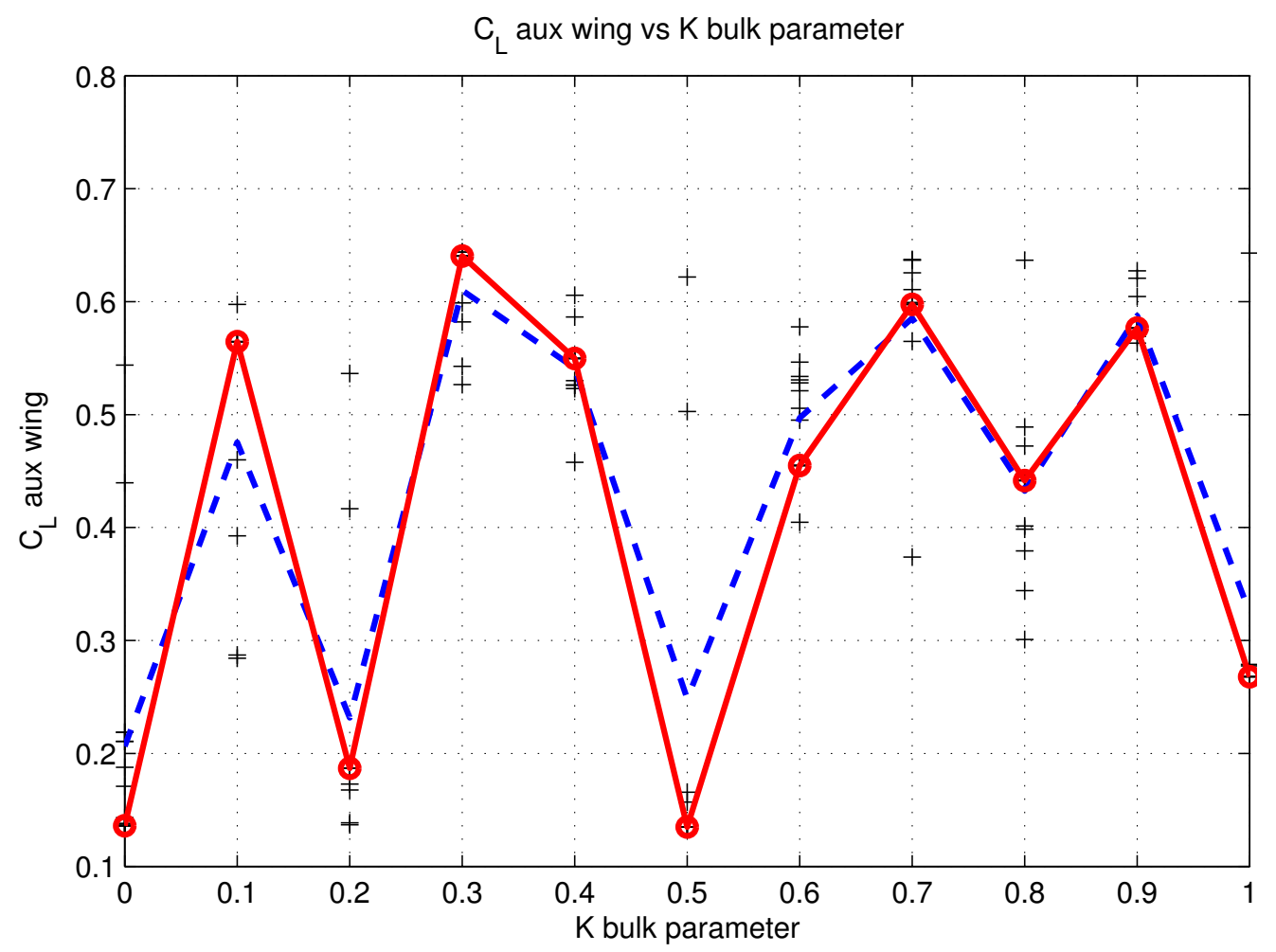

Figure 5.17. $C_{L}$ aux wing vs $K_{b u l k}$ parameter.

been reported (under the symbol "+") together with the average values, calculated and connected by the dash blue lines from Fig. 5.17 to Fig. 5.20.

The results is that the average and the optimum trends are close each other. Now, the $K_{\text {bulk }}$ parameter is set. It is chosen taking into account the following main features:

- Aerodynamic efficiency E (Fig. 5.18) has to be maximum.

- The bulk length (Lbulk) has to be limited to avoid buckling effects (Fig. 5.18).

- The lifts on the three wings have to be as equal as possible (Fig. 5.19).

- High wing loadings increase the performance but structural feasibility needs to be considered as well (Fig. 5.20).

- The optimizer tends to evenly distribute the lifting contributions by raising the $C_{L}$ of the auxiliary wing, but too high $C_{L}$ values have to be avoided (Fig. 5.17) owing to structural problems.

All the previous remarks lead to the choice of $K_{b u l k}=0.8$. 

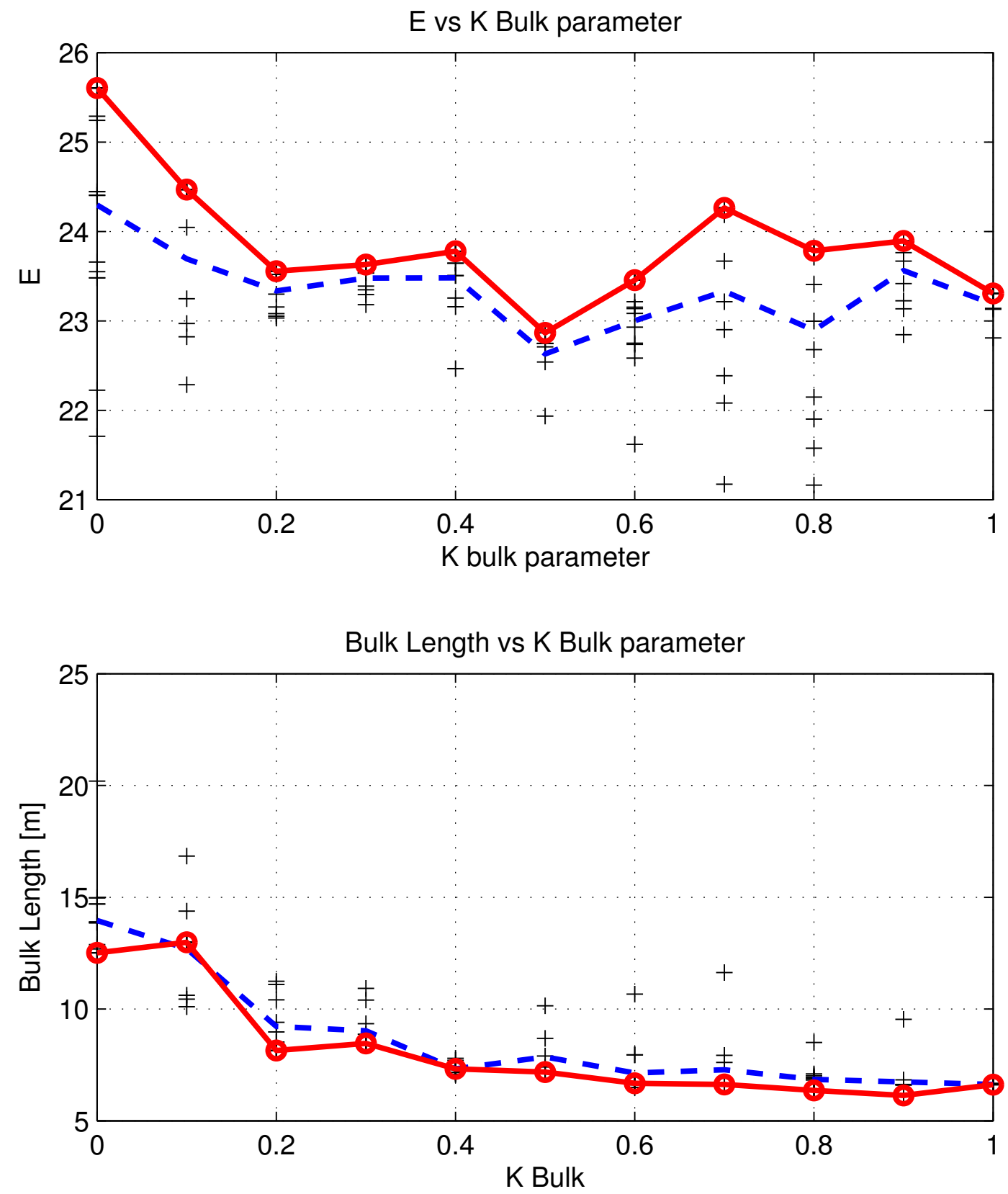

Figure 5.18. Efficiency and Bulk Length. 

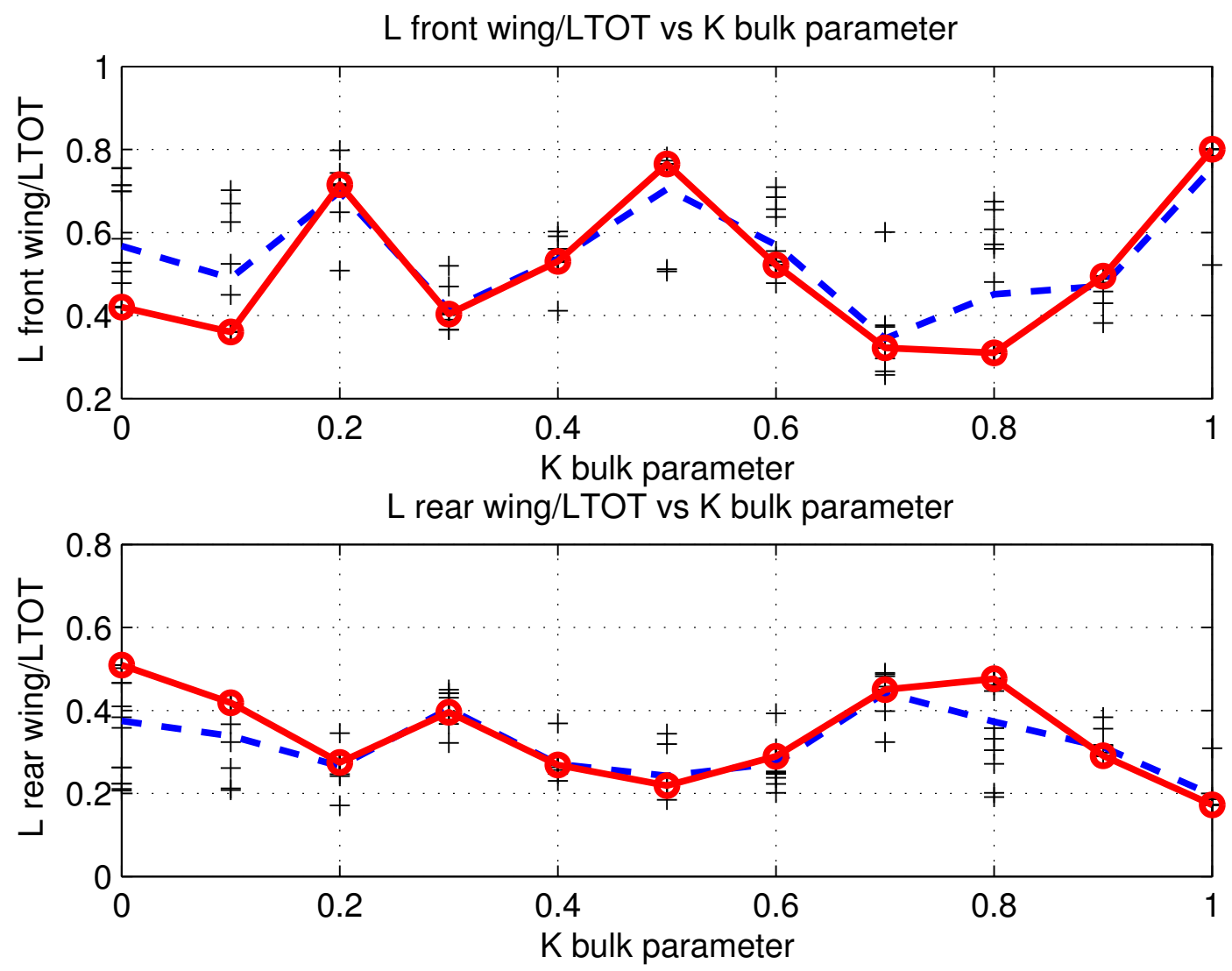

$\mathrm{L}$ aux wing/LTOT vs $\mathrm{K}$ bulk parameter

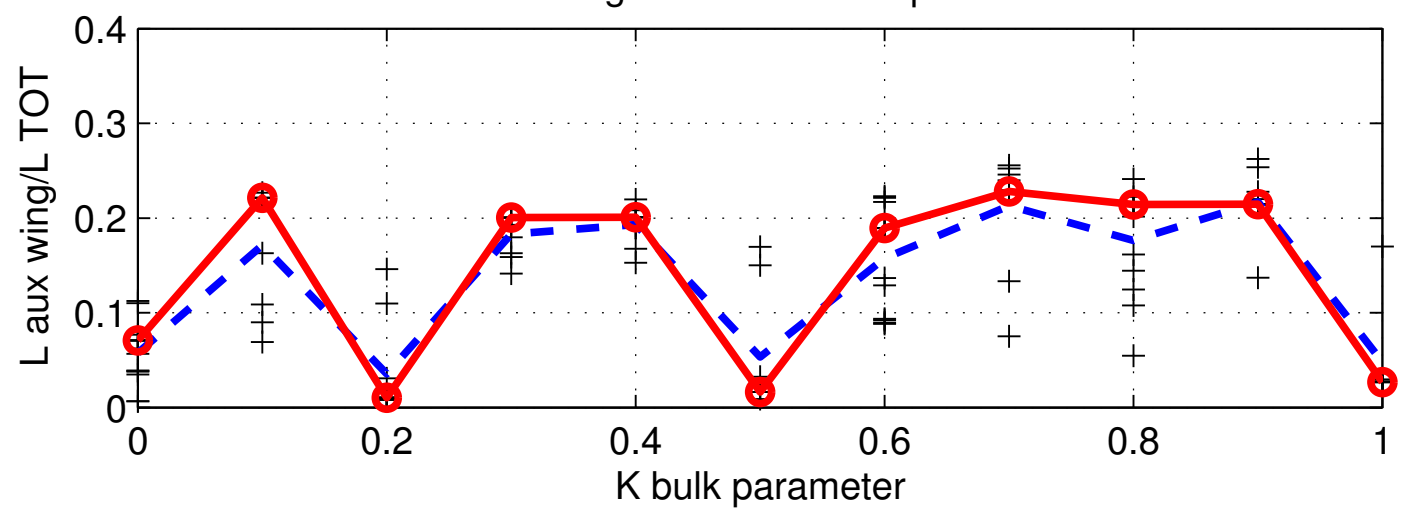

Figure 5.19. Lift Contribution vs $K_{b u l k}$ parameter. 


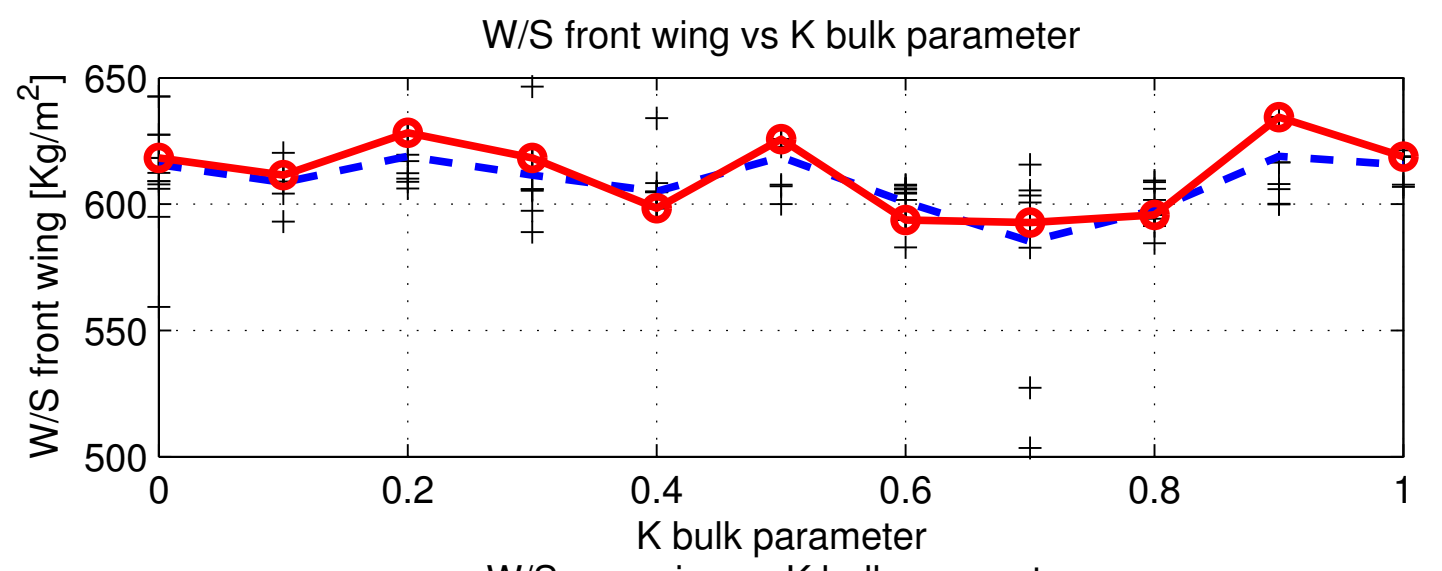

W/S rear wing vs $\mathrm{K}$ bulk parameter

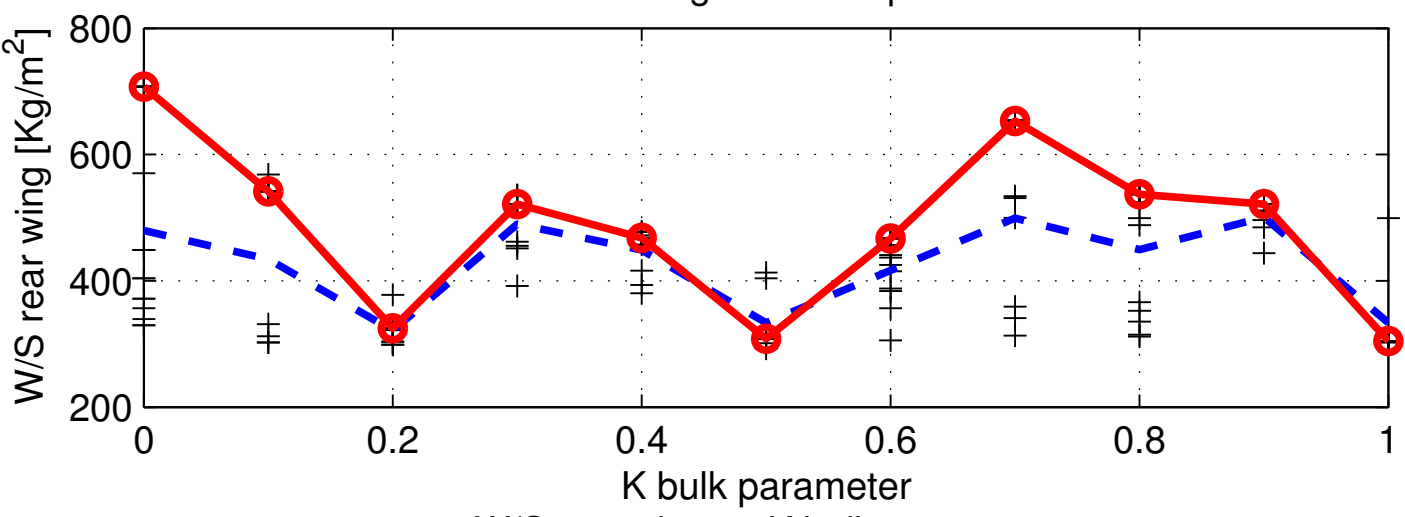

W/S aux wing vs $\mathrm{K}$ bulk parameter

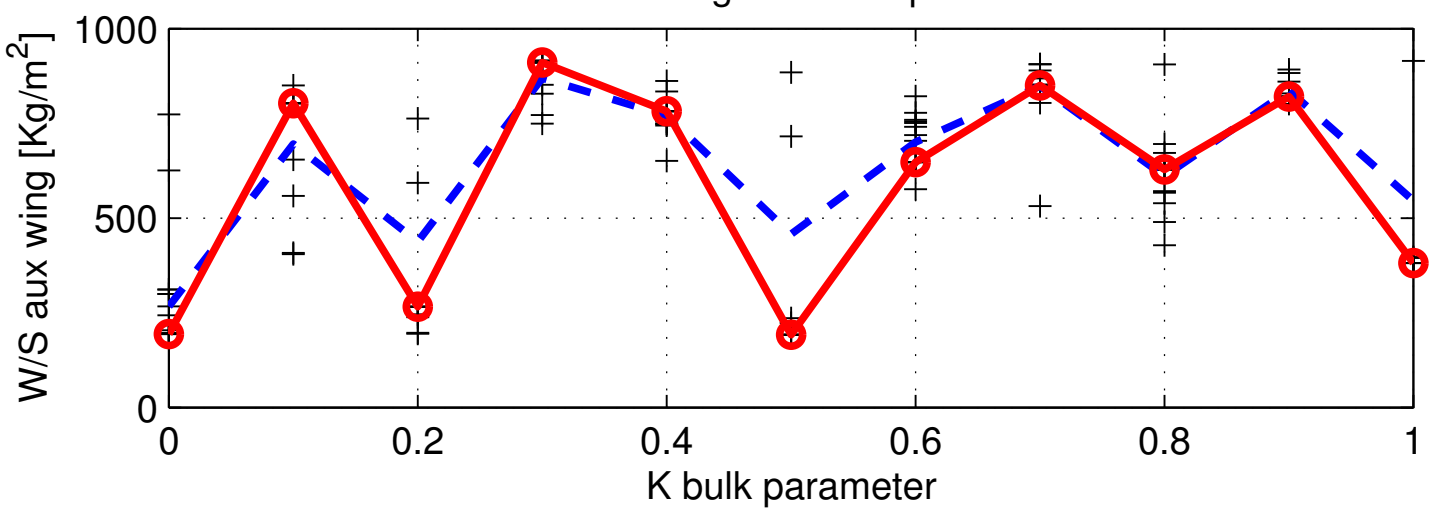

Figure 5.20. Wing Loading vs $K_{\text {bulk }}$ parameter. 


\subsection{Validation of the front wing loading study}

Once $K_{\text {bulk }}$ has been established, we can evaluate the influence of the different wing loadings on the overall properties of the aircraft. We start from the front wing loading. The same runs of paragraph 5.1 have been repeated, according to tab. 5.5. Changing the bulk parameter from 1 to 0.8 , no remarkable change is foreseen. The graphics about these analyses are reported for completeness and they all confirm that there is not any surprising behaviour: all the trends of paragraph 5.1 are confirmed (refer to Appendix A). The front wing loading study has been validated.

\begin{tabular}{ccc}
\hline Analysis & $(W / S)_{\text {front } \min }$ & $(W / S)_{\text {front } \max }$ \\
\hline 16 & 400 & 600 \\
\hline 17 & 500 & 700 \\
\hline 18 & 600 & 800 \\
\hline 19 & 700 & 900
\end{tabular}

Table 5.5. Front wing loading Lower Boundaries and Upper Boundaries $-K_{\text {bulk }}=0.8$.

\subsection{The rear wing loading}

The effects of the rear wing loading are now investigated. The aim of this section is to find out a suitable range of values for the rear wing loading to reach high efficiencies. Previous analyses (refer to section 5.1.2 and to Appendix A) show that the rear wing $C_{L}$ is not directly correlated to the rear wing loading, contrary to the front wing $C_{L}$ and the auxiliary wing $C_{L}$ (which are strictly related to the respective wing loadings). These basic considerations lead to the necessity of new analysis, that will be explained later. The ranges of variation for these analyses are given in Tab. 5.6 and in the following expressions:

\begin{tabular}{ccc}
\hline Analysis & $(W / S)_{\text {rear min }}$ & $(W / S)_{\text {rear max }}$ \\
\hline 20 & 300 & 600 \\
\hline 21 & 400 & 700 \\
\hline 22 & 500 & 800
\end{tabular}

Table 5.6. Rear wing loading Lower Boundaries and Upper Boundaries. 
Chapter 5. Research of preliminary optimum configurations

$$
\begin{gathered}
0 \%<M S<3 \% \\
\text { alpha }=0 \\
K_{\text {bulk }}=0.8 \\
400 \mathrm{~kg} / \mathrm{m}^{2}<\left(\frac{W}{S}\right)_{\text {front }}<600 \mathrm{~kg} / \mathrm{m}^{2} \\
200 \mathrm{~kg} / \mathrm{m}^{2}<\left(\frac{W}{S}\right)_{\text {winglet }}<900 \mathrm{~kg} / \mathrm{m}^{2}
\end{gathered}
$$

The results are reported from Fig. 5.21 to Fig. 5.27 and in Appendix B. The reliability of this analysis is checked in Fig. 5.21: using the same method of section 5.1.2, equation 5.7 is still valid, in which $A_{\text {graph }}=6.67 * 10^{-4} \mathrm{~m}^{2} / \mathrm{kg}$ and $A_{\text {theo }}=7.03 * 10^{-4} \mathrm{~m}^{2} / \mathrm{kg}$; the error is about $5 \%$, where

$$
C_{L}=\frac{1}{q}\left(\frac{W}{S}\right)_{g e n}=A\left(\frac{W}{S}\right)_{g e n}
$$

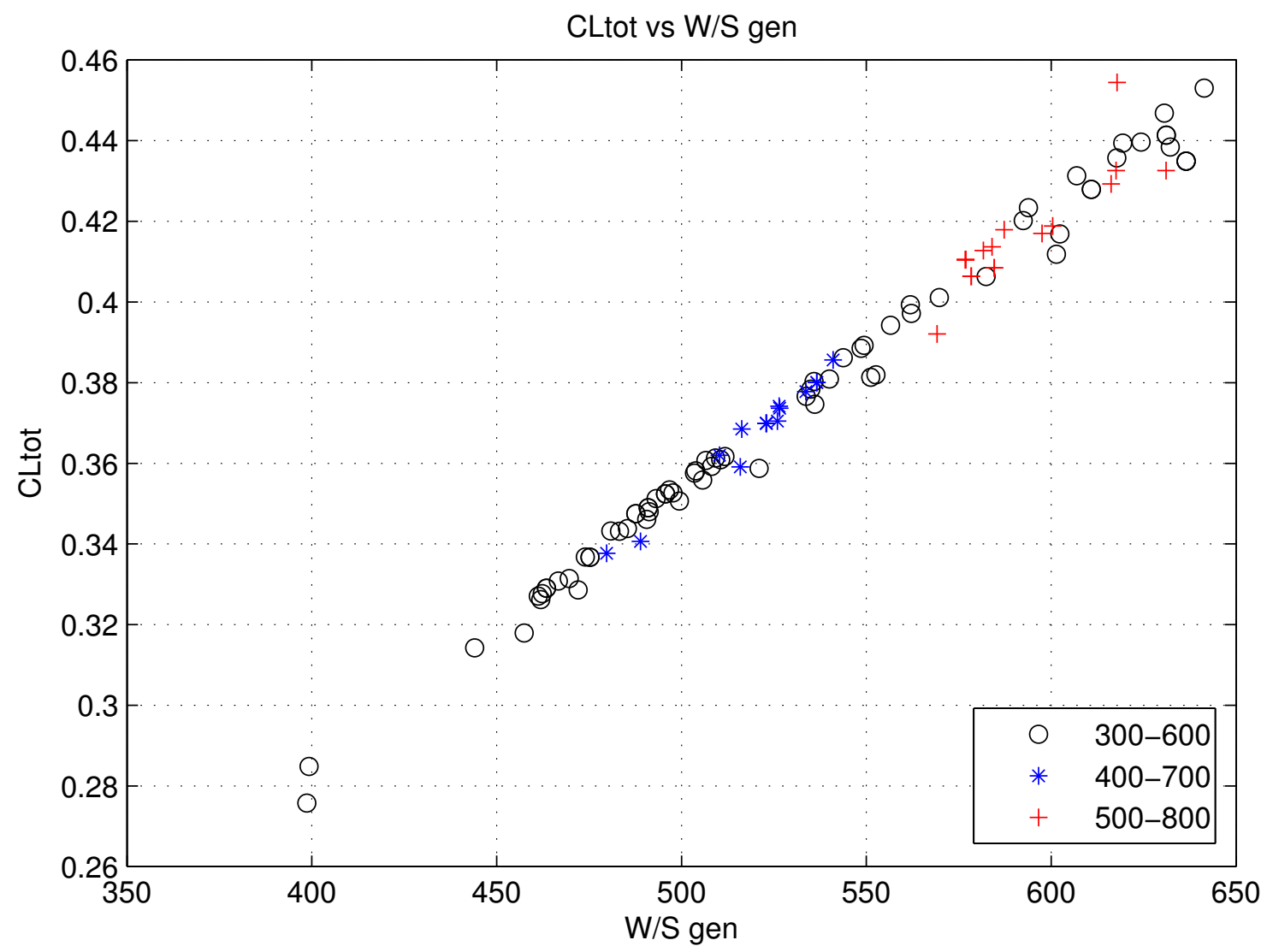

Figure 5.21. $C_{L}$ tot vs Global wing loading - Rear wing analysis. 
The aerodynamic efficiency is a slightly growing function of the rear wing loading (Fig. 5.23); besides it is affected by the global wing loading, as demonstrated in section 5.1.2 and shown in Fig. 5.22.

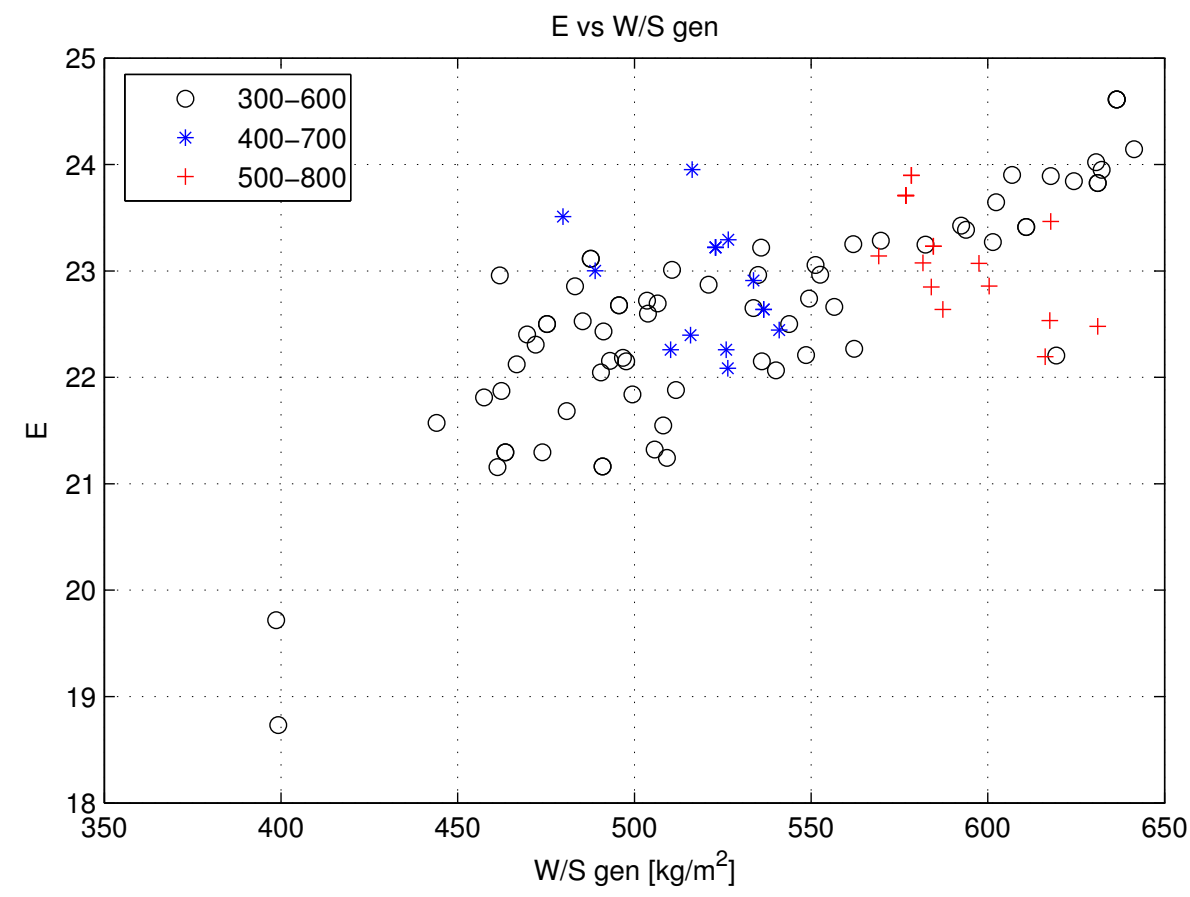

Figure 5.22. Efficiency vs Global wing loading - Rear wing analysis.

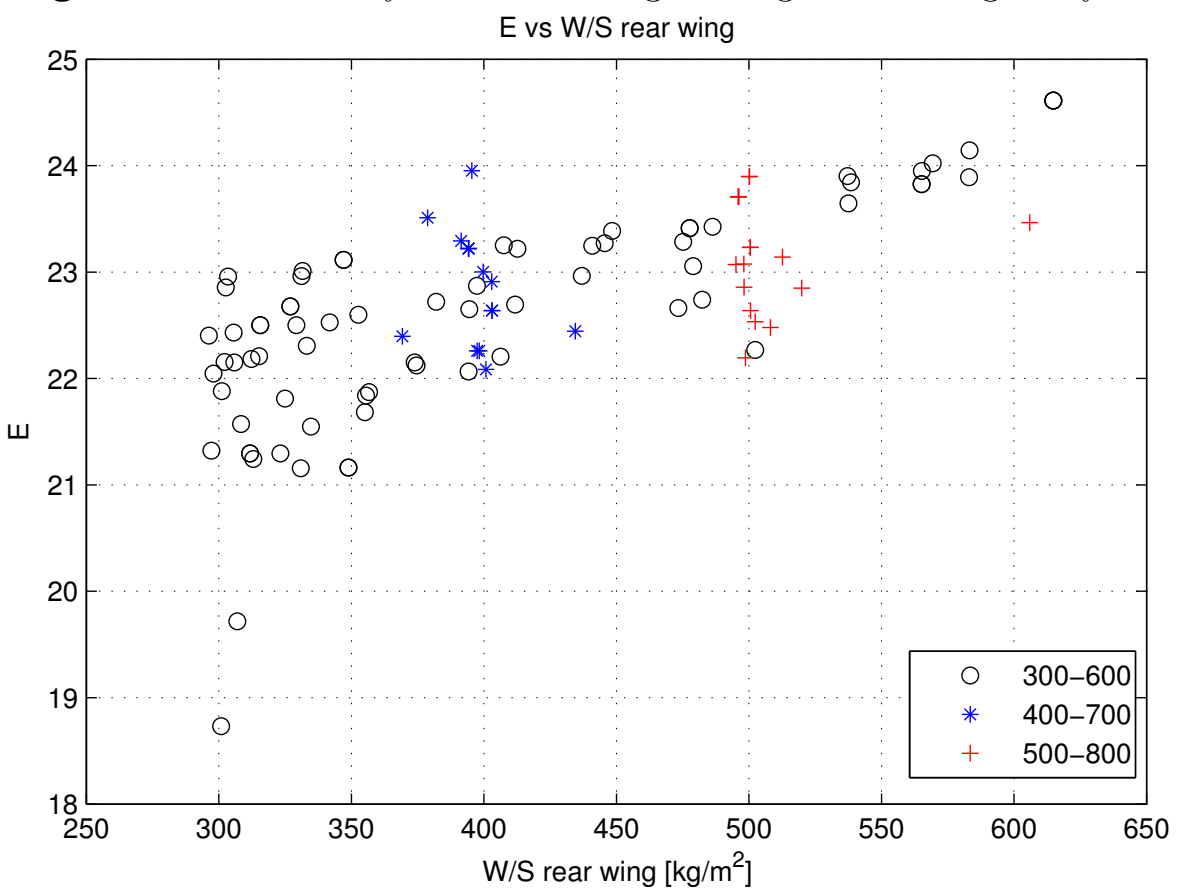

Figure 5.23. Efficiency vs Rear wing loading - Rear wing analysis. 
The margin of stability is not clearly influenced by the rear wing loading, as shown in Fig. 5.24, and, also, the constraint of the margin of stability is not always fulfilled.

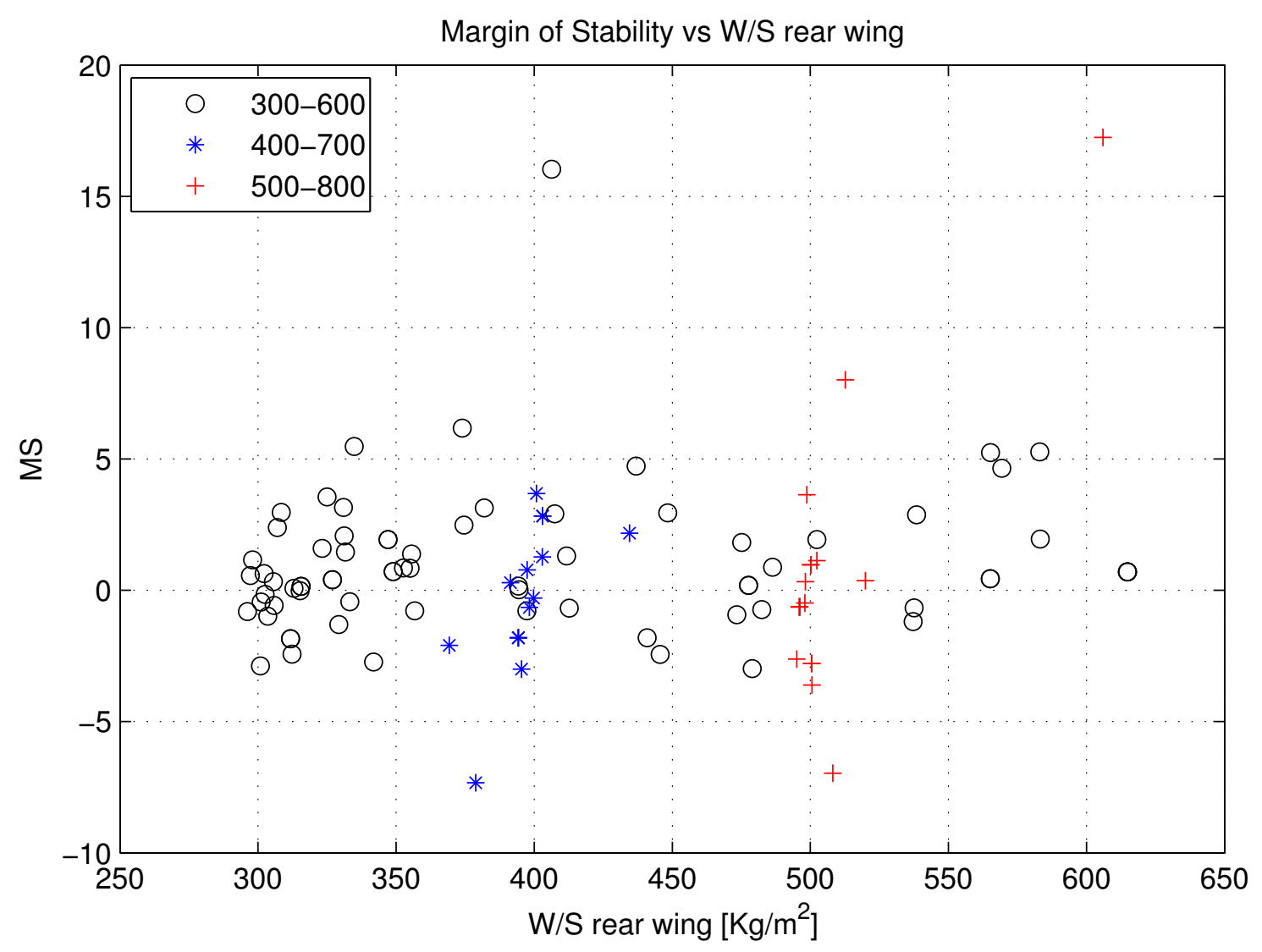

Figure 5.24. Margin of Stability vs Rear wing loading- Rear wing analysis.

The auxiliary wing seems to be independent from the rear wing characteristic. It has been verified that neither the lift of the auxiliary wing nor its surface exhibit a particular relation with the rear wing loading; the front wing is affected by the rear wing: it is evident that the front wing becomes smaller and the lift is reduced, as the rear wing loading increases (refer to Appendix B). The lack of lift on the front wing is recovered by the rear wing, whose lift increases. Although the trend is quite chaotic, it can be inferred that the rear surface increases its size. Hence, a higher rear wing loading implies an increasing lift and an increasing surface; thus, the rear lift rises more than the rear surface (refer to Appendix B).

A way to increase the lift on the rear wing is to move the root towards the centre of gravity; thus, the global neutral point moves forward, together its centre of gravity and, in conclusion, the margin of stability is not directly affected by the rear wing loading. Actually, it is not always true that, when the rear lift is high, the rear wing is closer to the global centre of gravity, according to Fig. 5.25. 


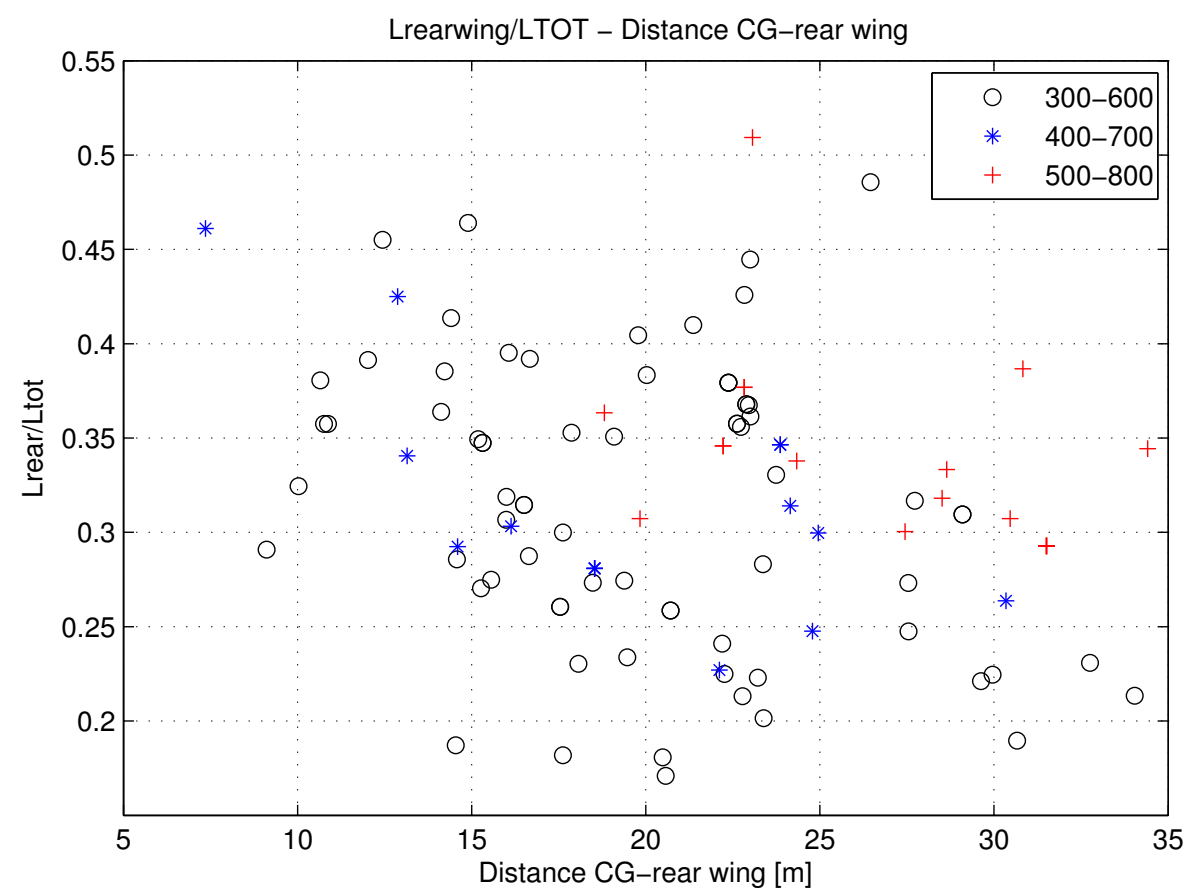

Figure 5.25. Rear Lift vs CG-rear wing distance - Rear wing analysis.

Fig. 5.26 shows a different situation in which the rear wing remains farther than the front wing from the centre of gravity.

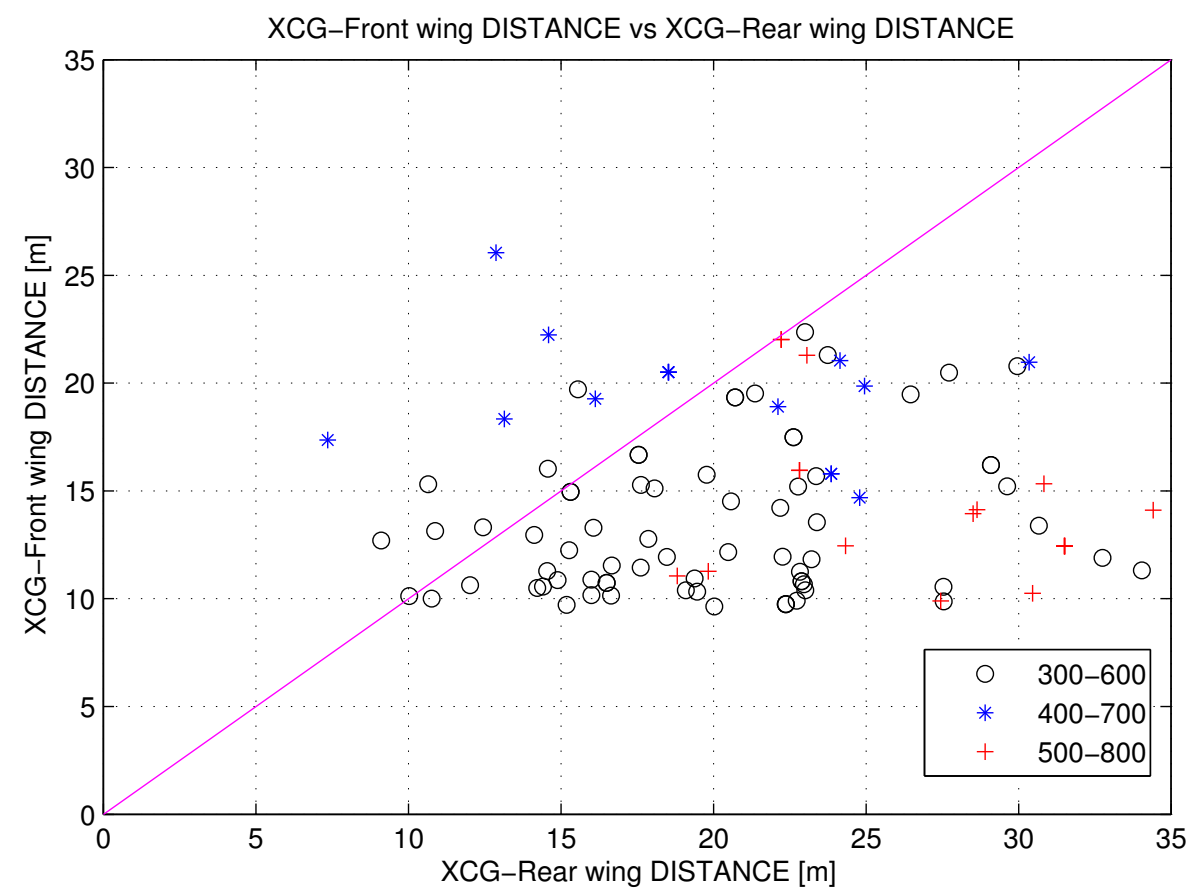

Figure 5.26. CG-front wing distance vs CG-rear wing distance - Rear wing analysis. 
The effects of the rear Aspect Ratio and the global Aspect Ratio are shown in Appendix B. The rear $A R$ has not a significant influence on the rear wing loading, due to the weak relationship between rear surface and rear wing loading. A small wing loading allows also solutions with very high rear $A R$; they are not admitted in case of a conventional monoplane configuration because of structural limitations, but in a PrandtlPlane ${ }^{\circledR}$ configuration a solution to structural static and dynamic problems (e.g. flutter) can be found by means of linked wings. The rear $A R$ does not affect the aerodynamic efficiency; instead, the general $A R$ is related to the rear wing loading. Moreover, the aerodynamic efficiency is affected by $A R$ and the relationship is very close to be linear. The last figure about this analysis deals with $C_{L}$ trends. The global $C_{L}$ trends are reported for completeness: the global $C_{L}$ is not conditioned by any wing loading and the only noticeable relationship is plotted in Fig. 5.21. The front $C_{L}$ and the auxiliary wing $C_{L}$ are strictly related to the respective wing loading while it does not happen for the rear wing. In this chaotic graphic about the rear $C_{L}$ the second way to improve the rear lift is followed: instead of getting closer to centre of gravity, the rear wing increases its lift contribution by means the rear aerodynamic angle of attack, hence by means the rear $C_{L}$.

The conclusion of this section is that the optimizer (AEROSTATE) prefers neither making the rear wing approach the centre of gravity nor increasing the rear $C_{L}$. Thus there is not a criterion to choose one way rather than another. It means that interesting configurations could occur in both situations. The results about the analyses of the rear wing loading are not encouraging, yet the lack of relationship between the rear wing and the aerodynamic efficiency let the designer free to think about high speed considering the front wing and the auxiliary wing, and to use the rear wing to satisfy both the stability and the low speed constraints: in fact, there are efficient solutions with high and low rear wing loading, with the rear wing near and far from the centre of gravity. This aspect underlines the capability of the PrandtlPlane ${ }^{\circledR}$ freighter to be elastic and versatile. Finally, the lower and the upper boundary of the rear wing loading for the reference analysis, that will be explained in section 5.8, are:

$$
400 \mathrm{~kg} / \mathrm{m}^{2}<\left(\frac{W}{S}\right)_{\text {rear }}<600 \mathrm{~kg} / \mathrm{m}^{2}
$$

where the lower boundary is $400 \mathrm{~kg} / \mathrm{m}^{2}$ because too low values imply less efficient configurations and the auxiliary wing lift coefficient has to be not too high; moreover, the upper boundary is not $700 \mathrm{~kg} / \mathrm{m}^{2}$ because the optimized solutions do not presents such a high rear wing loading. 

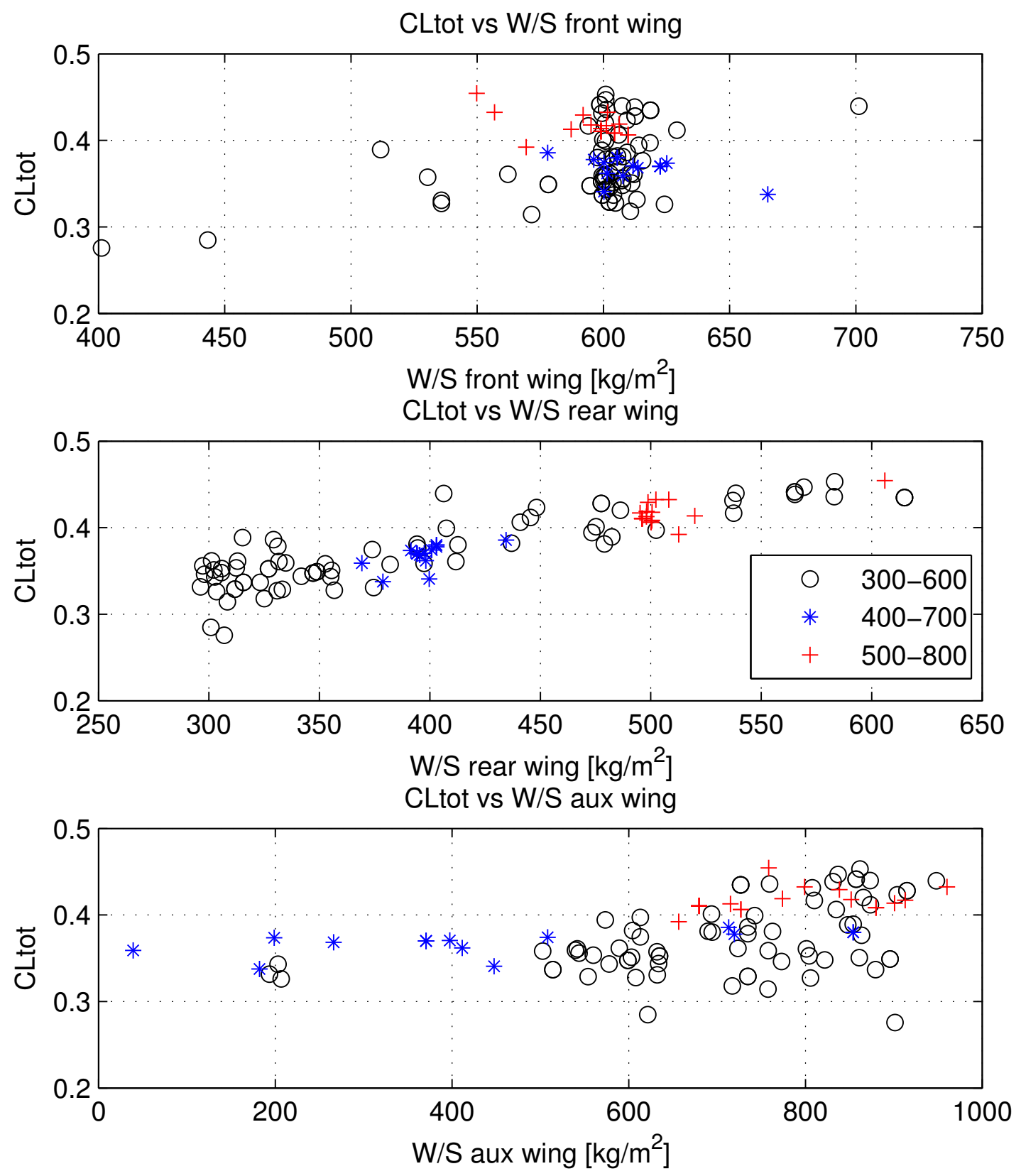

Figure 5.27. $C_{L}$ tot trends - Rear wing analysis. 


\subsection{Sensitivity to the wing span and to the fin height}

The previous analysis was performed with a given wingspan and a given height of fins. Now, we study how the optimum configurations are influenced by variations of these two parameters. The results are shown in the following figures with the same sequence shown before.

A reference analysis (Analysis 34) is used to compare the results obtained from wing span and fin height variations, with the conditions given in Tab. 5.7 and in eq. 5.14.

$$
\begin{gathered}
0<M S<0.03 \% \\
\text { alpha }=0 \text { deg } \\
K_{\text {bulk }}=0.8 \\
0 \mathrm{~kg} / \mathrm{m}^{2}<\left(\frac{W}{S}\right)_{\text {front }}<600 \mathrm{~kg} / \mathrm{m}^{2} \\
0 \mathrm{~kg} / \mathrm{m}^{2}<\left(\frac{W}{S}\right)_{\text {rear }}<600 \mathrm{~kg} / \mathrm{m}^{2} \\
200 \mathrm{~kg} / \mathrm{m}^{2}<\left(\frac{W}{S}\right)_{\text {winglet }}<600 \mathrm{~kg} / \mathrm{m}^{2}
\end{gathered}
$$

\begin{tabular}{ccc}
\hline${\text { Analisys } \mathbf{n}^{\circ}}^{\circ}$ & Wing span $[m]$ & Fin height $[m]$ \\
\hline 34 & 80 & 12 \\
\hline 35 & 80 & 11 \\
\hline 36 & 80 & 10 \\
\hline 37 & 75 & 12 \\
\hline 39 & 70 & 12 \\
\hline
\end{tabular}

Table 5.7. Wing span and fin height for each analyses.

\subsubsection{Fin height sensitivity}

A lower fin height reduces the manufacturing costs and improves the maintainability of the aircraft; the directional stability is an open question, but it can be obtained easily by improving the fins volume.

The effects of the height fin variation are shown from Fig. 5.28 to Fig. 5.36 and in Appendix C. 

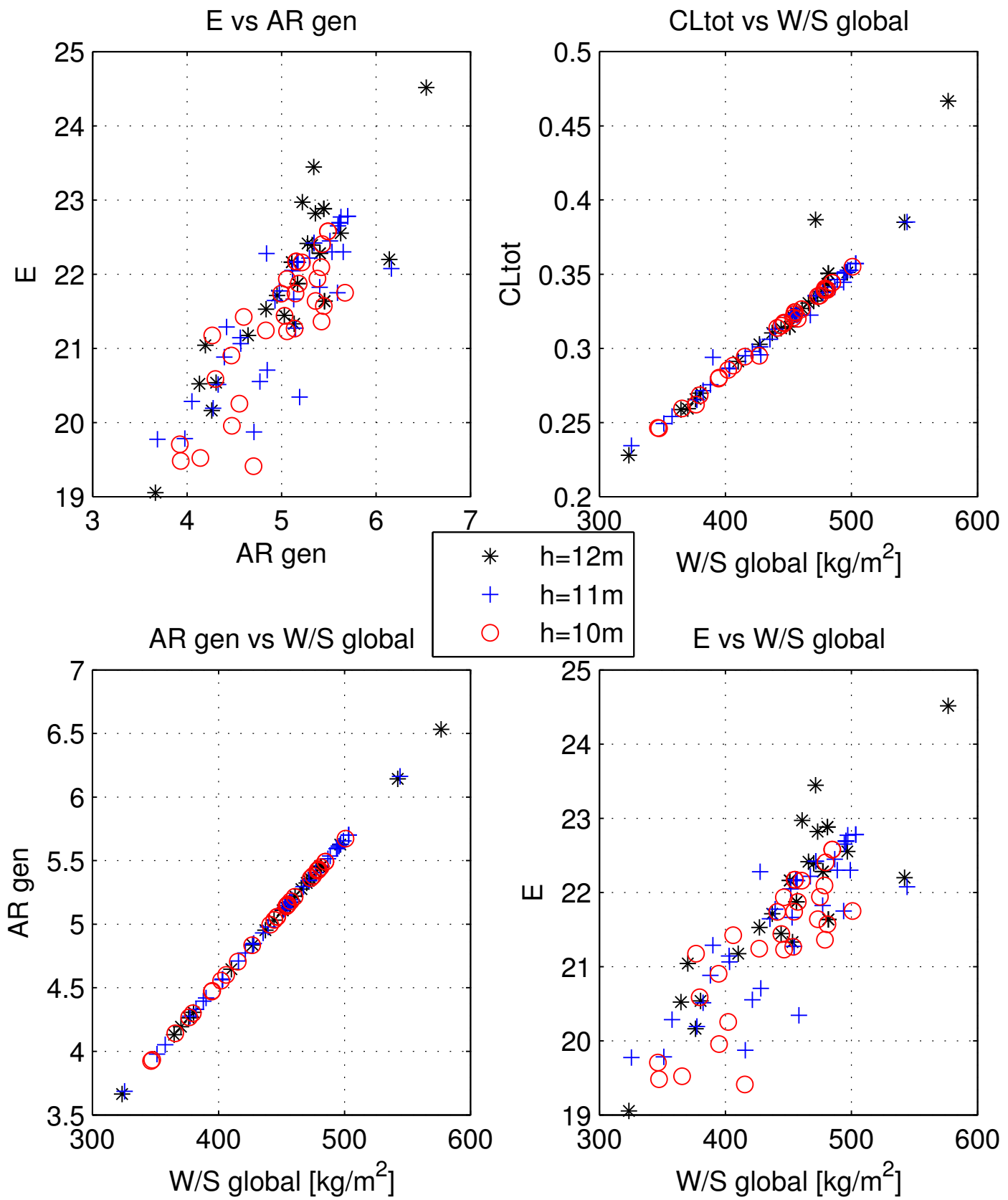

Figure 5.28. Generalities (part 1) - H sensitivity. 


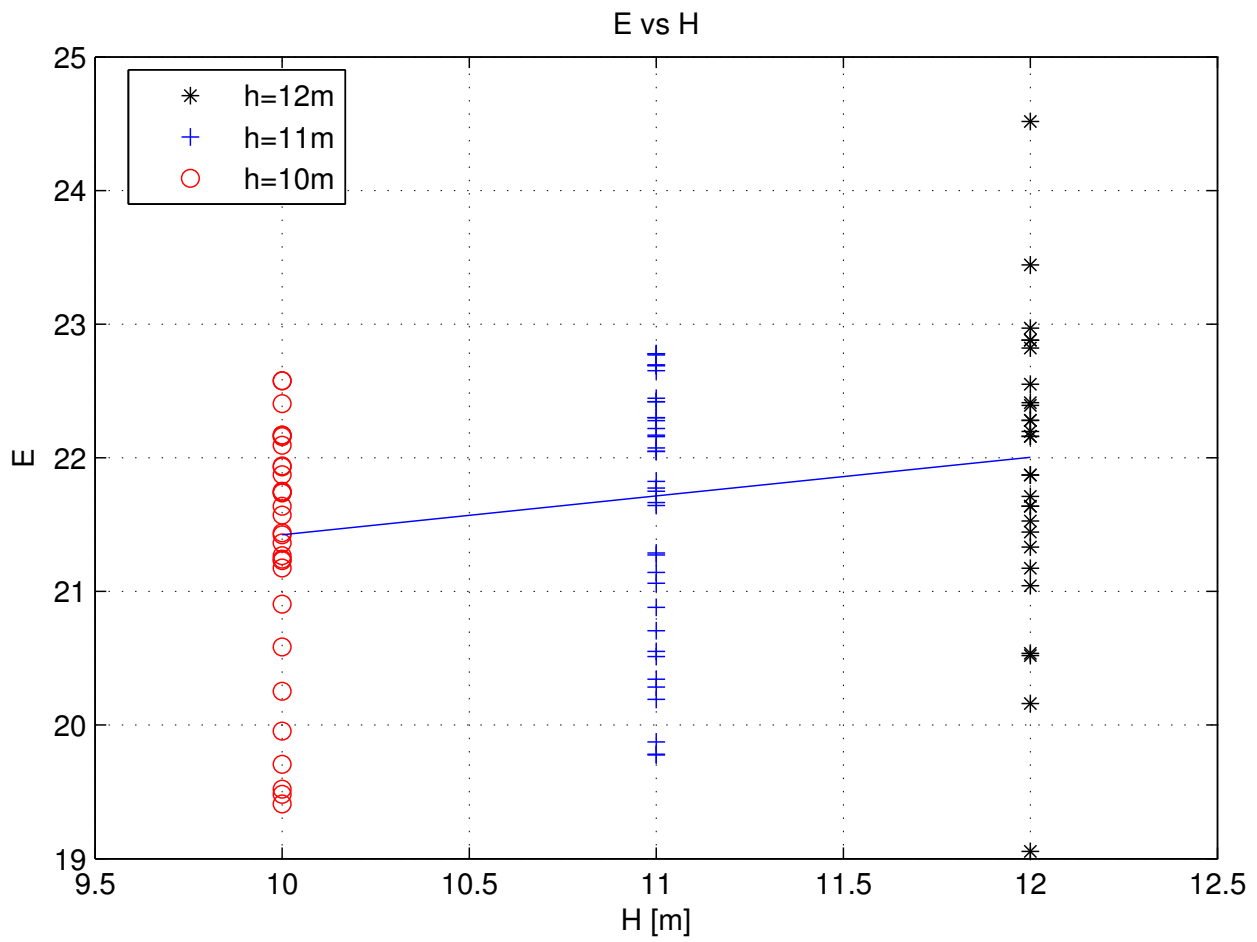

Figure 5.29. Efficiency vs $\mathrm{H}-\mathrm{H}$ sensitivity.

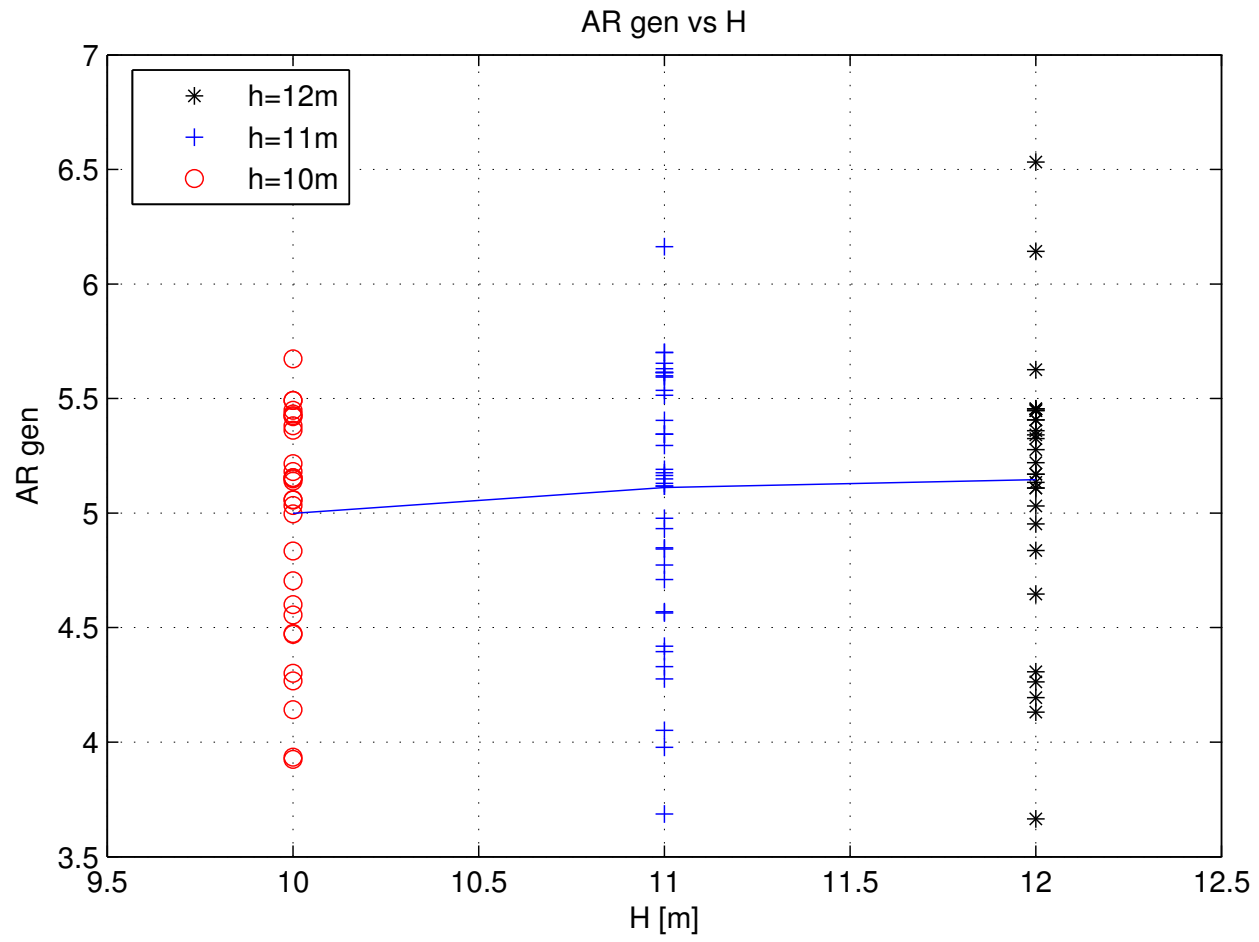

Figure 5.30. AR gen vs $\mathrm{H}-\mathrm{H}$ sensitivity. 

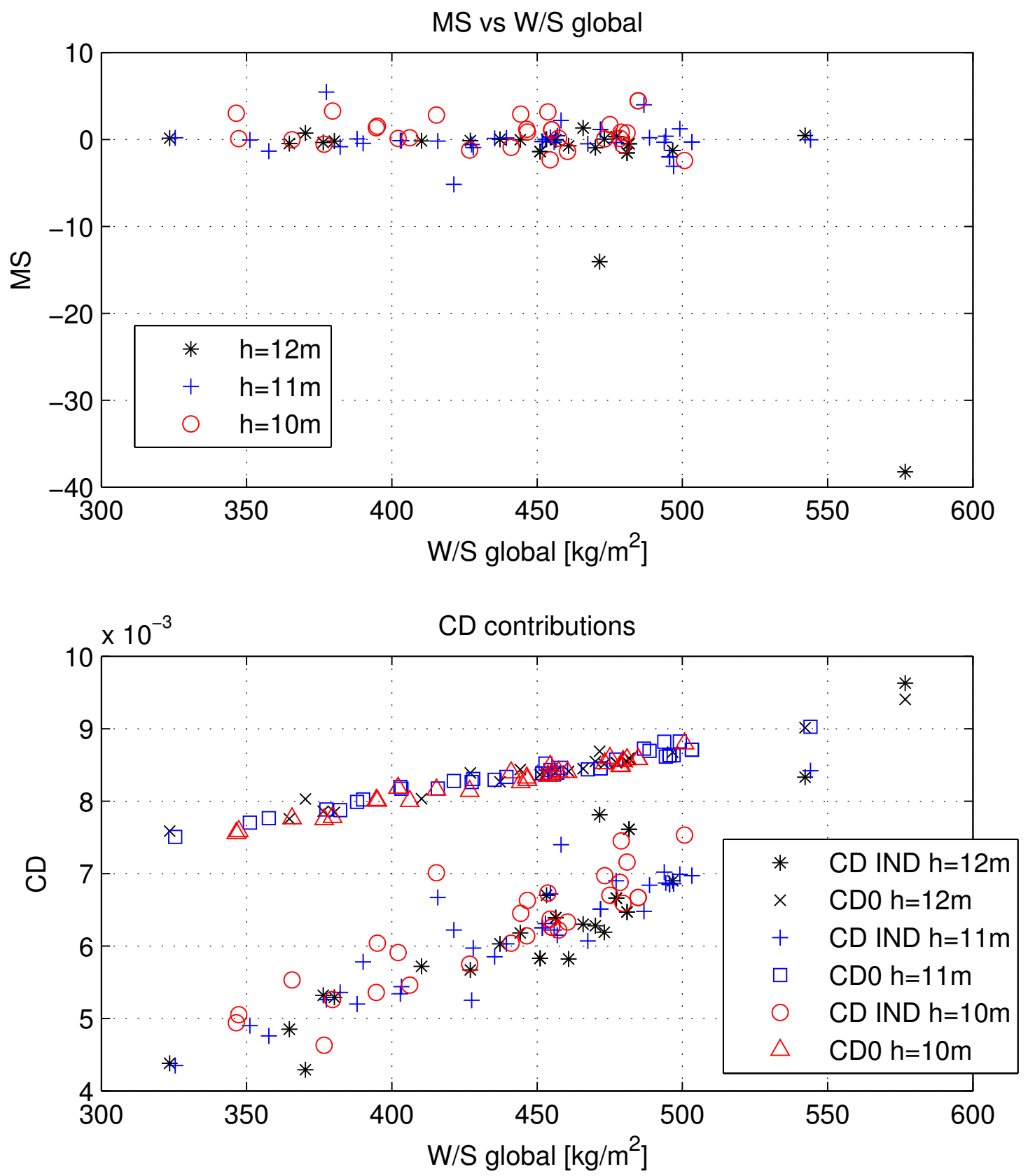

Figure 5.31. Generalities (part 2) - H sensitivity. 

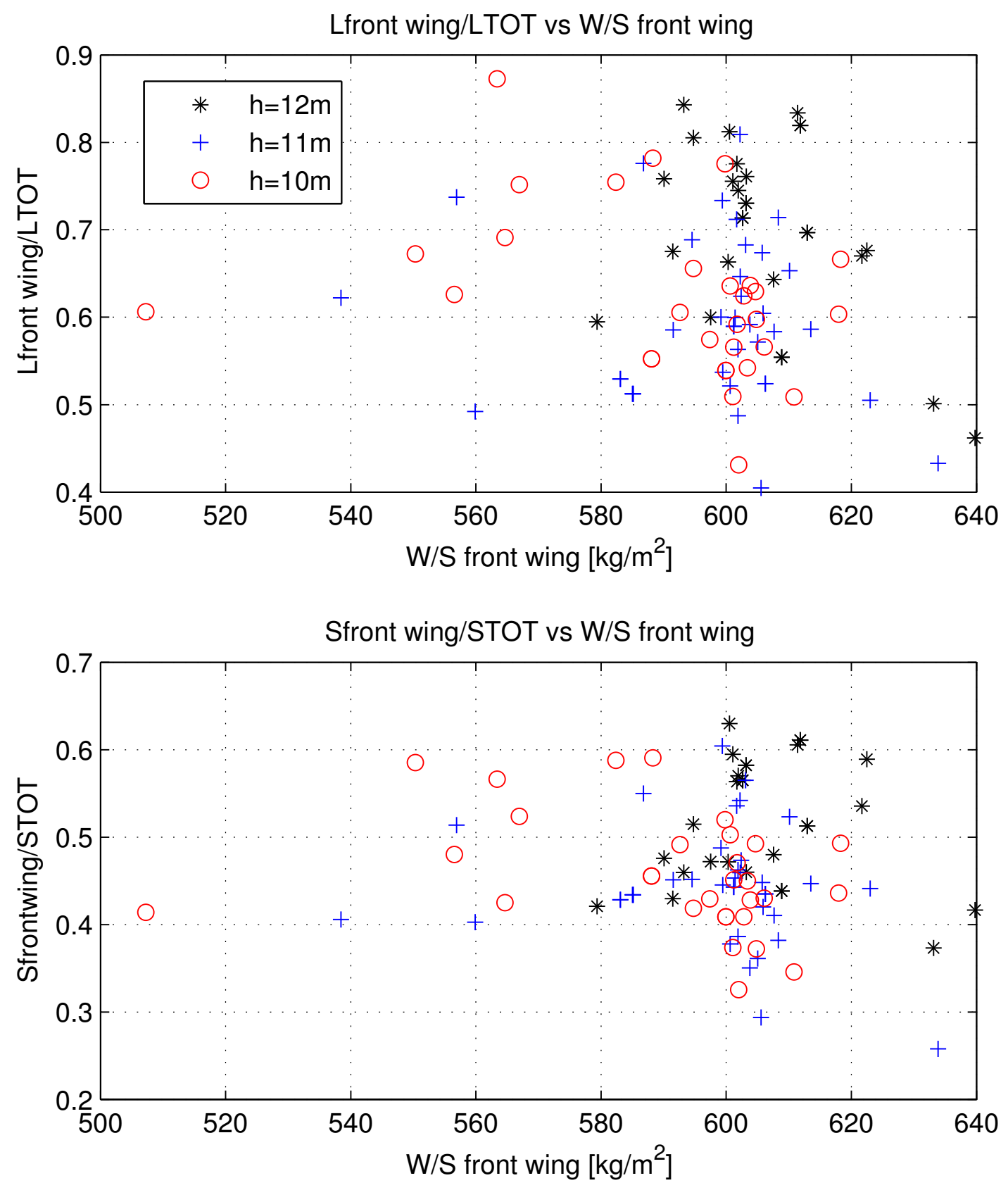

Figure 5.32. Front wing lift and surface - $\mathrm{H}$ sensitivity. 

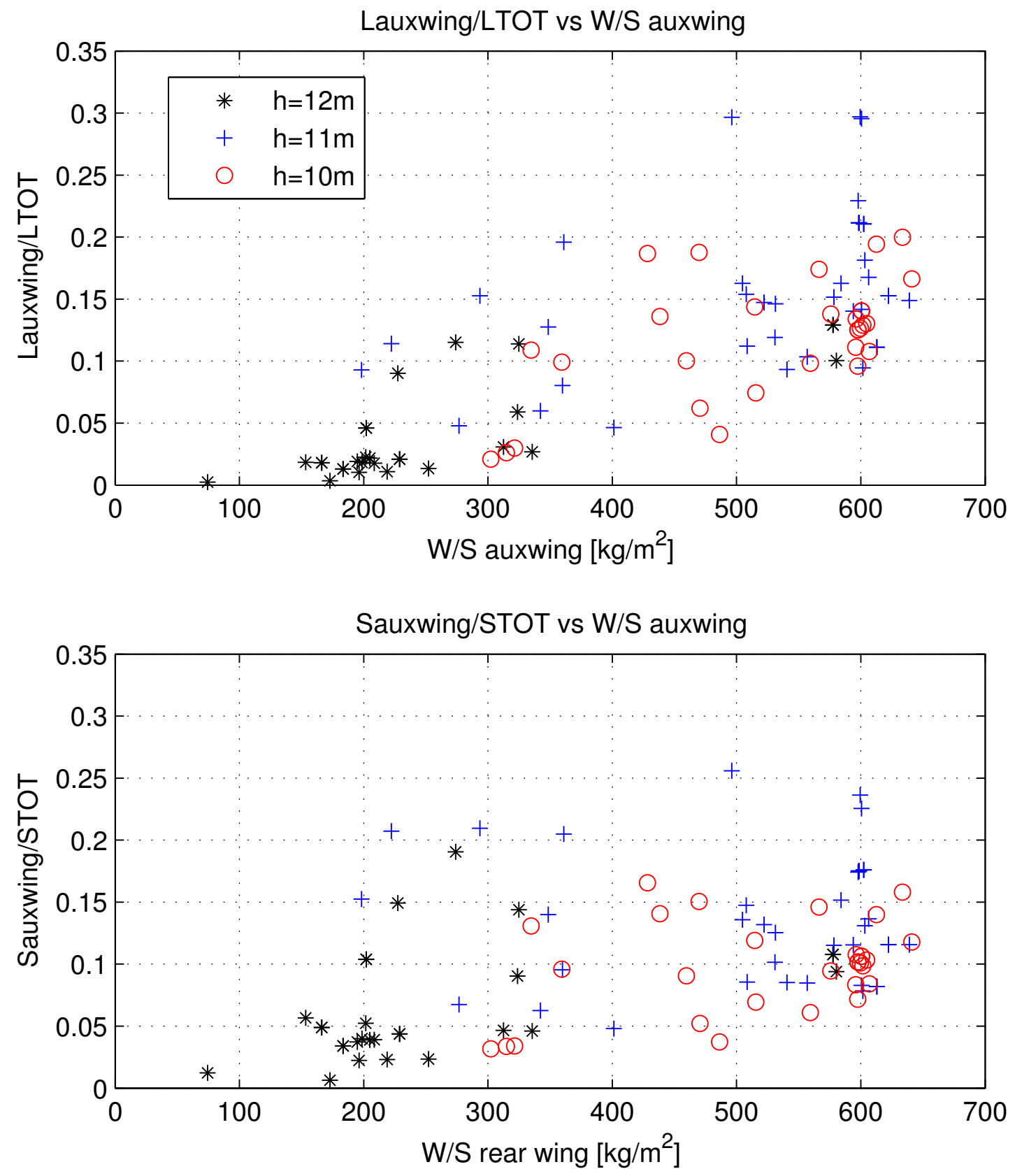

Figure 5.33. Auxiliary wing lift and surface - $\mathrm{H}$ sensitivity. 

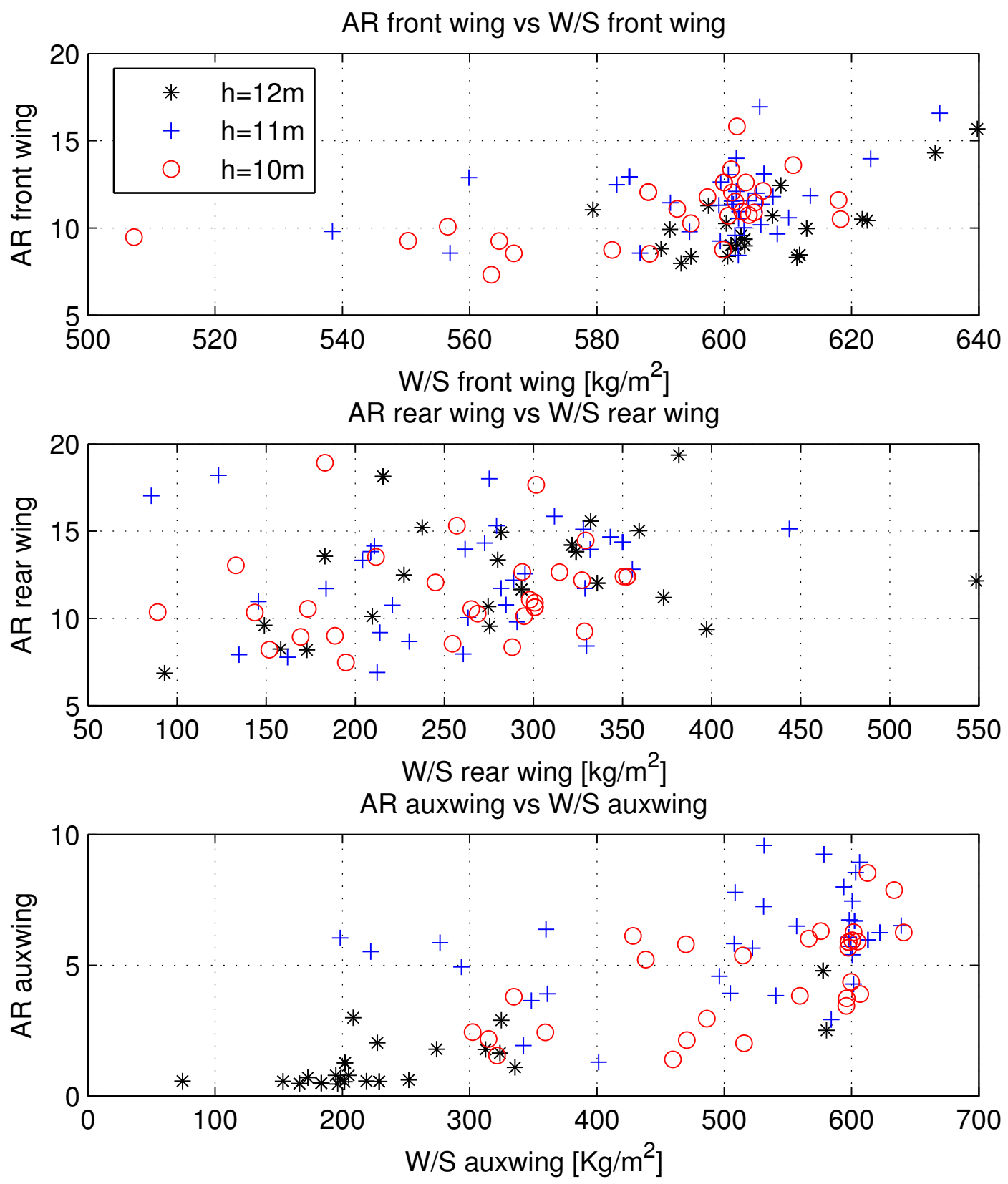

Figure 5.34. AR behaviour - $\mathrm{H}$ sensitivity. 

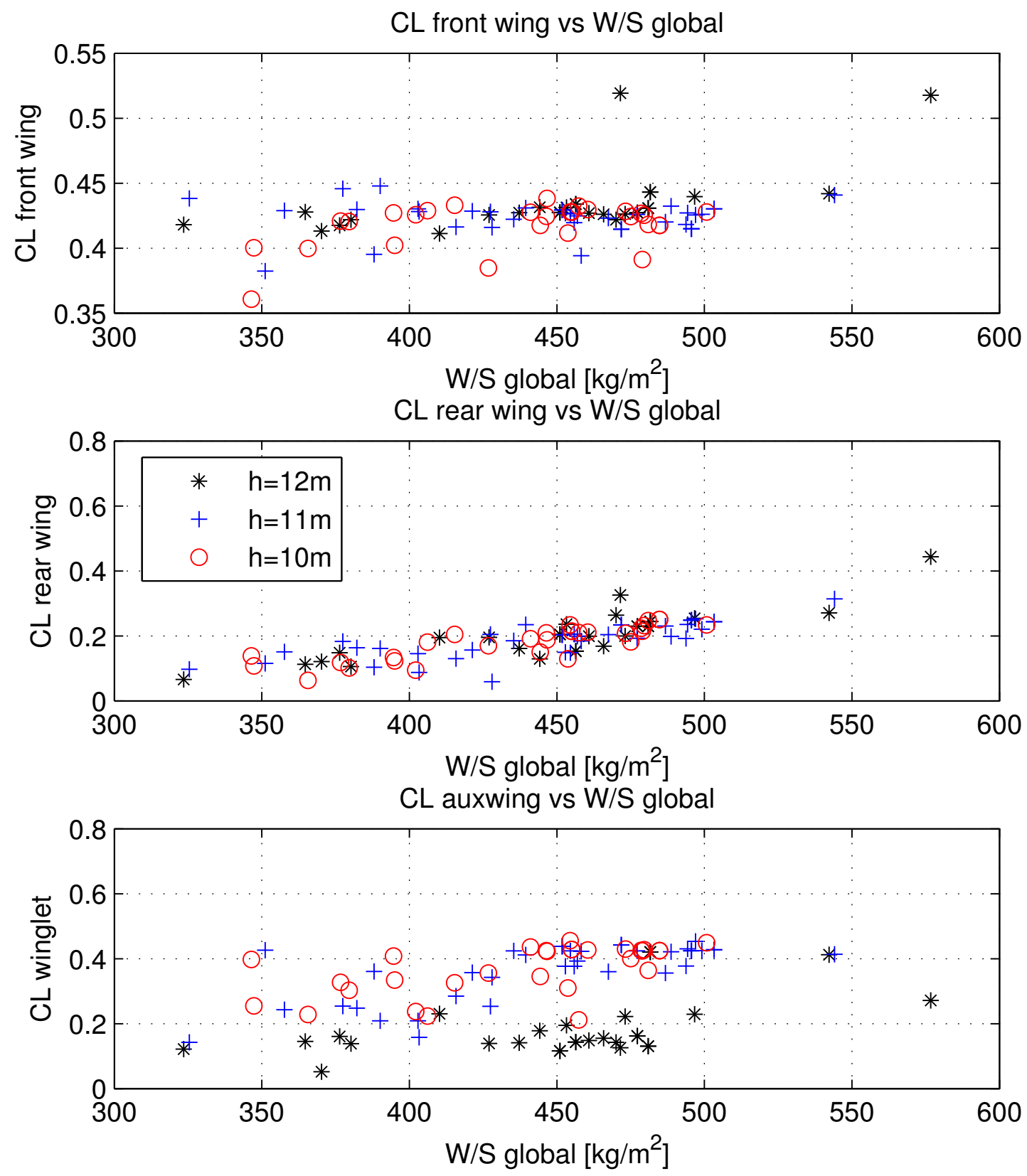

Figure 5.35. CL behaviour (part 1) - H sensitivity. 

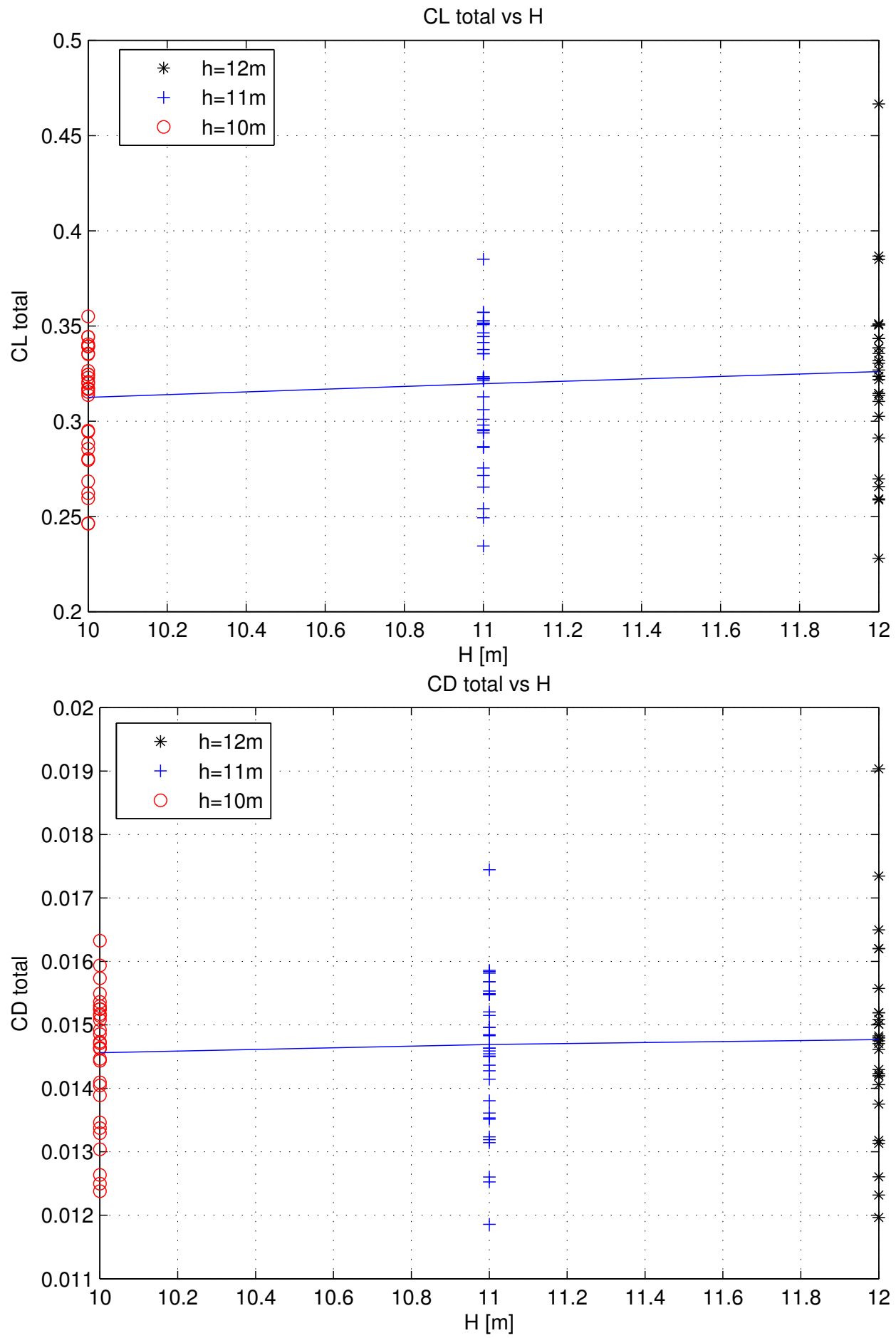

Figure 5.36. Total lift coefficient and total drag coefficient - H sensitivity. 
Fig. 5.29 and Fig. 5.30 show that the average aerodynamic efficiency increases if the fin height increases. This trend depends on the increase of efficiency due to the Prandtl's Best Wing System effect but, on the other hand, a reduction due to the larger wetted surface is also present (in every analysis the design of the fins is fixed). In total, the average efficiency variation is about $2,8 \%$. Indeed, if the wings are more distant, this aerodynamic system is more efficient. This trend is confirmed by both the behaviour of the optima efficiencies and the efficiency averages.

Fig. 5.33 shows that the auxiliary wing lift increases if the fin height decreases; similarly, the lift coefficient of the auxiliary wing (Fig. 5.35) shows a similar trend. Moreover, the aspect ratio of the auxiliary wing increases if the fin height decreases (Fig. 5.34).

In Fig. 5.36, is pointed out that the lift total coefficient and the drag total coefficient increase with the fin height growth: in any case, the efficiency increases.

The total lift coefficient and the total drag coefficients do not show significant average variations (Fig. 5.36), in accordance with the modest variation of efficiency shown before.

\subsubsection{Wing span sensitivity}

Another important parameter is the wing span which is limited by the hangar sizes and structural efficiency, in particular flutter; the efficiency trend with the wing span variation is analysed in this section. The results are plotted from Fig. 5.37 to Fig. D.5 and in Appendix D.

The most important (and expected) result is that the global efficiency of the airplane is reduced for smaller spans, as shown in Fig. 5.41 to Fig. 5.42; Fig. 5.44, accordingly, indicates that the induced drag coefficient and the viscosity drag coefficient decrease if the wing span increases. The influence of wing span over the margin of stability is not significant (Fig. 5.43).

The front lift coefficient is almost constant in all the configurations, as shown in Fig. 5.46; this effect causes a reduction of the front wing lift if the wing span decreases, with the consequence that the rear wing and the auxiliary wing increase their lift and their lift coefficients. Moreover, the front wing surface decreases because the wing span reduction is more important than the chords increase, as shown in Fig. 5.45. These effects cause an improvement of the lift on the auxiliary wing, as Fig. 5.46 shows.

The verification of results is made by Fig. 5.37 and Fig. 5.38 


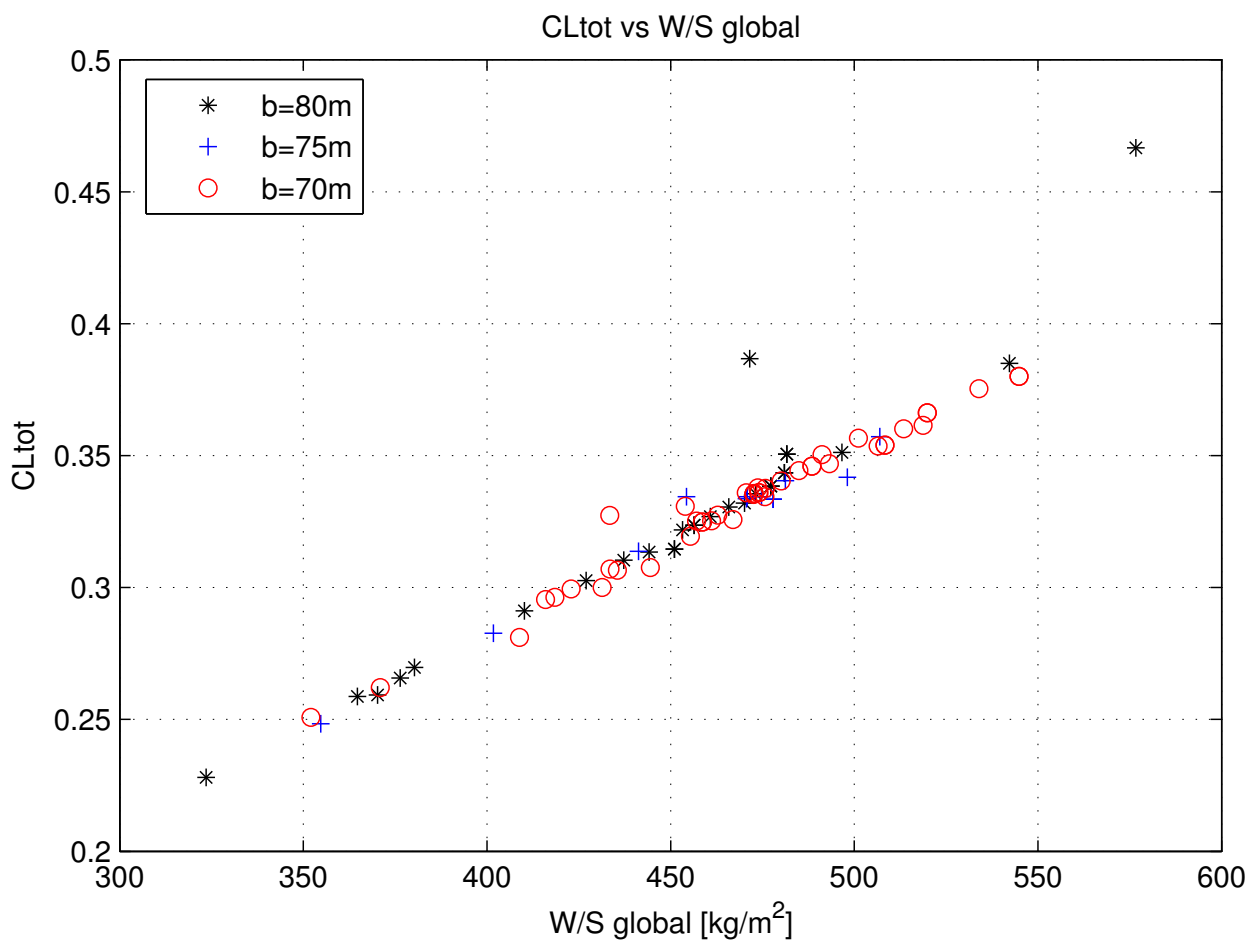

Figure 5.37. CL tot vs Global wing loading - B sensitivity W/S global vs AR gen

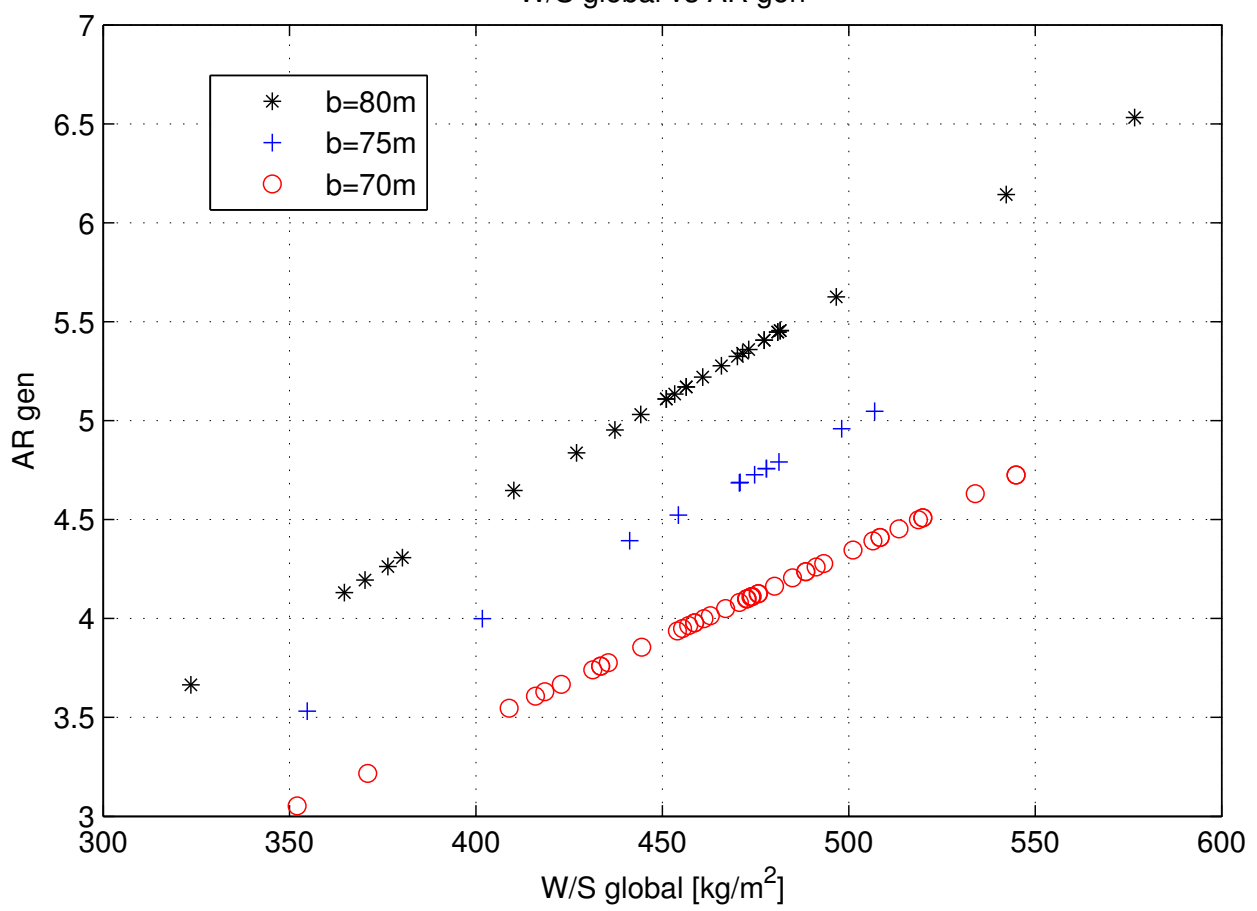

Figure 5.38. AR gen vs Global wing loading - B sensitivity 


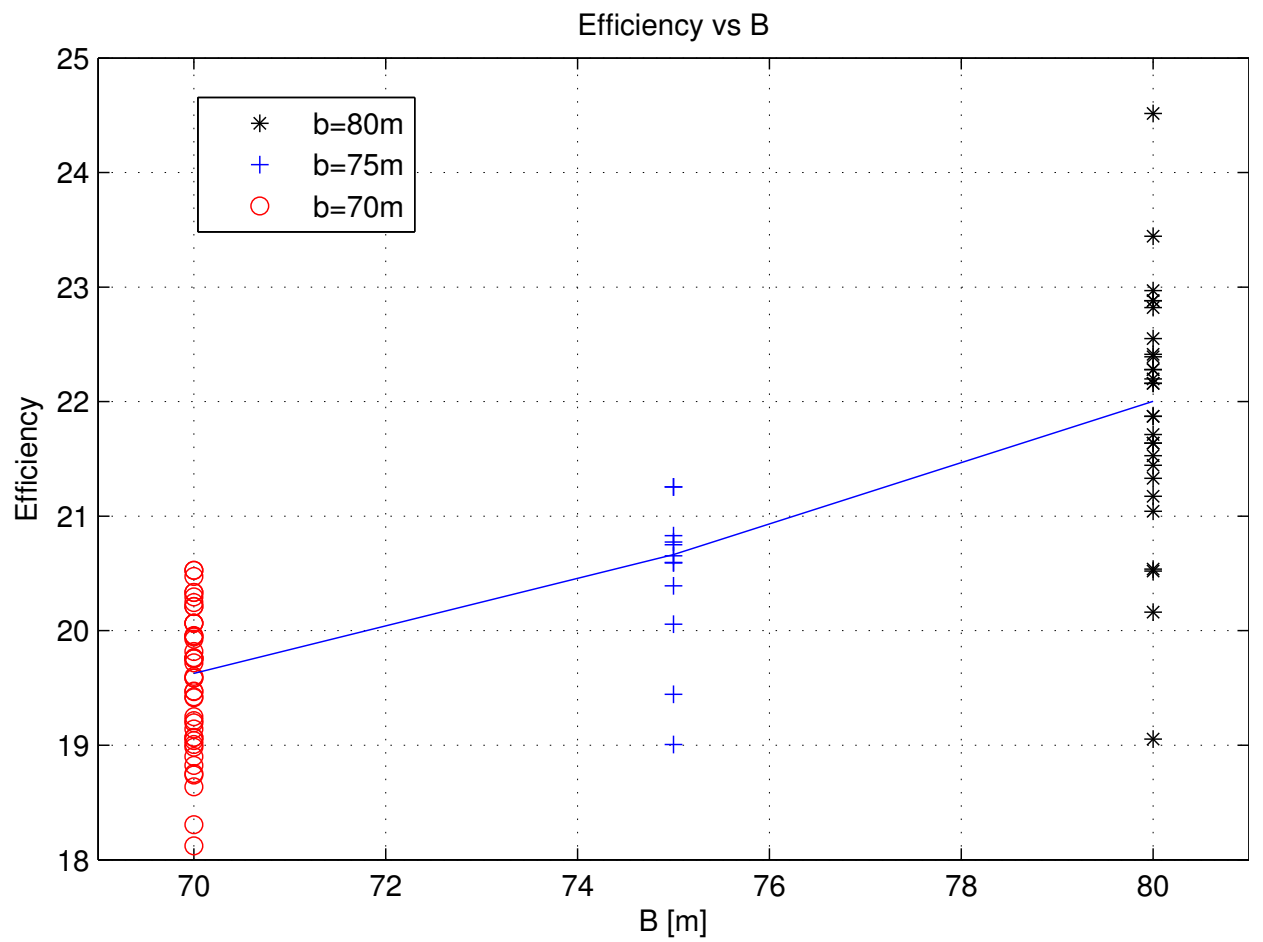

Figure 5.39. Efficiency - B sensitivity

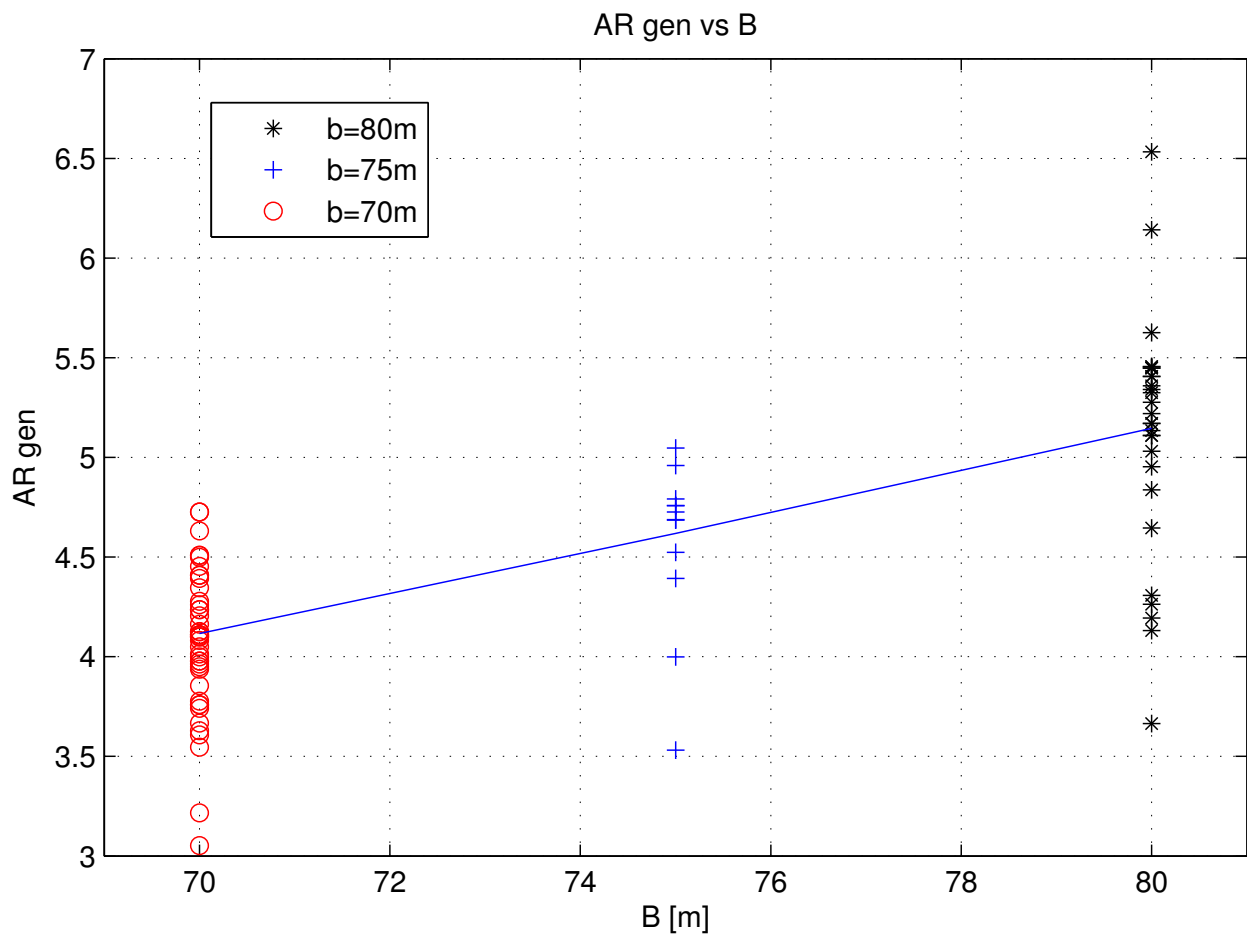

Figure 5.40. $A R$ gen - B sensitivity 


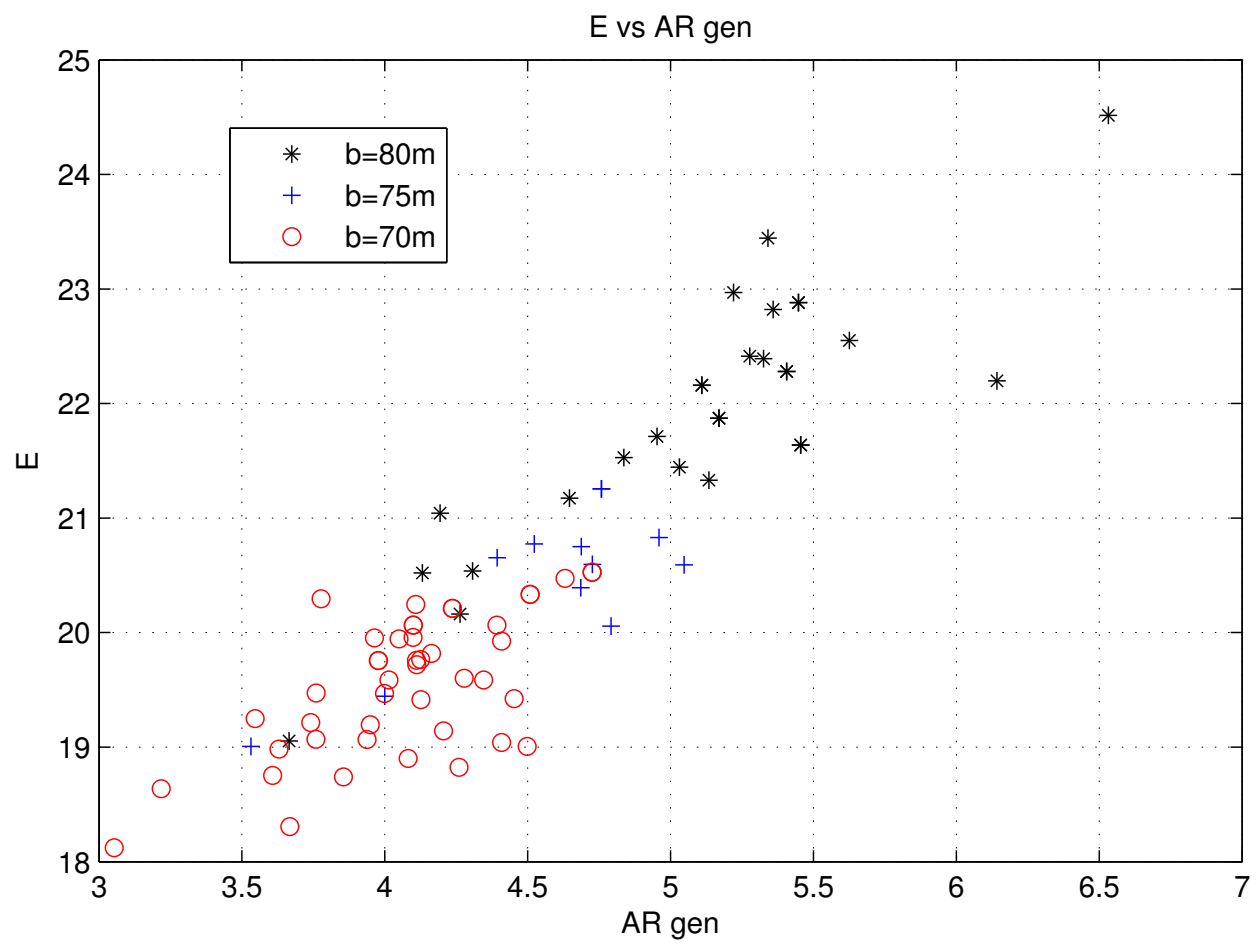

Figure 5.41. Efficiency vs $A R$ gen - B sensitivity

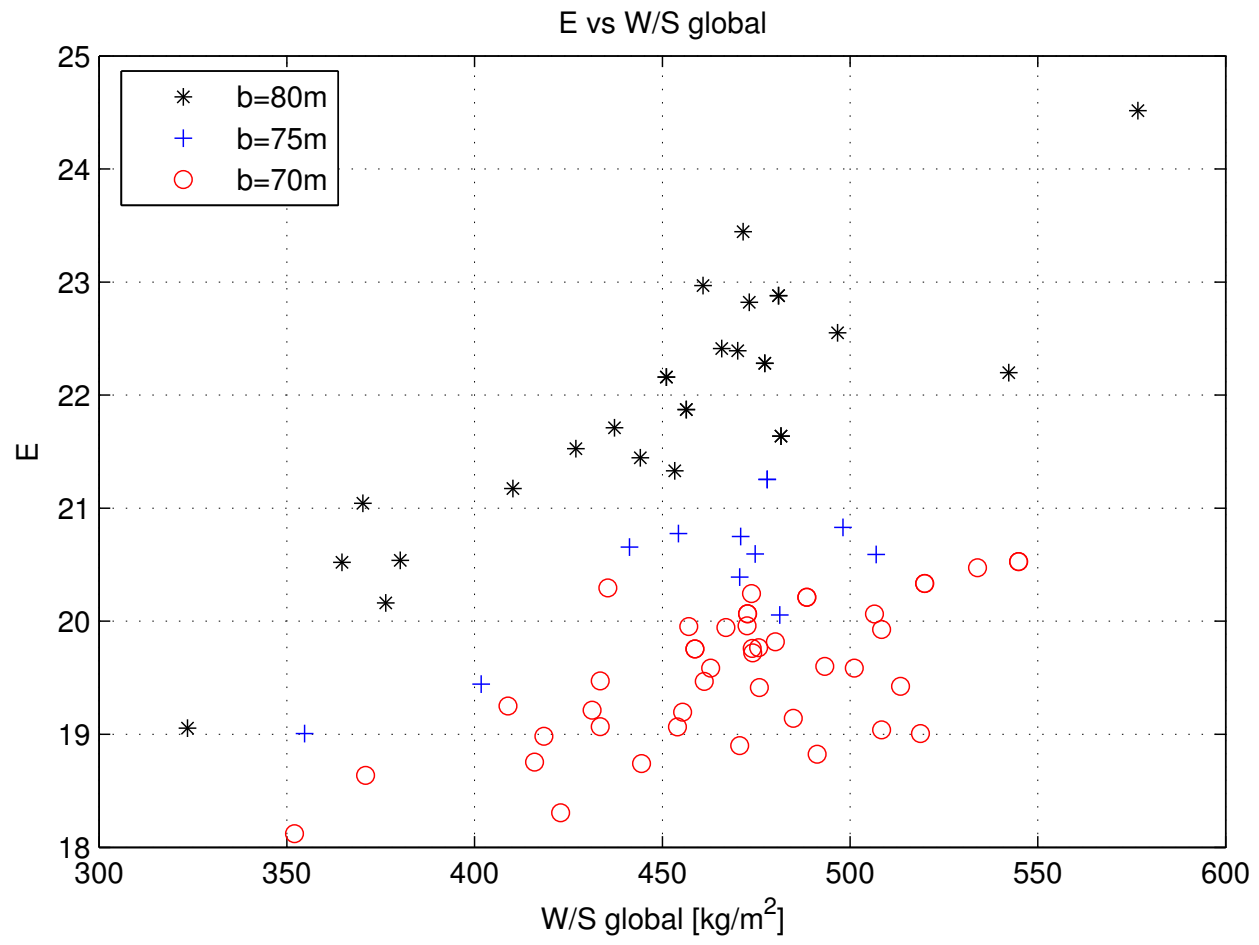

Figure 5.42. Efficiency vs Global wing loading - B sensitivity 


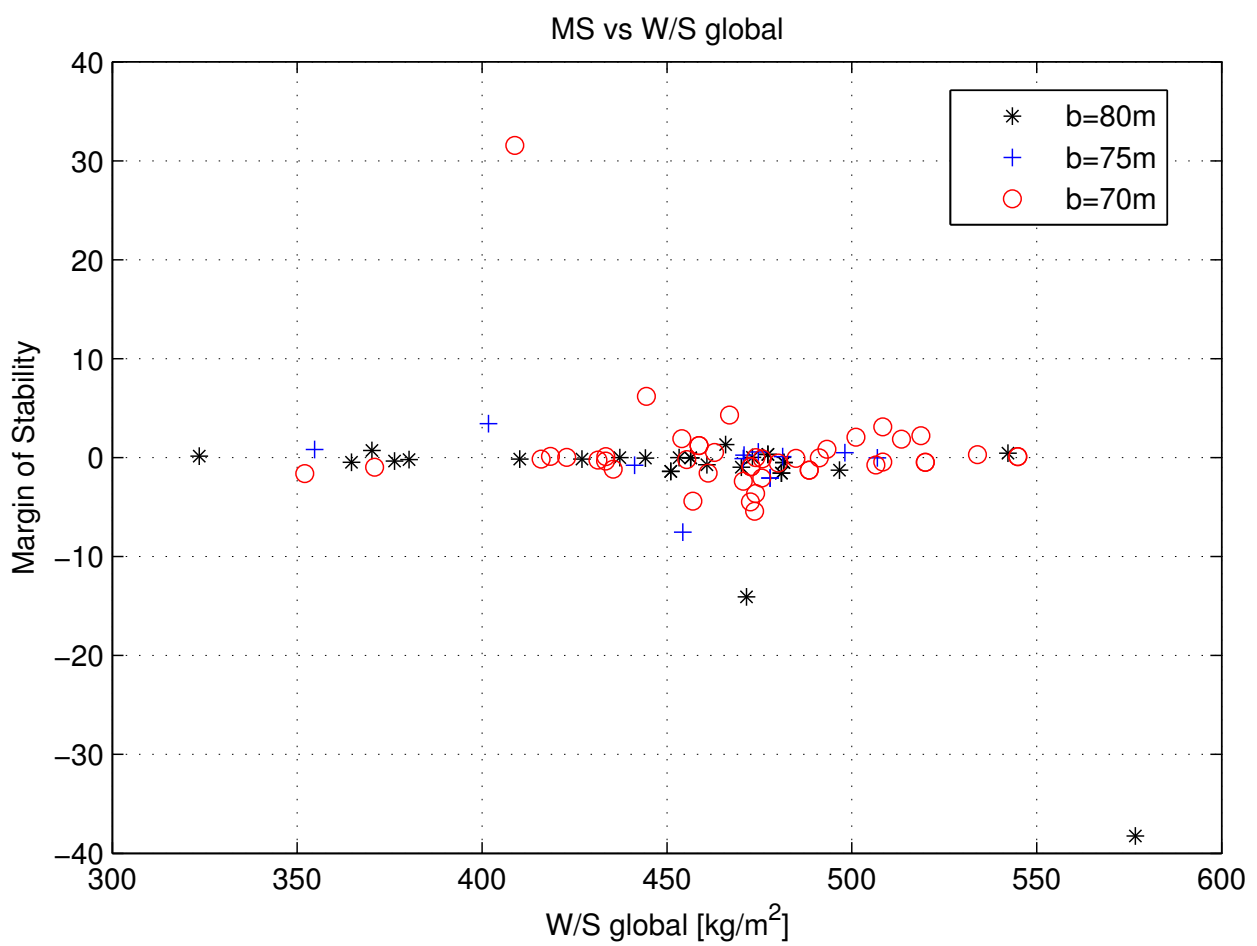

Figure 5.43. Margin of Stability vs Global wing loading - B sensitivity

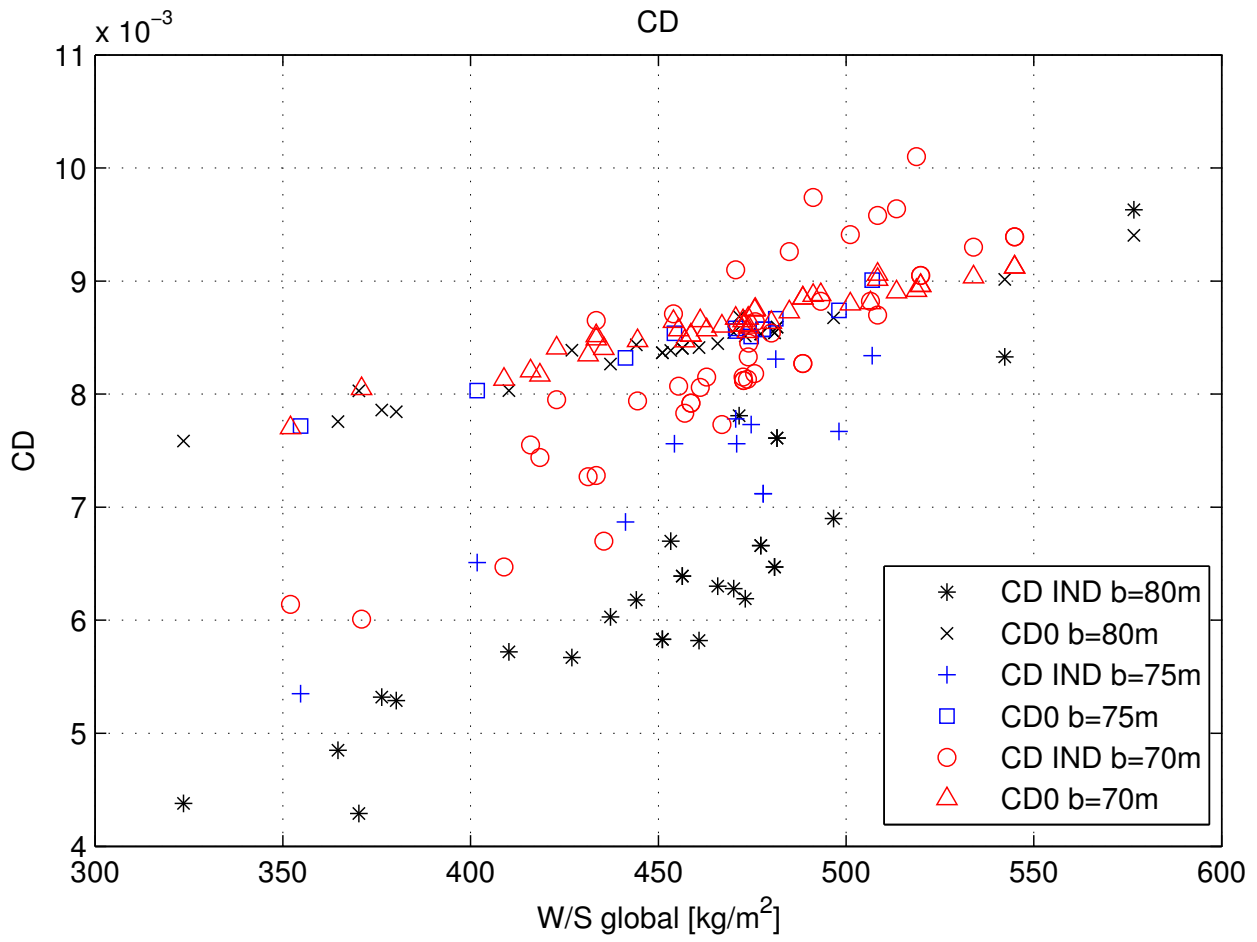

Figure 5.44. Drag coefficients - B sensitivity 

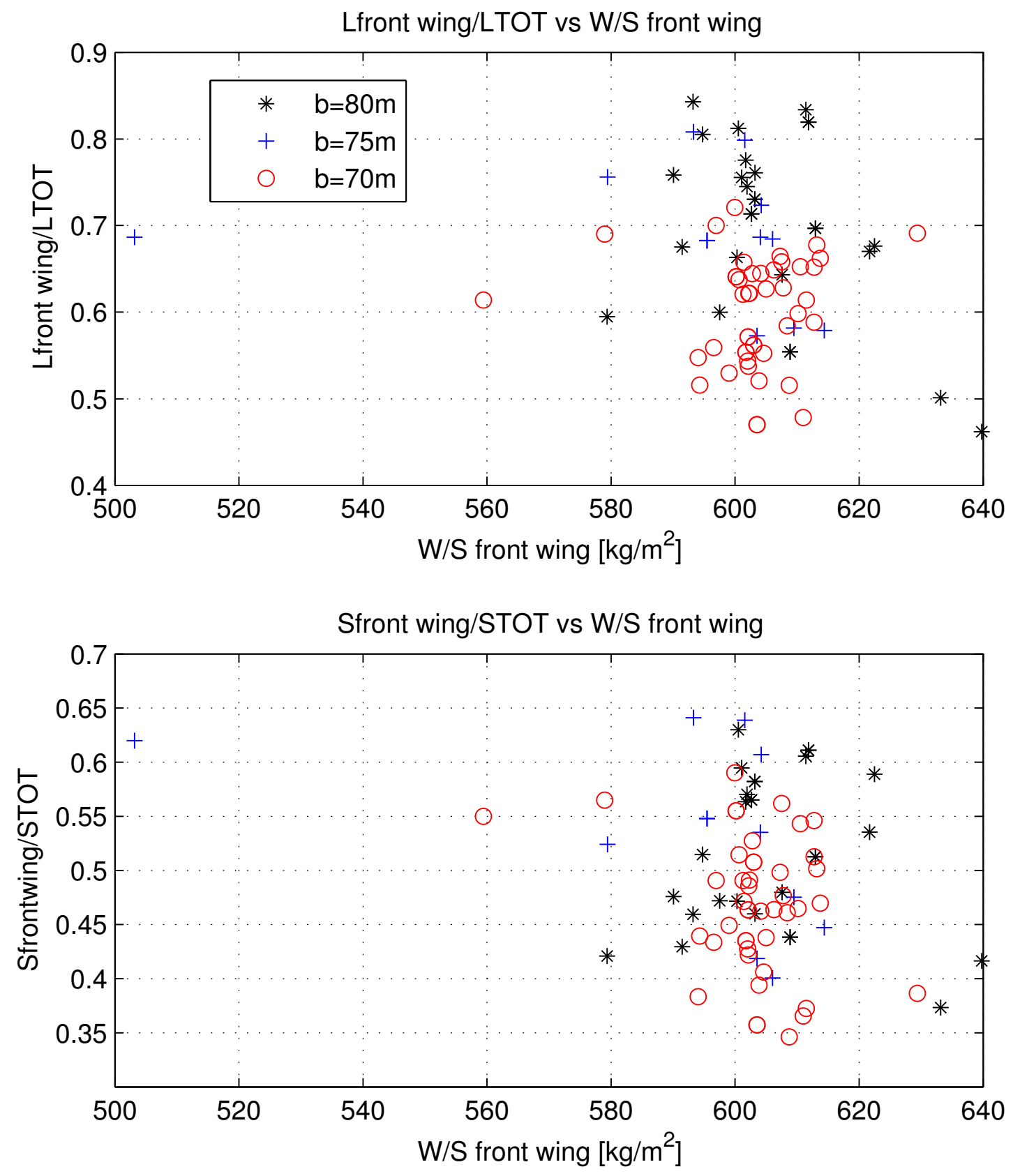

Figure 5.45. Front wing lift and surface - B sensitivity 

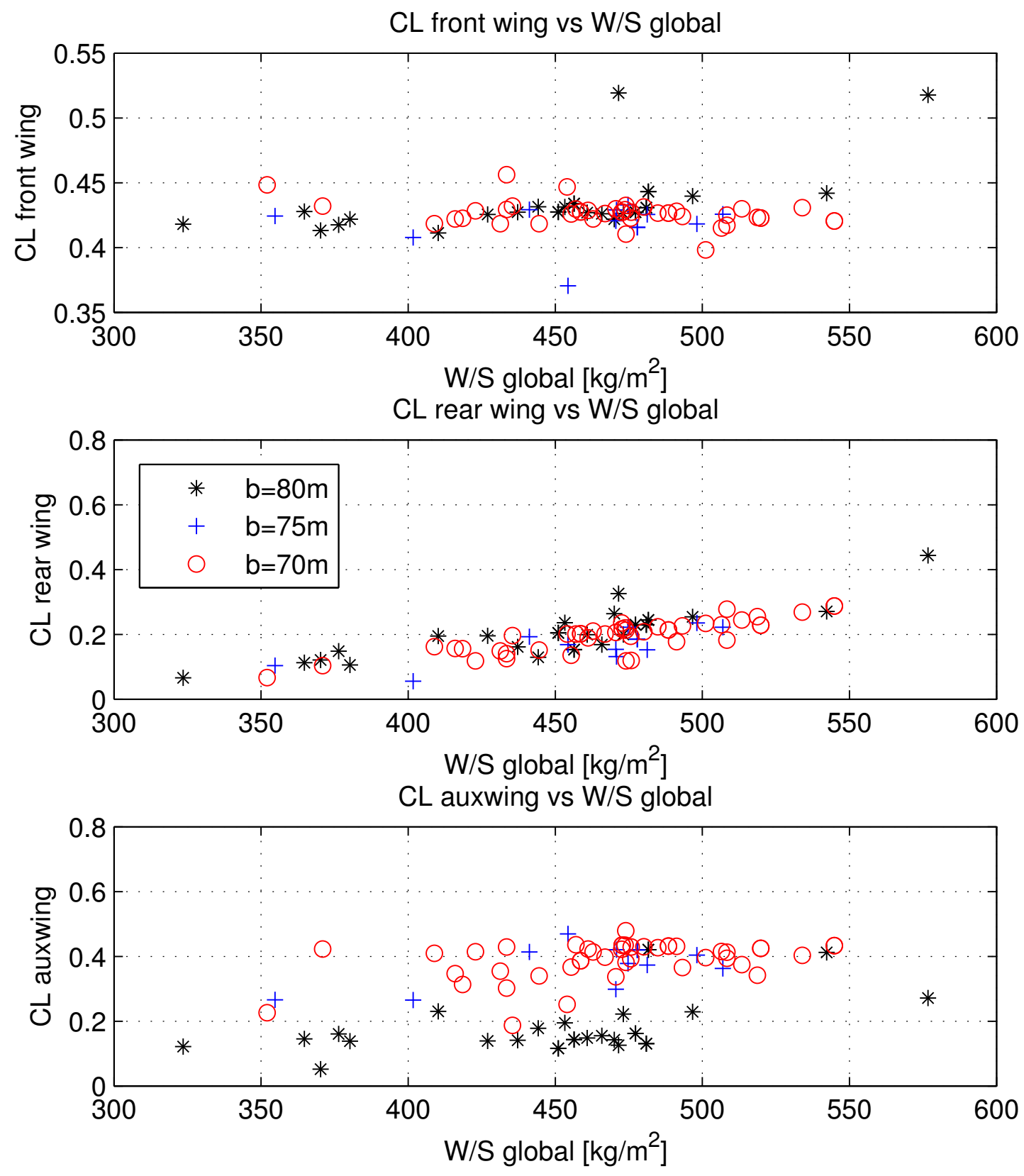

Figure 5.46. $C L$ behaviour - B sensitivity 

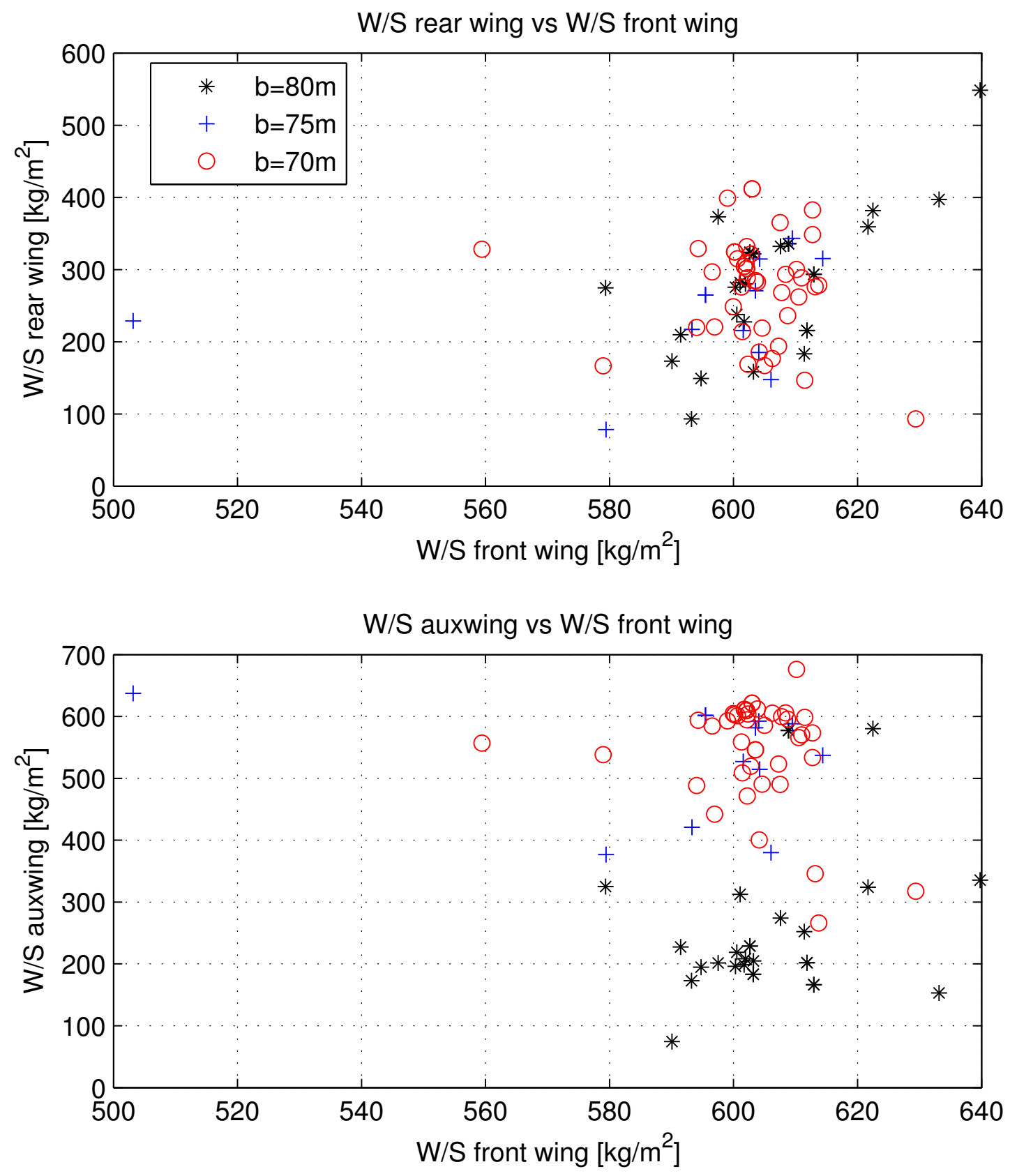

Figure 5.47. Wing loadings (part 2) - B sensitivity 


\subsection{The height-wingspan ratio}

The present section wants to explain how the solutions, provided by the optimizer, can change, keeping constant the height-wingspan ratio $\left(\frac{h}{b}\right)$.

Three analyses are compared: the first one is the same analysis of section 5.8; the second and the third analyses have been performed reducing both the wingspan and the fin height. Main information about the analyses are summarized in Tab. 5.8.

\begin{tabular}{cccc}
\hline Analysis Number & Wingspan $b[\mathbf{m}]$ & Fin Height $h[\mathbf{m}]$ & $\left(\frac{h}{b}\right)$ \\
\hline 31 & 80 & 12 & 0.150 \\
\hline 46 & 75 & 11 & 0.147 \\
\hline 47 & 70 & 10 & 0.143 \\
\hline
\end{tabular}

Table 5.8. Comparison between analysis 31,46 and 47 .

The results are shown from Fig. 5.48 to Fig. 5.59 and the reliability of these analyses is checked in Fig. 5.48 and in Fig. 5.49: the Fig. 5.49 emphasizes that the global Aspect Ratio and the global wing loading are linearly dependent, because the total span and the weight are fixed.

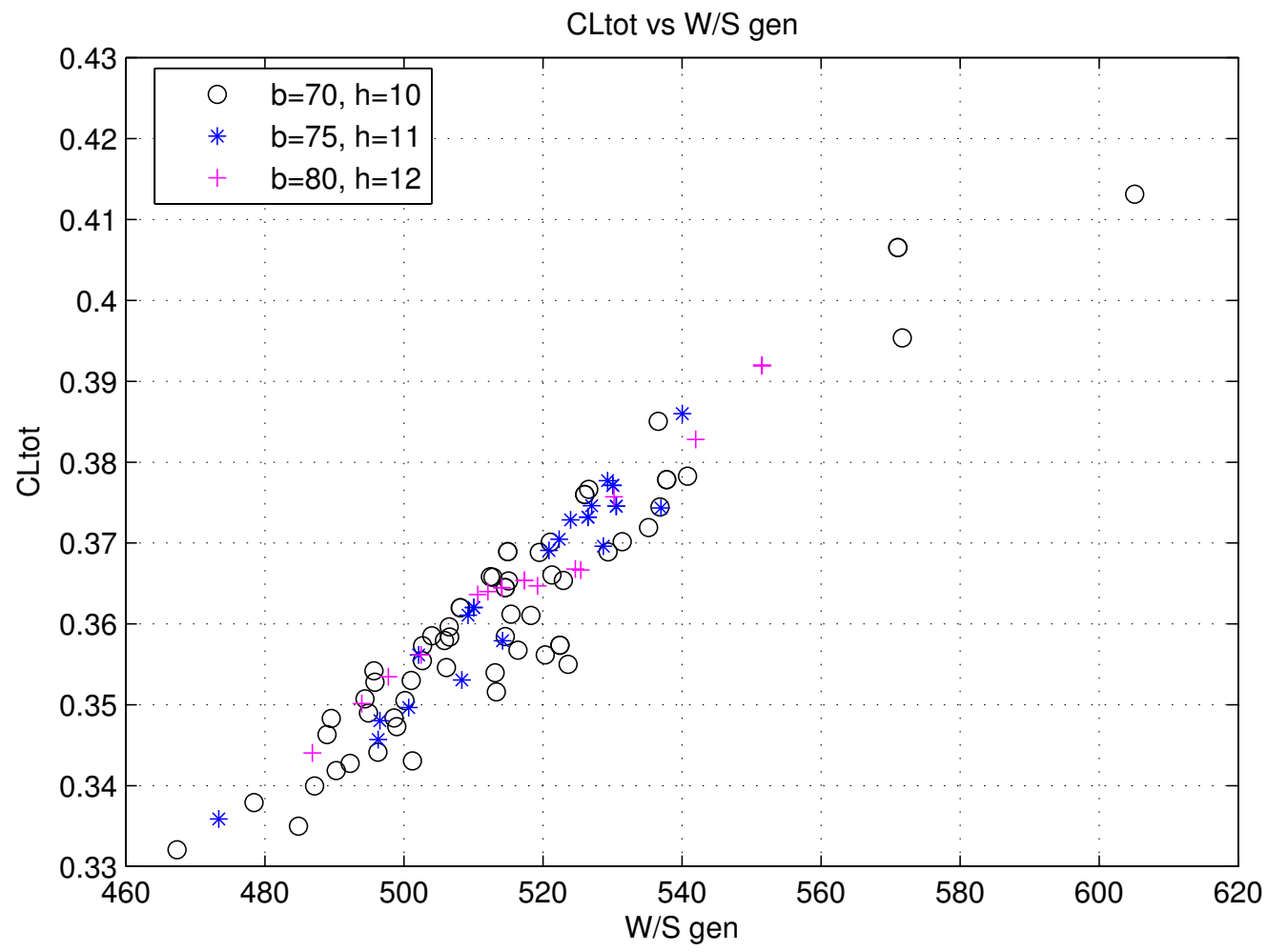

Figure 5.48. CLtot vs Global wing loading $-h / b$ constant 
Chapter 5. Research of preliminary optimum configurations

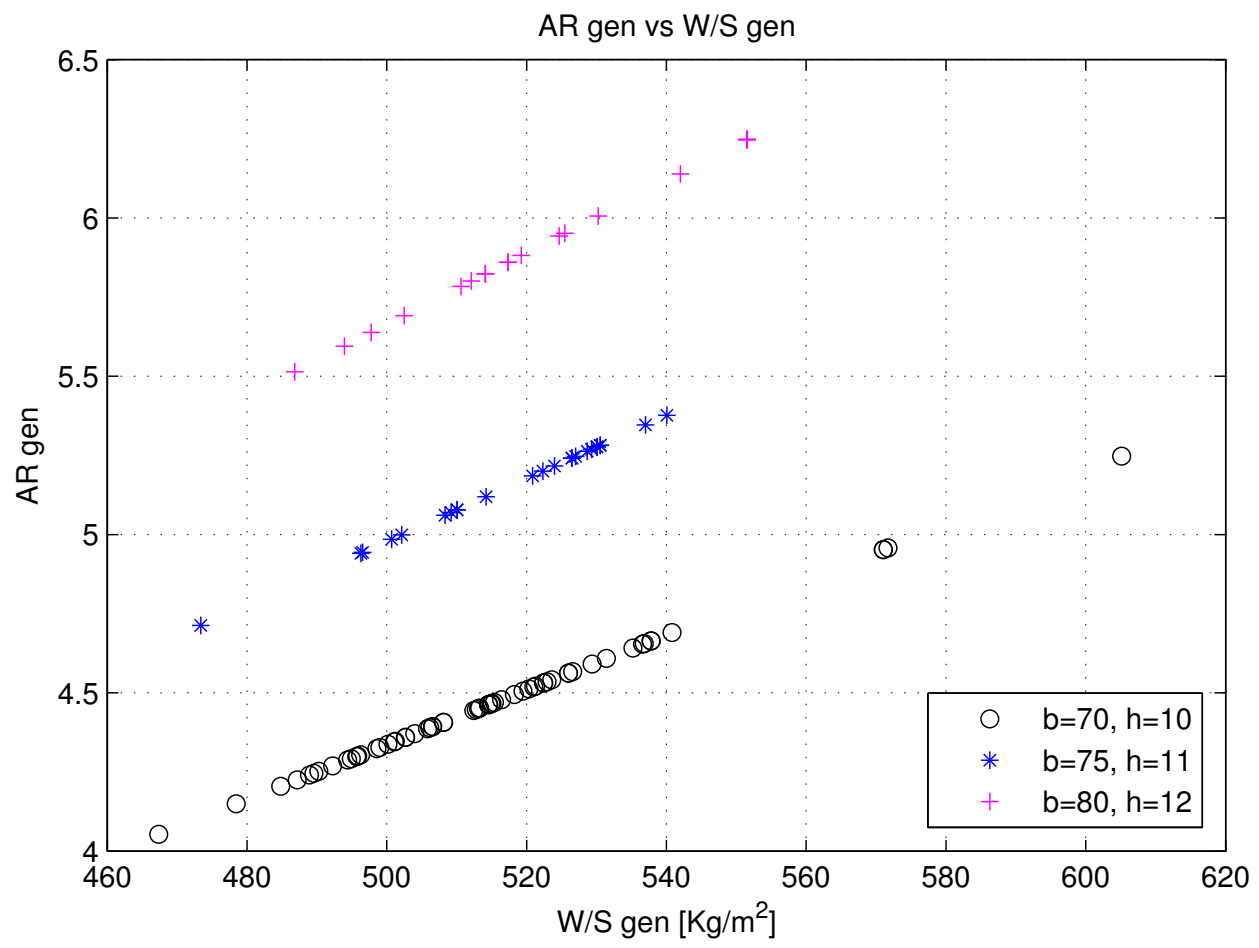

Figure 5.49. AR gen vs Global wing loading $-h / b$ constant E vs W/S gen

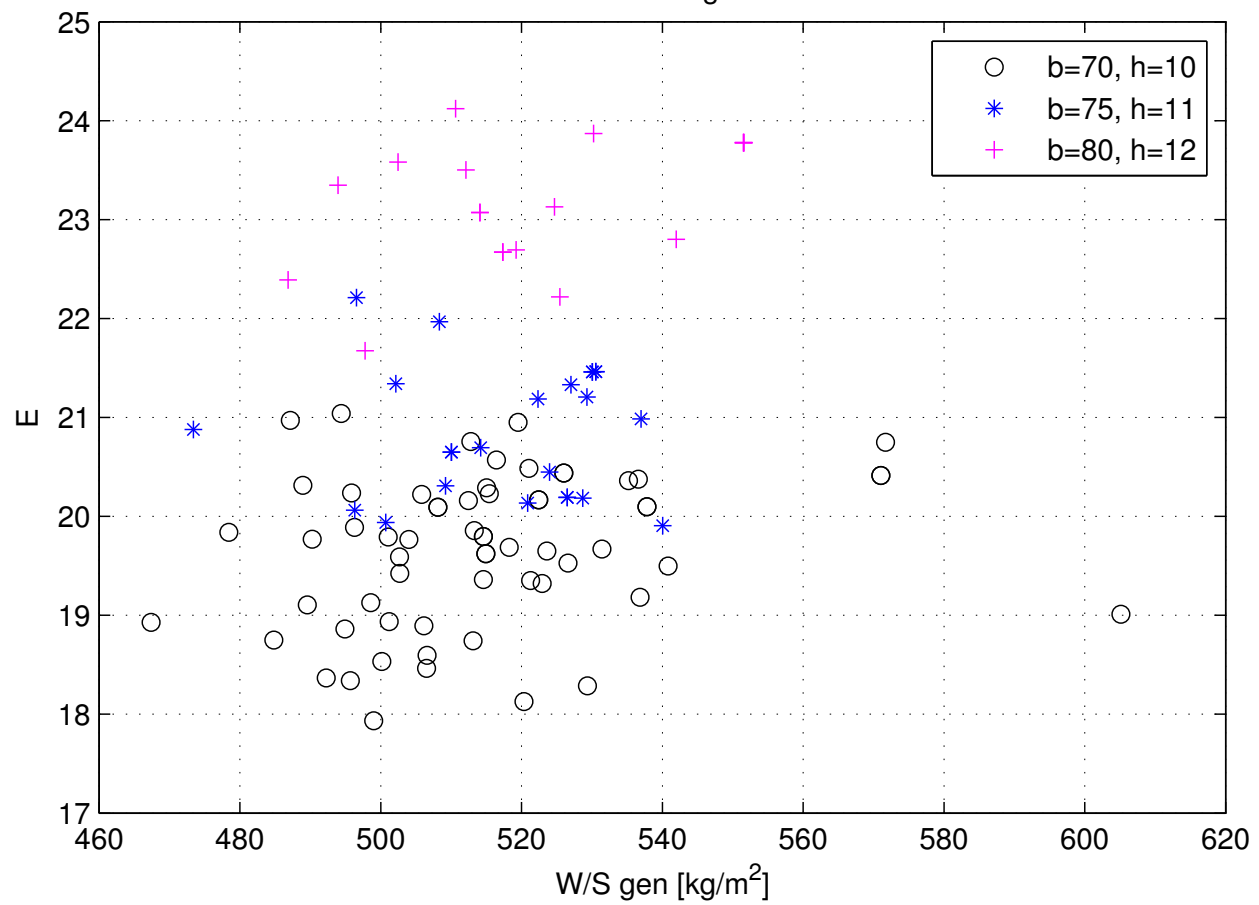

Figure 5.50. Efficiency vs Global wing loading - $h / b$ constant 
Fig. 5.50 and Fig. 5.51 show how the big configurations are more efficient than smaller ones. For a simplified box wing configuration (refer to the section 1), the induced drag is constant if $\left(\frac{h}{b}\right)$ is constant. In this case, despite the fin height - wingspan ratio is about constant, the induced drag is significantly affected by the wingspan, thus, the configurations with $b=75 \mathrm{~m}, h=11 \mathrm{~m}$ and $b=70 \mathrm{~m}, h=10 \mathrm{~m}$ undergo an increasing induced drag (refer to 5.52).

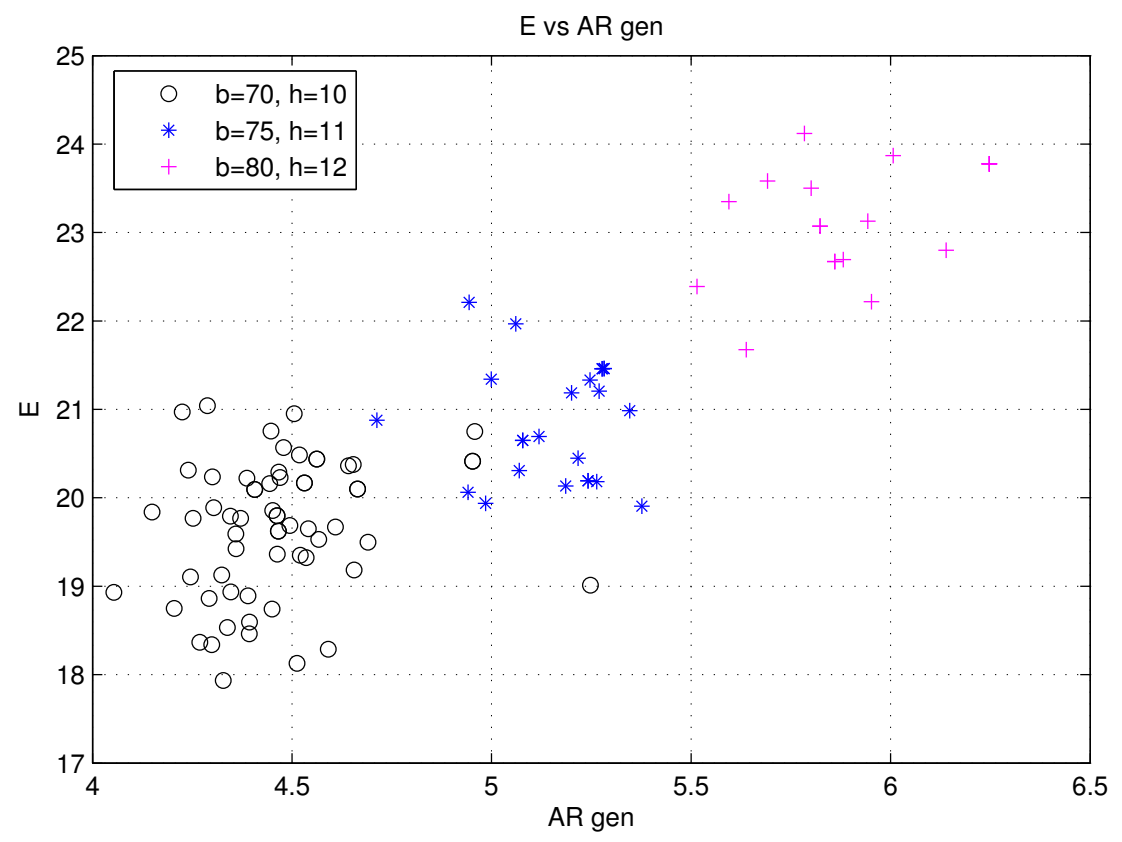

Figure 5.51. Efficiency vs ARgen - $h / b$ constant

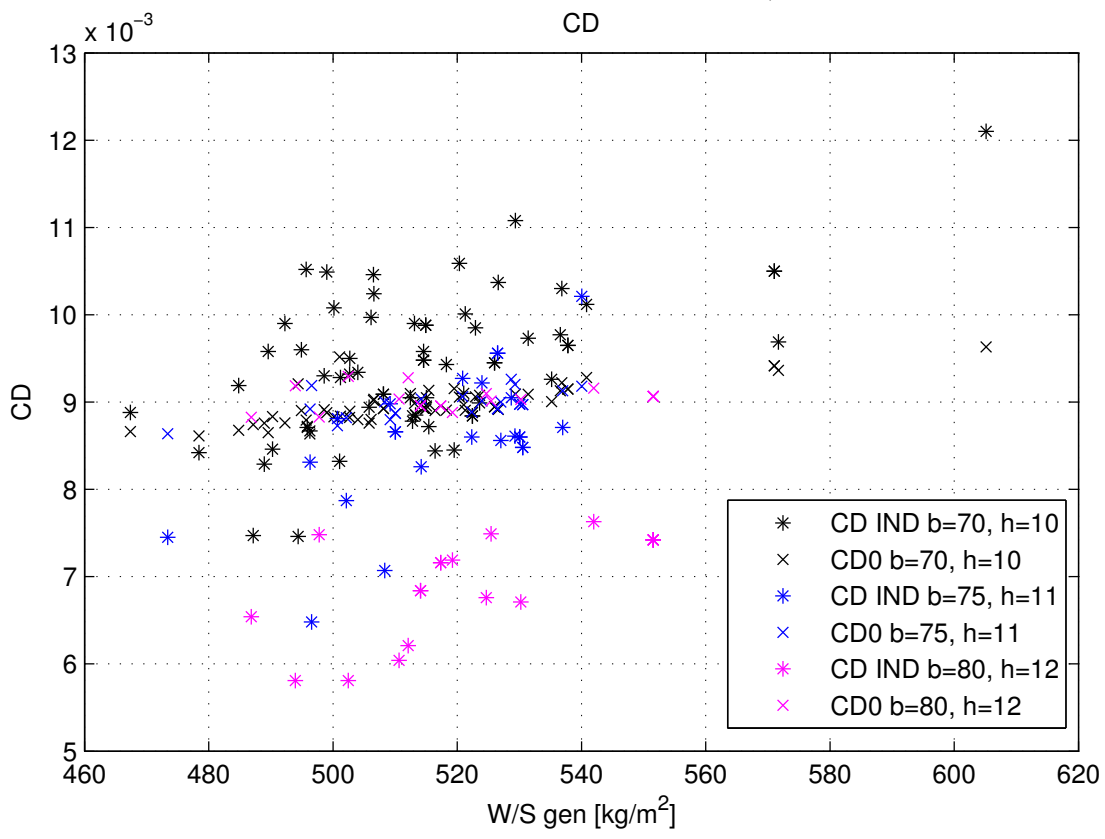

Figure 5.52. CD trends $-h / b$ constant 
Instead, the friction drag approximately does not change because the total surface does not change significantly for the three wings arrangements. If the wingspan and the fins are shorter, the chords are bigger and the two effects compensate each other. Then, the cut of efficiency in the small configuration is due to the higher induced drag. This unexpected fact probably occurs because, in these cases, the wing system is not simply a box. Wings have noticeable sweep angles and dihedral, which affect the induction of vortices and the $\left(\frac{h}{b}\right)$ parameter is not sufficient to dominate the phenomenon.

When the configuration has big wingspan and big fin height, the front wing can produce up to $70 \%$ of the total lift; if the wingspan and the fin height are reduced, the front wing develops a lower percentage of the total lift for nearly all the configurations (refer to Fig. $5.53)$.
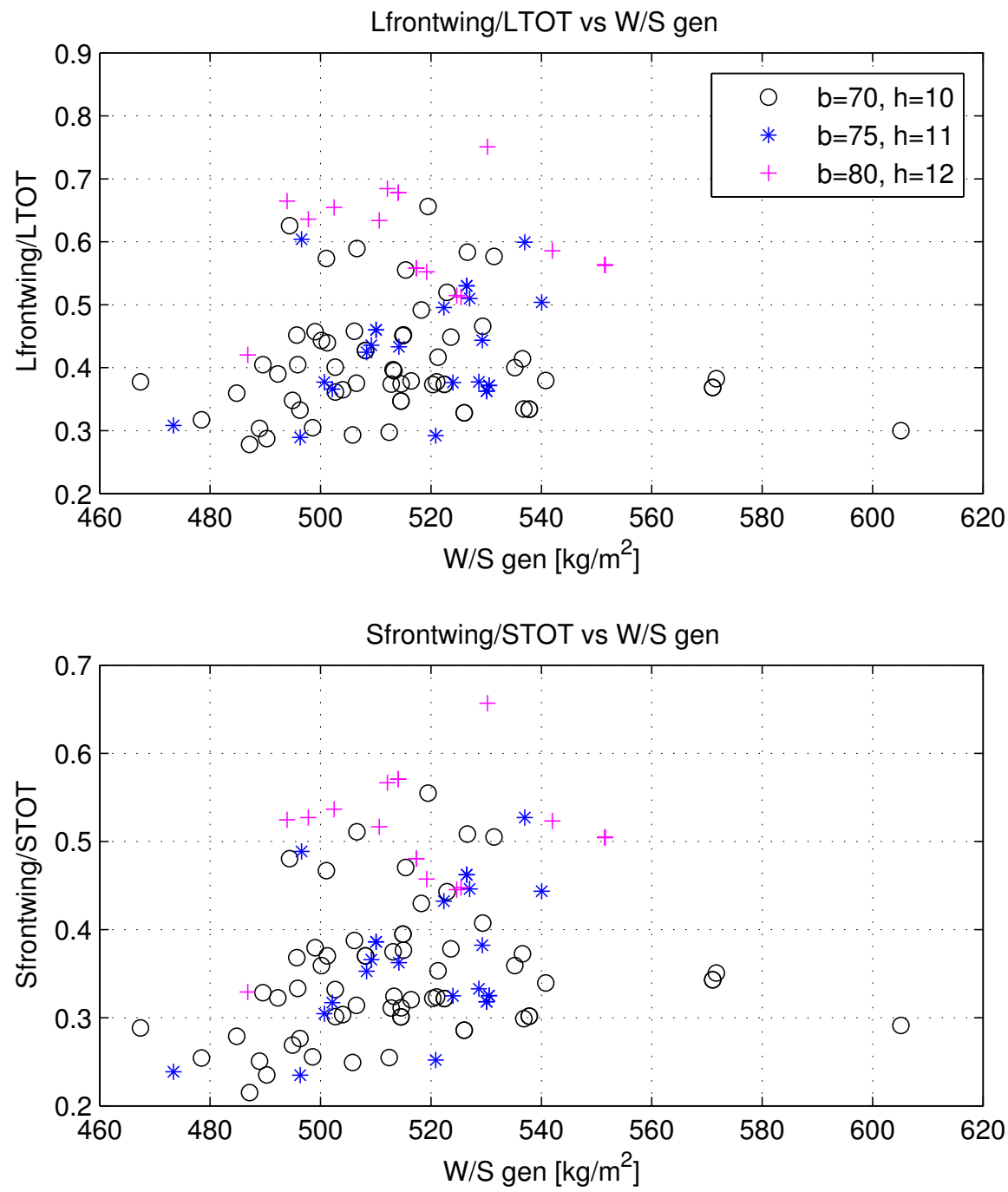

Figure 5.53. Front Wing behaviour $-h / b$ constant 
It is evident that, when the wingspan and the fin height are reduced, the rear wing and the auxiliary wing tend to lift more, Fig. 5.54 and Fig. 5.55): this behaviour is obtained by the decrease in lift of the front wing, which is compensate by a lift increase of rear and auxiliary wings.
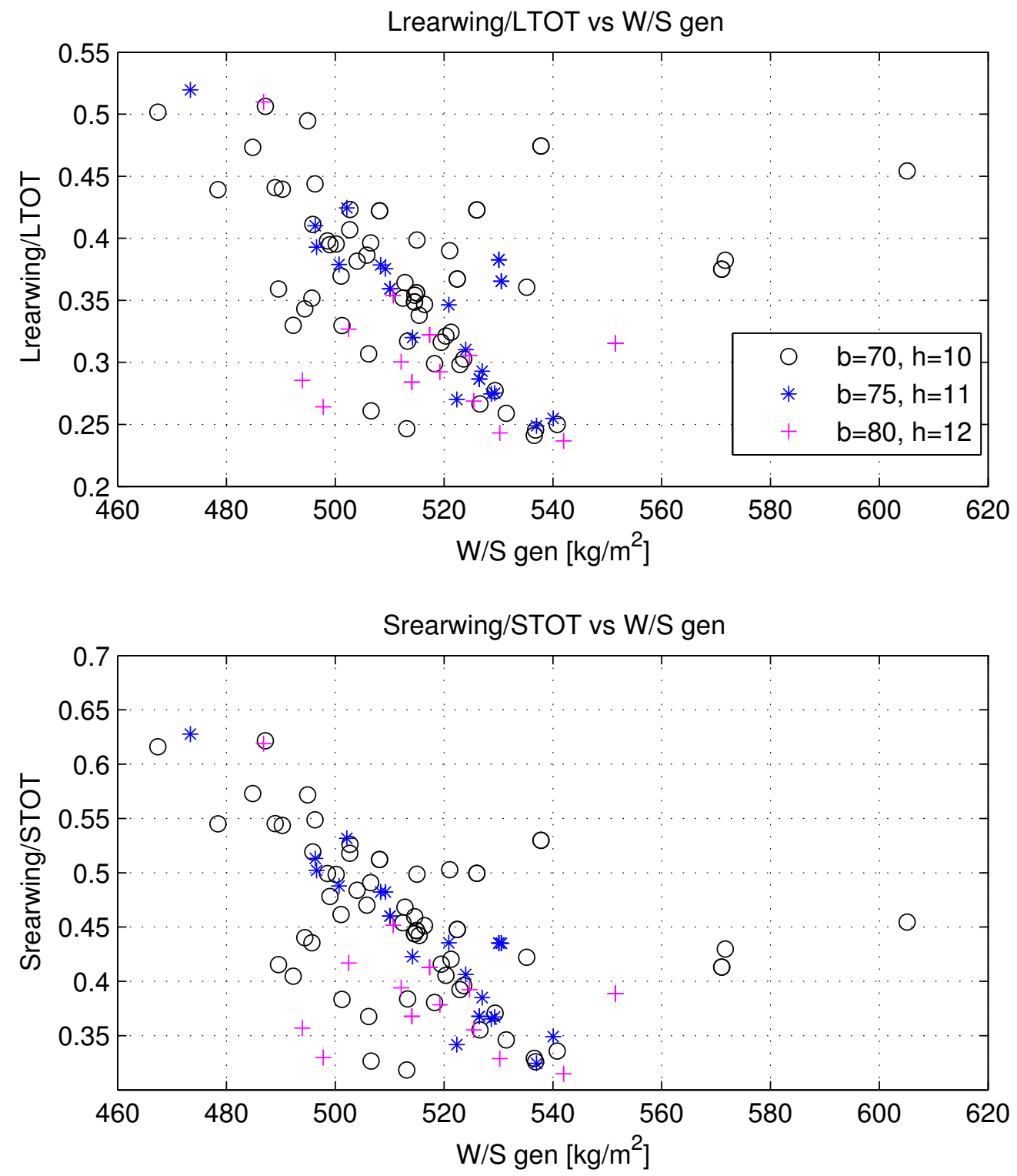

Figure 5.54. Rear Wing behaviour - $h / b$ constant 

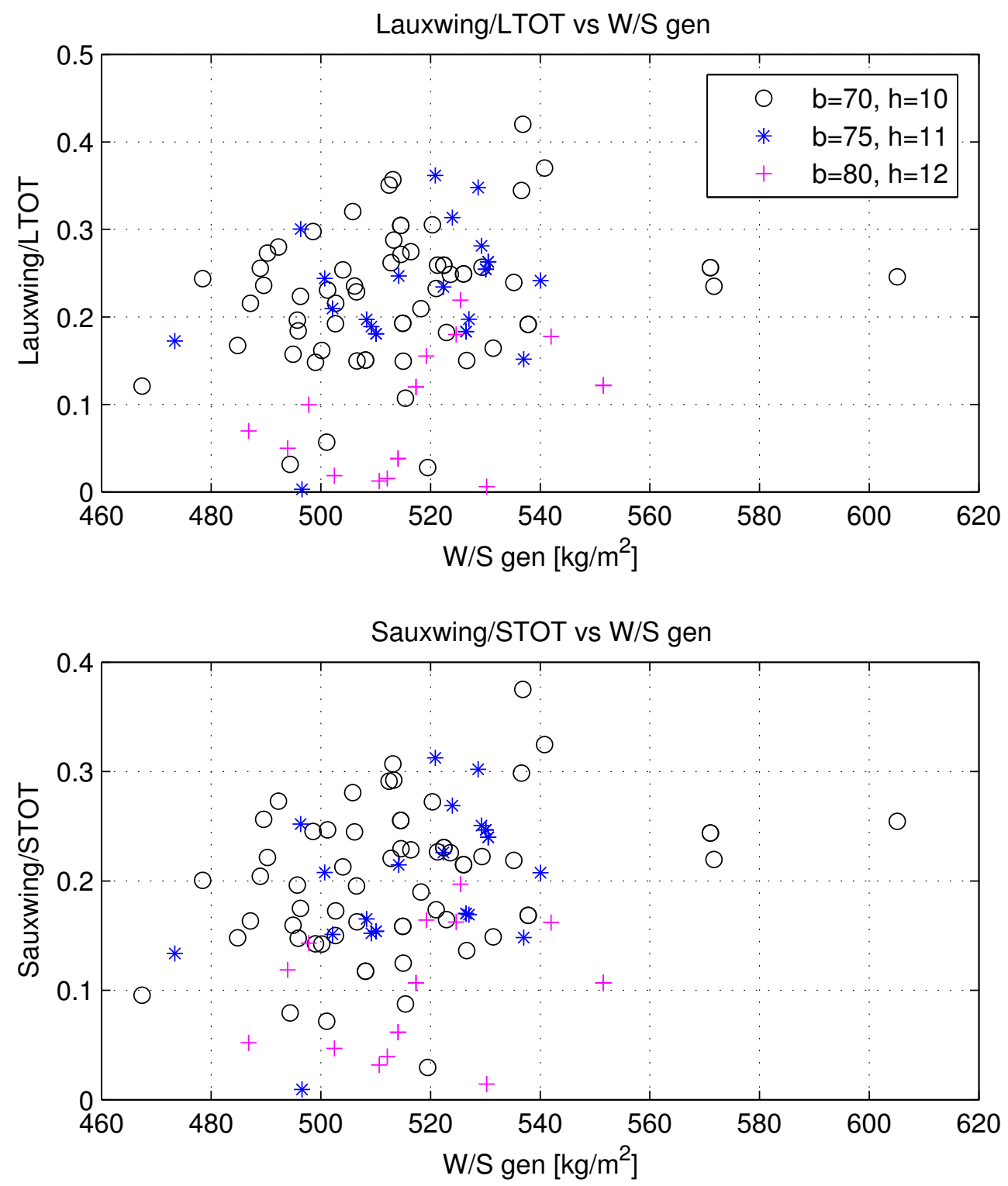

Figure 5.55. Auxiliary Wing behaviour $-h / b$ constant

Furthermore, the auxiliary wing surfaces are raised, with the purpose of generating the lift required: the optimizer chooses to generate this surface increasing both the auxiliary wingspan (Fig. 5.56) and the chords lenght, refer to Fig. 5.57, in most configurations. In this way, the aspect ratio of the auxiliary wing is about constant. 


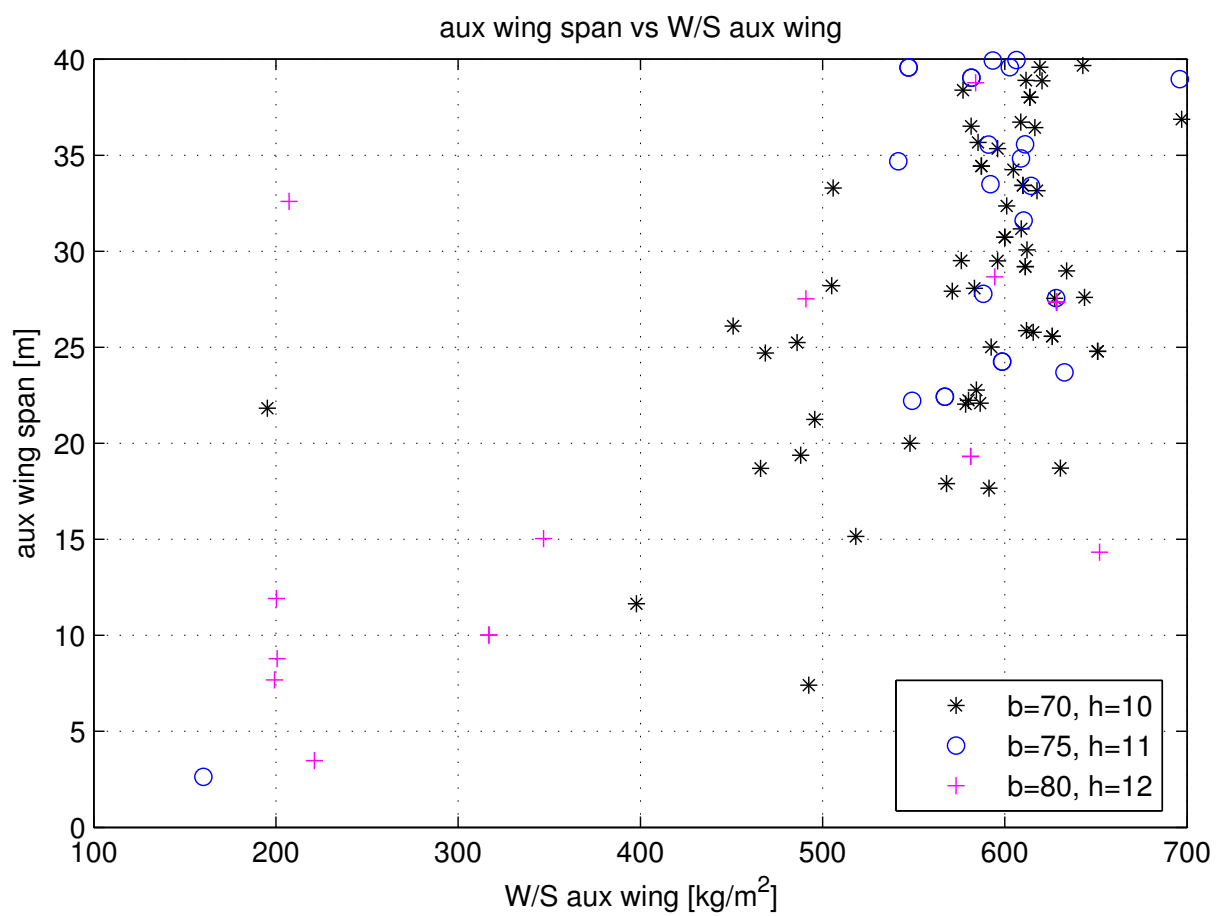

Figure 5.56. Auxiliary Wing wingspan - $h / b$ constant aux wing root chord vs W/S aux wing

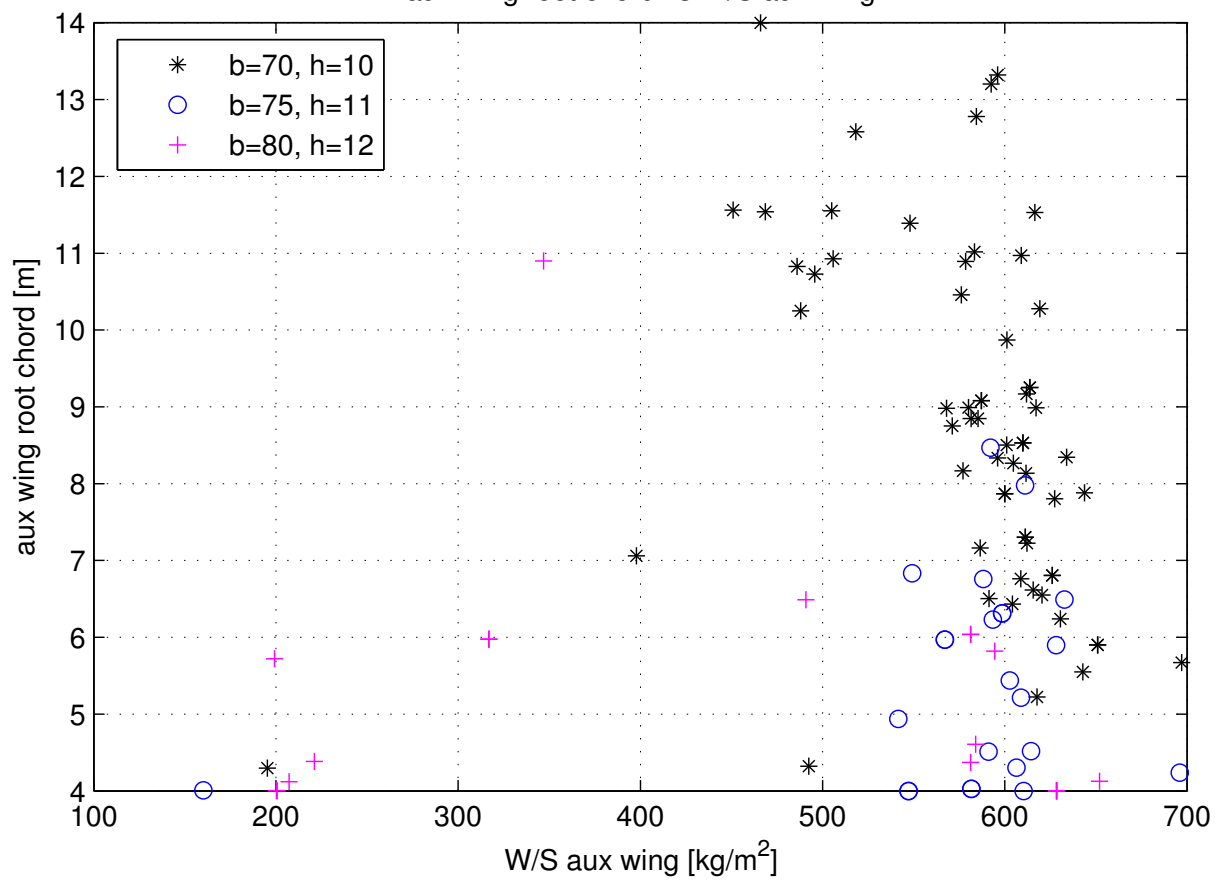

Figure 5.57. Auxiliary Wing root chord $-h / b$ constant

Fig. 5.58 shows how the global $C_{L}$ is not affected by any wing loading. Besides, it seems that the optimizer tries to rise the $C_{L}$ value but there is a threshold around 0.39 that is impossible to overcome. 

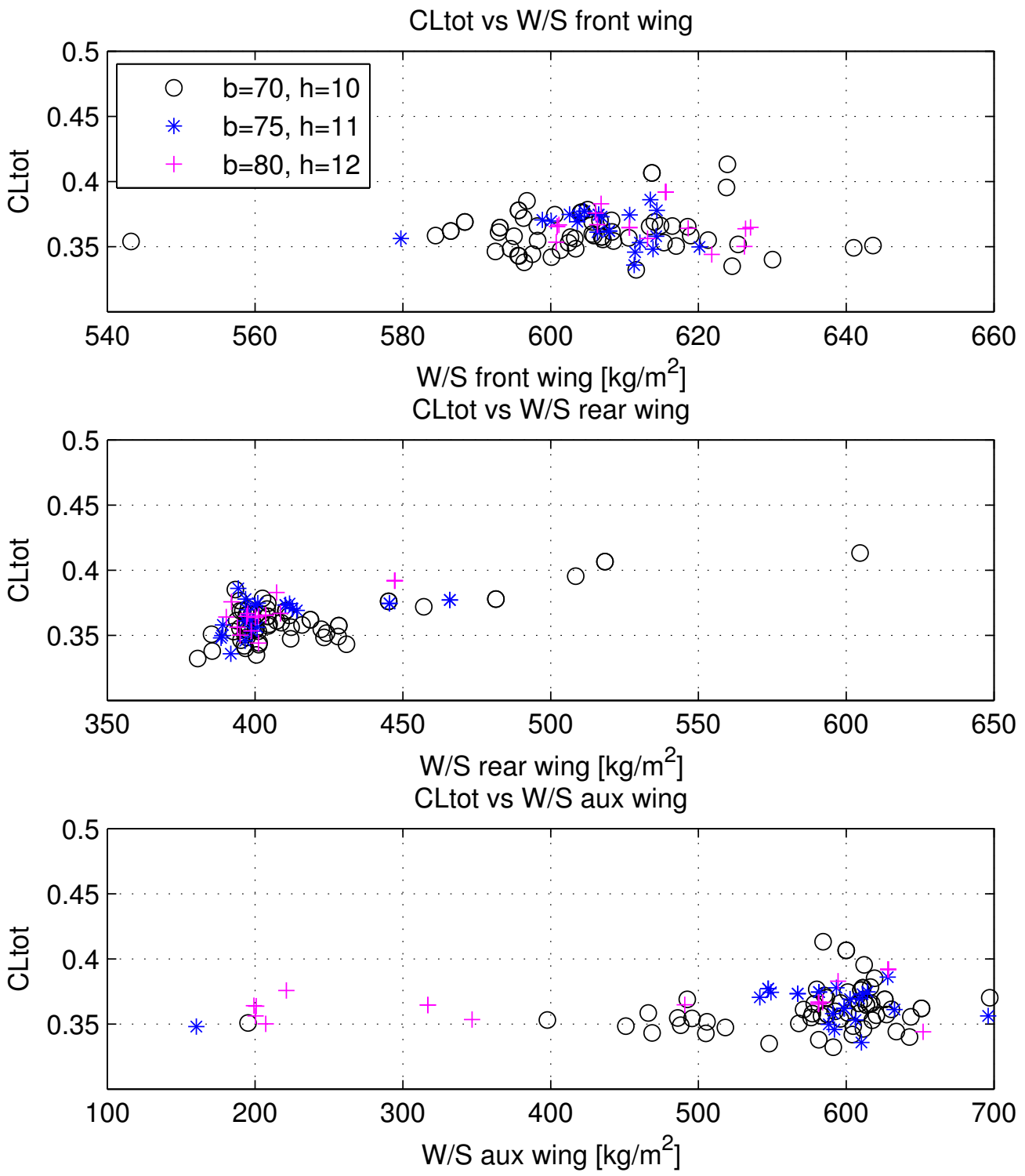

Figure 5.58. CLtot trends $-h / b$ constant 

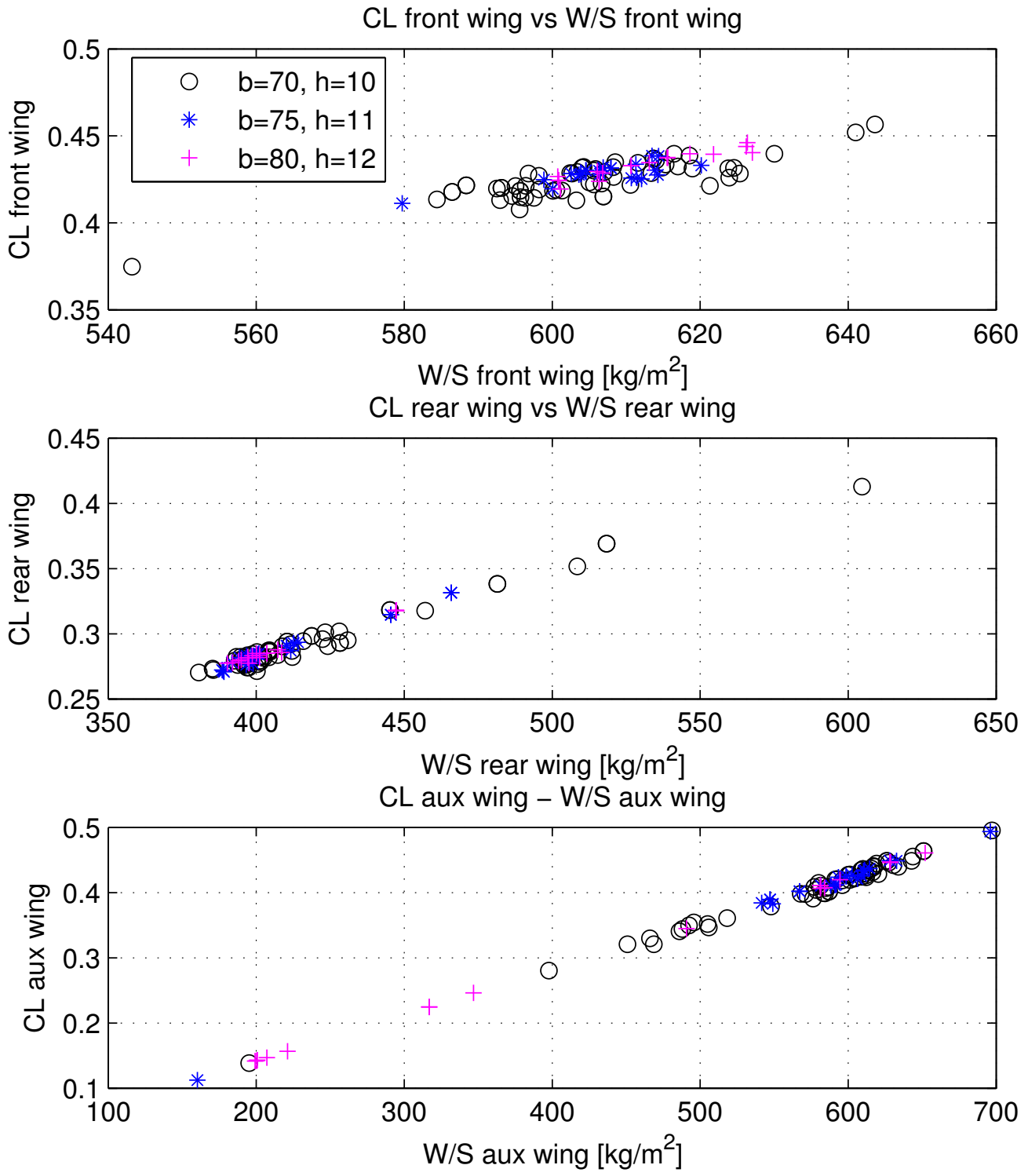

Figure 5.59. CL trends - $h / b$ constant 


\subsection{Advancement of the rear wing}

This section deals with the advancement of the rear wing along the fuselage axis, that is an effect connected with the rear wing loading variation and, in particular, an increase of this wing loading, already examined in section 5.4. Contrary to the previous analyses, the auxiliary wing does not remain ahead of the fuselage but moves after the rear wing.

One of the resultant configuration of this analysis is plotted in Fig. 5.60.

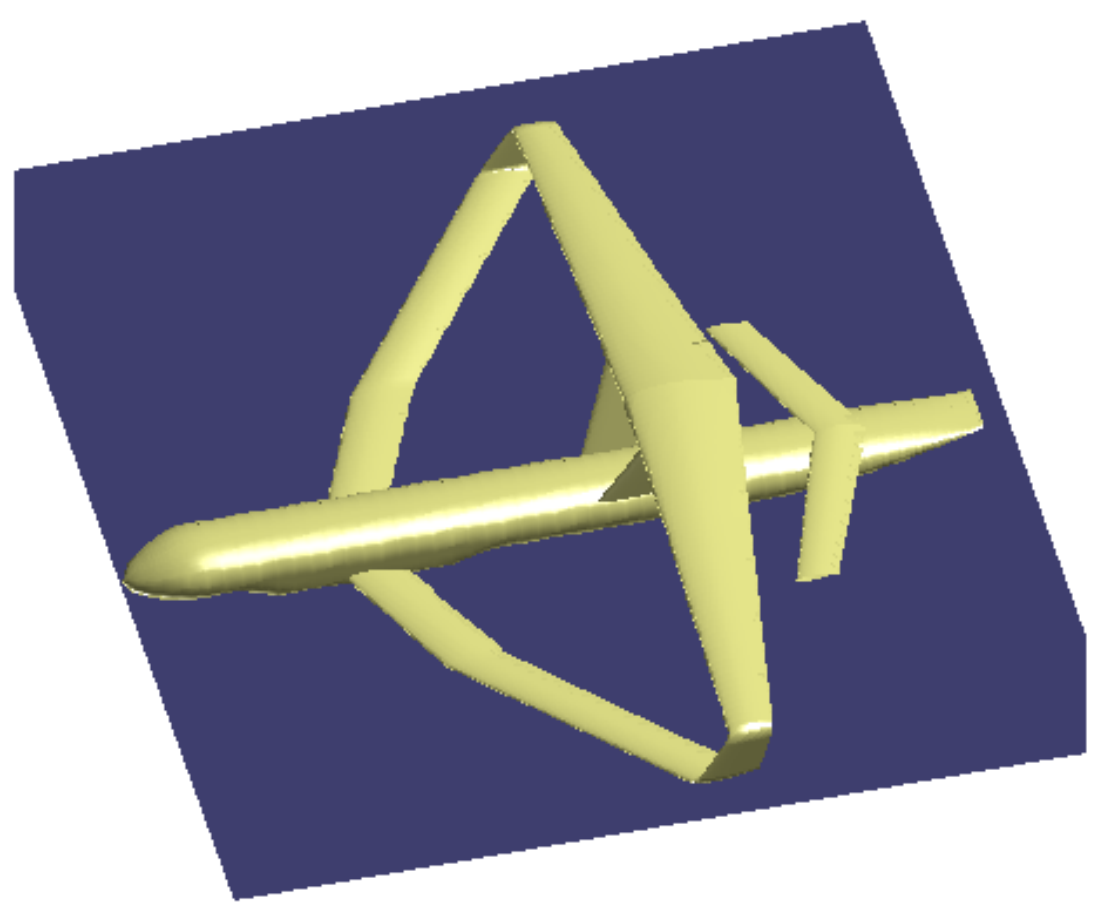

Figure 5.60. Example of configuration with rear wing advancement

The parameters setting of the current analysis is reported below:

$$
\begin{aligned}
B & =80 \mathrm{~m} \\
H & =11 \mathrm{~m} \\
\text { alpha } & =0 \\
K_{\text {bulk }} & =0.8 \\
0 \% & <M S<3 \% \\
400 \mathrm{~kg} / \mathrm{m}^{2} & <\left(\frac{W}{S}\right)_{\text {front }}<600 \mathrm{~kg} / \mathrm{m}^{2} \\
450 \mathrm{~kg} / \mathrm{m}^{2} & <\left(\frac{W}{S}\right)_{\text {rear }}<750 \mathrm{~kg} / \mathrm{m}^{2} \\
200 \mathrm{~kg} / \mathrm{m}^{2} & <\left(\frac{W}{S}\right)_{\text {aux }}<600 \mathrm{~kg} / \mathrm{m}^{2}
\end{aligned}
$$


The results of this analysis (reported from Fig. 5.61 to Fig. 5.67 and in Appendix E) are compared with the results of the analysis of section 5.4. The results are reported at varying the rear wing loading boundaries; so, "450 - 750" identifies configurations of the present analysis, while " $500-800 "$ identifies configurations of the previous analyses. Fig. 5.61 and Fig. 5.62 show where the rear wing and the auxiliary wing are located: in the present analysis the rear wing is approached to the centre of gravity in order to increase the lift. Meanwhile the auxiliary wing is translated at the rear of the fuselage: in fact the stability constraint has to be met together with the equilibrium condition. When the rear wing is very close the centre of gravity, the neutral point moves forward along the fuselage, so the margin of stability decreases; AEROSTATE tries to respect the $M S$ constraint by moving the auxiliary wing backwards. Despite this solution, many configurations do not satisfy the $M S$ constraint and are slightly unstable (refer to Fig. 5.63).

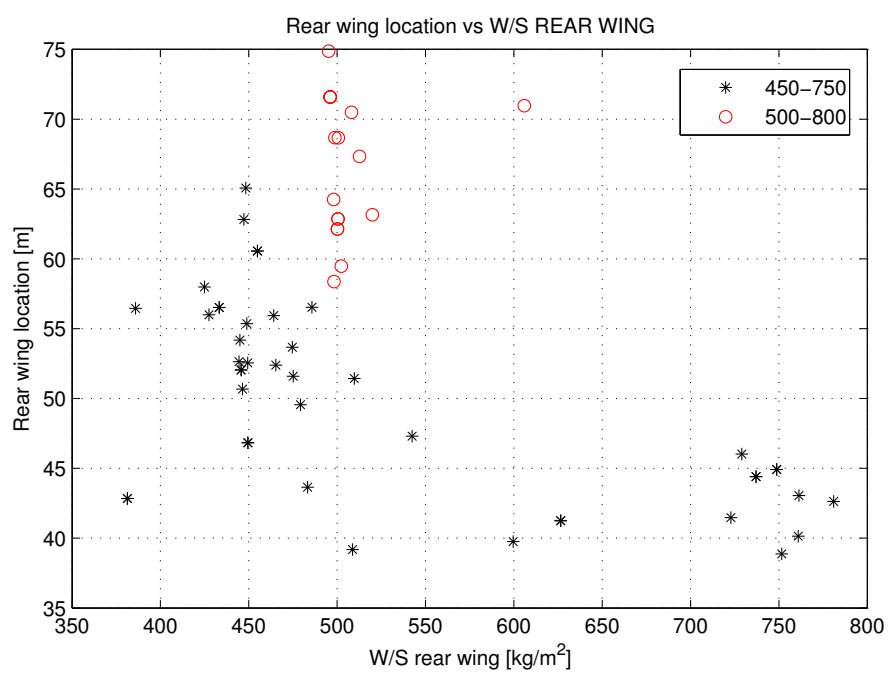

Figure 5.61. Rear wing location vs rear wing loading (rear wing advancement)

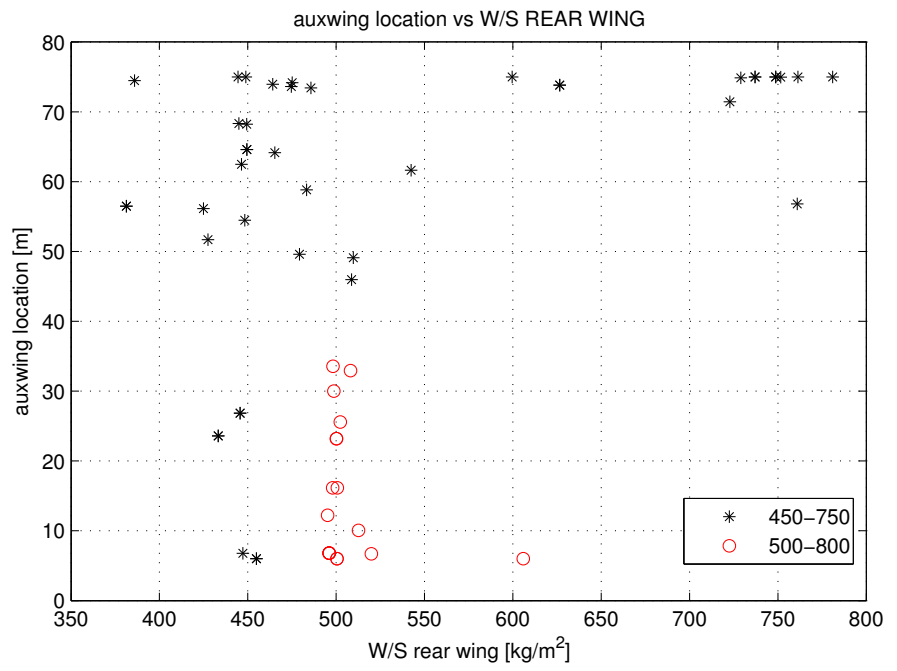

Figure 5.62. Auxiliary wing location vs rear wing loading (rear wing advancement) 


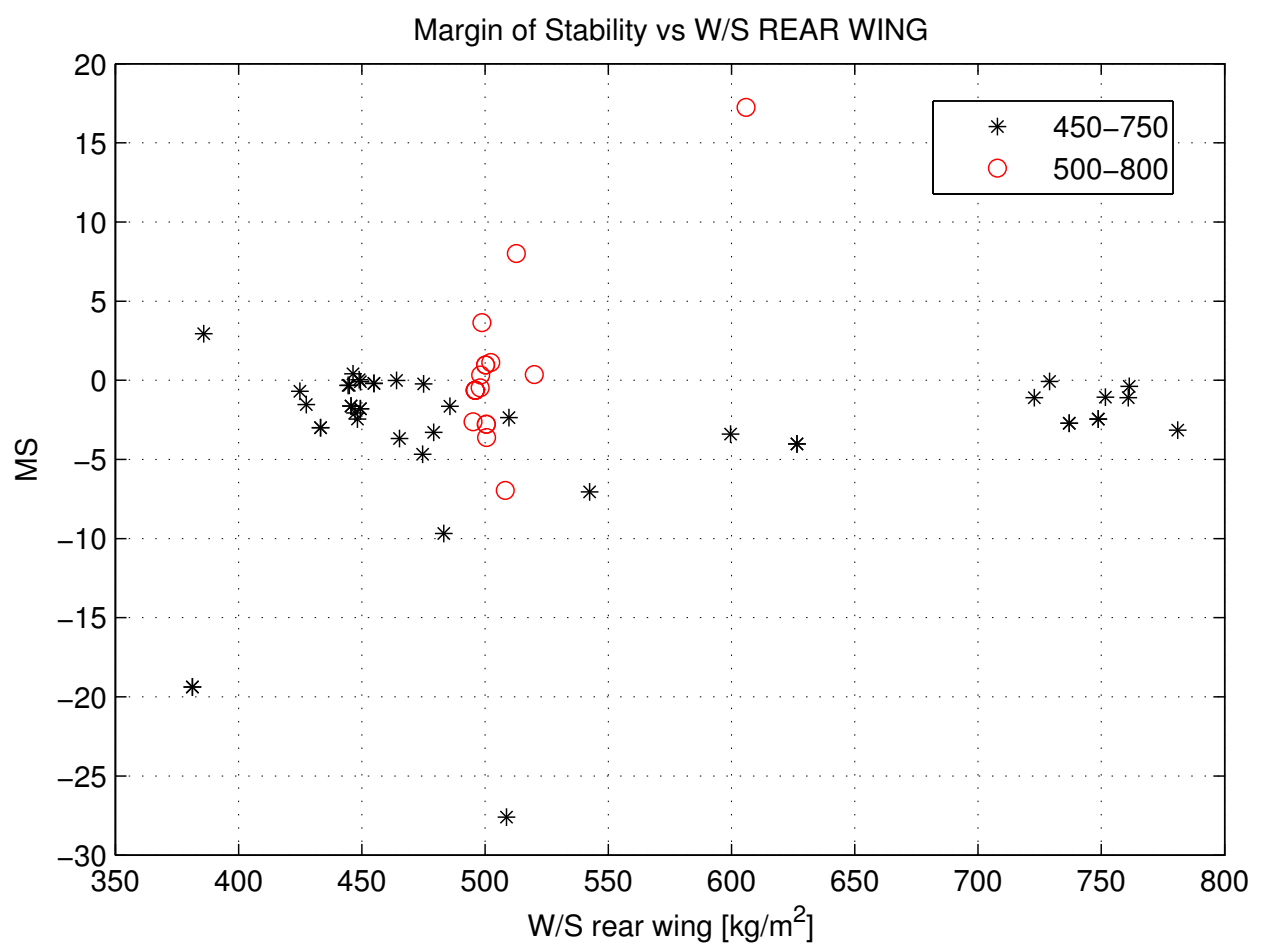

Figure 5.63. Margin of Stability vs rear wing loading (rear wing advancement)

It is appropriate to investigate the behaviour of the three wings (refer to Fig. 5.64 and to Fig. E.3).

The most remarkable difference between the two analyses is the role of the auxiliary wing and the rear wing. The auxiliary wing has the same percentage of the total surface in both the analyses, but in one case ("500-800") the auxiliary wing is very important to the lift because it contributes nearly up to $40 \%$ of the total lift.

This fact is connected to the different behaviour of the rear wing: in this analysis the auxiliary wing loading is limited to $600 \mathrm{~kg} / \mathrm{m}^{2}$ while in the previous it could rise to $900 \mathrm{~kg} / \mathrm{m}^{2}$.

Thus, the optimizer is pushed to make other surfaces lift, rather than the auxiliary wing. However the boundaries of the front wing loading are unchanged, then the only possibility, that AEROSTATE has to satisfy the equilibrium constraint, is to increase the lift contribution of the rear wing: hence, in the "500-800" analysis only the lower boundary of the rear wing loading is considered, while in the "450 - 750" analysis the optimizer finds efficient configurations in the whole given range.

Particularly, there are some configurations with high rear wing loading $\left(750 \mathrm{~kg} / \mathrm{m}^{2}\right)$ which significantly contribute to the lift. The different role of the auxiliary wing is emphasized in Fig. 5.65, where it is clear that the auxiliary wing is a very stressed structure in the "500 - 800" analysis.

It is very interesting to notice that the different way to load the wing system has evident effects on the arrangement of the wing system itself. 

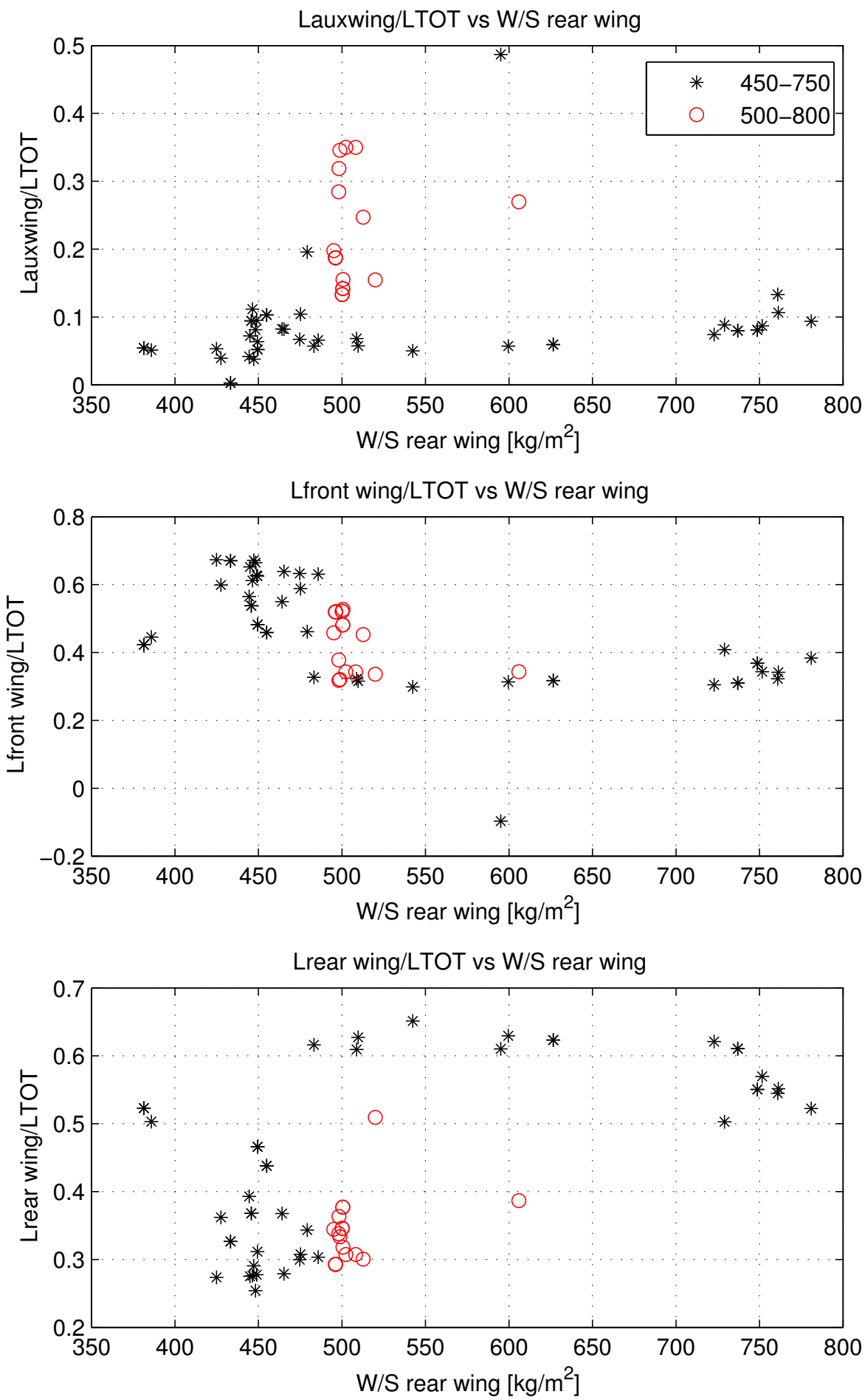

Figure 5.64. Lift contributions (rear wing advancement) 


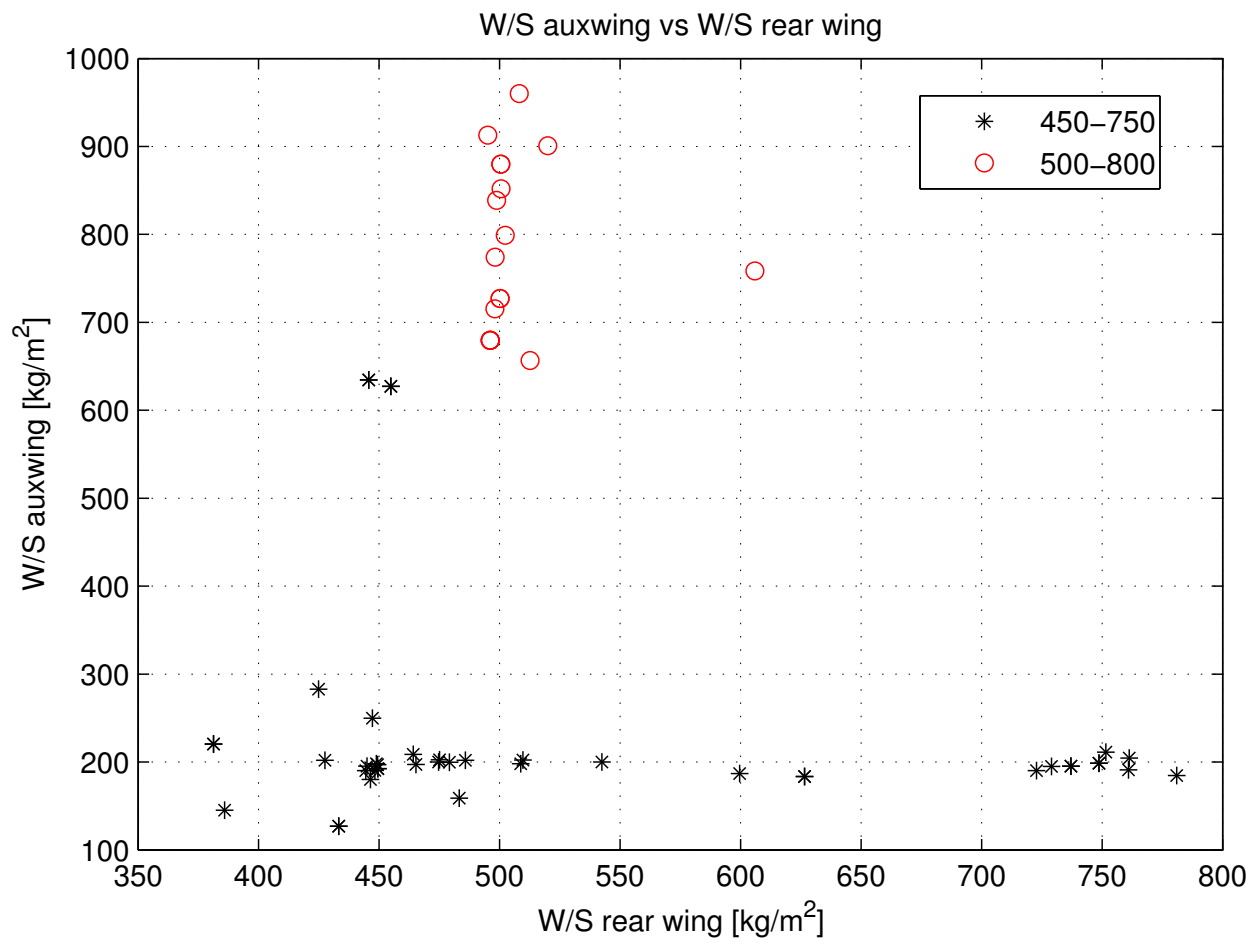

Figure 5.65. Auxiliary wing loading vs rear wing loading (rear wing advancement)

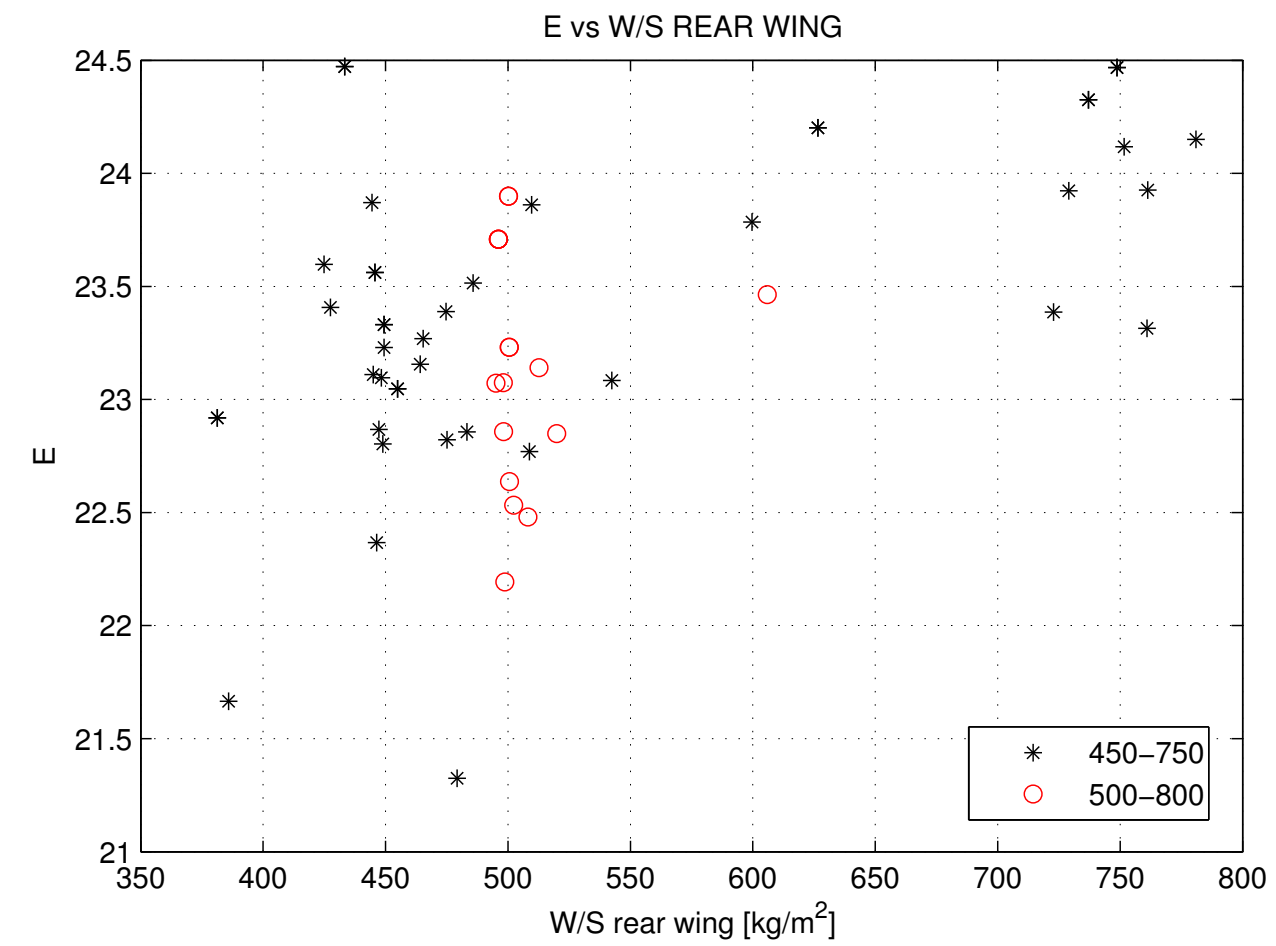

Figure 5.66. Efficiency vs rear wing loading (rear wing advancement) 
Fig. 5.66 and Fig. 5.67 show that when the optimizer tries to increase the rear wing loading, the global wing loading rises and the efficiency is positively influenced by the increased rear wing loading. Fig. E.1 and Fig. E.2 explain how the lifting coefficient $\left(C_{L}\right)$ is related to the wing loading for each wing and for the whole configuration. It is remarked that if the auxiliary wing is allowed to increase its wing loading (analysis "500 - 800"), it will work with high $C_{L}$ and also the global $C_{L}$ increases; instead, if the rear wing approaches the centre of gravity and the auxiliary wing can not increase its wing loading, the rear $C_{L}$ will increase and the global $C_{L}$ rises too.

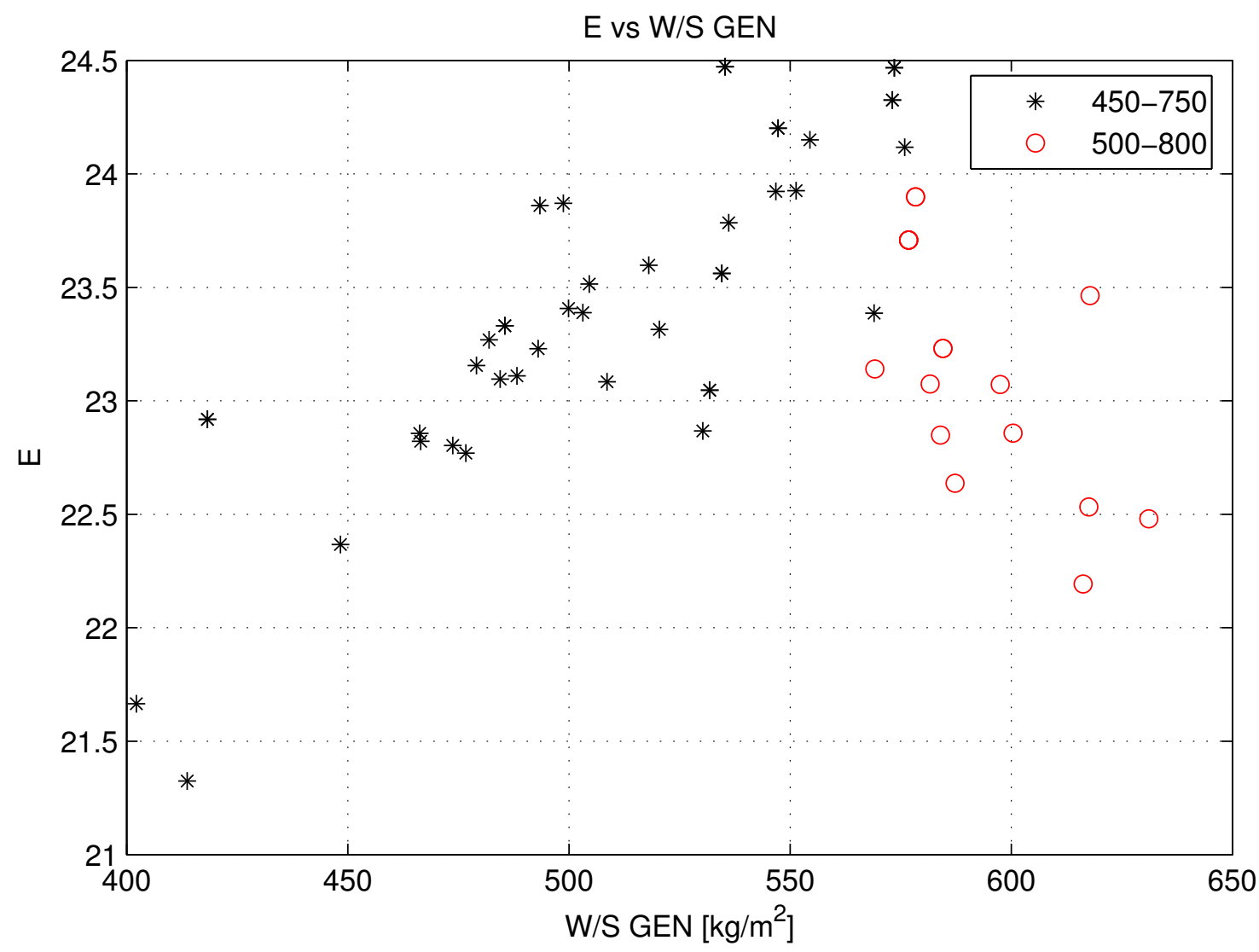

Figure 5.67. Efficiency vs global wing loading (rear wing advancement) 
Finally, the aim of this section is to emphasize that increasing the rear wing loading in a large PrandtlPlane ${ }^{\circledR}$ configuration is a very sensitive issue and it can strongly affect all the lifting system structure. The guidelines in the design process are very general and every configuration needs to be investigated. By the way, if the efficiency is the most important purpose, the task is to increase the rear wing loading because it is possible to get higher efficiency values. However, this decision probably leads to a unstable or nearly unstable configurations: this difficulty can be overcome by modifying the configuration with slight operations on the geometry (e.g. by moving backwards the rear wing just to have a positive Margin of Stability), without spoiling the aerodynamic efficiency. When the designer does not want to risk the Margin of Stability, the better solution is to limit both the rear wing loading and the auxiliary wing loading. When the auxiliary wing moves backwards and the rear wing approaches the centre of gravity, it probably occupy a central position on the fuselage axis. As the rear wing needs to be supported by two vertical fins, they probably are inefficient for the lateral stability because they are too near to the centre of gravity. Thus, the solution could be a " $\mathrm{H}$ " tail, with two vertical bulks at the tip of the auxiliary wing to improve the lateral stability.

\subsection{The reference analysis}

In this section, all the information about the PrandtlPlane ${ }^{\circledR}$ are collected to produce three-dimensional plots of the optimized configuration and of all the configurations corresponding to the local minima. The parameters setting of this new analysis is summarized below.

$$
\begin{gathered}
0 \%<M S<3 \% \\
\text { alpha }=0 \\
K_{\text {bulk }}=0.8 \\
400 \mathrm{~kg} / \mathrm{m}^{2}<\left(\frac{W}{S}\right)_{\text {front }}<600 \mathrm{~kg} / \mathrm{m}^{2} \\
400 \mathrm{~kg} / \mathrm{m}^{2}<\left(\frac{W}{S}\right)_{\text {rear }}<600 \mathrm{~kg} / \mathrm{m}^{2} \\
200 \mathrm{~kg} / \mathrm{m}^{2}<\left(\frac{W}{S}\right)_{\text {auxwing }}<600 \mathrm{~kg} / \mathrm{m}^{2}
\end{gathered}
$$

The upper boundary of the auxiliary wing loading has been limited in order to avoid structural problems and also because high wing loading implies high values of the auxiliary wing $C_{L}$ (refer to Fig. A.9 and Fig. B.6): if the auxiliary wing $C_{L}$ is too high, the configuration could be not trimmed in the low speed condition. Moreover, it is observed that the auxiliary wing is free to move along the fuselage axis, acting as a canard or a tail. In the rest of this section, a set of solutions are presented. All these solutions verify the aerodynamics and flight mechanics constraints. 


\begin{tabular}{|c|c|c|c|c|c|c|c|c|c|}
\hline \multirow{2}{*}{\begin{tabular}{|c|} 
FILE NAME \\
$\mathrm{E}$ \\
\end{tabular}} & \multicolumn{9}{|l|}{ test5trisCurro } \\
\hline & Cltot & \multicolumn{3}{|c|}{ CL_front } & \multicolumn{3}{|c|}{ CL_rear } & \multicolumn{2}{|c|}{ CL_auxwing } \\
\hline \multirow[t]{4}{*}{22,67} & 0,365 & \multicolumn{3}{|c|}{0,424} & \multicolumn{3}{|c|}{0,285} & \multicolumn{2}{|c|}{0,411} \\
\hline & MS [\%] & $\mathrm{W} / \mathrm{s} \mathrm{fr}$ & ont [Kg & $\left./ \mathrm{m}^{\wedge} 2\right]$ & $\mathrm{w} / \mathrm{s} \mathrm{re}$ & ear $[\mathrm{Kg}$ & $\left.3 / \mathrm{m}^{\wedge} 2\right]$ & $\mathrm{w} / \mathrm{s}$ aux & {$\left[\mathrm{Kg} / \mathrm{m}^{\wedge} 2\right]$} \\
\hline & 1,25 & \multicolumn{3}{|c|}{600,9} & \multicolumn{3}{|c|}{403,6} & \multicolumn{2}{|c|}{581,3} \\
\hline & Configuration & \multicolumn{3}{|c|}{ Front wing } & \multicolumn{3}{|c|}{ Rear wing } & \multicolumn{2}{|c|}{ Auxiliary wing } \\
\hline XCG [m] & 40,2 & \multicolumn{3}{|c|}{36,6} & \multicolumn{3}{|c|}{60,4} & \multicolumn{2}{|c|}{11,3} \\
\hline XPN [m] & 40,3 & \multicolumn{3}{|c|}{1} & \multicolumn{3}{|c|}{1} & \multicolumn{2}{|c|}{$\backslash$} \\
\hline $\mathrm{b}[\mathrm{m}]$ & 80,0 & \multicolumn{3}{|c|}{80,0} & \multicolumn{3}{|c|}{80,0} & \multicolumn{2}{|c|}{19,3} \\
\hline $\mathrm{S}\left[\mathrm{m}^{\wedge} 2\right]$ & 1092,1 & \multicolumn{3}{|c|}{524,7} & \multicolumn{3}{|c|}{450,9} & \multicolumn{2}{|c|}{116,6} \\
\hline MAC [m] & 6,85 & \multirow{2}{*}{\multicolumn{3}{|c|}{$\begin{array}{l}7,91 \\
12,20\end{array}$}} & \multicolumn{3}{|c|}{5,82} & \multicolumn{2}{|c|}{6,03} \\
\hline AR & 5,86 & \multicolumn{2}{|r|}{12,20} & & \multicolumn{3}{|c|}{14,20} & \multicolumn{2}{|c|}{3,20} \\
\hline $\mathrm{c}[\mathrm{m}]$ & 1 & 11,20 & 6,55 & 4,03 & 7,59 & 5,65 & 4,02 & 6,03 & 6,04 \\
\hline Pitch Angle [deg] & 1 & \multicolumn{3}{|c|}{2,64} & \multicolumn{3}{|c|}{0,71} & & \\
\hline Twist Angle [deg] & 1 & 0,65 & $-1,95$ & $-0,54$ & $-1,97$ & 1,97 & 0,67 & $-0,12$ & 1,10 \\
\hline Sweep Angle [deg] & 1 & 27,31 & & 31,03 & $-18,42$ & & $-21,43$ & & \\
\hline Dihedral [deg] & 1 & 4,75 & & 3,96 & $-0,04$ & & $-0,86$ & & \\
\hline Taper Ratio & 1 & 0,59 & & 0,62 & 0,74 & & 0,71 & & \\
\hline
\end{tabular}
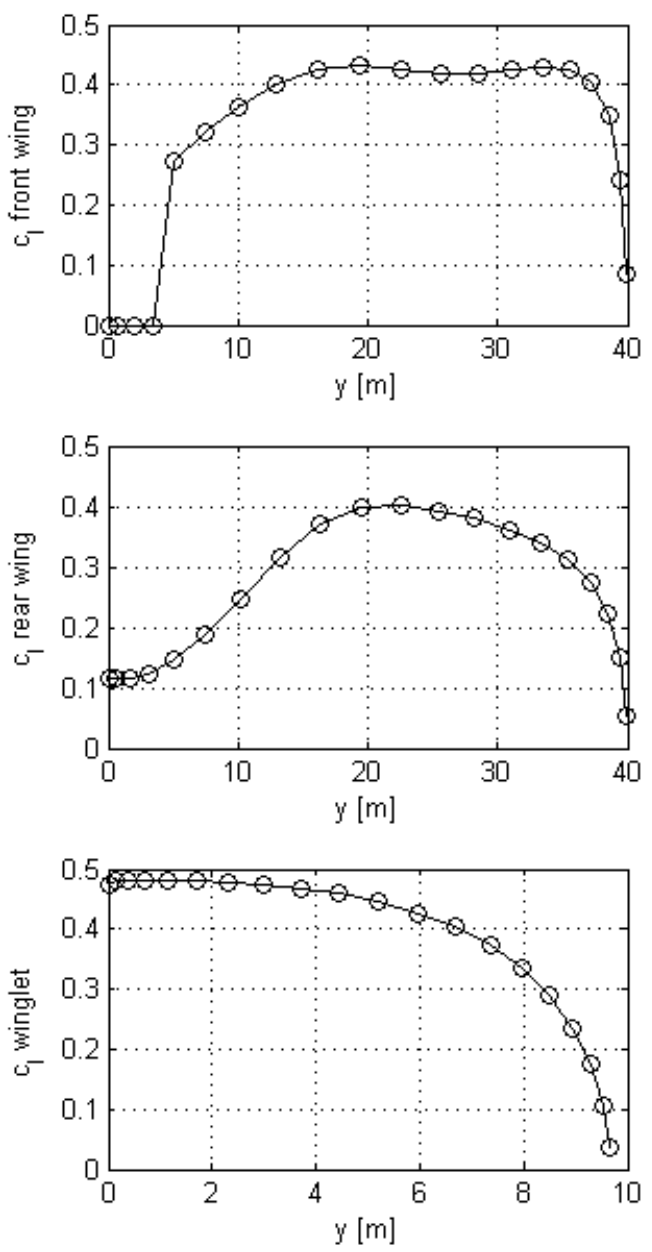

Table 5.9. Analysis Results: test5trisCurr0. 


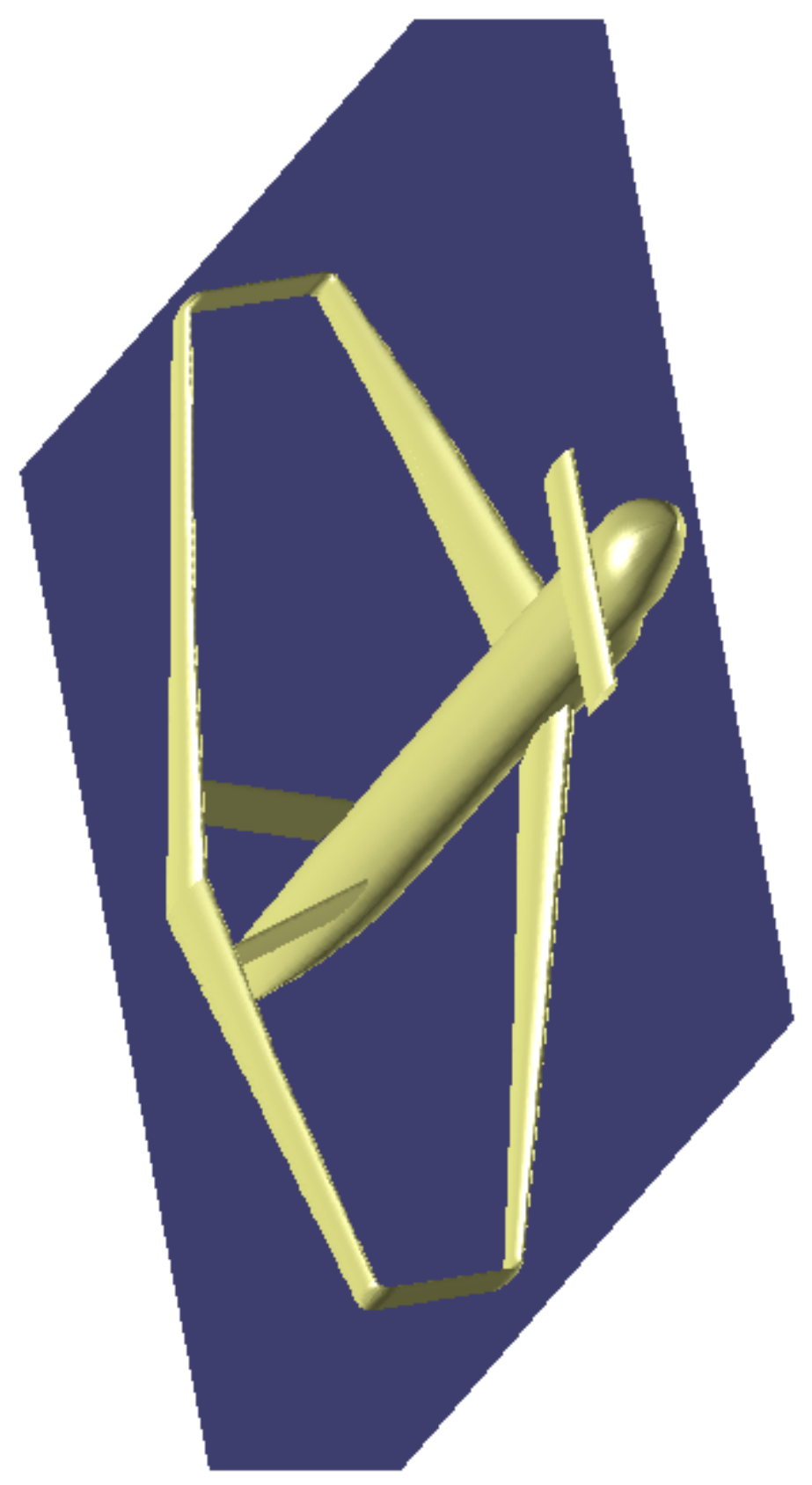

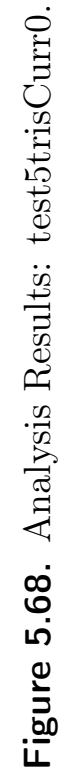




\begin{tabular}{|c|c|c|c|c|c|c|c|c|c|}
\hline \multirow{2}{*}{\begin{tabular}{|c|} 
FILE NAME \\
$\mathrm{E}$ \\
\end{tabular}} & \multicolumn{9}{|l|}{ test5trisCurr1 } \\
\hline & Cltot & \multicolumn{3}{|c|}{$\mathrm{CL}_{-}$front } & \multicolumn{3}{|c|}{ CL_rear } & \multicolumn{2}{|c|}{ CL_auxwing } \\
\hline \multirow[t]{4}{*}{23,07} & 0,364 & \multicolumn{3}{|c|}{0,433} & \multicolumn{3}{|c|}{0,282} & \multicolumn{2}{|c|}{0,225} \\
\hline & MS [\%] & \multicolumn{3}{|c|}{$\mathrm{W} / \mathrm{s}$ front $\left[\mathrm{Kg} / \mathrm{m}^{\wedge} 2\right]$} & \multicolumn{3}{|c|}{$\mathrm{W} / \mathrm{S}$ rear $\left[\mathrm{Kg} / \mathrm{m}^{\wedge} 2\right]$} & \multicolumn{2}{|c|}{$\mathrm{W} / \mathrm{S}$ auxwing $\left[\mathrm{Kg} / \mathrm{m}^{\wedge} 2\right]$} \\
\hline & $-0,09$ & \multicolumn{3}{|c|}{610,7} & \multicolumn{3}{|c|}{397,2} & \multicolumn{2}{|c|}{316,8} \\
\hline & Configuration & \multicolumn{3}{|c|}{ Front wing } & \multicolumn{3}{|c|}{ Rear wing } & \multicolumn{2}{|c|}{ Auxiliary wing } \\
\hline XCG [m] & 40,4 & \multicolumn{3}{|c|}{35,6} & \multicolumn{3}{|c|}{61,1} & \multicolumn{2}{|c|}{27,6} \\
\hline XPN [m] & 40,4 & \multicolumn{3}{|c|}{1} & \multicolumn{3}{|c|}{1} & \multicolumn{2}{|c|}{$\backslash$} \\
\hline$S\left[m^{\wedge} 2\right]$ & 1099,0 & \multicolumn{3}{|c|}{627,0} & \multicolumn{3}{|c|}{404,2} & \multicolumn{2}{|c|}{67,8} \\
\hline $\mathrm{b}[\mathrm{m}]$ & 80,0 & \multicolumn{3}{|c|}{80,0} & \multicolumn{3}{|c|}{80,0} & \multicolumn{2}{|c|}{10,0} \\
\hline MAC [m] & 7,37 & \multicolumn{3}{|c|}{8,80} & \multicolumn{3}{|c|}{5,24} & \multicolumn{2}{|c|}{6,80} \\
\hline AR & 5,82 & \multicolumn{3}{|c|}{10,21} & \multicolumn{3}{|c|}{15,83} & & \\
\hline $\mathrm{c}[\mathrm{m}]$ & 1 & 9,20 & 9,20 & 6,46 & 7,05 & 4,21 & 4,02 & 7,56 & 5,98 \\
\hline Pitch Angle [deg] & 1 & & 2,47 & & & 1,81 & & & \\
\hline Twist Angle [deg] & 1 & 2,00 & $-1,04$ & $-1,71$ & $-2,00$ & 0,75 & \begin{tabular}{|l|}
$-0,37$ \\
\end{tabular} & 1,33 & 1,64 \\
\hline Sweep Angle [deg] & 1 & 38,45 & & 39,82 & $-7,91$ & & $-16,81$ & & \\
\hline Dihedral [deg] & 1 & 6,00 & & 3,10 & $-5,79$ & & \begin{tabular}{|l|}
$-3,61$ \\
\end{tabular} & & \\
\hline Taper Ratio & 1 & 1,00 & & 0,70 & 0,60 & & 0,95 & & \\
\hline
\end{tabular}
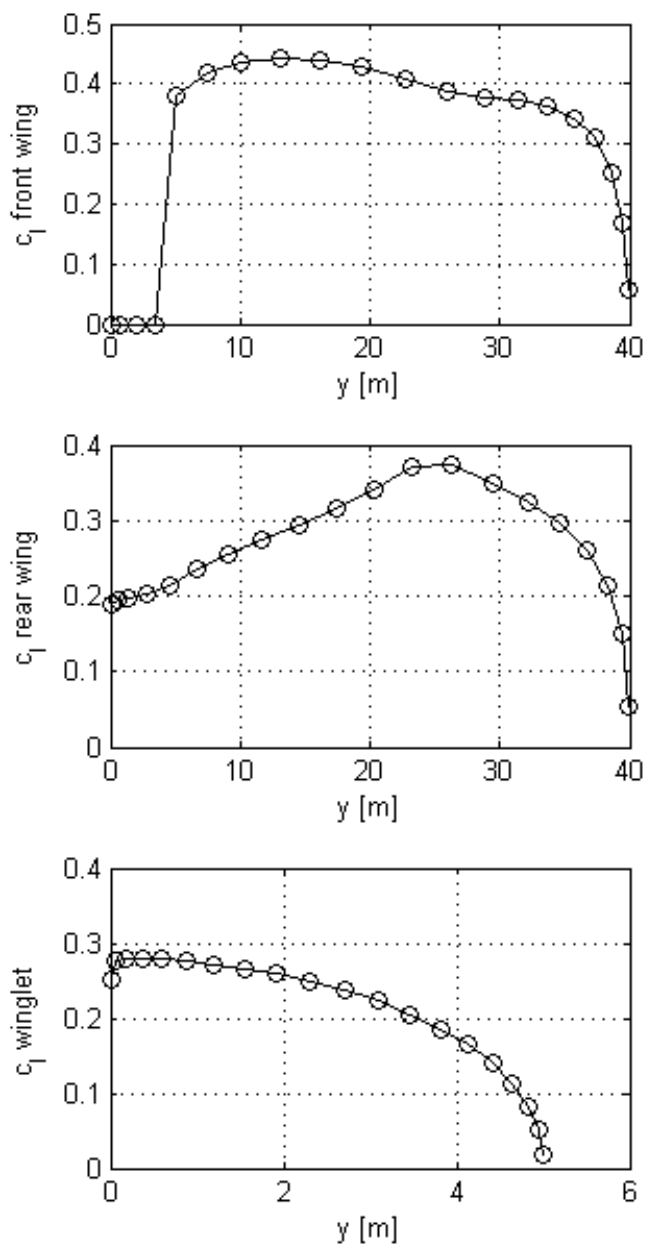

Table 5.10. Analysis Results: test5trisCurr1. 


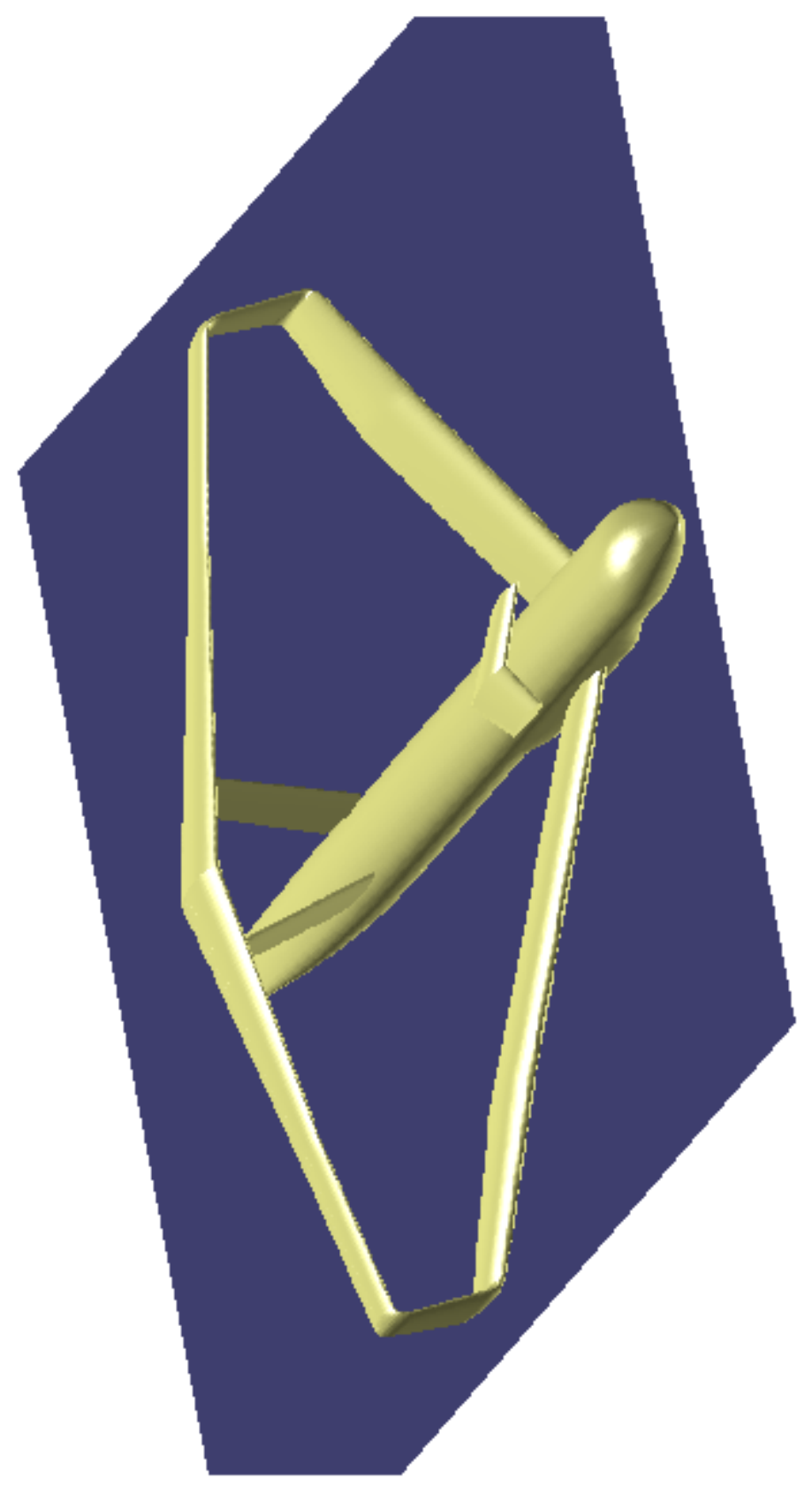

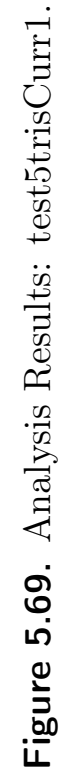




\begin{tabular}{|c|c|c|c|c|c|c|c|c|c|}
\hline \multirow{2}{*}{$\begin{array}{r}\text { FILE NAME } \\
\mathrm{E} \\
\end{array}$} & \multicolumn{9}{|l|}{ test5trisCurr3 } \\
\hline & Cltot & \multicolumn{3}{|c|}{$\mathrm{CL}_{-}$front } & \multicolumn{3}{|c|}{$\mathrm{CL}$ rear } & \multicolumn{2}{|c|}{ CL_auxwing } \\
\hline \multirow[t]{4}{*}{21,67} & 0,353 & \multicolumn{3}{|c|}{0,427} & \multicolumn{3}{|c|}{0,283} & \multicolumn{2}{|c|}{0,246} \\
\hline & MS [\%] & \multicolumn{3}{|c|}{$\mathrm{W} / \mathrm{s}$ front $\left[\mathrm{Kg} / \mathrm{m}^{\wedge} 2\right]$} & \multicolumn{3}{|c|}{$\mathrm{W} / \mathrm{S}$ rear $\left[\mathrm{Kg} / \mathrm{m}^{\wedge} 2\right]$} & \multicolumn{2}{|c|}{$\mathrm{w} / \mathrm{S}$ auxwing $\left[\mathrm{Kg} / \mathrm{m}^{\wedge} 2\right]$} \\
\hline & 0,60 & \multicolumn{3}{|c|}{600,8} & \multicolumn{3}{|c|}{398,7} & \multicolumn{2}{|c|}{346,8} \\
\hline & Configuration & \multicolumn{3}{|c|}{ Front wing } & \multicolumn{3}{|c|}{ Rear wing } & \multicolumn{2}{|c|}{ Auxiliary wing } \\
\hline XCG [m] & 39,8 & \multicolumn{3}{|c|}{37,3} & \multicolumn{3}{|c|}{61,3} & \multicolumn{2}{|c|}{12,1} \\
\hline XPN [m] & 39,8 & \multicolumn{3}{|c|}{1} & \multicolumn{3}{|c|}{1} & \multicolumn{2}{|c|}{1} \\
\hline $\mathrm{S}\left[\mathrm{m}^{\wedge} 2\right]$ & 1135,1 & \multicolumn{3}{|c|}{598,2} & \multicolumn{3}{|c|}{374,4} & \multicolumn{2}{|c|}{162,4} \\
\hline b [m] & 80,0 & \multicolumn{3}{|c|}{80,0} & \multicolumn{3}{|c|}{80,0} & \multicolumn{2}{|c|}{15,0} \\
\hline MAC [m] & 7,55 & \multicolumn{3}{|c|}{8,46} & \multicolumn{3}{|c|}{4,70} & \multicolumn{2}{|c|}{10,80} \\
\hline AR & 5,64 & \multicolumn{3}{|c|}{10,70} & \multicolumn{3}{|c|}{17,09} & & \\
\hline$c[\mathrm{~m}]$ & $\backslash$ & 8,84 & 8,59 & 4,00 & 4,88 & 4,88 & 4,00 & 10,71 & 10,90 \\
\hline Pitch Angle [deg] & $\backslash$ & & 2,26 & & & 0,93 & & & \\
\hline Twist Angle [deg] & 1 & 1,81 & $-2,00$ & $-1,73$ & $-1,94$ & 1,24 & 2,00 & 2,00 & 2,00 \\
\hline Sweep Angle [deg] & 1 & 26,84 & & 38,21 & $-17,79$ & & $-28,89$ & & \\
\hline Dihedral [deg] & 1 & 5,80 & & 3,64 & $-0,29$ & & $-3,37$ & & \\
\hline Taper Ratio & 1 & 0,97 & & 0,47 & 1,00 & & 0,82 & & \\
\hline
\end{tabular}
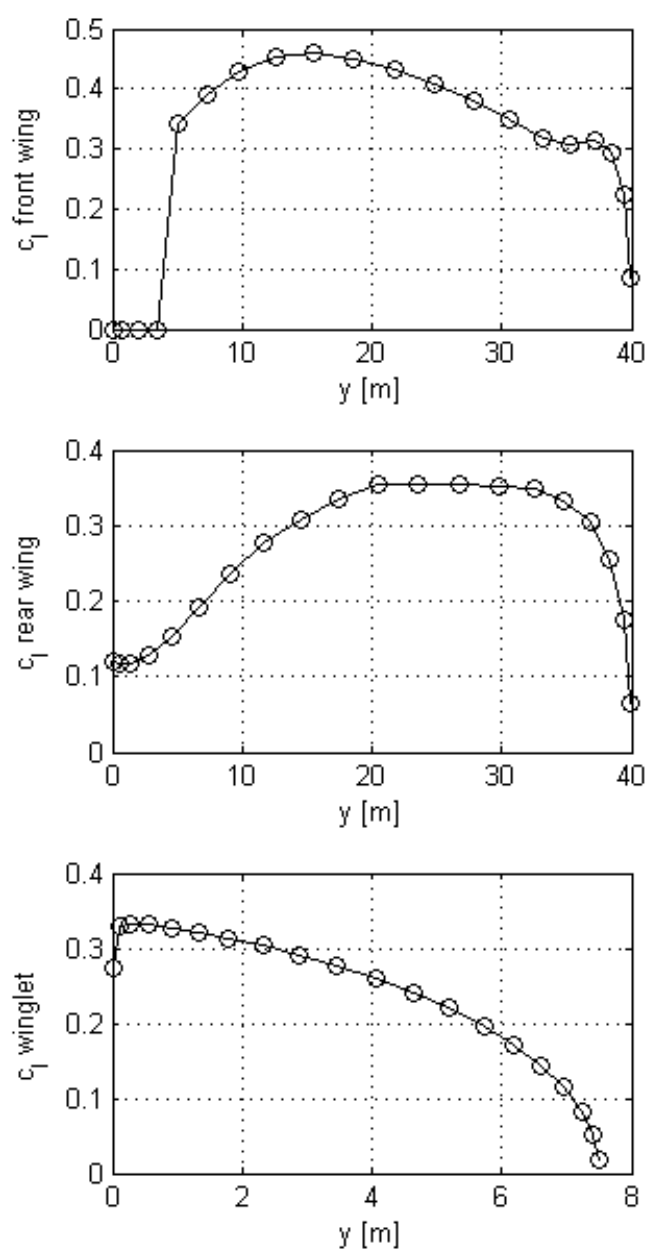

Table 5.11. Analysis Results: test5trisCurr3. 


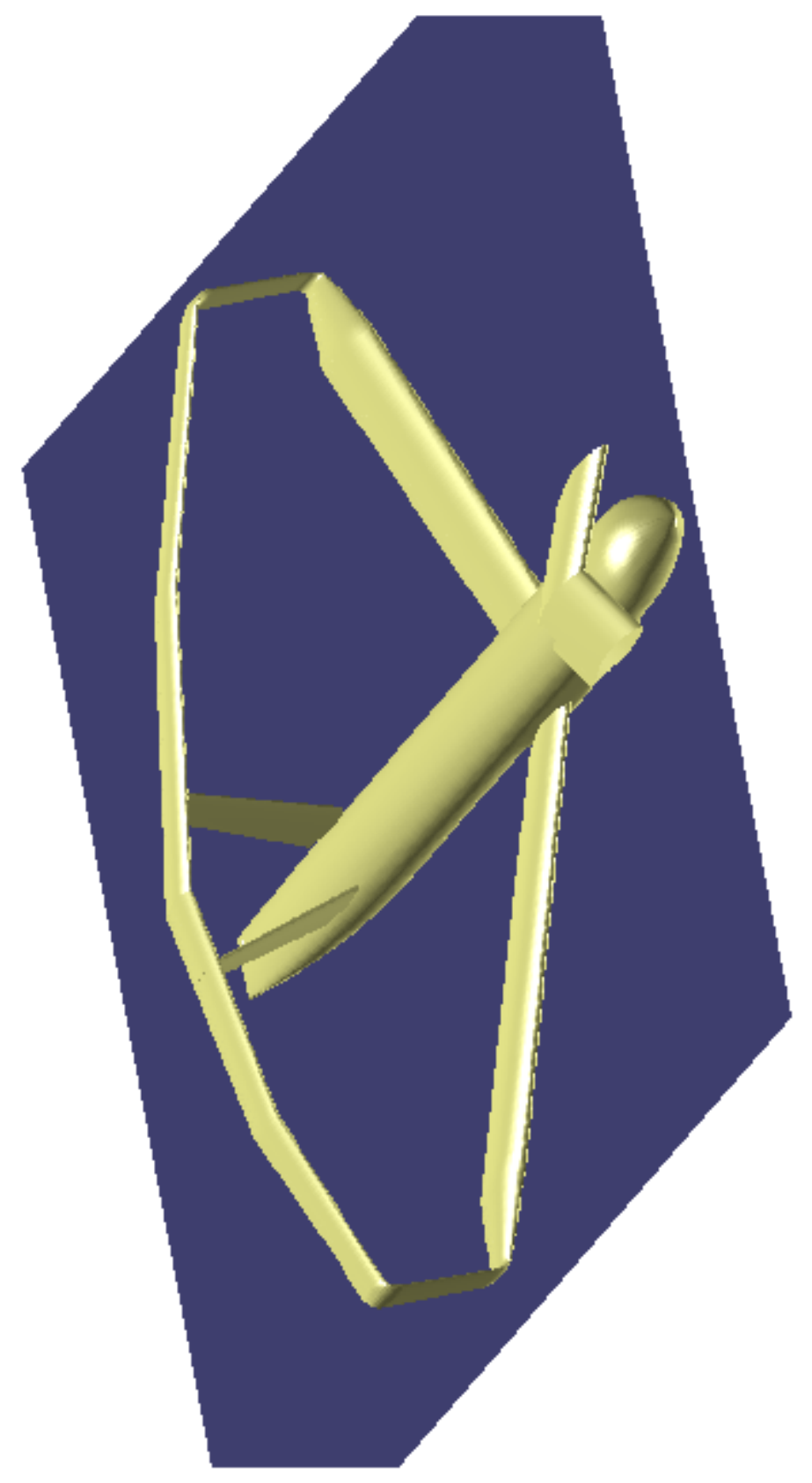

苛 


\begin{tabular}{|c|c|c|c|c|c|c|c|c|c|}
\hline FILE NAME & OPTIMUM & & & & & & & & \\
\hline$E$ & Cltot & & $C L \_$fron & & & CL_rea & & $\mathrm{CL}$ & ing \\
\hline 23,78 & 0,392 & & 0,437 & & & 0,318 & & & \\
\hline & MS [\%] & $\mathrm{w} / \mathrm{s} \mathrm{fr}$ & ront $\left[\mathrm{K}_{\mathrm{g}}\right.$ & $\left./ \mathrm{m}^{\wedge} 2\right]$ & $\mathrm{w} / \mathrm{s} \mathrm{r}$ & ear $\left[K_{\xi}\right.$ & $\left./ \mathrm{m}^{\wedge} 2\right]$ & $\mathrm{w} / \mathrm{s}$ aux & {$\left[\mathrm{Kg} / \mathrm{m}^{\wedge} 2\right]$} \\
\hline & $-4,03$ & & 615,6 & & & 447,2 & & & \\
\hline & Configuration & & ront wir & & & ear wi & & Auxi & wing \\
\hline XCG [m] & 39,7 & & 35,8 & & & 56,3 & & & \\
\hline XPN [m] & 39,5 & & 1 & & & 1 & & & \\
\hline$S\left[m^{\wedge} 2\right]$ & 1024,4 & & 516,7 & & & 398,2 & & & \\
\hline b [m] & 80,0 & & 80,0 & & & 80,0 & & & \\
\hline MAC [m] & 6,19 & & 7,55 & & & 5,02 & & & \\
\hline AR & 6,25 & & 12,39 & & & 16,07 & & & \\
\hline$c[\mathrm{~m}]$ & 1 & 9,36 & 7,87 & 4,00 & 5,41 & 5,41 & 4,00 & 4,00 & 4,00 \\
\hline Pitch Angle [deg] & 1 & & 3,51 & & & 0,72 & & & \\
\hline Twist Angle [deg] & $\backslash$ & 1,12 & $-2,00$ & $-2,00 \mid$ & $-0,64$ & 1,57 & 0,48 & $-1,26$ & 1,52 \\
\hline Sweep Angle [deg] & 1 & 35,49 & & 40,00 & $-9,55$ & & $-0,83$ & & \\
\hline Dihedral [deg] & 1 & 6,00 & & 5,25 & $-6,00$ & & $-6,00$ & & \\
\hline Taper Ratio & 1 & 0,84 & & 0,51 & 1,00 & & 0,74 & & \\
\hline
\end{tabular}
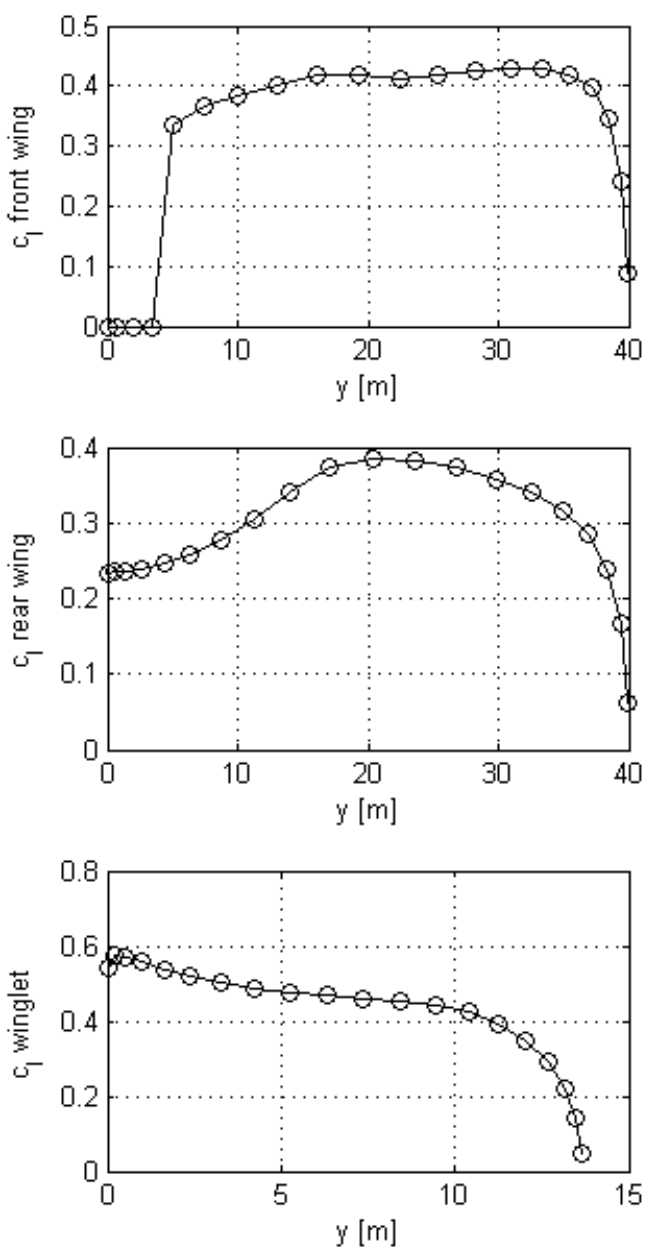

Table 5.12. Analysis Results: OPTIMUM. 


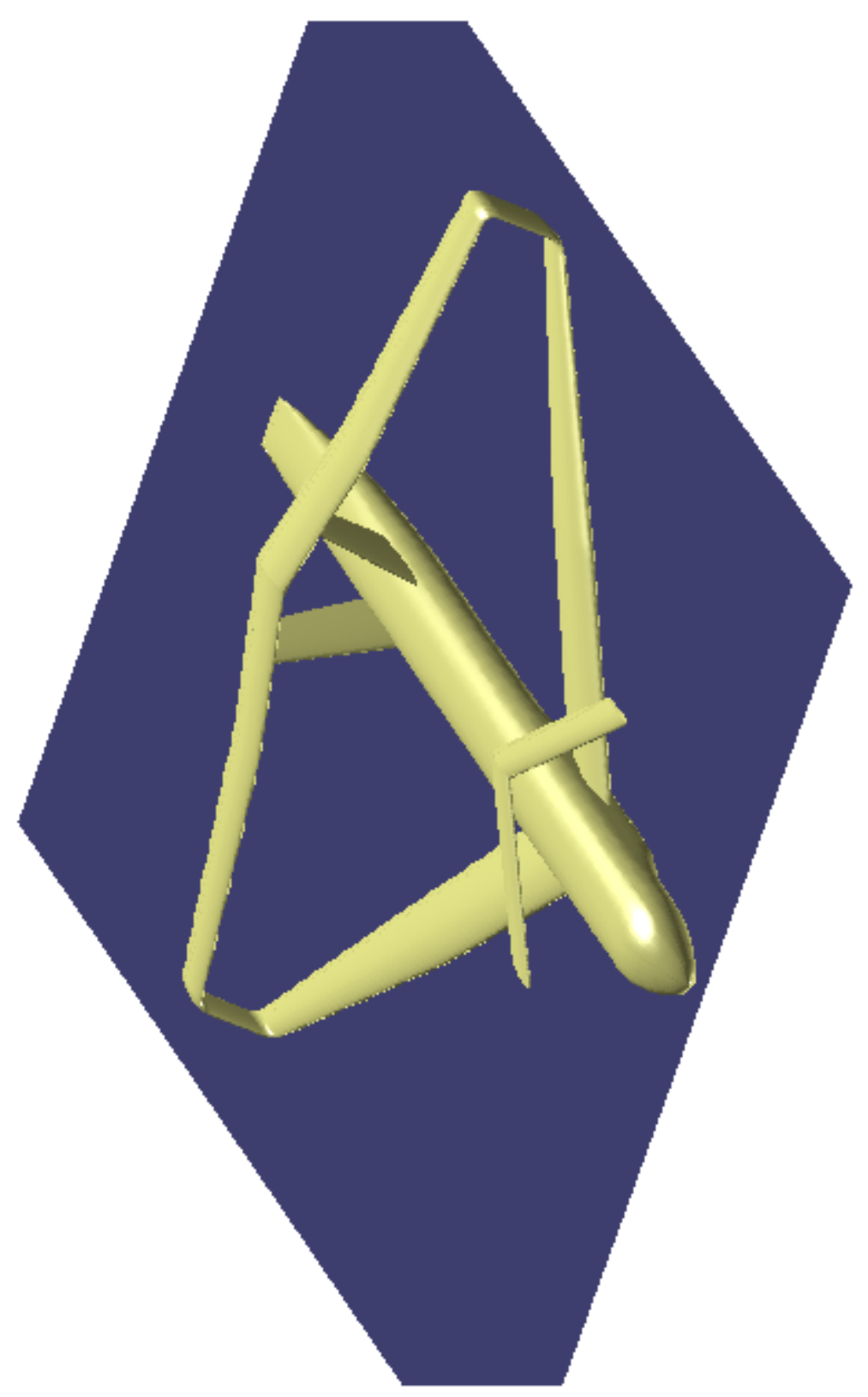

离 


\begin{tabular}{|c|c|c|c|c|c|c|c|c|c|}
\hline \multirow{2}{*}{\begin{tabular}{|c} 
FILE NAME \\
$\mathrm{E}$ \\
\end{tabular}} & \multicolumn{9}{|l|}{ test5trisCurr6 } \\
\hline & Cltot & \multicolumn{3}{|c|}{$\mathrm{CL}_{-}$front } & \multicolumn{3}{|c|}{ CL_rear } & \multicolumn{2}{|c|}{ CL_auxwing } \\
\hline \multirow[t]{4}{*}{23,35} & 0,350 & \multicolumn{3}{|c|}{0,444} & \multicolumn{3}{|c|}{0,280} & \multicolumn{2}{|c|}{0,147} \\
\hline & MS [\%] & \multicolumn{3}{|c|}{$\mathrm{W} / \mathrm{S}$ front $\left[\mathrm{Kg} / \mathrm{m}^{\wedge} 2\right]$} & \multicolumn{3}{|c|}{$\mathrm{W} / \mathrm{S}$ rear $\left[\mathrm{Kg} / \mathrm{m}^{\wedge} 2\right]$} & \multicolumn{2}{|c|}{$\mathrm{W} / \mathrm{S}$ auxwing $\left[\mathrm{Kg} / \mathrm{m}^{\wedge} 2\right]$} \\
\hline & $-2,37$ & \multicolumn{3}{|c|}{626,2} & \multicolumn{3}{|c|}{394,9} & \multicolumn{2}{|c|}{207,2} \\
\hline & Configuration & \multicolumn{3}{|c|}{ Front wing } & \multicolumn{3}{|c|}{ Rear wing } & \multicolumn{2}{|c|}{ Auxiliary wing } \\
\hline XCG [m] & 40,7 & \multicolumn{3}{|c|}{36,9} & \multicolumn{3}{|c|}{56,1} & \multicolumn{2}{|c|}{54,1} \\
\hline XPN [m] & 40,5 & \multicolumn{3}{|c|}{1} & \multicolumn{3}{|c|}{1} & \multicolumn{2}{|c|}{1} \\
\hline$S\left[\mathrm{~m}^{\wedge} 2\right]$ & 1143,9 & \multicolumn{3}{|c|}{599,8} & \multicolumn{3}{|c|}{408,4} & \multicolumn{2}{|c|}{135,7} \\
\hline b [m] & 80,0 & \multicolumn{3}{|c|}{80,0} & \multicolumn{3}{|c|}{80,0} & \multicolumn{2}{|c|}{32,6} \\
\hline MAC [m] & 6,77 & \multicolumn{3}{|c|}{8,35} & \multicolumn{3}{|c|}{5,30} & \multicolumn{2}{|c|}{4,16} \\
\hline AR & 5,59 & \multicolumn{3}{|c|}{10,67} & \multicolumn{3}{|c|}{15,67} & & \\
\hline $\mathrm{c}[\mathrm{m}]$ & 1 & 8,48 & 8,33 & 8,35 & 8,86 & 5,22 & 4,20 & 4,20 & 4,12 \\
\hline Pitch Angle [deg] & 1 & & 1,58 & & & $-0,02$ & & & \\
\hline Twist Angle [deg] & 1 & 2,00 & 2,00 & \begin{tabular}{|l|}
$-2,00$ \\
\end{tabular} & $-1,78$ & 1,47 & 1,84 & 0,13 & 1,59 \\
\hline Sweep Angle [deg] & $\backslash$ & 20,73 & & 32,39 & $-13,53$ & & $-3,24$ & & \\
\hline Dihedral [deg] & 1 & 5,49 & & 2,93 & $-2,29$ & & $-3,20$ & & \\
\hline Taper Ratio & 1 & 0,98 & & 1,00 & 0,59 & & 0,81 & & \\
\hline
\end{tabular}
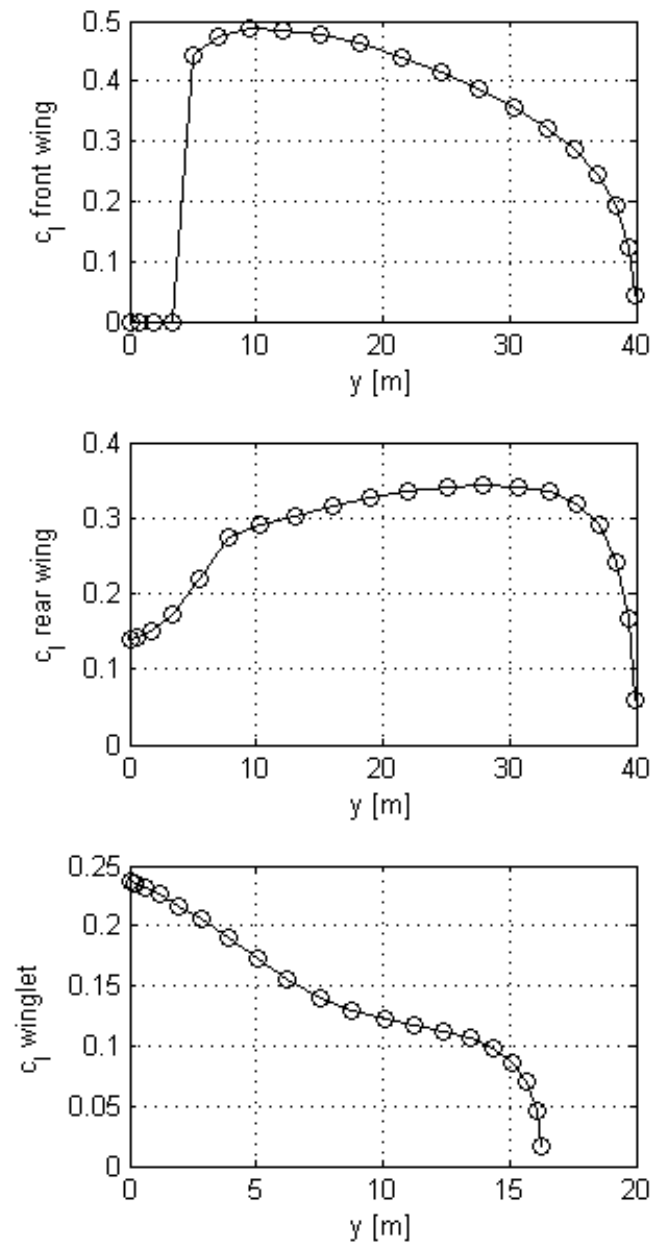

Table 5.13. Analysis Results: test5trisCurr6. 


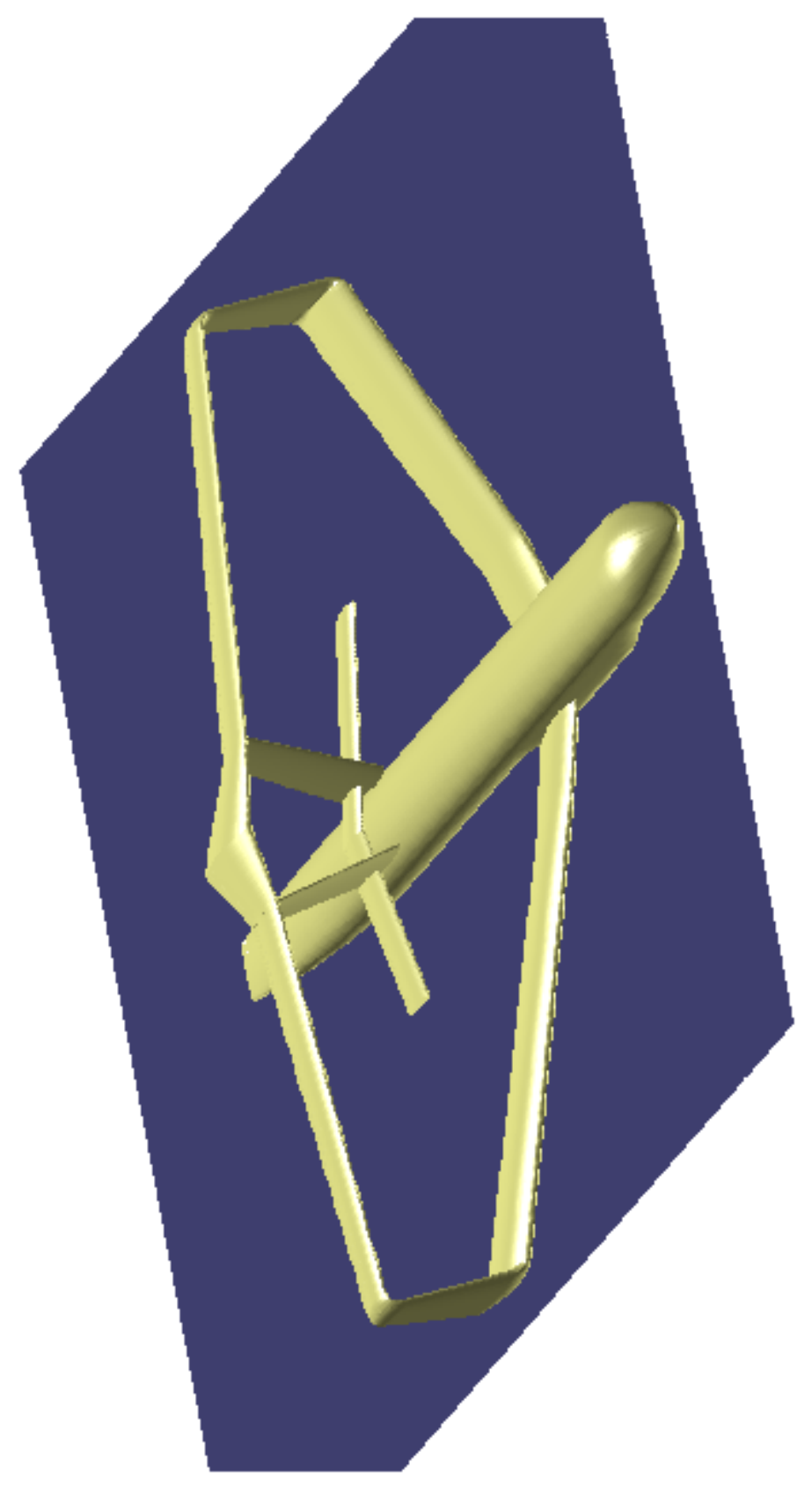

苛 


\begin{tabular}{|c|c|c|c|c|c|c|c|c|c|}
\hline \multirow{2}{*}{$\begin{array}{r}\text { FILE NAME } \\
\mathrm{E} \\
\end{array}$} & \multicolumn{9}{|l|}{ test5trisCurr7 } \\
\hline & Cltot & \multicolumn{3}{|c|}{ CL_front } & \multicolumn{3}{|c|}{ CL_rear } & \multicolumn{2}{|c|}{ CL_auxwing } \\
\hline \multirow[t]{4}{*}{23,50} & 0,364 & \multicolumn{3}{|c|}{0,440} & \multicolumn{3}{|c|}{0,277} & \multicolumn{2}{|c|}{0,142} \\
\hline & MS [\%] & \multicolumn{3}{|c|}{$\mathrm{W} / \mathrm{S}$ front $\left[\mathrm{Kg} / \mathrm{m}^{\wedge} 2\right]$} & \multicolumn{3}{|c|}{$\mathrm{W} / \mathrm{S}$ rear $\left[\mathrm{Kg} / \mathrm{m}^{\wedge} 2\right]$} & \multicolumn{2}{|c|}{ W/S auxwing $\left[\mathrm{Kg} / \mathrm{m}^{\wedge} 2\right]$} \\
\hline & $-1,30$ & \multicolumn{3}{|c|}{618,6} & \multicolumn{3}{|c|}{390,2} & \multicolumn{2}{|c|}{199,2} \\
\hline & Configuration & \multicolumn{3}{|c|}{ Front wing } & \multicolumn{3}{|c|}{ Rear wing } & \multicolumn{2}{|c|}{ Auxiliary wing } \\
\hline XCG [m] & 41,5 & \multicolumn{3}{|c|}{35,3} & \multicolumn{3}{|c|}{63,1} & \multicolumn{2}{|c|}{73,7} \\
\hline XPN [m] & 41,4 & \multicolumn{3}{|c|}{1} & \multicolumn{3}{|c|}{1} & \multicolumn{2}{|c|}{1} \\
\hline $\mathrm{S}\left[\mathrm{m}^{\wedge} 2\right]$ & 1103,3 & \multicolumn{3}{|c|}{625,0} & \multicolumn{3}{|c|}{434,8} & \multicolumn{2}{|c|}{43,5} \\
\hline b [m] & 80,0 & \multicolumn{3}{|c|}{80,0} & \multicolumn{3}{|c|}{80,0} & \multicolumn{2}{|c|}{7,7} \\
\hline MAC [m] & 7,32 & \multicolumn{3}{|c|}{8,74} & \multicolumn{3}{|c|}{5,44} & \multicolumn{2}{|c|}{5,67} \\
\hline AR & 5,80 & \multicolumn{3}{|c|}{10,24} & \multicolumn{3}{|c|}{14,72} & & \\
\hline $\mathrm{c}[\mathrm{m}]$ & 1 & 10,25 & 8,47 & 8,22 & 5,58 & 5,54 & 4,76 & 5,61 & 5,72 \\
\hline \begin{tabular}{|l} 
Pitch Angle [deg] \\
\end{tabular} & 1 & & 3,10 & & & 0,78 & & & \\
\hline Twist Angle [deg] & 1 & 1,53 & $-1,33$ & $-1,89$ & $-2,00$ & 2,00 & 1,31 & 0,74 & $-2,00$ \\
\hline Sweep Angle [deg] & 1 & 28,73 & & 37,40 & $-31,39$ & & $-8,75$ & & \\
\hline Dihedral [deg] & 1 & 6,00 & & 1,21 & 0,00 & & $-4,62$ & & \\
\hline Taper Ratio & 1 & 0,83 & & 0,97 & 0,99 & & 0,86 & & \\
\hline
\end{tabular}
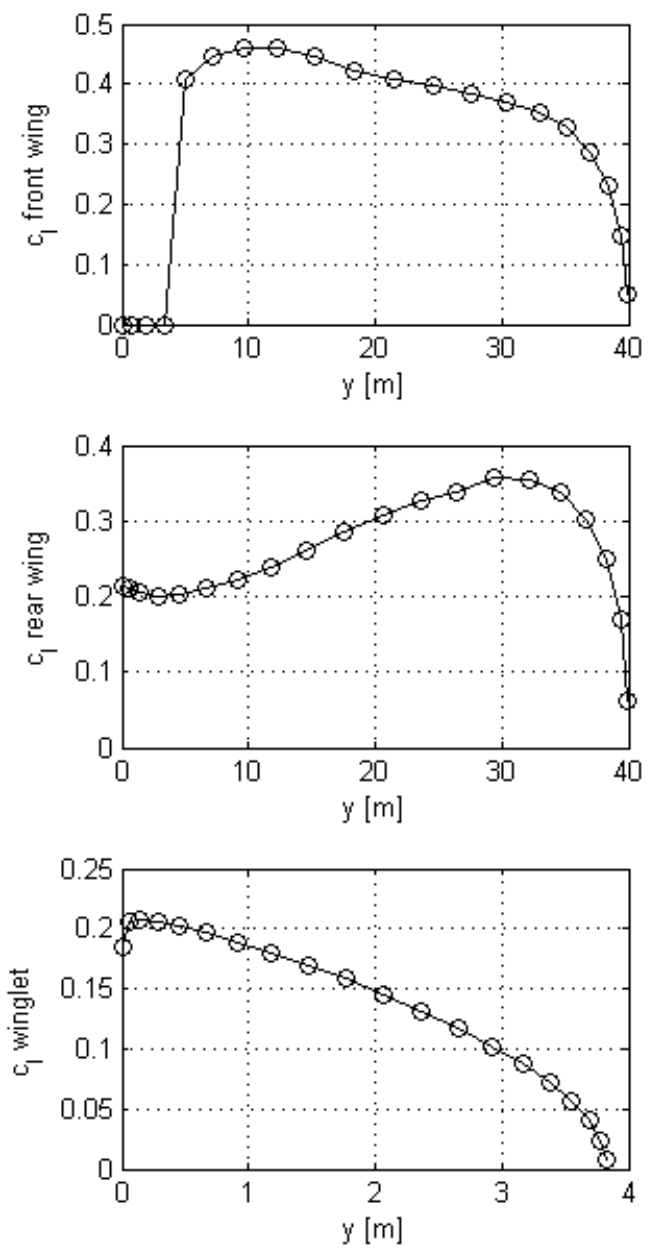

Table 5.14. Analysis Results: test5trisCurr7. 


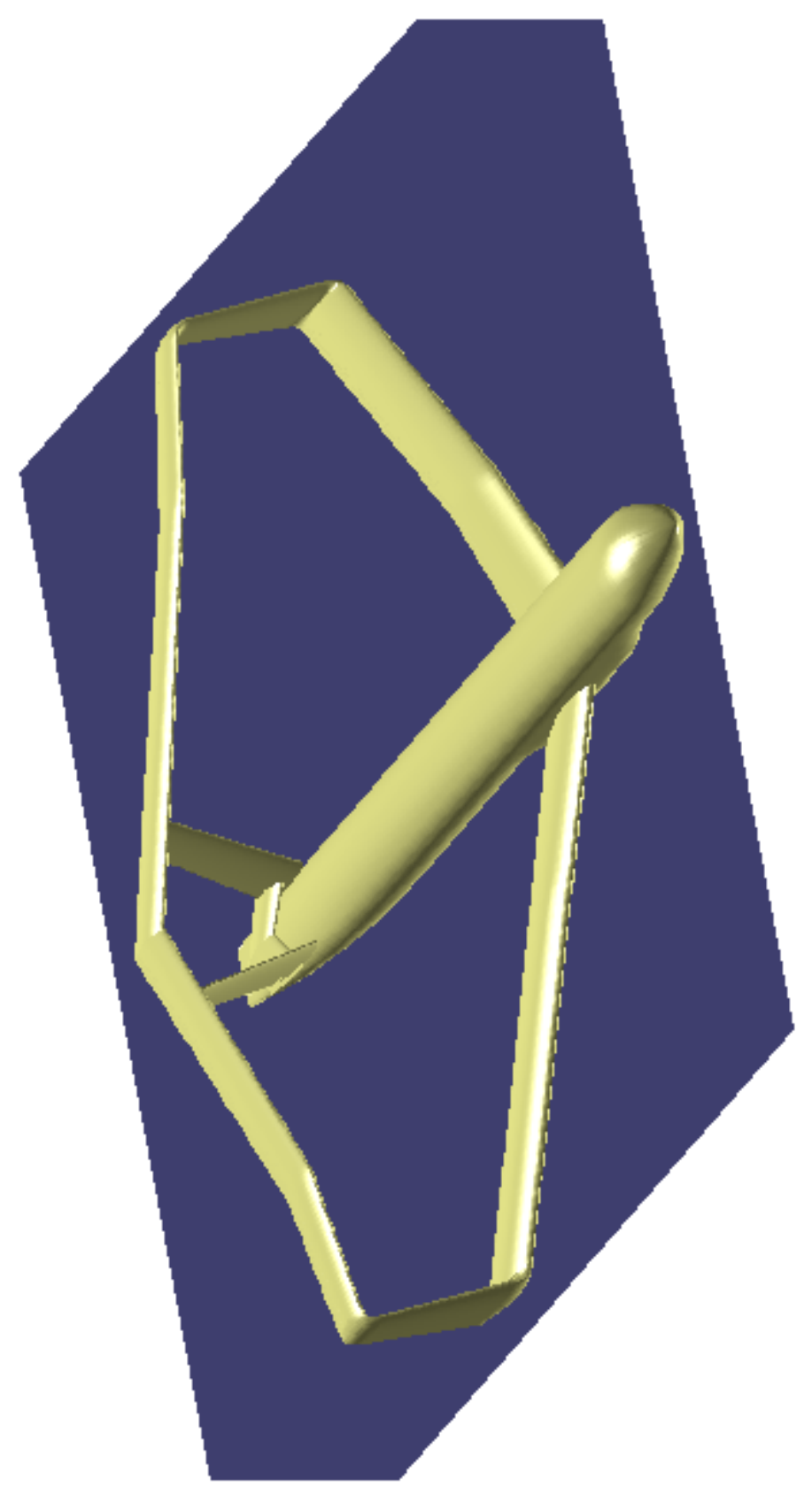

章 


\begin{tabular}{|c|c|c|c|c|c|c|c|c|c|}
\hline \multirow{2}{*}{\begin{tabular}{|c|} 
FILE NAME \\
$\mathrm{E}$ \\
\end{tabular}} & test5trisCurr8 & & & & & & & & \\
\hline & Cltot & \multicolumn{3}{|c|}{$\mathrm{CL}_{-}$front } & \multicolumn{3}{|c|}{$\mathrm{CL}_{-}$rear } & \multicolumn{2}{|c|}{ CL_auxwing } \\
\hline \multirow[t]{4}{*}{22,22} & 0,367 & \multicolumn{3}{|c|}{0,419} & \multicolumn{3}{|c|}{0,277} & \multicolumn{2}{|c|}{0,407} \\
\hline & MS [\%] & $\mathrm{W} / \mathrm{s}$ fr & ront $[\mathrm{Kg}$ & $\left./ \mathrm{m}^{\wedge} 2\right]$ & $\mathrm{w} / \mathrm{s} \mathrm{re}$ & ear $[\mathrm{Kg}]$ & $\left./ m^{\wedge} 2\right]$ & w/S aux & {$\left[\mathrm{Kg} / \mathrm{m}^{\wedge} 2\right]$} \\
\hline & $-0,72$ & \multicolumn{3}{|c|}{601,1} & \multicolumn{3}{|c|}{397,6} & \multicolumn{2}{|c|}{584,0} \\
\hline & Configuration & \multicolumn{3}{|c|}{ Front wing } & \multicolumn{3}{|c|}{ Rear wing } & \multicolumn{2}{|c|}{ Auxiliary wing } \\
\hline XCG [m] & 39,8 & \multicolumn{3}{|c|}{38,0} & \multicolumn{3}{|c|}{60,9} & \multicolumn{2}{|c|}{19,2} \\
\hline XPN [m] & 39,8 & \multicolumn{3}{|c|}{1} & \multicolumn{3}{|c|}{1} & \multicolumn{2}{|c|}{1} \\
\hline$S\left[m^{\wedge} 2\right]$ & 1075,2 & \multicolumn{3}{|c|}{481,3} & \multicolumn{3}{|c|}{382,0} & \multicolumn{2}{|c|}{211,9} \\
\hline b [m] & 80,0 & \multicolumn{3}{|c|}{80,0} & \multicolumn{3}{|c|}{80,0} & \multicolumn{2}{|c|}{38,8} \\
\hline MAC [m] & 6,27 & \multicolumn{3}{|c|}{7,49} & \multicolumn{3}{|c|}{5,14} & \multicolumn{2}{|c|}{5,51} \\
\hline AR & 5,95 & \multicolumn{3}{|c|}{13,30} & \multicolumn{3}{|c|}{16,75} & \multicolumn{2}{|c|}{7,10} \\
\hline $\mathrm{c}[\mathrm{m}]$ & 1 & 11,03 & 4,15 & 4,00 & 8,80 & 4,04 & 4,07 & 6,32 & 4,61 \\
\hline Pitch Angle [deg] & 1 & \multicolumn{3}{|c|}{3,51} & \multicolumn{3}{|c|}{1,31} & & \\
\hline Twist Angle [deg] & 1 & $-0,04$ & $-1,32$ & $-2,00$ & 1,57 & $-2,00$ & 2,00 & 2,00 & 2,00 \\
\hline Sweep Angle [deg] & 1 & 39,54 & & 38,04 & $-10,83$ & & $-4,70$ & & \\
\hline Dihedral [deg] & 1 & 6,00 & & 4,29 & $-6,00$ & & $-6,00$ & & \\
\hline Taper Ratio & 1 & 0,38 & & 0,96 & 0,46 & & 1,01 & & \\
\hline
\end{tabular}
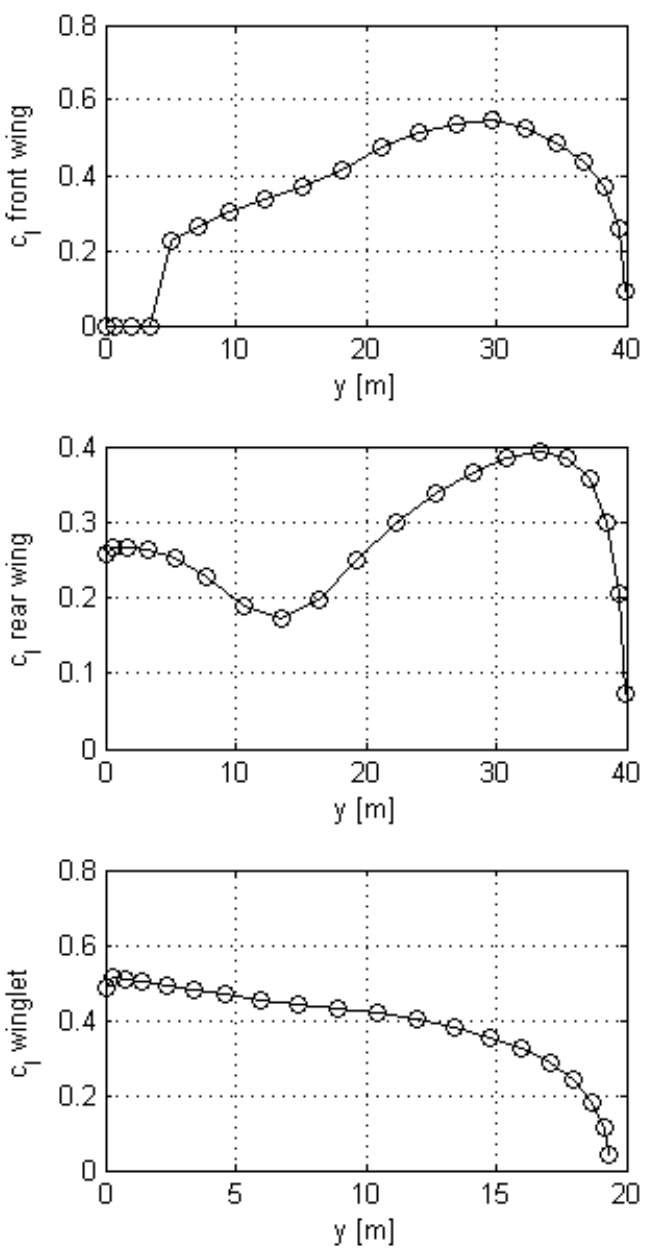

Table 5.15. Analysis Results: test5trisCurr8. 


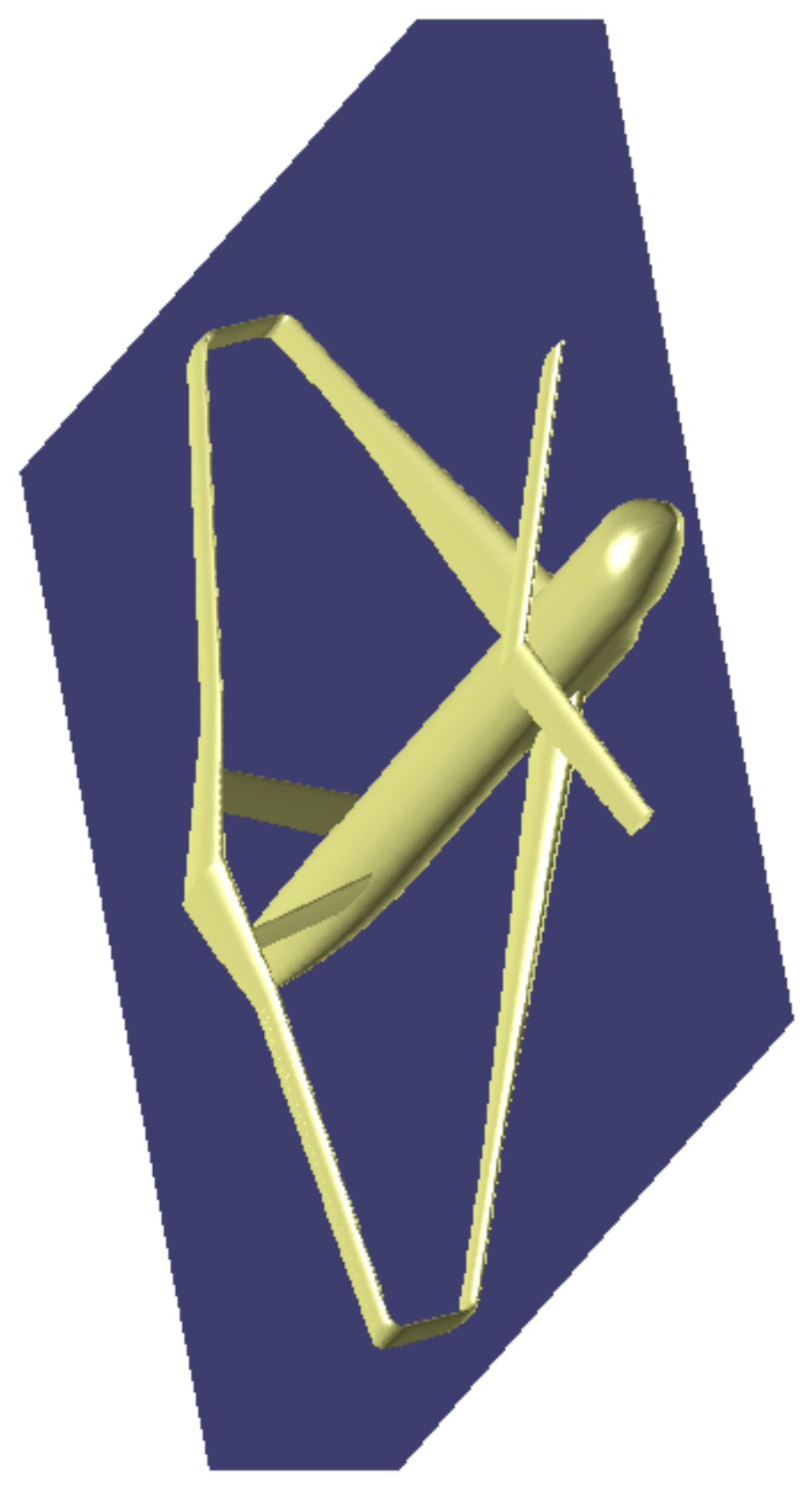

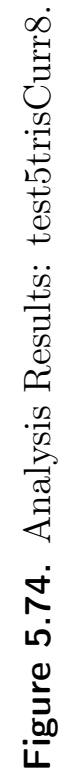




\begin{tabular}{|c|c|c|c|c|c|c|c|c|c|}
\hline \multirow{2}{*}{$\begin{array}{r}\text { FILE NAME } \\
\mathrm{E} \\
\end{array}$} & \multicolumn{9}{|l|}{ test5trisCurr9 } \\
\hline & Cltot & \multicolumn{3}{|c|}{ CL_front } & \multicolumn{3}{|c|}{ CL_rear } & \multicolumn{2}{|c|}{$\mathrm{CL}_{\text {_auxwing }}$} \\
\hline \multirow[t]{4}{*}{22,39} & 0,344 & \multicolumn{3}{|c|}{0,439} & \multicolumn{3}{|c|}{0,283} & \multicolumn{2}{|c|}{0,461} \\
\hline & MS [\%] & \multicolumn{3}{|c|}{$\mathrm{w} / \mathrm{s}$ front $\left[\mathrm{Kg} / \mathrm{m}^{\wedge} 2\right]$} & \multicolumn{3}{|c|}{$\mathrm{W} / \mathrm{S}$ rear $\left[\mathrm{Kg} / \mathrm{m}^{\wedge} 2\right]$} & \multicolumn{2}{|c|}{$\mathrm{W} / \mathrm{S}$ auxwing $\left[\mathrm{Kg} / \mathrm{m}^{\wedge} 2\right]$} \\
\hline & 0,03 & \multicolumn{3}{|c|}{621,8} & \multicolumn{3}{|c|}{401,1} & \multicolumn{2}{|c|}{652,0} \\
\hline & Configuration & \multicolumn{3}{|c|}{ Front wing } & \multicolumn{3}{|c|}{ Rear wing } & \multicolumn{2}{|c|}{ Auxiliary wing } \\
\hline XCG [m] & 40,0 & \multicolumn{3}{|c|}{30,3} & \multicolumn{3}{|c|}{54,1} & \multicolumn{2}{|c|}{14,2} \\
\hline XPN [m] & 40,0 & \multicolumn{3}{|c|}{1} & \multicolumn{3}{|c|}{$\perp$} & \multicolumn{2}{|c|}{1} \\
\hline $\mathrm{S}\left[\mathrm{m}^{\wedge} 2\right]$ & 1160,6 & \multicolumn{3}{|c|}{381,9} & \multicolumn{3}{|c|}{718,2} & \multicolumn{2}{|c|}{60,4} \\
\hline$b[\mathrm{~m}]$ & 80,0 & \multicolumn{3}{|c|}{80,0} & \multicolumn{3}{|c|}{80,0} & \multicolumn{2}{|c|}{14,3} \\
\hline MAC [m] & 7,84 & \multicolumn{3}{|c|}{5,33} & \multicolumn{3}{|c|}{9,47} & \multicolumn{2}{|c|}{4,21} \\
\hline AR & 5,51 & \multicolumn{3}{|c|}{16,76} & \multicolumn{3}{|c|}{8,91} & & \\
\hline $\mathrm{c}[\mathrm{m}]$ & $\backslash$ & 5,63 & 5,70 & 4,77 & 11,58 & 10,21 & 4,67 & 4,30 & 4,12 \\
\hline Pitch Angle [deg] & $\backslash$ & & 2,11 & & & 0,35 & & & \\
\hline Twist Angle [deg] & $\backslash$ & 2,00 & 1,15 & $-2,00$ & 0,10 & 1,15 & 0,44 & $-0,80$ & 1,03 \\
\hline Sweep Angle [deg] & 1 & 33,79 & & 38,39 & $-4,19$ & & $-10,12$ & & \\
\hline Dihedral [deg] & 1 & 4,85 & & 3,80 & $-5,27$ & & $-6,00$ & & \\
\hline Taper Ratio & 1 & 1,01 & & 0,84 & 0,88 & & 0,46 & & \\
\hline
\end{tabular}
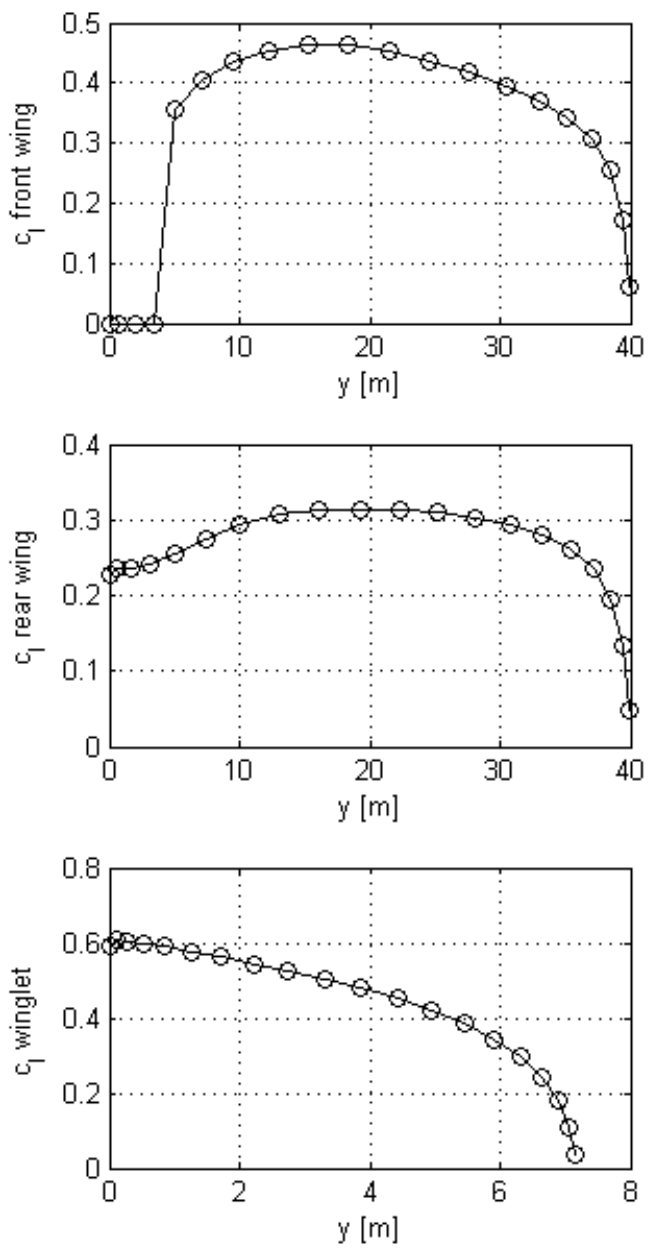

Table 5.16. Analysis Results: test5trisCurr9. 


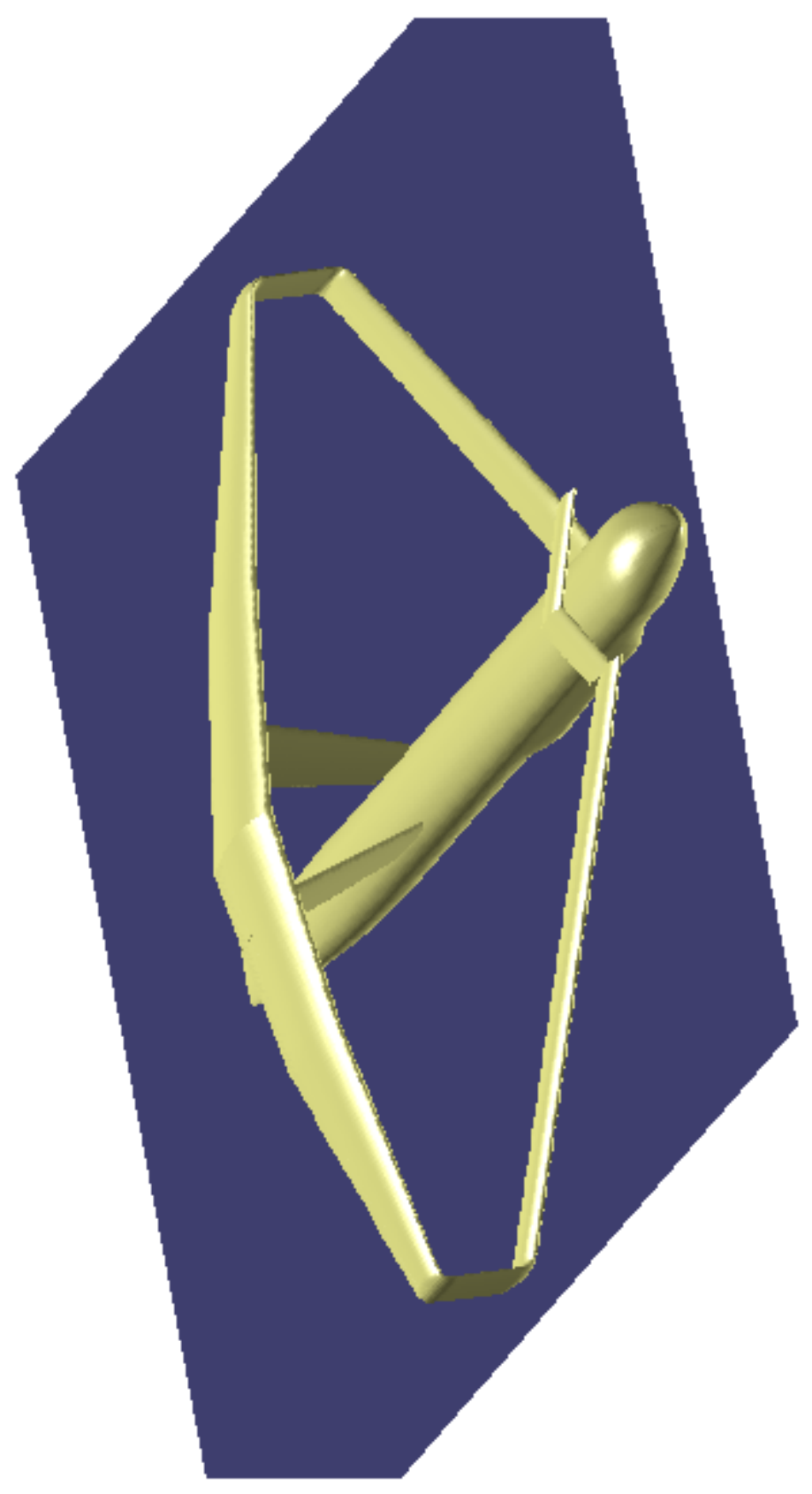

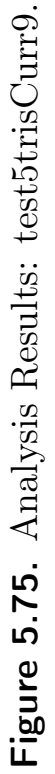




\begin{tabular}{|c|c|c|c|c|c|c|c|c|c|}
\hline FILE NAME & test5trisCurr10 & & & & & & & & \\
\hline $\mathrm{E}$ & Cltot & & CL_front & & & CL_rea & & $\mathrm{CL}$ & ing \\
\hline 23,13 & 0,367 & & 0,424 & & & 0,286 & & & \\
\hline & MS [\%] & $\mathrm{w} / \mathrm{s}$ frc & ont $[\mathrm{Kg}$ & $\left./ \mathrm{m}^{\wedge} 2\right]$ & $\mathrm{w} / \mathrm{s} \mathrm{r}$ & ear $[\mathrm{Kg}$ & $\left./ \mathrm{m}^{\wedge} 2\right]$ & W/s aux & {$\left[\mathrm{Kg} / \mathrm{m}^{\wedge} 2\right]$} \\
\hline & $-0,23$ & & 606,3 & & & 408,6 & & & \\
\hline & Configuration & & ont win & & & ear wir & & Aux & wing \\
\hline XCG [m] & 40,0 & & 36,4 & & & 56,2 & & & \\
\hline XPN [m] & 40,0 & & 1 & & & 1 & & & \\
\hline $\mathrm{S}\left[\mathrm{m}^{\wedge} 2\right]$ & 1076,9 & & 479,5 & & & 422,6 & & & \\
\hline b [m] & 80,0 & & 80,0 & & & 80,0 & & & \\
\hline MAC [m] & 5,83 & & 6,69 & & & 5,45 & & & \\
\hline AR & 5,94 & & 13,35 & & & 15,14 & & & \\
\hline$c[\mathrm{~m}]$ & 1 & 6,87 & 6,88 & 5,85 & 8,32 & 5,52 & 4,23 & 4,37 & 4,37 \\
\hline Pitch Angle [deg] & 1 & & 2,77 & & & 1,79 & & & \\
\hline Twist Angle [deg] & 1 & 2,00 & $-2,00$ & $-1,73$ & $-1,48$ & 0,52 & $-2,00$ & 0,23 & $-1,32$ \\
\hline Sweep Angle [deg] & 1 & 36,49 & & 40,00 & $-0,86$ & & $-0,58$ & & \\
\hline Dihedral [deg] & 1 & 6,00 & & 4,74 & $-3,43$ & & $-4,82$ & & \\
\hline Taper Ratio & 1 & 1,00 & & 0,85 & 0,66 & & 0,77 & & \\
\hline
\end{tabular}
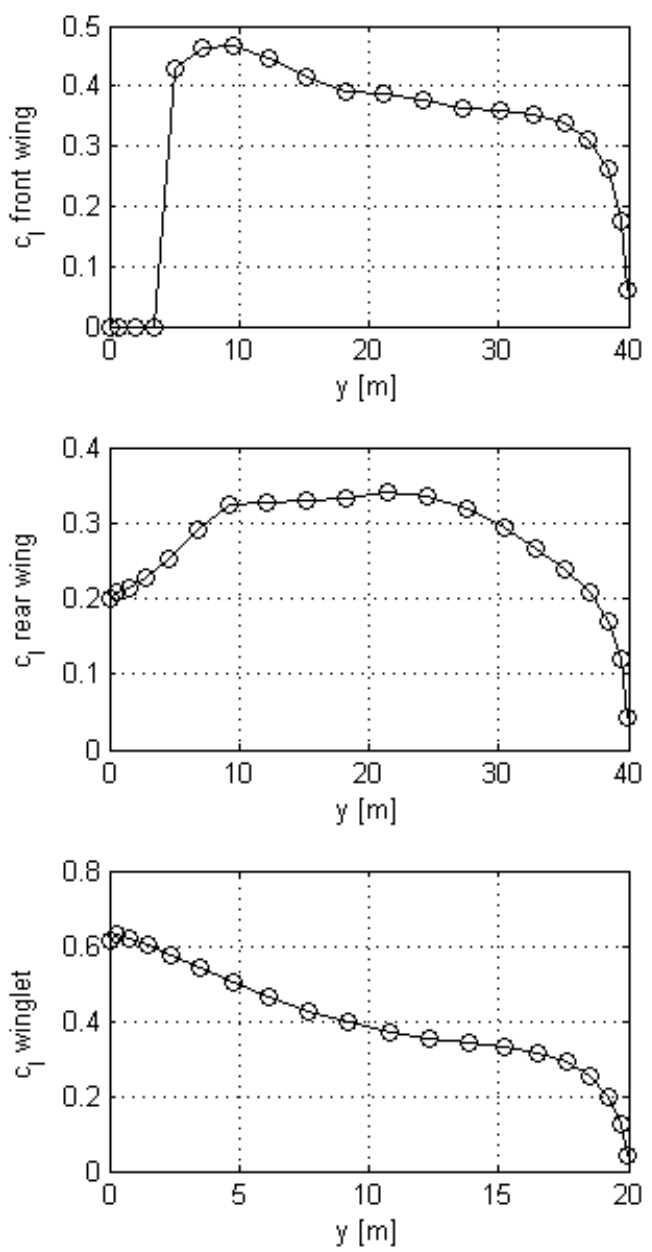

Table 5.17. Analysis Results: test5trisCurr10. 


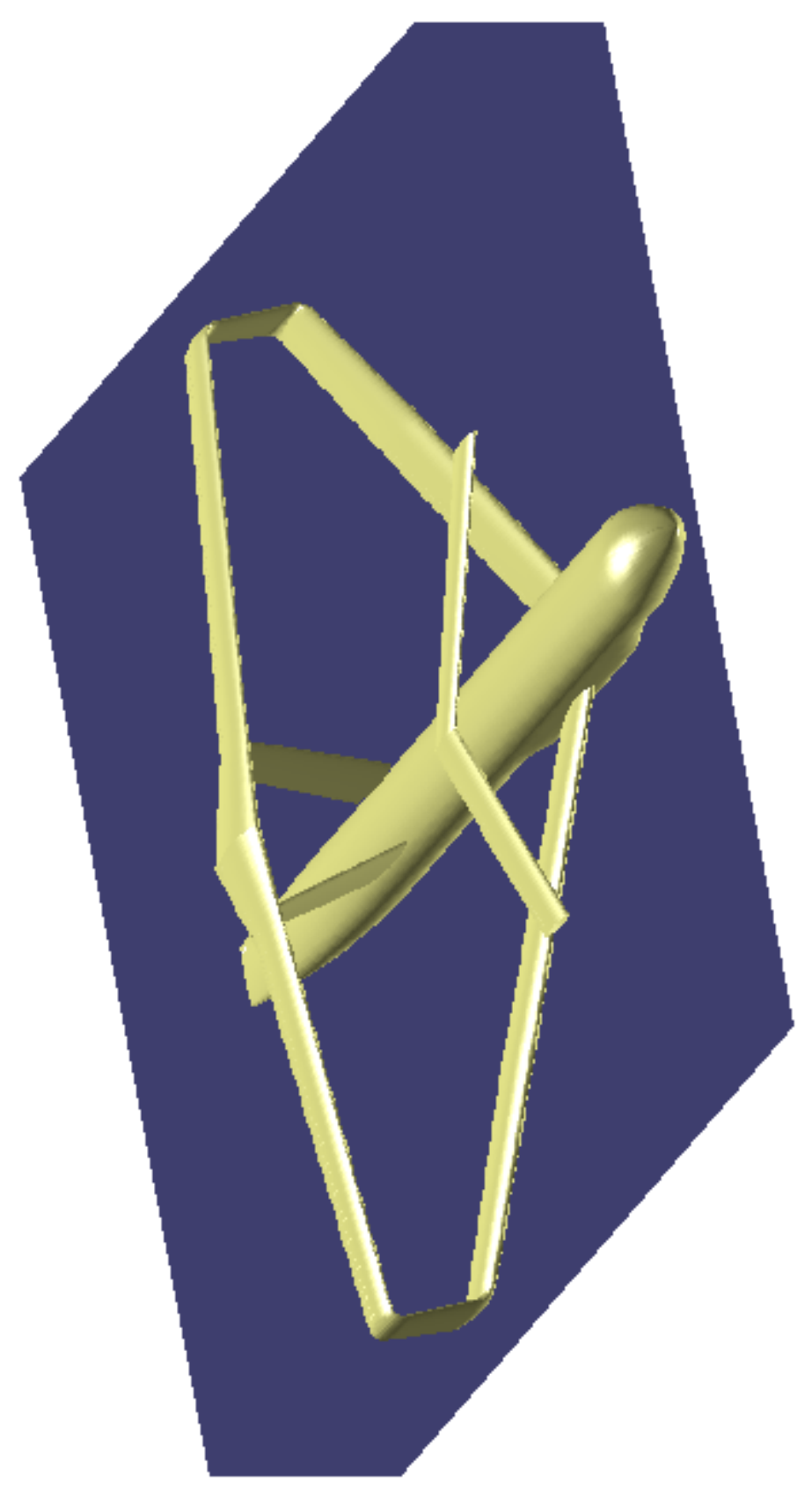

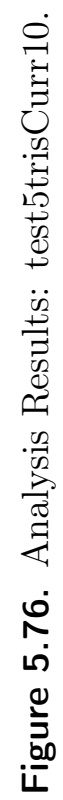




\begin{tabular}{|c|c|c|c|c|c|c|c|c|c|}
\hline \multirow{2}{*}{$\begin{array}{r}\text { FILE NAME } \\
\mathrm{E}\end{array}$} & \multicolumn{9}{|l|}{\begin{tabular}{|l|} 
test5trisCurr12 \\
\end{tabular}} \\
\hline & Cltot & \multicolumn{3}{|c|}{ CL_front } & \multicolumn{3}{|c|}{ CL_rear } & \multicolumn{2}{|c|}{ CL_auxwing } \\
\hline \multirow[t]{4}{*}{24,12} & 0,364 & \multicolumn{3}{|c|}{0,446} & \multicolumn{3}{|c|}{0,285} & \multicolumn{2}{|c|}{0,143} \\
\hline & MS [\%] & \multicolumn{3}{|c|}{$\mathrm{W} / \mathrm{s}$ front $\left[\mathrm{Kg} / \mathrm{m}^{\wedge} 2\right]$} & \multicolumn{3}{|c|}{$\mathrm{W} / \mathrm{S}$ rear $\left[\mathrm{Kg} / \mathrm{m}^{\wedge} 2\right]$} & \multicolumn{2}{|c|}{$\mathrm{W} / \mathrm{S}$ auxwing $\left[\mathrm{Kg} / \mathrm{m}^{\wedge} 2\right]$} \\
\hline & $-3,91$ & \multicolumn{3}{|c|}{626,4} & \multicolumn{3}{|c|}{400,1} & \multicolumn{2}{|c|}{200,7} \\
\hline & Configuration & \multicolumn{3}{|c|}{ Front wing } & \multicolumn{3}{|c|}{ Rear wing } & \multicolumn{2}{|c|}{ Auxiliary wing } \\
\hline XCG [m] & 40,2 & \multicolumn{3}{|c|}{35,7} & \multicolumn{3}{|c|}{54,2} & \multicolumn{2}{|c|}{51,9} \\
\hline XPN [m] & 39,9 & \multicolumn{3}{|c|}{$\backslash$} & \multicolumn{3}{|c|}{1} & \multicolumn{2}{|c|}{1} \\
\hline $\mathrm{S}\left[\mathrm{m}^{\wedge} 2\right]$ & 1106,5 & \multicolumn{3}{|c|}{571,6} & \multicolumn{3}{|c|}{499,8} & \multicolumn{2}{|c|}{35,1} \\
\hline b [m] & 80,0 & \multicolumn{3}{|c|}{80,0} & \multicolumn{3}{|c|}{80,0} & \multicolumn{2}{|c|}{8,8} \\
\hline MAC [m] & 7,21 & \multicolumn{3}{|c|}{8,02} & \multicolumn{3}{|c|}{6,51} & \multicolumn{2}{|c|}{4,00} \\
\hline AR & 5,78 & \multicolumn{3}{|c|}{11,20} & \multicolumn{3}{|c|}{12,81} & & \\
\hline$c[\mathrm{~m}]$ & 1 & 8,25 & 8,28 & 5,35 & 10,68 & 7,16 & 4,56 & 4,00 & 4,00 \\
\hline Pitch Angle [deg] & 1 & & 2,75 & & & $-0,41$ & & & \\
\hline Twist Angle [deg] & 1 & 2,00 & $-2,00$ & $-1,83$ & 2,00 & 1,28 & 2,00 & $-2,00$ & $-1,11$ \\
\hline Sweep Angle [deg] & 1 & 40,00 & & 28,36 & $-29,04$ & & $-0,24$ & & \\
\hline Dihedral [deg] & 1 & 4,05 & & 3,74 & $-2,83$ & & $-2,25$ & & \\
\hline Taper Ratio & 1 & 1,00 & & 0,65 & 0,67 & & 0,64 & & \\
\hline
\end{tabular}
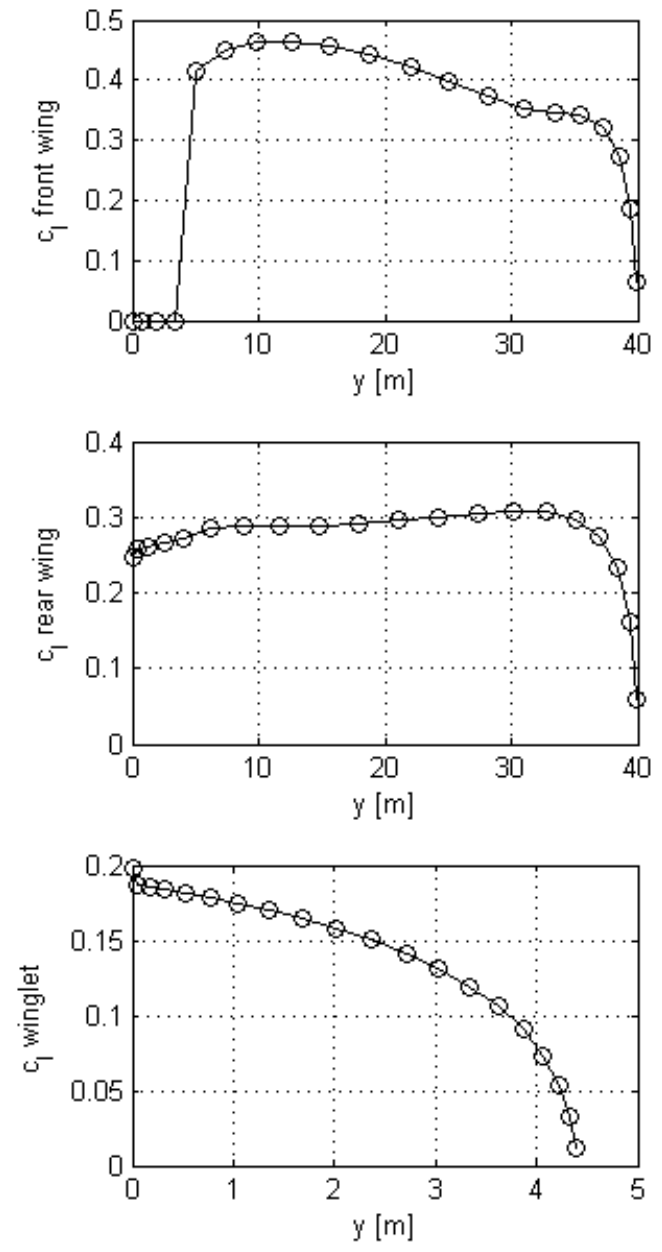

Table 5.18. Analysis Results: test5trisCurr12. 


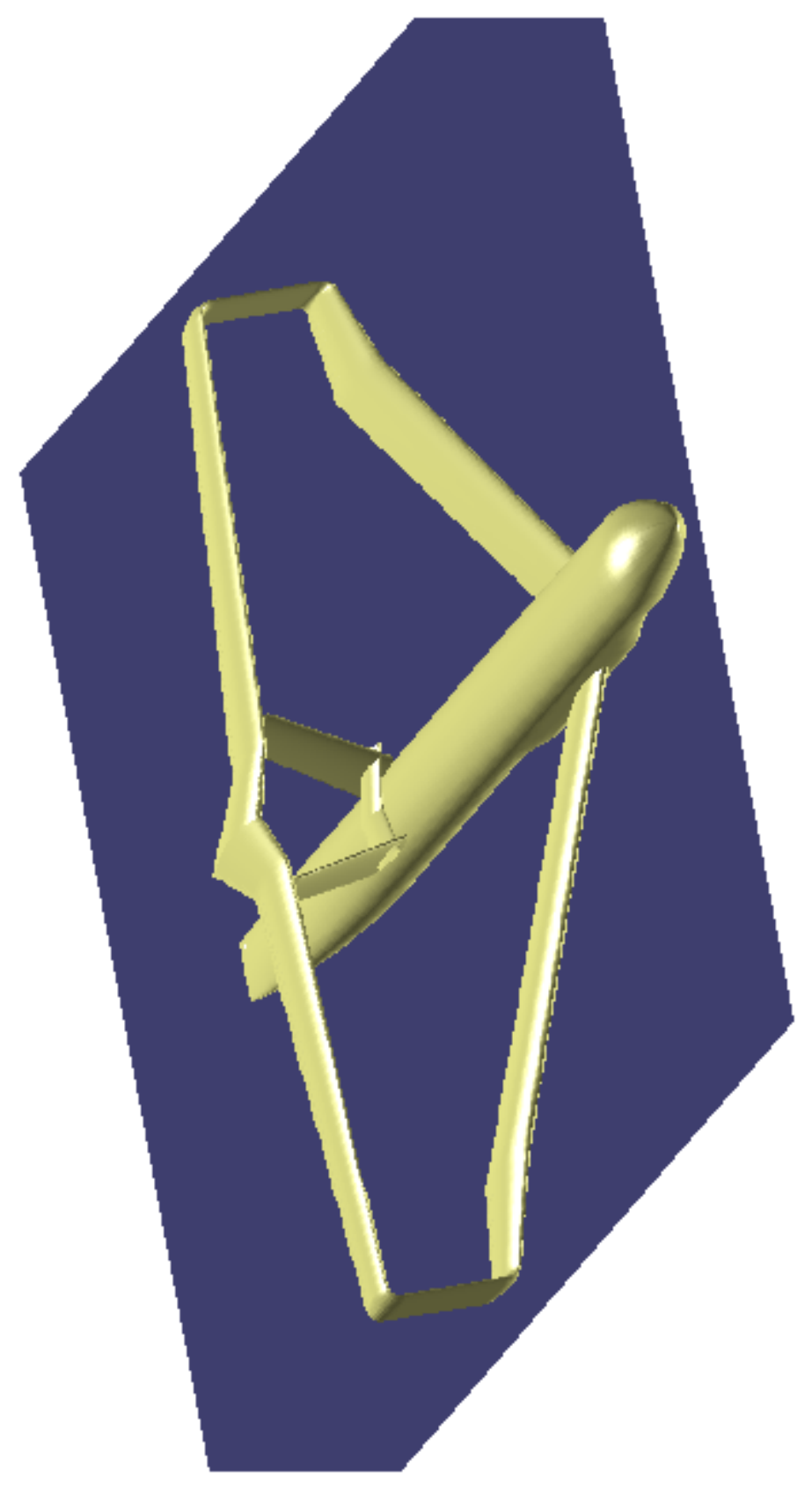

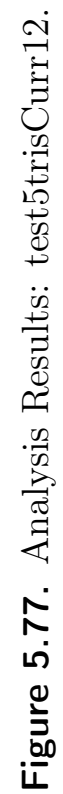




\begin{tabular}{|c|c|c|c|c|c|c|c|c|c|}
\hline FILE NAME & test5trisCurr13 & & & & & & & & \\
\hline $\mathrm{E}$ & Cltot & \multicolumn{3}{|c|}{$\mathrm{CL}_{-}$front } & \multicolumn{3}{|c|}{ CL_rear } & \multicolumn{2}{|c|}{ CL_auxwing } \\
\hline 23,58 & 0,356 & \multicolumn{3}{|c|}{0,435} & \multicolumn{3}{|c|}{0,279} & \multicolumn{2}{|c|}{0,142} \\
\hline & MS [\%] & \multicolumn{3}{|c|}{$\mathrm{W} / \mathrm{S}$ front $\left[\mathrm{Kg} / \mathrm{m}^{\wedge} 2\right]$} & \multicolumn{3}{|c|}{$\mathrm{W} / \mathrm{S}$ rear $\left[\mathrm{Kg} / \mathrm{m}^{\wedge} 2\right]$} & \multicolumn{2}{|c|}{$\mathrm{W} / \mathrm{s}$ auxwing $\left[\mathrm{Kg} / \mathrm{m}^{\wedge} 2\right]$} \\
\hline & 0,07 & \multicolumn{3}{|c|}{613,1} & \multicolumn{3}{|c|}{393,9} & \multicolumn{2}{|c|}{200,3} \\
\hline & Configuration & \multicolumn{3}{|c|}{ Front wing } & \multicolumn{3}{|c|}{ Rear wing } & \multicolumn{2}{|c|}{ Auxiliary wing } \\
\hline XCG [m] & 40,9 & \multicolumn{3}{|c|}{36,1} & \multicolumn{3}{|c|}{57,8} & \multicolumn{2}{|c|}{55,2} \\
\hline XPN [m] & 40,9 & \multicolumn{3}{|c|}{1} & \multicolumn{3}{|c|}{1} & \multicolumn{2}{|c|}{1} \\
\hline$S\left[m^{\wedge} 2\right]$ & 1124,4 & \multicolumn{3}{|c|}{603,2} & \multicolumn{3}{|c|}{468,6} & \multicolumn{2}{|c|}{52,6} \\
\hline $\mathrm{b}[\mathrm{m}]$ & 80,0 & \multicolumn{3}{|c|}{80,0} & \multicolumn{3}{|c|}{80,0} & \multicolumn{2}{|c|}{11,9} \\
\hline MAC [m] & 7,23 & \multicolumn{3}{|c|}{8,40} & \multicolumn{3}{|c|}{6,04} & \multicolumn{2}{|c|}{4,43} \\
\hline AR & 5,69 & \multicolumn{3}{|c|}{10,61} & \multicolumn{3}{|c|}{13,66} & \multicolumn{2}{|c|}{2,70} \\
\hline$c[\mathrm{~m}]$ & 1 & 8,84 & 8,32 & 8,36 & 7,51 & 7,22 & 4,04 & 4,82 & 4,00 \\
\hline Pitch Angle [deg] & 1 & \multicolumn{3}{|c|}{1,84} & \multicolumn{3}{|c|}{0,77} & & \\
\hline Twist Angle [deg] & 1 & 2,00 & 1,45 & $-2,00$ & 1,47 & \begin{tabular}{|l|}
$-0,08$ \\
\end{tabular} & 0,53 & $-1,27$ & $-0,46$ \\
\hline Sweep Angle [deg] & $\backslash$ & 12,86 & & 40,00 & $-23,58$ & & $-0,71$ & & \\
\hline Dihedral [deg] & $\backslash$ & 0,00 & & 3,93 & $-4,77$ & & $-1,01$ & & \\
\hline Taper Ratio & 1 & 0,94 & & 1,00 & 0,96 & & 0,56 & & \\
\hline
\end{tabular}
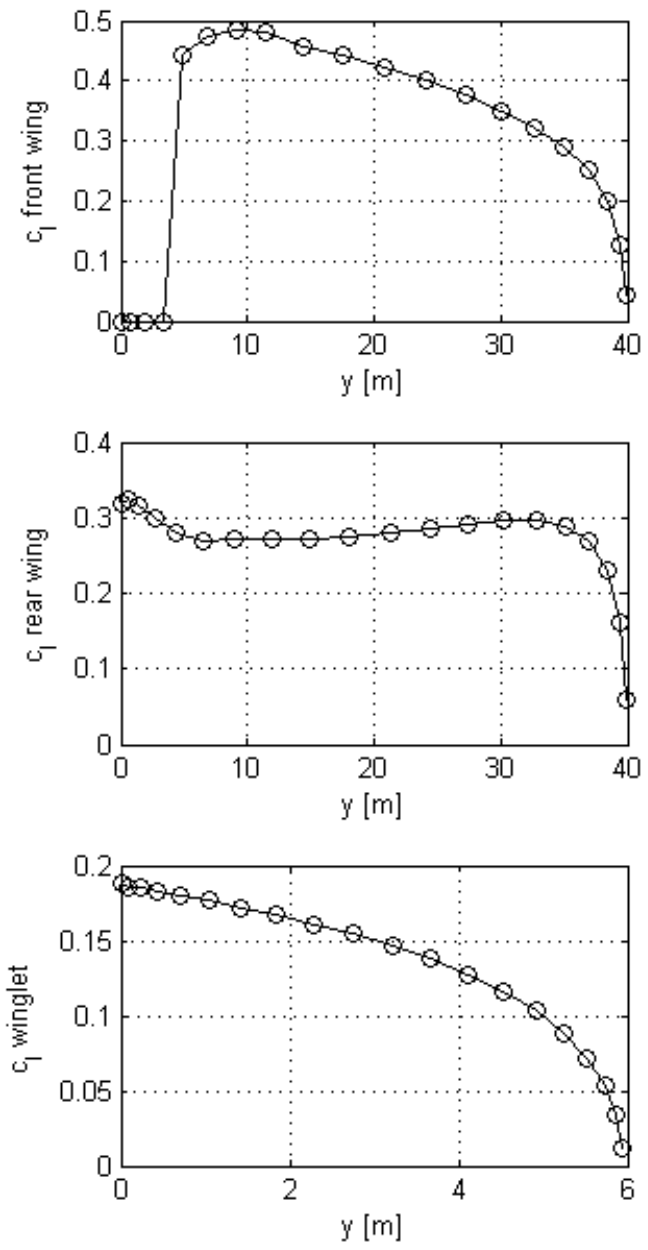

Table 5.19. Analysis Results: test5trisCurr13. 


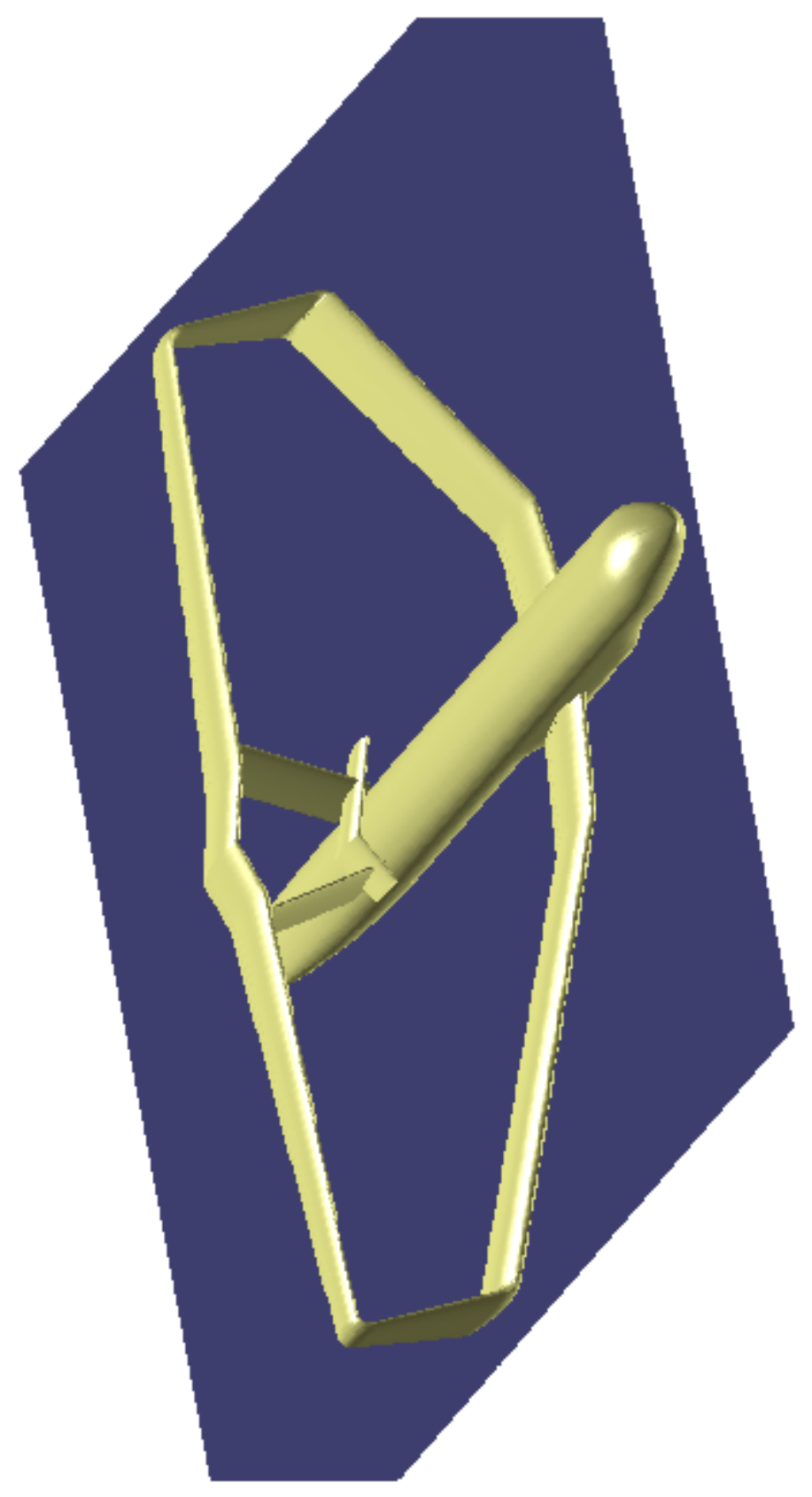

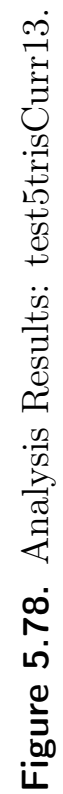




\begin{tabular}{|c|c|c|c|c|c|c|c|c|c|}
\hline FILE NAME & test5trisCurr14 & & & & & & & & \\
\hline $\mathrm{E}$ & Cltot & \multicolumn{3}{|c|}{ CL_front } & \multicolumn{3}{|c|}{ CL_rear } & \multicolumn{2}{|c|}{ CL_auxwing } \\
\hline 22,69 & 0,365 & \multicolumn{3}{|c|}{0,440} & \multicolumn{3}{|c|}{0,282} & \multicolumn{2}{|c|}{0,345} \\
\hline & MS [\%] & \multicolumn{3}{|c|}{$\mathrm{W} / \mathrm{S}$ front $\left[\mathrm{Kg} / \mathrm{m}^{\wedge} 2\right]$} & \multicolumn{3}{|c|}{$\mathrm{W} / \mathrm{S}$ rear $\left[\mathrm{Kg} / \mathrm{m}^{\wedge} 2\right]$} & \multicolumn{2}{|c|}{$\mathrm{W} / \mathrm{S}$ auxwing $\left[\mathrm{Kg} / \mathrm{m}^{\wedge} 2\right]$} \\
\hline & $-1,89$ & \multicolumn{3}{|c|}{627,1} & \multicolumn{3}{|c|}{401,1} & \multicolumn{2}{|c|}{490,8} \\
\hline & Configuration & \multicolumn{3}{|c|}{ Front wing } & \multicolumn{3}{|c|}{ Rear wing } & \multicolumn{2}{|c|}{ Auxiliary wing } \\
\hline XCG [m] & 39,9 & \multicolumn{3}{|c|}{35,0} & \multicolumn{3}{|c|}{57,8} & \multicolumn{2}{|c|}{29,1} \\
\hline XPN [m] & 39,7 & \multicolumn{3}{|c|}{1} & \multicolumn{3}{|c|}{1} & \multicolumn{2}{|c|}{1} \\
\hline$S\left[m^{\wedge} 2\right]$ & 1088,1 & \multicolumn{3}{|c|}{497,7} & \multicolumn{3}{|c|}{411,8} & \multicolumn{2}{|c|}{178,7} \\
\hline b [m] & 80,0 & \multicolumn{3}{|c|}{80,0} & \multicolumn{3}{|c|}{80,0} & \multicolumn{2}{|c|}{27,5} \\
\hline MAC [m] & 6,51 & \multicolumn{3}{|c|}{7,59} & \multicolumn{3}{|c|}{5,21} & \multicolumn{2}{|c|}{6,49} \\
\hline AR & 5,88 & \multicolumn{3}{|c|}{12,86} & \multicolumn{3}{|c|}{15,54} & \multicolumn{2}{|c|}{4,24} \\
\hline$c[\mathrm{~m}]$ & 1 & 12,80 & 8,06 & 4,00 & 5,70 & 5,66 & 4,00 & 6,49 & 6,49 \\
\hline Pitch Angle [deg] & 1 & \multicolumn{3}{|c|}{2,86} & \multicolumn{3}{|c|}{2,31} & & \\
\hline Twist Angle [deg] & 1 & 0,73 & $-0,21$ & $-1,60$ & $-2,00$ & $-1,15$ & 0,05 & $-1,47$ & 2,00 \\
\hline Sweep Angle [deg] & 1 & 24,11 & & 40,00 & $-20,73$ & & $-3,84$ & & \\
\hline Dihedral [deg] & 1 & 5,92 & & 5,86 & $-4,29$ & & $-5,73$ & & \\
\hline Taper Ratio & 1 & 0,63 & & 0,50 & 0,99 & & 0,71 & & \\
\hline
\end{tabular}
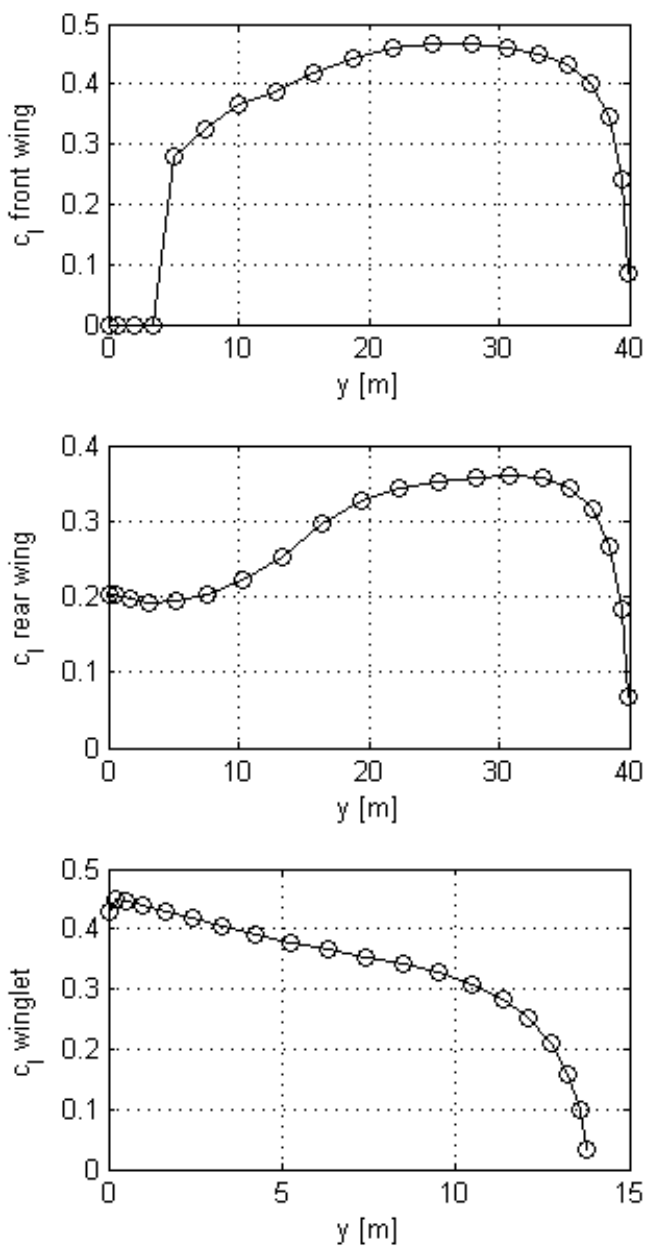

Table 5.20. Analysis Results: test5trisCurr14. 


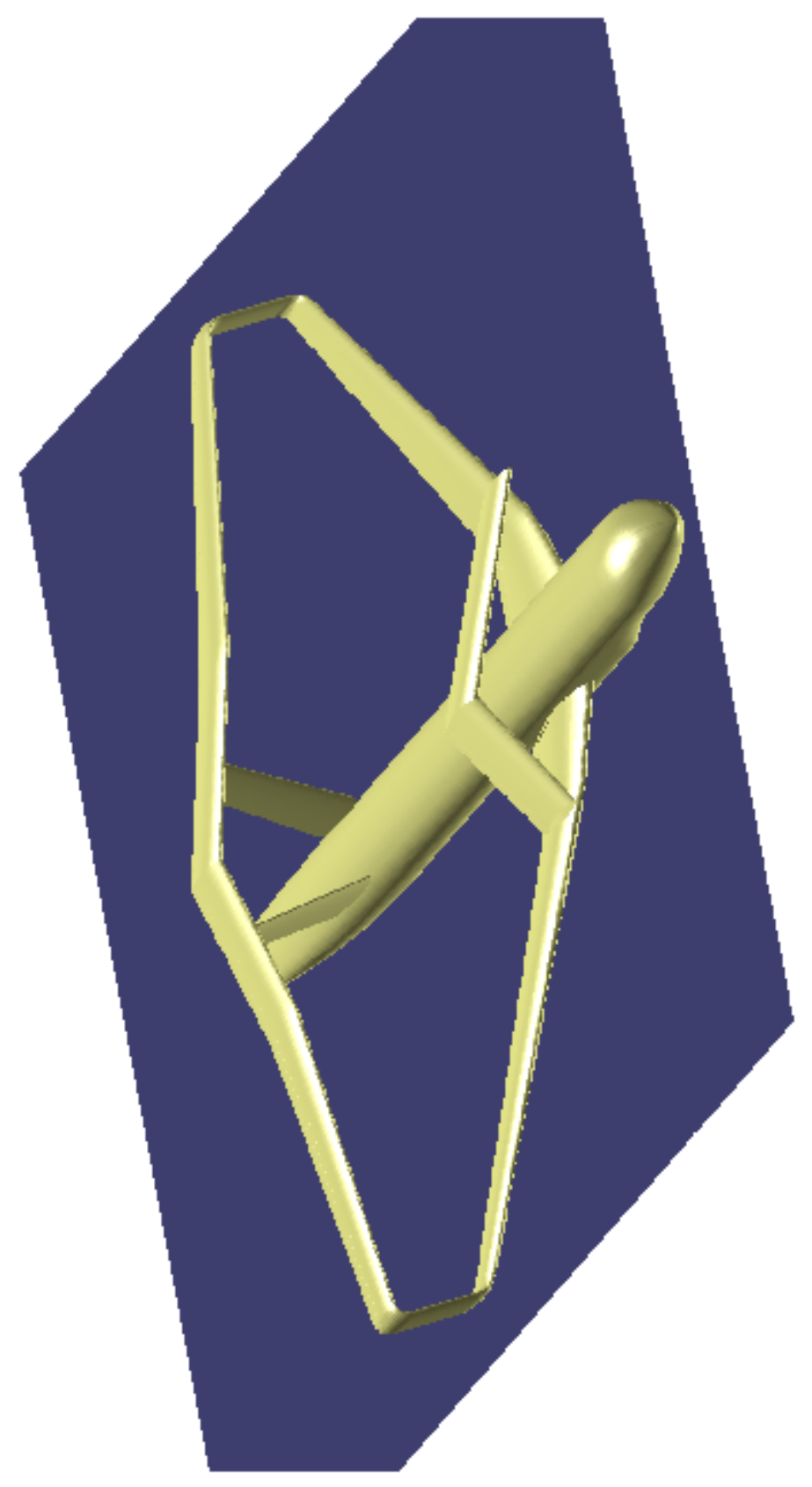

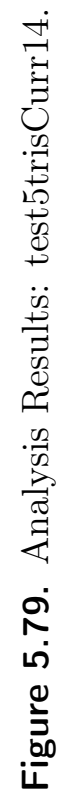




\begin{tabular}{|c|c|c|c|c|c|c|c|c|c|}
\hline \multirow{2}{*}{\begin{tabular}{|c|} 
FILE NAME \\
$\mathrm{E}$ \\
\end{tabular}} & \multicolumn{9}{|l|}{\begin{tabular}{|l|} 
test5trisCurr15 \\
\end{tabular}} \\
\hline & Cltot & \multicolumn{3}{|c|}{ CL_front } & \multicolumn{3}{|c|}{ CL_rear } & \multicolumn{2}{|c|}{ CL_auxwing } \\
\hline 22,80 & 0,383 & \multicolumn{3}{|c|}{0,429} & \multicolumn{3}{|c|}{0,288} & \multicolumn{2}{|c|}{0,420} \\
\hline & MS [\%] & \multicolumn{3}{|c|}{$\mathrm{W} / \mathrm{s}$ front $\left[\mathrm{Kg} / \mathrm{m}^{\wedge} 2\right]$} & \multicolumn{3}{|c|}{$\mathrm{W} / \mathrm{S}$ rear $\left[\mathrm{Kg} / \mathrm{m}^{\wedge} 2\right]$} & \multicolumn{2}{|c|}{$\mathrm{W} / \mathrm{S}$ auxwing $\left[\mathrm{Kg} / \mathrm{m}^{\wedge} 2\right]$} \\
\hline & $-0,53$ & \multicolumn{3}{|c|}{606,8} & \multicolumn{3}{|c|}{407,3} & \multicolumn{2}{|c|}{594,4} \\
\hline & Configuration & \multicolumn{3}{|c|}{ Front wing } & \multicolumn{3}{|c|}{ Rear wing } & \multicolumn{2}{|c|}{ Auxiliary wing } \\
\hline XCG [m] & 39,8 & \multicolumn{3}{|c|}{38,3} & \multicolumn{3}{|c|}{56,0} & \multicolumn{2}{|c|}{31,5} \\
\hline XPN [m] & 39,8 & \multicolumn{3}{|c|}{1} & \multicolumn{3}{|c|}{1} & \multicolumn{2}{|c|}{1} \\
\hline$S\left[m^{\wedge} 2\right]$ & 1042,5 & \multicolumn{3}{|c|}{545,4} & \multicolumn{3}{|c|}{328,3} & \multicolumn{2}{|c|}{168,7} \\
\hline b [m] & 80,0 & \multicolumn{3}{|c|}{80,0} & \multicolumn{3}{|c|}{80,0} & \multicolumn{2}{|c|}{28,7} \\
\hline MAC [m] & 6,29 & \multicolumn{3}{|c|}{7,73} & \multicolumn{3}{|c|}{4,11} & \multicolumn{2}{|c|}{5,89} \\
\hline AR & 6,14 & \multicolumn{3}{|c|}{11,73} & \multicolumn{3}{|c|}{19,49} & \multicolumn{2}{|c|}{4,87} \\
\hline $\mathrm{c}[\mathrm{m}]$ & 1 & 8,19 & 8,09 & 4,40 & 4,35 & 4,11 & 4,00 & 5,95 & 5,82 \\
\hline Pitch Angle [deg] & 1 & & 2,80 & & & 1,00 & & & \\
\hline Twist Angle [deg] & 1 & 1,72 & $-2,00$ & $-2,00$ & $-1,55$ & 0,09 & 1,57 & 1,03 & 1,05 \\
\hline Sweep Angle [deg] & $\backslash$ & 33,67 & & 33,22 & $-21,44$ & & $-3,89$ & & \\
\hline Dihedral [deg] & 1 & 5,63 & & 2,92 & $-1,48$ & & $-5,82$ & & \\
\hline Taper Ratio & 1 & 0,99 & & 0,54 & 0,95 & & 0,97 & & \\
\hline
\end{tabular}
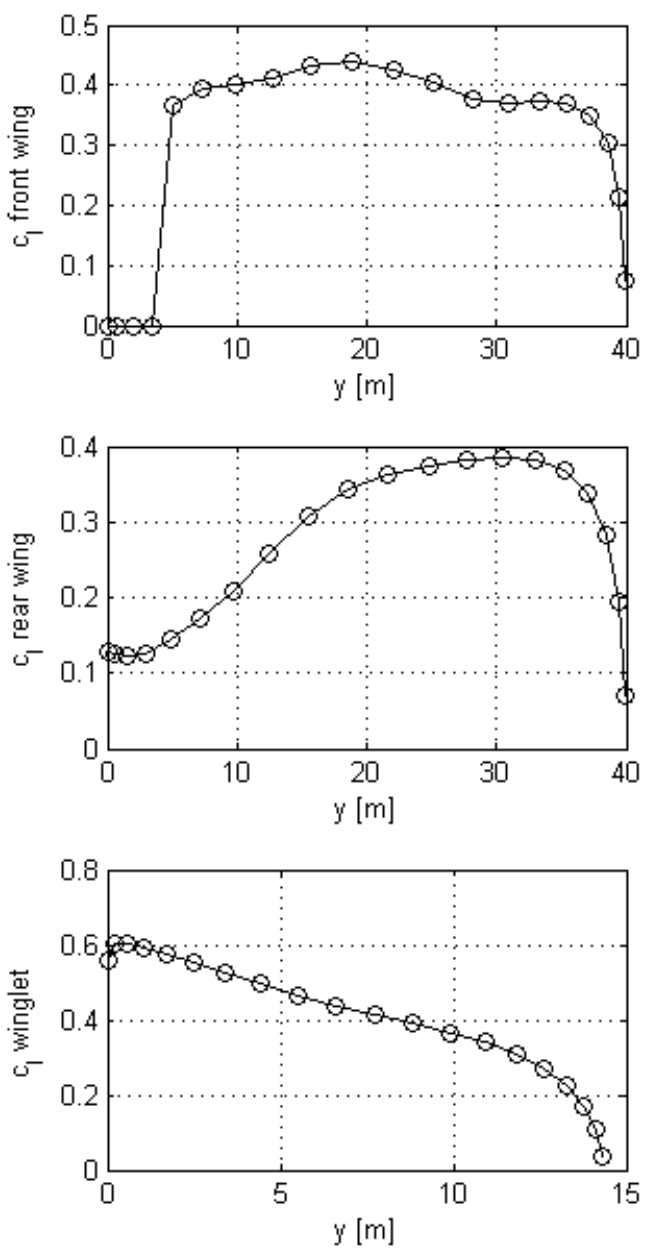

Table 5.21. Analysis Results: test5trisCurr15. 


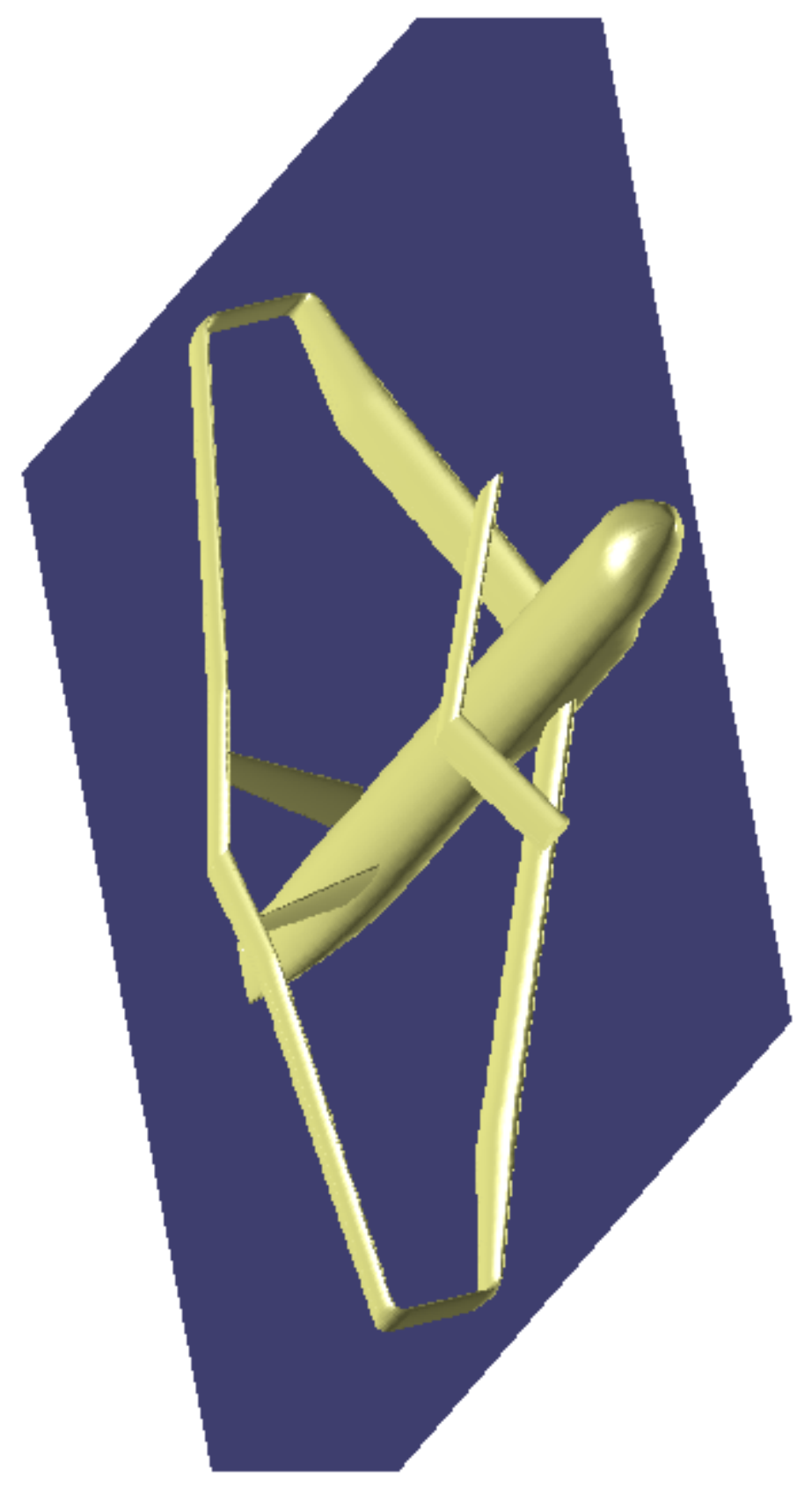

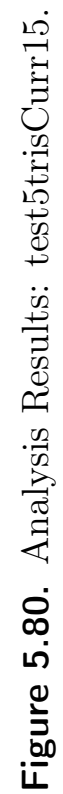




\begin{tabular}{|c|c|c|c|c|c|c|c|c|c|}
\hline FILE NAME & test5trisCurr16 & & & & & & & & \\
\hline $\mathrm{E}$ & Cltot & & L_front & & & CL_rea & & $\mathrm{CL}$ & ing \\
\hline 23,87 & 0,376 & & 0,429 & & & 0,278 & & & \\
\hline & MS [\%] & $\mathrm{w} / \mathrm{s}$ fro & ont $[\mathrm{Kg}$ & $\left./ \mathrm{m}^{\wedge} 2\right]$ & $\mathrm{w} / \mathrm{s} \mathrm{r}$ & ear $\left[K_{\xi}\right.$ & $\left./ \mathrm{m}^{\wedge} 2\right]$ & $\mathrm{W} / \mathrm{S}$ aux & {$\left[\mathrm{Kg} / \mathrm{m}^{\wedge} 2\right]$} \\
\hline & $-0,41$ & & 606,1 & & & 392,0 & & & \\
\hline & Configuration & & ont win & & & ear wi & & Auxi & wing \\
\hline XCG [m] & 40,6 & & 37,0 & & & 62,1 & & & \\
\hline XPN [m] & 40,6 & & 1 & & & 1 & & & \\
\hline$S\left[m^{\wedge} 2\right]$ & 1065,5 & & 700,0 & & & 350,4 & & & \\
\hline b [m] & 80,0 & & 80,0 & & & 80,0 & & & \\
\hline MAC [m] & 8,22 & & 10,22 & & & 4,39 & & & \\
\hline AR & 6,01 & & 9,14 & & & 18,27 & & & \\
\hline$c[\mathrm{~m}]$ & 1 & 12,74 & 10,40 & 5,43 & 5,01 & 4,58 & 4,00 & 4,38 & 4,38 \\
\hline Pitch Angle [deg] & 1 & & 3,00 & & & 0,71 & & & \\
\hline Twist Angle [deg] & 1 & 2,00 & $-2,00$ & $-2,00$ & 1,54 & 0,42 & 0,26 & $-0,60$ & 1,99 \\
\hline Sweep Angle [deg] & $\backslash$ & 40,00 & & 40,00 & $-3,11$ & & $-12,35$ & & \\
\hline Dihedral [deg] & 1 & 5,59 & & 4,75 & $-4,37$ & & $-5,24$ & & \\
\hline Taper Ratio & 1 & 0,82 & & 0,52 & 0,92 & & 0,87 & & \\
\hline
\end{tabular}
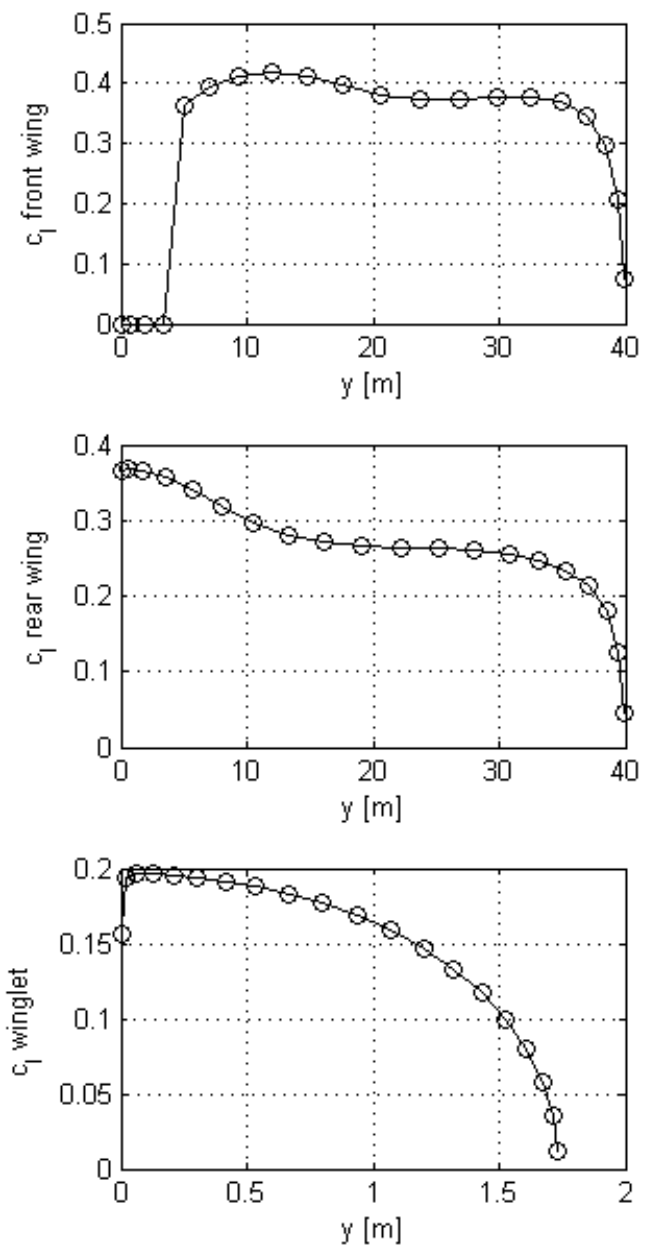

Table 5.22. Analysis Results: test5trisCurr16. 


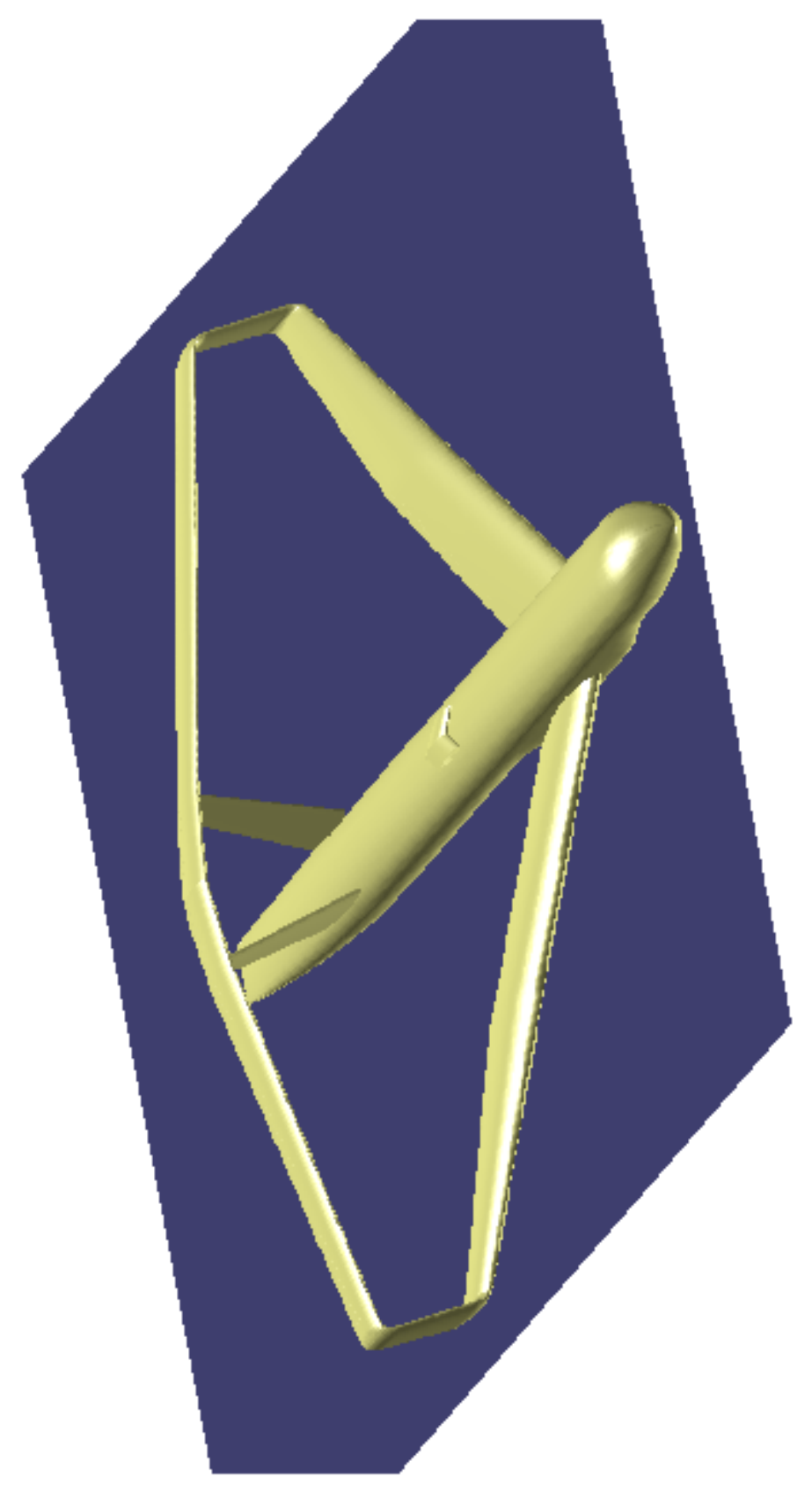

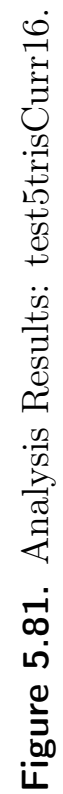





\section{Chapter 6}

\section{Low Speed Design}

The present chapter is dedicated to the low speed design of a large PrandtlPlane ${ }^{\circledR}$. Firstly, with reference to NASA CR 4746 requirement, the landing maximum lift condition is analysed. A possible solution is found and an arrangement of high lift devices and elevators is assumed and, then, the aircraft is verified to be able to land, meeting also trim and longitudinal stability conditions. Finally, the most remarkable results are reported in order to understand which are the key-parameters to control in low speed design; plots of the configurations with control surfaces, slats and flaps are provided.

It is worth to mention that the design of flaps and slats produces effects on the aerodynamics and on the wing structure; in particular, the effects of the high lift devices and the interactions between leading edge and trailing edge effects have to be optimized. In this preliminary phase, performances of flaps and slats are studied by applying theoretical and semi empirical formulas, provided by [8].

\subsection{Landing conditions - NASA CR 4746 method}

We consider the approach landing condition of the airplane; the approach speed $V_{3}$ is introduced.

$$
V_{3}=1.3 V_{S d y n},
$$

where $V_{S d y n}$ is the dynamic stall speed for a given flap configuration, as reported in [16]: indeed, regulations refer to a stalling speed achieved in manoeuvre.

In these analyses, we assume

$$
V_{S d y n}=0.94 V_{S 1 g}
$$

where $V_{S 1 g}$ is the stall speed of the aircraft in steady flight; the relation between $V_{S d y n}$ and $V_{S 1 g}$ is reported in [8].

In this way, the necessary lift coefficient of the whole configuration $C_{L S 1 g}$ is

$$
C_{L S 1 g}=(0.94)^{2}(1.3)^{2} C_{\text {Lapproach }}
$$


and

$$
C_{\text {Lapproach }}=\frac{W / S}{\frac{1}{2} \rho V_{L S}^{2}}
$$

where

$$
\begin{aligned}
& W=75 \% \text { MTOW } \\
& \text { MTOW }=624800 \mathrm{~kg} \\
& V_{L S}=145 \mathrm{kts} \\
& \rho=1,225 \mathrm{~kg} / \mathrm{m}^{3} \quad(\mathrm{~h}=0)
\end{aligned}
$$

\subsection{Low speed devices design}

In the following sections the procedure used to size the low speed devices is described, together with all the experimental and theoretical formulas for leading edge and trailing edge lift contributes.

\subsubsection{The low speed analytical procedure}

The purpose of these studies is to investigate about the feasibility of high lift devices on the optimized configurations.

The low speed method, described in this section, consists into satisfying the following conditions:

$$
\begin{aligned}
& C_{l \max F w} \leq C_{l_{\max F w T o r}} \\
& C_{l \max R w} \leq C_{l \max R w T o r} \\
& C_{l \max A w} \leq C_{l \max A w T o r}
\end{aligned}
$$

$C_{l \max F w}, C_{l_{\max R w}}$ and $C_{l \max A w}$ are the maximum airfoil lift coefficients (with or without high lift devices extracted) of each wing; $C_{l_{\max } \text { wTor }}, C_{l_{\max R w T o r}}$ and $C_{l_{\max } \text { AwTor }}$ are the corresponding maximum airfoil lift coefficients, calculated by the Torenbeek's method (refer to sec. 6.2.2 and sec. 6.2.3).

The $C_{l_{\max F w}}, C_{l_{\max R w}}$ and $C_{l_{\max A w}}$ are computed by using the $A V L$ software, in which all the aerodynamic surfaces and all the wing devices (elevators, trailing edge flaps and slats) are modelled; elevators, flaps and slats are represented in $A V L$ as plain flap and slat.

In this procedure, the angles of elevators, flaps and slats are established in the following way: 
1. The approach angle $\alpha$ (the angle between the unperturbed flow and the fuselage axis) is determined by an iterative cycle, until the local $C_{l \max A w}$ of the clean configuration is less than 1.2 (corresponding to the linearity loss in Fig. 5.2): then, $\alpha_{\text {approach }}$ is the angle at which the stall of the auxiliary wing airfoil occurs.

2. Once that $\alpha$ is chosen, the deflection angles of elevators and trailing edge flaps are obtained from the equilibrium equations (reference to the center of gravity):

$$
\left\{\begin{array}{l}
C_{L S 1 g}=C_{L 0}+C_{L \alpha} \alpha+C_{L \delta_{e}} \delta_{e}+C_{L \delta_{f}} \delta_{f} \\
0=C_{M 0}+C_{M \alpha} \alpha+C_{M \delta_{e}} \delta_{e}+C_{M \delta_{f}} \delta_{f}
\end{array}\right.
$$

$C_{L 0}$ and $C_{M 0}$ are the total lift coefficient and the total moment coefficient of the entire configuration, respectively, when $\alpha, \delta_{e}$ and $\delta_{f}$ are equal to zero; $\delta_{e}$ and $\delta_{f}$ are perturbations close to zero.

3. Now, a new aerodynamic calculation is performed by launching $A V L$, with the deflection angles $\delta_{e}, \delta_{f}$ which solve equations 6.9 and with the correct angle of approach $\alpha$; the results are the $C_{l}$ distributions along all the aerodynamic surfaces.

In particular, the $C_{l}$ perpendicular to the isobars are extrapolated and compared with the allowable data values that are obtained from the Torenbeek's method.

At this step, it is verified that:

$$
\frac{\left|C_{L S 1 g_{A V L}}-C_{L S 1 g}\right|}{C_{L S 1 g_{A V L}}} \leq 5 \%
$$

and

$$
C_{M t o t A V L}= \pm 0.03
$$

This step is necessary, because $\delta_{e}$ and $\delta_{f}$ are calculated under the hypothesis of linear aerodynamics and without considering the slats effects: in this way the vertical equilibrium and the moment equilibrium are confirmed within appropriate tolerances.

The effects of slats are not considered in 6.9, for simplicity sake: furthermore, the slat angle $\delta_{s}$ is set

$$
\delta_{s}=22,5^{\circ}
$$

because the usually slat deflection is between $20^{\circ}$ and $25^{\circ}$ (refer to [8]).

When the approach angle is too large, it is corrected by hand: this effect appears when the auxiliary wing is placed in the rear fuselage and subjected to downwash. 


\subsubsection{Leading edge high lift devices}

When a leading edge high lift device is used, the pressure peak suction moves towards the aerofoil nose, thereby delaying leading edge separation to a higher angle of attack.
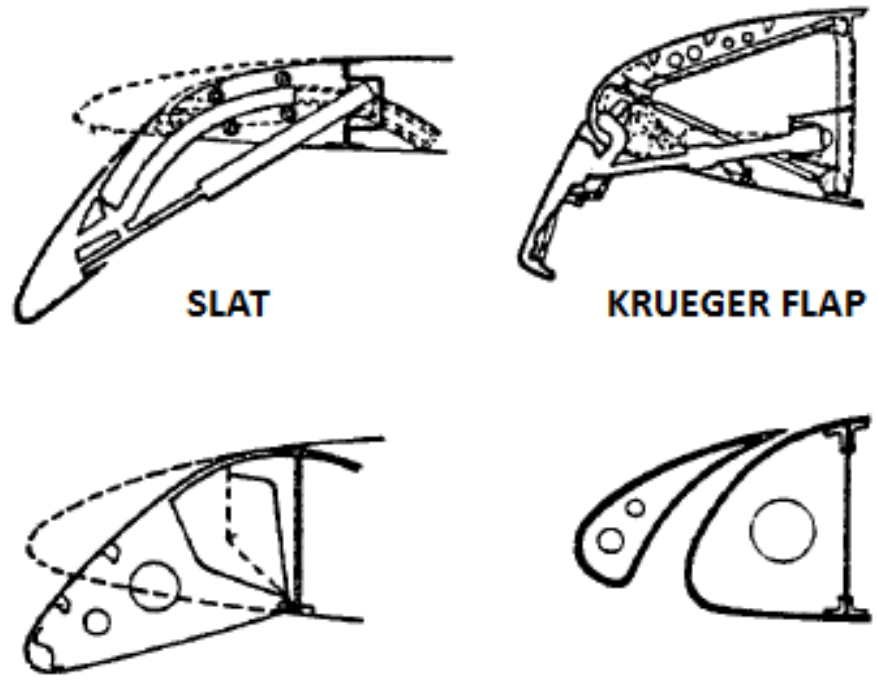

HINGED NOSE

FIXED SLOT

Figure 6.1. Lift edge high lift devices.

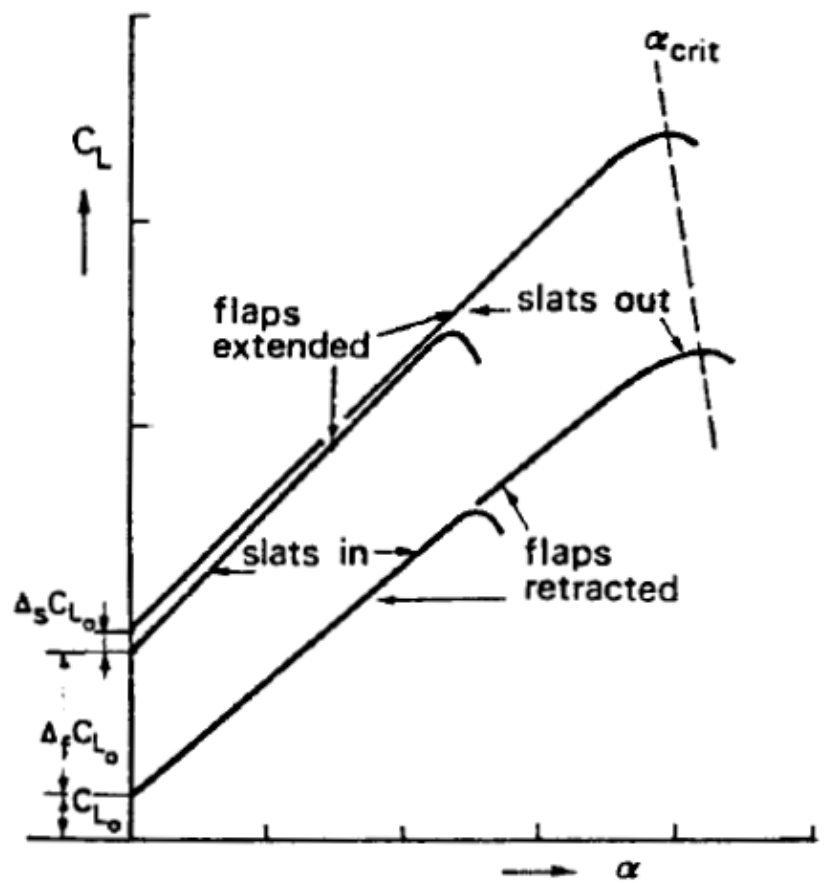

Figure 6.2. Lift curve with and without high lift devices. 
Airfoils with a deflected trailing edge flap normally exhibit a leading edge stall, even on relatively thick sections: leading edge devices suppress this stall, by increasing the stalling angle of attack and hence, increasing the maximum lift (6.2).

However, the slat wake may cause undesirable interference with flow around trailingedge flaps and the increment due to the slat with trailing edge flaps deflected is typically $15 \%$ lower than the $C_{l \max }$ increment of the unflapped airfoil.

Extension of a slat results in a slight decrement in lift at zero angle of attack $\left(\Delta_{s} C_{L 0}\right.$ in Fig. 6.2), caused by the nose droop. However, the slat increases the effective chord length and this causes a lift increment when $C_{l}$ is based on the original chord. Both effects are generally of the same order of magnitude and it is reasonable to assume that $\Delta_{s} C_{L 0}=0$; it is observed that the slope of the lift curve is not affected to any slat deflection.

The estimation of $C_{l_{\max }}$ with a deflected slat is influenced by a number of factor:

- For a given configuration of trailing edge flap deflected, the slat will be more effective in terms of $\Delta_{s} C_{L \max }$ on an airfoil with a sharp nose than on a well-rounded airfoil nose;

- For an optimized slat configuration, the stall may be of the trailing edge type, whereas without a slat is of the leading edge type;

- The gap between the airfoil and the slat has a very pronounced effect on the lift increment;

- Trailing edge flaps and the leading edge slats should be matched in order to obtain the highest performance;

- Compressibility effects may limit the performances of complex high lift systems: the maximum lift increment due to slats should be based on the condition that sonic flow sets a limit to the pressure peaks.

It can be shown that for a particular configuration, $\Delta_{s} C_{L \max }$ due to the slat may vary between 0.5 and 1.2. For this reason, it is possible to define a simple (non-optimized) high lift system and an optimized configuration.

Simple high lift devices configuration: the maximum lift increment with trailing edge flaps retracted is about

$$
\Delta_{s} C_{L \max }=2.2 \sqrt{\frac{c_{s}}{c}}
$$

and, with trailing edge flaps extracted is

$$
\Delta_{s} C_{L \max }=1.9 \sqrt{\frac{c_{s}}{c}}
$$

The corresponding slat deflection is about $25^{\circ}$. 
Optimized configuration: the high lift system (which is composed by the main airfoil, the trailing edge flap and slat) is designed to have a simultaneously condition of separation of all its parts, at high $C_{l}$. It is observed that these configurations achieve a critical angle of attack of about

$$
\alpha_{c}=27-2 C_{l}
$$

degrees, irrespective of the airfoil section shape and the high lift system. Eq. 6.16 is thus used to compute the $C_{l \max }$ of an optimized configuration:

$$
C_{l \max }=\left(1-k_{s}\right) \frac{C_{l 0}+\Delta_{f} C_{l 0}+0.47 C_{l_{\alpha}}}{1+0.035 C_{l \alpha}}
$$

where $C_{l \alpha}$ is in $\mathrm{rad}^{-1}$ and $k_{s}$ is a factor which takes the non linearity of the lift curve at high lift into account : in general, $k_{s}$ is between 0.03 and 0.15 , with $k_{s}=0.07$ a good average. $C_{l_{0}}$ and $C_{l_{\alpha}}$ are the lift coefficient at zero angle of attack and the slope of the lift coefficient curve for the airfoil with or without flaps deflected (refer to [8]).

It has been verified that simple high lift systems are not efficient enough to meet the low speed requirement, thus, the optimized configuration has been chosen for slats design.

\subsubsection{Trailing edge flaps}

The main effects of trailing edge flap deflection are shown in Fig. 6.3. The relationship between the airfoil lifting coefficient $\left(c_{l}\right)$ and the angle of attack $\alpha$ is linear, even with flaps extended; however, the slope of the curve changes $\left(c_{l \alpha}\right.$ becomes $\left.c_{l \alpha}^{\prime}\right)$ and $c_{l 0}$ (the lifting coefficient at null angle of attack) is increased by $\Delta c_{l 0}$. Also the maximum lifting coefficient $\left(c_{\text {lmax }}\right)$ is increased by $\Delta c_{\text {lmax }}$. The most remarkable disadvantage, occurring by the flaps deflection, is that the stall angle of attack decreases (refer to Fig. 6.3).

Firstly, it is necessary to evaluate $\Delta c_{l 0}$, because it directly affects the $\Delta c_{\text {lmax }}$, that is the key value for the low speed procedure. Instead, in the same procedure, it is not considered the lift curve slope modification, so we assume $c_{l \alpha}=2 \pi$ for the airfoil.

The flap efficiency is an important parameter and it gauges how the flap deflection $\left(\delta_{f}=\delta\right)$ affects the lift coefficient (defined by eq. 6.17$)$.

$$
c_{l \delta}=\left(\frac{\partial c_{l}}{\partial \delta}\right)_{\alpha}
$$

Another significant parameter is the rate of change of the zero-lift angle of attack with flap deflection, defined by eq. 6.18.

$$
\frac{\partial \alpha}{\partial \delta}=\alpha_{\delta}=\frac{\left(c_{l \delta}\right)_{\alpha}}{\left(c_{l \alpha}\right)_{\delta}}
$$

According to the Glauert linearised theory, $\alpha_{\delta}$ is given by eq. 6.19), where $\theta_{f}$ is computed in eq. 6.20 (refer to Fig. 6.4). 


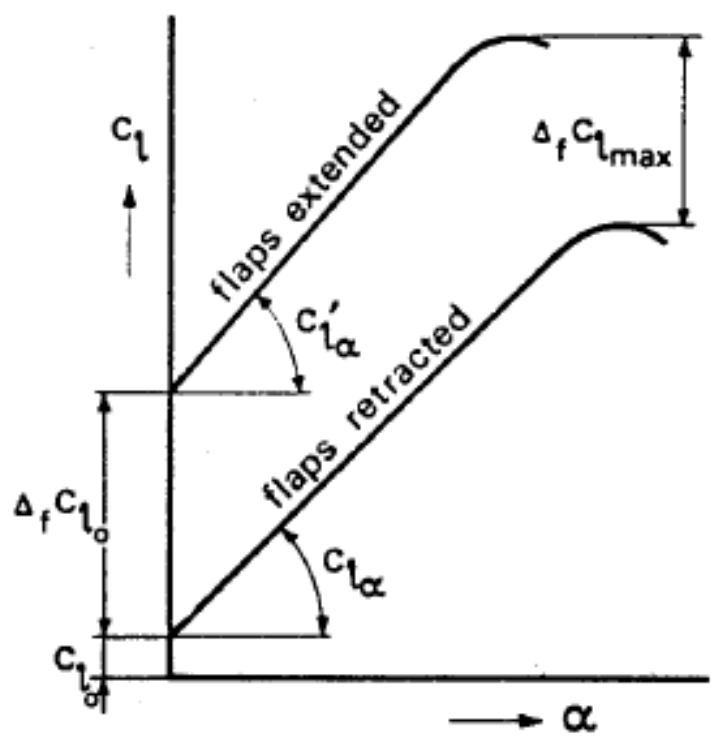

Figure 6.3. Flap deflection effects on the lift curve.

$$
\begin{gathered}
\alpha_{\delta}=1-\frac{\theta_{f}-\sin \left(\theta_{f}\right)}{\pi} \\
\theta_{f}=\cos ^{-1}\left(2 \frac{c_{f}}{c}-1\right)
\end{gathered}
$$

Then, the gain of the $\Delta c_{l 0}$ can be computed as follows:

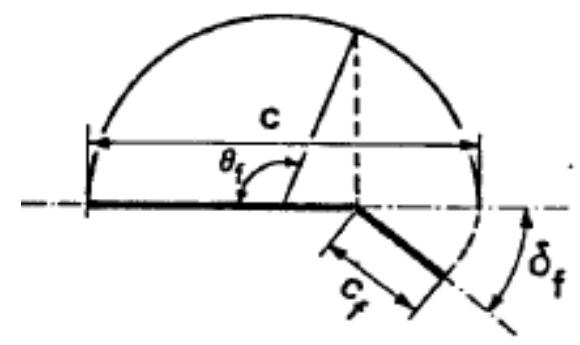

Figure 6.4. Parameters definition for flaps deflection.

$$
\Delta c_{l 0}=\left(c_{l \delta}\right)_{\alpha} \delta_{f}=c_{l \alpha} \alpha_{\delta} \delta_{f}
$$

Eq. 6.21 can not be used directly, because actually large flap deflections imply that the linearised theory is not still completely valid and the viscosity of the flow becomes a dominant parameter in the separation phenomenon (the separation starts at $10-15$ degrees for a plain flaps and at 20 - 35 degrees for a slotted flap, depending upon the design details). So, all these effects can be taken into account by considering an efficiency 
parameter $\left(\eta_{\delta}\right)$ : higher is the flap deflection, lower is $\eta_{\delta}$ (it can even go down to 0.5 if the flap deflection about 40 degrees or more). The new value of $\Delta c_{l 0}$ is given by eq. 6.22 .

$$
\Delta c_{l 0}=\eta_{\delta} c_{l \alpha} \alpha_{\delta} \delta_{f}
$$

The value of $\eta_{\delta}$ is provided by [8] with reference to empirical data. The choice of the flap kind for the PrandtlPlane ${ }^{\circledR}$ is not unique and it needs to be discussed for every configuration analysed: once the low speed constraint has been fulfilled, the leading criterion is to set as cheaper as possible high lift devices. Plain flaps, single slotted flaps, fowler flaps and double slotted flaps (ranked according increasing cost) have been considered and they are briefly described.

\section{Plain flap}

The plain flap is the simplest device to install but it is very sensitive to the boundary layer at the knuckle. Thus, the percentage of the flapped chord $\left(c_{f} / c\right)$ is a fundamental parameter. It has to be noticed that the gap between the flap and the other part of the airfoil has to be sealed. Refer to Fig. 6.5 and to Fig. 6.6 to have an overview on the simple kinematics and on the plain flap efficiency.

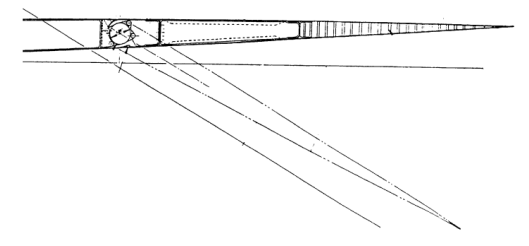

Figure 6.5. Plain flap.

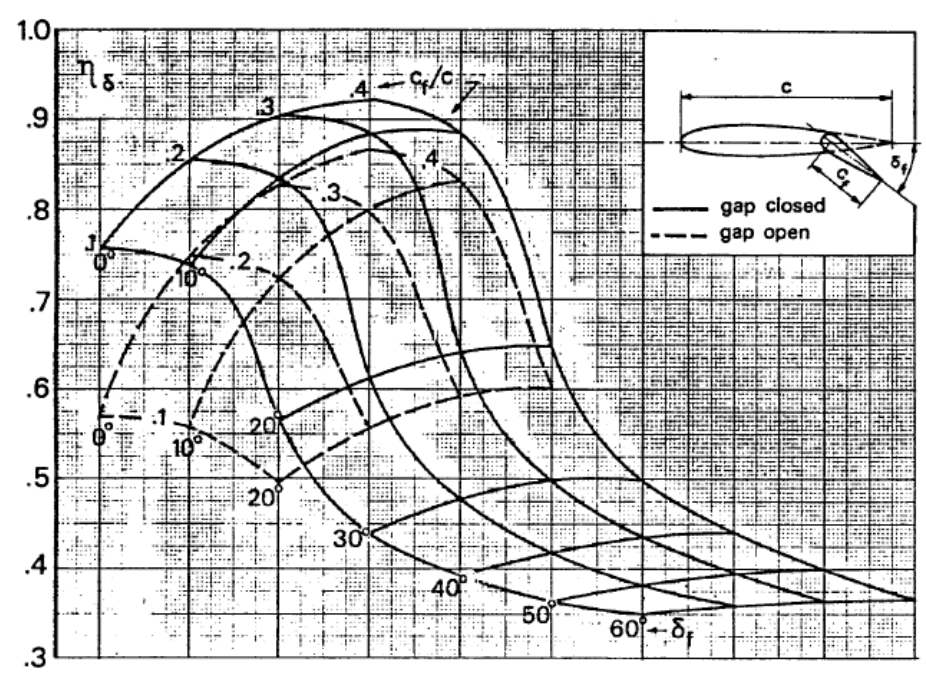

Figure 6.6. Plain flap efficiency. 


\section{Single Slotted flap and fowler flap}

The lifting coefficient is not affected by the boundary layer of the original airfoil but it depends on the new boundary layer due to the flap deflection; indeed, the efficiency of a single slotted flap (refer to Fig. 6.7) is heavily influenced by the flap and the slot geometry. Thus, a single slotted flap needs to have an optimized shape. Often the single slotted flap is supported by means a fixed hinge in order to simplify the structure: in this case the efficiency is sensitive to the hinge location.

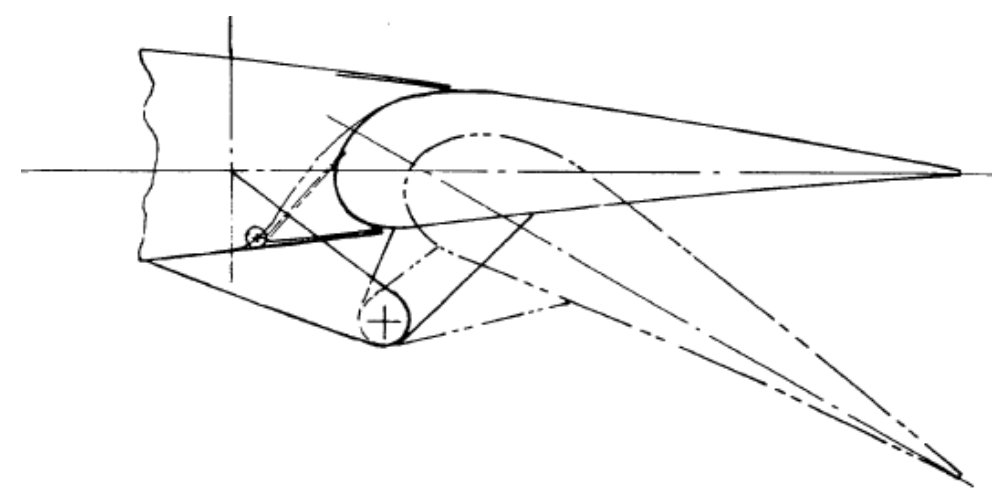

Figure 6.7. Single slotted flap.

Aerodynamically, the fowler flap (refer to Fig. 6.8) acts as a single slotted flap; the main difference is the increased chord extension. Furthermore, for angles of $30-35$ degrees, the fowler flap has a better efficiency than the single slotted flap, as it is shown in Fig. 6.9.
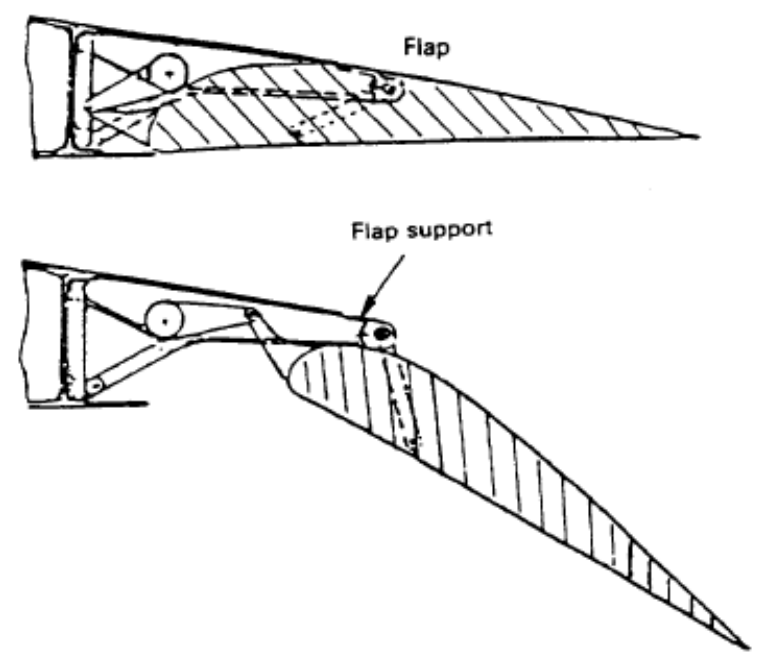

Figure 6.8. Fowler flap. 


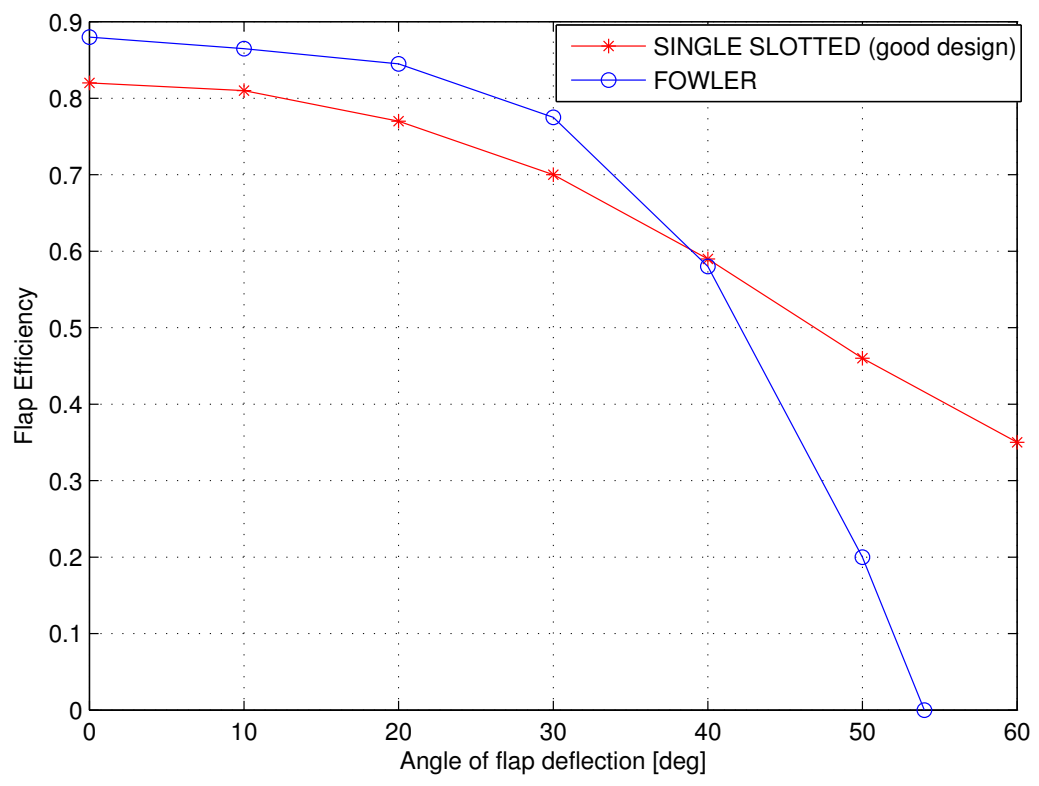

Figure 6.9. Single slotted flap and Fowler flap efficiency.

\section{Double slotted flap}

Double slotted flap is more complicated than single slotted flap and it is used to have an efficient trailing edge device also with high deflections (40 degrees or more). The double slotted flap can be considered as a single slotted flap with a turning vane in the slot to recover the flow turning effectiveness. The two main categories are the double slotted flap with fixed vane (Fig. 6.10) and with variable geometry (Fig. 6.11).

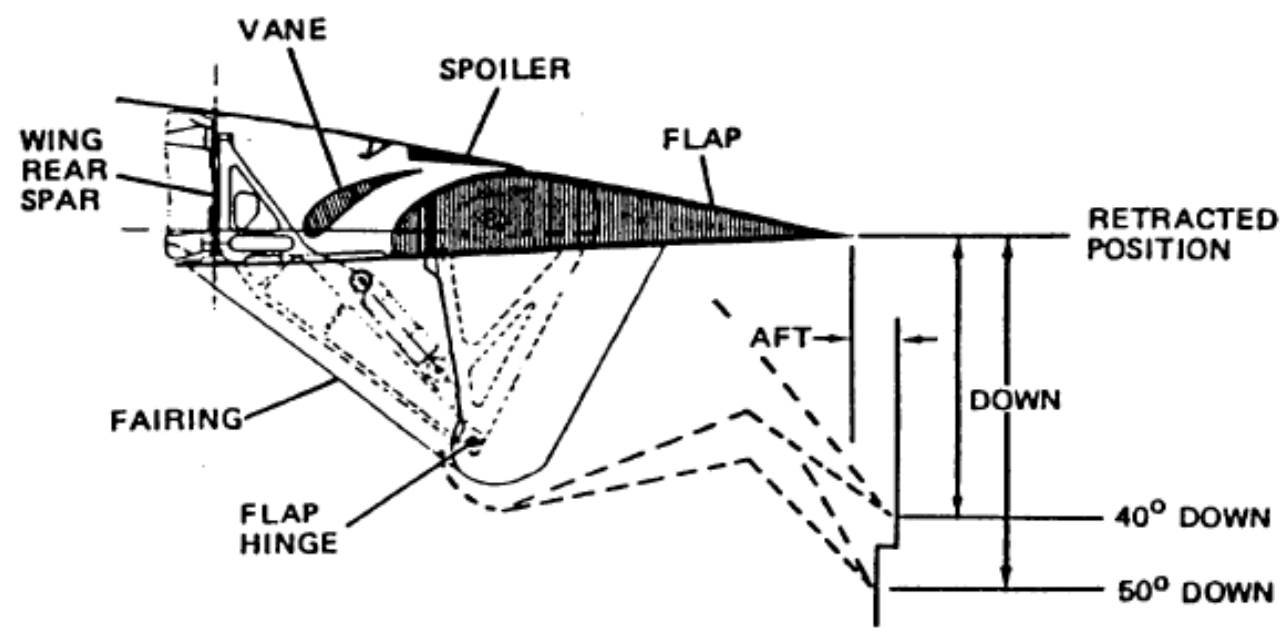

Figure 6.10. Double slotted flap with fixed vane. 


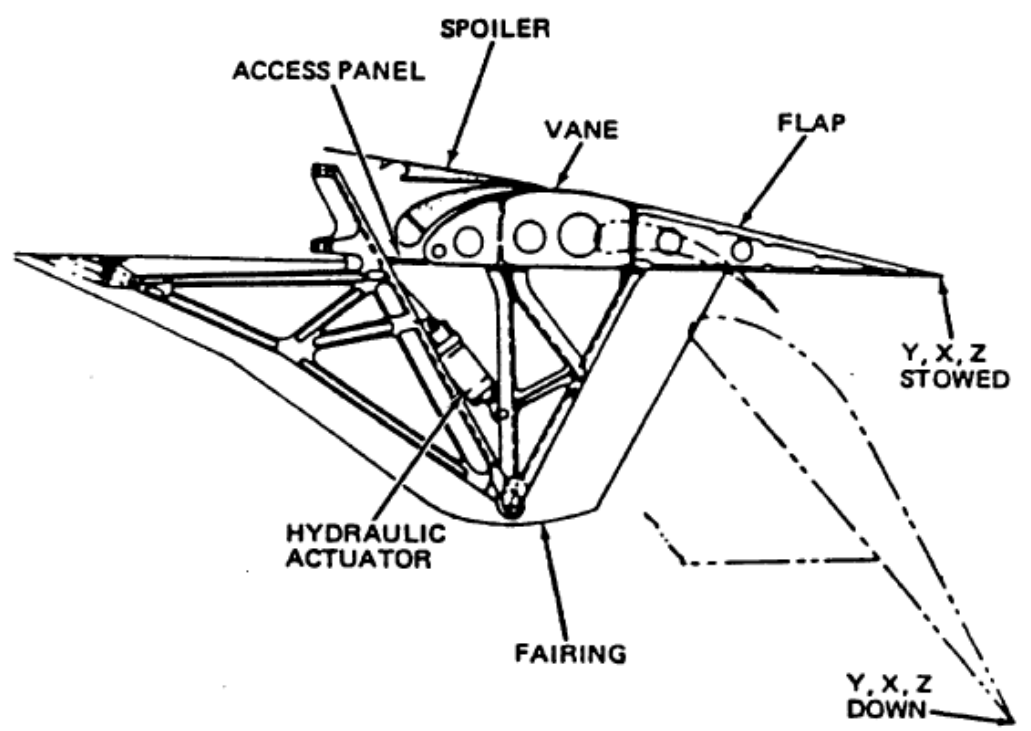

Figure 6.11. Double slotted flap with variable geometry.

The efficiency for the double slotted flap is reported in Fig. 6.12. The difference between a fixed vane and a variable geometry flap is not the efficiency but the chord extension, as it will be explained later on.

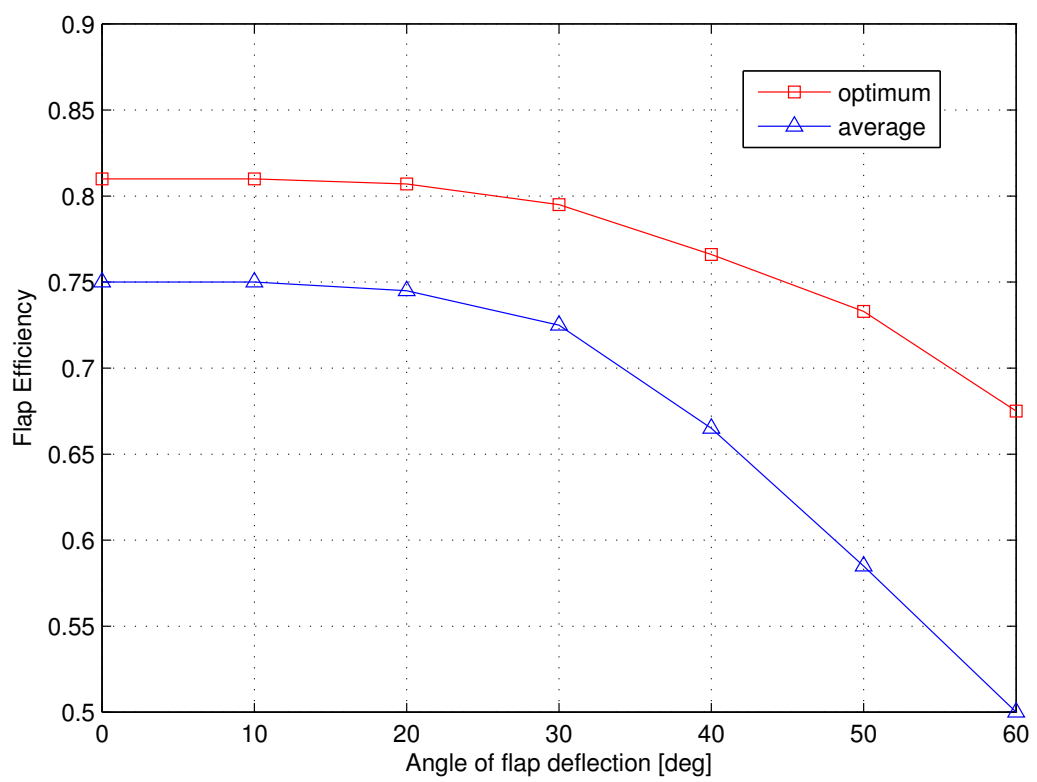

Figure 6.12. Double slotted flap efficiency. 
By using a single slotted flap, a fowler flap or a double slotted flap, the chord increases; hence, this leads to another beneficial aspect because $\Delta c_{l 0}$ can increase and this fact is related to an increasing $\Delta c_{\text {lmax }}$, as it will be explained. Let us call $c^{\prime}$ the extended chord and $c_{l}^{\prime}$ the lift coefficient relative to $c^{\prime}$. The following relation exists:

$$
c_{l}=c_{l}^{\prime}\left(\frac{c^{\prime}}{c}\right)
$$

It is assumed that the lift coefficient at null angle of attack for the clean configuration is the same; thus $c_{l 0}=c_{l 0}^{\prime}$. When flaps are deflected eq. 6.24 holds and, by rearranging its members, eq. 6.25 is obtained.

$$
\begin{aligned}
& \left(c_{l 0}+\Delta c_{l 0}\right)=\left(c_{l 0}^{\prime}+\Delta c_{l 0}^{\prime}\right)\left(\frac{c^{\prime}}{c}\right) \\
& \Delta c_{l 0}=\Delta c_{l 0}^{\prime}\left(\frac{c^{\prime}}{c}\right)+c_{l 0}\left(\frac{c^{\prime}}{c}-1\right)
\end{aligned}
$$

In the latter equation the value of $\Delta c_{l 0}^{\prime}$ is computed according to eq. 6.22 , but the new value of the chord $\left(c^{\prime}\right)$ has to be considered to compute $\alpha_{\delta}^{\prime}$.

Then, the value of the parameter $c^{\prime} / c$ is very important because it affects the flaps design, but in a preliminary design process it is unknown. In this case the graph of Fig. 6.13 can be used, where $\Delta c=c^{\prime}-c$ : this graph is very general and it summarizes informations for single slotted flaps, fowler flaps and double slotted flaps.

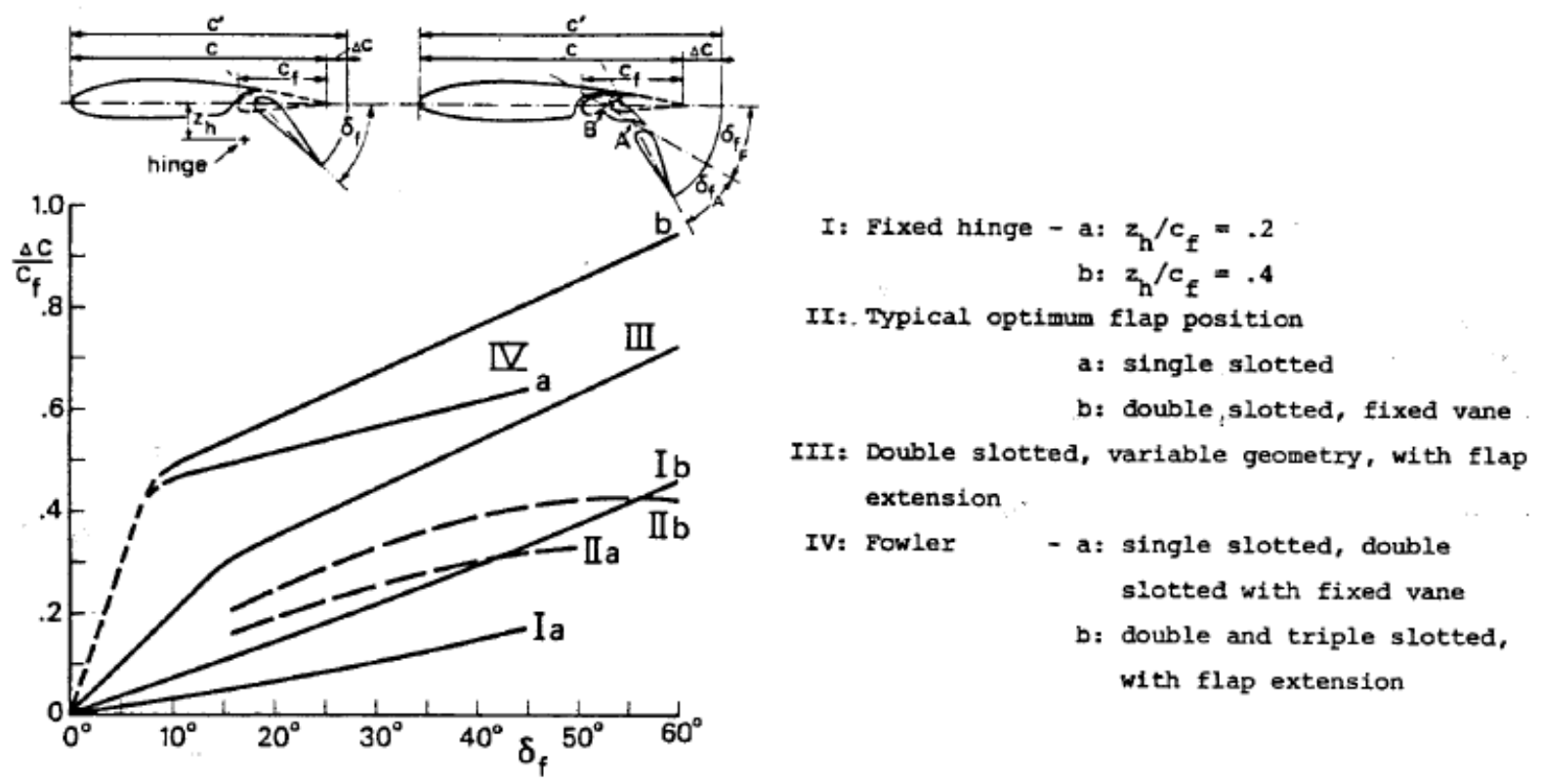

Figure 6.13. Chord extension. 
The double slotted flap with fixed vane has a maximum useful deflection up to $50-55$ degrees and its effects, in terms of $\Delta c_{l 0}$ and of $\Delta c_{l \max }$, can be computed as if it were a single slotted flap, using the correct value of $\eta_{\delta}$. The double slotted flap with variable geometry has to be considered in a different way: the flap is composed of a forward flap and a backward flap; typically, the forward flap is deflected at a maximum angle of 30 degrees, while the backward flap is deflected about 30-40 degrees relative to the forward flap. Thus, the total lift increment at zero angle of attack is given by two contributions (refer to eq. 6.26).

$$
\Delta c_{l 0}=\Delta_{1} c_{l 0}+\Delta_{2} c_{l 0}
$$

Each contribution of eq. 6.26 can be computed by using the formula 6.22 .

The flow conditions at the maximum lift coefficient depend on the viscous effects, so the prediction of the $\Delta c_{l m a x}$ is based on empirical methods. For airfoil sections with sharp noses the stall is determined by the flow conditions near the nose, and $\Delta c_{\text {max }}$ can be computed by the thin airfoil theory. In the present case, the airfoil is thick, so it displays a separation associated with the pressure gradient at the rear part of the airfoil. In this situation the trailing edge stall can be delayed by deflection of an effective trailing edge flap. The parameter that controls this phenomenon is the leading edge sharpness parameter, $\Delta y$, defined as follows:

$$
\frac{\Delta y}{c}=\frac{y_{6 \%}-y_{0.15 \%}}{c} * 100 \%
$$

For $\Delta y$ up to $1.2-1.5 \%$, the maximum lift is associated with the leading edge stall: there is a long bubble on the airfoil. For larger values of $\Delta y, c_{l m a x}$ increases proportionally with $\Delta y$; there is a short bubble on the airfoil up to a point (a certain value of $\Delta y$ ) where trailing edge stall begins to dominate. Consider now an airfoil with a trailing edge stall (as the NACA 642415) when the flap is retracted. It has been shown that small flap deflections clean up the flow near the trailing edge in such a way that the resulting maximum lift increment is of the same order of magnitude as the lift increment at small angles of attack. At a certain flap angle the load induced by the airfoil nose will increase up to a point at which leading edge stall will occur first. Then the criterion to predict the maximum lifting coefficient for considered flap (but not for the split flap) is simply the following: compute the maximum lift coefficient (it is based on the extended chord) using eq. 6.28 , where $R_{c}$ is the Reynolds number based on the original chord length, and eq. 6.29. Take the minimum of them.

$$
\begin{gathered}
c_{l \max }^{\prime}=0.533 \Delta y\left(\frac{R_{c}}{3 * 10^{6}}\right)^{0.08}+0.5\left(c_{l 0}+\Delta c_{l 0}^{\prime}\right) \\
c_{l \max }^{\prime}=\left(c_{\text {max }}\right)_{\delta=0}+\Delta c_{l 0}^{\prime}
\end{gathered}
$$

Convert the selected value to the not extended chord by using eq. 6.30 .

$$
c_{\operatorname{lmax}}=\left(\frac{c^{\prime}}{c}\right) c_{l \max }^{\prime}
$$




\subsection{Results}

In this section the results of the low speed procedure are shown. Different kinds of configurations are considered in order to show that the PrandtlPlane ${ }^{\circledR}$ freighter, provided of a third lifting surface, can satisfy the low speed constraint with many arrangements of the wing system. All the examined configurations have been already analysed in the cruise condition: the aerodynamic efficiency in high speed condition is reported in order to have a global evaluation of each configuration. For every aircraft a briefly geometric description is reported, together with aerodynamic derivatives due to the high lift devices.

\subsubsection{The main design quantities}

The main parameters and design choices are reported below:

1. The front wing devices are:

- Trailing edge double slotted flaps;

- Leading edge slats.

2. The rear wing devices are:

- Trailing edge single slotted flaps;

- Leading edge absent.

3. The elevator is modelled as a plain flap;

4. The flap chord is the $30 \%$ of the local chord;

5. The slat chord is the $15 \%$ of the local chord;

6. The gain deflection between front and rear flap is set to 1 ;

The gain deflection $K$ is defined as follows:

$$
K=\frac{\delta_{f_{\text {rear }}}}{\delta_{f_{\text {front }}}}
$$

Firstly, two similar configurations will be analysed; the auxiliary wing is a canard for both of them, but the box wing span is different: $75 \mathrm{~m}$ for the first, $80 \mathrm{~m}$ for the second. 


\section{Test075}

$$
\begin{gathered}
E_{\text {cruise }}=21.25 \\
b=75 \mathrm{~m} \\
S=1182.2 \mathrm{~m}^{2}
\end{gathered}
$$

\begin{tabular}{ccc}
\hline Type & Chord $[\mathrm{m}]$ & Surface $\left[\mathrm{m}^{2}\right]$ \\
\hline Front Wing & {$[13 ; 9.87 ; 4]$} & 647.8 \\
\hline Rear Wing & {$[6.1 ; 6.1 ; 4]$} & 422.6 \\
\hline Auxiliary Wing & {$[4.6 ; 4.4]$} & 111.8 \\
\hline \multicolumn{2}{c}{ Table 6.1. test5trisb75Curr0 - Geometry }
\end{tabular}

Table 6.1. test5trisb75Curr0 - Geometry

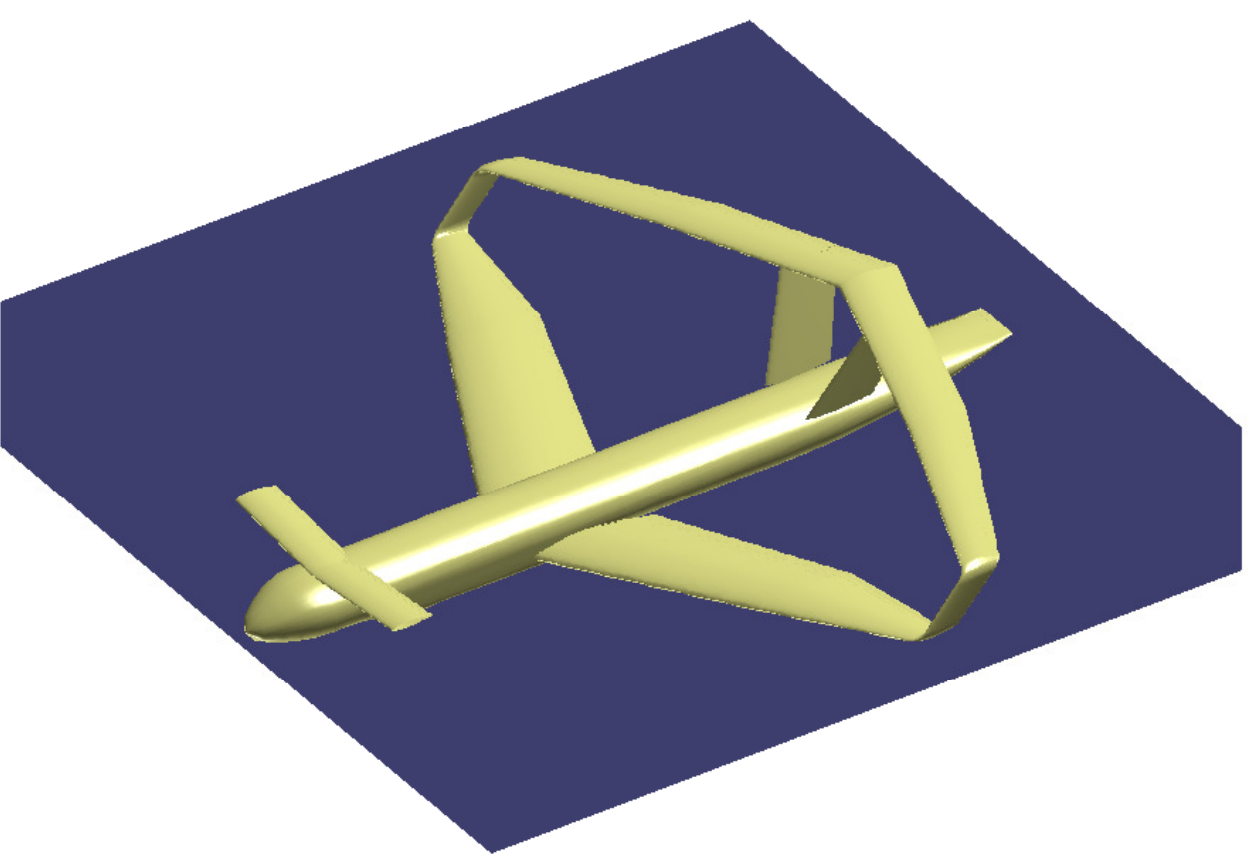

Figure 6.14. test5trisb75Curr0. 


\begin{tabular}{cccc}
\hline$\alpha[\mathrm{deg}]$ & $\delta_{f}[\mathrm{deg}]$ & $\delta_{e}[\mathrm{deg}]$ & $\delta_{s}[\mathrm{deg}]$ \\
\hline 6.0 & 22.9 & 16.8 & 22.5 \\
\hline$\left(C_{L}\right)_{\text {nec }}$ & $\left(C_{L}\right)_{\text {tot }}$ & $\epsilon[\%]$ & $\left(C_{M}\right)_{\text {tot }}$ \\
\hline 1.7034 & 1.6838 & 1.168 & 0.008 \\
\hline$C_{L \alpha}\left[\mathrm{rad}^{-1}\right]$ & $C_{L \delta f}\left[\mathrm{deg}^{-1}\right]$ & $C_{L \delta e}\left[\mathrm{deg}^{-1}\right]$ & $C_{L 0}$ \\
\hline 4.050$]$ & 0.038 & -0.003 & 0.405 \\
\hline$C_{M \alpha}\left[\mathrm{rad}^{-1}\right]$ & $C_{M \delta f}\left[\mathrm{deg}^{-1}\right]$ & $C_{M \delta e}\left[\mathrm{deg}^{-1}\right]$ & $C_{M 0}$ \\
\hline-0.035 & -0.016 & 0.022 & 0.030 \\
\hline
\end{tabular}

Table 6.2. test5trisb75Curr0 - Low Speed Analysis

As it can be inferred from Tab. 6.2, the angle of attack at landing is 6 degrees and the difference between the necessary $C_{L}$ and the available $C_{L}$ is less than $1.2 \%$ (the value of $\epsilon$ in the table). The global $C_{M}$ is very close to 0 , but not exactly null because it is determined by means an iterative procedure, as it has been explained in section 6.2.1. The disposition of all lifting surfaces, high lift devices and control surfaces is shown in Fig. 6.15. This configuration meets the low speed conditions because the trim is respected and the elevator angle does not present saturation problems.

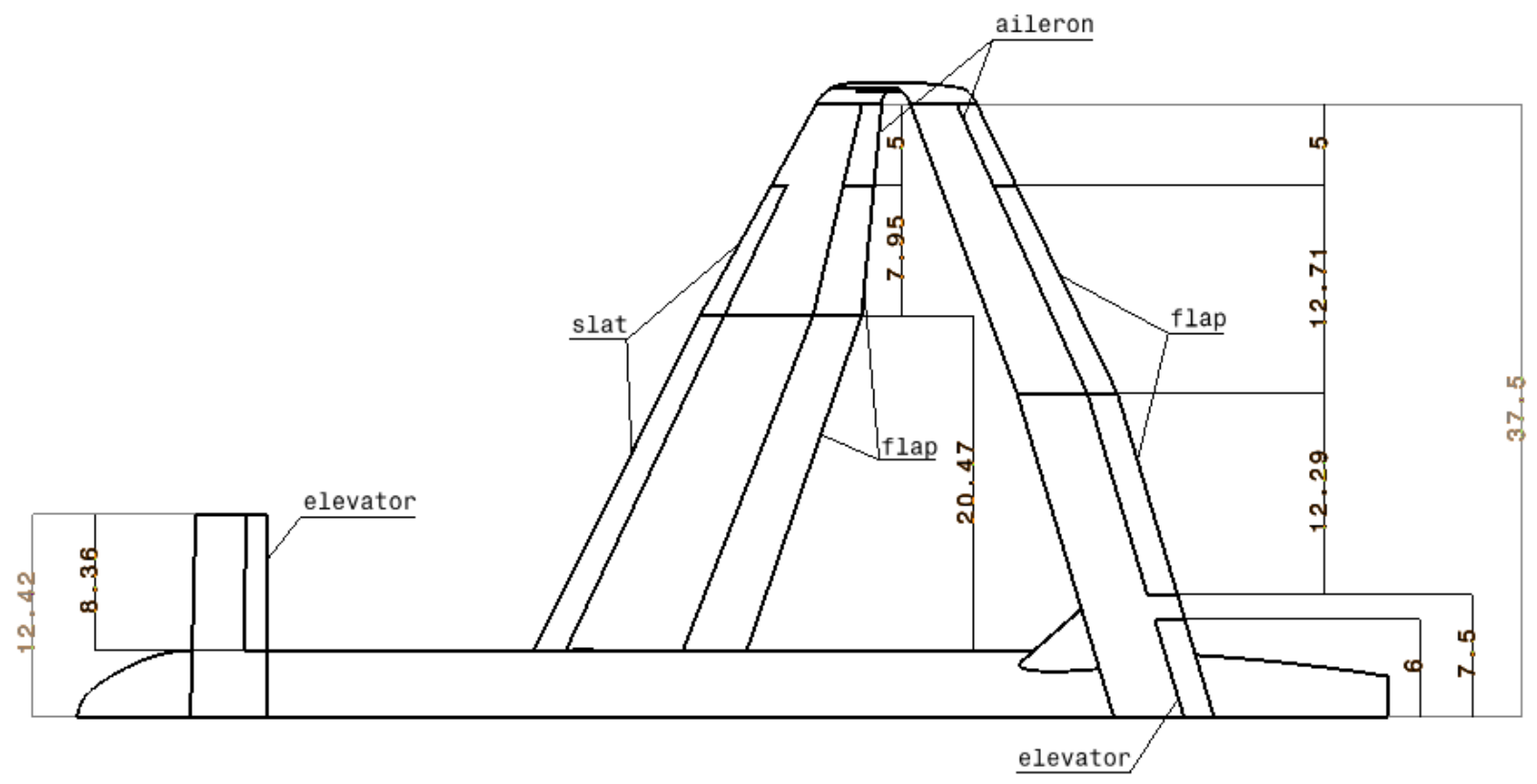

Figure 6.15. test5trisb75Curr0 - High lift devices. 


\begin{tabular}{ccccc}
\hline Type & $\left(c_{l}\right)_{\max }$ available & $\left(c_{l}\right)_{\max }$ necessary & $y$ at $\left(c_{l}\right)_{\max }[m]$ & \% Lift \\
\hline Front Wing & 3.58 & 3.14 & 31.35 & 71.0 \\
\hline Rear Wing & 2.74 & 1.63 & 18.15 & 20.0 \\
\hline Auxiliary Wing & 1.20 & 1.91 & 5.55 & 9.0 \\
\hline
\end{tabular}

Table 6.3. test5trisb75Curr0 - $\left(c_{l}\right)_{\max }$

Tab. 6.3 summarizes the comparison between the required $\left(c_{l}\right)_{\max }$ on each wing span, due to the trim in low speed condition, and the $\left(c_{l}\right)_{\max }$ that is provided by the high lift devices and it is computed as shown in the previous sections. The auxiliary wing seems to stall because the local $\left(c_{l}\right)_{\max }$ is higher than 1.2; however the elevator can act as a plain flap, so the $\left(c_{l}\right)_{\max }$ can be increased of 0.8 or more, as it can be inferred by [8]. Again, the present configuration satisfy all the low speed constraints. Fig. 6.16 shows the lifting distribution on the three wings and the highest values of the $c_{l}$ are emphasized.

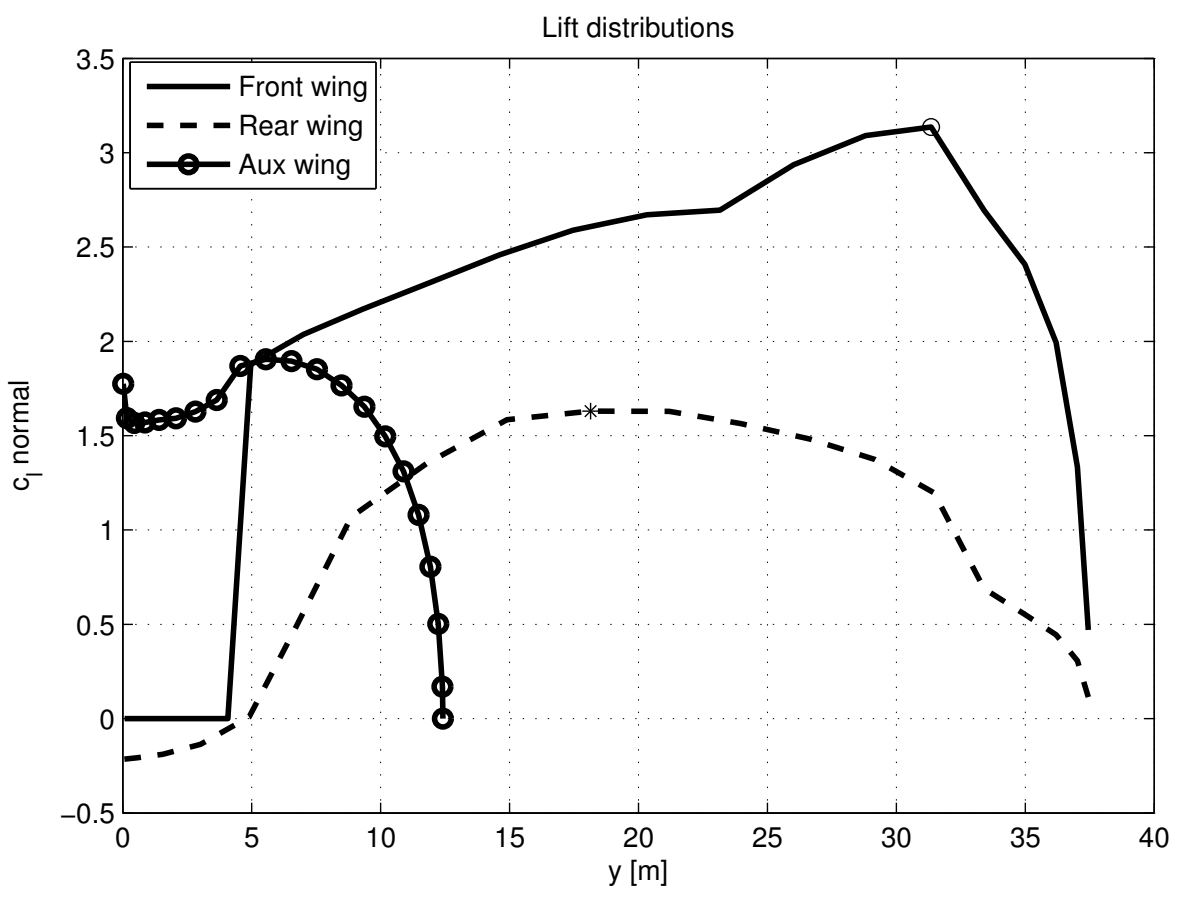

Figure 6.16. test5trisb75Curr0 - Lifting distributions. 


\section{Test080}

$$
\begin{gathered}
E_{\text {cruise }}=22.67 \\
b=80 \mathrm{~m} \\
S=1092.1 \mathrm{~m}^{2}
\end{gathered}
$$

\begin{tabular}{ccc}
\hline Type & Chord $[\mathrm{m}]$ & Surface $\left[\mathrm{m}^{2}\right]$ \\
\hline Front Wing & {$[11.2 ; 6.5 ; 4.0]$} & 524.7 \\
\hline Rear Wing & {$[7.6 ; 5.6 ; 4.0]$} & 450.8 \\
\hline Auxiliary Wing & {$[6.0 ; 6.0]$} & 116.6 \\
\hline
\end{tabular}

Table 6.4. test5trisCurr0 - Geometry

The present analysis has been performed to emphasize that the canard configuration is a good solution both for both the cruise condition (refer to Chapter 5) and for low speed condition: in fact, the configuration is not influenced by the wing span because the two analysed configurations produce similar results. Both the configurations Test075 and Test080 are designed with double slotted flaps on the front wing and single slotted flaps on the rear wing. Thus, it can be noticed that the front wing needs efficient and optimized high lift devices to satisfy the low speed constraint.

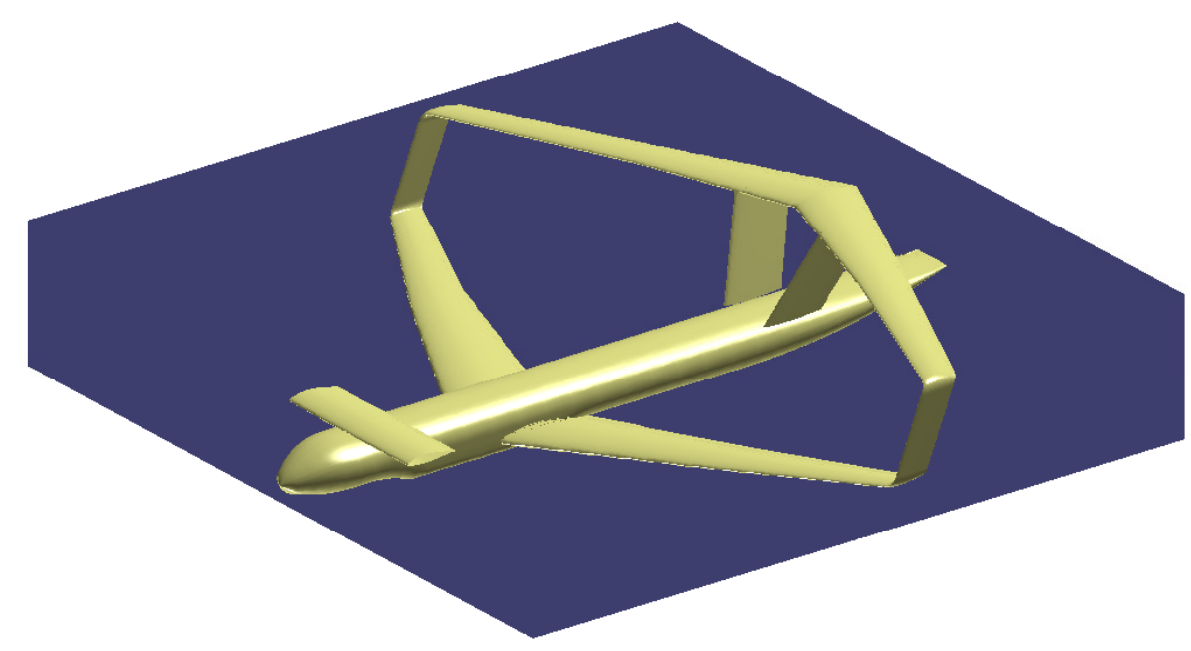

Figure 6.17. test5trisCurr0. 
Chapter 6. Low Speed Design

\begin{tabular}{cccc}
\hline$\alpha[\mathrm{deg}]$ & $\delta_{f}[\mathrm{deg}]$ & $\delta_{e}[\mathrm{deg}]$ & $\delta_{s}[\mathrm{deg}]$ \\
\hline 8.0 & 21.7 & 17.5 & 22.5 \\
\hline$\left(C_{L}\right)_{\text {nec }}$ & $\left(C_{L}\right)_{t o t}$ & $\epsilon[\%]$ & $\left(C_{M}\right)_{t o t}$ \\
\hline 1.8440 & 1.8464 & 0.128 & 0.004 \\
\hline$C_{L \alpha}\left[\mathrm{rad}^{-1}\right]$ & $C_{L \delta f}\left[\mathrm{deg}^{-1}\right]$ & $C_{L \delta e}\left[\mathrm{deg}^{-1}\right]$ & $C_{L 0}$ \\
\hline 4.191$]$ & 0.037 & -0.003 & 0.458 \\
\hline$C_{M \alpha}\left[\mathrm{rad}^{-1}\right]$ & $C_{M \delta f}\left[\mathrm{deg}^{-1}\right]$ & $C_{M \delta e}\left[\mathrm{deg}^{-1}\right]$ & $C_{M 0}$ \\
\hline-0.830 & -0.012 & 0.023 & -0.048 \\
\hline
\end{tabular}

Table 6.5. test5trisCurr0 - Low Speed Analysis

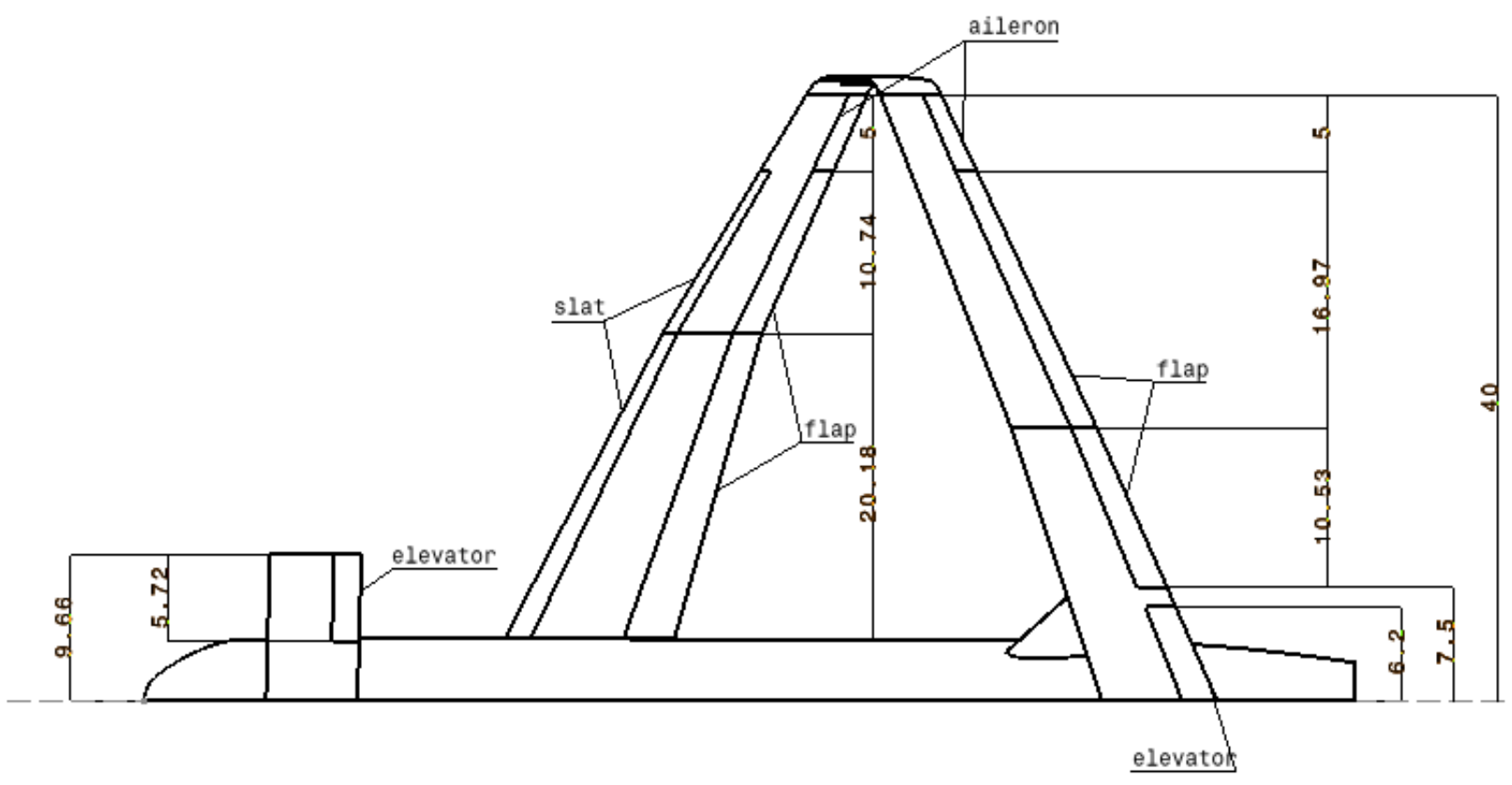

Figure 6.18. test5trisCurr0 - High lift devices.

\begin{tabular}{ccccc}
\hline Type & $\left(c_{l}\right)_{\max }$ available & $\left(c_{l}\right)_{\max }$ necessary & $y$ at $\left(c_{l}\right)_{\max }[m]$ & \% Lift \\
\hline Front Wing & 3.48 & 3.43 & 25.76 & 61.4 \\
\hline Rear Wing & 2.66 & 2.26 & 19.56 & 30.4 \\
\hline Auxiliary Wing & 1.20 & 1.65 & 4.45 & 8.2 \\
\hline
\end{tabular}

Table 6.6. test5trisCurr0 - $\left(c_{l}\right)_{\max }$ 
It is important to remark that the $\left(c_{l}\right)_{\max }$ of the auxiliary wing is not a real problem because, in any case, the elevator can give a sensitive contribution to the maximum $\left(c_{l}\right)$ that the auxiliary develops.

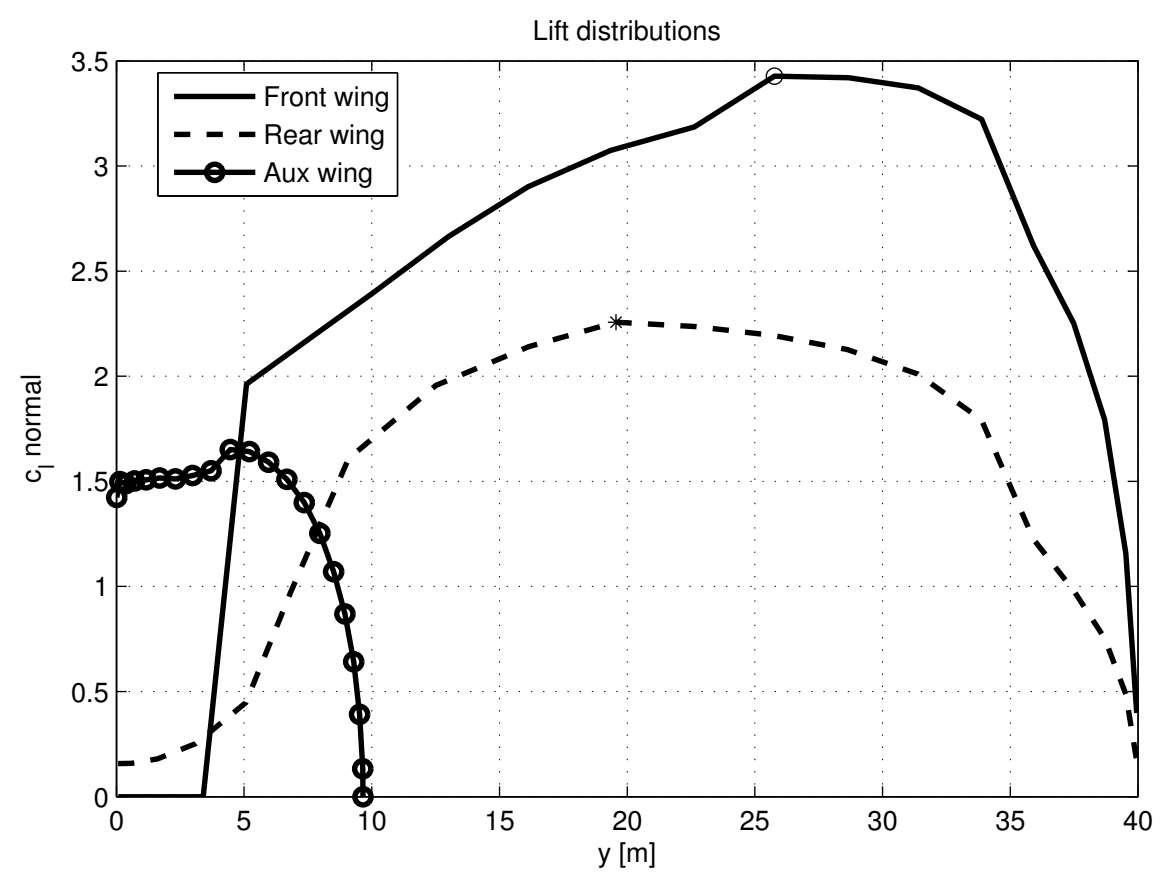

Figure 6.19. test5trisCurr0 - Lifting distributions.

Now, a very interesting observation can be formulated on the role of the auxiliary wing in low speed conditions: an elevator is installed on the auxiliary wing and it acts in opposition of phase with the elevator of the rear wing, so the elevator tends to pull up the aircraft and to contrast the increased pitching moment due to the flaps deflection. It can be shown that a configuration with inefficient elevators can not meet the landing conditions: if the elevator is placed too close to the centre of gravity, the resultant of the aerodynamic force is too near to it, hence, the pull-up moment is not high enough and the saturation of the elevator command occurs. The following analysis has been performed in order to verify the previous statements. 


\section{Test8}

$$
\begin{gathered}
E_{\text {cruise }}=22.20 \\
b=80 \mathrm{~m} \\
S=1075.2 \mathrm{~m}^{2}
\end{gathered}
$$

\begin{tabular}{ccc}
\hline Type & Chord $[\mathrm{m}]$ & Surface $\left[\mathrm{m}^{2}\right]$ \\
\hline Front Wing & {$[11.0 ; 4.1 ; 4.0]$} & 481.2 \\
\hline Rear Wing & {$[8.8 ; 4.0 ; 4.0]$} & 382.0 \\
\hline Auxiliary Wing & {$[6.3 ; 4.6]$} & 212.0 \\
\hline
\end{tabular}

Table 6.7. test5trisCurr8 - Geometry

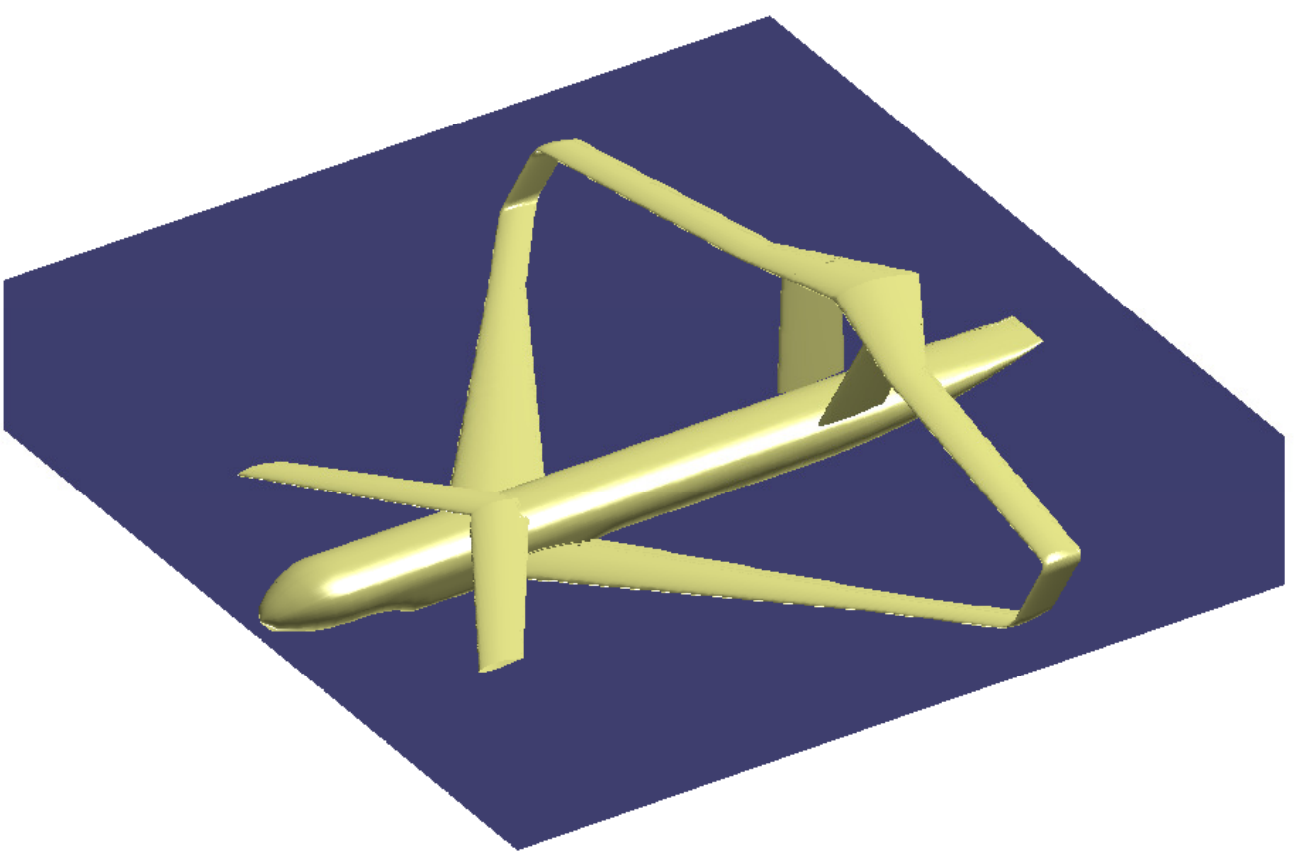

Figure 6.20. test5trisCurr8. 


\begin{tabular}{cccc}
\hline$\alpha[\mathrm{deg}]$ & $\delta_{f}[\mathrm{deg}]$ & $\delta_{e}[\mathrm{deg}]$ & $\delta_{s}[\mathrm{deg}]$ \\
\hline 4.0 & 36.2 & 30.0 & 22.5 \\
\hline$\left(C_{L}\right)_{\text {nec }}$ & $\left(C_{L}\right)_{t o t}$ & $\epsilon[\%]$ & $\left(C_{M}\right)_{t o t}$ \\
\hline 1.8729 & 1.8269 & 2.517 & 0.014 \\
\hline$C_{L \alpha}\left[\mathrm{rad}^{-1}\right]$ & $C_{L \delta f}\left[\mathrm{deg}^{-1}\right]$ & $C_{L \delta e}\left[\mathrm{deg}^{-1}\right]$ & $C_{L 0}$ \\
\hline 3.778$]$ & 0.031 & -0.001 & 0.447 \\
\hline$C_{M \alpha}\left[\mathrm{rad}^{-1}\right]$ & $C_{M \delta f}\left[\mathrm{deg}^{-1}\right]$ & $C_{M \delta e}\left[\mathrm{deg}^{-1}\right]$ & $C_{M 0}$ \\
\hline-0.990 & -0.026 & 0.034 & -0.020 \\
\hline
\end{tabular}

Table 6.8. test5trisCurr8 - Low Speed Analysis

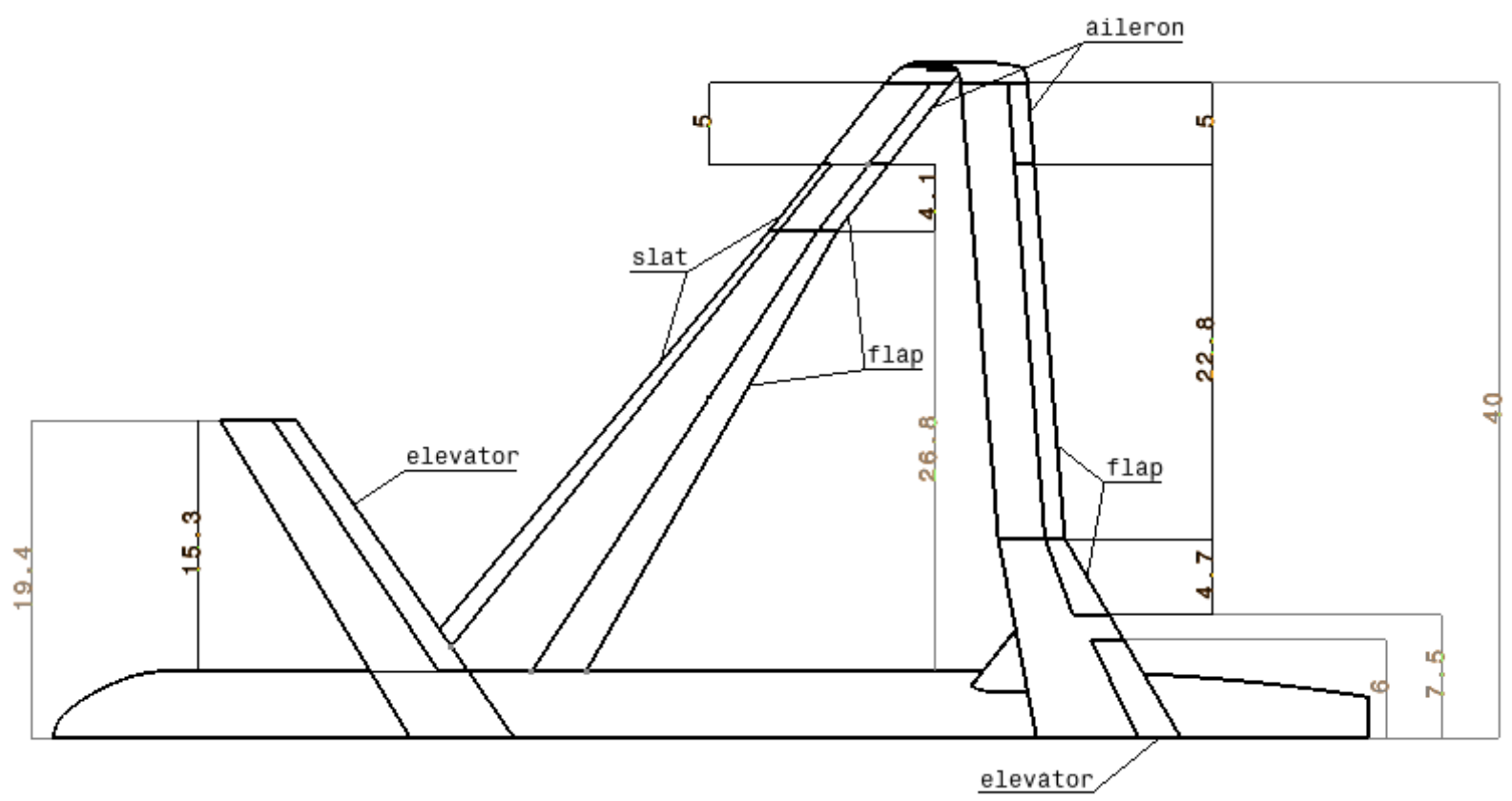

Figure 6.21. test5trisCurr8 - High lift devices.

\begin{tabular}{ccccc}
\hline Type & $\left(c_{l}\right)_{\max }$ available & $\left(c_{l}\right)_{\max }$ necessary & $y$ at $\left(c_{l}\right)_{\max }[\mathrm{m}]$ & \% Lift \\
\hline Front Wing & 4.19 & 4.95 & 29.65 & 50.6 \\
\hline Rear Wing & 3.32 & 2.79 & 25.68 & 28.6 \\
\hline Auxiliary Wing & 1.20 & 3.08 & 6.06 & 20.8 \\
\hline
\end{tabular}

Table 6.9. test5trisCurr8 - $\left(c_{l}\right)_{\max }$ 
It is evident that the elevator, in this configuration, is closer to the centre of gravity (refer to Fig. 6.21): the configuration tries to meet the trim condition by increasing the flap deflection. However, the angle of attack is very low for this configuration because the auxiliary wing undergoes stall (it is strongly loaded because it is near the centre of gravity) and this pushes to a higher flap deflection than usual. This fact has an important effect: the local $\left(c_{l}\right)_{\max }$ increases on the front wing, so the double slotted flap on the front wing is not sufficient any more (refer to Tab. 6.9). Moreover, the rear wing increases its aerodynamic load too and, thus, the pitching moment is very high. The elevator has to contrast the pitching moment by increasing the deflection $\delta_{e}$, hence the saturation is reached.

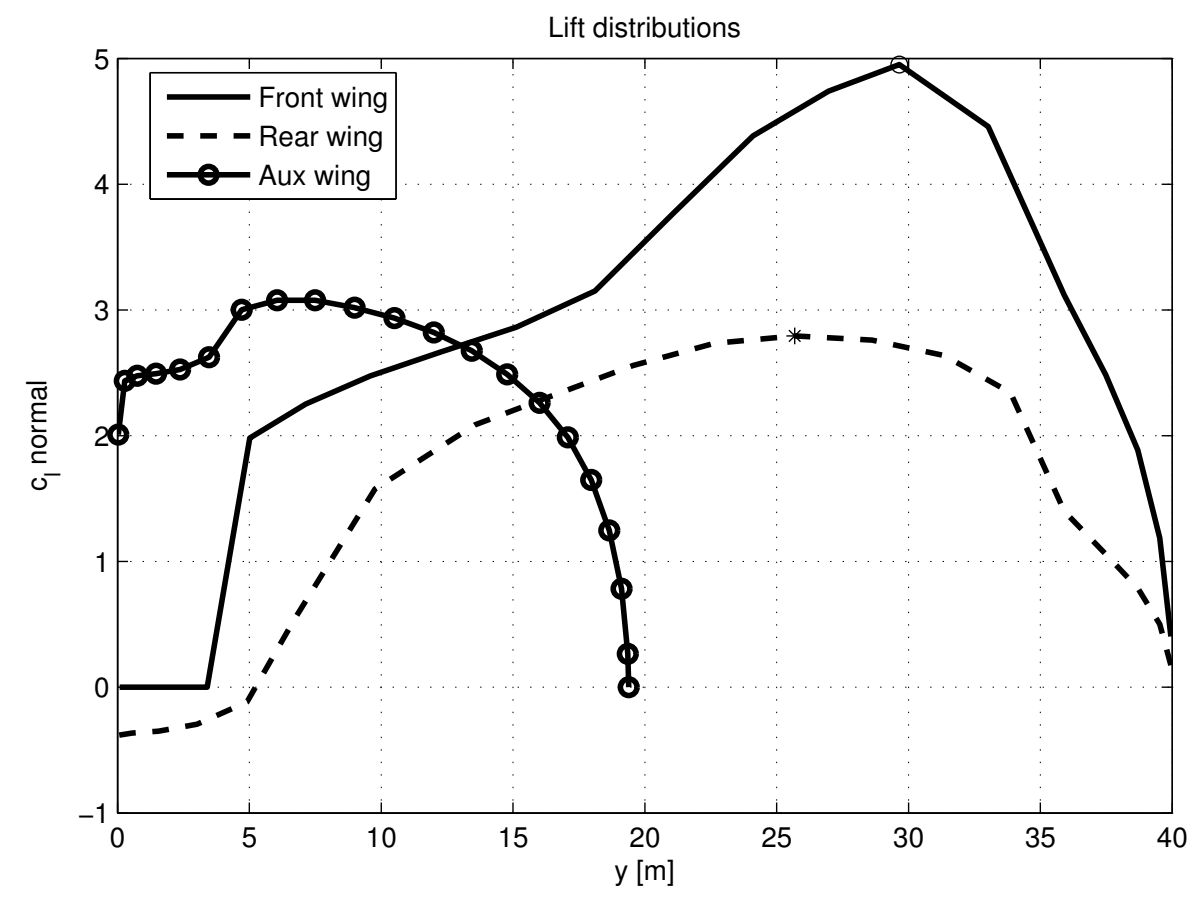

Figure 6.22. test5trisCurr8 - Lifting distributions. 


\section{Test6}

The present analysis has been performed in order to show that it is possible to meet the low speed constraint, even if the auxiliary wing moves backwards. As in the previous analyses, an elevator is installed on both the auxiliary wing and the rear wing, thus there are two elevators which work on phase to pull-up the aircraft.

$$
\begin{gathered}
E_{\text {cruise }}=23.35 \\
b=80 \mathrm{~m} \\
S=1144.0 \mathrm{~m}^{2}
\end{gathered}
$$

\begin{tabular}{ccc}
\hline Type & Chord $[\mathrm{m}]$ & Surface $\left[\mathrm{m}^{2}\right]$ \\
\hline Front Wing & {$[8.5 ; 8.3 ; 8.3]$} & 600.0 \\
\hline Rear Wing & {$[8.9 ; 5.2 ; 4.2]$} & 408.4 \\
\hline Auxiliary Wing & {$[4.2 ; 4.1]$} & 135.6 \\
\hline
\end{tabular}

Table 6.10. test5trisCurr6 - Geometry

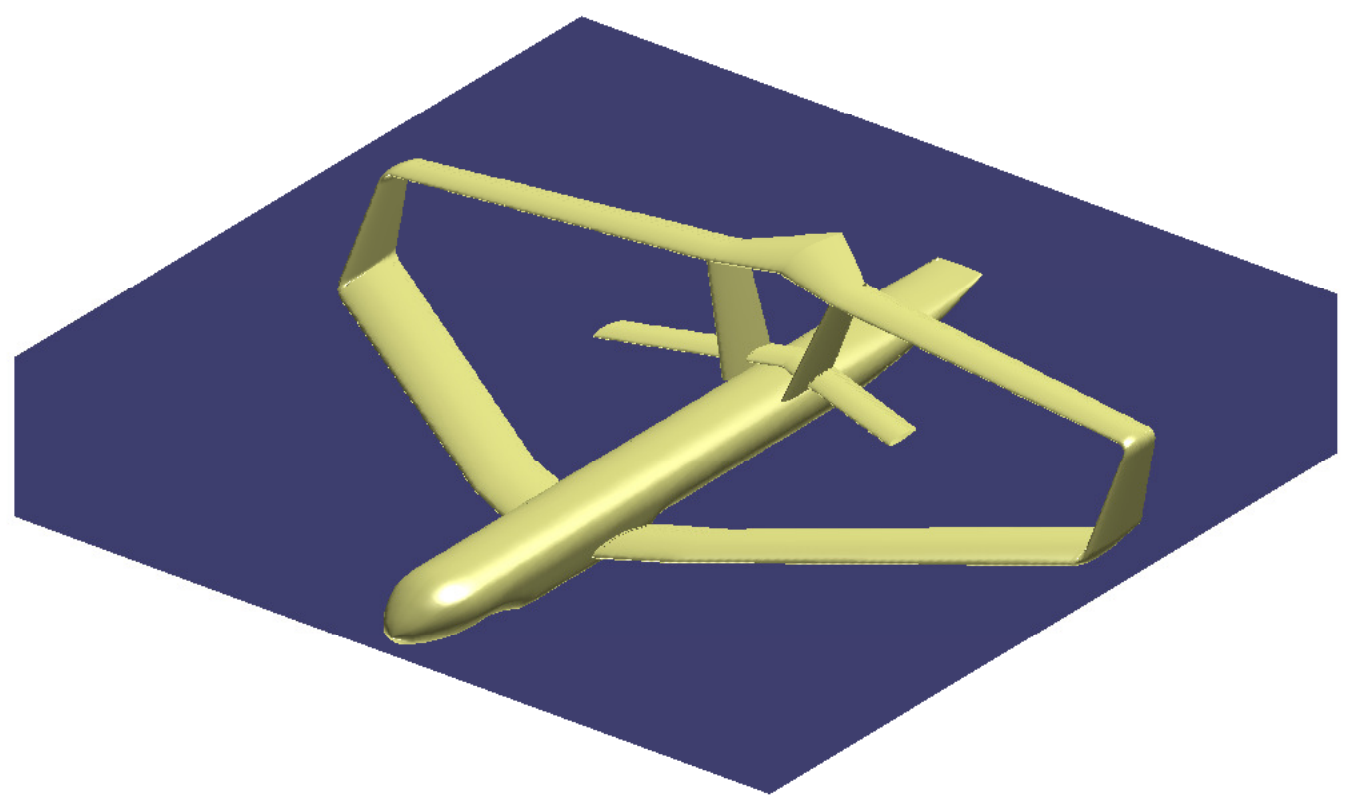

Figure 6.23. test5trisCurr6. 
Chapter 6. Low Speed Design

\begin{tabular}{cccc}
\hline$\alpha[\mathrm{deg}]$ & $\delta_{f}[\mathrm{deg}]$ & $\delta_{e}[\mathrm{deg}]$ & $\delta_{s}[\mathrm{deg}]$ \\
\hline 10.0 & 20.0 & 10.0 & 22.5 \\
\hline$\left(C_{L}\right)_{\text {nec }}$ & $\left(C_{L}\right)_{t o t}$ & $\epsilon[\%]$ & $\left(C_{M}\right)_{t o t}$ \\
\hline 1.7606 & 1.8111 & 2.789 & 0.021 \\
\hline$C_{L \alpha}\left[\mathrm{rad}^{-1}\right]$ & $C_{L \delta f}\left[\mathrm{deg}^{-1}\right]$ & $C_{L \delta e}\left[\mathrm{deg}^{-1}\right]$ & $C_{L 0}$ \\
\hline 4.230$]$ & 0.034 & -0.007 & 0.424 \\
\hline$C_{M \alpha}\left[\mathrm{rad}^{-1}\right]$ & $C_{M \delta f}\left[\mathrm{deg}^{-1}\right]$ & $C_{M \delta e}\left[\mathrm{deg}^{-1}\right]$ & $C_{M 0}$ \\
\hline-0.550 & -0.019 & 0.014 & -0.032 \\
\hline
\end{tabular}

Table 6.11. test5trisCurr6 - Low Speed Analysis
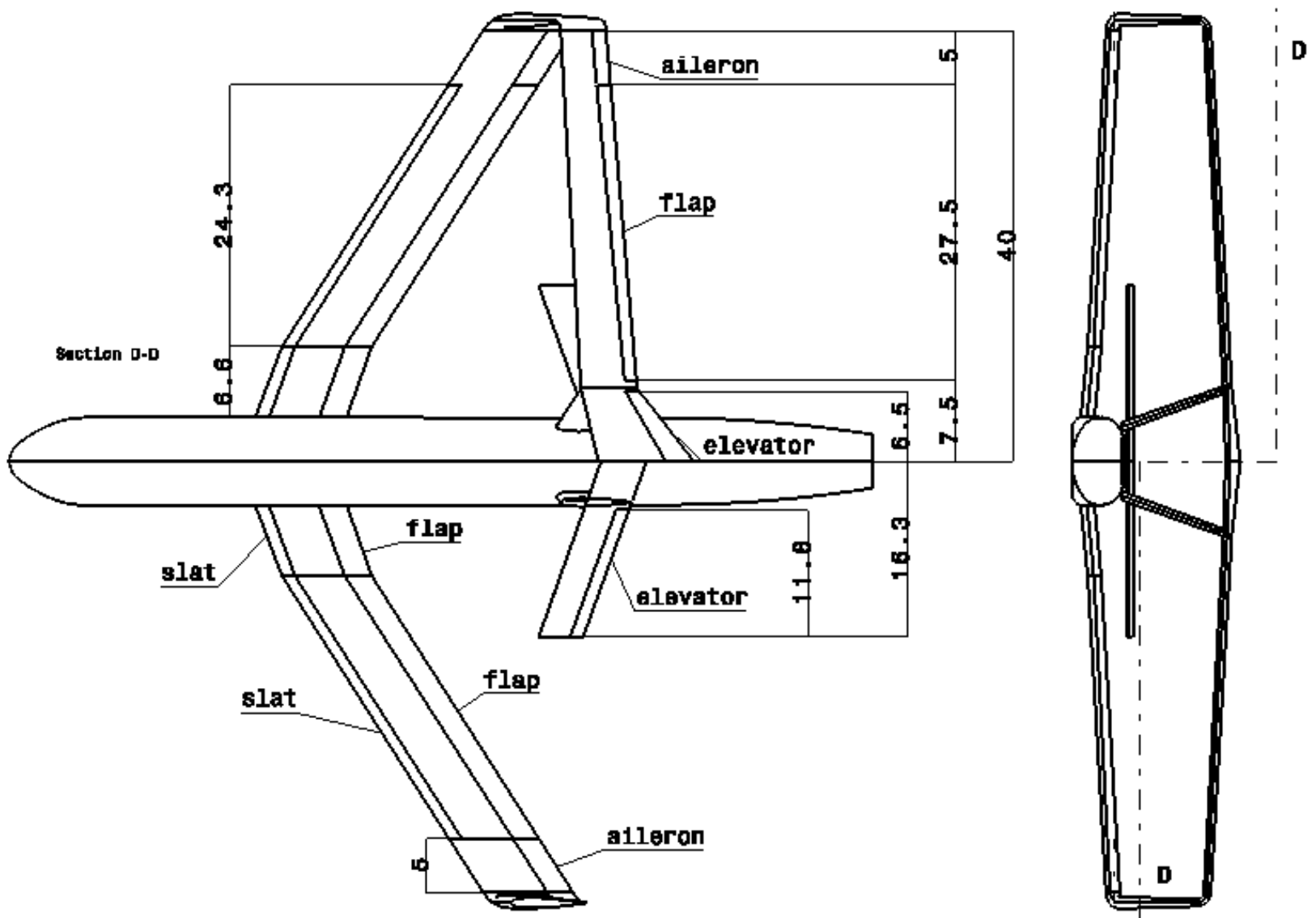

Figure 6.24. test5trisCurr6 - High lift devices. 


\begin{tabular}{ccccc}
\hline Type & $\left(c_{l}\right)_{\max }$ available & $\left(c_{l}\right)_{\max }$ necessary & $y$ at $\left(c_{l}\right)_{\max }[\mathrm{m}]$ & \% Lift \\
\hline Front Wing & 3.40 & 3.22 & 15.29 & 66.3 \\
\hline Rear Wing & 2.49 & 2.12 & 18.31 & 32.2 \\
\hline Auxiliary Wing & 1.20 & 0.98 & 0.03 & 1.5 \\
\hline
\end{tabular}

Table 6.12. test5trisCurr6 - $\left(c_{l}\right)_{\max }$

In this case the maximum angle of attack is not dominated by the stall of the auxiliary wing: in fact, the auxiliary wing undergoes the downwash of the front wing, so it tends to develop a low aerodynamic load. The low speed procedure provides $\alpha=14$ degrees as maximum angle of attack, but this choice would lead to very small flap deflections (about 10 degrees). Since double slotted flap and single slotted flap are employed, then, the value of $\alpha$ has been limited to 10 degrees and the auxiliary wing is not near to the stall condition, as in the previous configurations. This new arrangement allows efficient high lift devices to work properly and the low angle of attack helps the pilot's sight during landing.

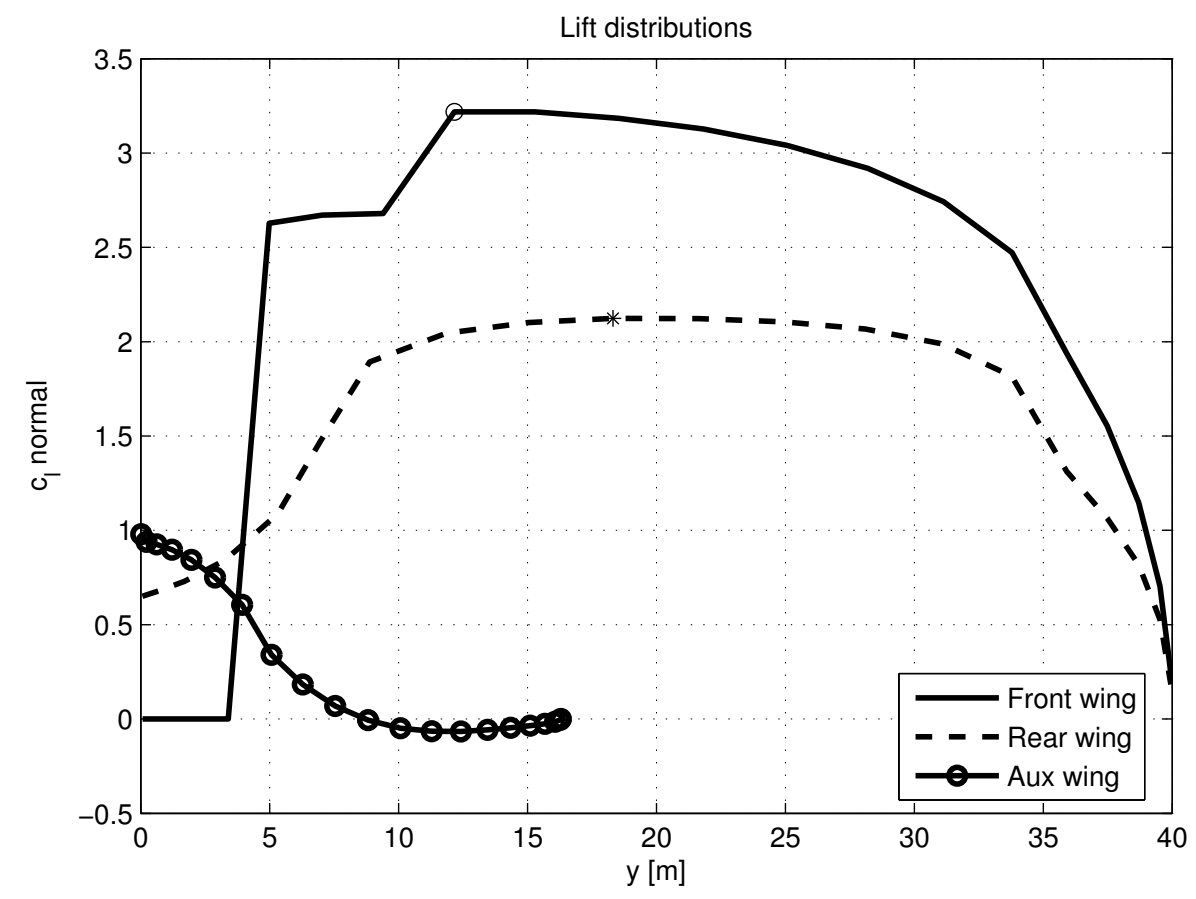

Figure 6.25. test5trisCurr6 - Lifting distributions. 


\section{Testh1118}

In this analysis the elevators do not work on phase and the auxiliary wing is positioned in the very rear part of the fuselage, acting as a conventional tail surface. Since its distance from the centre of gravity is high, an efficient solution to fulfill the low speed requirements is to install the elevators on the front wing and on the auxiliary wing: in fact, an elevator on the rear wing would be very inefficient because the rear wing is very close to the centre of gravity. In this case the rear wing holds only single slotted flaps for the most of the wing span, except the tip zone, that is dedicated to the ailerons.

$$
\begin{gathered}
E_{\text {cruise }}=23.87 \\
b=80 \mathrm{~m} \\
S=1132.8 \mathrm{~m}^{2}
\end{gathered}
$$

\begin{tabular}{ccc}
\hline Type & Chord $[m]$ & Surface $\left[\mathrm{m}^{2}\right]$ \\
\hline Front Wing & {$[7.4 ; 7.4 ; 4.5]$} & 508.8 \\
\hline Rear Wing & {$[8.6 ; 7.8 ; 4]$} & 499.5 \\
\hline Auxiliary Wing & {$[6.0 ; 5.3]$} & 124.5 \\
\hline
\end{tabular}

Table 6.13. test5trish11Curr18 - Geometry

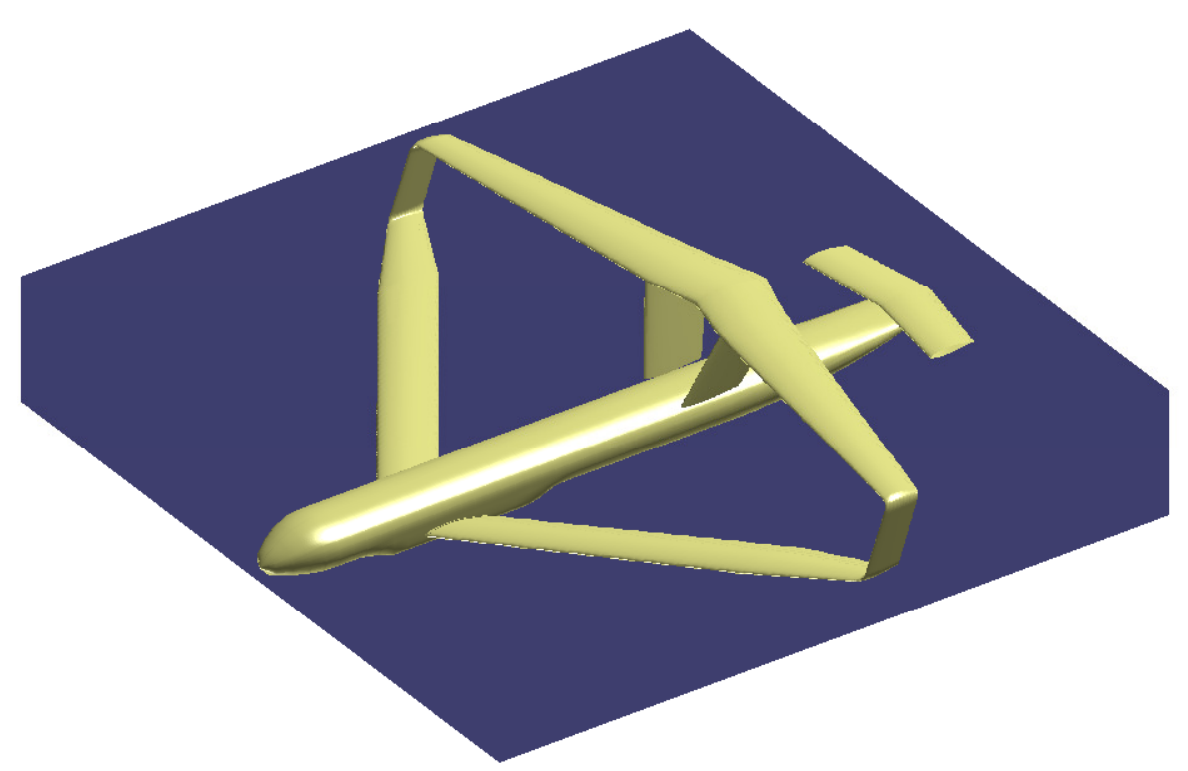

Figure 6.26. test5trish11Curr18. 


\begin{tabular}{cccc}
\hline$\alpha[\mathrm{deg}]$ & $\delta_{f}[\mathrm{deg}]$ & $\delta_{e}[\mathrm{deg}]$ & $\delta_{s}[\mathrm{deg}]$ \\
\hline 8.0 & 18.5 & 12.5 & 22.5 \\
\hline$\left(C_{L}\right)_{\text {nec }}$ & $\left(C_{L}\right)_{\text {tot }}$ & $\epsilon[\%]$ & $\left(C_{M}\right)_{\text {tot }}$ \\
\hline 1.7778 & 1.777 & 0.004 & 0.029 \\
\hline$C_{L \alpha}\left[\mathrm{rad}^{-1}\right]$ & $C_{L \delta f}\left[\mathrm{deg}^{-1}\right]$ & $C_{L \delta e}\left[\mathrm{deg}^{-1}\right]$ & $C_{L 0}$ \\
\hline 4.361$]$ & 0.035 & -0.0002 & 0.460 \\
\hline$C_{M \alpha}\left[\mathrm{rad}^{-1}\right]$ & $C_{M \delta f}\left[\mathrm{deg}^{-1}\right]$ & $C_{M \delta e}\left[\mathrm{deg}^{-1}\right]$ & $C_{M 0}$ \\
\hline-0.926 & -0.016 & 0.031 & -0.002 \\
\hline
\end{tabular}

Table 6.14. test5trish11Curr18 - Low Speed Analysis

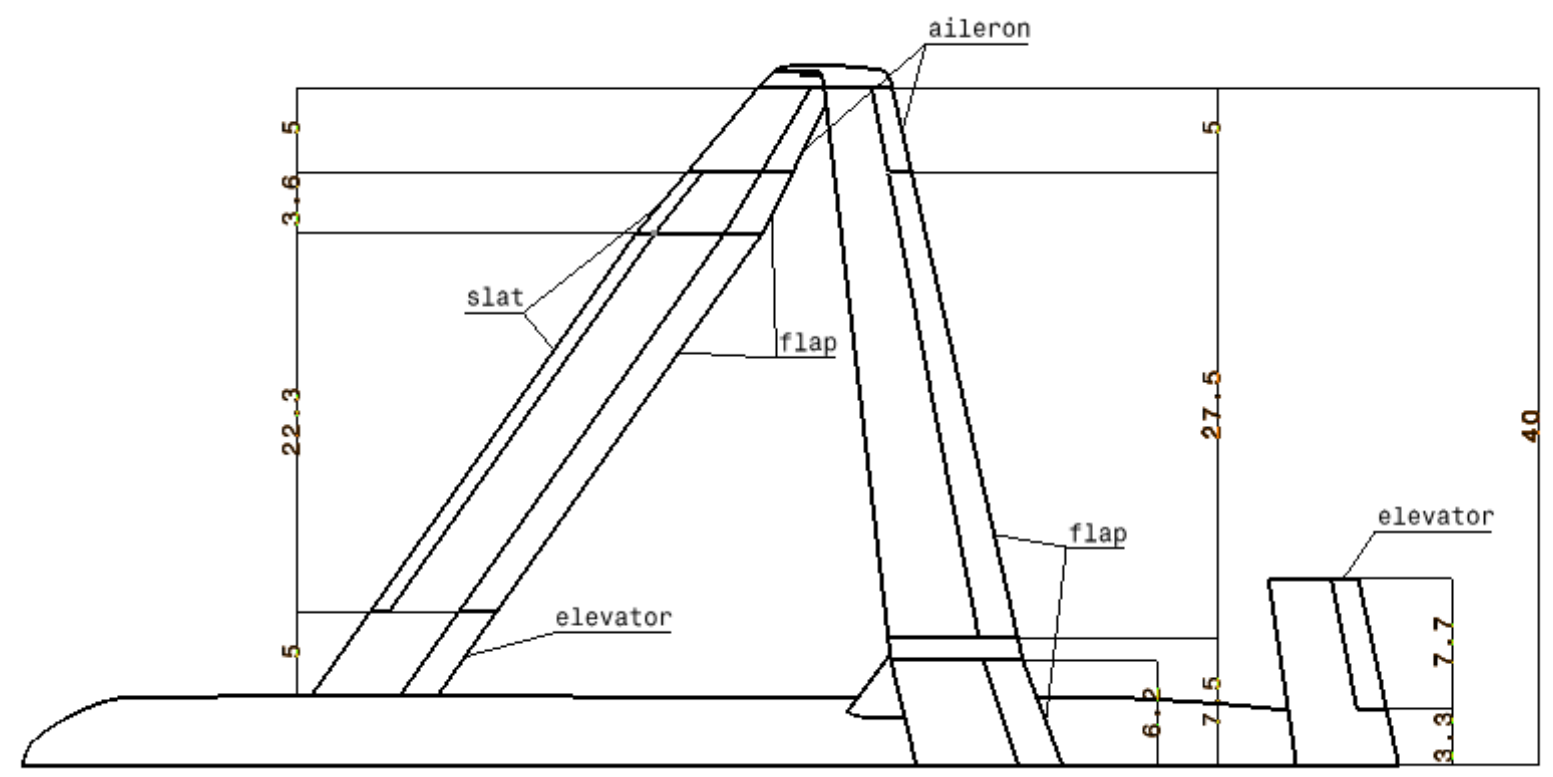

Figure 6.27. test5trish11Curr18 - High lift devices.

\begin{tabular}{ccccc}
\hline Type & $\left(c_{l}\right)_{\max }$ available & $\left(c_{l}\right)_{\max }$ necessary & $y$ at $\left(c_{l}\right)_{\max }[\mathrm{m}]$ & \% Lift \\
\hline Front Wing & 3.37 & 3.07 & 15.81 & 52.9 \\
\hline Rear Wing & 2.45 & 2.33 & 0.06 & 47.6 \\
\hline Auxiliary Wing & 1.20 & 0.19 & 0.02 & -0.5 \\
\hline
\end{tabular}

Table 6.15. test5trish11Curr18 - $\left(c_{l}\right)_{\max }$ 


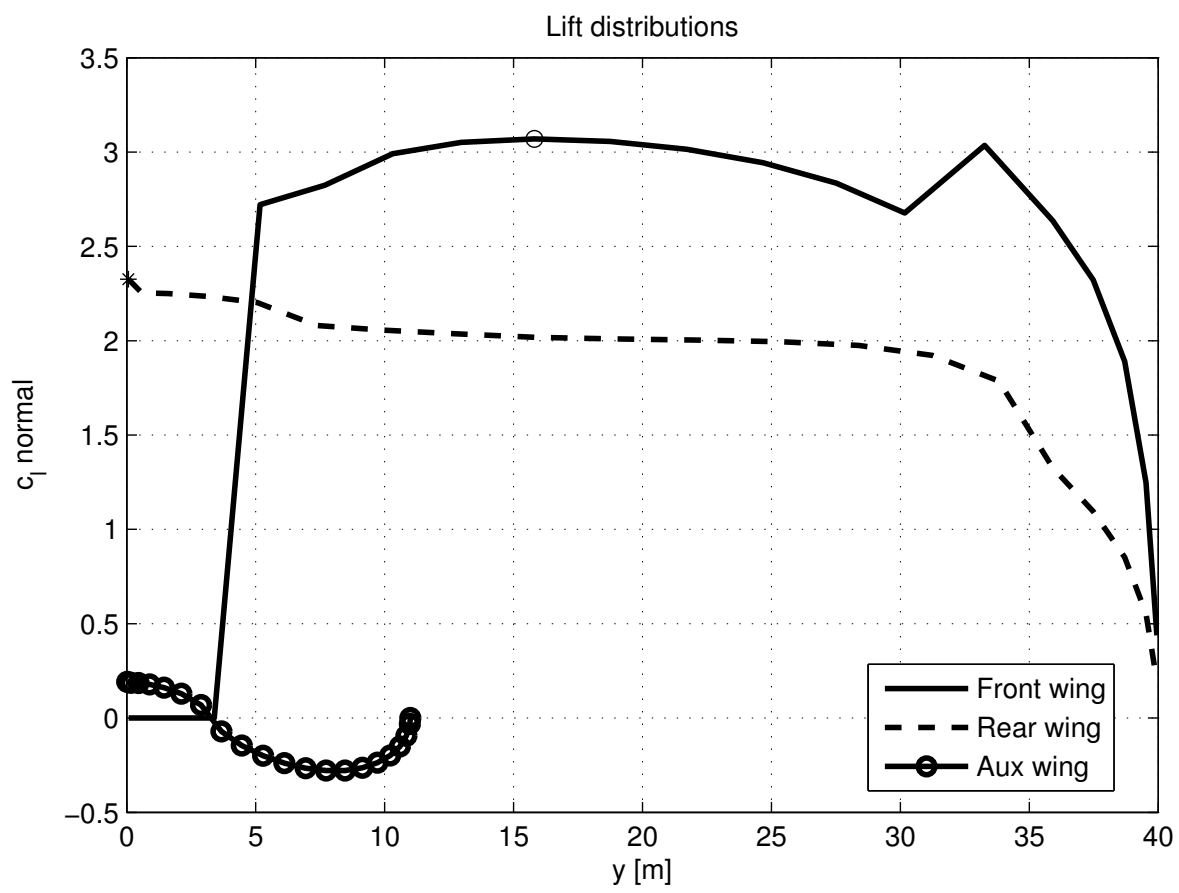

Figure 6.28. test5trish11Curr18 - Lifting distributions.

Finally, from the previous analyses, it can be deduced that the PrandtlPlane ${ }^{\circledR}$ freighter can land with many arrangements of the wing system, from a preliminary point of view. An important remark deals with the role of the auxiliary wing: it is evident how it is important to manage the pitching moment in trim operations. 



\section{Conclusions}

An optimization procedure has been set up in this thesis in order to evaluate optima wing planforms that minimize the lift-to-drag ratio. Proper constraints have been considered to take structural requirements and flight mechanics into account. In particular, the wing loading, deriving from both regulation on the landing and from structural reasons, appears the most significant parameter which affects the wing planform.

It has been verified that a high front wing loading improves the aerodynamic efficiency $E$ but, at the same time, it makes the front wing overloaded (refer to section 5.1): when the front wing loading is over $700 \mathrm{~kg} / \mathrm{m}^{2}$, the auxiliary wing is very inefficient, with a small surface and a low $C_{L}$. Thus, the fuselage has two supports, instead of three, and the bending moment in the fuselage increases.

The rear wing loading has been investigated too, and results underline that when the rear wing loading increases, the response of the aircraft is not unique (refer to section 5.4): on one hand, the rear wing is moved towards the centre of gravity (but in this case many configurations do not meet the constraint on the Margin of Stability); on the other hand, the rear $C_{L}$ can be improved. The previous two situations refer to analyses where the auxiliary wing was a canard and its wing loading was limited between $200 \mathrm{~kg} / \mathrm{m}^{2}$ and $900 \mathrm{~kg} / \mathrm{m}^{2}$. If the upper boundary of the auxiliary wing is cut to $600 \mathrm{~kg} / \mathrm{m}^{2}$, the optimizer provides new configurations, where the auxiliary wing is a tail and the rear wing is heavily moved towards the centre of gravity and the Margin of Stability condition is met by positioning the auxiliary wing backwards, far from the centre of gravity (refer to section 5.7). In this case the lateral stability is difficult to be managed with two vertical fins, because they are very close to the centre of gravity. Then, the tail can have a " $\mathrm{H}$ " shape in order to locate two rudders. Anyway, the efficiency $E$ increases when the rear wing loading increases.

In general, it can be inferred that the most efficient configurations should have three slender lifting surfaces: in fact, it is not sufficient to have a front or rear high Aspect Ratio to improve $E$. It is necessary to reduce all the surfaces when the wingspan $b$ is given, because the generalized Aspect Ratio directly affects the efficiency.

The bulks length has been taken into account in the objective function by means the parameter $K_{\text {bulk }}$ (refer to section 5.2). Bulks have to be sufficiently short because they can undergo buckling. It has been chosen $K_{\text {bulk }}=0.8$ because it is a compromise value between high efficiency, short bulks, homogeneous distribution of the aerodynamic load on the three wings and not too high $C_{L}$.

It has been provided that the height of the two fins $h$ does not affect the efficiency 
in a sensitive manner. Reducing $h$ from $12 m$ to $11 m$ or $10 m$ seems a good solution because the structure is more compact, maintainability is simpler and the wet surface is decreased. However, if the rear wing is closer to the front wing, the downwash effect is stronger and the flow on the rear wing can be slightly spoiled (refer to section 5.5.1). The aerodynamic efficiency is very sensitive to the wingspan because, when $b$ increases, both the induced drag and the friction drag decrease. Besides, changing the wingspan, the margin of stability is not influenced (refer to section 5.5.2). The $h / b$ parameter has been considered in section 5.6 and it is observed that the Prandtl's aerodynamic theory does not comply with the results: in particular, the efficiency is not constant, but it decreases if the wingspan decreases. Again, the wingspan effects are prominent.

In Chapter 6 , it has been verified that the auxiliary wing is actually necessary to meet low speed conditions for the PrandtlPlane ${ }^{\circledR}$ freighter. The auxiliary wing works as an elevator that guarantees the trim condition during landing. The elevator needs to be very efficient, then the auxiliary wing has to be positioned as further as possible from the centre of gravity: in fact, when the auxiliary wing is positioned between the front wing and the rear wing, the configuration does not fulfill the low speed condition. It should be remarked that the low speed procedure is very preliminary: flaps and slats need to be optimized, taking into account interaction effects. Moreover, in this work, only slotted flaps have been considered. If more expensive fowler flaps are employed, low speed performances will increase and the two elevators can be less stressed. It is important to remark that many different wings arrangements allow the freighter to land and to meet the low speed constraint. This is a key point in the design process because it demonstrates that the PrandtlPlane ${ }^{\circledR}$ freighter with three lifting surfaces is a very versatile aircraft.

Finally, three macro-solutions have been provided by the high speed analysis (optimization process in cruise conditions); they all meet the low speed requirement too. The most remarkable geometric difference is the position of the auxiliary wing along the fuselage.

Canard configuration The canard configuration provides many efficient solution in cruise condition. Aerodynamic efficiency can nearly rise up to 23, but longitudinal stability needs to be strictly controlled. The low speed requirement is met by means low flaps deflections and suitable elevators angles, avoiding saturation.

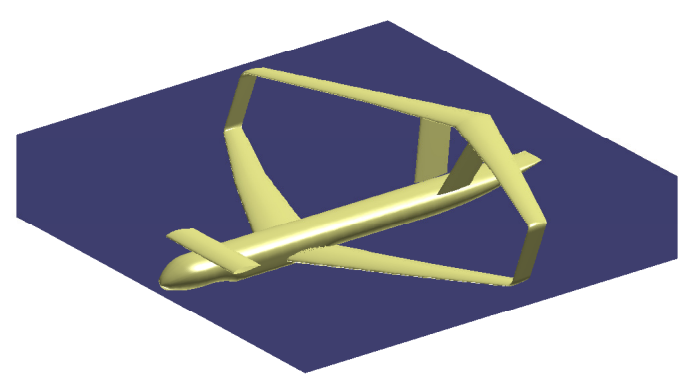


Intermediate configuration The aerodynamic efficiency is higher than 23 but the position of the auxiliary wing has to be corrected after the optimization process because this configuration tends to be unstable. Moreover, the fuselage can not enjoy three supports any more. It seems easy to trim this kind of aircraft in low speed condition because high lift devices and elevators need small deflections; however, the auxiliary wing is unloaded. The position of the auxiliary wing, in this case, could provide suitable supports for engines. The auxiliary wing interferes with the two vertical fins, so the structure is more complicated.

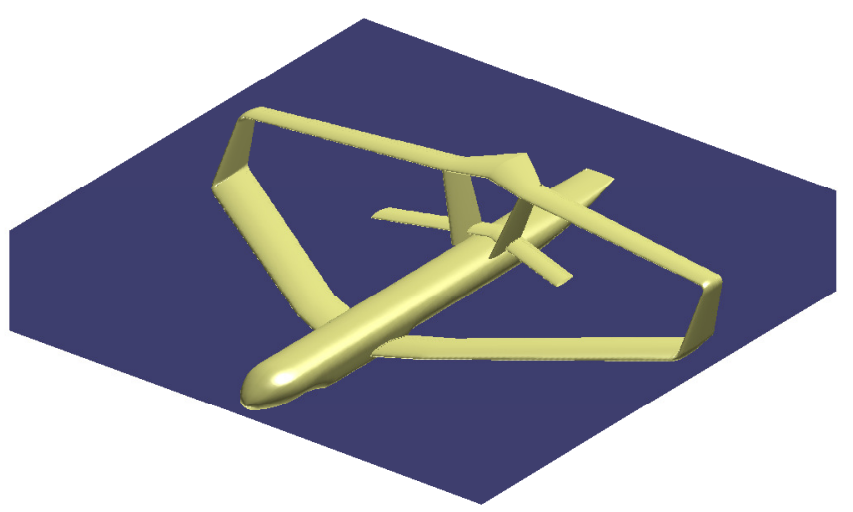

Tail configuration This kind of PrandtlPlane ${ }^{\circledR}$ freighter reaches high aerodynamic efficiency (up to 23.9), the margin of stability is nearly null and all the three lifting surfaces give a positive contribution to the total lift in cruise. The low speed requirement is met with not elevated flaps and elevators deflections; the auxiliary wing gives a slightly negative contribution to the lift to contribute in balancing the increasing pitching moment due to the flaps and slats deflections. By the way, the contribution of the auxiliary wing to the total lift is negligible and the aerodynamic load is equally distributed on the front wing and on the rear wing.

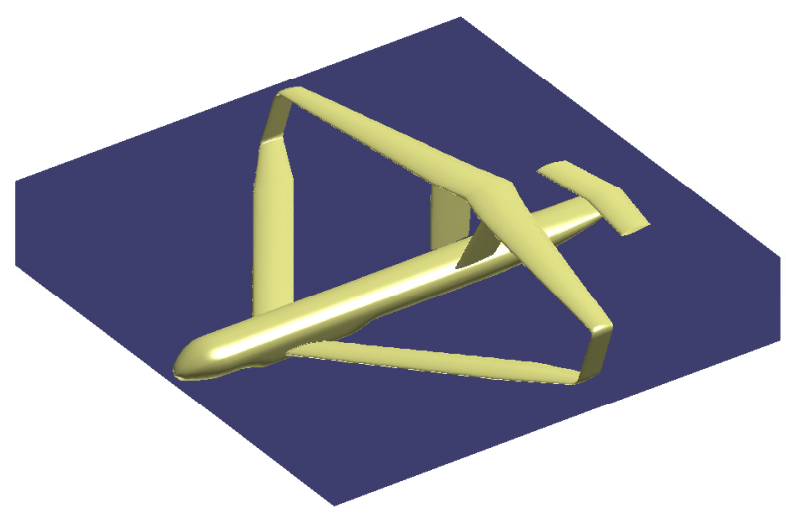




\section{Future Developments}

The PrandtlPlane ${ }^{\circledR}$ freighter requires specific and deeper studies, in order to select a configuration and to proceed with the detail design.

AEROSTATE needs to be improved by means a new weights model. Furthermore, a structural model of the aircraft and a preliminary low speed model can be implemented, as a post-processor.

The lateral stability has been neglected in this thesis because it is a preliminary study and because four ailerons and two big rudders are supposed suitable enough to control roll and yaw; however, lateral stability needs to be investigated.

All the information provided by this thesis can be collected to choice a configuration to start a detail design. Power plant installation should be considered too.

Once the configuration has been chosen, a suitable structural model of the box wing and of the auxiliary wing is necessary to investigate the stress on the upper panel and on the lower panel. Materials have to be chosen and the auxiliary wing has to be connected to the whole structure. Aeroelastic computations are basic for this large structure: the structural stiffness has to be designed to avoid divergence, reverse command and flutter phenomena.

An automated procedure has been set up in order to obtain the CAD of a configuration (refer to Chapter 4). This tool is used in the preliminary design to evaluate the effectiveness of the solution provided by the optimization process. Furthermore, CAD sketches that are carried out from INTERFACE and from ASD can be easily used and adopted to further investigation by means of CFD codes for both high and low speed. 


\section{Appendix A}

The results of the validation of the front wing loading analysis with $K_{\text {bulk }}=0.8$ are shown (refer to section 5.3).

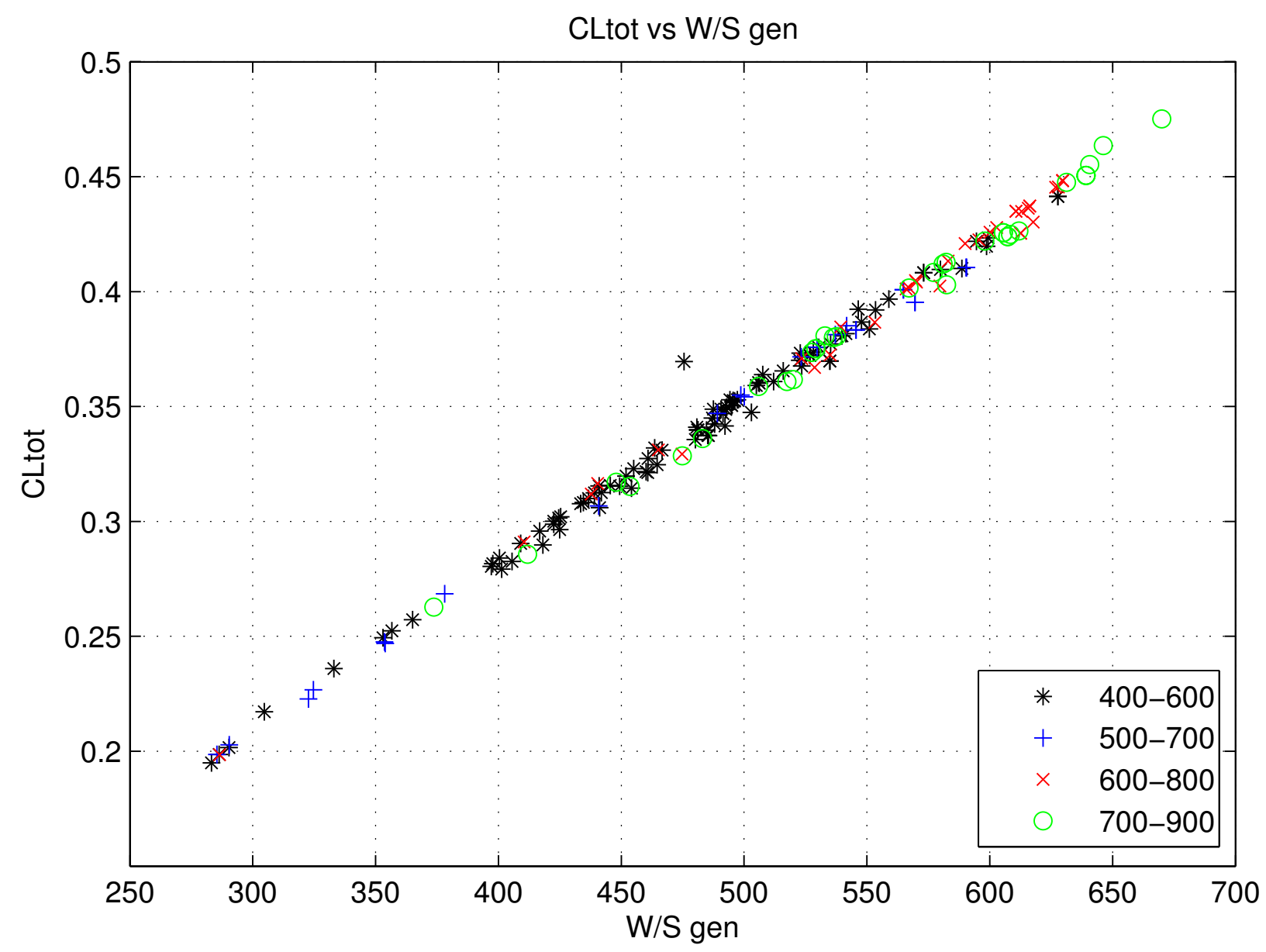

Figure A.1. $C_{L}$ tot vs Global wing loading $-K_{b u l k}=0.8$. 


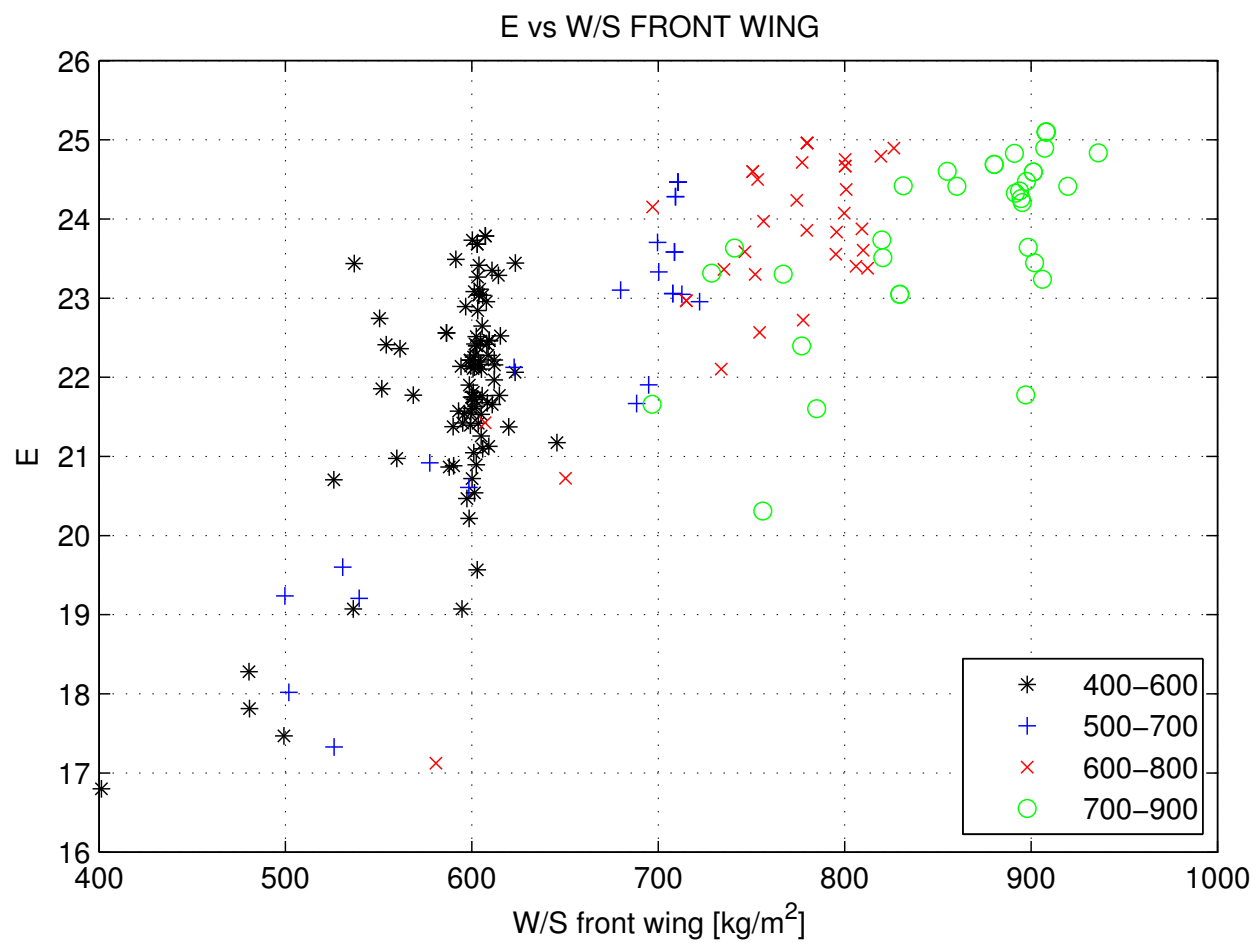

Figure A.2. Efficiency vs Front wing loading $-K_{b u l k}=0.8$.

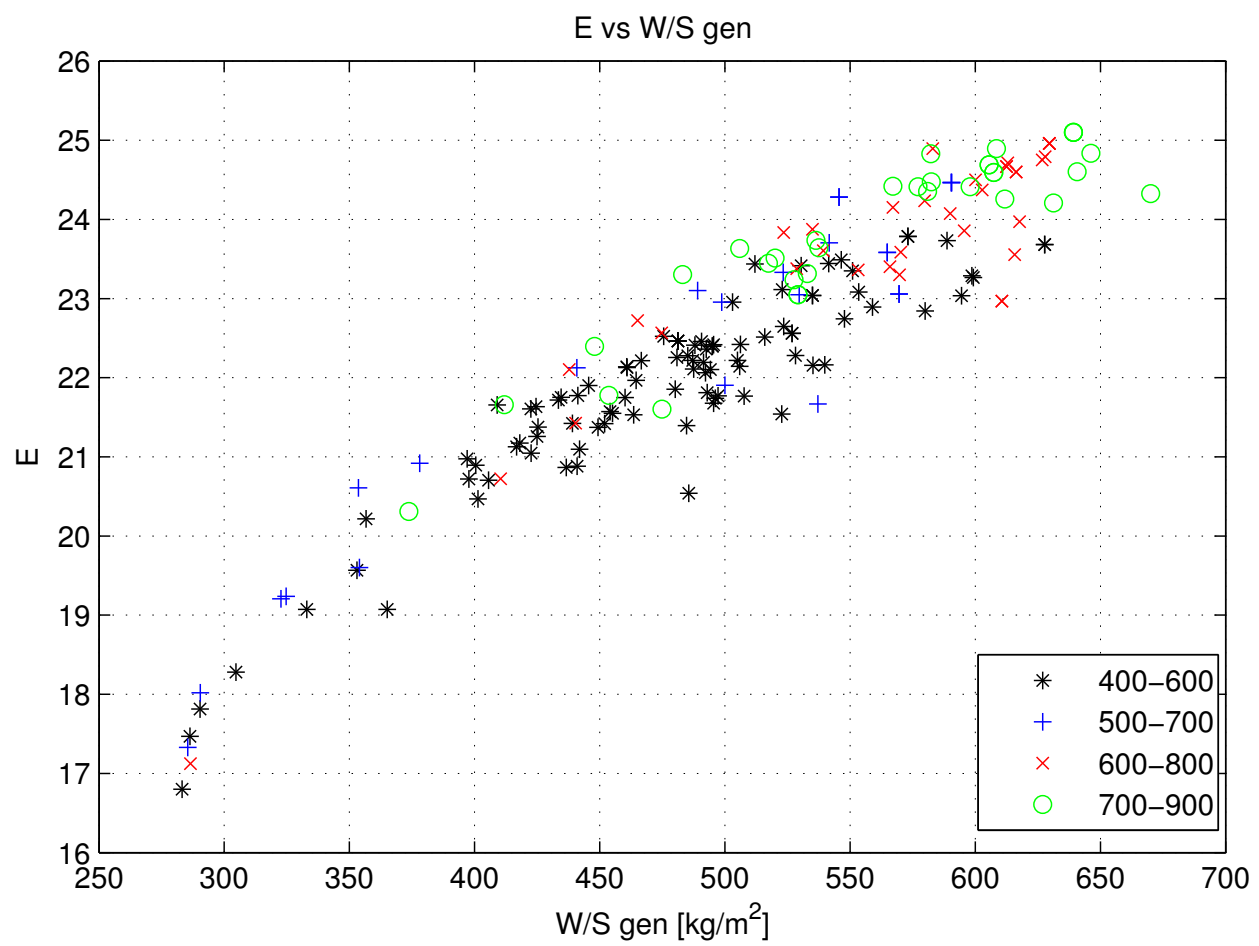

Figure A.3. Efficiency vs Global wing loading $-K_{b u l k}=0.8$. 

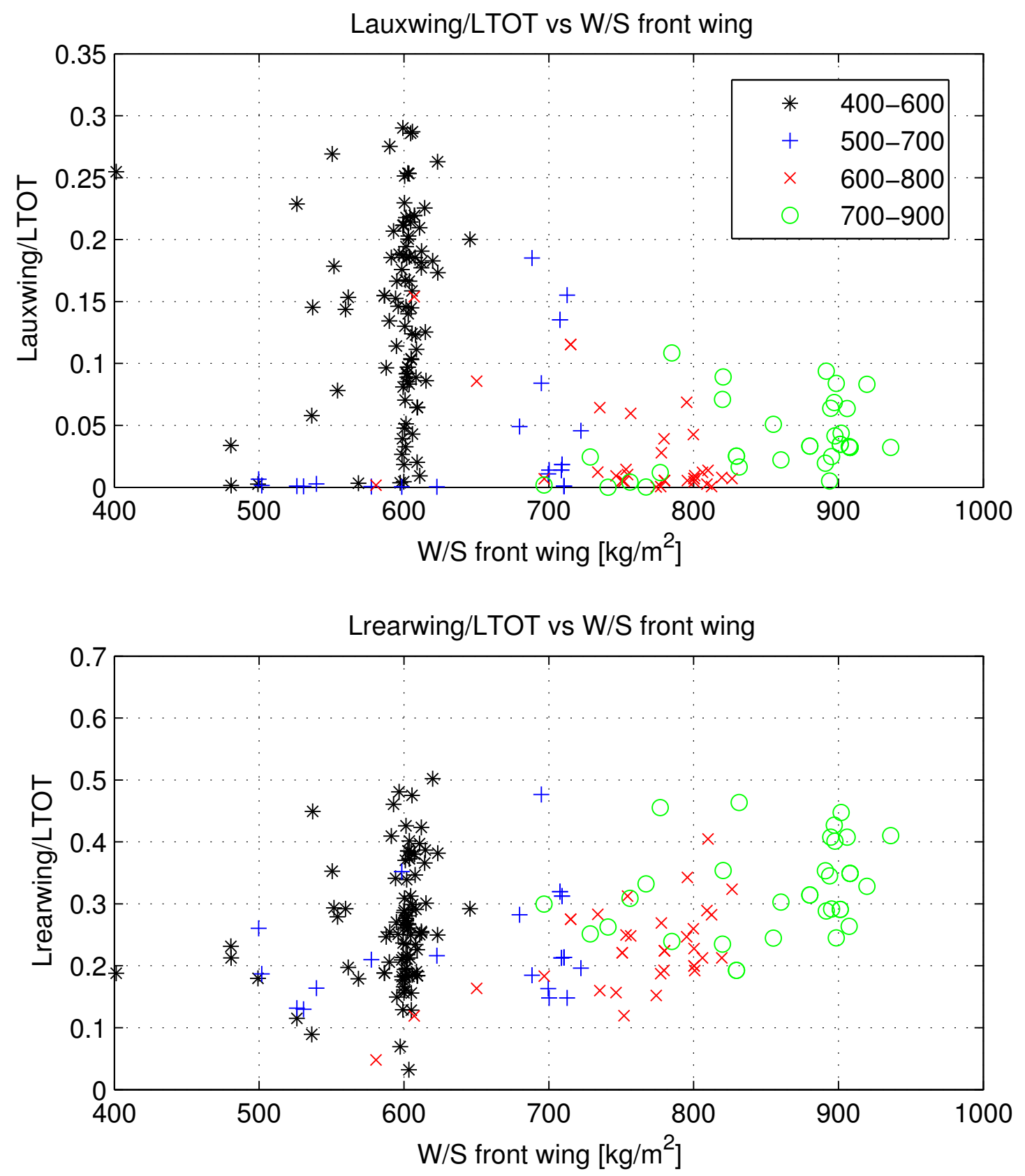

Figure A.4. Lift of rear wing and auxiliary wing $-K_{b u l k}=0.8$. 


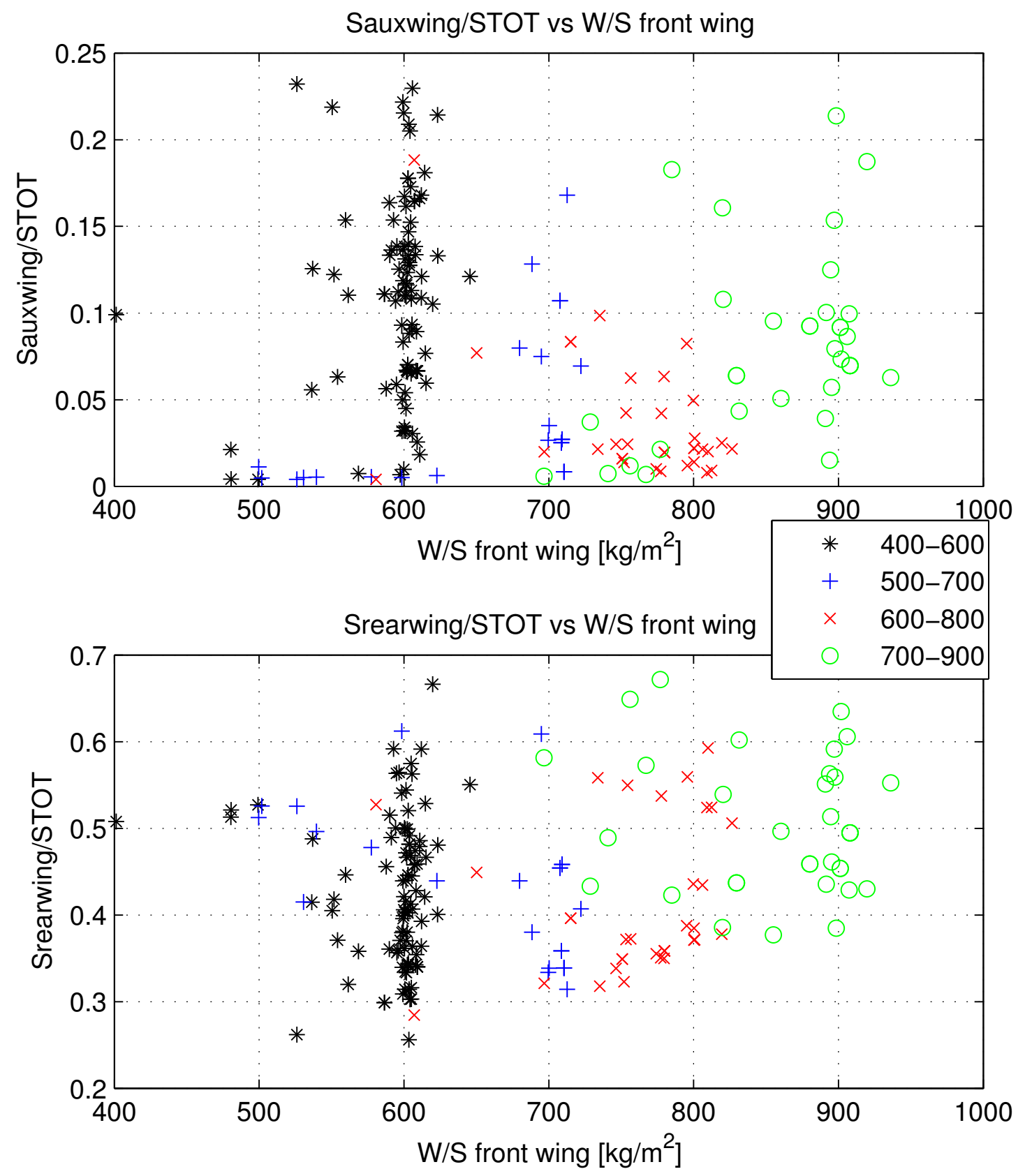

Figure A.5. Surface of rear wing and auxiliary wing $-K_{b u l k}=0.8$. 


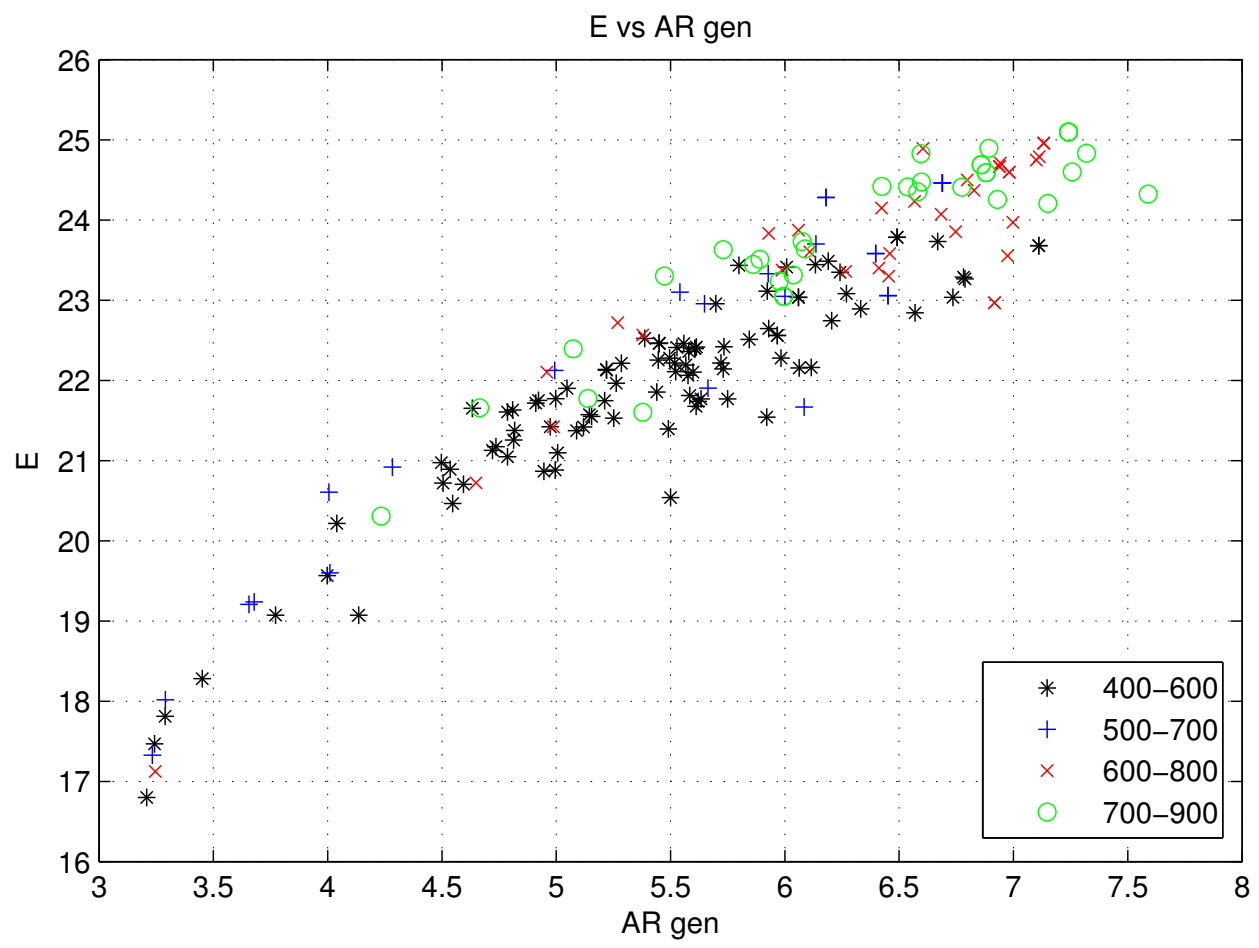

Figure A.6. Efficiency vs Global aspect ratio $-K_{b u l k}=0.8$.

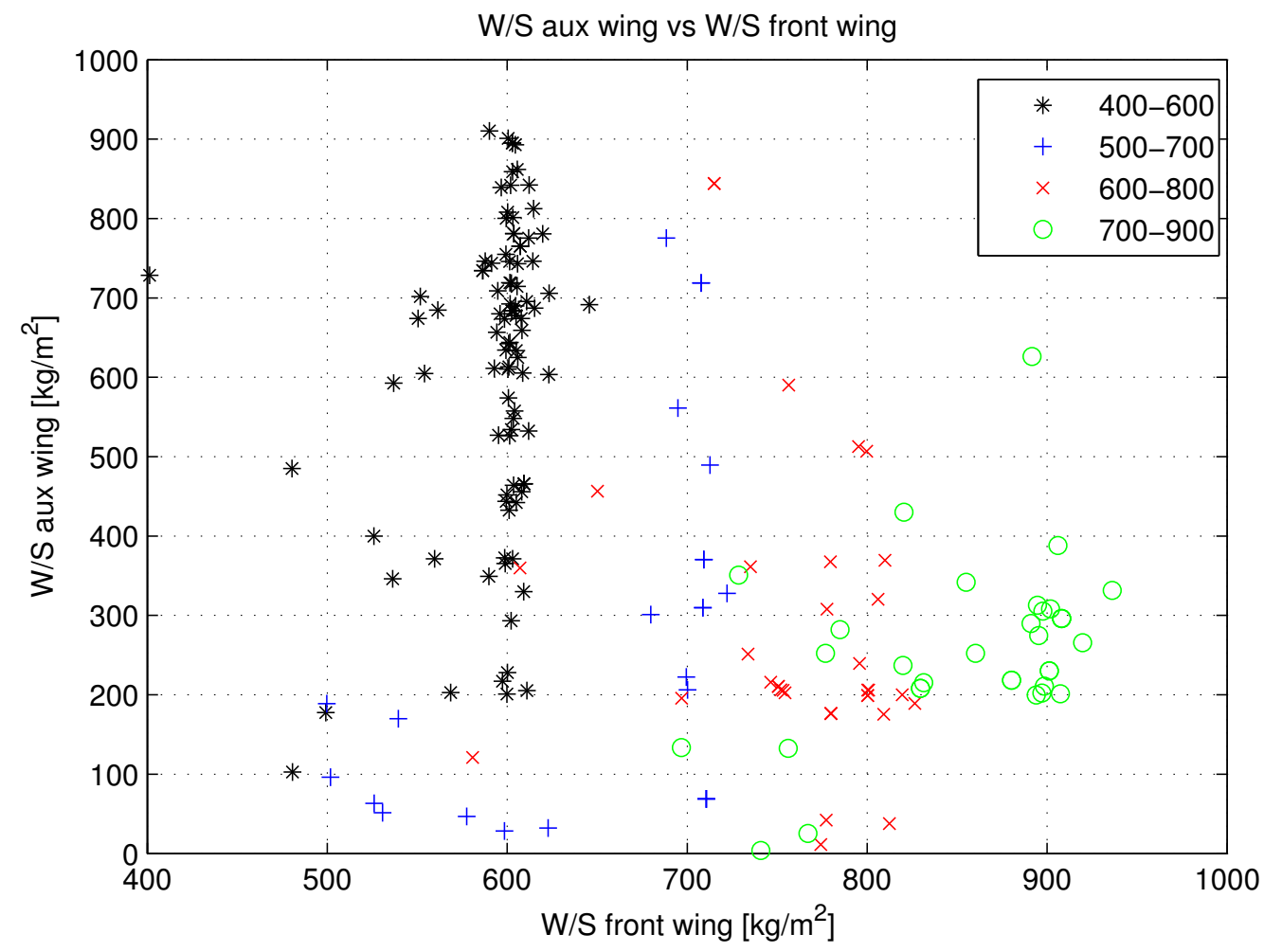

Figure A.7. Auxiliary wing loading vs Front wing loading $-K_{b u l k}=0.8$. 

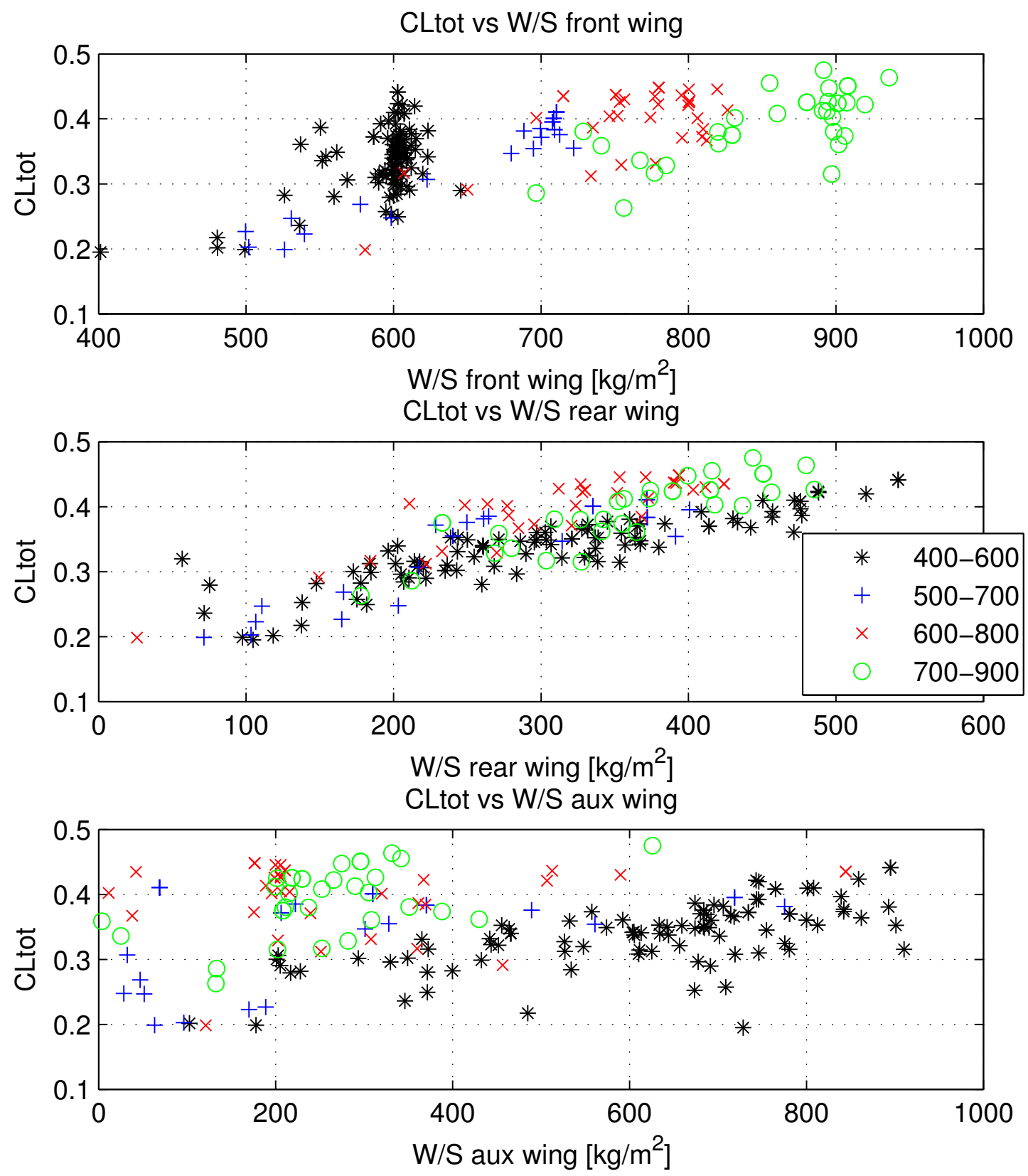

Figure A.8. $C_{L}$ tot trends $-K_{b u l k}=0.8$. 

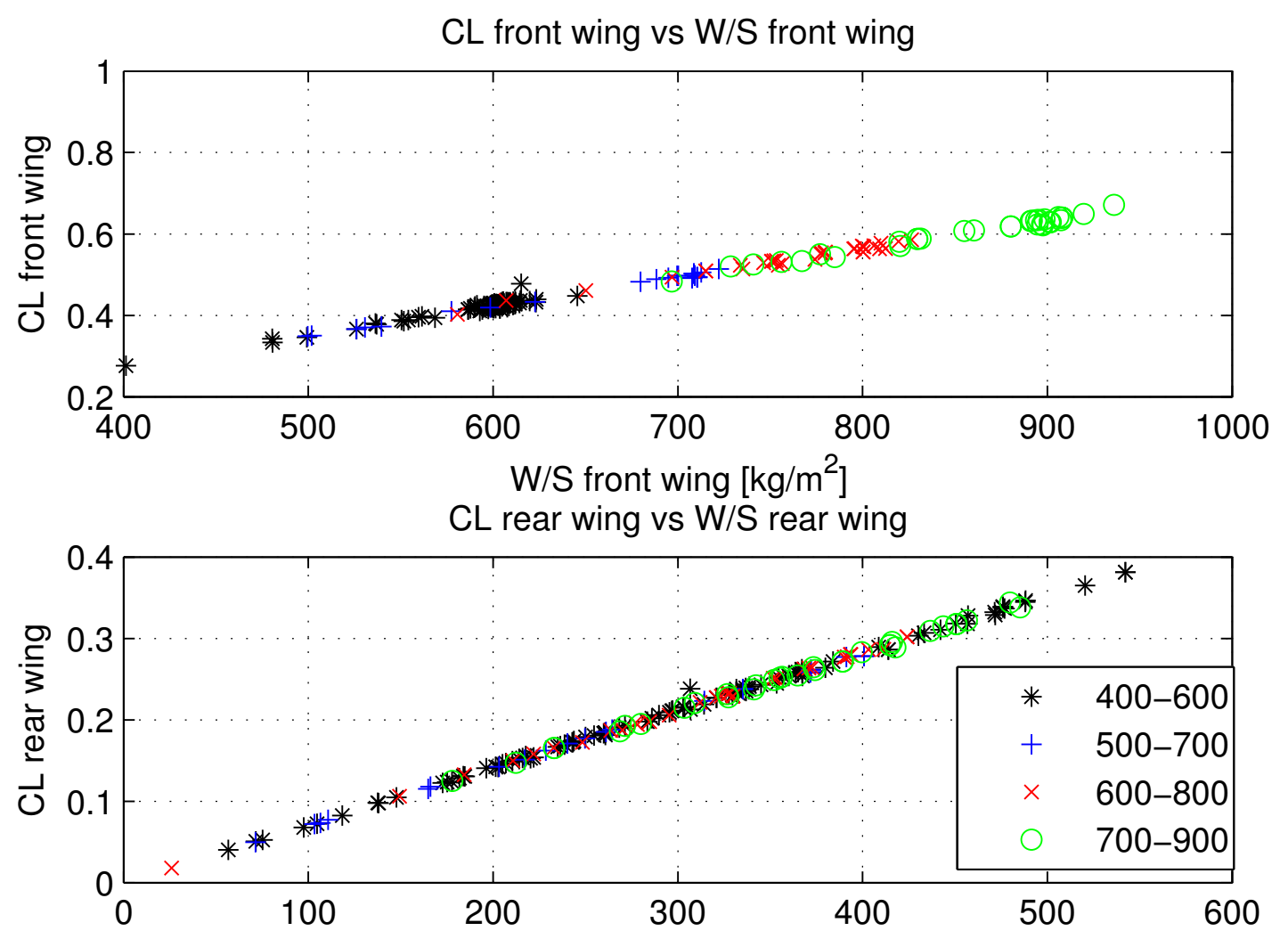

W/S rear wing $\left[\mathrm{kg} / \mathrm{m}^{2}\right]$

CL aux wing vs W/S aux wing

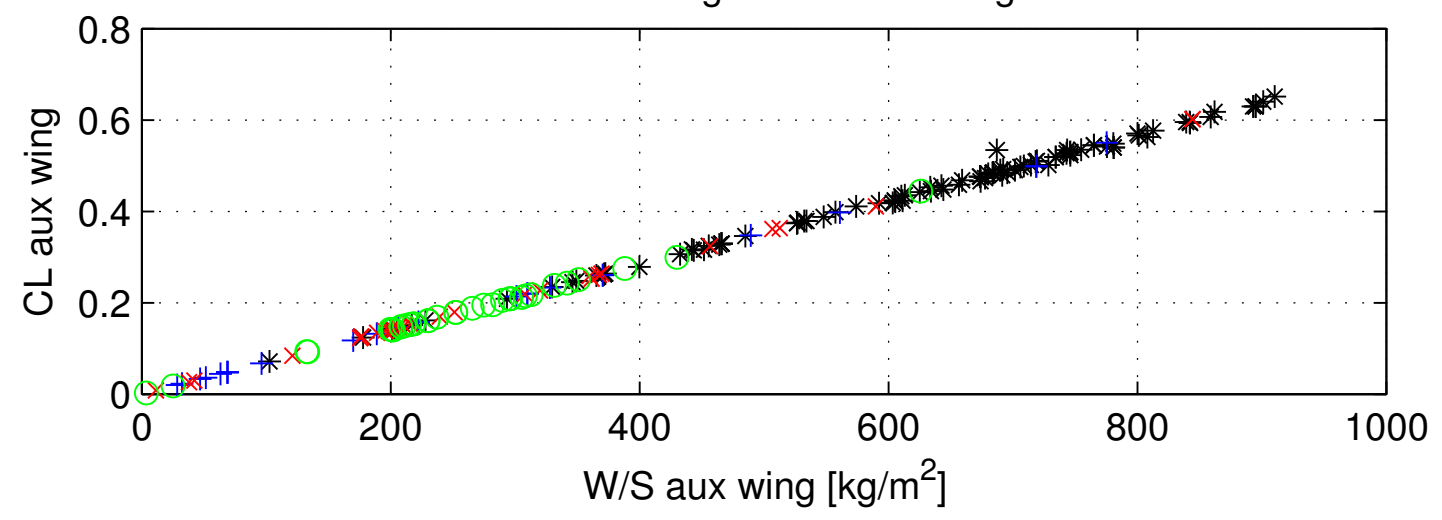

Figure A.9. $C_{L}$ trends $-K_{b u l k}=0.8$. 


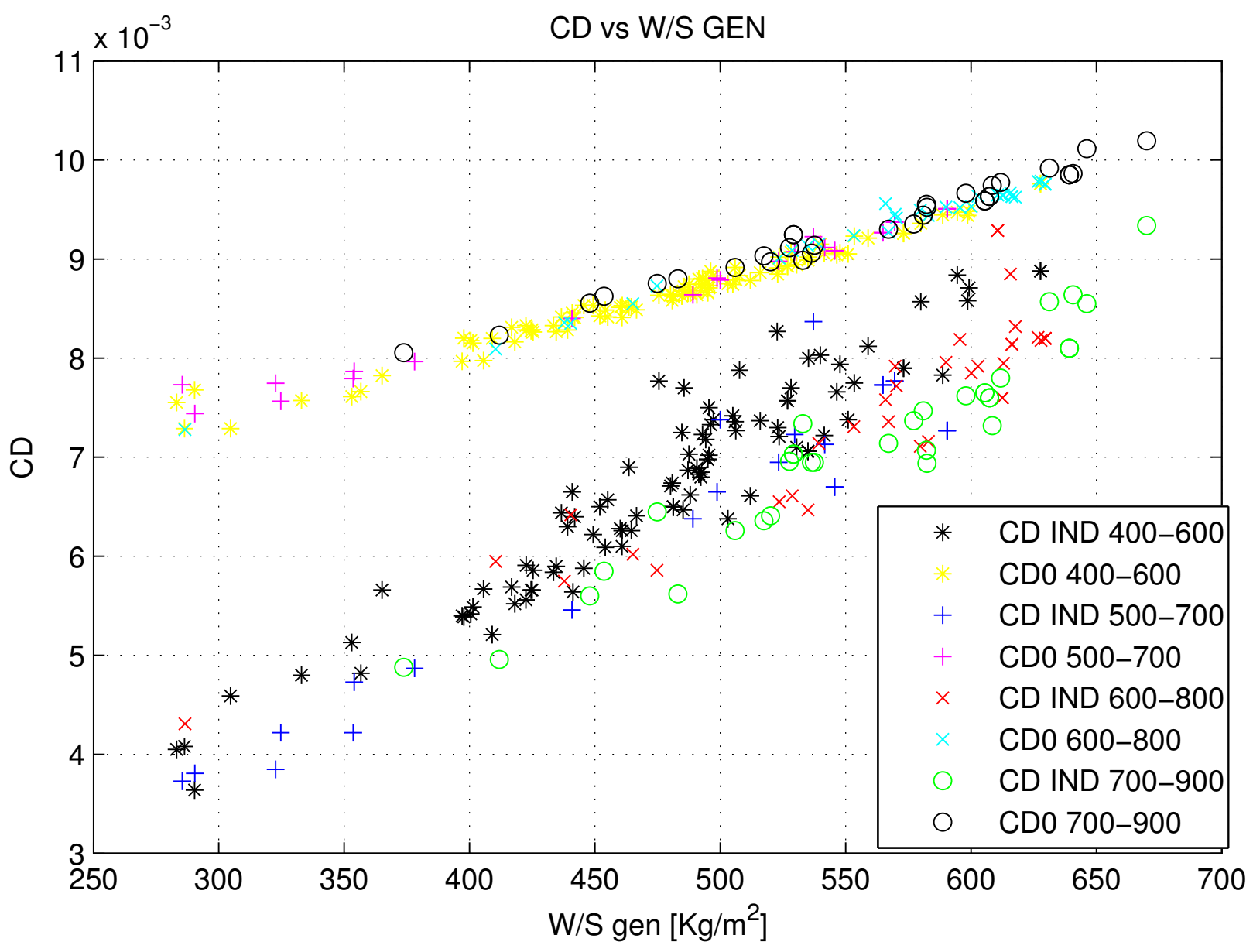

Figure A.10. $C_{D}$ contributions $-K_{b u l k}=0.8$. 


\section{Appendix B}

The results of the rear wing loading analyses are shown (refer to section 5.4).
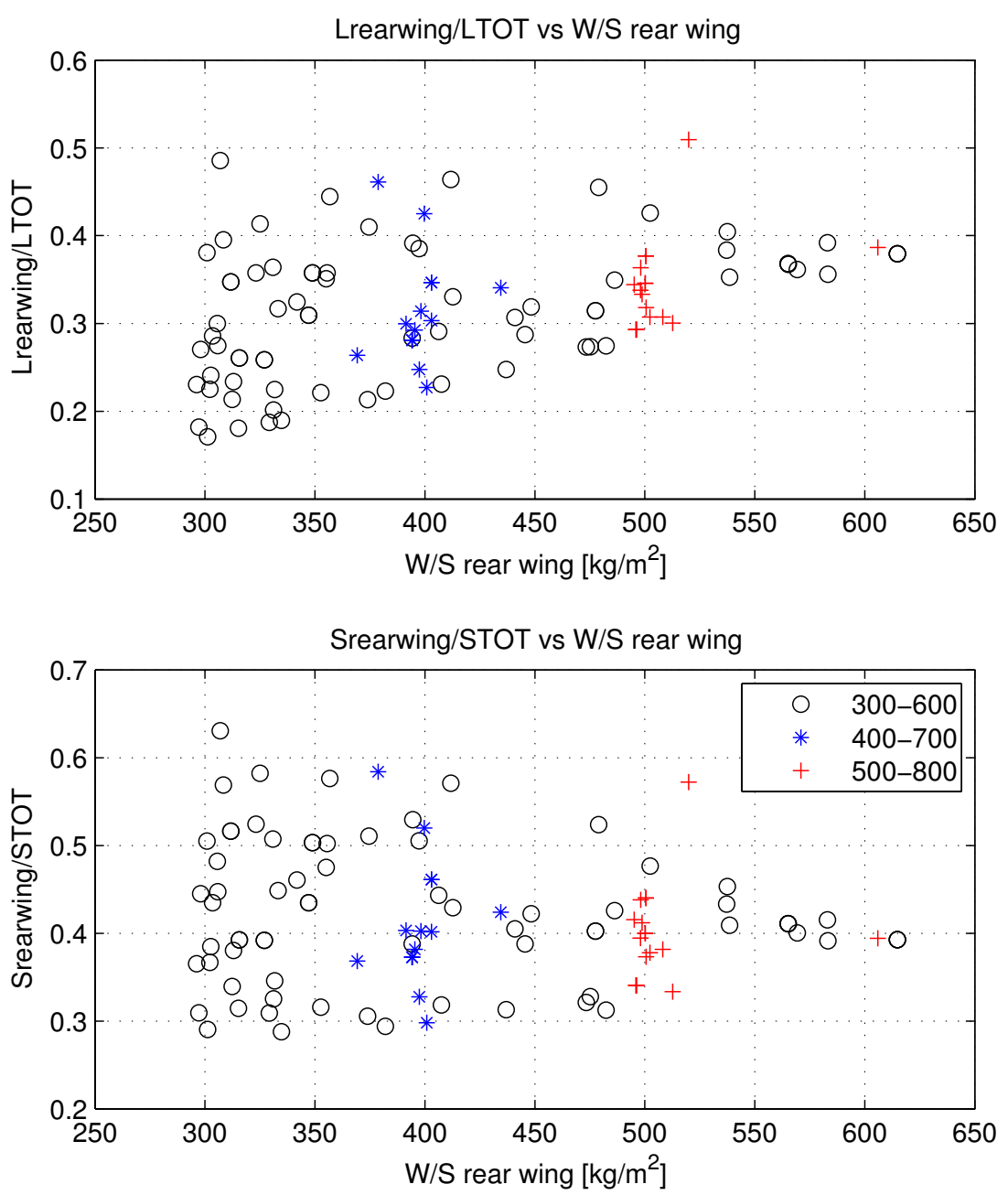

Figure B.1. Rear wing behaviour - Rear wing analysis. 

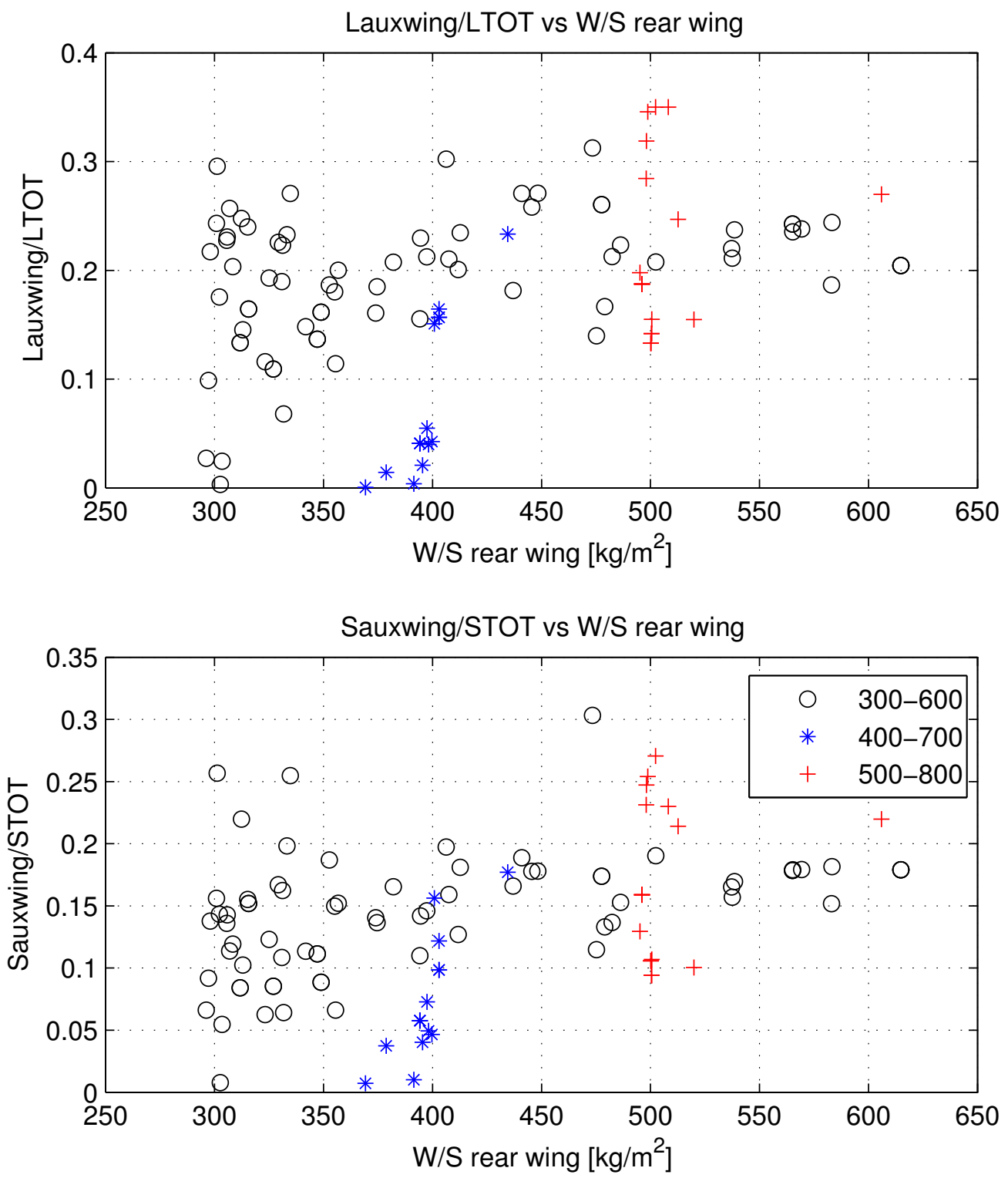

Figure B.2. Auxiliary wing behaviour - Rear wing analysis. 

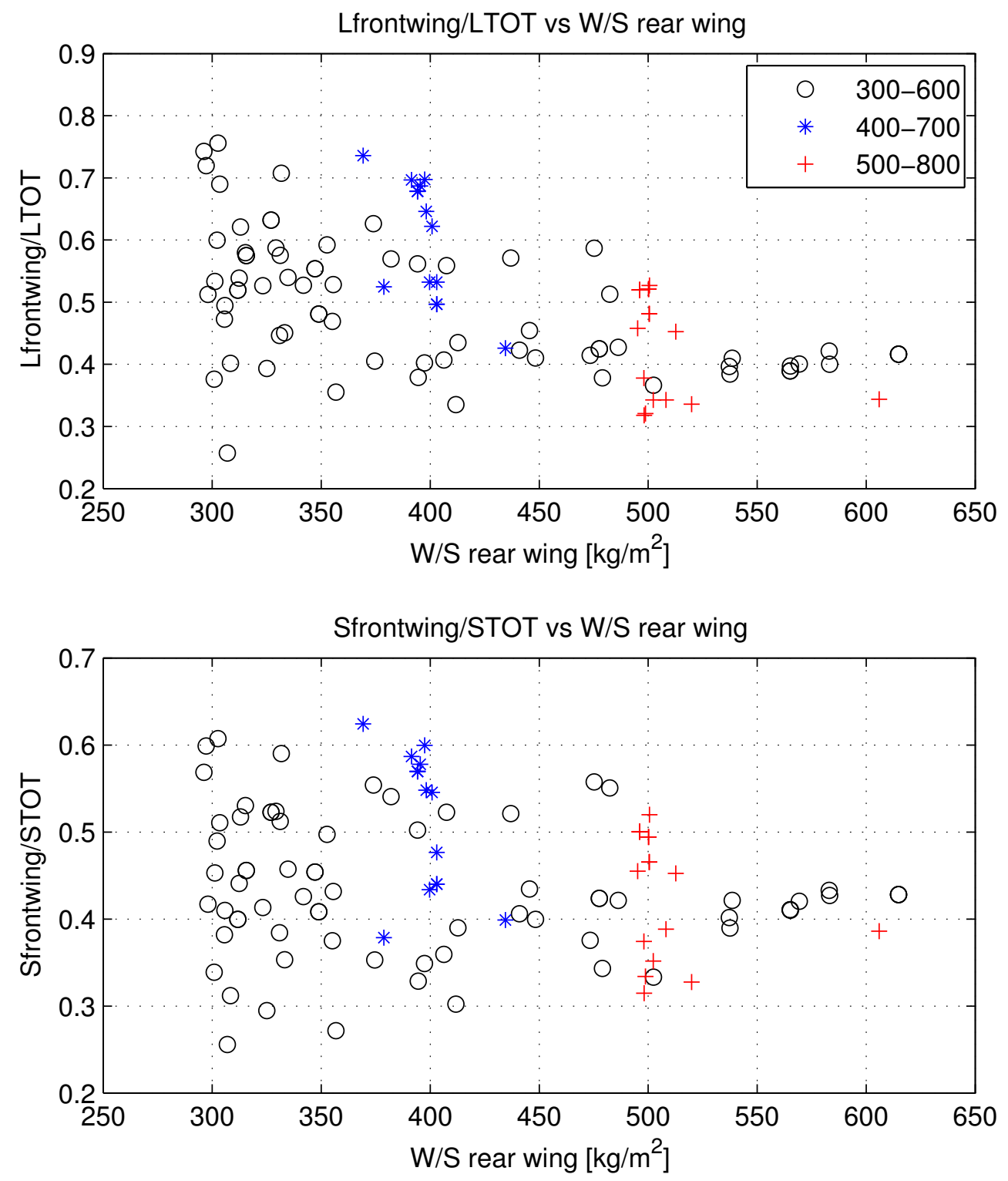

Figure B.3. Front wing behaviour - Rear wing analysis. 

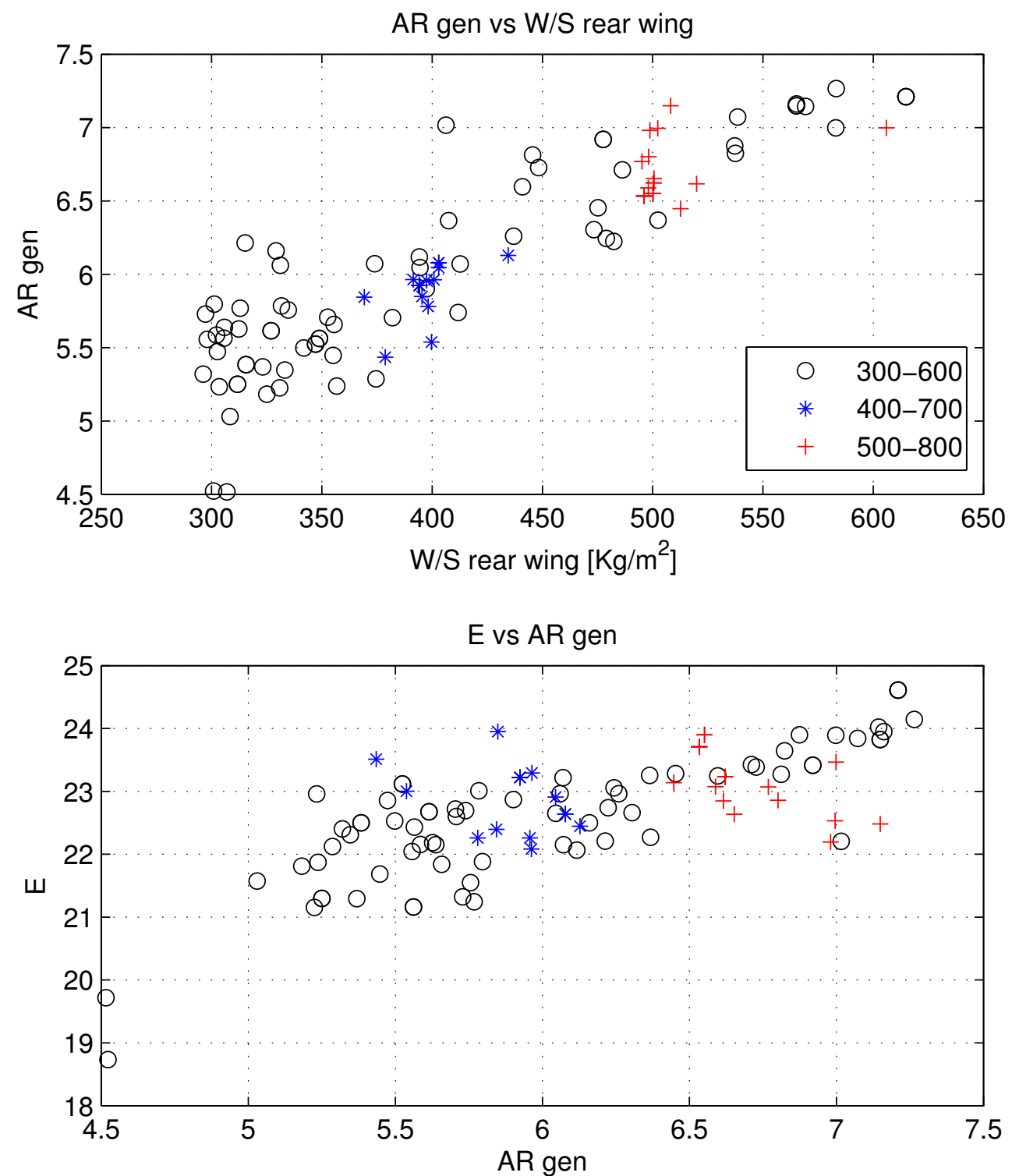

Figure B.4. Global Aspect Ratio effects - Rear wing analysis. 

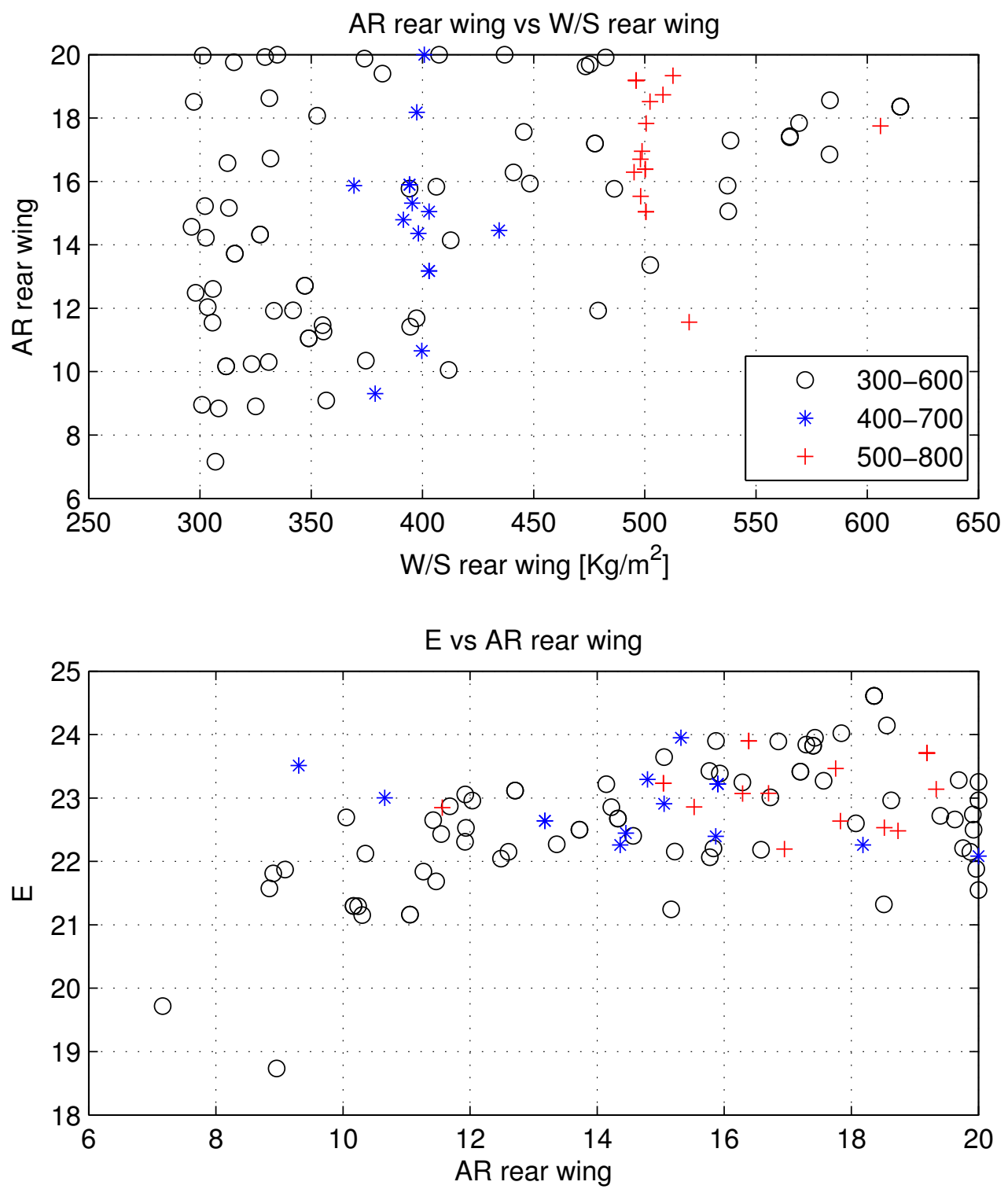

Figure B.5. Rear Aspect Ratio effects - Rear wing analysis. 


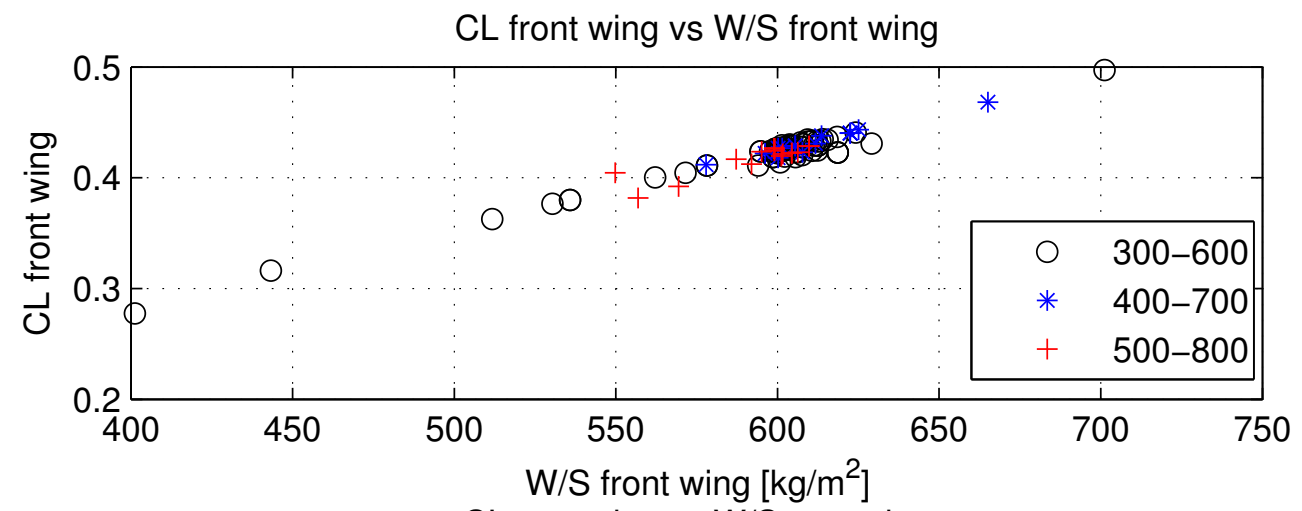

$\mathrm{CL}$ rear wing vs W/S rear wing
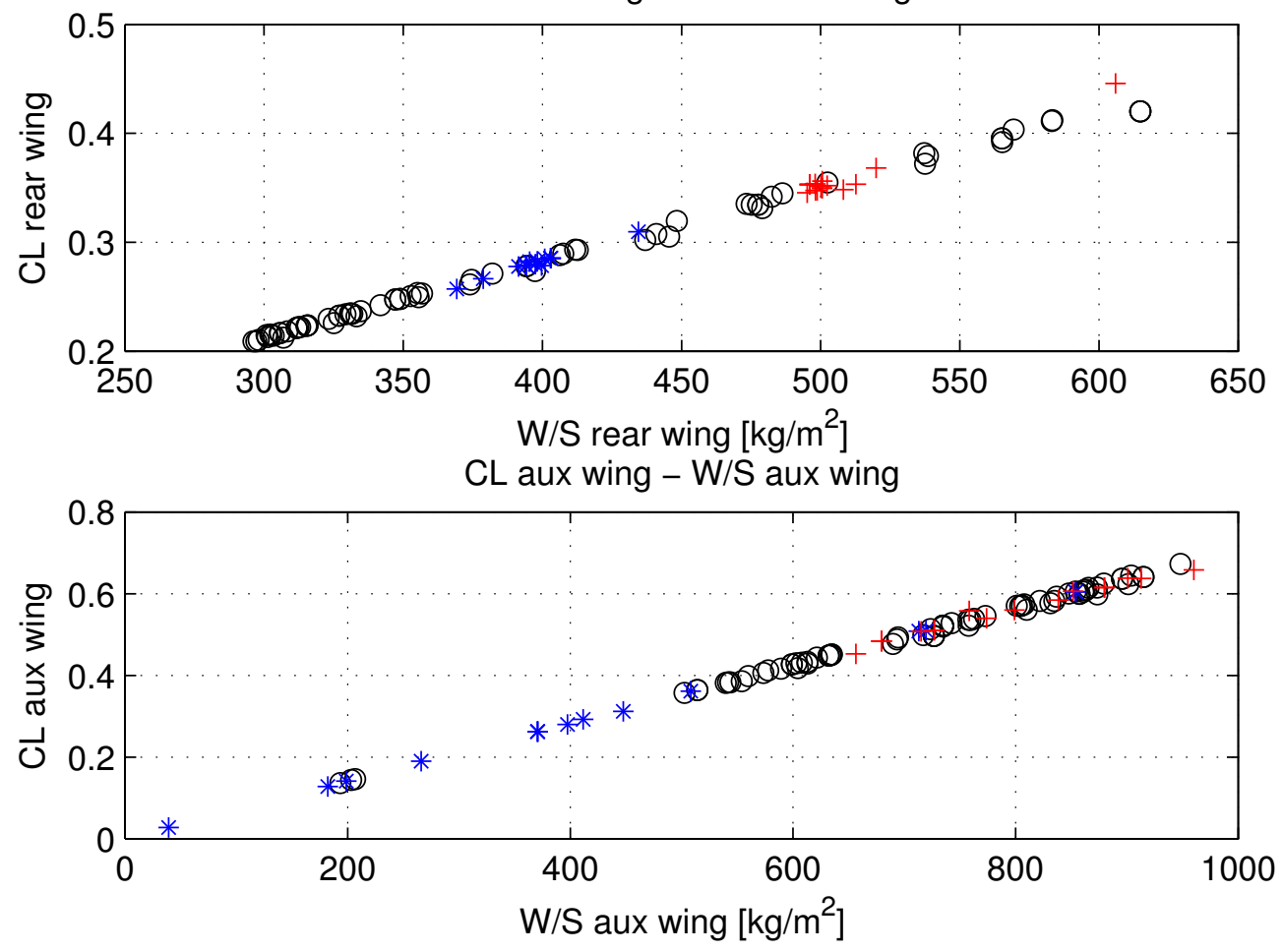

Figure B.6. $C_{L}$ trends - Rear wing analysis. 


\section{Appendix C}

The results of the fin height sensitivity analyses are shown (refer to section 5.5.1).
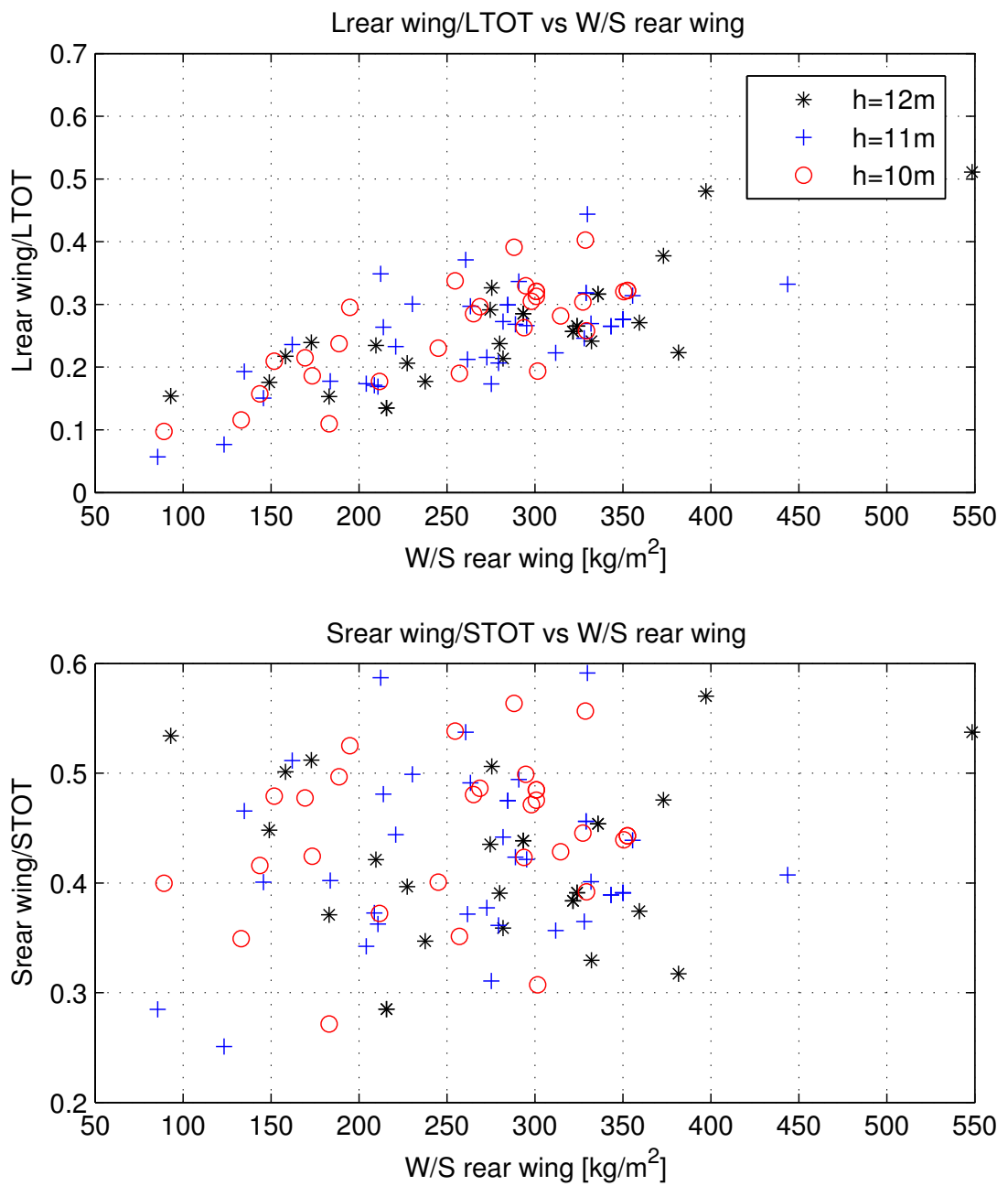

Figure C.1. Rear wing lift and surface - H sensitivity. 

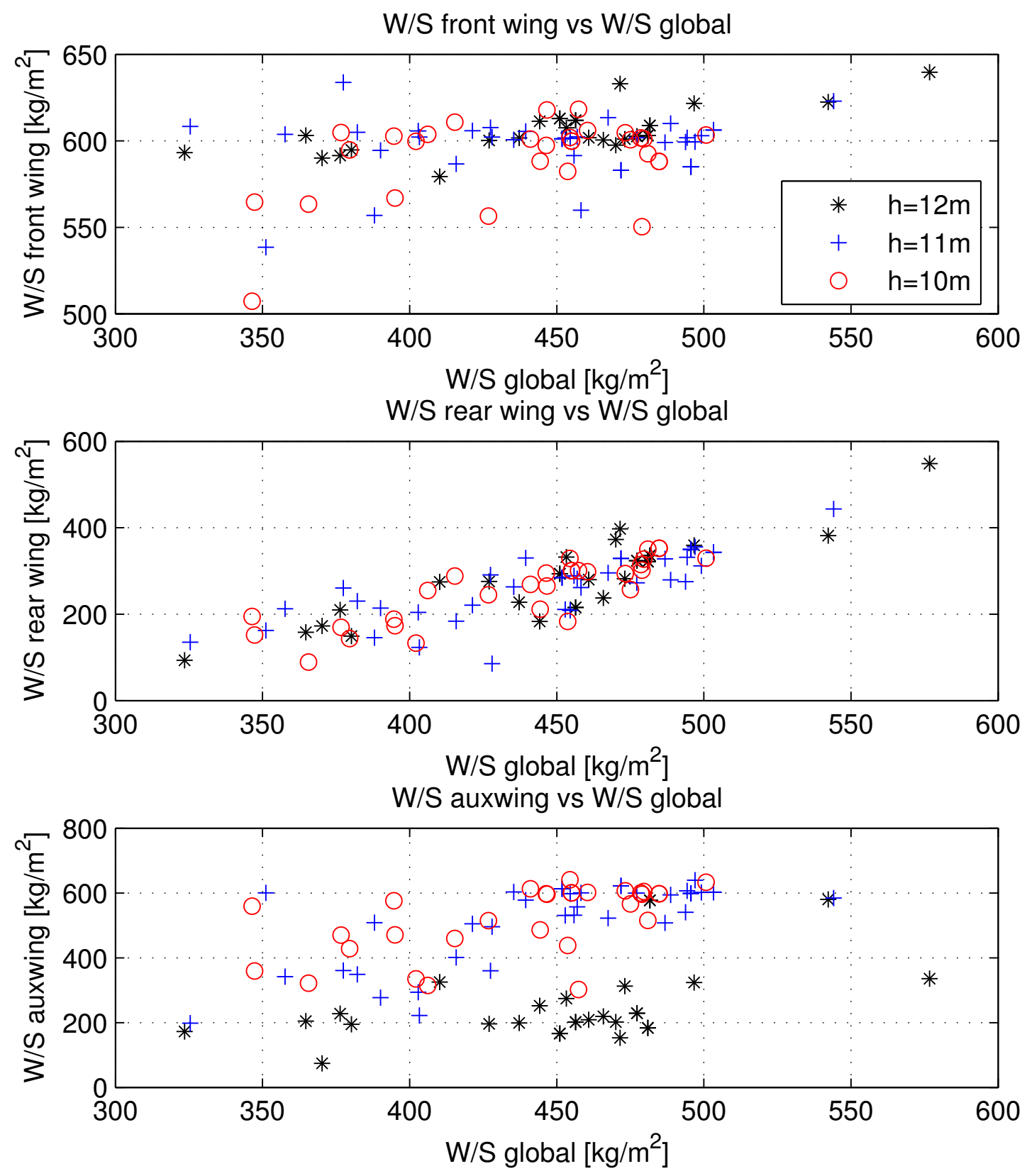

Figure C.2. Wing loadings - H sensitivity. 

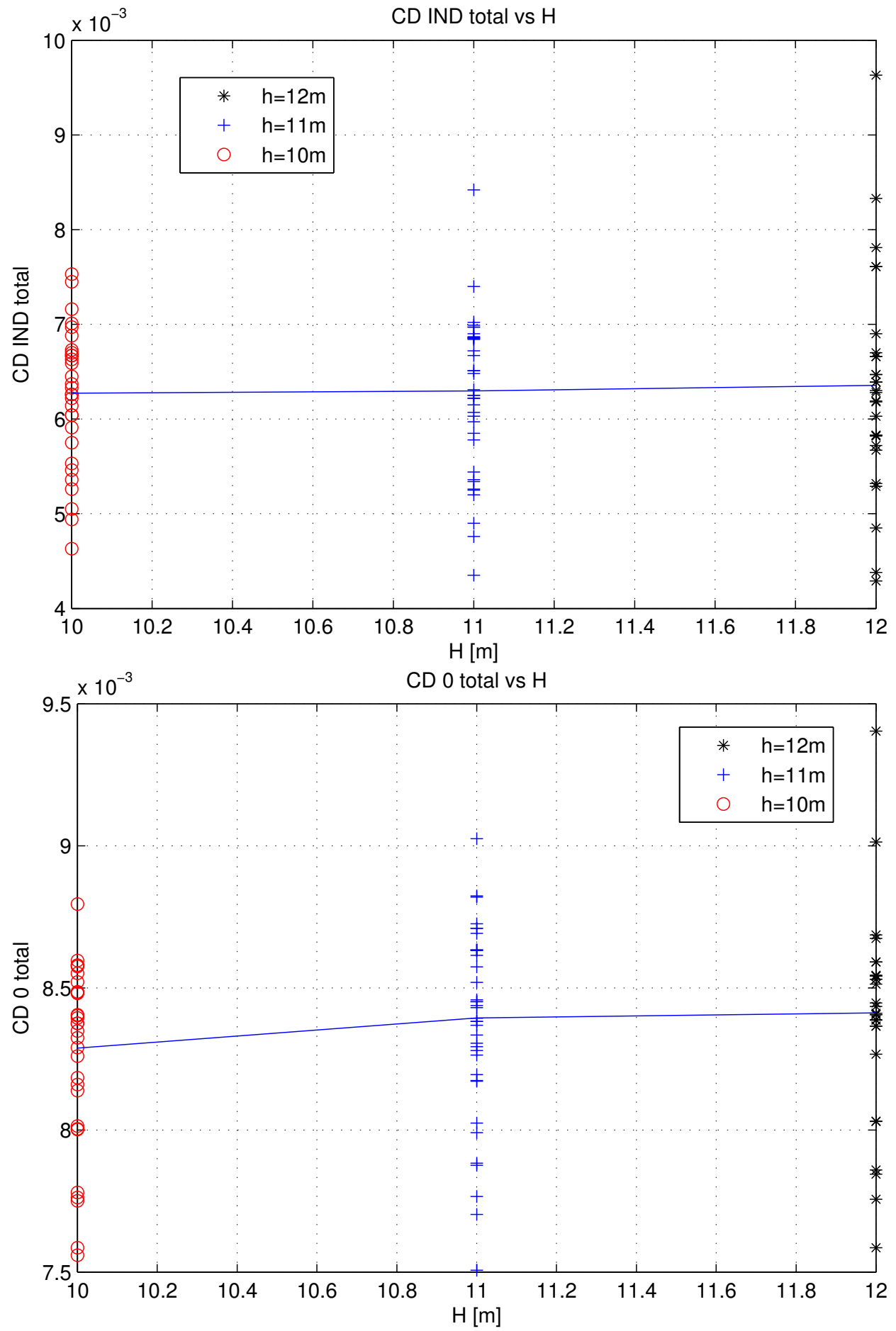

Figure C.3. Induced drag coefficient and viscosity drag coefficient - H sensitivity. 

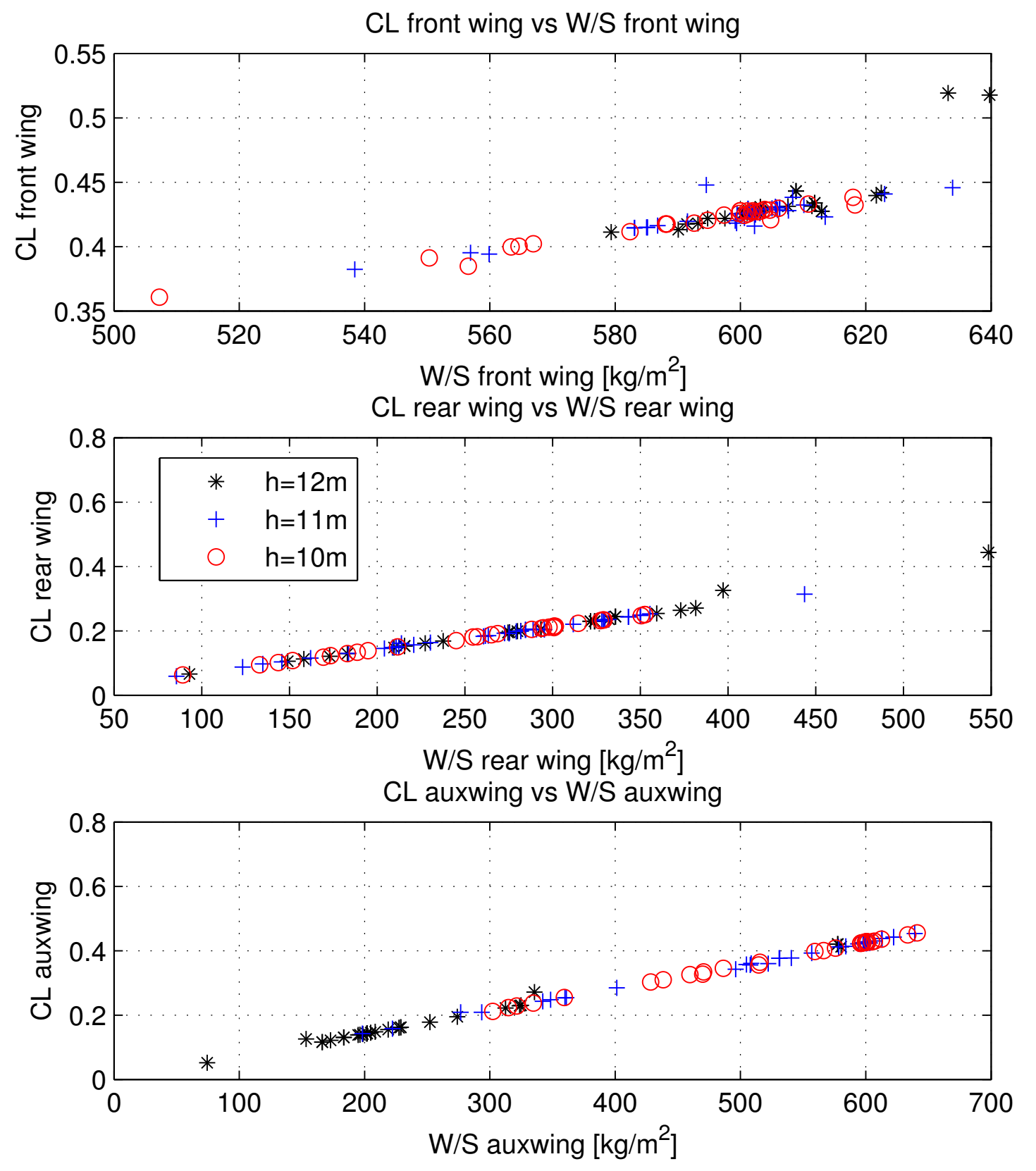

Figure C.4. CL behaviour (part 2) - H sensitivity. 


\section{Appendix D}

The results of the wing span analyses are shown (refer to section 5.5.2).
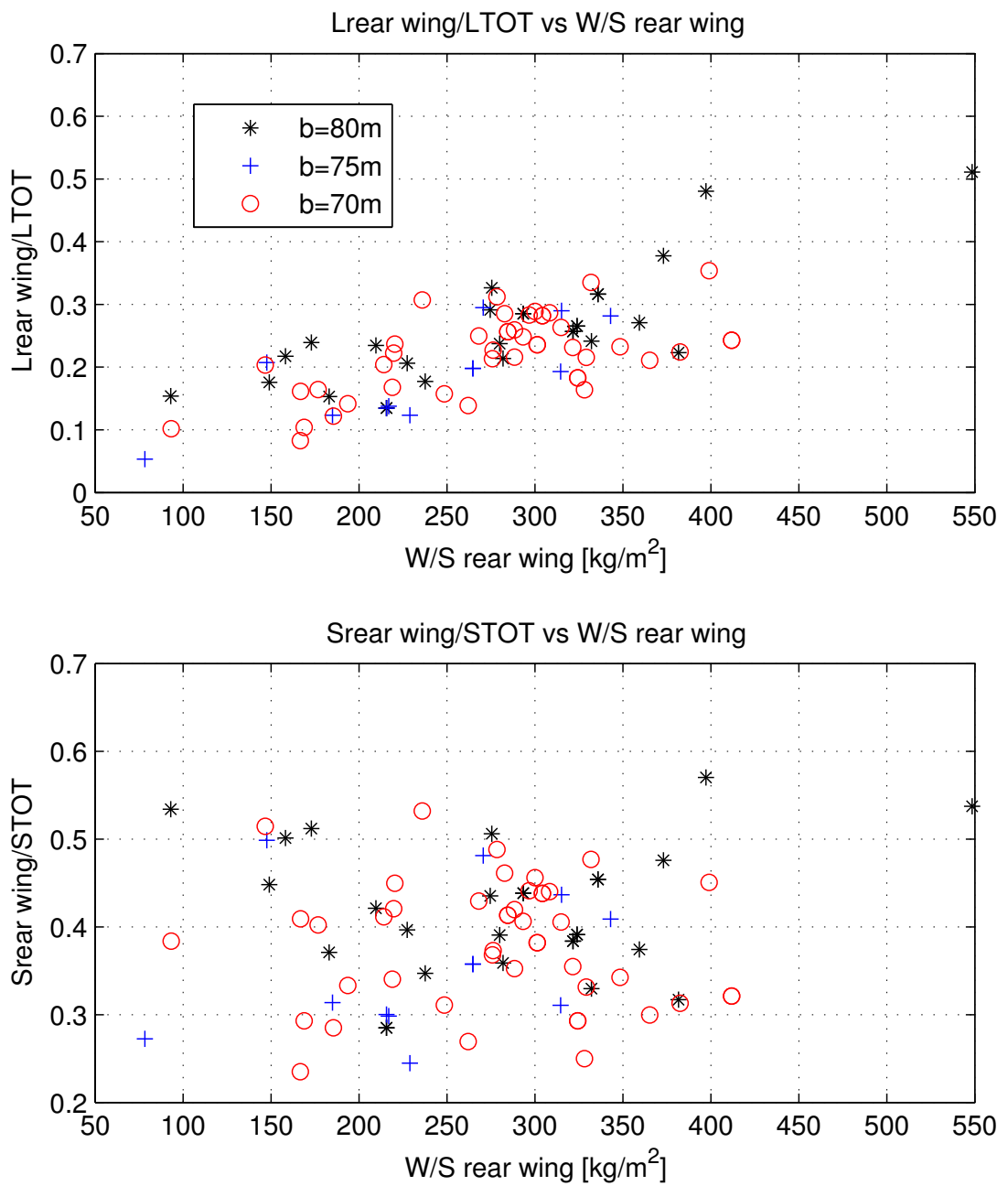

Figure D.1. Rear wing lift and surface - B sensitivity 

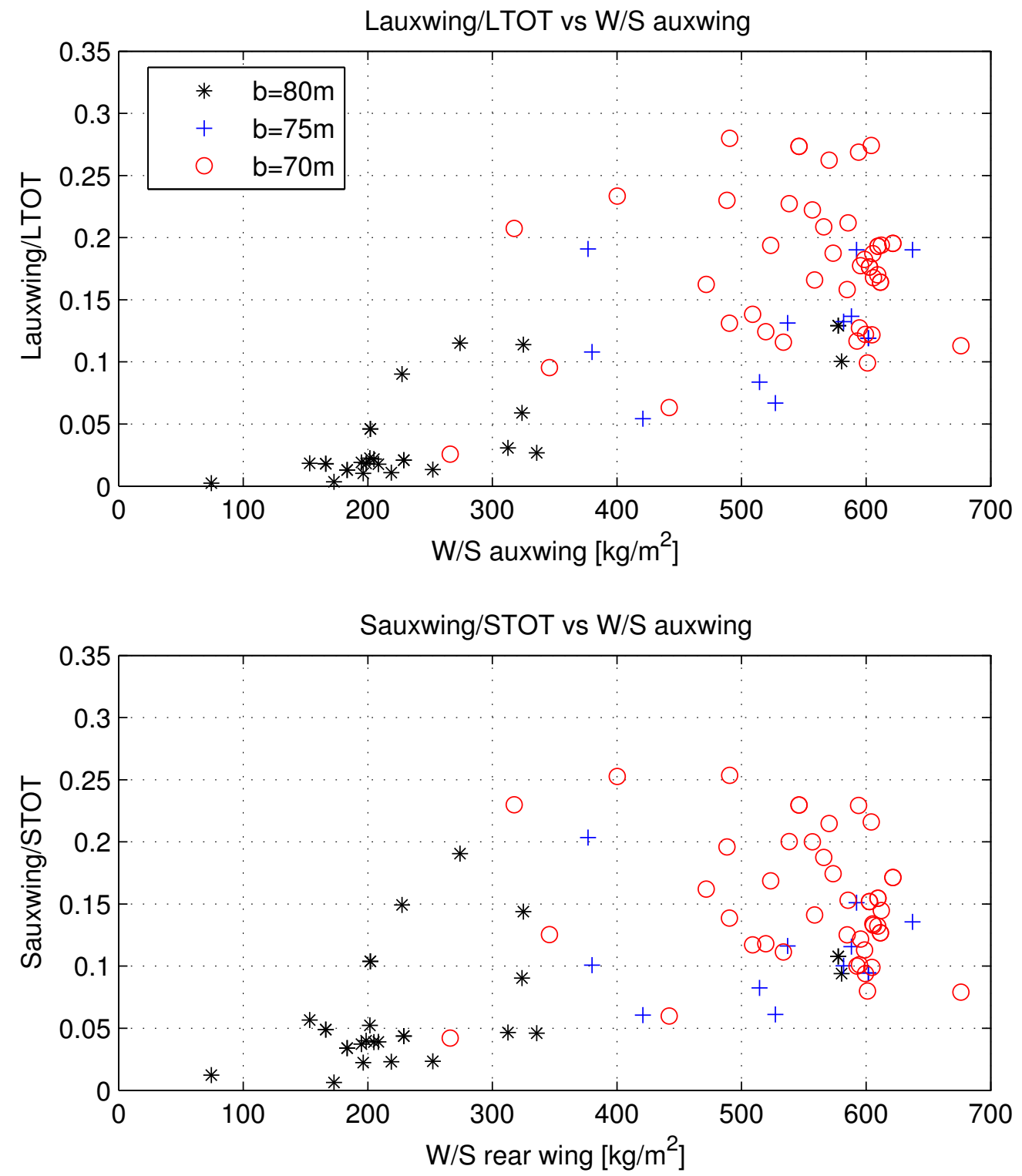

Figure D.2. Auxiliary wing lift and surface - B sensitivity 

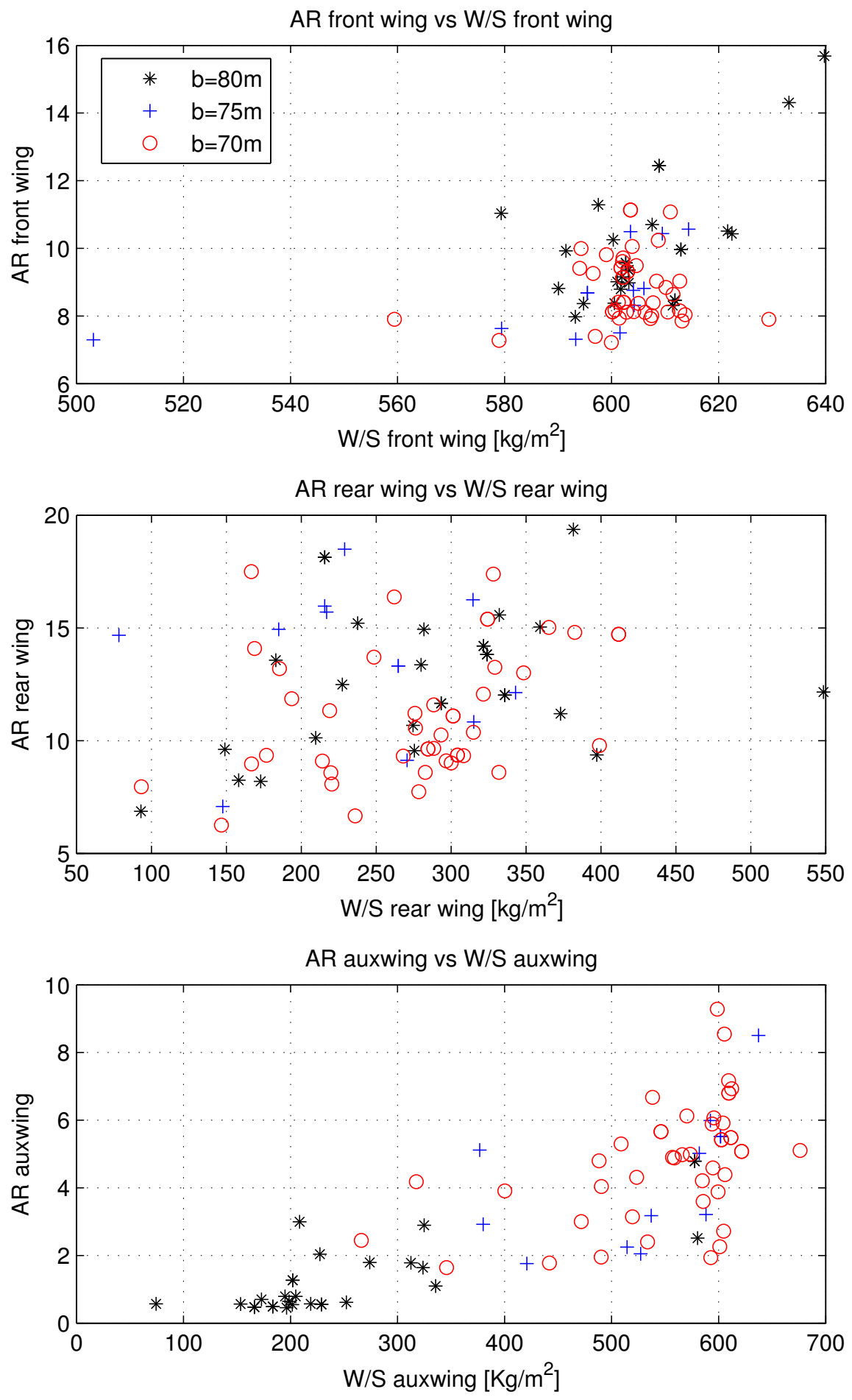

Figure D.3. AR behaviour - B sensitivity 

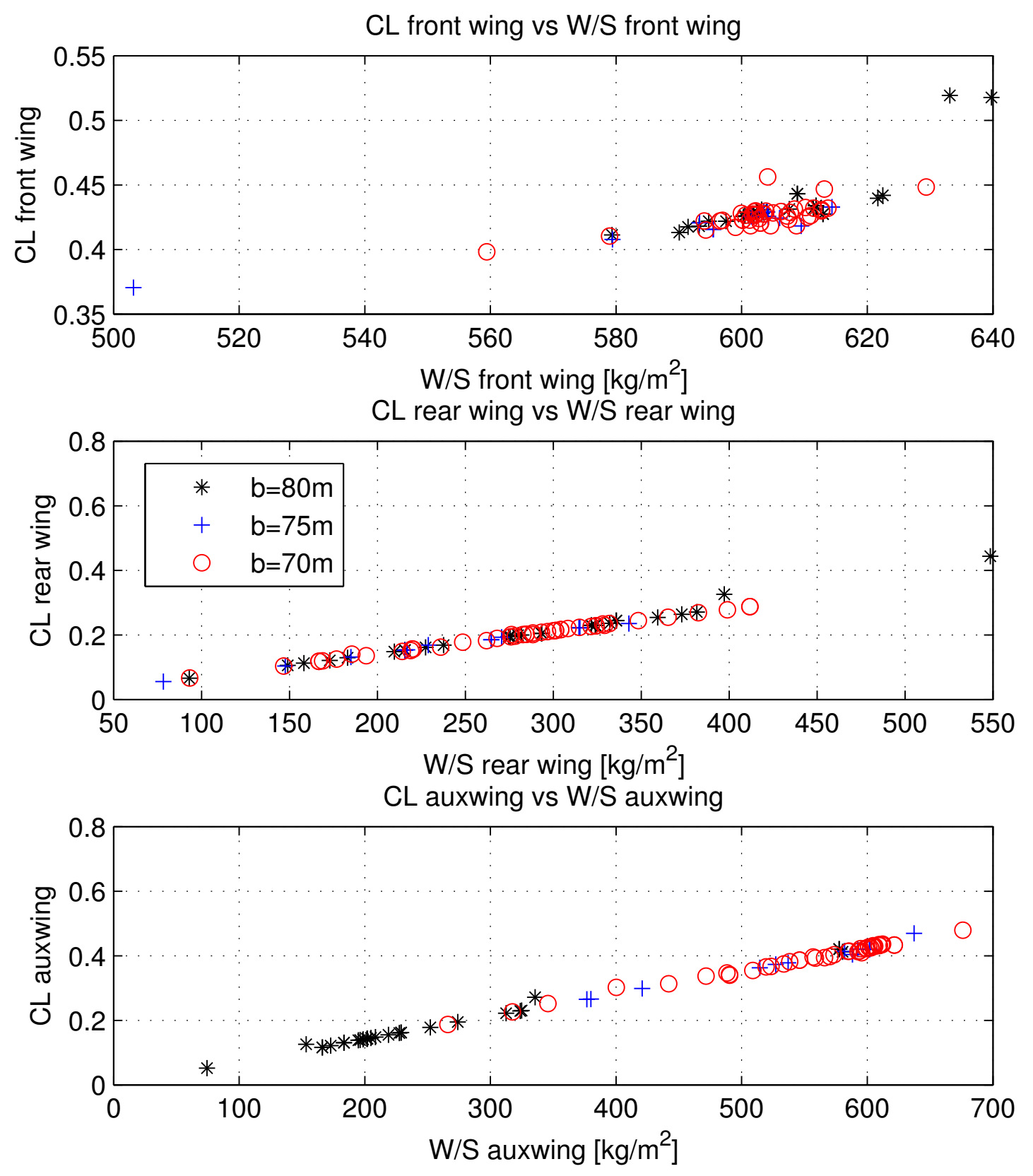

Figure D.4. $C L$ behaviour (part 2) - B sensitivity 

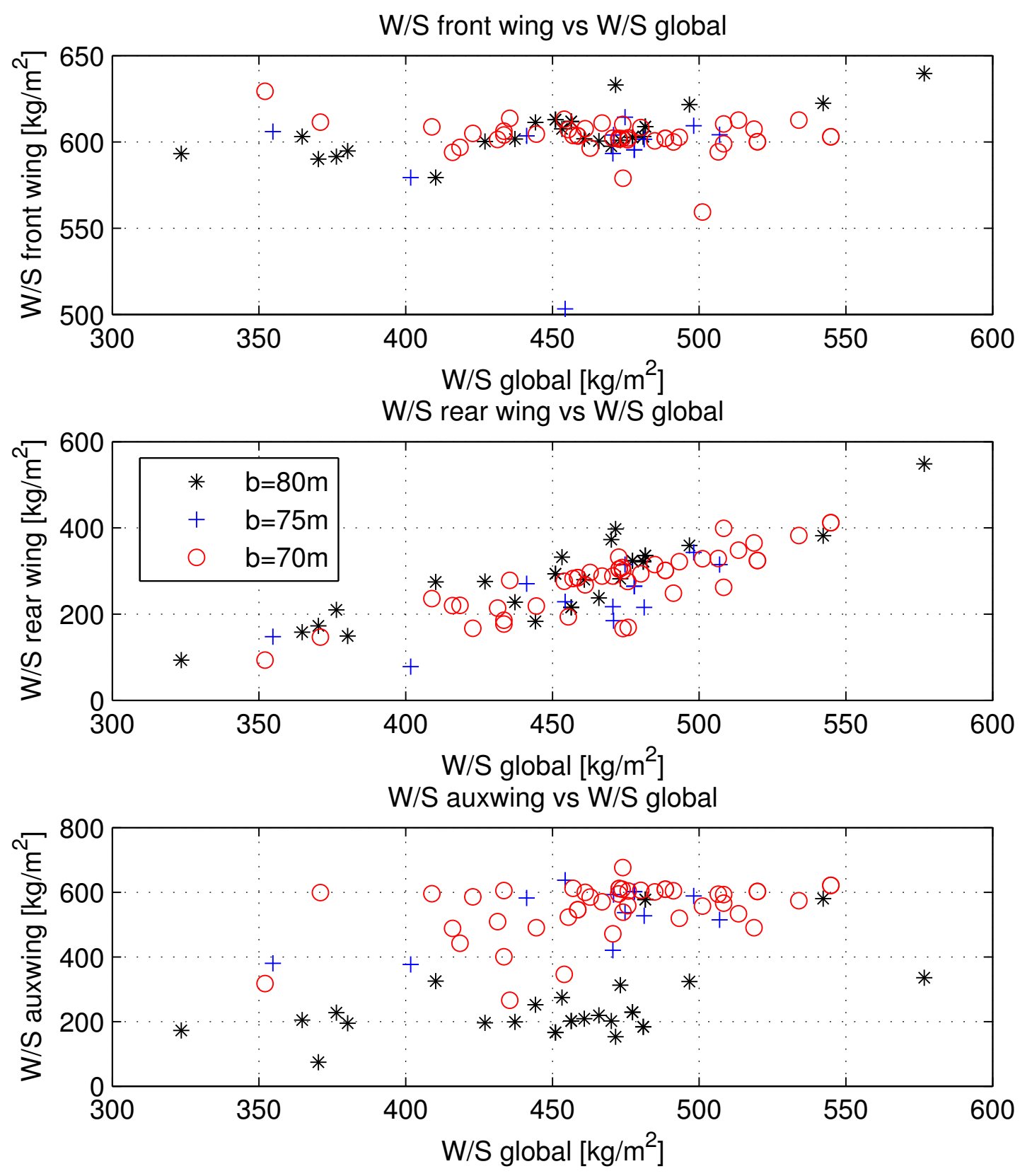

Figure D.5. Wing loadings (part 1) - B sensitivity 


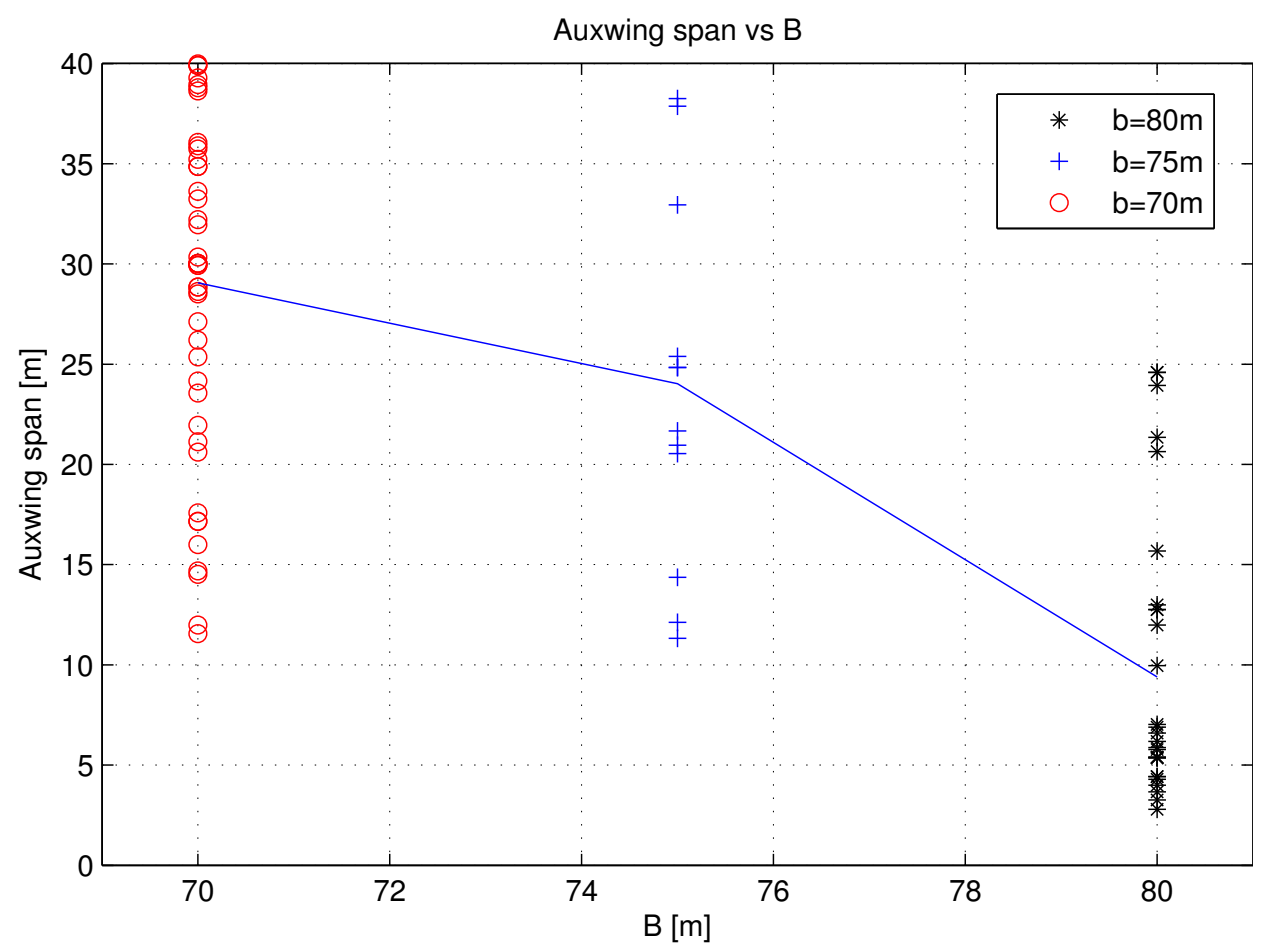

Figure D.6. Auxiliary wing span - B sensitivity

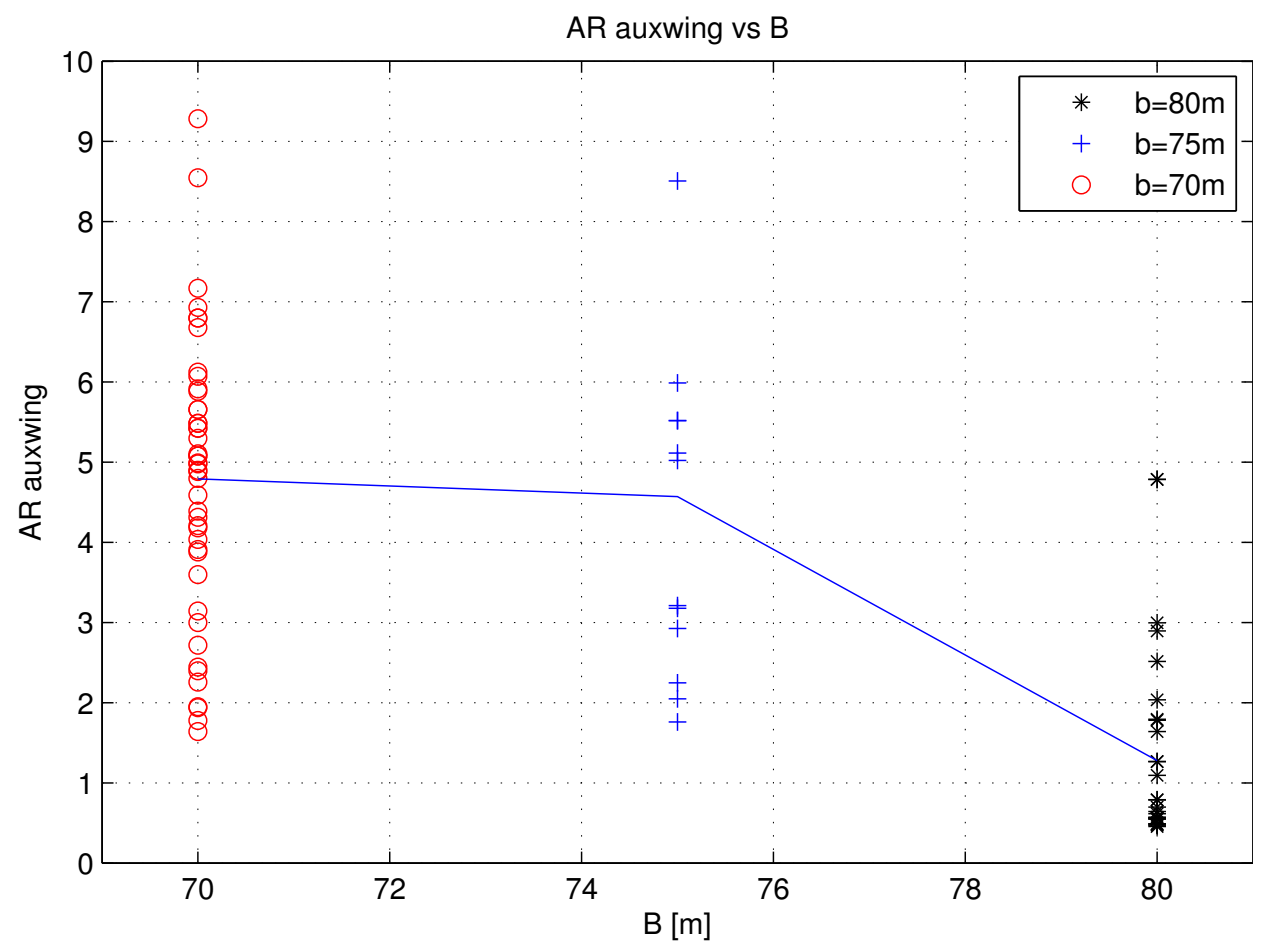

Figure D.7. Auxiliary wing $A R$ - B sensitivity 


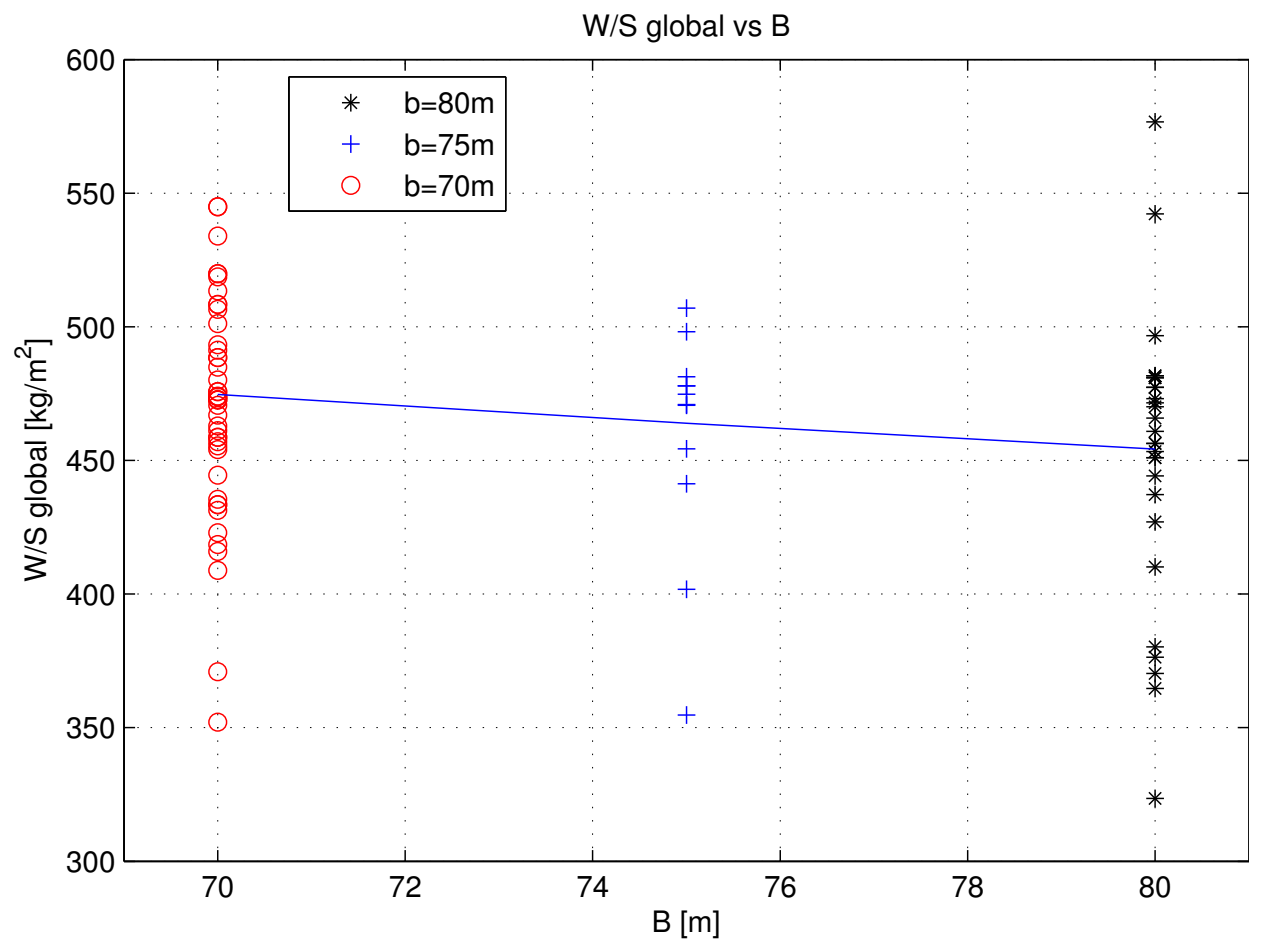

Figure D.8. Global wing loading - B sensitivity

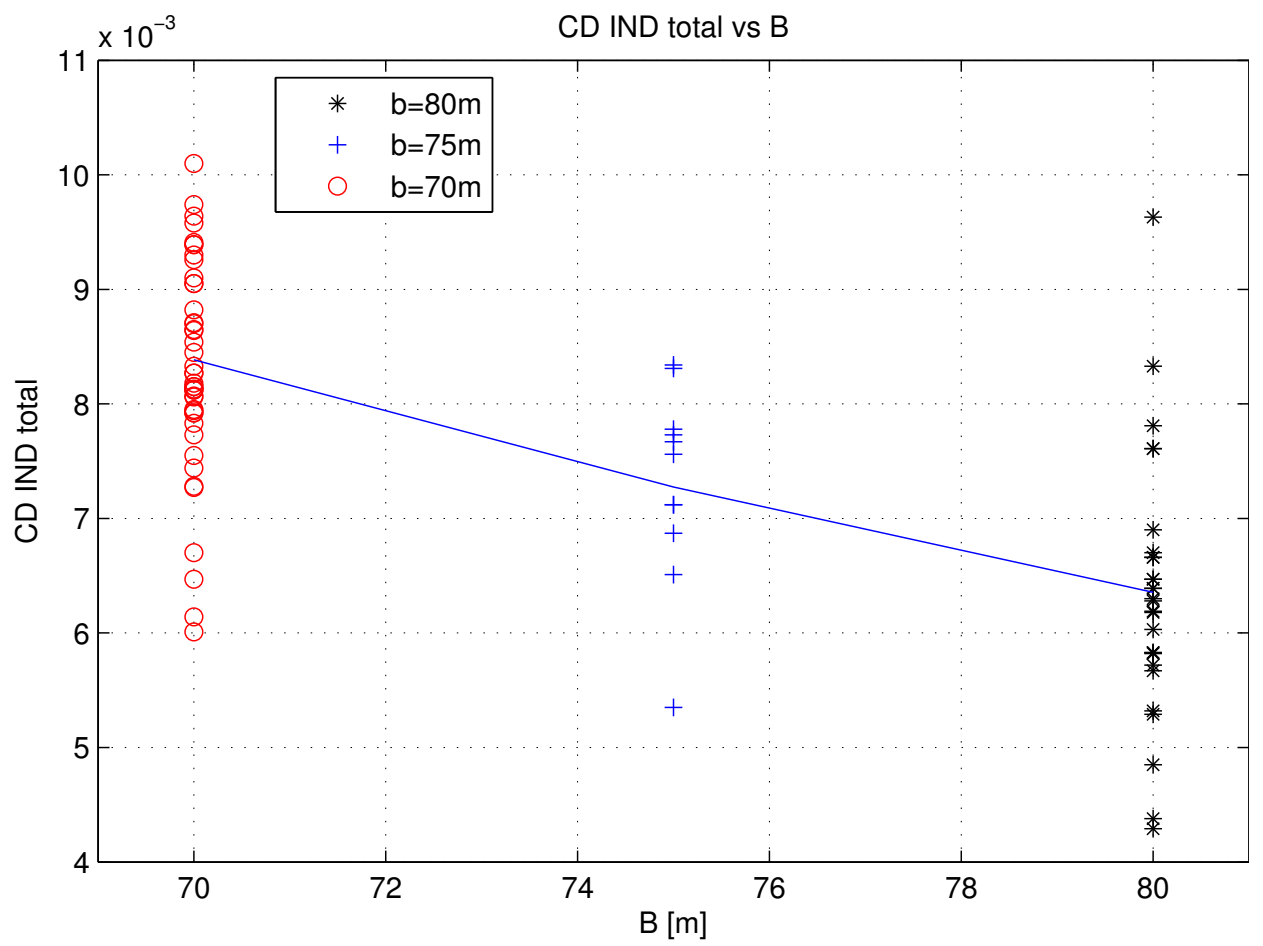

Figure D.9. Induced drag coefficient - B sensitivity 


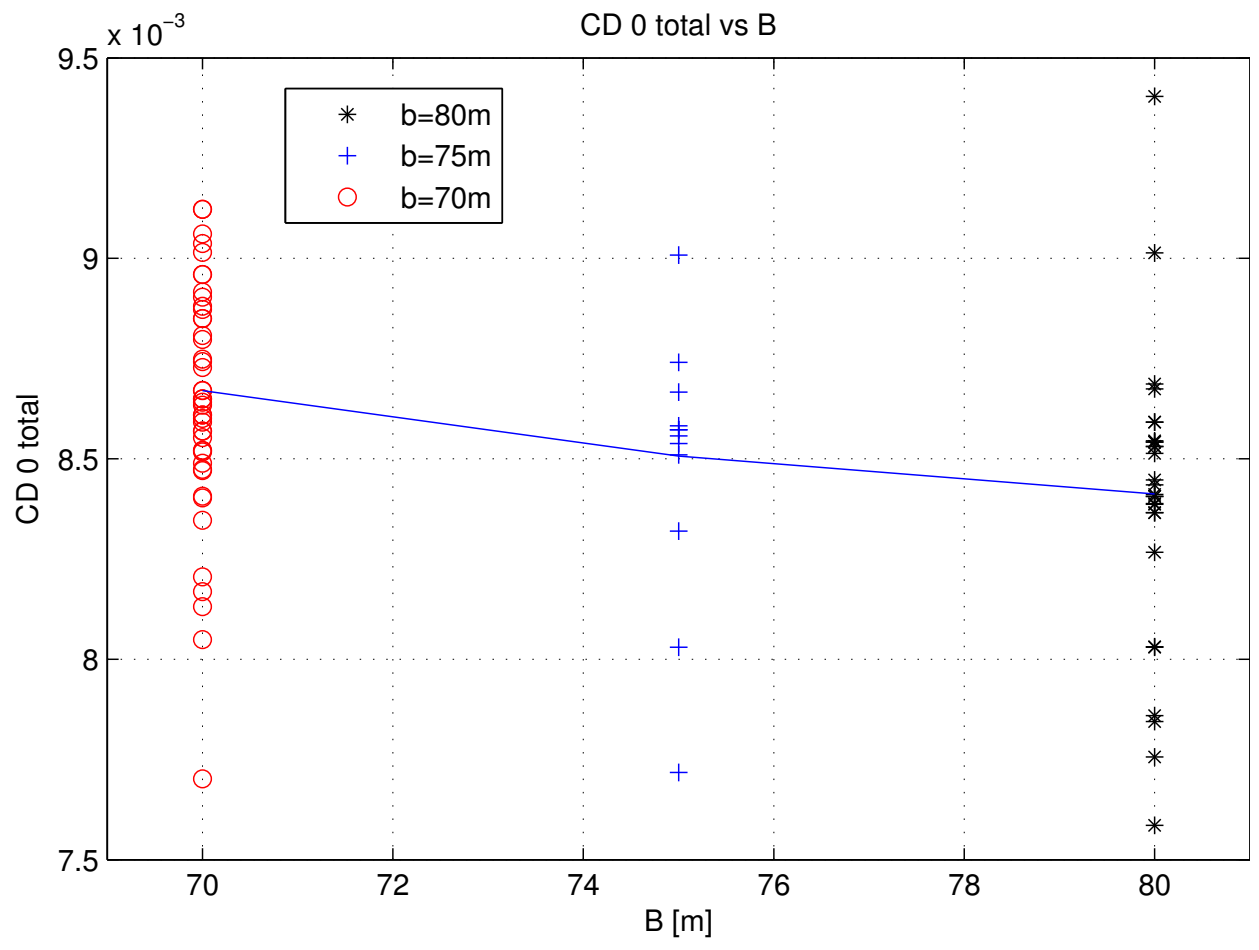

Figure D.10. Viscosity drag coefficient - B sensitivity 


\section{Appendix E}

The results of the advancement of the rear wing analyses are shown (refer to section 5.7).
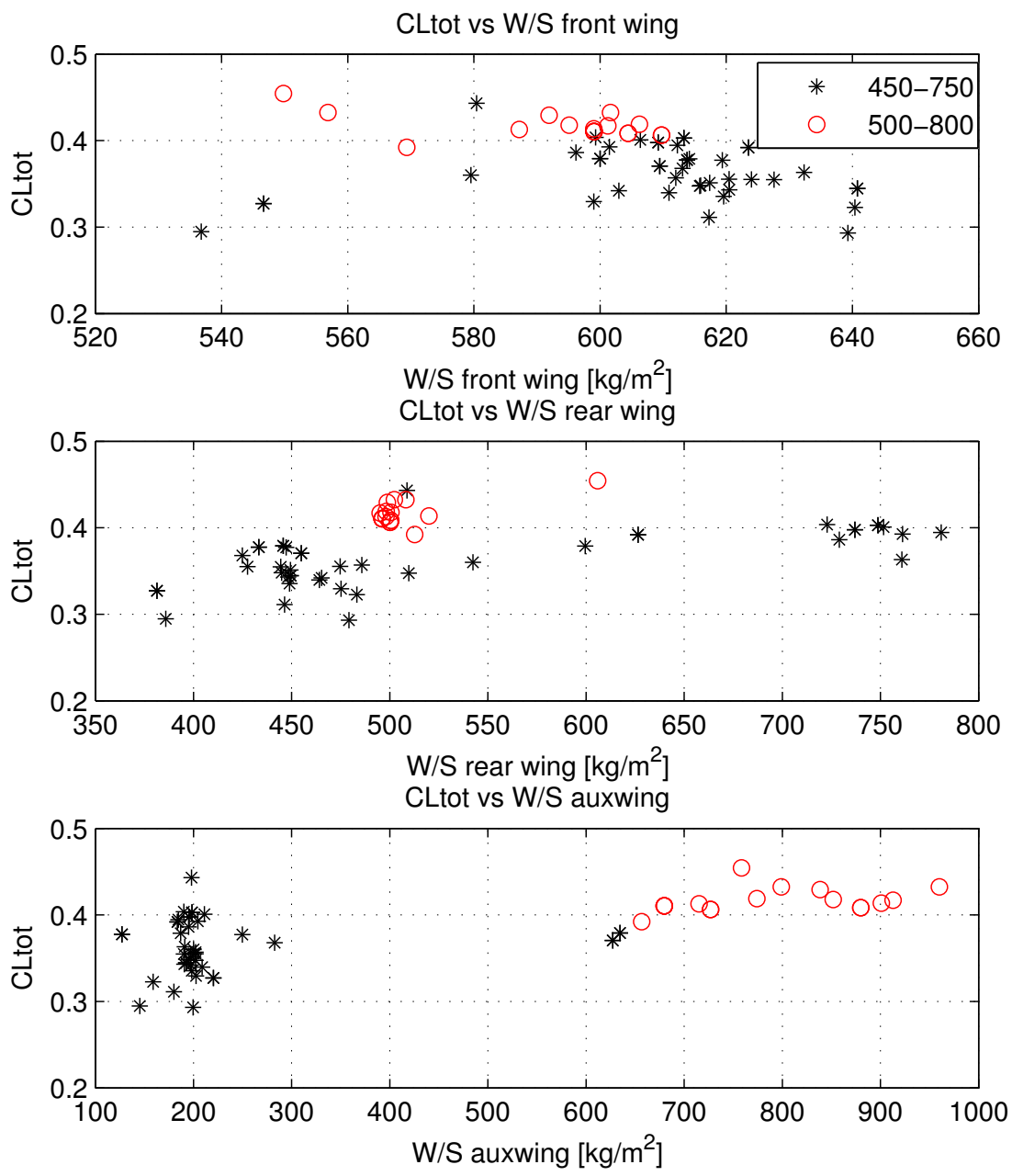

Figure E.1. CLtot trends (rear wing advancement) 


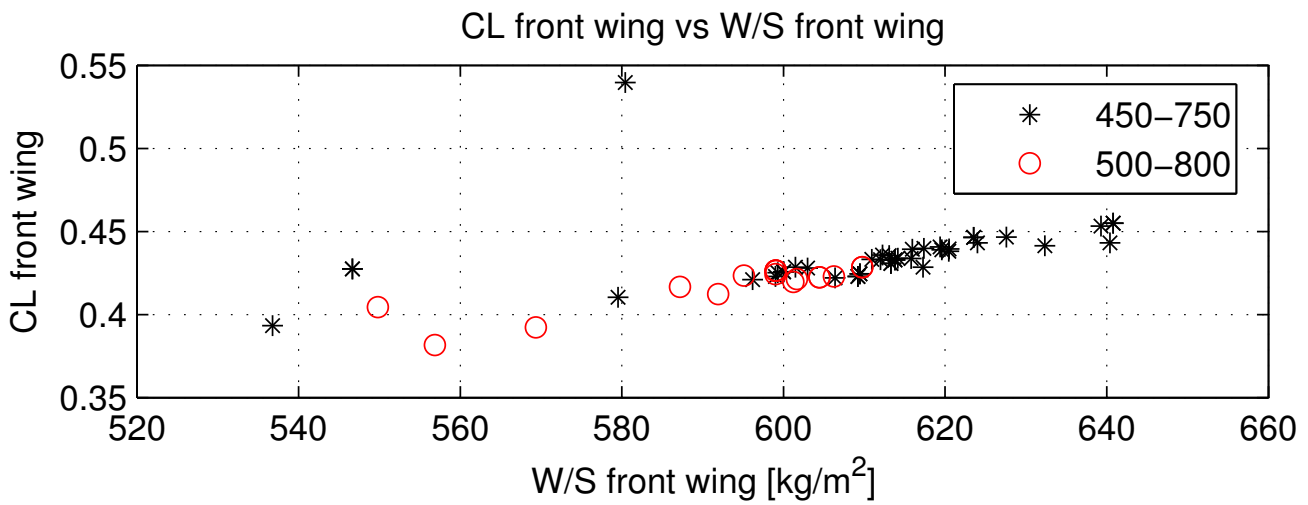

$\mathrm{CL}$ rear wing vs W/S rear wing

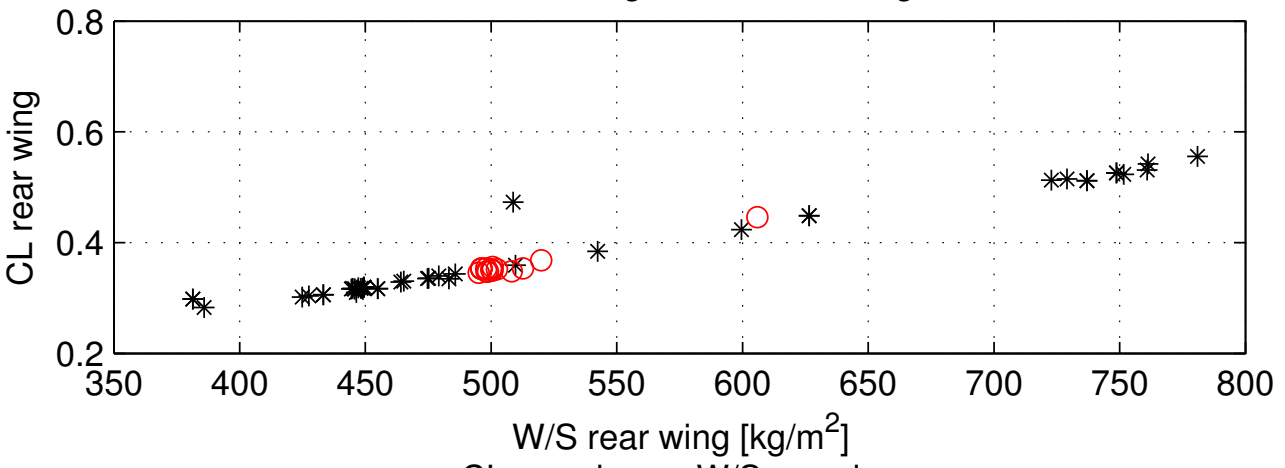

$\mathrm{CL}$ auxwing vs W/S auxwing

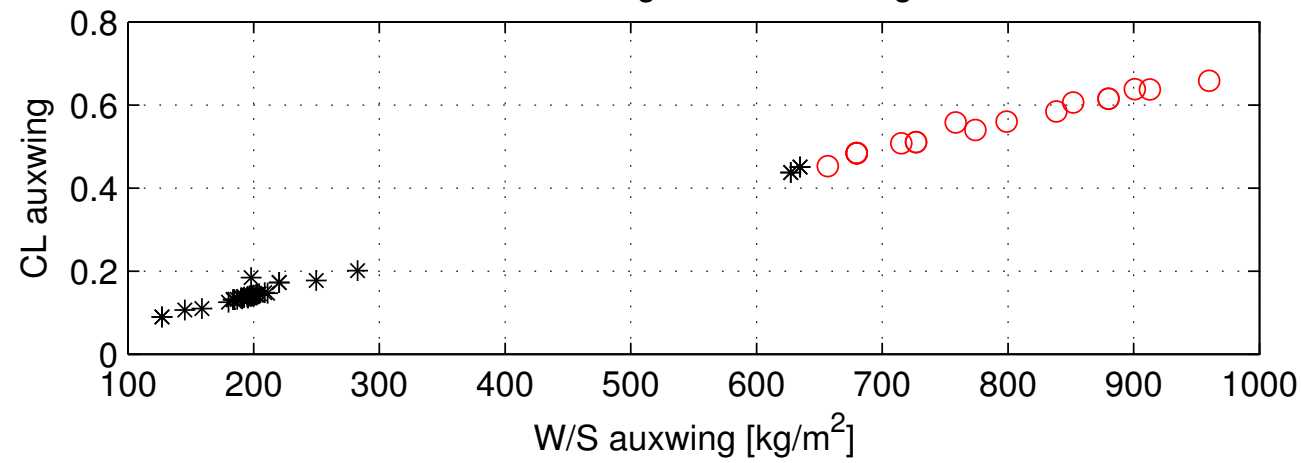

Figure E.2. CL trends (rear wing advancement) 

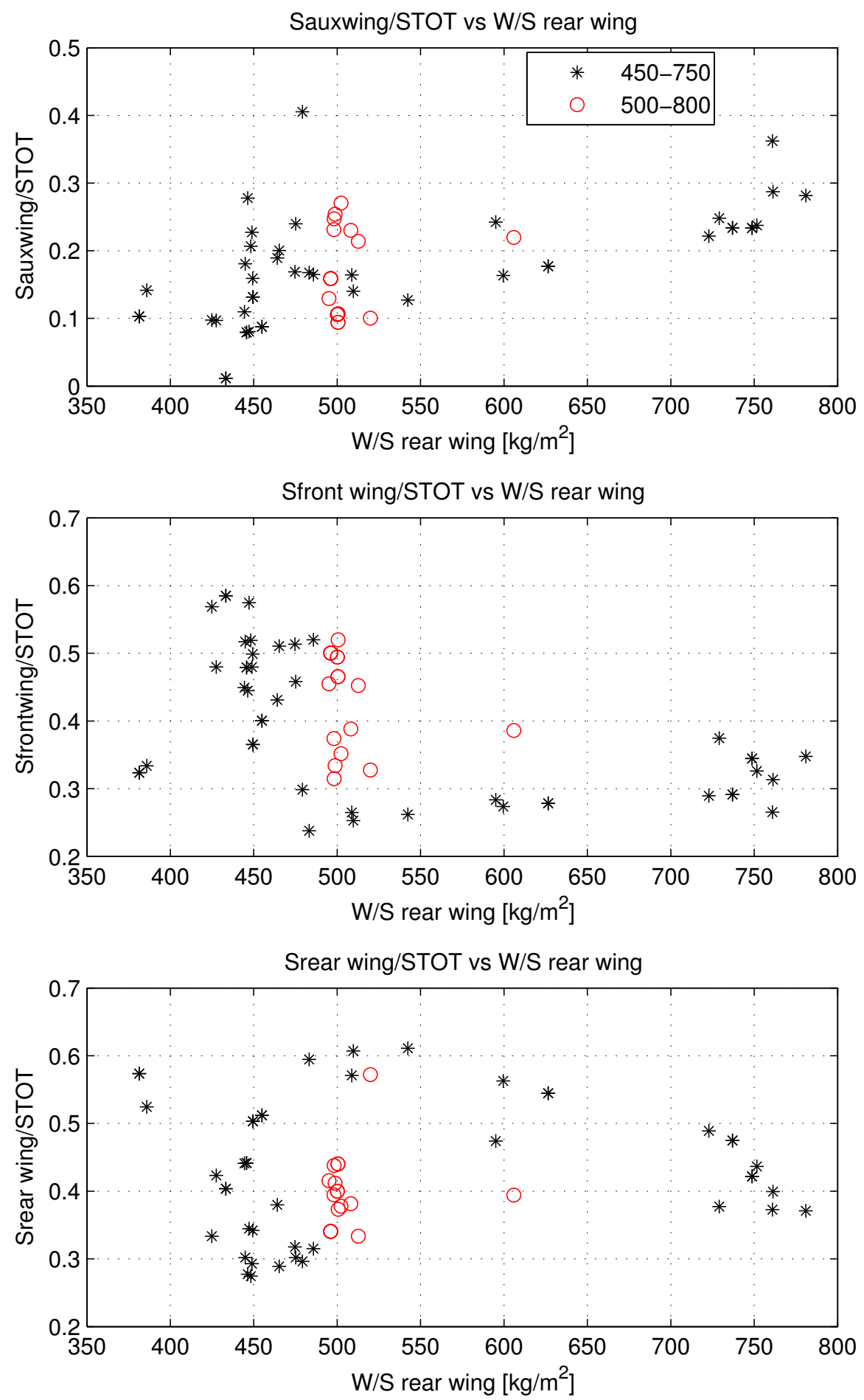

Figure E.3. Surface trends (rear wing advancement) 



\section{Bibliography}

[1] A. Rimondi Generazione di configurazioni aerodinamiche mediante NURBS. (NonUniform Rational B-Splines), Tesi di Laurea - Dipartimento Ingegneria Aerospaziale, Università di Pisa, 2004.

[2] F. Petri, Sviluppo del codice A.S.D. per la generazione parametrica di superfici aerodinamiche mediante N.U.R.B.S. (Non-Uniform Rational B-Spline), Tesi di Laurea Dipartimento Ingegneria Aerospaziale, Università di Pisa, 2005.

[3] A. Rimondi, ASD v. 1.0 - Manuale Utente, Pisa, 2005.

[4] V. Cipolla, Utilizzo di codici a pannelli nel progetto preliminare di velivoli PrandtlPlane ultraleggeri; applicazione a nuove configurazioni, Tesi di Laurea - Dipartimento Ingegneria Aerospaziale, Università di Pisa, 2006.

[5] F. Oliviero, Conceptual design of a large PrandtlPlane ${ }^{\circledR}$ freighter, Tesi di Laurea Dipartimento Ingegneria Aerospaziale, Università di Pisa, 2010.

[6] L. Prandtl, "Induced drag of multiplanes", NACA TN 182, 1924.

[7] A. Frediani, G. Montanari, "The Prandtl's Best Wing System", 1999.

[8] E. Torenbeek, "Synthesis of subsonic Airplane Design" Kluwer Boston Inc., Hingham, 1982.

[9] E. Rizzo, Optimization Methods Applied to the preliminary design of innovative, non conventional aircraft configurations., Doctorate Thesis - Scuola di Dottorato "Leonardo da Vinci" of Università di Pisa, Pisa, 2007.

[10] A. Frediani, G. Buttazzo, Variational Analysis and Aerospace Engineering, Pisa, 2008.

[11] R. Martí, MULTI-START METHODS Cap. 12 in Handbook of Metaheuristics, F. Glover, G. A. Kochenberger, Kluwer Academic Publishers Group, 2003.

[12] W. H. Mason, Applied Computational Aerodynamics, Chapter 6, Volume 1 - Virginia Tech Department of Aerospace and Ocean Engineering, 1998.

[13] M. Drela, H. Youngren AVL 3.30 User Primer, 2010. 
[14] D. Zanetti, Studio preliminare della dinamica libera e delle qualità di volo della configurazione PrandtlPlane ${ }^{\circledR}$, Tesi di Laurea - Dipartimento Ingegneria Aerospaziale, Università di Pisa, 2013.

[15] G. Iezzi, PrandtlPlane High Lift System Preliminary Aerodynamic Design, Tesi di Laurea - Dipartimento Ingegneria Aerospaziale, Università di Pisa, 2006.

[16] Peter K. C. Rudolph, High-Lift Systems on Commercial Subsonic Airliners, NASA Contractor Report 4746, 1996. 